\title{
A Primer on Resurgent Transseries and Their Asymptotics
}

\author{
Inês Aniceto, ${ }^{a, b}$ Gökçe Bașar, ${ }^{c}$ Ricardo Schiappa ${ }^{a, d}$ \\ ${ }^{a}$ Kavli Institute for Theoretical Physics, University of California, \\ Santa Barbara, CA 93106, United States of America \\ ${ }^{b}$ Institute of Physics, Jagiellonian University, ul. Eojasiewicza 11, 30-348 Kraków, Poland \\ ${ }^{c}$ Department of Physics, University of Illinois, Chicago, IL 6060\%, United States of America \\ ${ }^{d}$ CAMGSD, Departamento de Matemática, Instituto Superior Técnico, \\ Universidade de Lisboa, 1049-001 Lisboa, Portugal \\ E-mail: ines@th.if.uj.edu.pl, gbasar@uic.edu, \\ schiappa@math.tecnico.ulisboa.pt
}

ABstract: The computation of observables in general interacting theories, be them quantum mechanical, field, gauge or string theories, is a non-trivial problem which in many cases can only be addressed by resorting to perturbative methods. In most physically interesting problems these perturbative expansions result in asymptotic series with zero radius of convergence. These asymptotic series then require the use of resurgence and transseries in order for the associated observables to become nonperturbatively well-defined. Resurgence encodes the complete largeorder asymptotic behaviour of the coefficients from a perturbative expansion, generically in terms of (multi) instanton sectors and for each problem in terms of its Stokes constants. Some observables arise from linear problems, and have a finite number of instanton sectors and associated Stokes constants; some other observables arise from nonlinear problems, and have an infinite number of instanton sectors and Stokes constants. By means of two very explicit examples, and with emphasis on a pedagogical style of presentation, this work aims at serving as a primer on the aforementioned resurgent, large-order asymptotics of general perturbative expansions. This includes discussions of transseries, Stokes phenomena, generalized steepest-descent methods, Borel transforms, nonlinear resonance, and alien calculus. Furthermore, resurgent properties of transseries - usually described mathematically via alien calculus - are recast in equivalent physical languages: either a "statistical mechanical" language, as motions in chains and lattices; or a "conformal field theoretical" language, with underlying Virasoro-like algebraic structures.

Keywords: Resurgence, Transseries, Perturbation Theory, (Multi) Instantons, Renormalons, Nonperturbative Path Integrals, Large-Order Behaviour, Asymptotics, Stokes Phenomena, Stokes Constants, Lefschetz Thimbles, Complex Saddles, Borel Transform, Resonance, Alien Calculus 


\section{Contents}

1 Introduction 2

2 Resurgent Analysis of a Quartic-Potential Integral $\quad 6$

2.1 Phase Diagram from Stokes Phenomena 8

2.2 Basic Formulae for Resurgent Analysis $\quad 12$

2.3 Asymptotics and Large-Order Behaviour: Partition Function 18

2.4 First Steps Towards Nonlinear Resurgent Analysis 20

2.5 Stokes Constants of Partition Function versus Free Energy 37

2.6 Asymptotics and Large-Order Behaviour: Free Energy 43

3 Lefschetz Thimbles and Linear Problems $\quad 56$

4 Borel Transforms and Nonlinear Problems $\quad 65$

5 Physical Resurgence: From Lattices to Virasoro Algebras $\quad 77$

6 Resurgent Analysis of an Elliptic-Potential Integral 100

6.1 Phase Diagram from Stokes Phenomena 102

6.2 Basic Formulae for Resurgent Analysis 107

6.3 Asymptotics and Large-Order Behaviour: Partition Function 113

6.4 First Steps Towards Nonlinear Resurgent Analysis 116

6.5 Stokes Constants of Partition Function versus Free Energy 124

6.6 Asymptotics and Large-Order Behaviour: Free Energy 128

6.7 Nonlinear Resonance and Transseries Structures 136

6.8 Linear Resonant-Asymptotics: Partition Function 139

6.9 First Steps Towards Nonlinear Resonant Analysis 140

6.10 Stokes Constants of Partition Function versus Free Energy: Resonance 148

6.11 Nonlinear Resonant-Asymptotics: Free Energy 150

$\begin{array}{lr}\text { A Further Details on Alien Calculus } & 157\end{array}$

B Stokes Lines Within the WKB Expansion 168

C On the Nature of Strong-Coupling Expansions 171

$\begin{array}{ll}\text { D Recurrence Relations for Free Energies } & 174\end{array}$

$\begin{array}{ll}\text { E Further Details on Borel Transforms } & 177\end{array}$ 


\section{Introduction}

Exactly-solvable interacting quantum theories, from quantum mechanics and field theory through gauge and string theories, are sparse. This naturally implies that, in practice, most problems are hard to solve. In principle, one would like to compute (generating functions for) all possible correlation functions, involving both local and non-local observables. But, generically, even focusing on the simplest local observables such as the partition function or the free energy may already present hard and daunting calculations if not for resorting to perturbation theory. At first this may strike as a reasonable course of action, after all perturbation theory can be applied to a broad range of different systems. The non-trivial question, however, asks what exactly is computable from the resulting series (in the interaction weak coupling-constant). This, of course, will very much depend on the convergence properties of such series, determining if any results may be extracted from it, and how far may one go into the strong-coupling region. Unfortunately, in most interesting interacting quantum theories, perturbative expansions for large classes of observables have vanishing radius of convergence (and are dubbed asymptotic).

With hindsight this should not come as a surprise. As discussed long ago by Dyson [108] a given theory may have dramatically different physics when considering positive versus negative coupling; in particular being unstable at negative coupling - and thus the physical requirement of finding a null radius of convergence. To be more specific, let us consider some observable $\Phi:=\langle\mathcal{O}\rangle$ and denote by $\Phi_{k}^{(0)}$ the $k$-loop coefficients of its perturbative expansion (in some adequate coupling constant). That this perturbative series is asymptotic translates to a (leading) factorial growth of these coefficients, $\Phi_{k}^{(0)} \sim k$ ! at large order $k$. In many cases this is precisely due to the factorial growth of Feynman diagrams relevant at each order $k$ of perturbation theory, see, e.g., [233, 171]. But what can be said about the next-to-leading growth of the coefficients $\Phi_{k}^{(0)}$ ?

This is where the theory of resurgence first appears in full glory. Of course early research on instantons had already made clear that at next-to-leading order one finds $\Phi_{k}^{(0)} \sim k ! A^{-k}$, with $A$ the instanton action, and at next-to-next-to-leading order one will start seeing the appearance the resurgence - of the perturbative series around the one-instanton background, $\Phi_{\ell}^{(1)}$ (a list of references will follow shortly). But one has to deploy resurgence in order to take this story to its full conclusion; where it tells us exactly what are all the different components appearing in the exact growth/behaviour of the $k$-sequence $\Phi_{k}^{(0)}$. The remarkable feature is that only, and in fact all, multi-instanton sequences appear, $\Phi_{\ell}^{(n)}$ (where here $\Phi_{\ell}^{(n)}$ are the coefficients of the perturbative loop-expansions around the $n$-instanton sectors). Schematically, the corresponding resurgence large-order relation is of the form

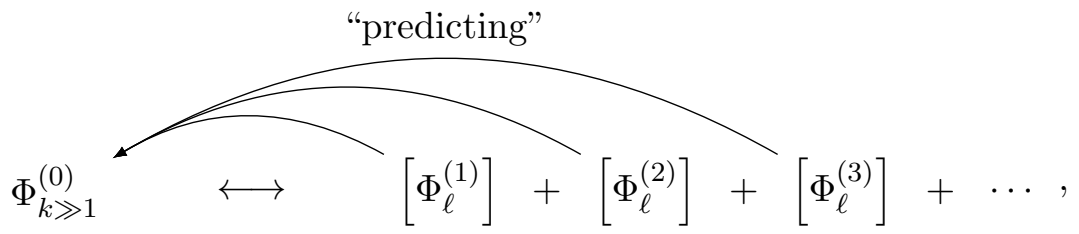

where we illustrate via the solid arrows how resurgence works: if one knows all multi-instanton data-including the instanton action $A$ and all perturbative coefficients around all instanton sectors $\Phi_{\ell}^{(n)}$ - then one can predict the exact behaviour of the perturbative coefficients. Initially written as a large-order relation (thus the subscript $k \gg 1$ ), the more nonperturbative sectors one gets a handle of, the less of a large-order and the more of an exact relation one obtains. 
While the relations implied by (1.1) are usually taken as expectable, it is sometimes the case that one forgets that the converse is also true. One can in fact invert the direction of the arrows in (1.1) and extract the complete nonperturbative content of a given observable out of its exact perturbative expansion, i.e., the perturbative series already encodes all other nonperturbative series, even if deep in its large-order behaviour. As such, decomposing the sequence $\Phi_{k}^{(0)}$ into its different components, be them polynomial $\sim k^{-\ell}$ or exponential $\sim n^{-k}$ or else, one can read the resurgence large-order relation instead as

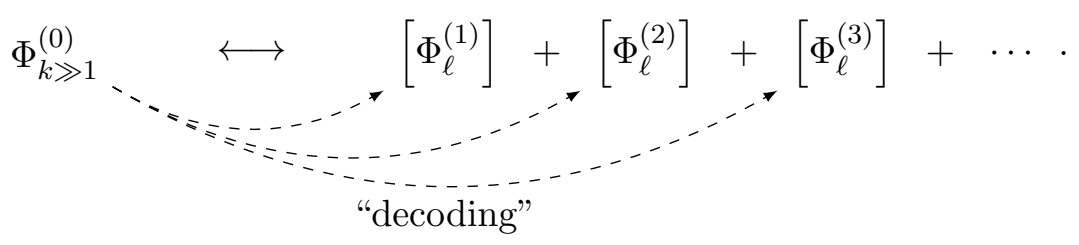

This makes it clear how the asymptotics of perturbative expansions encode a lot of physics!

Resurgence relations such as (1.1) or (1.2) may in fact be written for any instanton ${ }^{1}$ sector, where on the left-hand-side one now finds the growth of any (multi) instanton $k$-sequence $\Phi_{k}^{(n)}$ and, on the right-hand-side, the different components describing its complete behaviour; of course now themselves expressible in terms of all other instanton sectors alongside the perturbative expansion. This is in fact the reason for the name resurgence: picking any sector of the theory, by digging deep enough into its large-order behaviour one will eventually find the "resurgence" of all other sectors. Having seen some initial physical developments within quantum mechanics, e.g. $[38,39,36,67,215,23,24,222,45,232,234,147,235,223,236,212,211,86,87,224-$ 226, 88, 90, 228] (see [233] for further earlier references) and (non-critical) string theory, e.g., $[136,51,210,134,113,114]$ (see [119] for further earlier references), the modern (physical) version of resurgence theory has itself recently resurged in many areas, from quantum mechanics $[8-10,237-239,157,7,156,158,101,27,15,129,102,183,126,122,127,216,107,165,208,65$, $28,123,209,124,137,66,144]$ through gauge and field theories $[213,179,175,180,195,178$, 206, 16, 201, 20, 21, 176, 99, 100, 205, 60, 15, 61, 62, 14, 33, 80, 25, 98, 146, 151, 161, 103, 12, $95,26,29,19,162,148,184,92,149,140,122,22,139,107,202,34,182,32,63,49,231,74$, $2,1,150,94,47,152,58,172,181]$ to string theory $[174,179,175,131,180,112,132,195,178$, $130,163,97,16,176,205,15,77,135,78,221,145,76,81,79,65,137,66,64]$ and cosmology $[53,116,117,96,118,52]$. The modern (mathematical) version of the theory of resurgence (and name) arises in the seminal work of Écalle [109]. For earlier reviews on resurgence see, e.g., [55, 56, 69-71, 50, 91, 207]. More modern reviews include, e.g., [192, 193, 83, 203, 176, 204, 93, $84,104,177,105,106,185,167,85,42,35]$; see also [110] for a review specifically on transseries. Our (novel) aim in this work sets on an elementary introduction to these mathematical ideas, within physical contexts, and with an emphasis on their relation to asymptotics (itself mostly absent in the aforementioned reviews).

While in the main body of the paper we shall be quantitatively precise on the exact nature of the resurgence relations (1.1) or (1.2), for the moment let us note that they have a somewhat universal character. This universal structure depends upon the nature of the transseries and of the bridge equations. Briefly, transseries enlarge power series by the inclusion of non-analytic terms and, as such, allow for the construction of a single object (the transseries itself) which

\footnotetext{
${ }^{1}$ While in this paper we shall mainly stick to the use of the word "instanton" to denote generic nonperturbative (exponentially non-analytic) contributions, and will thus be a bit loose on its use, of course in many quantum field theory examples this should be more precisely replaced by the word "renormalon"; see, e.g., [40].
} 
gathers all different nonperturbative contributions to whatever observable we are computing (where by "all" we mean all contributions having already appeared in the resurgence relations). The simplest way to think about this is to imagine the "trans" in transseries as encompassing all powers of the non-analytic monomial $\exp \left(-\frac{1}{x}\right)$, and generalizations thereof, with $x$ the (small) coupling constant of the problem under consideration. Further, the bridge equations essentially encode the resurgent nature of each transseries component, i.e., they make precise the resurgence link between a chosen sector $(n)$, and all other sectors $(m)$ associated to $(n)$ via resurgence as in (1.1) or (1.2). In this way, many different problems may have the same transseries structure and the same bridge equations. So what tells them apart? For once, obviously, the perturbative and multi-instanton data appearing in (1.1) or (1.2). But this is not all. There is also a (possibly infinite) set of constants, the Stokes constants, $S_{n}$, which are distinct for each different problem. They already appear in the resurgence relations: to be more precise (1.1) and (1.2) above should have been written in the schematic form

$$
\Phi_{k \gg 1}^{(0)} \longleftrightarrow \boldsymbol{S}_{\mathbf{1}}\left[\Phi_{\ell}^{(1)}\right]+\boldsymbol{S}_{\mathbf{2}}\left[\Phi_{\ell}^{(2)}\right]+\boldsymbol{S}_{\mathbf{3}}\left[\Phi_{\ell}^{(3)}\right]+\cdots .
$$

In this way it should be clear that, without having the Stokes constants, any resurgence statement will be quantitatively limited. So how do we compute them? This is generically a hard problem, specially for nonlinear systems, and one which we shall discuss at length in this paper.

The origin of these Stokes constants predates resurgence by over one hundred years: they of course arise with the discovery of Stokes phenomena [214] (see, e.g., the excellent review [41] in a closely related context). When the transseries is first assembled, all its nonperturbative content $\Phi^{(n)}$ is exponentially suppressed; in fact the higher the instanton number the greater the exponential damping. This means that already the one-instanton contribution is invisible from the point-of-view of the perturbative power series - at least as long as the (overall) argument of the exponential is negative. But as one changes the couplings or other parameters in the theory along the complex plane, this argument may vary and consequentially these exponentially suppressed contributions may change dominance. In fact they can become of the same order as the perturbative series and, eventually, even exponentially enhanced as compared to $\Phi^{(0)}$. This is the essence of Stokes phenomena: small, suppressed exponentials, hidden behind perturbation theory, may actually grow to become the dominant contribution. The transseries formalism already incorporates Stokes phenomena in the sense that it assembles all non-analytic exponential contributions together. The Stokes constants then precisely control how the transseries changes as one varies the couplings across their complex domains and different types of Stokes phenomena may occur. As we shall show later, this is accomplished by having the transseries parameters $\boldsymbol{\sigma}$ transform at the Stokes lines, with these transformations explicitly dependent upon the Stokes constants "attached" to the corresponding Stokes line (in the simplest cases these are just shifts $\boldsymbol{\sigma} \rightarrow \boldsymbol{\sigma}+\boldsymbol{S}_{\mathbf{1}}$, such that if one starts off with the perturbative series alone, $\boldsymbol{\sigma}=\mathbf{0}$, upon crossing a Stokes line the exponential contributions become "turned on" with their weight measured by $\boldsymbol{S}_{\mathbf{1}}$ ). How general transseries incorporate Stokes phenomena was recently discussed in [15].

The fundamental role played by the Stokes constants in the nonperturbative construction of a given observable should be now clear. They allow for precise, quantitative statements concerning the large-order resurgent behaviour of its different sectors; and they precisely quantify Stokes phenomena, allowing for a fully nonperturbative exploration of the whole parameter (coupling) space. Yet, computing them may be very hard. For instance, in the examples of the Painleve I $[179,130,16]$ or the Painlevé II $[175,205]$ equations, out of a possibly infinite set of Stokes constants only one (in each problem) was accessible to analytic calculation. All others had to 
be determined numerically. Even worse, most of these resulting numbers seemed to be transcendental. Having a first principles guide to the computation of Stokes constants is thus a critical step towards achieving analytical control over this type of nonperturbative constructions.

There is one more feature which may be found in general resurgent asymptotics and we still wish to mention: resonance. While we have been talking about a single instanton action, in many scenarios one may in fact have several instanton (or renormalon) actions. The number of nonperturbative sectors then multiplies accordingly. For simplicity, let us assume there are only two instanton actions, $A_{1}$ and $A_{2}$, and the nonperturbative sectors are organized as $(n, m)$ corresponding to an exponential factor of the form $\sim n A_{1}+m A_{2}$. The reader immediately realizes that a door is now open towards new phenomena: indeed, if the two actions happen to be $\mathbb{Z}$-linearly dependent, then there is $n^{\star}, m^{\star}$ such that $n^{\star} A_{1}+m^{\star} A_{2}=0$ and the nonperturbative exponential contributions "collapse" to order one, no longer suppressed or enhanced. Such phenomena have appeared recurrently in recent string theoretic examples [130, 16, 205]. In these cases both the large-order resurgence relations as well as the transseries structure will change, in order to incorporate new (nonperturbative) resonant sectors, and logarithms may appear throughout. Because general physical problems may depend upon many parameters, it is conceivable that resonance is "tunable" and may generically appear in many examples. We shall later discuss in a very explicit example how this may come about and what are its implications.

These lectures are organized as follows. We begin in section 2 by considering a toy model for an interacting quantum theory: a one-dimensional integral representing the partition function of a system with quartic potential. Because this partition function satisfies a "Schwinger-Dyson" linear differential equation, this allows us to start the analysis from simple saddle-point arguments and, in the process of computation, introduce many basic resurgence methods and ideas. As we turn to the free energy of this system, the problem becomes nonlinear and full-fledged resurgence is then required. We discuss its asymptotics, both analytically and via high-precision numerical studies, and show how Stokes constants from the linear problem may be used to find the Stokes constants of the nonlinear problem. Most numerical tools used in the asymptotic analyses are always introduced as needed. Section 2 also aims at being selfcontained, and enough material for the first-time reader who is only interested in the basics of resurgence. Having grasped the basics of resurgent analysis, we then start addressing its diverse generalizations. Section 3 discusses a generalization of saddle-point analysis (previously used in section 2) to higher dimensions, replacing one-dimensional steepest-descent contours with multi-dimensional Lefschetz thimbles, and how these are adequate tools to use when addressing linear problems. Nonlinear problems require heavier machinery and in section 4 we discuss it by addressing general results on Borel transforms and their associated resurgence singularities. These linear and nonlinear discussions lead up to section 5, where we describe the (more complicated) general structure of resurgent transseries, involving arbitrary instanton actions, and always having in mind a physical point-of-view. In particular, "physical interpretations" of alien calculus are suggested, either via statistical mechanics or via conformal field theory. Ensemble, these sections yield an overview of many technical ideas and concepts pertaining to resurgence and transseries. Section 6 then reconsiders a more complicated toy model where resonance may also appear: a one-dimensional integral representing the partition function of a system with an elliptic-function potential. Modularity now enters the game and the instanton actions depend upon a modulus, which may be chosen so that the system resonates. In both linear (partition function) and nonlinear (free energy) systems we analyze how resonance appears in the asymptotics, and how it changes as the elliptic modulus is varied. Sections 2 and $\mathbf{6}$ thus introduce and review, in very explicit examples, most working methods of resurgent asymptotics. There are also 
a few appendices, collecting some technicalities and directions of further study. A more mathematical approach to alien calculus, alongside further technical details, appears in appendix A. Then, appendix B discusses (anti) Stokes lines and their wall crossing in quantum mechanics, while appendix $\mathbf{C}$ discusses resummations and strong-coupling expansions - together, these two appendices complement some discussions from section 2. Technicalities concerning generating high-order data, used in sections $\mathbf{2}$ and $\mathbf{6}$, are collected in appendix $\mathbf{D}$; while some other further details on Borel transforms, complementing section 4, may be found in appendix $\mathrm{E}$. Finally, it is worth pointing out two interesting topics which are not included in the present work. One of these topics is the resummation of transseries (albeit briefly discussed in appendix C). This involves not only the classical idea of Borel resummation, alongside its practical implementation as Borel-Padé resummation, but mainly its newer version - as it applies to transseries with multi-instanton sectors and occurrence of Stokes phenomena - in the form of Borel-Padé-Écalle resummation. We refer the reader to, e.g., the recent papers $[175,135,80,12,79,66,64]$ for nice illustrations of resummations within gauge and string theoretic contexts. The one other topic which is left absent deals with reorganizations of transseries, by exchanging the order of the sums in the perturbative and multi-instanton indexes. This actually leads to a myriad of interesting results connecting to, e.g., modular properties [46, 111, 112, 178, 17, 18], transasymptotics [72, 73] and its "nonlinear" generalizations [17, 18] (all relating back to modularity [46, 111, 112]), and (generalized) partition-function phases $[17,18]$. The interested reader is encouraged to proceed into the aforementioned references, given the basis acquired in the present lectures.

\section{Resurgent Analysis of a Quartic-Potential Integral}

Let us begin by setting up some notation and definitions which will be needed in order to develop the resurgence analyses that follow. As mentioned earlier, there are a few reviews on the theory of resurgence and its associated transseries and alien calculus, but on what concerns an introduction to resurgent asymptotics we are not aware of much beyond section 2 of [16]. We begin precisely by recalling a few formulae from that paper. Consider an observable $\Phi:=\langle\mathcal{O}\rangle$ and its perturbative computation in some coupling constant, denoted by $x$, which as usual we shall take around $x \sim 0$.

The perturbative series

$$
\Phi^{(0)}(x) \simeq \sum_{g=0}^{+\infty} \Phi_{g}^{(0)} x^{g+1}
$$

is asymptotic with zero radius of convergence if its coefficients grow as $\Phi_{g}^{(0)} \sim g$ !. But if this power-series does not converge for any non-zero value of $x$, then how can one compute the observable $\Phi$ as a function of the coupling? One of the most powerful frameworks to extract information out of this type of asymptotic series is Borel analysis, where one starts with the Borel transform

$$
\mathcal{B}\left[x^{\alpha+1}\right](s)=\frac{s^{\alpha}}{\Gamma(\alpha+1)} .
$$

This produces a new series, $\mathcal{B}\left[\Phi^{(0)}\right](s)$, out of the asymptotic series $(2.1)$, which has finite non-zero convergence radius, as its coefficients no longer grow factorially but instead only exponentially fast. It may thus be analytically continued throughout $s \in \mathbb{C}$, into a function, albeit having singularities and branch cuts. Once this is done, and chosen a direction $\theta$ in the complex $s$-plane along which $\mathcal{B}\left[\Phi^{(0)}\right](s)$ has no singularities, the Borel resummation (or inverse Borel transform)

$$
\mathcal{S}_{\theta} \Phi^{(0)}(x)=\int_{0}^{\mathrm{e}^{\mathrm{i} \theta} \infty} \mathrm{d} s \mathcal{B}\left[\Phi^{(0)}\right](s) \mathrm{e}^{-\frac{s}{x}}
$$


finally associates a finite value to the divergent sum (2.1), for each $x$. If the Borel transform was an entire function this would pretty much be the end of the story. But that can only happen if the original series was not asymptotic to begin with; being asymptotic, then $\mathcal{B}\left[\Phi^{(0)}\right](s)$ will have singularities - and this is instead the beginning of a fascinating story. Indeed, if $\mathcal{B}\left[\Phi^{(0)}\right](s)$ has singularities along $\theta$, this (singular) direction is now known as a Stokes line, where (2.3) is no longer well-defined: there is an ambiguity concerning along which direction should these singularities be avoided by the integration contour. But if the end-result of this process of resummation might be ambiguous, it would seem we still cannot compute the observable $\Phi$ as a function of $x$ ? In order to solve this issue, one first defines lateral Borel resummations, $\mathcal{S}_{\theta^{ \pm}} \Phi^{(0)}(x)$, which avoid all singularities via a contour just to the left (to the right) of the direction $\theta$. They naturally lead to different results and the one seemingly simple question which actually sparks many features of resurgence is: are these left- and right-lateral resummations related somehow?

As it turns out, and as we shall pedagogically discuss at greater length in this section-and, in a more rigorous mathematical language, in appendix $\mathrm{A}-$, the answer to the above question is "yes". The lateral Borel resummations lead to distinct sectorial resummations of our original asymptotic series, but they are nonetheless connected via the so-called Stokes automorphism $\underline{\mathfrak{S}}_{\theta}$,

$$
\mathcal{S}_{\theta^{+}}=\mathcal{S}_{\theta^{-}} \circ \underline{\mathfrak{S}}_{\theta} .
$$

In words, there is an operator, $\mathfrak{S}_{\theta}$, relating both resummations (and essentially encoding all the singular structure along the Stokes direction $\theta$ ), and which we shall explain below how to construct starting from a simple example. This is also the point where alien calculus kicks in, as the Stokes automorphism may be computed in terms of the alien derivative, $\Delta_{\omega}$, a differential operator encoding the singular behaviour of the Borel transform at the point $\omega$. We shall also explain in the following how this alien derivative may be very simply read from the Borel structure of a given asymptotic series. Furthermore, it turns out that $\underline{\mathfrak{S}}_{\theta} \sim \exp \left(\mathrm{e}^{-\frac{\omega}{x}} \Delta_{\omega}\right)$ with $\omega$ in the $\theta$-direction (more on all this below), a relation which also implies that the aforementioned ambiguity is nonperturbative (or non-analytic), essentially of order $\sim \mathrm{e}^{-\frac{1}{x}}$. Clearly, if we know all possible Stokes automorphisms associated to all Stokes lines, or, equivalently, all possible alien derivatives along these same singular directions, then we will know how to match all possible sectorial resummations and it is thus possible to reconstruct the nonperturbative solution of the problem at hand, anywhere in the coupling-constant complex-plane. Because each of these matches is non-analytic in $\sim \mathrm{e}^{-\frac{1}{x}}$, this further implies that the nonperturbative solution we are trying to construct must involve all possible such (exponential-type) instanton contributions. This naturally leads to our next ingredient.

As we shall see, the calculation of alien derivatives is undissociated from transseries, general solutions to nonlinear problems, going beyond the realm of power series. For example, a transseries ansatz for our resurgent function, depending on a single parameter $\sigma$, could be

$$
\Phi(x, \sigma)=\sum_{n=0}^{+\infty} \sigma^{n} \Phi^{(n)}(x),
$$

where $\Phi^{(0)}(x)$ is the formal asymptotic power series $(2.1)$, and where the $\Phi^{(n)}(x)$ are the $n$ instanton contributions,

$$
\Phi^{(n)}(x) \simeq \mathrm{e}^{-n \frac{A}{x}} \sum_{g=1}^{+\infty} \Phi_{g}^{(n)} x^{g+\beta_{n}},
$$


with $A$ the instanton action and $\beta_{n}$ a characteristic exponent. The exponentials in (2.6) are non-analytic. In this way, indeed the transseries (2.5) includes all possible (exponential-type) instanton contributions, going beyond the realm of power-series and organizing itself as a double series: for each $n$ as an asymptotic series, and for the sum in $n$ as a "power series" in the nonanalytic term $\mathrm{e}^{-\frac{A}{x}}$. The transseries parameter $\sigma$ is an instanton counting parameter, associated to the instanton action $A$, and it parameterizes different choices of boundary conditions for whatever problem one is addressing (e.g., (2.5) would be an appropriate solution ansatz for a first-order nonlinear ordinary differential equation, representing a one-parameter family of solutions for unspecified $\sigma$ ). Generically one should consider transseries depending on multiple parameters; e.g., a two-parameter transseries was fundamental in the solution to the Painlevé I equation and the quartic matrix model in $[130,16]$, or the solution to the Painlevé II equation in [205]. Even more complicated multi-parameter transseries solutions seem to appear within the topological string theory context, e.g., $[97,77,78,76]$. In particular, note that a problem whose transseries solution involves $k$-parameters will have multiple instanton sectors, labeled by a set of integers $\boldsymbol{n}=\left(n_{1}, \ldots, n_{k}\right) \in \mathbb{N}^{k}$, as $\Phi^{(\boldsymbol{n})}(x)$. The $\boldsymbol{n}=(0, \ldots, 0)$ sector is the perturbative sector, the $\boldsymbol{n}=\left(n_{1}, 0, \ldots, 0\right)$ sector is a typical instanton sector associated to the instanton action $A_{1}$, but most sectors will be mixed and one may even find new ("unusual") sectors associated to complex instantons as in, e.g., [215, 23, 24, 223, 130, 16, 205, 27, 135, 78, 79, 66].

Finally, let us mention that the reason why the automorphism $\underline{\mathfrak{S}}_{\theta}$ in (2.4) is known as the Stokes automorphism is, of course, because it precisely describes Stokes phenomena-which we already explained qualitatively in the introduction. We may now be a bit more quantitative by considering the one-parameter transseries (2.5), for which $\theta=0$ is a singular direction of the Borel transform, i.e., it is a Stokes line (for $A>0$ ). As we shall explain later, applying the Stokes automorphism (2.4) one finds

$$
\mathcal{S}_{+} \Phi(x, \sigma)=\mathcal{S}_{-} \Phi\left(x, \sigma+S_{1}\right),
$$

where $S_{1}$ is a Stokes constant as in (1.3). One immediately realizes that this expression, (2.7), precisely describes the Stokes phenomena of classical asymptotics within the resurgence framework: starting out with $\sigma=0$, all that the transseries (2.5) includes is the original perturbative series (2.1). But once we cross a Stokes line, signaled by the fact that the Borel resummation (2.3) is no longer yielding correct results, then (2.7) above will make appear a series of exponentially suppressed terms, as in (2.6), which correct the Borel resummation of the perturbative expansion and which may eventually grow and take over the dominance of the transseries (2.5).

\subsection{Phase Diagram from Stokes Phenomena}

Let us now describe these ideas in greater detail as they appear in a simple example, that of a partition-function toy-model described by a one-dimensional integral with quartic potential. We will set up this analysis within the resurgence framework, identifying Stokes and anti-Stokes lines, and constructing all different asymptotic expansions which hold on different sides of the Stokes lines. For pedagogical purposes, we shall start over familiar ground by considering simple saddle-point analysis and steepest-descent contours in the present subsection.

Consider the "quartic partition function", described by

$$
Z(\hbar)=\frac{1}{2 \pi} \int_{\Gamma} \mathrm{d} z \mathrm{e}^{-\frac{1}{\hbar} V(z)},
$$

where $V(z)$ is the quartic potential

$$
V(z)=\frac{1}{2} z^{2}-\frac{\lambda}{24} z^{4}
$$




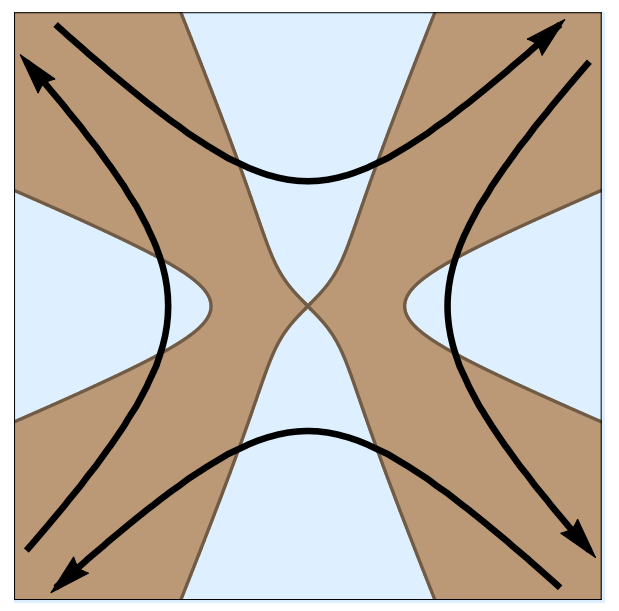

Figure 1. The quartic potential (2.9) in the complex $z$-plane, with $\operatorname{Re} \lambda>0$ ( $\lambda=1$ in the plot). The brown (darker) region is where $\mathbb{R e} V(z)>0$, while the blue (lighter) region is where $\mathbb{R e} V(z)<0$. The four contours are the admissible contours, leading to three homologically independent integration paths. The $\mathbb{Z}_{2}$ symmetry $V(z)=V(-z)$ of the quartic potential (2.9) further narrows down the number of independent results one may compute over homologically distinct contours.

with coupling constant $\lambda$, and $\Gamma$ is a contour to be specified. Not every contour is admissible: there are only four directions going off to infinity with $\mathbb{R e} V(z)>0$ as $z \rightarrow \infty$, naturally leading to three homologically-independent admissible integration paths. This is illustrated in figure 1 when both $\mathbb{R e} \hbar>0$ and $\mathbb{R e} \lambda>0$. As these conditions change, so will the contours.

With a simple change of variables the partition function (2.8) may be written as

$$
Z(x)=\frac{\sqrt{\hbar}}{2 \pi} \int_{\Gamma} \mathrm{d} z \exp \left(-\frac{1}{2} z^{2}+\frac{x}{24} z^{4}\right)
$$

which explicitly illustrates how all non-trivial dependence occurs through the variable $x \equiv \lambda \hbar$, and this is what we shall study in the following. One way to evaluate this integral as an asymptotic expansion is through the method of steepest descents (see, e.g., [37]). First, one computes the saddle-points $z_{0}^{*}=0, z_{ \pm}^{*}= \pm \sqrt{\frac{6}{x}}$, with $V\left(z_{0}^{*}\right)=0$ and $V\left(z_{ \pm}^{*}\right)=\frac{3}{2 x}$. Their leading contribution to the quartic partition function is thus given by $\exp \left(-V\left(z^{*}\right)\right)$ and which is the dominant saddle will of course depend on the value of $\arg x$. Next, chosen a reference saddle $z^{*}$, one deforms the contour of integration into the infinite, oriented path of steepest descent through $z^{*}$, defined as

$$
\operatorname{Im}\left(V(z)-V\left(z^{*}\right)\right)=0
$$

and increasing away from $z^{*}$. For both "perturbative" saddle at the origin, $z_{0}^{*}$, and "instanton" saddles, $z_{ \pm}^{*}$, steepest descent (and steepest ascent) contours are depicted in figure 2, for different values of $\theta=\arg x$. We have also plotted the regions where the quartic potential is positive (negative): comparing with figure 1 , we now see how these regions change with $\theta$. In particular, this allows us to distinguish steepest descent ${ }^{2}$ from steepest ascent contours. Let us explain the plotting details of figure 2: for each value of $\theta$ we have plotted the leading saddle(s) with a solid disk, while the subleading saddle(s) were plotted with a circle. Regardless of dominance,

\footnotetext{
${ }^{2}$ Departing off to infinity along brown (darker) regions, ensuring convergence of the integral.
} 


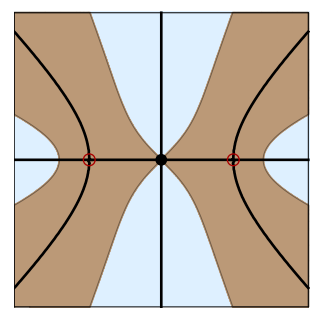

$\theta=0$

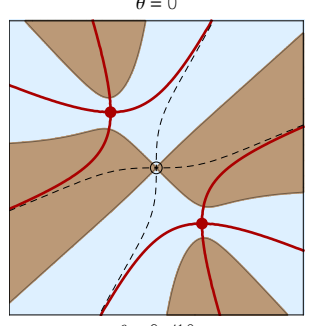

$\theta=9 \pi / 16$

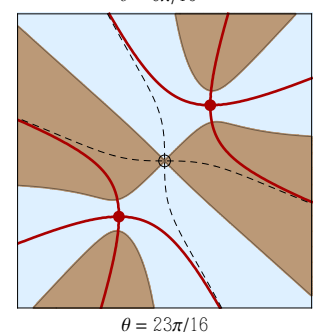

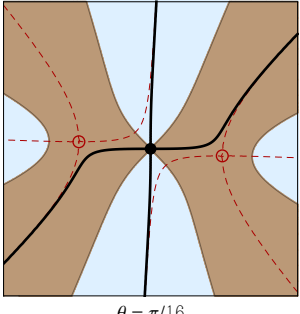

$\theta=\pi / 16$

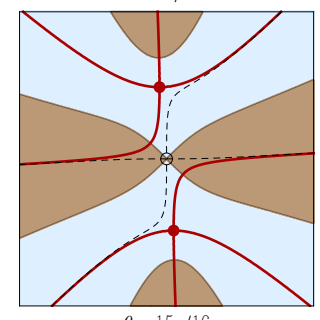

$\theta=15 \pi / 16$

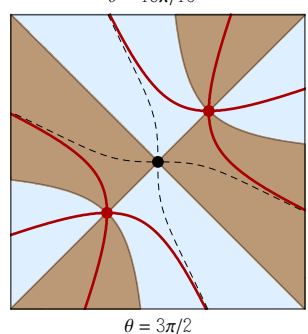

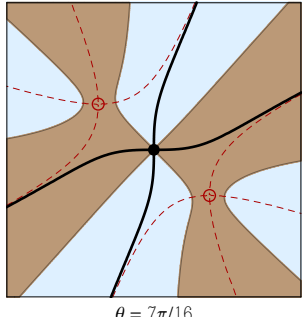

$\theta=7 \pi / 16$

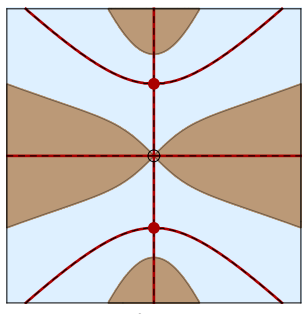

$\theta=\pi$

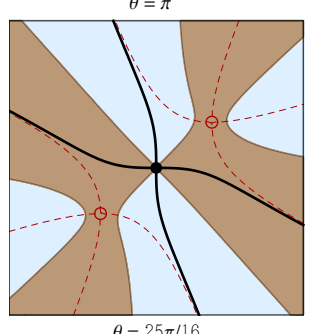

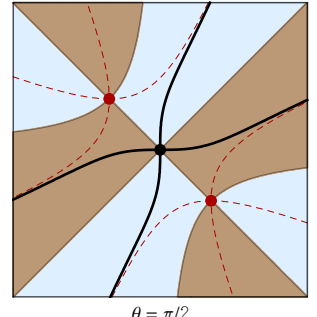
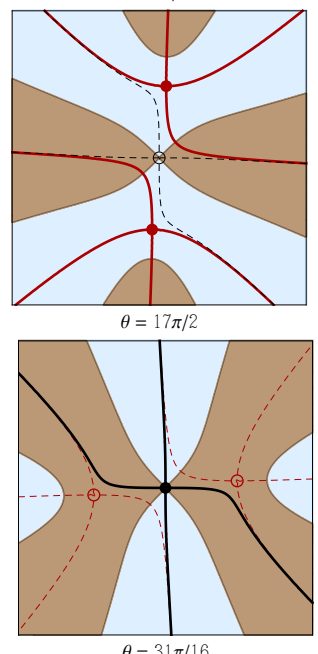

Figure 2. Steepest descent (ascent) contours for the quartic integral, through the several saddles (black for the "perturbative" saddle and red for the "instanton" saddle), along with the positivity of the potential, for different values of $\theta=\arg x(|x|=1$ in the plot). This makes clear which are Stokes and anti-Stokes lines: Stokes lines at $\arg x=0, \pi$ when the steepest descent contours hit the three saddles; anti-Stokes lines at $\arg x=\frac{\pi}{2}, \frac{3 \pi}{2}$. See the main text for a full discussion of the plot sequence.

the "perturbative" saddle is always plotted in black, with the "instanton" saddles in red. Then, solid contours are steepest descent (ascent) contours associated with the leading saddle(s), while dashed contours are steepest descent (ascent) contours associated with the subleading saddle(s).

What we immediately learn from figure 2 is that, generically, a steepest-descent contour goes through a single saddle - but this is not always the case. Indeed, this procedure yields welldefined asymptotic expansions as long as the path of steepest descent used in their calculation does not pass through a second saddle. This will occur when

$$
\operatorname{Im}\left(V\left(z_{ \pm}^{*}\right)-V\left(z_{0}^{*}\right)\right)=0 \quad \Leftrightarrow \quad \operatorname{Im} \frac{1}{x}=0
$$

corresponding to $\arg x=0, \pi$ (these lines are very distinctive in figure 2). These $\operatorname{are}^{3}$ the Stokes lines for the quartic partition-function, where, because other (suppressed) saddles start contributing to the evaluation of the integral, subleading exponentials likewise start contributing to the asymptotics. At $\theta=0$ the leading saddle is $z_{0}^{*}$, while $z_{ \pm}^{*}$ lead to exponentially suppressed contributions (in fact, purely exponential contributions along the Stokes direction). As $\theta$ keeps

\footnotetext{
${ }^{3}$ Note that the definitions of Stokes and anti-Stokes lines are usually reversed between the mathematical and physical literatures. In this work we are using the appropriate definitions within the resurgence framework. As such, the reader who has been exposed to these ideas before should pay attention to our precise definitions.
} 

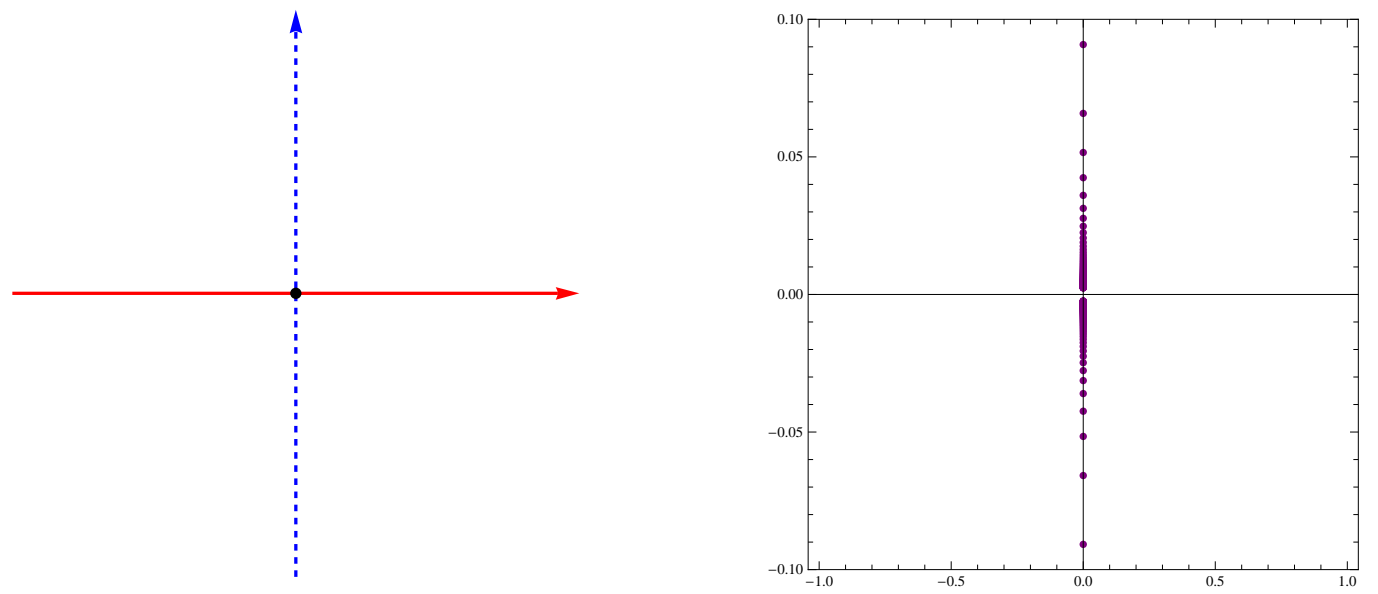

Figure 3. The first image shows the coupling constant $x$-plane, representing the phase diagram of the quartic partition function. Stokes lines are in red (solid), at $\arg x=0, \pi$, while anti-Stokes lines are in blue (dashed), at $\arg x=\frac{\pi}{2}, \frac{3 \pi}{2}$. The second image shows the Lee-Yang accumulation of zeros of the modified Bessel function describing the quartic partition-function, signaling a phase transition. One can clearly see that indeed the anti-Stokes lines correspond to phase boundaries.

increasing, these exponentially subleading contributions grow and, when they becomes of the same magnitude as the leading term, we reach what is known as an anti-Stokes line. Different exponential contributions are of the same magnitude when both saddles have the same real part,

$$
\mathbb{R e}\left(V\left(z_{ \pm}^{*}\right)-V\left(z_{0}^{*}\right)\right)=0 \quad \Leftrightarrow \quad \mathbb{R e} \frac{1}{x}=0
$$

corresponding to $\arg x=\frac{\pi}{2}, \frac{3 \pi}{2}$ (these lines are also shown in figure 2). At $\theta=\frac{\pi}{2}$ the subleading saddles $z_{ \pm}^{*}$ have become of the same magnitude as $z_{0}^{*}$ and one thus finds an oscillatory asymptotic behaviour along this direction. As we keep increasing $\theta$ beyond $\frac{\pi}{2}$ one sees that the different saddles have reversed roles: it is now the $z_{0}^{*}$ contribution which is exponentially suppressed with respect to that of $z_{ \pm}^{*}$. This situation is maintained all the way through the Stokes line at $\theta=\pi$, where the $z_{0}^{*}$ contribution, albeit suppressed, starts "growing" again (as expected upon crossing a Stokes line), until we reach the anti-Stokes line at $\theta=\frac{3 \pi}{2}$. Here all saddles recovered the same magnitude and one finds oscillatory asymptotic behaviour once again. Past this line, the leading contribution is again given by $z_{0}^{*}$ all the way back to $\theta=2 \pi$. The (very simple) "phase diagram" of the quartic partition function, describing the aforementioned behaviour, is shown in figure 3 .

What we have just described is the Stokes phenomenon: in an asymptotic approximation, different asymptotic formulae hold, on different sectors, for the same analytic function. As we shall see, transseries precisely capture all these possible asymptotic formulae in a single go. In the meantime, it is also interesting to understand the physical interpretation where Stokes phenomenon describes phase transitions [198], building upon the Lee-Yang construction $[168,169]$ (see [141] as well). In the Lee-Yang set-up, the finite-size grand-canonical partition function is first written as a polynomial. A phase transition then arises if, in the thermodynamic limit, the zeros of this polynomial condense onto lines describing phase boundaries. In the case of the quartic partition function (where the asymptotic approximation plays the role of the thermodynamic limit), it is simple to see that phase transitions occur at the anti-Stokes lines, from the "one-saddle" phase to the "two-saddle" phase and back again. This follows from an 
explicit Lee-Yang analysis: one can actually exactly compute the integral (2.10) as a modified Bessel function of the first kind (up to an exponential piece and a change of variables), whose zeros $^{4}$ precisely condense upon the imaginary axis as shown in figure 3 . We shall next describe how resurgent analysis unfolds, but for the moment just note that the picture we have unveiled for the phase diagram of the quartic partition function ${ }^{5}$ is the following: in the complex $x$-plane, the real axis is a Stokes line, while the imaginary axis is an anti-Stokes line/phase boundary.

\subsection{Basic Formulae for Resurgent Analysis}

Having understood the steepest-descent contour structure of the integral (2.10) we still need to explicitly evaluate it, as an asymptotic series. This is most simply done by first noticing that this integral satisfies a second-order linear ordinary differential equation ${ }^{6}$ (ODE),

$$
16 x^{2} Z^{\prime \prime}(x)+(32 x-24) Z^{\prime}(x)+3 Z(x)=0 .
$$

An ansatz of the form $Z(x)=\mathrm{e}^{-A / x} \Phi(x)$, with $\Phi(x)$ a power series, yields

$$
A=0 \quad \text { or } \quad A=\frac{3}{2}
$$

as expected from our earlier saddle-point analysis; alongside the corresponding asymptotic series

$$
\begin{array}{ll}
\Phi_{0}(x) \simeq \sum_{n=0}^{+\infty} Z_{n}^{(0)} x^{n}, & Z_{n}^{(0)}=\left(\frac{2}{3}\right)^{n} \frac{(4 n) !}{2^{6 n}(2 n) ! n !} Z_{0}^{(0)} \\
\Phi_{1}(x) \simeq \sum_{n=0}^{+\infty} Z_{n}^{(1)} x^{n}, & Z_{n}^{(1)}=(-1)^{n}\left(\frac{2}{3}\right)^{n} \frac{(4 n) !}{2^{6 n}(2 n) ! n !} Z_{0}^{(1)} .
\end{array}
$$

The "instanton action" $A=\frac{3}{2}$ is evident in the growth of the coefficients $Z_{n}^{(0)}, Z_{n}^{(1)} \sim A^{-n} n$ !. Notice that one still has to evaluate the constant ( $x$-independent) contributions, $Z_{0}^{(0)}$ and $Z_{0}^{(1)}$, sometimes denoted by residual coefficients in the resurgence literature. This is done by associating each of the above asymptotic expansions to distinct steepest-descent contours. For the perturbative saddle, in the "zero-instanton" sector, it is simple to evaluate $Z(0)=\sqrt{\frac{\hbar}{2 \pi}} \equiv Z_{0}^{(0)}$ from (2.10); while for the two subleading saddles, in the "one-instanton" sector, the integral over the appropriate contour ${ }^{7}$ may be evaluated in terms of modified Bessel functions whose asymptotic expansion then yields $Z_{0}^{(1)}=-\frac{\mathrm{i}}{\sqrt{2}} Z_{0}^{(0)}$. The asymptotic evaluation of the quartic partition function thus boils down to

$$
\begin{aligned}
& Z^{(0)}(x) \simeq \sqrt{\frac{\hbar}{2 \pi}} \sum_{n=0}^{+\infty}\left(\frac{2}{3}\right)^{n} \frac{(4 n) !}{2^{6 n}(2 n) ! n !} x^{n} \\
& Z^{(1)}(x) \simeq-\frac{\mathrm{i}}{\sqrt{2}} \sqrt{\frac{\hbar}{2 \pi}} \mathrm{e}^{-\frac{3}{2 x}} \sum_{n=0}^{+\infty}(-1)^{n}\left(\frac{2}{3}\right)^{n} \frac{(4 n) !}{2^{6 n}(2 n) ! n !} x^{n} .
\end{aligned}
$$

\footnotetext{
${ }^{4}$ Rigorously, this depends upon a choice of contour, yielding appropriate linear combinations of two Bessel functions. We are plotting the zeros of each of these building blocks (not exactly the same, but extremely similar).

${ }^{5} \mathrm{~A}$ short extension of this analysis, from the present realm of partition-function toy models to the full framework of one-dimensional quantum mechanics, is included in appendix B for the benefit of the interested reader.

${ }^{6}$ As mentioned, peeling off an exponential piece and changing variables, this is the modified Bessel equation.

${ }^{7}$ This is one of the "side" contours in figure 1, only picking-up one "instanton" saddle.
} 
Note how one may also obtain the Stokes and anti-Stokes lines directly from the exponential structure of the above solutions; by looking at their instanton structure. Stokes lines are given by

$$
\operatorname{Im}\left(\frac{3}{2 x}\right)=0
$$

such that the exponential in (2.19) is as damped (enhanced) as possible; while anti-Stokes lines correspond to

$$
\mathbb{R e}\left(\frac{3}{2 x}\right)=0
$$

such that the exponential in (2.19) is of order one, and (2.18) and (2.19) are of the same order. This obviously yields the precise same results as discussed earlier and depicted in figure 3.

Having constructed two linearly independent solutions ${ }^{8}(2.18)$ and (2.19) to the linear secondorder ODE (2.14), the general solution follows as their linear combination. This introduces two integration constants, parameterizing boundary conditions. One finds:

$$
\mathcal{Z}\left(x, \sigma_{0}, \sigma_{1}\right)=\sigma_{0} Z^{(0)}(x)+\sigma_{1} Z^{(1)}(x) .
$$

This is in fact the transseries solution to the problem, in the sense that it incorporates all saddles. The transseries parameters are $\sigma_{0}$ and $\sigma_{1}$, and it is also clear how they are parameterizing boundary conditions or contours of integration, depending upon the point-of-view. This is, of course, a trivial statement in elementary (linear) differential equations theory. Further, when comparing this explicit result (2.22) with the general form of a (one-parameter) transseries (2.5), in here we only find a finite number of instanton sectors. Again, this is due to linearity of the present problem. As we shall explicitly see in an upcoming subsection, only when considering nonlinear problems do we find an infinite number of (multi) instanton sectors.

In order to illustrate the ideas of resurgence in an elementary setting, we now turn to the computation of the Borel transforms (2.2), for the asymptotic power series $\Phi_{0}(x)$ and $\Phi_{1}(x)$. It is straightforward to obtain ${ }^{9}$

$$
\begin{aligned}
& \mathcal{B}\left[\Phi_{0}\right](s)=\frac{1}{8} \sqrt{\frac{\hbar}{2 \pi}}{ }_{2} F_{1}\left(\frac{5}{4}, \frac{7}{4}, 2 \mid \frac{2 s}{3}\right), \\
& \mathcal{B}\left[\Phi_{1}\right](s)=\frac{\mathrm{i}}{8 \sqrt{2}} \sqrt{\frac{\hbar}{2 \pi}}{ }_{2} F_{1}\left(\frac{5}{4}, \frac{7}{4}, 2 \mid-\frac{2 s}{3}\right),
\end{aligned}
$$

where ${ }_{2} F_{1}(a, b, c \mid z)$ is the hypergeometric function, with a branch cut on the complex $z$-plane running from $z=1$ to infinity. Thus, $\mathcal{B}\left[\Phi_{0}\right](s)$ has a singularity at $s=\frac{3}{2} \equiv A$ and $\mathcal{B}\left[\Phi_{1}\right](s)$ has a singularity at $s=-\frac{3}{2} \equiv-A$, implying that both have non-zero radius of convergence around $s=0$. If one next asks what is the nature of these singularities, it is not too hard to find the following expansions ${ }^{10}$ near $s= \pm A$,

$$
\mathcal{B}\left[\Phi_{0}\right](s)=(+2) \times \frac{Z_{0}^{(1)}}{2 \pi \mathrm{i}(s-A)}+(+2) \times \mathcal{B}\left[\Phi_{1}\right](s-A) \frac{\log (s-A)}{2 \pi \mathrm{i}}+\text { holomorphic }
$$

\footnotetext{
${ }^{8}$ Respectively associated to each of the two linearly/homologically independent steepest-descent contours of integration in figure 1 ; i.e., one for the perturbative saddle and another for the nonperturbative saddle (due to the $\mathbb{Z}_{2}$ symmetry of the potential, both $\pm \sqrt{6 / x}$ saddles obviously yield the same result).

${ }^{9}$ Note that the Borel transform (2.2) is undefined for the residual coefficient, with $\alpha+1=0$. This is simply handled by dropping this coefficient and only adding it back-in at the end - its contribution is trivial to include at any stage. Our Borel transforms thus follow from (2.16) and (2.17) with the asymptotic series starting at $n=1$.

${ }^{10}$ Note how the aforementioned residual coefficients are again visible in here; attached to the simple poles.
} 


$$
\mathcal{B}\left[\Phi_{1}\right](s)=(-1) \times \frac{Z_{0}^{(0)}}{2 \pi \mathrm{i}(s+A)}+(-1) \times \mathcal{B}\left[\Phi_{0}\right](s+A) \frac{\log (s+A)}{2 \pi \mathrm{i}}+\text { holomorphic }
$$

The first thing one notices in these results is the origin of the name "resurgence": in fact, at the singularity of $\mathcal{B}\left[\Phi_{0}\right](s)$ one finds the "reappearance" or resurgence of the $\Phi_{1}$ sector, via its Borel transform; while at the singularity of $\mathcal{B}\left[\Phi_{1}\right](s)$ one finds the resurgence of the $\Phi_{0}$ sector. This reappearance includes the full asymptotic series, with the residual coefficient attached to the simple pole and the rest of the coefficients appearing next to the logarithmic branch-cut.

This property of "reappearance" turns out to be rather generic and it is in fact at the basis of the theory of resurgence. If we forget for one moment that it was uncovered by looking at the functional nature of the Borel singularities, or even that these singularities may be poles or logarithmic branch-cuts or else, what (2.25) and (2.26) are expressing are relations in-between the asymptotic series $\Phi_{0}(x)$ and $\Phi_{1}(x)$, the building blocks of the transseries (2.22). Such a level of abstraction may be easily reachable from (2.25) and (2.26) by first considering the difference between left and right Borel resummations, (2.4), along the appropriate Stokes directions, $\theta=0$ and $\theta=\pi$ respectively,

$$
\begin{aligned}
& \left(\mathcal{S}_{0^{+}}-\mathcal{S}_{0^{-}}\right) \Phi_{0}(x)=(+2) \times\left(-Z_{0}^{(1)}-\Phi_{1}^{\star}(x)\right) \mathrm{e}^{-\frac{A}{x}}=(-2) \times \Phi_{1}(x) \times \mathrm{e}^{-\frac{A}{x}}, \\
& \left(\mathcal{S}_{\pi^{+}}-\mathcal{S}_{\pi^{-}}\right) \Phi_{1}(x)=(-1) \times\left(-Z_{0}^{(0)}-\Phi_{0}^{\star}(x)\right) \mathrm{e}^{+\frac{A}{x}}=(+1) \times \Phi_{0}(x) \times \mathrm{e}^{+\frac{A}{x}} .
\end{aligned}
$$

After the first equality, we momentarily used the notation $\Phi^{\star}(x)$ to explicitly denote the asymptotic series which follow from $\Phi(x)$ by dropping their residual coefficients (recall the discussion leading up to (2.23) and (2.24); in particular footnote 9). It is very interesting to observe how precisely these coefficients are being added back-in by the simple-pole contributions in (2.25) and (2.26), which then lead to the very neat final equalities. As just discussed, we are aiming at a level of abstraction where we can do without looking at the particular functional nature of the Borel transforms. That is finally obtained by defining a linear operator, $\Delta_{\omega}$, with $\omega$ a point in the complex $s$-plane, which acts on the transseries building blocks $\Phi_{n}$ (the asymptotic series), and which describes the basic structure encoded in (2.25) and (2.26). In this particular linear example, a definition for such an operator naturally follows from the previous two equations, as ${ }^{11}$

$$
\Delta_{\omega}:=\mathrm{e}^{\frac{\omega}{x}}\left(\mathcal{S}_{\theta^{+}}-\mathcal{S}_{\theta^{-}}\right),
$$

leading to the (for the moment) abstract structure formalizing the property of resurgence:

$$
\begin{aligned}
& \Delta_{+A} \Phi_{0}=(-2) \times \Phi_{1}, \\
& \Delta_{-A} \Phi_{1}=\Phi_{0} .
\end{aligned}
$$

Our departure equations may here be recovered by reading these expressions as follows: the Borel transform of $\Phi_{0}$ has a singularity at $\omega=+A$ which is given by the Stokes coefficient $S_{1}=-2$, multiplied by the Borel transform of $\Phi_{1}$ (and "attached" to the logarithmic ${ }^{12}$ branch-cut). The

\footnotetext{
${ }^{11}$ It is very important to keep in mind that this definition only holds in the present linear case. It will not hold for nonlinear problems, where it will require (substantial) modification. More on this in the following.

${ }^{12}$ If all singularities are simple poles alongside logarithmic branch-cuts, this implies we are in the class of so-called simple resurgent functions, but we will get back to this point in section 4 .
} 
second line is read similarly. Furthermore, this algebraic structure closes upon itself,

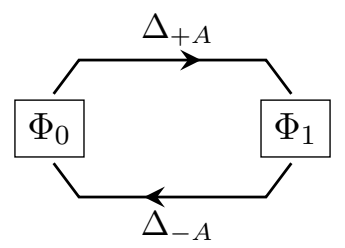

as, in particular, $\Delta_{\omega} \Phi_{n}=0$ if the Borel transform of $\Phi_{n}$ is not singular at $\omega$. This linear operator is known as the alien derivative as it is in fact a linear differential operator, satisfying the Leibniz rule (see appendix A). We refer the reader to the reviews [55, 56, 91, 207, 203, 204] and references therein for the proof that $\Delta_{\omega}$ is a derivation, and subsequently the construction of alien calculus.

In our spirit of introducing the main ideas of resurgence in a simple example, let us proceed and address the Stokes automorphism (2.4). One of the reasons to introduce alien calculus, as sketched above, is that the Stokes automorphism is essentially given by the exponential of the alien derivative (why this is so will hopefully be motivated by the end result that follows),

$$
\underline{\mathfrak{S}}_{\theta}=\exp \left(\sum_{\left\{\omega_{\theta}\right\}} \mathrm{e}^{-\frac{\omega_{\theta}}{x}} \Delta_{\omega_{\theta}}\right) .
$$

Now, from (2.30) and (2.31), it is easy to see that $\Delta_{+A} \Delta_{+A} \Phi_{0}=0=\Delta_{-A} \Delta_{-A} \Phi_{1}$, implying that the above exponential collapses to a finite sum for both the $\theta=\arg (+A)=0$ and $\theta=$ $\arg (-A)=\pi$ Stokes rays. The only non-trivial relations are thus:

$$
\underline{\mathfrak{S}}_{0} \Phi_{0}=\exp \left(\mathrm{e}^{-\frac{A}{x}} \Delta_{+A}\right) \Phi_{0}=\Phi_{0}-2 \mathrm{e}^{-\frac{3}{2 x}} \Phi_{1} \quad \text { and } \quad \underline{\mathfrak{S}}_{\pi} \Phi_{1}=\Phi_{1}+\mathrm{e}^{\frac{3}{2 x}} \Phi_{0},
$$

or, equivalently,

$$
\underline{\mathfrak{S}}_{0} Z^{(0)}(x)=Z^{(0)}(x)-2 Z^{(1)}(x), \quad \text { and } \quad \underline{\mathfrak{S}}_{\pi} Z^{(1)}(x)=Z^{(1)}(x)+Z^{(0)}(x) .
$$

That $\Phi_{0}$ only has one Stokes line along $\theta=0$ on the Borel plane, and $\Phi_{1}$ only one along $\theta=\pi$, is of course already dictated by the alien calculus (2.30) and (2.31) (or, earlier on, by (2.25) and (2.26)). The above expressions now completely encode the Stokes phenomena (2.7) across all Stokes lines, where, in this problem, singular directions on the Borel plane equally relate with Stokes lines on the complex $x$-plane. Using (2.22) one thus finds that the Stokes automorphism (2.4) at $\theta=0$ is

$$
\mathcal{S}_{0^{+}} \mathcal{Z}\left(x, \sigma_{0}, \sigma_{1}\right)=\mathcal{S}_{0^{-}} \mathcal{Z}\left(x, \sigma_{0}, \sigma_{1}-2 \sigma_{0}\right),
$$

while at $\theta=\pi$ it is

$$
\mathcal{S}_{\pi^{+}} \mathcal{Z}\left(x, \sigma_{0}, \sigma_{1}\right)=\mathcal{S}_{\pi^{-}} \mathcal{Z}\left(x, \sigma_{0}+\sigma_{1}, \sigma_{1}\right) .
$$

It is interesting to notice that if we move twice around the origin, $\left(\underline{\mathfrak{S}}_{\pi} \underline{\mathfrak{S}}_{0}\right)^{2}$, the transseries parameters change as $\left(\sigma_{0}, \sigma_{1}\right) \rightarrow\left(-\sigma_{0},-\sigma_{1}\right)$, while if we move four times around the origin we recover the identity,

$$
\left(\underline{\mathfrak{S}}_{\pi} \underline{\mathfrak{S}}_{0}\right)^{4}=\mathbf{1} .
$$

This expression tells us what is the monodromy encoded in the transseries solution to the quartic integral, which is thus best understood as a multi-sheeted Riemann surface ${ }^{13}$.

\footnotetext{
${ }^{13}$ See [15] for a discussion on the relation between Stokes transitions and the monodromy of transseries solutions.
} 
At this stage, it is instructive to take a step back and re-derive the above monodromy relation (2.38) directly from our discussion of steepest-descent contours in the previous subsection. Recall that we had three saddle-points, $z_{0}^{*}$ and $z_{ \pm}^{*}$, which led to three steepest-descent contours for $\Gamma$ in the evaluation of the partition function (2.10), and which we shall now respectively denote by $\mathcal{J}_{0}$ and $\mathcal{J}_{ \pm}$(we shall discuss the nature of such steepest-descent contours in further detail later-on, in section 3). The asymptotic series $\Phi_{0}$ and $\Phi_{1}$, which we computed in (2.16) and (2.17), are obviously also generated by the integration (2.10) along these contours. Now, what we wish to point out is that these contours will acquire discontinuities at the Stokes rays $\theta=0$ and $\pi$, as shown in figure 2. In fact, in order to instead ensure the continuity of each asymptotic series $\Phi_{i}$ along these Stokes rays, one needs to make sure that the contour has the same asymptotics for, e.g., $\theta=0^{-}$and $\theta=0^{+}$(i.e., the tails of the contour lie in the same brown regions of figure 2 for both $\theta=0^{-}$and $\theta=0^{+}$). This continuity condition implies that the steepest-descent contours need to change as $\mathcal{J}_{0} \rightarrow \mathcal{J}_{0}+\mathcal{J}_{-}-\mathcal{J}_{+}$as one rotates from $\theta=0^{-}$to $0^{+}$(compare the last and second plots in figure 2). On the other hand, $\mathcal{J}_{ \pm}$need not acquire any jumps at $\theta=0$. These conditions may be compactly expressed as

$$
\left(\begin{array}{c}
\mathcal{J}_{0} \\
\mathcal{J}_{+} \\
\mathcal{J}_{-}
\end{array}\right) \rightarrow S_{\circlearrowleft}^{(0)}\left(\begin{array}{c}
\mathcal{J}_{0} \\
\mathcal{J}_{+} \\
\mathcal{J}_{-}
\end{array}\right)=\left(\begin{array}{ccc}
1 & -1 & 1 \\
0 & 1 & 0 \\
0 & 0 & 1
\end{array}\right)\left(\begin{array}{c}
\mathcal{J}_{0} \\
\mathcal{J}_{+} \\
\mathcal{J}_{-}
\end{array}\right),
$$

where $S_{\circlearrowleft}^{(0)}$ is the "Stokes matrix" associated to $\theta=0$. Essentially the same reasoning goes through around $\theta=\pi$, where the continuity conditions may now be obtained by comparing the sixth and eight plots in figure 2 . The reader can check that one will now obtain

$$
\left(\begin{array}{c}
\mathcal{J}_{0} \\
\mathcal{J}_{+} \\
\mathcal{J}_{-}
\end{array}\right) \rightarrow S_{\circlearrowleft}^{(\pi)}\left(\begin{array}{c}
\mathcal{J}_{0} \\
\mathcal{J}_{+} \\
\mathcal{J}_{-}
\end{array}\right)=\left(\begin{array}{ccc}
1 & 0 & 0 \\
1 & 1 & 0 \\
-1 & 0 & 1
\end{array}\right)\left(\begin{array}{c}
\mathcal{J}_{0} \\
\mathcal{J}_{+} \\
\mathcal{J}_{-}
\end{array}\right) .
$$

If we finally define the monodromy matrix $\mathfrak{M}:=S_{\circlearrowleft}^{(\pi)} \cdot S_{\circlearrowleft}^{(0)}$ it is simple to check that $\mathfrak{M}^{4}=\mathbf{1}$, which is the steepest-descent equivalent of the monodromy relation (2.38).

Having understood the basics of the Stokes automorphism, and how Stokes transitions may be implemented, let us ask what occurs at a Stokes line, say at $\arg x=0$ (equivalently, along the singular direction $\theta=0$ ). For our transseries $(2.22)$, one starts off at $\arg x=0^{-}$with $\sigma_{1}=0$ and $\sigma_{0} \neq 0$, which we may always normalize to $\sigma_{0}=1$, i.e., we start off with $\mathcal{Z}(x, 1,0)$ which is simply an adequate Borel resummation of the (perturbative) asymptotic expansion (2.18). As we cross the Stokes line via (2.36) we obtain the transseries

$$
\mathcal{Z}(x, 1,2)=\mathcal{S}_{\theta>0} \Phi_{0}(x)+2 \mathrm{e}^{-\frac{3}{2 x}} \mathcal{S}_{\theta>0} \Phi_{1}(x) .
$$

Increasing $\theta$ until we reach the positive imaginary axis where $x=+\mathrm{i}|x|$, this becomes, to leading order,

$$
\mathcal{Z}(\mathrm{i}|x|, 1,2) \simeq \sqrt{\frac{\hbar}{2 \pi}}\left(1+\sqrt{2} \sin \left(\frac{3}{2|x|}\right)-\mathrm{i} \sqrt{2} \cos \left(\frac{3}{2|x|}\right)\right)+\cdots,
$$

yielding the expected asymptotic oscillatory behaviour to be found at anti-Stokes lines.

Of course the transseries construction includes much more information beyond finding this oscillatory behaviour. As one keeps rotating in the complex plane, successively crossing Stokes lines, the transseries solution displays its full multi-sheeted structure encoded in (2.38). Let us explore this particular point. An exact evaluation of the quartic-potential integral around the 

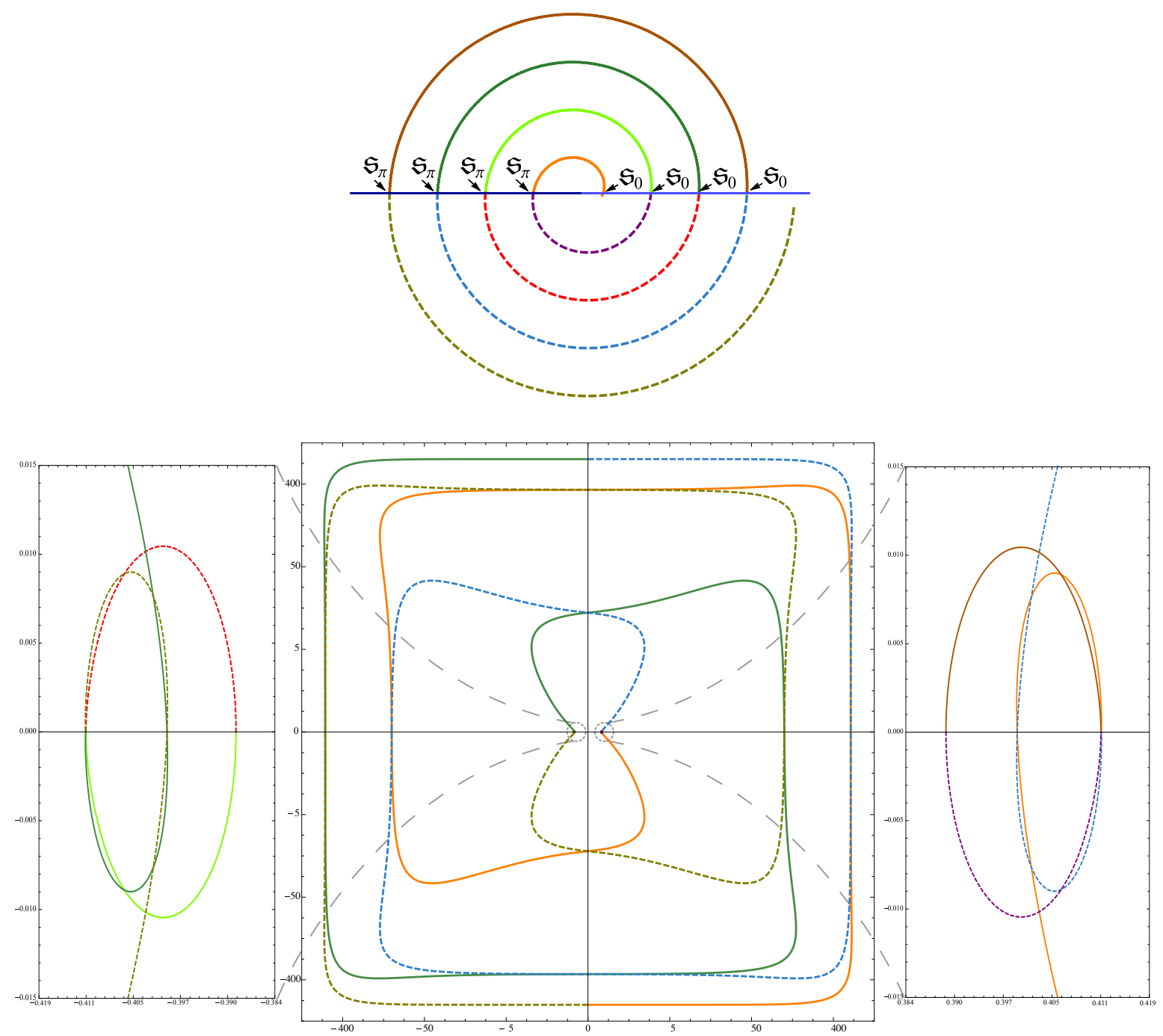

Figure 4. The monodromy of the transseries solution to the quartic integral. We start at $\arg x=0^{-}$ with $\mathcal{Z}\left(0.21 \mathrm{e}^{\mathrm{i} \theta}, 1,0\right)$, and rotate $\theta$ four times around the origin according to (2.38). This is illustrated in the upper plot, with different colors/types signaling different regions (on the Borel plane) in-between Stokes transitions. For instance, at $2 \pi n$, the Stokes transition (2.36) takes place, while at $\pi+2 \pi n$ it is (2.37) instead ( $n=0,1,2,3)$. In this way, each color/type occurs in an interval of length $\pi$ and the complete sequence yields the full $8 \pi$ rotation as shown. As we translate this rotation back to the $x$ plane (illustrated in the lower plots, with matching colors/types as in the upper plot), it is clear how the transseries trajectory closes upon itself, fulfilling the predicted monodromy and showing how indeed the transseries encodes the full nonperturbative solution. A word on numerics: we have resummed the asymptotic series with Padé approximants, and chosen relatively small $|x|$ for improved accuracy. This, however, results in the different scales appearing in the lower plots due to the exponential factor. The central figure is plotted with a logarithmic scale and also gives an (almost complete) overview of the trajectory. The exponential factor makes it hard to understand what happens closer to the origin and, thus, the lateral images zoom-in onto the small "loops" which are already visible in the central plot.

"elementary" contours of figure 1 is possible, with the different results being written in terms of 
modified Bessel functions of the first kind; concretely as linear combinations of ${ }^{14}$

$$
\sqrt{\frac{3}{4 x}} \mathrm{e}^{-\frac{3}{4 x}} I_{ \pm \frac{1}{4}}\left(\frac{3}{4 x}\right)
$$

However, all such results have branch cuts along the negative real axis and the question remains on how to explicitly implement the appropriate analytic continuation yielding a fully nonperturbative solution to our problem, in its correct domain of validity. This is one of the points encoded in the Stokes transitions (2.36) and (2.37): they already automatically yield this analytic continuation with the prescribed monodromy - the only point left to address being an adequate resummation of the different asymptotic series, which is now just a numerical question. To illustrate this point, let us again start off at $\arg x=0^{-}$with $\mathcal{Z}(x, 1,0)$, let us fix $|x|$, and let us rotate $\arg x$ around the origin four times, according to the monodromy (2.38). The result is shown in figure 4, where we have set $|x|=0.21$, resummed the asymptotic series with (appropriately chosen) Padé approximants, and rotated as described: in this way, via Stokes transitions, the transseries describes a smooth trajectory over the multi-sheeted solution to our problem.

This brief overview of the quartic-potential integral introduced some of the ideas of resurgence in a very simple setting, and also illustrated how resurgent analysis is particularly straightforward for linear systems (i.e., with a finite number of instanton sectors and Stokes constants). It is of course important to point out that the story is very different for generic nonlinear systems, with multi-instanton sectors and even resonance, where trying to build the correct analytic continuation as described above is already an extremely non-trivial problem (see [15] for the full Stokes transitions generalizing (2.36) and (2.37) and a glimpse at the general monodromy operator (2.38)). The interested reader may find more details on resurgent calculus in appendix A.

\subsection{Asymptotics and Large-Order Behaviour: Partition Function}

As we already made clear in the introduction, one very important physical outcome of resurgence is the capability it gives us to completely understand the asymptotics of some given perturbative expansion. Let us go back to (2.4),

$$
\mathcal{S}_{\theta^{+}}=\mathcal{S}_{\theta^{-}} \circ \underline{\mathfrak{S}}_{\theta},
$$

which quantifies how different asymptotic expansions hold, on different sides of a Stokes line, for the same function. This implies that there is a discontinuity of the asymptotic expansions ${ }^{15}$, $\operatorname{Disc}_{\theta}$, which must relate to the Stokes automorphism as

$$
\operatorname{Disc}_{\theta}=\mathbf{1}-\underline{\mathfrak{S}}_{\theta},
$$

so that $\mathcal{S}_{\theta^{+}}-\mathcal{S}_{\theta^{-}}=-\mathcal{S}_{\theta^{-}} \circ \operatorname{Disc}_{\theta}$ (see figure 5 for a sketch of this discontinuity).

Now given a function $F(x)$, with a discontinuity along some ray starting from the origin on the complex plane along the direction $\theta$, Cauchy's theorem translates to

$$
F(x)=-\frac{1}{2 \pi \mathrm{i}} \int_{0}^{\mathrm{e}^{\mathrm{i} \theta} \infty} \mathrm{d} w \frac{\operatorname{Disc}_{\theta} F(w)}{w-x},
$$

at least if there is no contribution around infinity - which is usually the case in the large-order context; e.g., [38, 39, 67, 233]. If we apply this relation to the perturbative asymptotic expansion

\footnotetext{
${ }^{14}$ One way to obtain this result is via a strong-coupling analysis, as in appendix C.

${ }^{15}$ So that the nature of this discontinuity is completely clear, let us repeat what we wrote earlier concerning Stokes phenomenon: in an asymptotic approximation, different asymptotic formulae hold, on different sectors, for the same analytic function. In this way, it is this discontinuity which then relates them to each other.
} 


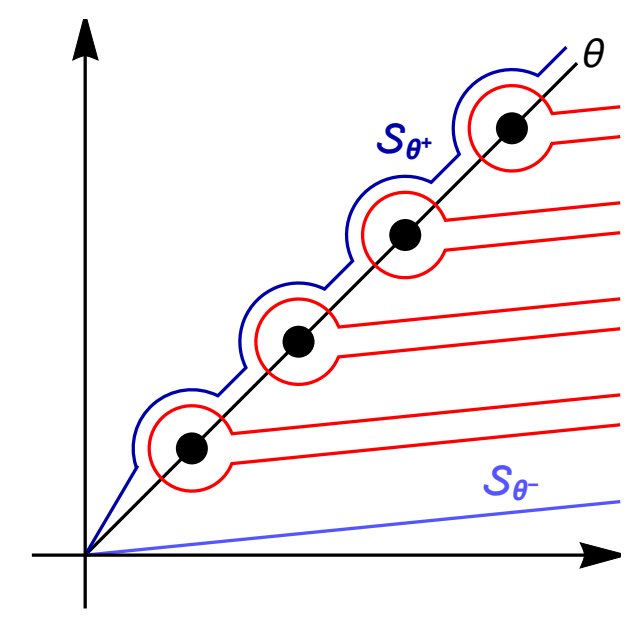

Figure 5. The left Borel resummation $\mathcal{S}_{\theta^{+}}$crosses the singular Stokes line along $\theta$, to yield the right Borel resummation $\mathcal{S}_{\theta^{-}}$alongside the Stokes discontinuity Disc $\theta$. This discontinuity has a geometrical representation as sum over the (red) Hankel contours associated to each (multi-instanton) singularity.

for $\Phi_{0}(x)$, given in (2.16), which will in fact have a discontinuity along $\theta=0$ due to the nontriviality of the Stokes automorphism in (2.34), it first follows ${ }^{16}$

$$
\sum_{n=0}^{+\infty} Z_{n}^{(0)} x^{n} \simeq-\frac{1}{2 \pi \mathrm{i}} \int_{0}^{+\infty} \mathrm{d} w \frac{2}{w-x} \mathrm{e}^{-\frac{3}{2 w}} \sum_{k=0}^{+\infty} Z_{k}^{(1)} w^{k} .
$$

This immediately implies ${ }^{17}$

$$
Z_{n}^{(0)} \simeq-\frac{2}{2 \pi \mathrm{i}} \frac{\Gamma(n)}{A^{n}} \sum_{k=0}^{+\infty} \frac{\Gamma(n-k)}{\Gamma(n)} Z_{k}^{(1)} A^{k}
$$

One may regard this expression as a large-order relation (see figure 6),

$$
Z_{n}^{(0)} \simeq-\frac{2}{2 \pi \mathrm{i}} \frac{(n-1) !}{A^{n}}\left(Z_{0}^{(1)}+\frac{Z_{1}^{(1)} A}{n-1}+\frac{Z_{2}^{(1)} A^{2}}{(n-1)(n-2)}+\cdots\right) ;
$$

or else make use of the exact coefficients $Z_{k}^{(1)}$, given in (2.17), to reobtain (without surprise) the exact coefficients (2.16). Indeed, replacing (2.17) in the above expression,

$$
Z_{n}^{(0)} \simeq \frac{1}{\sqrt{2} \pi}\left(\frac{2}{3}\right)^{n} \sum_{k=0}^{n-1}(-1)^{k} \frac{(4 k) !(n-k-1) !}{2^{6 k}(2 k) ! k !} Z_{0}^{(0)}
$$

and recalling that it only holds as a large-order expression, i.e., in a regime where $n \gg k$ and where subsequently one has

$$
\lim _{n \rightarrow+\infty} \frac{2^{6 n}(2 n) ! n !}{(4 n) !}(n-k-1) !=\sqrt{2} \pi \delta_{k 0},
$$




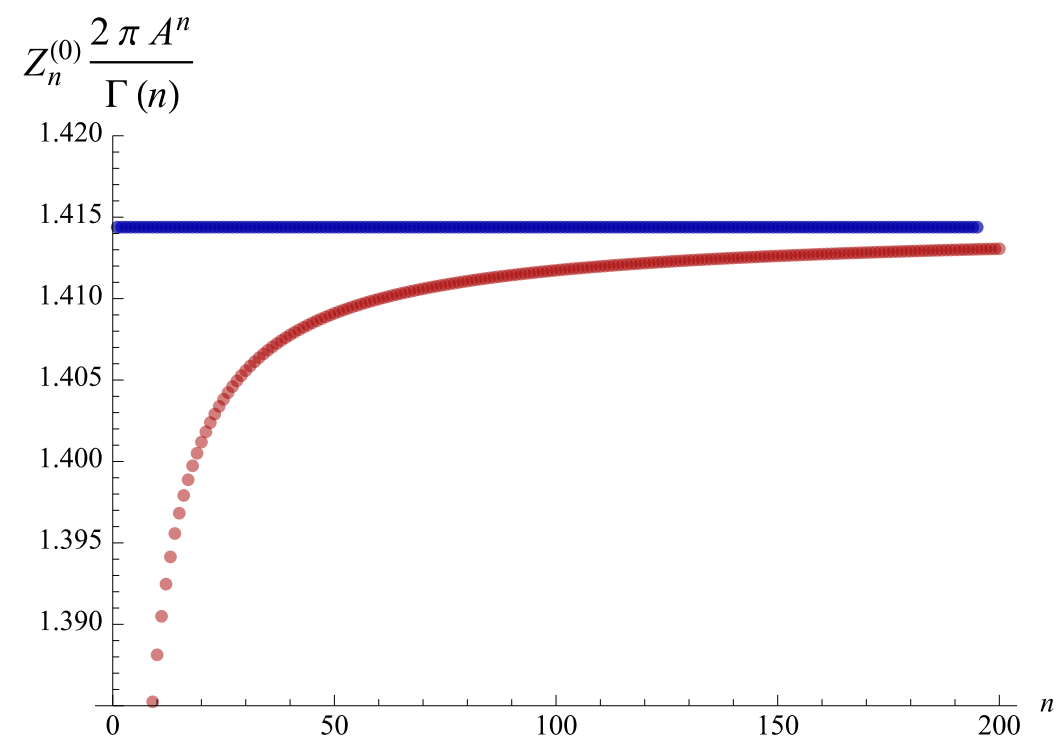

Figure 6. Numerical plot of the coefficients $Z_{n}^{(0)}$, weighted by the growth factor $\frac{2 \pi A^{n}}{\Gamma(n)}$ and up to 200 terms. The coefficients of the original series are shown in red, and in blue we plot their corresponding fifth Richardson transform, denoted by $\mathrm{RT}_{0}(0, n, 5)$ (details on these numerical acceleration schemes will appear in a following subsection). The value to which the sequence is converging can be read directly from (2.49) to be $2 \mathrm{i} Z_{0}^{(1)}=\sqrt{2}$ (where in this test we have set $\hbar=2 \pi$ ). The numerics fully validate the (leading) resurgence large-order relation $(2.49)$, up to an extremely small error of order $\sim \mathcal{O}\left(10^{-12}\right)$. Similar tests may be done to validate the subleading components of the growth, as predicted by resurgence.

one then directly recovers (2.16). Naturally, an exactly analogue procedure goes through when analyzing the large-order behaviour of the instanton asymptotic expansion (2.17) instead.

\subsection{First Steps Towards Nonlinear Resurgent Analysis}

Resurgence only shows its full powers when addressing nonlinear problems, i.e., problems where most popular (nonperturbative) techniques might be of little use. At the same time, in many quantum theoretic settings, it is often not the partition function which is most amenable to calculations, but rather the free energy. With this dual motivation in mind, let us now address the "quartic free energy", $F=\log Z$. Unlike the quartic-potential partition function in (2.14), this time around the quartic-potential free energy satisfies a nonlinear second-order ODE,

$$
16 x^{2} F^{\prime \prime}(x)+16 x^{2}\left(F^{\prime}(x)\right)^{2}+(32 x-24) F^{\prime}(x)+3=0 .
$$

An asymptotic perturbative solution $F(x) \simeq \sum_{n=0}^{+\infty} F_{n}^{(0)} x^{n}$ yields the recursion (determining it)

$$
F_{n+1}^{(0)}=\frac{2}{3}\left(n F_{n}^{(0)}+\frac{1}{n+1} \sum_{k=1}^{n-1} k(n-k) F_{k}^{(0)} F_{n-k}^{(0)}\right),
$$

with $F_{1}^{(0)}=\frac{1}{8}$ and $F_{0}^{(0)}$ an integration constant. Imagine we knew nothing about $F$ (although we can know a lot, as we have already analyzed the partition function thoroughly!), and let us

\footnotetext{
${ }^{16}$ Using asymptotic series on both sides of the equality, and changing summation and integration signs, the resulting expression only holds formally and thus the "asymptotic equality" sign meaning it holds at large order.

${ }^{17}$ Note that convergence of the integral requires $n>k$, again signaling the large-order nature of this relation.
} 
thus proceed with a large-order analysis of this recursion ${ }^{18}$. To very high numerical precision (for instance, using Richardson transforms - which we will describe in detail in a following subsection) it is possible to show that the leading large-order behaviour of the perturbative coefficients in the quartic free energy is

$$
F_{n}^{(0)} \sim \frac{S_{1} F_{0}^{(1)}}{2 \pi \mathrm{i}} \frac{\Gamma(n)}{A^{n}}+\cdots,
$$

with $^{19}$

$$
A \approx \frac{3}{2} \quad \text { and } \quad S_{1} F_{0}^{(1)} \approx \mathrm{i} \sqrt{2} .
$$

This behaviour points to the existence of instanton corrections, with action $A=\frac{3}{2}$. Now, in the study of the partition function, we saw that we only needed two sectors: the perturbative expansion and a single nonperturbative instanton sector, both solutions to a linear differential equation. On the other hand, in the present case the free energy obeys a nonlinear differential equation, and we thus expect to have a tower of multi-instanton sectors alongside the perturbative $\operatorname{series}^{20}$. Let us try to derive the above "experimental" results by considering an anstäz for the solution to the quartic free-energy equation (2.52), of the form

$$
F(x) \simeq \mathrm{e}^{-\frac{\ell A}{x}} x^{\ell \beta} \sum_{n=0}^{+\infty} F_{n}^{(\ell)} x^{n},
$$

where, besides the multi-loop multi-instanton coefficients $F_{n}^{(\ell)}$, we also have to determine the instanton action $A$ and a characteristic exponent $\beta$. Plugging this ansatz back into (2.52), one finds that a solution exists if one chooses

$$
A=0, \frac{3}{2}, \quad \beta=0 .
$$

We find two possible values for the instanton action, but only one non-zero. The first reasonable guess in order to compute the free energy is then to assume that the above zero instanton action is in fact part of the perturbative sector, and to expect to describe the complete (and most general!) solution to the free energy of the quartic-potential as the following one-parameter transseries

$$
F(x, \sigma)=\sum_{\ell=0}^{+\infty} \sigma^{\ell} \mathrm{e}^{-\frac{\ell A}{x}} \Phi_{\ell}(x) .
$$

Here $\sigma$ is the transseries instanton-counting parameter (an integration constant parameterizing boundary conditions), and the $\Phi_{\ell}(x)$ are the transseries building blocks, i.e., the asymptotic expansions associated to each (multi) instanton sector,

$$
\Phi_{\ell}(x) \simeq \sum_{n=0}^{+\infty} F_{n}^{(\ell)} x^{n} .
$$

\footnotetext{
${ }^{18}$ For the reader who is less familiar with such techniques, we shall discuss in detail such type of analyses in a following subsection. For the moment, take this in the spirit of what was just done in the previous subsection.

${ }^{19}$ Where $S_{1} F_{0}^{(1)}$ is the "scale invariant" combination carrying physical information; see [16].

${ }^{20}$ This is intuitively obvious. When searching for solutions, the nonlinear term in (2.52) essentially grabs any (non-analytic) exponential contribution and iteratively induces all possible powers of this exponential. In this way, a solution ansatz involving a single-instanton must then necessarily involve all possible multi-instanton contributions.
} 
Just like for the perturbative sector in (2.53), the coefficients of these instanton expansions also obey recursive relations, obtained by keeping track of equal powers of $\mathrm{e}^{-\frac{\ell A}{x}}$ and of $x^{n}$ when substituting the full transseries ansatz, (2.58) and (2.59), into the differential equation (2.52). These recursive relations are now more intricate ${ }^{21}$ than (2.53), and they may be found in appendix D. From these recursive relations one can determine enough coefficients in the expansions of any (multi) instanton sector to find that the expansions $\Phi_{\ell}$ are indeed asymptotic, as they diverge factorially ${ }^{22}$

$$
F_{n}^{(\ell)} \sim \frac{\Gamma(n)}{A^{n}}
$$

We shall now illustrate how resurgence extends to this one-parameter transseries solution of a nonlinear differential equation. Akin to what was done in earlier sections, within the linear problem, one starts by addressing the Borel transforms of each multi-instanton sector $\Phi_{\ell}$,

$$
\mathcal{B}\left[\Phi_{\ell}\right](s)=\sum_{n=1}^{+\infty} \frac{F_{n}^{(\ell)}}{\Gamma(n)} s^{n-1} .
$$

Of course one immediately stumbles upon a major difference as compared to the linear case: now, one no longer has closed-form expressions for the multi-loop multi-instanton coefficients, $F_{n}^{(\ell)}$, and so it is no longer possible to analytically compute the Borel transforms (as was done in the linear problem in (2.23) and (2.24)). Recall that such calculations led us to understand the singularity structure of the Borel transforms, (2.25) and (2.26), which opened the door to the introduction of the resurgence framework in (2.30) and (2.31). Nevertheless, given their recursive construction, it is straightforward to computationally calculate several of these coefficients - for any sector $\Phi_{(\ell)}$ of the one-parameter transseries - and one can then numerically approximate the analytic function we are interested in, e.g., via Padé approximants (see, e.g., [37], for a classical treatment, or, e.g., [11], for very recent applications; but more on this below). Being applied to the computation of Borel transforms, $\mathcal{B}\left[\Phi_{n}\right]$, we shall denote this approximation scheme as the Borel-Padé approximation method, written as $\mathrm{BP}_{\ell}\left[\Phi_{n}\right]$ for a (diagonal) order- $\ell$ Padé approximant. For the moment all one needs to know is that this method approximates the Borel transform by a rational function -in fact a ratio of two polynomials - whose singularity structure is then encoded in its poles (immediately computed as the zeroes of the polynomial in the denominator). As it turns out, in the case of resurgent functions these poles condense to form cuts which start at the positions of the instanton actions on the Borel plane.

As an example, we show in figure 7 the poles of the Padé approximants for the Borel transforms of perturbative, $\Phi_{0}$, and one-instanton, $\Phi_{1}$, asymptotic series in the quartic free energy. It is rather evident how these poles condense into either one or two branch-cuts, starting at the values of the instanton actions on the Borel plane, $s= \pm \frac{3}{2}$. The respective Borel transforms thus have a non-zero radius of convergence around $s=0$. Using the recursions in appendix D it is straightforward to plot further examples and to study numerically the nature of the resulting singularities and branch-cuts, which we leave as an exercise for the reader.

\footnotetext{
${ }^{21}$ In particular, they keep on demanding for the need of extra (higher) multi-instanton sectors, coupled to the previous ones (and eventually to the perturbative series), in such a way that in the end one indeed needs for an infinite number of multi-instanton sectors as initially put forward in the general transseries ansatz (2.58).

${ }^{22}$ The simplest way to find this factorial growth is to plot $\frac{F_{n}^{(\ell)}}{F_{n+1}^{(\ell)}}$, and notice that this quotient converges to zero. Had it converged to any other value than zero, one would need to extend the ansatz used, beyond the one-parameter transseries, and this will be thoroughly discussed below.
} 
Poles of $\mathrm{BP}_{60}\left[\Phi_{0}\right]$

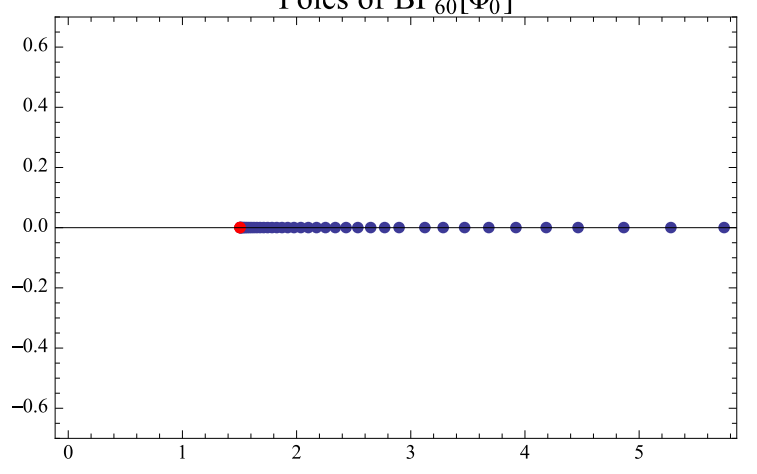

Poles of $\mathrm{BP}_{60}\left[\Phi_{1}\right]$

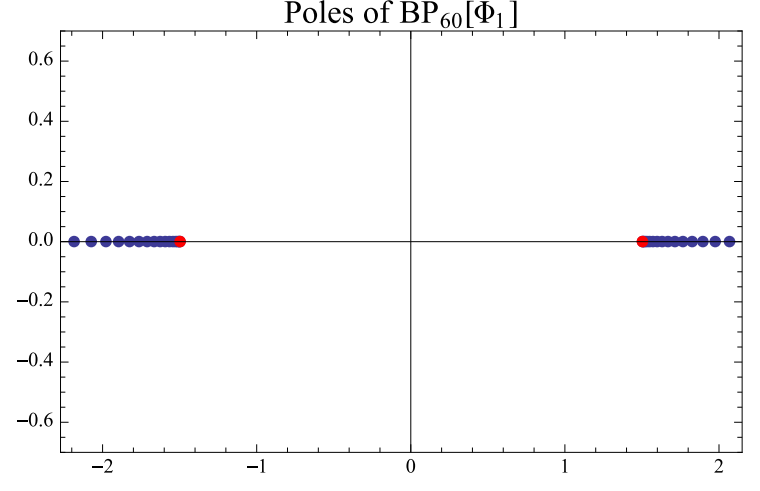

Figure 7. Poles of the (diagonal) order-60 Padé approximants for the Borel transforms of perturbative $\left(\mathrm{BP}_{60}\left[\Phi_{0}\right]\right)$ and one-instanton $\left(\mathrm{BP}_{60}\left[\Phi_{1}\right]\right)$ sectors in the free energy of the quartic potential. We have truncated the series $\Phi_{0}$ and $\Phi_{1}$ at order $N=120$, and used the built-in Mathematica command PadeApproximant at order 60 (typically the order of the approximant is half of the cut-off order, $N$, for a diagonal Padé approximant). One can easily see that the poles are all on the positive real line; for the perturbative expansion $\mathcal{B}\left[\Phi_{0}\right]$ condensing upon a branch-cut starting at $A=\frac{3}{2}$ (shown in red); and for the one-instanton series $\mathcal{B}\left[\Phi_{1}\right]$ condensing upon two separate branch-cuts, starting at either $\pm A$ (also in red).

One very important feature of resurgence is its universality: it is a rather general property of arbitrary complex functions. It is thus quite reasonable to expect that the nature of the Borel singularities associated to each multi-instanton sector of our one-parameter transseries (2.58) ought to be of similar nature to what was found in the linear problem, (2.25) and (2.26), and we shall take this as granted in the following. The novelty of the nonlinear problem will amount to an infinite number of (multi) instanton sectors, implying that the Borel transforms may generically have many more singularities than it was the case in the linear problem. Indeed, and to make a long-story short, it turns out that for the present one-parameter transseries, at a given fixed sector with instanton number $n$, the Borel transform will have singularities at $s=k A$, with $k \neq 0$ and $k \geq-n$ (the transseries does not include any sectors $\Phi_{n}$ with $n<0$ ). Up to holomorphic content, the expansions near these singularities are of the form ${ }^{23}$ [109]

$$
\left.\mathcal{B}\left[\Phi_{n}\right](s)\right|_{s=k A}=\mathrm{S}_{n \rightarrow n+k} \times \mathcal{B}\left[\Phi_{n+k}\right](s-k A) \frac{\log (s-k A)}{2 \pi \mathrm{i}}, \quad k \neq 0 .
$$

Near the singularity $s=k A$ of $\mathcal{B}\left[\Phi_{n}\right](s)$ one finds the resurgence of the $\Phi_{n+k}$ sector, through its corresponding Borel transform $\mathcal{B}\left[\Phi_{n+k}\right](s)$. These singularities are logarithmic ${ }^{24}$ branch-cuts (for simple resurgent functions), while the Borel transform of the "reappearing" sector is being evaluated around the origin - thus being locally regular. One also finds a proportionality constant $\mathrm{S}_{n \rightarrow n+k}$, the "Borel residue", numerically characterizing each distinct logarithmic singularity.

Being a very general statement about complex functions, and as we have already discussed in the linear case, resurgence is best expressed at an algebraic level of abstraction. In order to

\footnotetext{
${ }^{23}$ One could also easily include a simple pole in this expression; the discussion that follows would remain unchanged. But, as seen before, the role of the simple poles is solely to carry through with the residual coefficients and, as such, they are a bit secondary to the main analysis. We leave it to the reader to include them as fit.

${ }^{24}$ This is not necessarily always the case. However, as we shall later discuss in section 4, generically there is an adequate "representative" of the asymptotic series where indeed the branch cuts are logarithmic, as in the equation above (this is known as the simple representative). The main interest of using the representative with logarithms is that the properties of resurgence are most easily read using the logarithms branch points, as opposed to other types of singularities (but, again, this will be discussed in detail in section 4).
} 


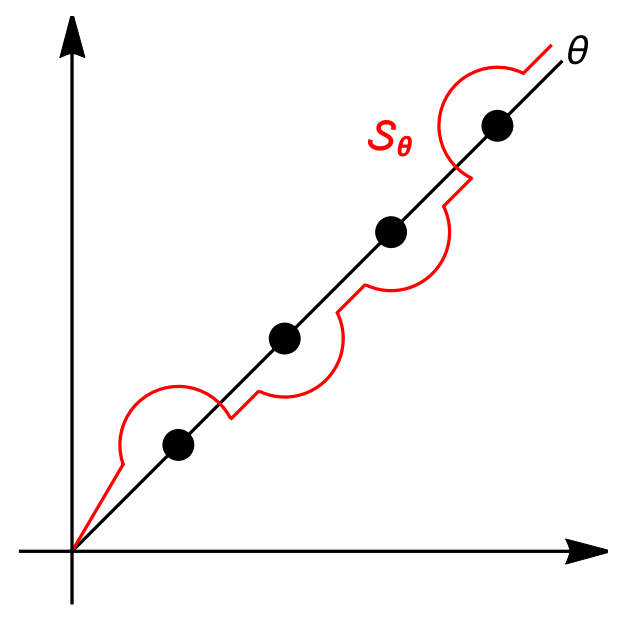

Figure 8. One of the many convoluted paths that we could choose for resummation, when in the presence of multiple instanton singularities. The alien derivative is a weighted-average over all these possible paths.

uncover algebraic properties behind the singularity structure encoded in (2.62), let us thus move beyond the functional nature of the Borel transforms. As discussed earlier, this is possible via the use of a linear differential operator, the alien derivative $\Delta_{\omega}$, which directly express any relations in-between different sectors in the transseries. However, unfortunately, the simple definition of alien derivative that we used in the linear case, (2.29), no longer works in the present nonlinear scenario. The reason why is simple. Indeed, while in the linear case there were only two possible distinct resummations to consider in (2.29), in the current nonlinear situation we will have many more due to the proliferation of Borel singularities. Of course that the original paths for lateral Borel resummations in (2.29) are still present, as in, e.g., figure 5; but now many others (and some quite convoluted) may be considered - see figure 8 for one such example. In the nonlinear case, the definition of alien derivative (2.29) must thus be extended to accommodate all these new possibilities: its definition [109] turns out to be an adequate, weighted-average over all these possible paths - but it is also too technical for the introductory and pedagogical goals of these lectures (nonetheless, see appendix A for further details and references). Note, however, how this caveat is also a blessing: one reason why the alien derivative is such a powerful concept is because it includes, inside it, all possible paths of resummation.

The one remarkable result in alien calculus is that, in spite of its intricate definition, the final result for the alien derivative is surprisingly simple. To some extent, $\Delta_{\omega}$ acts on the transseries building blocks, the asymptotic series $\Phi_{n}$, simply according to what is encoded in (2.62): it is only non-zero if the corresponding Borel transform has a singularity at $\omega$, in which case its action is schematically given by

$$
\Delta_{k A} \Phi_{n} \propto \Phi_{n+k} .
$$

For any other value of $\omega \in \mathbb{C}, \Delta_{\omega} \Phi_{n}=0$ (e.g., as aforementioned the transseries does not include any sectors $\Phi_{n}$ with $n<0$, in which case if $n+k<0$ then $\left.\Delta_{k A} \Phi_{n}=0\right)$. At this stage, we are still lacking the proportionality coefficients, i.e., the Stokes constants. In the linear case, (2.30) and (2.31), they matched the Borel residues up to an overall sign (see (2.27) and (2.28)). But this can no longer hold true in the nonlinear setting. In fact, due to the intertwining paths of resummation, Borel residues and Stokes constants, albeit obviously related, will not be equal. 
For the moment, we shall conveniently parameterize the Stokes constants $S_{k}$ as $^{25}$ :

$$
\Delta_{k A} \Phi_{n}=S_{k}(n+k) \Phi_{n+k}, \quad k \leq 1 \text { and } k \neq 0 .
$$

These resurgence relations follow directly from the so-called bridge equations of [109], and that is one way to derive them (in fact, contrary to what we have done here, they are usually the starting point in the discussions of resurgence). These bridge equations, which we describe at greater length in appendix A, are also behind the reason why ${ }^{26}$ alien-derivative singularities truncate to $k \leq 1$ in (2.64) (while Borel singularities did not in (2.62)).

The resulting structure in (2.64) is clearly the same as in the Borel singularities (2.62), but the proportionality constants $\mathrm{S}_{n \rightarrow n+k}$ and $S_{k}$ must still be related to each other. Hopefully, an intuition for the relations expressed in the following formulae will be developed by the reader as we discuss "alien chains" and Stokes automorphisms in the next couple of pages. Let us display some of these relations. When moving forward in the "chain" of singularities, one finds for example

$$
\begin{aligned}
& \mathrm{S}_{n \rightarrow n+1}=-(n+1) S_{1}, \\
& \mathrm{~S}_{n \rightarrow n+2}=-\frac{1}{2}(n+1)(n+2) S_{1}^{2}, \\
& \mathrm{~S}_{n \rightarrow n+3}=-\frac{1}{6}(n+1)(n+2)(n+3) S_{1}^{3},
\end{aligned}
$$

to which corresponds the closed-form expression ${ }^{27}$ :

$$
\mathrm{S}_{n \rightarrow n+k}=-\frac{1}{k !} \frac{(n+k) !}{n !} S_{1}^{k} .
$$

These formulae are also simply invertible, allowing to write the "more abstract" Stokes constant $S_{1}$ as a function of the "more numerical" Borel residue $S_{n \rightarrow n+1}$. When, instead, moving backwards along the "chain" of singularities, one finds for example

$$
\begin{aligned}
& \mathrm{S}_{n \rightarrow n-1}=-(n-1) S_{-1}, \\
& \mathrm{~S}_{n \rightarrow n-2}=-(n-2)\left(S_{-2}+\frac{1}{2}(n-1) S_{-1}^{2}\right), \\
& \mathrm{S}_{n \rightarrow n-3}=-(n-3)\left(S_{-3}+\frac{1}{2}(2 n-3) S_{-1} S_{-2}+\frac{1}{6}(n-2)(n-1) S_{-1}^{3}\right),
\end{aligned}
$$

to which corresponds the closed-form expression ${ }^{28}$

$$
\mathrm{S}_{n \rightarrow n-k}=-\sum_{s=1}^{k} \frac{1}{s !} \sum_{\substack{\left\{\ell_{1}, \ldots, \ell_{s} \geq 1\right\} \\ \sum \ell_{i}=k}} \prod_{a=1}^{s}\left(n-\sum_{j=1}^{a} \ell_{j}\right) \prod_{b=1}^{s} S_{-\ell_{b}}, \quad 1 \leq k<n .
$$

\footnotetext{
${ }^{25}$ At this stage, the reader may want to compare back to (2.30) and (2.31) in the linear case.

${ }^{26}$ The reason being intimately related to the one-parameter ansatz for the transseries (2.58).

${ }^{27}$ While this expression could be rewritten using a binomial coefficient, the present form is more natural in light of the upcoming vectorial generalization for multi-parameter transseries (to be discussed in section 5).

${ }^{28}$ This expression could be rewritten using sums over partitions (many closed-form formulae of this type may be found in $[16,15])$. However, in light of the upcoming vectorial generalization for multi-parameter transseries (to be discussed in section 5), it is more natural not to embark in such discussion at this stage.
} 
Although more intricate, also in this case inverse formulae are available; allowing to write the "more abstract" Stokes constants as functions of the "more numerical" Borel residues. For example, inverting equations (2.69) through (2.71) yields

$$
\begin{aligned}
S_{-1}= & -\frac{1}{n-1} \mathrm{~S}_{n \rightarrow n-1}, \\
S_{-2}= & -\frac{1}{n-2}\left(\mathrm{~S}_{n \rightarrow n-2}+\frac{1}{2} \mathrm{~S}_{n \rightarrow n-1} \mathrm{~S}_{n-1 \rightarrow n-2}\right), \\
S_{-3}= & -\frac{1}{n-3}\left(\mathrm{~S}_{n \rightarrow n-3}+\frac{1}{2} \mathrm{~S}_{n \rightarrow n-1} \mathrm{~S}_{n-1 \rightarrow n-3}+\frac{1}{2} \mathrm{~S}_{n \rightarrow n-2} \mathrm{~S}_{n-2 \rightarrow n-3}+\right. \\
& \left.+\frac{1}{3} \mathrm{~S}_{n \rightarrow n-1} \mathrm{~S}_{n-1 \rightarrow n-2} \mathrm{~S}_{n-2 \rightarrow n-3}\right),
\end{aligned}
$$

to which corresponds the closed-form expression:

$$
S_{-k}=-\frac{1}{n-k} \sum_{s=1}^{k} \frac{1}{s} \sum_{\substack{\left\{\ell_{1}, \ldots, \ell_{s} \geq 1\right\} \\ \sum \ell_{i}=k}} \mathrm{~S}_{n \rightarrow n-\ell_{1}} \mathrm{~S}_{n-\ell_{1} \rightarrow n-\ell_{1}-\ell_{2}} \cdots \mathrm{S}_{n-k+\ell_{s} \rightarrow n-k}, \quad 1 \leq k<n
$$

Note how the Borel residues $\mathrm{S}_{n \rightarrow n+k}$ depend on "departure" and "arrival" sectors, but how the Stokes constants $S_{k}$ only depend on the specific singularity being probed on the complex Borel plane. Furthermore, because $\Delta_{-n A} \Phi_{n}=0$, one can show that

$$
\mathrm{S}_{n \rightarrow 0}=0 .
$$

Finally, the truncation $k \leq 1$ in (2.64), or, equivalently, $\Delta_{k A} \Phi_{n}=0$ for $k \geq 2$, which does not occur in (2.62), implies that the Borel residues must satisfy the following consistency condition:

$$
\mathrm{S}_{n \rightarrow n+k}=\frac{(-1)^{k-1}}{k !} \prod_{\ell=0}^{k-1} \mathrm{~S}_{n+\ell \rightarrow n+\ell+1} .
$$

In the following we shall mostly work with the Stokes constants (attached to the specific $s=k A$ singularity, regardless of "departure" $\Phi_{n}$ and "arrival" $\Phi_{n+k}$ sectors), but sometimes the Borel residues turn out to be more useful, simplifying some formulae.

Let us briefly summarize what we have uncovered so far. For the nonlinear one-parameter transseries (2.58), the Borel transform of each of its asymptotic series $\Phi_{n}$ has logarithmic branchcut singularities at $s=k A$, which are essentially given by the corresponding Borel residue $\mathrm{S}_{n \rightarrow n+k}$, multiplied by the Borel transform of $\Phi_{n+k}$. This is completely analogous to what happened for the linear transseries (2.22), the only difference being that we have now moved from a finite to an infinite number of building blocks or nodes in the transseries. This will have a dramatic consequence: while the resurgence relations in the linear problem, (2.30) and (2.31), led to a simple algebraic structure which closed upon itself, (2.32), the resurgence relations for the nonlinear problem, (2.64), will instead lead to a much more intricate algebraic structure. In fact, one can now keep on connecting different transseries nodes by acting several times with the alien derivative, just like in a one-dimensional chain as shown in figure 9.

It is at this point that we depart from the standard descriptions or introductions to resurgent analysis. In fact, either having arrived at the resurgence relations (2.64) or starting out from 


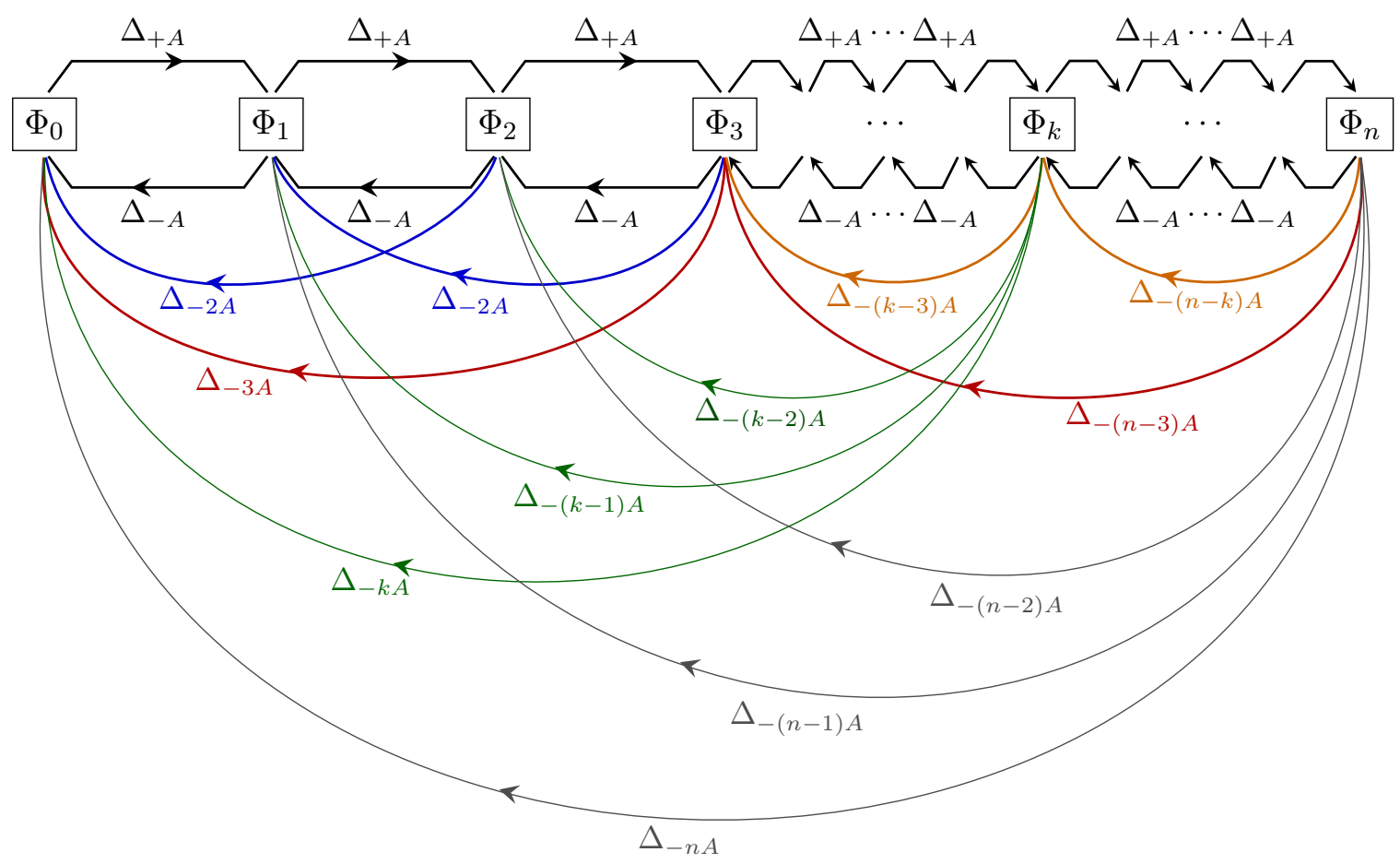

Figure 9. The alien chain: a pictorial representation of the (nonlinear) transseries (2.58), where each node in the chain corresponds to each of the transseries building blocks (each of the asymptotic series); and of the resurgence relations (2.64), herein represented as possible motions in-between the nodes of this infinite one-dimensional chain. The arrows in the figure represent allowed actions of the alien derivative on the (multi) instanton sectors $\Phi_{k}$, with different colors encoding different possible actions. Forward motions along the chain (i.e., motions from left to right) can only be achieved by the action of $\Delta_{+A}$, in which case to connect two different instanton sectors $\Phi_{m}$ and $\Phi_{\ell}$ with $\ell>m$ we need to act with this alien derivative $\ell-m$ times. But due to (2.64), backward motions have much more freedom. To move from right to left, one finds several more ways to connect $\Phi_{\ell}$ to $\Phi_{m}$ : in fact any left-moving paths are allowed with any combination of instanton sectors $\Phi_{k}$ with $m<k<\ell$ as mid-nodes. For example, to connect $\Phi_{3}$ to $\Phi_{0}$ we have four possible paths: act with $\Delta_{-A}$ three times (the black links $\Phi_{3} \rightarrow \Phi_{2} \rightarrow \Phi_{1} \rightarrow \Phi_{0}$ ); act with $\Delta_{-A}$ (the black link $\Phi_{3} \rightarrow \Phi_{2}$ ) followed by $\Delta_{-2 A}$ (the blue link $\Phi_{2} \rightarrow \Phi_{0}$ ); act with $\Delta_{-2 A}$ (the blue link $\Phi_{3} \rightarrow \Phi_{1}$ ) followed by $\Delta_{-A}$ (the black link $\Phi_{1} \rightarrow \Phi_{0}$ ); or act with $\Delta_{-3 A}$ (the red link $\Phi_{3} \rightarrow \Phi_{0}$ ).

them, most texts on this subject proceed into rather abstract algebraic discussions of their implications, fully developing alien calculus in this process. Herein, we wish to use a more physical language and pathway towards resurgence, and as such will use the one-dimensional alien chain of figure 9, with its set of nodes and allowed motions in-between these nodes, in order to proceed with a more physical, "statistical mechanical" approach to resurgence. Besides being a new point-of-view on resurgence, we also believe it is more intuitive and pedagogical.

The first obvious step is to reinterpret the (nonlinear) one-parameter transseries (2.58) as an (infinite) one-dimensional chain, with the asymptotic series which make up the transseries understood as the nodes of this chain. The resurgence relations (2.64) are then reinterpreted as defining an allowed set of resurgence motions in-between nodes of the chain. In this language, the content of figure 9 is obvious. The resurgence-relations constraint $k \leq 1$ (and $k \neq 0$ ) implies that the alien derivatives at each node $\Phi_{n}$ only have one singularity on the positive real axis, at 


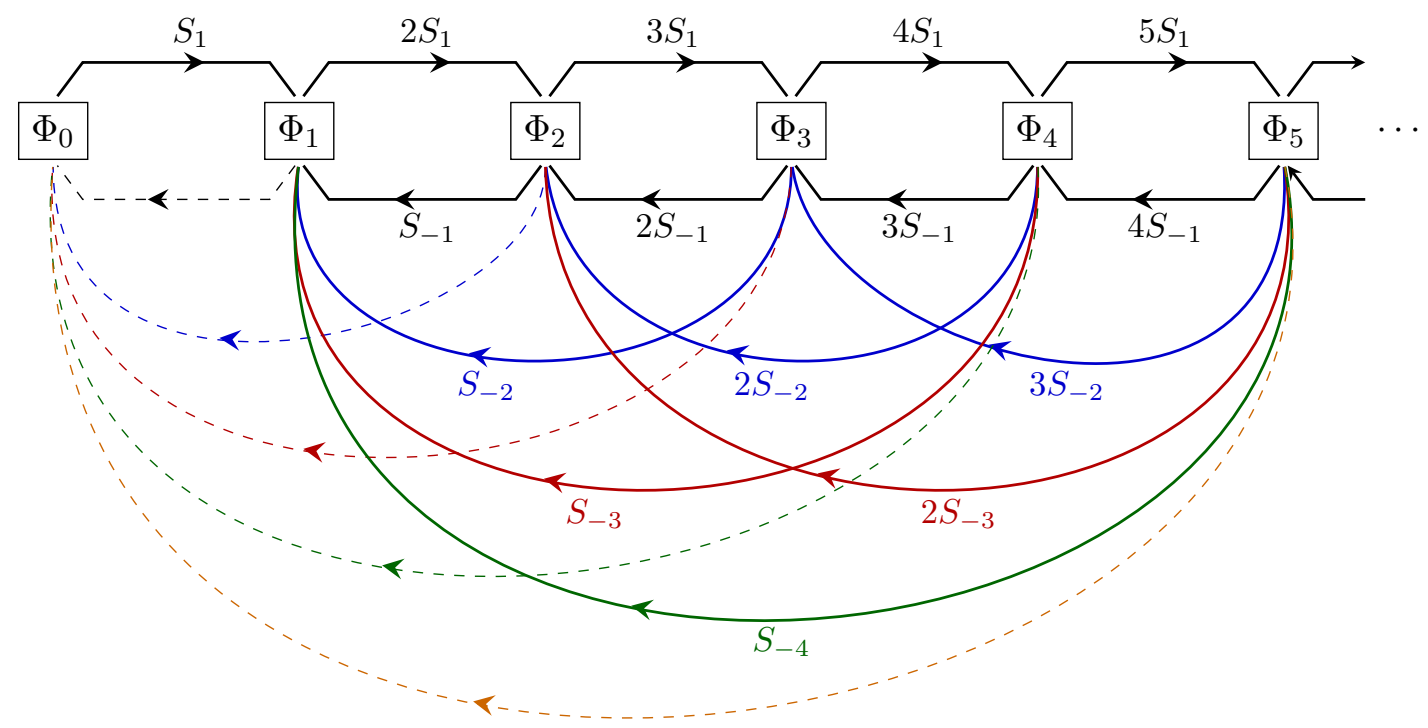

Figure 10. The alien chain revisited: pictorial representation of the action of the alien derivative (the right-hand-side of the resurgence relations (2.64)) upon the perturbative and first five instanton sectors of the alien chain. Different single arrows correspond to different steps, where each step has an associated weight as dictated by (2.64). The black links corresponds to the action of $\Delta_{+A}$ if the arrow is pointing forward (to the right); and to the action of $\Delta_{-A}$ if the arrow is pointing backward (to the left). Then the blue links correspond to the action of $\Delta_{-2 A}$, the red links to the action of $\Delta_{-3 A}$, the green ones to $\Delta_{-4 A}$, and finally the orange link refers to $\Delta_{-5 A}$. All colored links are backward motions. The dashed links refer to cases where the action of the alien derivative precisely yields zero, and thus invalidates such would-be motion. As one moves backward along the chain one finds different paths connecting the same chosen two nodes, each composed of different steps. The weight of each step is written next to the corresponding arrow. Further note that the action of two different alien derivatives does not commute (the mid-steps will be different), and this is seen explicitly as one takes weights into account. For example, if one starts off at $\Phi_{4}$ and acts with $\Delta_{-2 A}$ followed by $\Delta_{-A}$ (the blue link followed by the black one, with mid-step on $\left.\Phi_{2}\right)$, one obtains a total path weight of $2 S_{-2} S_{-1} \Phi_{1}$. But if instead one first acts with $\Delta_{-A}$ followed by $\Delta_{-2 A}$ (the black link followed by the blue one, with mid-step on $\Phi_{3}$ ), one rather obtains $3 S_{-1} S_{-2} \Phi_{1}$.

$s=+A$, allowing for a single type of forward motions on the chain (iterations still allowed). On the other hand, upon the negative real axis one finds logarithmic branch-cuts starting at every point $s=-|k| A$, with $k \geq 1$ (but truncating at $k=n$ as one cannot go further left in the chain than $\Phi_{0}$ ), allowing for many different types of backward motions on the chain. For example, to arrive at a node $\Phi_{\ell}$, starting at another node $\Phi_{m}$, the resurgence relations tell us that one needs to act with alien derivatives $\Delta_{\ell_{i} A}, \ell_{i} \neq 0$, consecutively as in $\Delta_{\ell_{N} A} \cdots \Delta_{\ell_{2} A} \Delta_{\ell_{1} A}$, and such that $\sum_{i=1}^{N} \ell_{i}=(\ell-m)$. Now if we are moving forward along the chain, i.e., $\ell>m$, there is only the $\Delta_{+A}$ action, and one must thus use this operator $\ell-m$ times. But if on the other hand we are moving backwards along the chain, $\ell<m$, there are now several possible paths to arrive at the desired node. Such paths can be composed of many steps, and go through any number of intermediary nodes. Each step is defined by a single link, one arrow connecting two nodes. In other words, in this situation one may use all possible combinations $\Delta_{-\ell_{i} A}, \ell_{i}>0$, with the same starting and ending nodes. One example of a choice of paths is given in the caption of figure 9 .

Above we were mostly interested in understanding resurgence motions on the alien chain 
as dictated by the left-hand-side of (2.64). We will now further include the information coming from its right-hand-side, in particular make the Stokes data explicit in all these motions ${ }^{29}$. This simply leads to the rewriting of the alien chain as shown in figure 10. With this in mind, we may finally introduce a set of "statistical mechanical" definitions, quantitatively describing motions on the alien chain and allowing for a more physical understanding of many upcoming resurgence formulae:

- Step $\mathcal{S}$ : any single link connecting two nodes on the chain

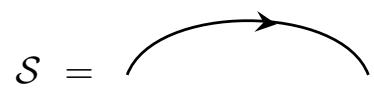

- Weight of a step $\mathcal{S}, w(\mathcal{S})$ : the product of Stokes coefficient and numerical factor associated with the step, which can be read directly from (2.64). In figure 10 the weights of the steps are written right next to the colored arrows. For a step connecting nodes $\Phi_{m}$ and $\Phi_{k}$

$$
w(\mathcal{S}(m \rightarrow k))=k S_{k-m}
$$

- Path $\mathcal{P}$ : a trajectory composed by any number of steps, connecting two nodes $\Phi_{m}$ to $\Phi_{k}$

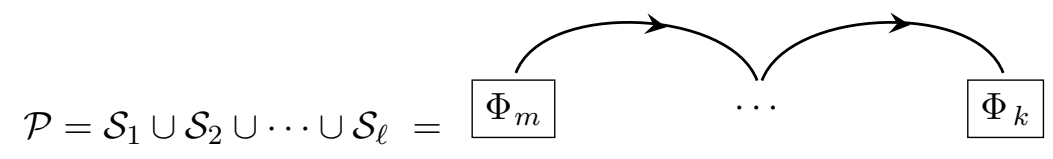

- Length of a path $\mathcal{P}, \ell(\mathcal{P})$ : the number of steps composing the path (where any step is always considered to have length one)

$$
\ell(\mathcal{P})=\#\left\{\mathcal{S}_{i} \in \mathcal{P}\right\}
$$

- Weight of a path $\mathcal{P}, w(\mathcal{P})$ : the product of the weights of every step composing the path

$$
w(\mathcal{P})=\prod_{i=1}^{\ell(\mathcal{P})} w\left(\mathcal{S}_{i}\right) .
$$

- Combinatorial factor of path $\mathcal{P}, \mathrm{CF}(\mathcal{P})$ : the division by the permutations of $\left\{\mathcal{S}_{i} \in \mathcal{P}\right\}$

$$
\mathrm{CF}(\mathcal{P})=\frac{1}{(\ell(\mathcal{P})) !}
$$

\footnotetext{
${ }^{29}$ This is another reason why to work with Stokes constants more often than with Borel residues. In some sense, the Stokes constants $S_{k}$ live on the complex Borel plane, but they are attached to the singular points regardless of which (instanton) sector we are considering. Borel residues $S_{n \rightarrow n+k}$, on the other hand, live on the specific complex Borel plane describing the singular nature of each particular (instanton) sector. This is already made clear by their labeling. But now, there are also subsequent differences in the language of motions on the alien chain. Stokes constants are "translational invariant" on the chain, in the sense that they only depend on the "distance vector" $k$ in-between nodes. But Borel residues are not: they need clear specification of both departure and arrival nodes.
} 
The last two points to notice concerning figure 10 are the following. First, we have plotted some dashed backward steps. While these are in principle allowed, they show no singularity, i.e., have zero weight. For example, the node $\Phi_{5}$ does not have a singularity at $s=-5 A$, as the alien derivative vanishes $\Delta_{-5 A} \Phi_{5}=0$. More generally, the Borel transform of $\Phi_{n}$ does not have a singularity at $s=-n A$, i.e., $\Delta_{-n A} \Phi_{n}=0$. Second, also noteworthy is the non-commutativity of alien derivatives which translates to a non-commutativity of different paths. Take for example $m, k>0$ and $m+k<n$ to compute:

$$
\begin{aligned}
& \Delta_{-m A} \Delta_{-k A} \Phi_{n}=S_{-k}(n-k) \Delta_{-m A} \Phi_{n-k}=S_{-k} S_{-m}(n-k)(n-k-m) \Phi_{n-k-m} \\
& \Delta_{-k A} \Delta_{-m A} \Phi_{n}=S_{-m}(n-m) \Delta_{-k A} \Phi_{n-m}=S_{-m} S_{-k}(n-m)(n-m-k) \Phi_{n-m-k}
\end{aligned}
$$

The start and end nodes are the same, but the multiplicative factors are different as long as $k \neq m$. This happens because the mid-step is different $\left(\Phi_{n-k}\right.$ for the first case and $\Phi_{n-m}$ for the second). In particular,

$$
\begin{aligned}
{\left[\Delta_{-m A}, \Delta_{-k A}\right] \Phi_{n} } & =S_{-m} S_{-k}(m-k)(n-m-k) \Phi_{n-m-k}= \\
& =\frac{S_{-m} S_{-k}}{S_{-m-k}}(m-k) \Delta_{-(m+k) A} \Phi_{n} .
\end{aligned}
$$

This means that two given paths made of smaller steps will not commute, as long as there is a single-step path between the departure and arrival nodes.

Recall that the discussion of the partition function in subsection 2.2 was much simpler. In fact, the alien chain in that linear problem only had two nodes, (2.32), and the corresponding algebraic structure was elementary. It was nonetheless required in order to study Stokes discontinuities and large-order asymptotics later on. The same is true in the present nonlinear case. Having discussed the alien chain for the transseries describing nonlinear problems, let us next see how to calculate the Stokes automorphism (2.4), or, more specifically, the related Stokes discontinuities (2.45) (illustrated in figure 5) for each of the asymptotic expansions (chain nodes) $\Phi_{n}$. This is a required step, in the present one-parameter transseries set-up, in order to finally fully understand the asymptotics of all multi-instanton sectors in the quartic free energy.

The Stokes discontinuities along some direction $\theta$ on the Borel plane, defined in (2.45) and depicted in figure 5, arise from the Stokes automorphism, which itself relates to the alien derivative via exponentiation (2.33). The motions on the alien chain we have just discussed dictate that each node of the chain, $\Phi_{n}$, only has two singular directions (i.e., Stokes lines; directions with Borel singularities), forward and backward or $\theta=0$ and $\pi$. Now, along the positive real line $\theta=0$ the alien derivative of any node $\Phi_{n}$ only has one single singularity, $s=A$; while along the negative real line $\theta=\pi$ there are many singularities at $s=-k A$ for $0<k<n$ (recall (2.64)). Via (2.33) applied to these directions, one finds

$$
\begin{aligned}
& \underline{\mathfrak{S}}_{0} \Phi_{n}=\exp \left(\mathrm{e}^{-\frac{A}{x}} \Delta_{A}\right) \Phi_{n}, \\
& \underline{\mathfrak{S}}_{\pi} \Phi_{n}=\exp \left(\sum_{k=1}^{n} \mathrm{e}^{\frac{k A}{x}} \Delta_{-k A}\right) \Phi_{n} .
\end{aligned}
$$

The discontinuities for each node then immediately follow as $\operatorname{Disc}_{\theta} \Phi_{n}=\Phi_{n}-\underline{\mathfrak{S}}_{\theta} \Phi_{n}$.

In order to understand the Stokes discontinuities in terms of motions and data on the alien chain, let us first discuss the above two formulae, (2.88) and (2.89), in a couple of examples. Stokes discontinuities will have several terms, each with two components: a "functional" component, 
comprising of the non-analytic exponential factor $\mathrm{e}^{k A / x}$ alongside the nodes (which are themselves asymptotic series as in (2.59)); and a "statistical" component, arising from a subset of paths on the alien chain in figure 10, and comprising their weights and combinatorial factors.

- What is the Stokes automorphism on the positive real line, when acting upon the node $\Phi_{2}$ ? One simply expands the exponential (2.88) to obtain ${ }^{30}$ :

$$
\begin{aligned}
\underline{\mathfrak{S}}_{0} \Phi_{2} & =\left(1+\mathrm{e}^{-\frac{A}{x}} \Delta_{A}+\frac{1}{2 !}\left(\mathrm{e}^{-2 \frac{A}{x}} \Delta_{A} \Delta_{A}\right)+\frac{1}{3 !}\left(\mathrm{e}^{-3 \frac{A}{x}} \Delta_{A} \Delta_{A} \Delta_{A}\right)+\cdots\right) \Phi_{2}= \\
& =\Phi_{2}+3 S_{1} \mathrm{e}^{-\frac{A}{x}} \Phi_{3}+\frac{12}{2 !}\left(S_{1}\right)^{2} \mathrm{e}^{-2 \frac{A}{x}} \Phi_{4}+\frac{60}{3 !}\left(S_{1}\right)^{3} \mathrm{e}^{-3 \frac{A}{x}} \Phi_{5}+\cdots \\
& =\Phi_{2}-\mathrm{S}_{2 \rightarrow 3} \mathrm{e}^{-\frac{A}{x}} \Phi_{3}-\mathrm{S}_{2 \rightarrow 4} \mathrm{e}^{-2 \frac{A}{x}} \Phi_{4}-\mathrm{S}_{2 \rightarrow 5} \mathrm{e}^{-3 \frac{A}{x}} \Phi_{5}-\cdots \\
& =\Phi_{2}-\mathrm{S}_{2 \rightarrow 3} \mathrm{e}^{-\frac{A}{x}} \Phi_{3}+\frac{1}{2 !} \mathrm{S}_{2 \rightarrow 3} \mathrm{~S}_{3 \rightarrow 4} \mathrm{e}^{-2 \frac{A}{x}} \Phi_{4}-\frac{1}{3 !} \mathrm{S}_{2 \rightarrow 3} \mathrm{~S}_{3 \rightarrow 4} \mathrm{~S}_{4 \rightarrow 5} \mathrm{e}^{-3 \frac{A}{x}} \Phi_{5}+\cdots
\end{aligned}
$$

The result may be written with either Stokes constants or, equivalently, Borel residues, via $^{31}(2.68)$ and (2.78). Let us compare this action of $\underline{\mathfrak{S}}_{0}$ on $\Phi_{2}$ with the allowed forward resurgence motions out of the node $\Phi_{2}$, on the alien chain of figure 10 . The coefficients of the several terms in the expansion above, (2.90), have a simple "physical" origin:

$-\Phi_{2} \rightarrow \Phi_{2}$ : this simply leaves the node invariant;

- $\Phi_{2} \rightarrow \Phi_{3}$ : there is a single path, of length $\ell=1$ and weight $w=3 S_{1}$, leading to a combinatorial factor $\mathrm{CF}=\frac{1}{1 !}$ and nonperturbative contribution $\mathrm{e}^{-\frac{A}{x}}$;

$-\Phi_{2} \rightarrow \Phi_{4}$ : there is a single path (made of two steps), with length $\ell=2$ and weight $w=12\left(S_{1}\right)^{2}$, leading to $\mathrm{CF}=\frac{1}{2 !}$ and nonperturbative contribution $\mathrm{e}^{-2 \frac{A}{x}}$;

$-\Phi_{2} \rightarrow \Phi_{5}$ : again there is a single path (with three steps), of length $\ell=3$ and weight $w=60\left(S_{1}\right)^{3}$, leading to $\mathrm{CF}=\frac{1}{3 !}$ and nonperturbative contribution $\mathrm{e}^{-3 \frac{A}{x}}$.

- What is the Stokes automorphism on the negative real line, when acting upon the node $\Phi_{4}$ ? Now we expand the exponential (2.89) instead:

$$
\begin{aligned}
\underline{\mathfrak{S}}_{\pi} \Phi_{4} & =\left(1+\sum_{k=1}^{4} \mathrm{e}^{\frac{k A}{x}} \Delta_{-k A}+\frac{1}{2 !}\left(\sum_{k=1}^{4} \mathrm{e}^{\frac{k A}{x}} \Delta_{-k A}\right)^{2}+\frac{1}{3 !}\left(\sum_{k=1}^{4} \mathrm{e}^{\frac{k A}{x}} \Delta_{-k A}\right)^{3}+\cdots\right) \Phi_{4}= \\
& =\Phi_{4}+3 S_{-1} \mathrm{e}^{\frac{A}{x}} \Phi_{3}+\left(2 S_{-2}+\frac{6}{2 !} S_{-1}^{2}\right) \mathrm{e}^{\frac{2 A}{x}} \Phi_{2}+\left(S_{-3}+\frac{5}{2 !} S_{-1} S_{-2}+\frac{6}{3 !} S_{-1}^{3}\right) \mathrm{e}^{\frac{3 A}{x}} \Phi_{1} \\
& =\Phi_{4}-\mathrm{S}_{4 \rightarrow 3} \mathrm{e}^{\frac{A}{x}} \Phi_{3}-\mathrm{S}_{4 \rightarrow 2} \mathrm{e}^{\frac{2 A}{x}} \Phi_{2}-\mathrm{S}_{4 \rightarrow 1} \mathrm{e}^{\frac{3 A}{x}} \Phi_{1} .
\end{aligned}
$$

The result may be written with either Stokes constants or, equivalently, Borel residues, $\operatorname{via}^{32}$ (2.69), (2.70) and (2.71) (or, more generally, via (2.72)). This action of $\underline{\mathfrak{S}}_{\pi}$ on $\Phi_{4}$ should now be compared with the allowed backward resurgence motions out of the node

\footnotetext{
${ }^{30}$ Note that the alien derivative does not act on the (non-analytic) exponential; but only upon asymptotic series.

${ }^{31}$ Or else, if we did not know equations (2.68) and (2.78), one could also take each exponential term in (2.90) as the defining equations for the Borel residues as functions of the Stokes constants.

${ }^{32}$ Again, if we did not know equation (2.72), one could also take each exponential term in (2.91) as the defining equations for the Borel residues as functions of the Stokes constants.
} 
$\Phi_{4}$, on the alien chain of figure 10. As already mentioned, we now have many more paths to consider. But, once again, the coefficients of the several terms in the expansion (2.91) have a simple "physical" origin:

- $\Phi_{4} \rightarrow \Phi_{4}$ : this simply leaves the node invariant;

- $\Phi_{4} \rightarrow \Phi_{3}$ : there is a single path, of length $\ell=1$ and weight $w=3 S_{-1}$, leading to a combinatorial factor $\mathrm{CF}=\frac{1}{1 !}$ and nonperturbative contribution $\mathrm{e}^{+\frac{A}{x}}$;

$-\Phi_{4} \rightarrow \Phi_{2}$ : there are now two paths. One consists of a single step (the blue link in figure 10), with length $\ell=1$, weight $w=2 S_{-2}$ and $\mathrm{CF}=\frac{1}{1 !}$; the other consists of two steps (the black links in figure 10), with length $\ell=2$, weight $w=6\left(S_{-1}\right)^{2}$ and $\mathrm{CF}=\frac{1}{2 !}$. They both lead to a nonperturbative contribution $\mathrm{e}^{+2 \frac{A}{x}}$;

$-\Phi_{4} \rightarrow \Phi_{1}$ : there are now four paths. One consists of a single step (the red link in figure 10), with length $\ell=1$, weight $w=S_{-3}$ and $\mathrm{CF}=\frac{1}{1 !}$; the second consists of two steps (first the blue link, followed by the black one), with length $\ell=2$, weight $w=2 S_{-1} S_{-2}$ and $\mathrm{CF}=\frac{1}{2 !}$; the third similarly consisting of two steps (now first a black link, followed by a blue one), with length $\ell=2, w=3 S_{-1} S_{-2}$ and $\mathrm{CF}=\frac{1}{2 !}$; and the fourth consists of three steps (the black links in figure 10), with length $\ell=3$, weight $w=6\left(S_{-1}\right)^{3}$ and $\mathrm{CF}=\frac{1}{3 !}$. They all have nonperturbative contribution $\mathrm{e}^{+3 \frac{A}{x}}$;

- $\Phi_{4} \rightarrow \Phi_{0}$ : all paths arriving at node $\Phi_{0}$ have zero weight. Further, one cannot move backwards from this node, in which case the expansion necessarily truncates here.

The pattern should be rather clear. In fact, one may now define the Stokes discontinuities, Disc $\theta$, purely in terms of motions and data on the alien chain ${ }^{33}$ :

Stokes discontinuity on positive real line: $\operatorname{Disc}_{0} \Phi_{n}$ is given by a sum over all forward paths, linking nodes to the right $\Phi_{m>n}$.

Stokes discontinuity on negative real line: $\operatorname{Disc}_{\pi} \Phi_{n}$ is given by a sum over all backward paths, linking nodes to the left $\Phi_{m<n}$.

In both cases each term in this sum $\left(\Phi_{n} \rightarrow \Phi_{m}\right)$ can be decomposed into two factors:

- Functional component, dictated solely by beginning and end nodes:

$$
-\mathrm{e}^{-(m-n) \frac{A}{x}} \Phi_{m} .
$$

- Statistical component, sum over all the allowed paths $\mathcal{P}(n \rightarrow m)$ linking the nodes as in figure 10:

$$
\mathrm{SF}_{(n \rightarrow m)} \equiv \sum_{\mathcal{P}(n \rightarrow m)} \mathrm{CF}(\mathcal{P}) w(\mathcal{P}) .
$$

For our present purposes, this will be our definition of Stokes discontinuities (and automorphism). It is a simple graphical and combinatorial procedure to understand how the structure

\footnotetext{
${ }^{33}$ One may also think of the statistical component below, $\mathrm{SF}_{(n \rightarrow m)}$, as a "statistical-mechanical" definition of the Borel residues (up to a sign).
} 
of Borel singularities in (2.62) translates to discontinuities of the asymptotic expansions within our transseries. One can also write general closed-form formulae, for all these quantities, based on sums over partitions, but we refer the interested reader to section 2 of [16] for those.

Let us now get back into our starting problem in this subsection. Recall that in trying to compute the quartic free energy (assuming no knowledge of the partition function), we were faced with a nonlinear differential equation (2.52) whose perturbative series diverged factorially (2.54). Trying to solve this nonlinear differential equation with a transseries ansatz led to an infinite number of multi-instanton sectors, as described in appendix D, where the multi-loop expansions of all these nonperturbative sectors also diverged factorially, (2.60). These growths translate to intricate singularity structures on the Borel plane, which may be studied numerically as in figure 7, and such analyses motivate general resurgence relations at the level of Borel transforms as in (2.62). The Stokes discontinuities which are created by this singular Borel structure then may be described as above, as motions and data upon the alien chain representing the transseries, and depicted in figure 10. Many of the results described above are in fact valid for the resurgent structure of general nonlinear problems solved by one-parameter transseries. But as we focus back upon the quartic free energy, and proceeding in analogy to what was earlier done for the (linear) partition function in subsection 2.3, we would now want to use the above Stokes discontinuities in order to write down large-order resurgent relations which would allow us to check (or predict) the multi-instanton content of the free energy. However, there is still one subtlety, particular to the quartic free energy and having its origin in (2.57), that we have to consider.

Take a closer look at the first two sectors in the transseries; the $\Phi_{0}$ and $\Phi_{1}$ nodes in the chain. As shown in figure 7, the Borel transform of the perturbative sector has a branch-cut starting at $s=A$. Figure 10 then shows how this is correctly reproduced by our transseries ansatz: there are no singularities along the negative real axis because $\Phi_{0}$ is the first node in the chain, and there is indeed one singularity at $s=A$ because $\Delta_{A} \Phi_{0} \propto \Phi_{1}$. But figure 7 also illustrates how the one-instanton sector has two branch-cuts, one similarly starting at $s=A$, but another at $s=-A$ along the negative real axis. As is made very clear in figure 10, this cannot happen within the one-parameter transseries set-up: in this case there should be a single singularity at $s=A$ and none at $s=-A$, because $\Delta_{-A} \Phi_{1}=0$ (i.e., one has $\left.\mathrm{S}_{1 \rightarrow 0}=0\right)$ ! We are then forced to conclude that the one-parameter transseries ansatz is not enough to solve our problem, and more transseries parameters may be required. This is in fact a generic feature as many systems have diverse instanton (or renormalon) actions; even by simply considering higher-order differential equations one will need to parameterize a larger space of boundary conditions with more transseries parameters.

As we already alluded to, the origin of this problem may be found in (2.57). Indeed when we plugged the multi-instanton ansatz (2.56) into the nonlinear differential equation for the quartic free energy, we obtained two possible values for the instanton action: $A=0$ and $A=\frac{3}{2}$. The one-parameter ansatz we then chose was implicitly assuming that the solution $A=0$ was just part of the perturbative solution. What we are now realizing is that this is not the case, the action $A=0$ must be considered as part of a truly independent sector, beyond the perturbative $\ell=0$ sector of the $A=\frac{3}{2}$ solution. In this case, the two-parameter transseries that fully describes the quartic free energy should be

$$
F\left(x, \sigma_{1}, \sigma_{2}\right)=\sum_{n=0}^{+\infty} \sum_{m=0}^{+\infty} \sigma_{1}^{n} \sigma_{2}^{m} \mathrm{e}^{-\frac{n A}{x}} \Phi_{(n, m)}(x) .
$$

Now do note that this is actually a simplified version of what a general two-parameter transseries would look like, as it might allow for two (independent) non-zero actions and thus a generic 
exponential contribution of the form $\sim \mathrm{e}^{-\left(n A_{1}+m A_{2}\right) \frac{1}{x}}$ (we shall discuss such systems later on). As usual, the sectors $\Phi_{(n, m)}$ are themselves asymptotic series,

$$
\Phi_{(n, m)}(x) \simeq \sum_{k=0}^{+\infty} F_{k}^{(n, m)} x^{k},
$$

with their coefficients growing factorially. Plugging this two-parameter transseries ansatz as a solution to the free-energy nonlinear differential equation, we obtain new recursive relations for the multi-loop multi-instanton coefficients $F_{k}^{(n, m)}$. Fortunately, given that one of the instanton actions is actually zero, we will be able to reuse much of what was previously done for the oneparameter case. Indeed, looking back at (2.92), it turns out that one can "hide" the second parameter into the definition of new "hatted" coefficients as

$$
F\left(x, \sigma_{1}, \sigma_{2}\right)=\sum_{n=0}^{+\infty} \sigma_{1}^{n} \mathrm{e}^{-\frac{n A}{x}} \widehat{\Phi}_{n}(x),
$$

where

$$
\widehat{\Phi}_{n}(x) \simeq \sum_{k=0}^{+\infty} \widehat{F}_{k}^{(n)} x^{k}, \quad \widehat{F}_{k}^{(n)}:=\sum_{m=0}^{+\infty} \sigma_{2}^{m} F_{k}^{(n, m)} .
$$

Above, we have rewritten our new ansatz such as to look like the earlier one-parameter transseries, where the newly defined multi-loop multi-instanton coefficients now obey the same recursive relations as those found in appendix $\mathrm{D}$. To reiterate, this is only possible within our example as one of the instanton actions identically vanishes; we shall loosely refer to this as having a "one-and-a-half" transseries. Of course that in a general two-parameter transseries this would not be the case.

But having rewritten the two-parameter ansatz as a one-parameter transseries, one is led to ask why did out first attempt at using a one-parameter transseries did not work? The rough answer is that even though we were able to reorganize our ansatz as to look like a one-parameter transseries, the "hidden" second parameter will still be seen by the resurgence relations.

Let us see how this comes about, for our "1.5"-parameter transseries. As it will turn out, the full transseries (2.92) is not even needed; restricting to the sectors $\Phi_{(n, 0)}, n \geq 0$, and $\Phi_{(0,1)}$ is enough to (finally!) fully describe the quartic free energy . Moreover, this last sector is not even an asymptotic series; it is just a constant:

$$
\Phi_{(0,1)}(x)=F_{0}^{(0,1)} .
$$

The main reason why one can restrict our two-parameter transseries into the form of a oneparameter transseries with an added single sector is the same reason why we were able to "hide" the second parameter in (2.95): one can check that the sectors associated to $\Phi_{(n, m)}$ with $m$ non-zero will not be asymptotic; with all asymptotic behaviour being restricted to the $\Phi_{(n, 0)}$. Still, our solution to the ODE does tell us that one ${ }^{34}$ extra sector needs to be included (albeit non-asymptotic), associated to the action $A=0$-and (2.96) is it. Notice that these asymptotic statements have a rather natural explanation ${ }^{35}$ by looking at the quartic free-energy second-order

\footnotetext{
${ }^{34}$ In principle one could include several sectors $\Phi_{(0, m)}$, but they would all be constant and indistinguishable, which in the end does amount to a single contribution.

${ }^{35} \mathrm{By}$ this we mean an explanation going beyond the fact that resurgence dictates them (our emphasis in these lectures), i.e., that our ansatz is indeed strongly validated by the associated large-order relations arising from resurgence - which we shall get to discuss in detail a bit later on, in subsection 2.6.
} 
ODE (2.52). Indeed, this equation has no dependence on $F(x)$ itself, but only on derivatives of $F(x)$. It can then be trivially integrated into a first-order ODE by the redefinition $\mathfrak{F}(x) \equiv F^{\prime}(x)$, where the new sector which we have just uncovered can then be identified with the integration constant associated to this redefinition. Another natural explanation will soon be obtained when we directly compare linear and nonlinear problems (i.e., the quartic partition function and free energy) in the next subsection. To make all these statements a bit more clear, let us finally rewrite the transseries solution as

$$
F\left(x, \sigma_{1}, \sigma_{2}\right)=\sum_{n=0}^{+\infty} \sigma_{1}^{n} \mathrm{e}^{-\frac{n A}{x}} \Phi_{(n, 0)}(x)+\sigma_{2} F_{0}^{(0,1)} .
$$

The local structure of Borel singularities may likewise be obtained having resurgence in mind. Although the sector $\Phi_{(0,1)}$ is not asymptotic, all the other sectors in (2.97) are, and their Borel transforms will have associated singularities and branch-cuts. One expects only mild corrections to (2.62) and performing similar calculations as in, e.g., [16], it is simple to obtain

$$
\mathcal{B}\left[\Phi_{(n, 0)}\right](s+k A)=\left(\mathrm{S}_{n \rightarrow n+k} \times \mathcal{B}\left[\Phi_{(n+k, 0)}\right](s)+\delta_{n+k} \widetilde{\mathrm{S}}_{n \rightarrow n+k} \times \mathcal{B}\left[\Phi_{(0,1)}\right](s)\right) \frac{\log s}{2 \pi \mathrm{i}}, \quad k \neq 0,
$$

where now $\widetilde{\mathrm{S}}_{n \rightarrow 0} \neq 0$. Of course as $\Phi_{(0,1)}(x)$ is just a constant (and not an asymptotic series), its Borel transform will not have any singularities ${ }^{36}$. The resurgence relations which follow from this resurgent singularity-structure are simply

$$
\Delta_{k A} \Phi_{(n, 0)}=S_{k}(n+k) \Phi_{(n+k, 0)}+\delta_{n+k} \widetilde{S}_{-n} \Phi_{(0,1)}, \quad k \leq 1 \text { and } k \neq 0,
$$

all other combinations vanishing 37 (in particular, $\Delta_{k A} \Phi_{(0,1)}=0$ ). The pictorial representation of these algebraic equations, as motions on the alien chain associated to the new "one-and-a-half" transseries, are depicted in figure 11. Do notice that this time around the chain is not strictly one-dimensional, having an extra "orthogonal" node which we placed just below the perturbative node $\Phi_{(0,0)}$. On what concerns forward motions, the chain still starts at the original perturbative series $\Phi_{(0,0)}$, but on what concerns backward motions, now the "last" node becomes $\Phi_{(0,1)}$.

Having a clear picture of this "1.5-dimensional" alien chain, describing the transseries solution to the quartic free energy, we can proceed and compute the Stokes discontinuities along the singular directions $\theta=0, \pi$ just like before (the rules are the same, either for calculating functional or statistical factors for each term; only the set of allowed motions is different). For example when evaluating $\operatorname{Disc}_{\pi} \Phi_{(n, 0)}$, which in the present case should be done with the motions depicted in figure 11, the last node on backward motions to the left is now $\Phi_{(0,1)}$. Further, this node $\Phi_{(0,1)}$ does not correspond to an asymptotic series, in which case

$$
\operatorname{Disc}_{0, \pi} \Phi_{(0,1)}=0 \text {. }
$$

Let us next write a few examples of discontinuities one may compute (we choose them as they will be useful in what follows). These should not be too hard to obtain, and we leave it as an exercise for the reader to prove them.

\footnotetext{
${ }^{36}$ To be precise, Borel transforms are not defined for constant functions (residual coefficients). Nevertheless one may define them as an identity on the Borel plane and perform calculations in this way; see [93] for some examples.

${ }^{37}$ Recall that we are taking $\Phi_{(n, m)}=0$ if either $n$ or $m$ are negative.
} 


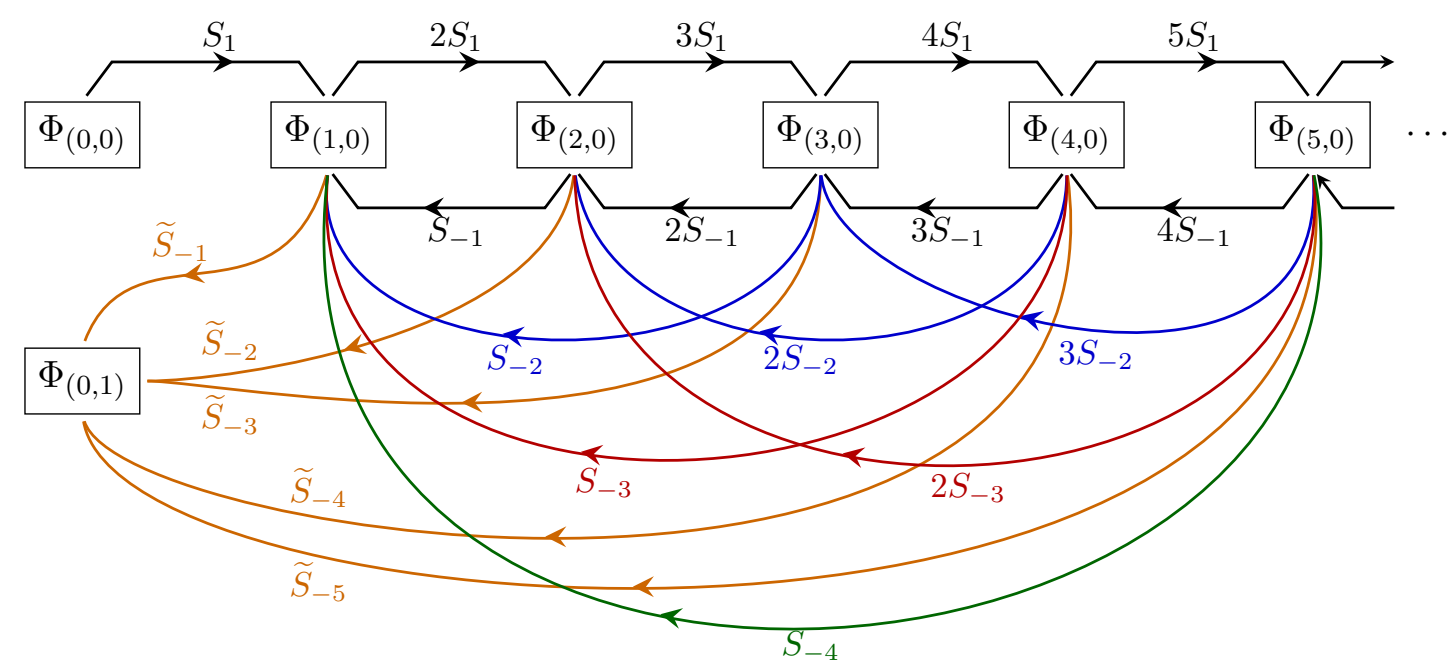

Figure 11. The alien chain for the "1.5"-parameter transseries: a pictorial representation of the action of the alien derivative upon the perturbative and first five instanton sectors, $\Phi_{(k, 0)}$, with $k=0, \ldots, 5$; alongside the extra "orthogonal" sector $\Phi_{(0,1)}$. As before, different single arrows correspond to different steps, and each step has an associated weight as dictated by the new resurgence relations (2.99). For simplicity, we have only drawn steps which have non-zero weight. One obtains Stokes discontinuities for this problem applying the same rules as before, now having in mind the set of motions illustrated above.

- The Stokes discontinuity along the positive real line is unchanged in this new chain, as there are no new forward motions. It is actually straightforward to find a general expression for all sectors, as

$$
\begin{aligned}
\operatorname{Disc}_{0} \Phi_{(n, 0)}(x) & =-\sum_{k=1}^{+\infty}\left(\begin{array}{c}
n+k \\
n
\end{array}\right) S_{1}^{k} \mathrm{e}^{-\frac{k A}{x}} \Phi_{(n+k, 0)}(x) \\
& =\sum_{k=1}^{+\infty} \mathrm{S}_{n \rightarrow n+k} \mathrm{e}^{-\frac{k A}{x}} \Phi_{(n+k, 0)}(x) .
\end{aligned}
$$

- The Stokes discontinuities along the negative real line will now have contributions from paths ending on the new node $\Phi_{(0,1)}=F_{0}^{(0,1)}$. A few examples are:

$$
\begin{aligned}
\operatorname{Disc}_{\pi} \Phi_{(1,0)}(x)= & -\widetilde{S}_{-1} \mathrm{e}^{\frac{A}{x}} \Phi_{(0,1)}=\widetilde{\mathrm{S}}_{1 \rightarrow 0} \mathrm{e}^{\frac{A}{x}} \Phi_{(0,1)}, \\
\operatorname{Disc}_{\pi} \Phi_{(2,0)}(x)= & -S_{-1} \mathrm{e}^{\frac{A}{x}} \Phi_{(1,0)}(x)-\left(\widetilde{S}_{-2}+\frac{1}{2 !} S_{-1} \widetilde{S}_{-1}\right) \mathrm{e}^{\frac{2 A}{x}} \Phi_{(0,1)} \\
= & \mathrm{S}_{2 \rightarrow 1} \mathrm{e}^{\frac{A}{x}} \Phi_{(1,0)}(x)+\widetilde{\mathrm{S}}_{2 \rightarrow 0} \mathrm{e}^{\frac{2 A}{x}} \Phi_{(0,1)}, \\
\operatorname{Disc}_{\pi} \Phi_{(3,0)}(x)= & -2 S_{-1} \mathrm{e}^{\frac{A}{x}} \Phi_{(2,0)}(x)-\left(\frac{2}{2 !} S_{-1}^{2}+S_{-2}\right) \mathrm{e}^{\frac{2 A}{x}} \Phi_{(1,0)}(x)- \\
& -\left(\frac{2}{3 !} S_{-1}^{2} \widetilde{S}_{-1}+\frac{1}{2 !} S_{-2} \widetilde{S}_{-1}+\frac{2}{2 !} S_{-1} \widetilde{S}_{-2}+\widetilde{S}_{-3}\right) \mathrm{e}^{\frac{3 A}{x}} \Phi_{(0,1)} \\
= & \mathrm{S}_{3 \rightarrow 2} \mathrm{e}^{\frac{A}{x}} \Phi_{(2,0)}(x)+\mathrm{S}_{3 \rightarrow 1} \mathrm{e}^{\frac{2 A}{x}} \Phi_{(1,0)}(x)+\widetilde{\mathrm{S}}_{3 \rightarrow 0} \mathrm{e}^{\frac{3 A}{x}} \Phi_{(0,1)} .
\end{aligned}
$$


At this stage, let us make a few remarks. First, this may seem like an awful lot of work to finally construct a complete solution to the quartic free energy, when we had already computed its partition function and it simply would have followed that $F=\log Z$. Of course we took this route in choosing a very simple example to illustrate what resurgence is and how it works. There are of course much more complicated examples where all one has in hand is a nonlinear system, without any possibility to bypass the nonlinearities via an easier problem. For example, this is the case concerning Painlevé equations which connect to $2 \mathrm{~d}$ (super) gravity. In fact, a similar albeit more involved analysis was followed for the Painlevé I equation, describing $2 \mathrm{~d}$ gravity, in $[179,180,130,16]$ and for the Painlevé II equation, describing $2 \mathrm{~d}$ supergravity, in [175, 205].

Further, one may wonder if this resurgent transseries we have constructed is in fact the correct and complete solution to the (nonlinear) quartic free energy. One interesting feature of these resurgence constructions is that they may be checked via large-order analyses: one may use the recursive relations to compute multi-loop multi-instanton data in the transseries, $F_{k}^{(n, 0)}$, and then resurgence yields very intricate relations in-between all these coefficients and their asymptotics which may be tested numerically. However, there is one point to handle before this may be done. The construction above was rather general on Stokes constants; in fact it had nothing to say about what are their exact, numerical values. If one wants to be more specific and quantitative, one still needs to determine the Stokes constants for the quartic free energy, and this is what we shall turn to now.

\subsection{Stokes Constants of Partition Function versus Free Energy}

Now we wish to make contact between the results in the previous subsection, concerning the quartic free energy $F$, and our earlier (analytical) results on the quartic partition function $Z$. This will serve a dual purpose of both checking the general structure of the two-parameter transseries for the quartic free energy, alongside determining its Stokes constants from first principles (rather than numerically, as in many cases; e.g., $[130,16,205])$. For example, recalling the (exact) perturbative expansion of the partition function (2.18), it is immediate to compute (and check) the perturbative expansion of the free energy. Using $F=\log Z$ one simply obtains

$$
F^{(0)}=\log Z^{(0)} \simeq \frac{1}{2} \log \frac{\hbar}{2 \pi}+\frac{1}{8} x+\frac{1}{12} x^{2}+\frac{11}{96} x^{3}+\frac{17}{72} x^{4}+\frac{619}{960} x^{5}+\cdots,
$$

which is exactly the same expansion as the one produced by (2.53) (and further fixing the integration constant $F_{0}^{(0)}$ ). Similar checks may be done concerning the nonperturbative sectors. For example, crossing a Stokes line to turn-on $Z^{(1)}$, as in (2.36), will induce a subsequent change in (2.105) above as

$$
\mathcal{F} \equiv \log \mathcal{Z}=\log \left(Z^{(0)}(x)+2 Z^{(1)}(x)\right) .
$$

Expanding the ratio of (2.19) with (2.18), one obtains

$$
\mathcal{F} \simeq F^{(0)}-\sum_{n=1}^{+\infty}(\mathrm{i} \sqrt{2})^{n} \mathrm{e}^{-n \frac{3}{2 x}}\left(\frac{1}{n}-\frac{1}{4} x+\frac{n}{32} x^{2}+\cdots\right) .
$$

One immediately finds $A=\frac{3}{2}$ and $S_{1} F_{0}^{(1)}=\mathrm{i} \sqrt{2}$, as expected from earlier numerical results. In particular, this expression also fixes all coefficients in the one-parameter transseries (2.56).

Let us make these checks more precise and more systematic, by translating resurgent properties of the partition function into resurgence results for the free energy. In particular, let us 
compute the Stokes constants of the free energy, given the Stokes constants of the partition function. The comparison of the structure of the transseries for partition function and free energy starts as above, but which we now write a bit more formally. First, the partition function transseries (2.22) is slightly rewritten as

$$
\mathcal{Z}\left(x, \sigma_{0}, \sigma_{1}\right)=\sigma_{0} \Phi_{0}(x)+\sigma_{1} \mathrm{e}^{-\frac{3}{2 x}} \Phi_{1}(x)=\sigma_{0} \Phi_{0}(x)\left(1+\zeta \mathrm{e}^{-\frac{3}{2 x}} \frac{\Phi_{1}(x)}{\Phi_{0}(x)}\right),
$$

where we have introduced $\zeta \equiv \frac{\sigma_{1}}{\sigma_{0}}$. Next, we write the free energy as the logarithm of the partition function, understood as a formal expansion of the function $\log (1+z)$. It follows:

$$
\mathcal{F}\left(x, \sigma_{0}, \sigma_{1}\right)=\log \sigma_{0}+\log \Phi_{0}+\sum_{n=1}^{+\infty} \zeta^{n} \mathrm{e}^{-n \frac{A}{x}} F^{(n)}(x),
$$

where the nonperturbative sectors $F^{(n)}$ may be written in terms of the original sectors of the partition function as

$$
F^{(n)}:=\frac{(-1)^{n+1}}{n}\left(\frac{\Phi_{1}}{\Phi_{0}}\right)^{n}
$$

On the other hand, and as discussed at length in the previous subsection, we also expect that the free energy will be given by a two-parameter ${ }^{38}$ transseries, $^{-}$

$$
F\left(x, \tau_{1}, \tau_{2}\right)=\sum_{n=0}^{+\infty} \sum_{m=0}^{+\infty} \tau_{1}^{n} \tau_{2}^{m} \mathrm{e}^{-\frac{n A_{1}+m A_{2}}{x}} \Phi_{(n, m)}(x),
$$

where $A_{1}=A \equiv \frac{3}{2}$ and $A_{2}=0$, and the nonperturbative sectors $\Phi_{(n, m)}(x)$ are given by the asymptotic series (2.93). If we now compare this expression for the free energy with the previous one, i.e., compare the two-parameter transseries (2.111) with the transseries following from the partition function (2.109), one may obtain the following identifications ${ }^{39}$

$$
\begin{aligned}
\tau_{1} & =\frac{\sigma_{1}}{\sigma_{0}}, \quad \tau_{2}=\log \sigma_{0}, \\
\Phi_{(0,0)} & =\log \Phi_{0}, \\
\Phi_{(n, 0)} & =F^{(n)}, \\
\Phi_{(0,1)} & =1, \\
\Phi_{(n, m)} & =0, \quad \text { if } m>1 \vee(m=1 \wedge n>0) .
\end{aligned}
$$

In particular, this identification is now validating our "1.5"-parameter transseries ansatz in (2.97).

Let us next see if we can make similar identifications at the level of resurgence relations and, eventually, at the level of Stokes data. Recall that the resurgence relations for the partition function, with sectors $\Phi_{0}$ and $\Phi_{1}$, were very simple, (2.30) and (2.31) (implying Stokes constants ${ }^{40}$

\footnotetext{
${ }^{38}$ Here we have changed notation for the parameters of the transseries, as compared to (2.97), in order not to confuse transseries parameters of the partition function and of the free energy.

${ }^{39}$ Obviously assuming $\sigma_{0} \neq 0$.

${ }^{40}$ In this subsection we shall label Stokes constants with a superscript $Z$ or $F$, depending on whether they are associated to the quartic partition function or to the quartic free energy, respectively.
} 
$S_{1}^{Z}=-2$ and $S_{-1}^{Z}=1$, respectively). The Stokes automorphism (2.33) was then very simply written as (2.34), implying (2.36) and (2.37), which we reproduce in here $\operatorname{as}^{41}$

$$
\begin{aligned}
\underline{\mathfrak{S}}_{0} \mathcal{Z}\left(x, \sigma_{0}, \sigma_{1}\right) & :=\exp \left(\mathrm{e}^{-\frac{A}{x}} \Delta_{+A}\right) \mathcal{Z}\left(x, \sigma_{0}, \sigma_{1}\right)=\mathcal{Z}\left(x, \sigma_{0}, \sigma_{1}+S_{1}^{Z} \sigma_{0}\right)= \\
& \equiv \exp \left\{S_{1}^{Z} \sigma_{0} \frac{\partial}{\partial \sigma_{1}}\right\} \mathcal{Z}\left(x, \sigma_{0}, \sigma_{1}\right) \\
\underline{\mathfrak{S}}_{\pi} \mathcal{Z}\left(x, \sigma_{0}, \sigma_{1}\right) & :=\exp \left(\mathrm{e}^{+\frac{A}{x}} \Delta_{-A}\right) \mathcal{Z}\left(x, \sigma_{0}, \sigma_{1}\right)=\mathcal{Z}\left(x, \sigma_{0}+S_{-1}^{Z} \sigma_{1}, \sigma_{1}\right)= \\
& \equiv \exp \left\{S_{-1}^{Z} \sigma_{1} \frac{\partial}{\partial \sigma_{0}}\right\} \mathcal{Z}\left(x, \sigma_{0}, \sigma_{1}\right) .
\end{aligned}
$$

As already briefly discussed earlier, one way to summarize the action of the Stokes automorphisms is to say that transseries parameters jump when crossing Stokes lines (see, e.g., [15] for further details). In this way, crossing the Stokes line at $\theta=0$ entails $\sigma_{0} \rightarrow \sigma_{0}$ and $\sigma_{1} \rightarrow \sigma_{1}+S_{1}^{Z} \sigma_{0}$; while crossing the Stokes line at $\theta=\pi$ entails $\sigma_{0} \rightarrow \sigma_{0}+S_{-1}^{Z} \sigma_{1}$ and $\sigma_{1} \rightarrow \sigma_{1}$. We may now use these results to directly determine the Stokes discontinuities associated to the free-energy transseries (2.111). Its associated transseries parameters need to satisfy (2.112), which immediately implies their "jumps". As such, crossing the Stokes line at $\theta=0$ will now entail $\tau_{1} \rightarrow \tau_{1}+S_{1}^{Z}$ and $\tau_{2} \rightarrow \tau_{2}$; while crossing the Stokes line at $\theta=\pi$ will entail $\tau_{1} \rightarrow \frac{\tau_{1}}{1+S_{-1}^{Z} \tau_{1}}$ and $\tau_{2} \rightarrow \tau_{2}+\log \left(1+S_{-1}^{Z} \tau_{1}\right)$ (again, see [15] for first-principle derivations of formulae like these). It is then straightforward to write the analogues of (2.117) and (2.118) for the free energy as

$$
\begin{aligned}
& \underline{\mathfrak{S}}_{0} F\left(x, \tau_{1}, \tau_{2}\right)=F\left(x, \tau_{1}+S_{1}^{Z}, \tau_{2}\right), \\
& \underline{\mathfrak{S}}_{\pi} F\left(x, \tau_{1}, \tau_{2}\right)=F\left(x, \frac{\tau_{1}}{1+S_{-1}^{Z} \tau_{1}}, \tau_{2}+\log \left(1+S_{-1}^{Z} \tau_{1}\right)\right) .
\end{aligned}
$$

The two equations above, (2.119) and (2.120), should be equally obtainable by direct action of the Stokes automorphism (2.33) upon the free-energy transseries (2.111), computed via the resurgence relations (2.99) (i.e., via explicit use of alien derivatives). This calculation is rather straightforward. Along $\theta=0$ the only non-vanishing alien derivative is $\Delta_{+A}$, in which case ${ }^{42}$

$$
\begin{aligned}
\mathrm{e}^{-\frac{A}{x}} \Delta_{+A} F & =\sum_{n=0}^{+\infty} \tau_{1}^{n} \mathrm{e}^{-(n+1) \frac{A}{x}} \Delta_{+A} \Phi_{(n, 0)}=S_{1}^{F} \sum_{n=0}^{+\infty} \tau_{1}^{n}(n+1) \mathrm{e}^{-(n+1) \frac{A}{x}} \Phi_{(n+1,0)}= \\
& =S_{1}^{F} \frac{\partial}{\partial \tau_{1}} F
\end{aligned}
$$

immediately leading to the action of the Stokes automorphism (2.88) as

$$
\underline{\mathfrak{S}}_{0} F\left(x, \tau_{1}, \tau_{2}\right)=\exp \left\{S_{1}^{F} \frac{\partial}{\partial \tau_{1}}\right\} F\left(x, \tau_{1}, \tau_{2}\right) .
$$

A completely analogous calculation holds along the direction $\theta=\pi$. One finds (with $k>0$ )

$$
\mathrm{e}^{k \frac{A}{x}} \Delta_{-k A} F=S_{-k}^{F} \sum_{n=k}^{+\infty} \tau_{1}^{n}(n-k) \mathrm{e}^{-(n-k) \frac{A}{x}} \Phi_{(n-k, 0)}+\widetilde{S}_{-k}^{F} \tau_{1}^{k} \Phi_{(0,1)}=
$$

\footnotetext{
${ }^{41}$ Notice in this simple example how alien derivatives, which act upon transseries nodes, may be translated to regular derivatives, which are now acting upon transseries parameters. This is the essence of the bridge equations: establishing a "bridge" between alien and ordinary calculus; see appendix A for further details.

${ }^{42}$ Recall that $\Delta_{+A} \Phi_{(0,1)}=0$, as this sector is not even asymptotic (it is just a constant).
} 


$$
=\left(S_{-k}^{F} \tau_{1}^{k+1} \frac{\partial}{\partial \tau_{1}}+\widetilde{S}_{-k}^{F} \tau_{1}^{k} \frac{\partial}{\partial \tau_{2}}\right) F
$$

now leading to the action of the Stokes automorphism (2.89) as

$$
\underline{\mathfrak{S}}_{\pi} F\left(x, \tau_{1}, \tau_{2}\right)=\exp \left\{\sum_{k=1}^{+\infty}\left(S_{-k}^{F} \tau_{1}^{k+1} \frac{\partial}{\partial \tau_{1}}+\widetilde{S}_{-k}^{F} \tau_{1}^{k} \frac{\partial}{\partial \tau_{2}}\right)\right\} F\left(x, \tau_{1}, \tau_{2}\right) .
$$

This is all the needed information in order to compare Stokes constants associated to the partition function and to the free energy. In fact, (2.119) and (2.122) should yield the same result, as should (2.120) and (2.124). Making sure such identifications hold will automatically compute the Stokes constants. For instance, from (2.122) follows

$$
\underline{\mathfrak{S}}_{0} F\left(x, \tau_{1}, \tau_{2}\right)=F\left(x, \tau_{1}+S_{1}^{F}, \tau_{2}\right),
$$

which, when compared to (2.119), immediately yields the Stokes constant $S_{1}^{F}$

$$
S_{1}^{F}=S_{1}^{Z} \quad(=-2) .
$$

For the second comparison, that of (2.120) and (2.124), the action of the exponential in (2.124) is now more intricate and we begin by expanding it as $^{43}$ (using the explicit transseries in, e.g., (2.111))

$$
\begin{gathered}
\exp \left\{\sum_{k=1}^{+\infty}\left(S_{-k}^{F} \tau_{1}^{k+1} \frac{\partial}{\partial \tau_{1}}+\widetilde{S}_{-k}^{F} \tau_{1}^{k} \frac{\partial}{\partial \tau_{2}}\right)\right\} F\left(x, \tau_{1}, \tau_{2}\right)=\tau_{2}+\sum_{\ell=1}^{+\infty} \frac{1}{\ell !}\left(\sum_{k=1}^{+\infty} S_{-k}^{F} \tau_{1}^{k+1} \frac{\partial}{\partial \tau_{1}}\right)^{\ell-1} \times \\
\times \sum_{k^{\prime}=1}^{+\infty} \widetilde{S}_{-k^{\prime}}^{F} \tau_{1}^{k^{\prime}}+\sum_{n=0}^{+\infty} \sum_{\ell=0}^{+\infty} \frac{1}{\ell !}\left(\sum_{k=1}^{+\infty} S_{-k}^{F} \tau_{1}^{k+1} \frac{\partial}{\partial \tau_{1}}\right)^{\ell} \tau_{1}^{n} \mathrm{e}^{-\frac{n A}{x}} \Phi_{(n, 0)}(x) .
\end{gathered}
$$

Clearly, in order to compare back to the resulting (shifted) transseries in (2.120), we will also need to expand that expression. One simply obtains

$$
\begin{aligned}
F\left(x, \frac{\tau_{1}}{1+S_{-1}^{Z} \tau_{1}}, \tau_{2}+\right. & \left.\log \left(1+S_{-1}^{Z} \tau_{1}\right)\right)= \\
& =\tau_{2}+\log \left(1+S_{-1}^{Z} \tau_{1}\right)+\sum_{n=0}^{+\infty}\left(\frac{\tau_{1}}{1+S_{-1}^{Z} \tau_{1}}\right)^{n} \mathrm{e}^{-\frac{n A}{x}} F^{(n)}(x) .
\end{aligned}
$$

The above two expressions will match if, upon the standard identification (2.114), one further has

$$
\begin{aligned}
\log \left(1+S_{-1}^{Z} \tau_{1}\right) & =\sum_{k=1}^{+\infty} \widetilde{S}_{-k}^{F}\left(\sum_{\ell=1}^{+\infty} \frac{1}{\ell !}\left\{\sum_{k^{\prime}=1}^{+\infty} S_{-k^{\prime}}^{F} \tau_{1}^{k^{\prime}+1} \frac{\partial}{\partial \tau_{1}}\right\}^{\ell-1}\right) \tau_{1}^{k}, \\
\frac{\tau_{1}^{n}}{\left(1+S_{-1}^{Z} \tau_{1}\right)^{n}} & =\left(\sum_{\ell=0}^{+\infty} \frac{1}{\ell !}\left\{\sum_{k=1}^{+\infty} S_{-k}^{F} \tau_{1}^{k+1} \frac{\partial}{\partial \tau_{1}}\right\}^{\ell}\right) \tau_{1}^{n}, \quad \forall n \geq 1 .
\end{aligned}
$$

\footnotetext{
${ }^{43}$ Note that $\widetilde{S}_{-k}^{F} \tau_{1}^{k} \frac{\partial}{\partial \tau_{2}}$ only acts once upon the transseries, as its $\tau_{2}$-dependence is purely linear.
} 
While at first these may seem as intricate conditions, we shall see in the following that there is a very simple set of values for the Stokes constants $S_{-k}^{F}$ and $\widetilde{S}_{-k}^{F}, k \geq 1$, which solve both conditions. Let us exemplify how this works starting with (2.130) and computing the $S_{-k}^{F}$. For $n=1$, a power-series expansion of the left-hand side, together with the recursive action of the differential operator on the right-hand side, leads to

$$
\begin{aligned}
\sum_{\ell=0}^{+\infty}\left(-S_{-1}^{Z}\right)^{\ell} \tau_{1}^{\ell+1} & =\tau_{1}+\sum_{k_{1}=1}^{+\infty} S_{-k_{1}}^{F} \tau_{1}^{k_{1}+1}+\frac{1}{2 !} \sum_{k_{1}, k_{2}=1}^{+\infty}\left(k_{1}+1\right) S_{-k_{1}}^{F} S_{-k_{2}}^{F} \tau_{1}^{k_{1}+k_{2}+1}+ \\
& +\frac{1}{3 !} \sum_{k_{1}, k_{2}, k_{3}=1}^{+\infty}\left(k_{1}+1\right)\left(k_{1}+k_{2}+1\right) S_{-k_{1}}^{F} S_{-k_{2}}^{F} S_{-k_{3}}^{F} \tau_{1}^{\sum_{i=1}^{3} k_{i}+1}+\cdots
\end{aligned}
$$

Let us compare equal powers of $\tau_{1}^{\ell+1}$. For $\ell=0$ we get an identity; for the next few $\ell$ 's we find

$$
\begin{aligned}
& \ell=1: \quad-S_{-1}^{Z}=S_{-1}^{F}, \\
& \ell=2:\left(-S_{-1}^{Z}\right)^{2}=S_{-2}^{F}+\frac{1}{2 !}(1+1)\left(S_{-1}^{F}\right)^{2}, \\
& \ell=3:\left(-S_{-1}^{Z}\right)^{3}=S_{-3}^{F}+\frac{1}{2 !}(2+3) S_{-1}^{F} S_{-2}^{F}+\frac{1}{3 !}(2 \times 3)\left(S_{-1}^{F}\right)^{3} .
\end{aligned}
$$

The first equation above, (2.132), immediately yields $S_{-1}^{F}=-S_{-1}^{Z}$. Recursively solving the following equations one sees that (2.133) then yields $S_{-2}^{F}=0$ and (2.134) $S_{-3}^{F}=0$. This is a pattern which generalizes to higher powers of $\tau_{1}$. Indeed, the first time the Stokes constant $S_{-k}^{F}$ will enter the game is when we write down the equation associated to $\ell=k$. But, at this stage, such equation will also include a term just in $\left(S_{-1}^{F}\right)^{k}$, while all other terms will be products of Stokes constants which always include at least one ${ }^{44} S_{-k^{\prime}}^{F}$ with $1<k^{\prime}<k$. For a general $\ell$ this looks like

$$
\left(-S_{-1}^{Z}\right)^{\ell}=S_{-\ell}^{F}+\cdots+\frac{1}{\ell !} \ell !\left(S_{-1}^{F}\right)^{\ell},
$$

where the dots indicate terms involving Stokes constants $S_{-k}^{F}, 1<k<\ell$, which were already set to zero from previous conditions. By induction, it follows that $S_{-k}^{F}=0$, for all $k>1$. Finally, it is straightforward to show that this solution also solves the initial constraint (2.130) for any other value of $n \geq 1$. This is indeed the case as

$$
\sum_{\ell=0}^{+\infty} \frac{1}{\ell !}\left(S_{-1}^{F}\right)^{\ell}\left(\tau_{1}^{2} \frac{\partial}{\partial \tau_{1}}\right)^{\ell} \tau_{1}^{n}=\tau_{1}^{n} \sum_{\ell=0}^{+\infty} \frac{(n+\ell-1) !}{\ell !(n-1) !}\left(S_{-1}^{F} \tau_{1}\right)^{\ell}=\frac{\tau_{1}^{n}}{\left(1-S_{-1}^{F} \tau_{1}\right)^{n}}
$$

A completely analogous procedure can be used to solve the remaining constraint (2.129) (where one also has to use the above solution for the $S_{-k}^{F}$ ), which we leave as an exercise for the reader. At the end of the day, one finally obtains

$$
S_{-1}^{F}=-S_{-1}^{Z} \quad(=-1),
$$

${ }^{44}$ The $m$-term contribution to the sum in (2.130) is given by

$$
\frac{1}{m !} \prod_{i=1}^{m}\left(\sum_{k_{i}=1}^{+\infty}\left(\sum_{j=1}^{i-1} k_{j}+1\right) S_{-k_{i}}^{F}\right) \tau_{1}^{\sum_{j=1}^{m} k_{j}+1} .
$$




$$
\begin{array}{rlrl}
\widetilde{S}_{-1}^{F} & =S_{-1}^{Z} & ( & =1), \\
S_{-m}^{F} & =0, & m \geq 2, \\
\widetilde{S}_{-m}^{F} & =0, & m \geq 2 .
\end{array}
$$

These results tell us that a large number of Stokes constants for the free energy actually vanish. This will be numerically checked via large-order analysis in the next subsection. For the moment let us just note that this implies that some of the resurgence relations allowed for in (2.99) are in fact trivial, e.g.,

$$
\Delta_{n A} \Phi_{(m, 0)}(x) \equiv 0, \quad n \neq-1,1 .
$$

That this is the case could have been somewhat expected from the results concerning the linear problem, where the only non-zero alien operators already were $\Delta_{ \pm A}$. In fact, from the transseries comparisons of (2.109) and (2.111) we learnt that the $\Phi_{(n, 0)}(x)$ sectors are given by the combinations $F^{(n)}(x)$ in (2.110), and these are ratios of two asymptotic series. Now, Borel transforms for these types of contributions are known to be given by convolutions of the Borel transforms of the original asymptotic series: as the inverse Borel transform is a Laplace transform, it is simple to show that the Borel of the product is a convolution, i.e., that $\mathcal{B}\left[\Phi_{1} \times \Phi_{2}\right]=\mathcal{B}\left[\Phi_{1}\right] * \mathcal{B}\left[\Phi_{2}\right]$, with * the convolution of functions. This allows us to relate Borel singularities of both $\Phi_{1}$ and $\Phi_{2}$ with Borel singularities of their product. In fact (see, e.g, [204]), the convolution will inherit the original singularities of each of the asymptotic series in the principal branch of the Borel plane, together with combinations of singularities, but which will fall on different sheets of the Borel plane, thus not contributing directly to the Stokes automorphism ${ }^{45}$.

Another way to understand the discussion in the above paragraph is the following. As we have the solution for the non-vanishing Stokes constants in hand, we may now rewrite the Stokes automorphism (2.124) in a simpler form, as

$$
\begin{aligned}
\underline{\mathfrak{S}}_{\pi} F\left(x, \tau_{1}, \tau_{2}\right) & =\exp \left\{S_{-1}^{F} \tau_{1}^{2} \frac{\partial}{\partial \tau_{1}}+\widetilde{S}_{-1}^{F} \tau_{1} \frac{\partial}{\partial \tau_{2}}\right\} F\left(x, \tau_{1}, \tau_{2}\right)= \\
& =\exp \left\{-\tau_{1}^{2} \frac{\partial}{\partial \tau_{1}}+\tau_{1} \frac{\partial}{\partial \tau_{2}}\right\} F\left(x, \tau_{1}, \tau_{2}\right) .
\end{aligned}
$$

Alongside (2.122), this equation is telling us something very interesting. They show how the Stokes automorphisms for the free energies, (2.122) and (2.143), turn out to be the very same operators as those acting at the level of the partition function, (2.117) and (2.118). This is immediately made explicit once one uses the respective relation between transseries parameters (2.112), to find

$$
\begin{aligned}
\sigma_{0} \frac{\partial}{\partial \sigma_{1}} & =\frac{\partial}{\partial \tau_{1}}, \\
\sigma_{1} \frac{\partial}{\partial \sigma_{0}} & =-\tau_{1}^{2} \frac{\partial}{\partial \tau_{1}}+\tau_{1} \frac{\partial}{\partial \tau_{2}} .
\end{aligned}
$$

\footnotetext{
${ }^{45}$ If $\Phi_{1}$ and $\Phi_{2}$ are asymptotic series with singularities on the Borel plane at $s=\omega_{1}$ and $s=\omega_{2}$, respectively, then the product $\Phi_{1} \times \Phi_{2}$ will have singularities on the Borel plane at $s=\omega_{1}, \omega_{2}, \omega_{1}+\omega_{2}$. Nevertheless, only the original singularities $s=\omega_{1}, \omega_{2}$ will be on the principal branch in the Borel plane; the one at $s=\omega_{1}+\omega_{2}$ will fall on a different sheet, and thus the alien derivative acting on the product will not see this extra singularity.
} 
There is yet another way to see how all this is not a big surprise, but rather a straightforward consequence $^{46}$ of the fact that $\Delta_{\omega}$ is a derivation. If $\Delta_{\omega}$ is a derivation and at some specific point $\omega$ one has $\Delta_{\omega} Z=0$, then it must be the case that at this same point one finds $\Delta_{\omega} F=0$, as $F=\log Z$. But then all the possibilities encoded in (2.123) must immediately simplify, as $\Delta_{-k A} Z=0$ for $k \geq 2$. In fact, this condition sets $\Delta_{-k A} F=0$ for $k \geq 2$, which further yields $S_{-k}^{F}=0$ and $\widetilde{S}_{-k}^{F}=0$ for $k \geq 2$ (indeed, as was later found in (2.140) and (2.141)). In other words, the resurgence relations for the partition function, (2.30) and (2.31), alongside the fact that $\Delta_{\omega}$ is a derivation, are enough to tell us that the only non-zero result in (2.123) must be

$$
\mathrm{e}^{\frac{A}{x}} \Delta_{-A} F=\left(S_{-1}^{F} \tau_{1}^{2} \frac{\partial}{\partial \tau_{1}}+\widetilde{S}_{-1}^{F} \tau_{1} \frac{\partial}{\partial \tau_{2}}\right) F .
$$

But this is precisely what we just had in (2.143) above, as expected.

\subsection{Asymptotics and Large-Order Behaviour: Free Energy}

Having discussed all basic ideas and constructed a transseries solution for the free energy, displaying an infinite number of (multi) instanton sectors, we may finally turn to a rather interesting feature of resurgent transseries: the fact that these constructions may be very explicitly tested, by means of resurgent large-order numerical analysis. In particular the tests we shall present next will very precisely confirm the structure we have put forward in the previous subsections.

The numerical tests we wish to perform are tests on the asymptotics of the different mutliinstantonic sectors, as predicted by the resurgent structure of the transseries we have proposed, and given known coefficients associated to other sectors than the one we are testing (e.g., computed by the recursive formulae in appendix D). But before diving into numerics, one must pay attention to a couple of details concerning these coefficients. Comparison of the actual quartic free-energy transseries (2.97) with its "one-parameter" rewriting (2.94) and (2.95), tells that the multi-loop multi-instanton coefficients $F_{k}^{(n, 0)}$ will obey the same recursive equations as the $F_{k}^{(n)}$ coefficients. However, for this match to remain precise, one also has to keep in mind that for instance the coefficient $F_{0}^{(0)}$ should actually be thought of as $F_{0}^{(0,0)}+\sigma_{2} F_{0}^{(0,1)}$, and that none of these will be fixed by the recursion relations. Instead, they belong to the set of initial conditions one needs to account for, in order to specify a solution to our differential equation (2.52). It turns out things are slightly simpler as we may directly compare free energy and partition function. For instance, the comparisons (2.113) to (2.115) immediately yield a relation between initial data for the free energies and the coefficients $Z_{0}^{(0)}$ and $Z_{0}^{(1)}$ of the partition function. In particular,

$$
F_{0}^{(1,0)}=\frac{Z_{0}^{(1)}}{Z_{0}^{(0)}}=-\frac{\mathrm{i}}{\sqrt{2}},
$$

where we used the relation $Z_{0}^{(1)}=-\frac{\mathrm{i}}{\sqrt{2}} Z_{0}^{(0)}$. We shall use this value for the coefficient $F_{0}^{(1,0)}$ in all the large-order tests carried out in this subsection.

Now, in order to understand the nature of the aforementioned resurgent, large-order, numerical tests, let us go back to the Stokes discontinuities (2.45) depicted in figure 5. As discussed in subsection 2.4, the resurgent structure of the quartic transseries (2.97) tells us that the sectors which build-it-up will have either: no Stokes discontinuities (the case of the sector $\Phi_{(0,1)}$, as in $(2.100)$ ); or else one single Stokes discontinuity along $\theta=0$ (the case of $\Phi_{(0,0)}$ ); or else two

\footnotetext{
${ }^{46} \mathrm{In}$ all rigour, this is more of a consistency check that $\Delta_{\omega}$ is a derivation, as we have not addressed such proof.
} 
Stokes discontinuities, along both $\theta=0$ and $\pi$ (for every other sector, as in (2.101) and, e.g., (2.102) or (2.103) or (2.104)). Next, as already discussed in subsection 2.3, given a function $F(x)$ with discontinuities along some rays starting from the origin on the complex plane, say along directions $\theta=0$ and $\theta=\pi$, Cauchy's theorem translates to (assuming no contributions around infinity, also as already discussed in subsection 2.3)

$$
F(x)=-\frac{1}{2 \pi \mathrm{i}} \int_{0}^{+\infty} \mathrm{d} w \frac{\operatorname{Disc}_{0} F(w)}{w-x}-\frac{1}{2 \pi \mathrm{i}} \int_{0}^{-\infty} \mathrm{d} w \frac{\operatorname{Disc}_{\pi} F(w)}{w-x} .
$$

For the function $F$ above one selects any sector within the transseries, $\Phi_{(n, m)}$ (of course in the case of the perturbative sector there is only one discontinuity, and the second term disappears). As should be very clear by now, we only know these sectors expressed as asymptotic series. We do know their Stokes discontinuities, but also these are given as sums over weighted sectors (e.g., (2.101)-(2.104)), themselves only known as asymptotic series. So when comparing both sides of (2.149) we will be comparing asymptotic series, resulting in large-order relations.

Let us begin with the perturbative sector $F=\Phi_{(0,0)}$. Using the Stokes discontinuity (2.101) in the Cauchy formula (2.149), and making asymptotic series explicit,

$$
\sum_{k=0}^{+\infty} F_{k}^{(0,0)} x^{k} \simeq \sum_{n=1}^{+\infty} \frac{S_{1}^{n}}{2 \pi \mathrm{i}} \int_{0}^{+\infty} \mathrm{d} w \frac{\mathrm{e}^{-n \frac{A}{w}}}{w-x} \sum_{h=0}^{+\infty} F_{h}^{(n, 0)} w^{h} .
$$

Formally expanding the denominator on the right-hand-side in powers of $x$, and performing the resulting $w$-integrations, one may compare equal powers of $x$ on both sides to obtain (after a slight rearrangement) $)^{47}$

$$
\begin{aligned}
F_{k}^{(0,0)} \frac{2 \pi \mathrm{i} A^{k}}{\Gamma(k)} \simeq & S_{1}\left(F_{0}^{(1,0)}+\frac{A}{k-1} F_{1}^{(1,0)}+\frac{A^{2}}{(k-1)(k-2)} F_{2}^{(1,0)}+\cdots\right)+ \\
& +2^{-k} S_{1}^{2}\left(F_{0}^{(2,0)}+\frac{2 A}{k-1} F_{1}^{(2,0)}+\frac{(2 A)^{2}}{(k-1)(k-2)} F_{2}^{(2,0)}+\cdots\right)+\mathcal{O}\left(3^{-k}\right) .
\end{aligned}
$$

Holding at large $k$, this expression ${ }^{48}$ is a large-order relation. With the aforementioned slight rearrangement, we made explicit both the leading factorial growth and the subleading exponential growths. In fact a very distinctive feature in this type of expressions are the $\sim n^{-k}$ contributions: in the first line above one finds the leading (one-instanton) terms with $n=1$, while in the second line one already finds exponentially-suppressed contributions with the two-instanton damping $\sim 2^{-k}$, and then on. In this way, large-order power-law corrections are associated to multi-loops, at fixed instanton number; while large-order exponentially-suppressed corrections are associated to the different multi-instanton numbers.

As they follow from the Stokes discontinuities via simple application of Cauchy's theorem, these large-order relations may also be directly written down starting-off by analyzing allowed motions on our alien chain (which dictated the resurgent structure of the transseries). In fact

\footnotetext{
${ }^{47}$ In here Stokes coefficients and Borel residues are essentially the same, up to a trivial transformation (2.68).

${ }^{48} \mathrm{It}$ is also simple to obtain the closed-form expression

$$
F_{k}^{(0,0)} \simeq \sum_{n=1}^{+\infty} \frac{\Gamma(k)}{(n A)^{k}} \frac{S_{1}^{n}}{2 \pi \mathrm{i}} \sum_{h=0}^{+\infty} \frac{\Gamma(k-h)}{\Gamma(k)} F_{h}^{(n, 0)}(n A)^{h} .
$$

Validity for $k \gg h$ reveals the large-order nature of this relation. Note that the ratio of Gamma-functions appearing in the second sum is subleading: it grows as $\sim k^{-h}$. See, e.g., [16] for more closed-form large-order relations.
} 
the "statistical mechanical" rules for writing down large-order relations will be very similar to the ones for writing down Stokes discontinuities in subsection 2.4, and equally rather general. In our present example we are dealing with the chain depicted in figure 11, and the "functional component" appearing in the Stokes discontinuity will now be essentially replaced by its integral, which we shall denote as the large-order factor (the "statistical component" will be essentially the same). For a path on the chain, connecting the nodes $\Phi_{(n, 0)} \rightarrow \Phi_{\left(m, c_{m}\right)}$, this is

$$
\chi_{(n \rightarrow m)}(k) \equiv \frac{1}{(m-n)^{k}} \sum_{h=0}^{+\infty} \frac{\Gamma(k-h)}{\Gamma(k)} F_{h}^{\left(m, c_{m}\right)}((m-n) A)^{h}, \quad c_{m}=\left\{\begin{array}{ll}
1, & m=0 \\
0, & m>0
\end{array} .\right.
$$

Using this definition, one may now write down generic large-order relations purely in terms of motions and data on the alien chain:

Large-order relations: The large-order (large $k$ ) behaviour of the coefficients $F_{k}^{(n, 0)}$, associated to the node $\Phi_{(n, 0)}$, is given by a sum over all forward and backward paths, linking nodes to the right $\Phi_{(m>n, 0)}$ and to the left $\Phi_{(m<n, 0)}$, in addition to a sum over paths to the extra "orthogonal" node in the chain, $\Phi_{(0,1)}$.

Each term in this sum $\left(\Phi_{(n, 0)} \rightarrow \Phi_{(m, \ell=0,1)}\right)$ can be decomposed into three factors:

- Leading growth-factor, dictated solely by the order $k$ of the coefficient:

$$
\frac{\Gamma(k)}{2 \pi \mathrm{i} A^{k}}
$$

- Large-order factor (2.153), dictated solely by beginning and end nodes:

$$
\chi_{(n \rightarrow m)}(k) .
$$

- Statistical factor, sum over all the allowed paths $\mathcal{P}(n \rightarrow m)$ linking the nodes as in figure 11:

$$
\mathrm{SF}_{(n \rightarrow m)} \equiv \sum_{\mathcal{P}(n \rightarrow m)} \mathrm{CF}(\mathcal{P}) w(\mathcal{P})
$$

Spelled out in writing, the result is:

$$
F_{k}^{(n, 0)} \frac{2 \pi \mathrm{i} A^{k}}{\Gamma(k)} \simeq \sum_{m \neq n} \mathrm{SF}_{(n \rightarrow m)} \chi_{(n \rightarrow m)}(k) .
$$

Let us make a few remarks concerning the above large-order relation. First, as the statistical factors do not depend upon the order, $k$, and once the leading growth is factored out, the (subleading) large-order behaviour will be fully dictated by the large-order factors (2.153) implying that in some sense this is where all the non-trivial large-order growth is included. Now equation (2.153) very sharply tells us that the further away two nodes are, the more exponentially suppressed the corresponding large-order factor will be, due to the term $(m-n)^{-k}$. One other interesting feature is how forward and backward motions appear at large order. When walking 
forward along the chain, $m-n>0$, but when walking backwards $m-n<0$ leading to an oscillatory behaviour as $(-1)^{k}$ at large $k$. Finally, note that the ratio of Gamma functions in (2.153) may be easily expanded in powers of $k^{-1}$. This precisely corresponds to expanding the fractions inside the brackets in (2.151). In fact, rewriting the large-order relation for the perturbative series in this light, we find

$$
\begin{aligned}
F_{k}^{(0,0)} \frac{2 \pi \mathrm{i} A^{k}}{\Gamma(k)} & \simeq \mathrm{SF}_{(0 \rightarrow 1)}\left(F_{0}^{(1,0)}+\frac{F_{1}^{(1,0)} A}{k}+\frac{F_{1}^{(1,0)} A+F_{2}^{(1,0)} A^{2}}{k^{2}}+\cdots\right)+ \\
& +2^{-k} \mathrm{SF}_{(0 \rightarrow 2)}\left(F_{0}^{(2,0)}+\frac{F_{1}^{(2,0)}(2 A)}{k}+\frac{F_{1}^{(2,0)}(2 A)+F_{2}^{(2,0)}(2 A)^{2}}{k^{2}}+\cdots\right)+\mathcal{O}\left(3^{-k}\right) .
\end{aligned}
$$

The statistical factors are read directly from figure 11, as $\mathrm{SF}_{(0 \rightarrow 1)}=S_{1}$ and $\mathrm{SF}_{(0 \rightarrow 2)}=S_{1}^{2}$. The agreement with (2.151) is then exact.

We may now go back to the discussion at the beginning of the present subsection, in particular the two main questions which are still open. First, having introduced so many mathematical tools to calculate discontinuities and such, how can we be absolutely sure that the structures we have put forward precisely describe our problem? Second, all statistical factors, defined using weights for the steps in each path, they all depend on a set of a priori unknown coefficients - the Stokes coefficients - and we still need to make sure that they may be determined (either via the partition function as in the previous subsection, or else). As already suggested, one may now settle both these issues by performing numerical checks of the previous asymptotic relations. Because such formulae have their origin in the resurgent structure associated to the transseries we started off from, then if they hold so will all that lays behind them hold. In order to understand what types of numerical tests may be performed, let us consider the expression above, (2.155). Having moved the leading growth-factor to the left-hand side, the right-hand side is now composed of a series of sequences, each one more and more exponentially suppressed. Each of these sequences is given by a number (the statistical factor) times the actual sequence itself, in powers of $\frac{1}{k}$. At very large order, both exponential and power suppressed contributions fade away, and the right-hand side above approaches a constant value (which in here is $\mathrm{SF}_{(0 \rightarrow 1)} F_{0}^{(1,0)}$ ). Can we test this?

In order to check (2.155), one first needs data. Using the recursive equations listed in the appendix, it is computationally straightforward to generate coefficients for both perturbative and one-instanton sectors, $F_{k}^{(0,0)}$ and $F_{k}^{(1,0)}$, and we have done this for $k \leq 120$. Let us begin with the perturbative data. Plotting the left-hand side of (2.155), the larger the value $k$ the closer we expect that this sequence will approach the constant ${ }^{49} S_{1} F_{0}^{(1,0)}$. In turn, this should allow us to determine, at least numerically, the value of the Stokes coefficient $S_{1}$. This is depicted in figure 12, where the red curve shows the coefficients of the left-hand side of (2.155) for growing $k$, and the purple line shows the constant value they are converging towards.

The first thing we notice is that this convergence is very slow! This brings us to our first parenthesis in this subsection, where we wish to digress a bit and discuss a simple numerical method to accelerate convergence of sequences. The type of sequences we are interested in are generically of the type appearing in (2.155), i.e., a leading constant term followed by a whole series in powers of $\frac{1}{k}$. A simple and powerful numerical method to accelerate convergence of this sort of sequences is that of Richardson transforms (mentioned earlier; but see, e.g., [37]).

\footnotetext{
${ }^{49}$ Recall that even though $F_{0}^{(1,0)}$ is unknown from the perspective of the differential equation (it belongs to the initial conditions), we have set its value, via the partition function coefficients, to be $F_{0}^{(1,0)}=-\frac{\mathrm{i}}{\sqrt{2}}$.
} 

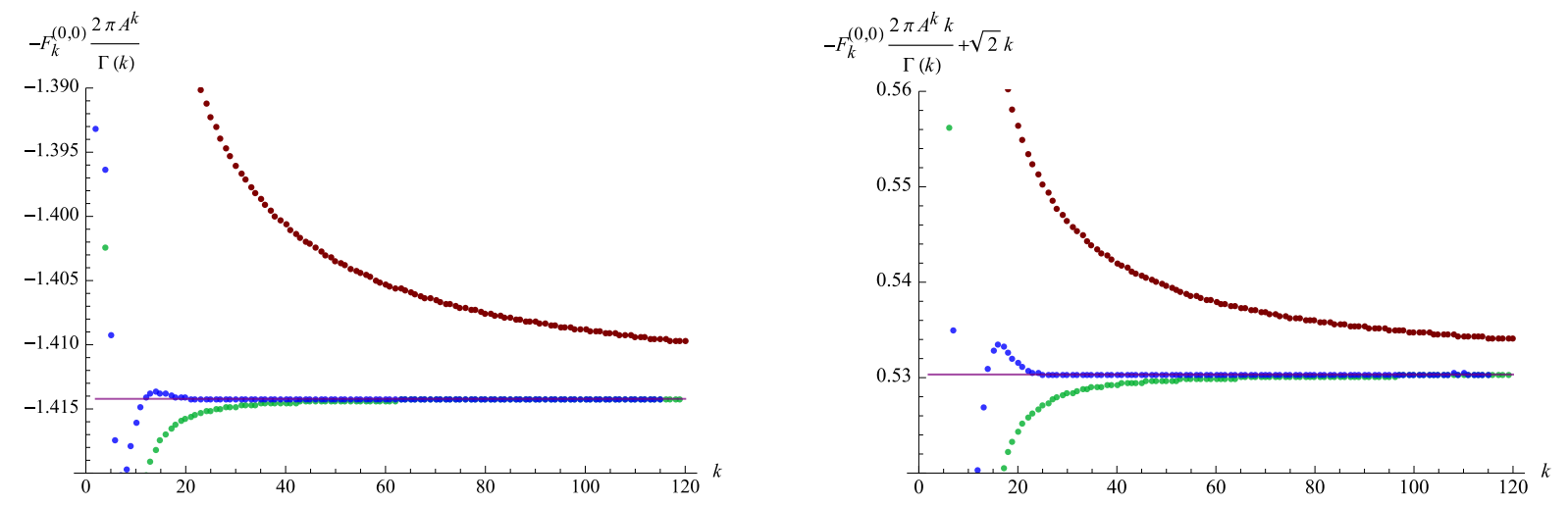

Figure 12. Plot of the coefficients of leading (left) and $1 / k$ (right) behaviour of the perturbative sector, $F_{k}^{(0,0)}$, weighted by the leading growth-factor. In red we plot the original sequences, while in green and blue we plot Richardson transforms $\mathrm{RT}_{0}(r, k, 1)$ and $\mathrm{RT}_{0}(r, k, 5)$, respectively $(r=0$ for the left plot and $r=1$ for the right plot). The purple lines show the constant values that these sequences are converging to: for the case of the left plot i $S_{1} F_{0}^{(1,0)}=-\sqrt{2}$; while for the case of the right plot i $S_{1} F_{1}^{(1,0)} A=\frac{3}{8} \sqrt{2}$ (both correct up to extremely small numerical errors). These plots also nicely illustrate the improved rate of convergence of each initial sequence, the higher the Richardson transform which was subsequently used.

Richardson extrapolation starts with a $k$-sequence

$$
\mathbb{S}(k) \simeq s_{0}+\frac{s_{1}}{k}+\frac{s_{2}}{k^{2}}+\cdots
$$

from where its $N^{\text {th }}$-Richardson transform, herein denoted by $\operatorname{RT}_{\mathbb{S}}(0, k, N)$, is a new $k$-sequence which can be recursively defined as ${ }^{50}$

$$
\begin{aligned}
\operatorname{RT}_{\mathbb{S}}(0, k, 0) & =\mathbb{S}(k) \\
\operatorname{RT}_{\mathbb{S}}(0, k, N) & =\mathrm{RT}_{\mathbb{S}}(0, k+1, N-1)+\frac{k}{N}\left(\operatorname{RT}_{\mathbb{S}}(0, k+1, N-1)-\mathrm{RT}_{\mathbb{S}}(0, k, N-1)\right)
\end{aligned}
$$

Loosely speaking, the Richardson transforms cancel the subleading terms in the original sequence $\mathbb{S}(k)$ up to order $k^{-N}$. This results in a much better numerical convergence towards the first term of the original sequence (denoted by $s_{0}$ above), the larger both $k$ and $N$ are. A couple of words on notation. First, the apparently "silent" zero appearing in the definition above simply refers to the fact that the Richardson transforms we have set up are zooming-in on the $s_{0}$ coefficient of the original sequence. Second, we will be using Richardson extrapolation to check large-order relations involving multi-instanton coefficients, $F_{k}^{(n, 0)}$. It will thus prove convenient to make explicit which series are we addressing, by using the notation $\operatorname{RT}_{n}(0, k, N)$ to signal Richardson transforms of the leading large-order of the $n$-instanton sector.

\footnotetext{
${ }^{50} \mathrm{~A}$ closed form expression for the $N^{\text {th }}$-Richardson transform is
}

$$
\mathrm{RT}_{\mathbb{S}}(0, k, N)=\sum_{n=0}^{N}(-1)^{n+N} \frac{(k+n)^{N}}{n !(N-n) !} \mathbb{S}(k+n) .
$$


Let us now go back to the analysis of the perturbative sector, considering the case $\mathbb{S}(k) \equiv$ $F_{k}^{(0,0)} \frac{2 \pi \mathrm{i} A^{k}}{\Gamma(k)}$, as in the left-hand side of (2.155). The left image in figure 12 depicts the first and fifth Richardson transforms (RTs) of this sequence, closing-in on the constant $\mathrm{SF}_{(0 \rightarrow 1)} F_{0}^{(1,0)}=$ $S_{1} F_{0}^{(1,0)}$. It is rather clear how the more RTs one considers, the faster the rate of convergence one observes. The best estimate we have produced for the value of this constant was upon taking the 5 -RT, at order $k=115$. It so happens that in this case the value of the constant is also very easy to pinpoint as $\mathrm{SF}_{(0 \rightarrow 1)} F_{0}^{(1,0)}=S_{1} F_{0}^{(1,0)}=\mathrm{i} \sqrt{2}$. Comparison with $\mathrm{RT}_{0}(0,115,5)$ yields an extremely small error,

$$
\frac{\mathrm{RT}_{0}(0,115,5)-\mathrm{i} \sqrt{2}}{\mathrm{i} \sqrt{2}} \approx 1.211978 \times 10^{-10} .
$$

Finally note that this value of $S_{1} F_{0}^{(1,0)}=\mathrm{i} \sqrt{2}$ precisely corresponds to a value for the Stokes coefficient of $S_{1}^{F}=-2$, which is in complete agreement with the predicted value (2.126).

This numerical check has confirmed the first (leading) term in the large-order expansion for the perturbative sector (2.155). But we would further like to check the subsequent terms - for the moment, at least at leading exponential order. This would verify that the exponentially-leading large-order behaviour is indeed dictated by all the coefficients of the one instanton sector, $F_{h}^{(1,0)}$, as predicted by resurgence. Such checks follow in very similar fashion to the one above. In fact the first line of (2.155) is precisely of the form (2.156), and it is rather simple to check the value of $s_{1}$ in this latter sequence. All one has to do is to repeat the procedure above, this time applied to the (trivially) modified sequence

$$
\mathbb{S}_{1}(k) \equiv\left(\mathbb{S}(k)-s_{0}\right) k \simeq s_{1}+\frac{s_{2}}{k}+\frac{s_{3}}{k^{2}}+\cdots .
$$

The RT is defined in a completely similar way. More generally, we can recursively check every coefficient in the sequence (2.156) simply by defining

$$
\mathbb{S}_{r}(k) \equiv\left(\mathbb{S}_{r-1}(k)-s_{r-1}\right) k \simeq s_{r}+\frac{s_{r+1}}{k}+\frac{s_{r+2}}{k^{2}}+\cdots, \quad r \geq 1,
$$

and the corresponding RTs

$$
\begin{aligned}
\operatorname{RT}_{\mathbb{S}}(r, k, 0) & =\mathbb{S}_{r}(k), \\
\operatorname{RT}_{\mathbb{S}}(r, k, N) & =\mathrm{RT}_{\mathbb{S}}(r, k+1, N-1)+\frac{k}{N}\left(\operatorname{RT}_{\mathbb{S}}(r, k+1, N-1)-\operatorname{RT}_{\mathbb{S}}(r, k, N-1)\right),
\end{aligned}
$$

One may now proceed in the exact same way as above, checking each coefficient in (2.155). The right image in figure 12 is checking the coefficient $r=1$, where we have obtained a convergence towards the expected value of $s_{1} \equiv \mathrm{SF}_{(0 \rightarrow 1)} F_{1}^{(1,0)} A=-\frac{3 \mathrm{i}}{8} \sqrt{2}$, with extremely small error

$$
\frac{\mathrm{RT}_{0}(1,115,5)-s_{1}}{s_{1}} \approx-3.142630 \times 10^{-9} .
$$

Checking the subsequent coefficients may be easily done in the very same way, albeit sometimes one does need to use a higher RT to keep good accuracy. For instance, at six-loops the convergence towards $s_{5}=-\frac{6767133 \mathrm{i}}{131072 \sqrt{2}}$ (do recall we are starting with the sequence $\mathbb{S}(k) \equiv F_{k}^{(0,0)} \frac{2 \pi \mathrm{i} A^{k}}{\Gamma(k)}$ ) again yields an extremely small error

$$
\frac{\mathrm{RT}_{0}(5,110,8)-s_{5}}{s_{5}} \approx 1.818376 \times 10^{-8} .
$$


One word of caution on the nature of these numerical tests should still be said. As per definition, the coefficients in the sequence $\mathbb{S}(k)$ from $(2.155)$ grow factorially - this is simply due to the appearance of the one-instanton series, $F_{h}^{(1,0)}$. As such, this can lead to large numerical errors when checking higher multi-loop coefficients, unless one is careful from scratch in implementing an elevated numerical accuracy in the calculations.

Proceeding along these lines, one eventually arrives at a stage where one has very strong checks on the validity of the first line in (2.155), and thus the fact that it was correctly predicted by the resurgent structure of the transseries we started off with. This check corresponds to verifying the correctness of the term $\mathrm{SF}_{(0 \rightarrow 1)} \chi_{(0 \rightarrow 1)}$ in (2.154), which is the exponentially leading motion in the large-order relations of the perturbative sector. But one may likewise naturally wonder if it is possible to check all other motions, even if exponentially suppressed? In other words, may one also check, say, the (exponentially suppressed) second line in (2.155)? The first thing to notice is that in order to probe the $2^{-k}$ behaviour, we first need to subtract out of the original series all contribution coming from the $\mathrm{SF}_{(0 \rightarrow 1)} \chi_{(0 \rightarrow 1)}$ motion, i.e., to subtract out the first line in (2.155). But this line corresponds to an asymptotic expansion on its own (more generically, do recall (2.153)), and in order to subtract out something which is actually meaningful one first needs to re-sum this asymptotic series into some number.

This brings us to our second parenthesis in this subsection, where we wish to digress a bit and discuss resummations of asymptotic series. The most straightforward method to re-sum asymptotic series is that of optimal truncation, where the series is cut-off essentially at the point where its coefficients start growing. While this method may sometimes yield reasonable accuracy, for the purposes of this review it does not reach the required accuracy for our tests, and we will not dwell on it any further ${ }^{51}$. A more intricate resummation method, which in fact reaches the accuracy we are looking for, is the method of Borel-Padé approximants. As already discussed in an earlier subsection, given an asymptotic series $\mathbb{S}(k)$, with zero radius of convergence, its Borel transform

$$
\mathcal{B}[\mathbb{S}](t)=\sum_{m=0}^{+\infty} \frac{s_{m}}{\Gamma(m+1)} t^{m}
$$

will instead have a (finite) non-zero radius of convergence. Of course as long as there are no closed-form expressions for all the coefficients $s_{m}$, one cannot precisely pinpoint the above analytic function. However, using the recursion relations for, e.g., the free energy coefficients listed in the appendix, we have computationally calculated them in our example up to $2 N=120$. If we make sole use of these resulting coefficients, what we have managed to compute is

$$
\mathcal{B}[\mathbb{S}](t) \approx \sum_{m=0}^{2 N} \frac{s_{m}}{\Gamma(m+1)} t^{m} .
$$

Now instead of immediately "inverting" this result via Borel resummation (2.3) (which at this point would only allow us to recover these first $2 N$ terms of the asymptotic expansion $\mathbb{S}(k)$ ), we shall first approximate the above truncated Borel transform by an order- $N$ diagonal Padé approximant, as in:

$$
\mathrm{BP}_{N}[\mathbb{S}](t)=\frac{\sum_{\ell=0}^{N} a_{n} t^{n}}{\sum_{\ell=0}^{N} b_{n} t^{n}},
$$

\footnotetext{
${ }^{51}$ The reader who might be interested to see this in an example may take a look at the study of Painlevé I solutions in [16]. In this reference and within that example it was shown how the accuracy of the optimal truncation method was exactly of the same order as the first exponentially-suppressed term, and would thus be blind to the full large-order structure of the $k$-sequences associated to any exponentially suppressed contributions.
} 

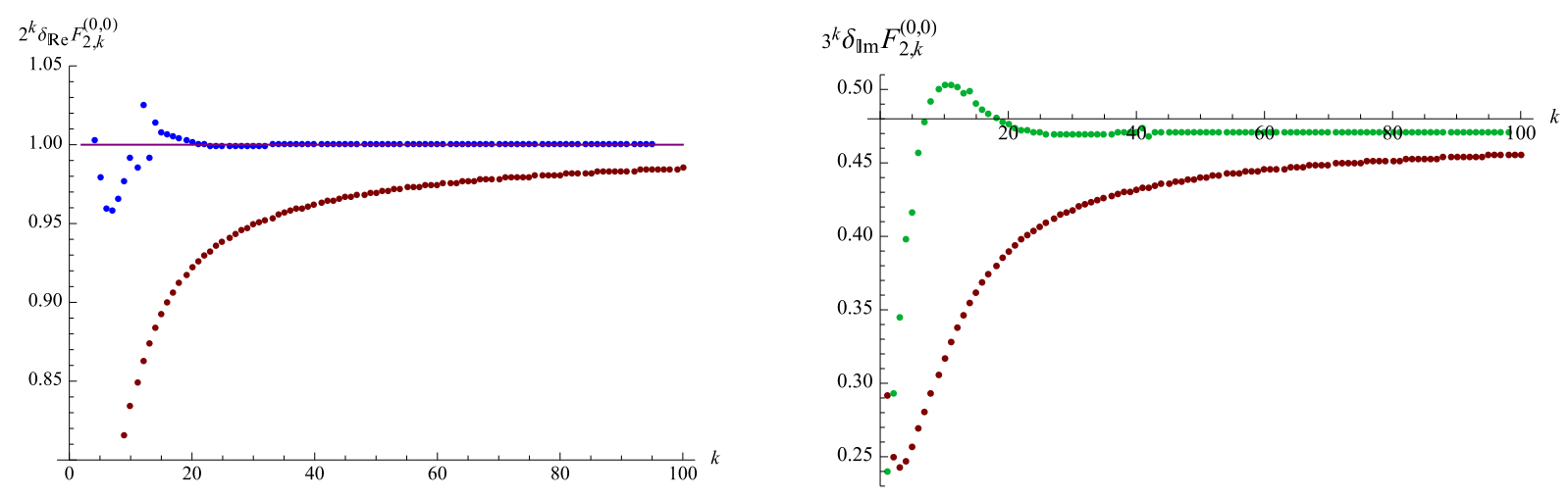

Figure 13. The image on the left, plots the leading behaviour of the coefficients $2^{k} \delta_{\mathbb{R e}} F_{2, k}^{(0,0)} \equiv$ $-2^{k} \mathrm{SF}_{(0 \rightarrow 1)} \times \mathcal{S}_{\mathbb{R e}} \mathrm{BP}_{60}\left[\chi_{(0 \rightarrow 1)}\right](k)$. In red we plot the original sequence, while in blue we plot its $\mathrm{RT}_{0,2}(0, k, 5)$ Richardson transform. The purple line shows the constant value that this sequence is converging towards, $S_{1}^{2} F_{0}^{(2,0)}=1$. The image on the right, plots the leading behaviour of the coefficients $3^{k} \delta_{\mathbb{I m}} F_{2, k}^{(0,0)} \equiv \operatorname{Im}\left(F_{k}^{(0,0)} \frac{2 \pi \mathrm{i}(3 A)^{k}}{\Gamma(k)}\right)-3^{k} \mathrm{SF}_{(0 \rightarrow 1)} \times \mathcal{S}_{\mathbb{I m}} \mathrm{BP}_{60}\left[\chi_{(0 \rightarrow 1)}\right](k)$, in red, and their first Richardson transform, in green. It is very clear how this sequence is converging towards a constant value of order $\sim \mathcal{O}(1)$, which immediately validates the expected $3^{-k}$ decay.

where the coefficients $a_{n}, b_{n}$ are chosen to match the ones in the expansion of the Borel transform in a small- $t$ expansion. The main reason for choosing this rational function instead of the expansion (2.168) is that, while for small $t$ they are basically the same, for large $t$ the rational function converges to a constant and is much better behaved ${ }^{52}$. Moreover, the poles of this rational function will also prove to be very good approximations (upon "condensation") to the branch-cuts of the Borel transform. It is then to the rational function (2.169) which we apply Borel resumation, along the positive real axis (as the original variable $k$ is the order of the coefficients, and is thus positive),

$$
\mathcal{S}_{0} \mathrm{BP}_{N}[\mathbb{S}](k)=\int_{0}^{+\infty} \mathrm{d} t \mathrm{BP}_{N}[\mathbb{S}]\left(\frac{t}{k}\right) \mathrm{e}^{-t} .
$$

As should be familiar by now, this integration can only be performed when there are no poles of $\mathrm{BP}_{N}[\mathbb{S}](t)$ along the positive real axis of the Borel $t$-plane. However, this is very many times the case, and in such situations one needs to shift the integration contour off the real axis, say by performing a lateral Borel resummation, e.g., $\mathcal{S}_{0^{-}} \mathrm{BP}_{N}[\mathbb{S}](k)$. Imaginary contributions may then appear, and one has to be careful handling them as we shall further discuss below.

Let us now go back to our ongoing analysis of the perturbative large-order growth, and use the method described above to check exponentially-suppressed contributions to its asymptotics. If we start by assuming that there will be no poles upon the real axis, and that the Laplacetype integral (2.170) can thus be evaluated without any shift in the integration contour, then the procedure should be rather clear. Applying the Borel-Padé (BP) resummation to the asymptotic series $\chi_{(0 \rightarrow 1)}(k)$, we expect to obtain a resulting meaningful quantity which may be subtracted

\footnotetext{
${ }^{52}$ When performing Borel resummation (2.3), we need to integrate over the range of both $t$ small and large!
} 
out of the original perturbative sequence $F_{k}^{(0,0)} \frac{2 \pi \mathrm{i} A^{k}}{\Gamma(k)}$. Looking back at (2.155), one writes ${ }^{53}$

$$
\begin{aligned}
F_{k}^{(0,0)} & \frac{2 \pi \mathrm{i} A^{k}}{\Gamma(k)}-\mathrm{SF}_{(0 \rightarrow 1)} \times \mathcal{S}_{0} \mathrm{BP}_{N}\left[\chi_{(0 \rightarrow 1)}\right](k) \simeq \\
& \simeq 2^{-k} \mathrm{SF}_{(0 \rightarrow 2)}\left(F_{0}^{(2,0)}+\frac{F_{1}^{(2,0)}(2 A)}{k}+\frac{F_{1}^{(2,0)}(2 A)+F_{2}^{(2,0)}(2 A)^{2}}{k^{2}}+\cdots\right)+\mathcal{O}\left(3^{-k}\right),
\end{aligned}
$$

and the analysis would now proceed akin to what we did earlier. But, as we said, in many situations, including the case we are now addressing, the BP approximant to $\chi_{(0 \rightarrow 1)}$ will indeed have poles along the positive real axis. We shall deal with them by simply performing a lateral resummation, $\mathcal{S}_{0^{-}} \mathrm{BP}_{N}\left[\chi_{(0 \rightarrow 1)}\right](k)$, which integrates right below the real line. This will then produce a result with both real and imaginary components. As it turns out, the real contribution will be similar to above, and can be compared to the exponential order $2^{-k}$ of the large-order behaviour of the perturbative series, just as in (2.171). But the imaginary contribution, on the other hand, cancels with the perturbative coefficients up to order $3^{-k}$ and is thus irrelevant at the order we are interested in (see also [16]). Let us make this explicit. Writing

$$
\mathcal{S}_{0^{-}} \mathrm{BP}_{N}\left[\chi_{(0 \rightarrow 1)}\right](k)=\mathcal{S}_{\mathbb{R e}} \mathrm{BP}_{N}\left[\chi_{(0 \rightarrow 1)}\right](k)+\mathrm{i} \mathcal{S}_{\mathbb{I m}} \mathrm{BP}_{N}\left[\chi_{(0 \rightarrow 1)}\right](k),
$$

then, splitting imaginary (first line) and real (second and third lines) components, what we find is

$$
\begin{aligned}
& F_{k}^{(0,0)} \frac{2 \pi A^{k}}{\Gamma(k)}- \mathrm{SF}_{(0 \rightarrow 1)} \times \mathcal{S}_{\mathbb{I m}} \mathrm{BP}_{N}\left[\chi_{(0 \rightarrow 1)}\right](k) \simeq \mathcal{O}\left(3^{-k}\right), \\
& \quad-\mathrm{SF}_{(0 \rightarrow 1)} \times \mathcal{S}_{\mathbb{R e} \mathrm{BP}_{N}\left[\chi_{(0 \rightarrow 1)}\right](k) \simeq} \\
& \simeq 2^{-k} \mathrm{SF}_{(0 \rightarrow 2)}\left(F_{0}^{(2,0)}+\frac{F_{1}^{(2,0)}(2 A)}{k}+\frac{F_{1}^{(2,0)}(2 A)+F_{2}^{(2,0)}(2 A)^{2}}{k^{2}}+\cdots\right)+\mathcal{O}\left(3^{-k}\right) .
\end{aligned}
$$

That this is indeed the resulting behaviour can be tested numerically, and is illustrated in figure 13 for a $\mathrm{BP}$ of $N=60$. On its left image, we plot the convergence of the real part of the coefficients (do note that they are already properly weighted by the exponential term $2^{k}$ )

$$
2^{k} \delta_{\mathbb{R e}} F_{2, k}^{(0,0)} \equiv-2^{k} \mathrm{SF}_{(0 \rightarrow 1)} \times \mathcal{S}_{\mathbb{R e}} \mathrm{BP}_{N}\left[\chi_{(0 \rightarrow 1)}\right](k)
$$

towards the leading term within the two-instanton contribution, $\mathrm{SF}_{(0 \rightarrow 2)} F_{0}^{(2,0)}=S_{1}^{2} F_{0}^{(2,0)}=1$, which is achieved with great precision. Let us use the notation $\mathrm{RT}_{n, \ell}(0, k, N)$ to denote the $N^{\text {th }}$ RT associated to the $\ell^{-k}$ exponentially-suppressed behaviour of the large-order relation obeyed by the $n$-instanton coefficients $F_{k}^{(n, 0)}$, when zooming-in on the leading contribution, $s_{0}$, and as obtained via the resummation procedure described above (thus $\mathrm{RT}_{n}(0, k, N)$ defined previously is just $\mathrm{RT}_{n, 1}(0, k, N)$ in the new notation). Then, in the present case, we find that the convergence error is once again extremely small,

$$
\frac{\mathrm{RT}_{0,2}(0,95,5)-1}{1} \approx 3.40814 \times 10^{-8} .
$$

\footnotetext{
${ }^{53}$ Recall that $\mathrm{SF}_{(0 \rightarrow 2)}=S_{1}^{2}$.
} 


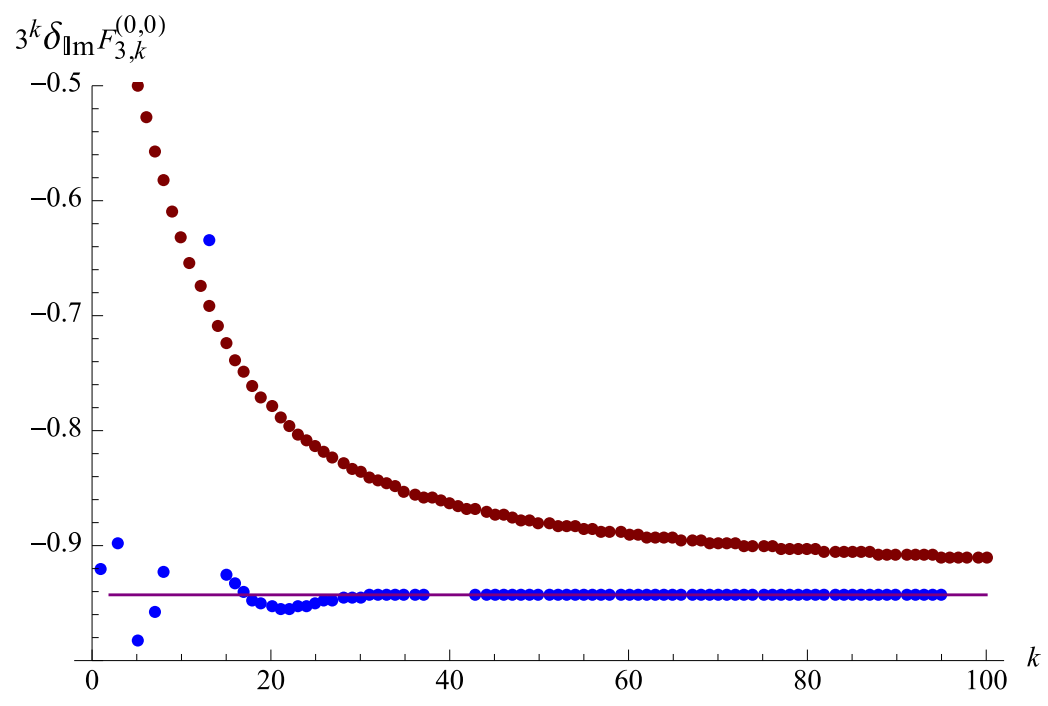

Figure 14. Plot of the large-order behaviour of the coefficients $3^{k} \delta_{\mathbb{I m}} F_{3, k}^{(0,0)}$. The original sequence is shown in red, while in blue we plot its $\mathrm{RT}_{0,3}(0, k, 5)$ Richardson transform. The purple line shows the constant value this sequence is converging towards, $-\mathrm{i} S_{1}^{3} F_{0}^{(3,0)}=-\frac{2 \sqrt{2}}{3}$.

On the right image of figure 13, we plot the imaginary part (again, already properly weighted by the corresponding exponential term $3^{k}$ )

$$
3^{k} \delta_{\mathbb{I m}} F_{2, k}^{(0,0)} \equiv F_{k}^{(0,0)} \frac{2 \pi(3 A)^{k}}{\Gamma(k)}-3^{k} \mathrm{SF}_{(0 \rightarrow 1)} \times \mathcal{S}_{\mathbb{I m}} \mathrm{BP}_{N}\left[\chi_{(0 \rightarrow 1)}\right](k) .
$$

The plot clearly shows that indeed the imaginary part of this resummation is of order $3^{-k}$, and will mix with the next exponentially-suppressed order in the large-order relations.

The procedure above can of course keep being applied to study more and more exponentiallysuppressed contributions to the perturbative asymptotics, in a completely straightforward fashion. Nonetheless, due to the aforementioned mixing of real and imaginary contributions arising upon BP resummation, let us still schematically work out the next step, illustrating how to re-sum the order $2^{-k}$ contributions to the large-order behaviour of the perturbative sequence $F_{k}^{(0,0)}$ in $(2.155)$. As expected, one may once again verify that the BP of $\chi_{(0 \rightarrow 2)}$ will have poles upon the positive real axis, and we need to perform a lateral resummation. We shall do this consistently with the previous choice of resummation direction, and will thus re-sum below the poles obtaining both a real and an imaginary contribution, as in

$$
\mathcal{S}_{0^{-}} \mathrm{BP}_{N}\left[\chi_{(0 \rightarrow 2)}\right](k)=\mathcal{S}_{\mathbb{R e}_{\mathrm{e}}} \mathrm{BP}_{N}\left[\chi_{(0 \rightarrow 2)}\right](k)+\mathrm{i} \mathcal{S}_{\mathbb{I m}} \mathrm{BP}_{N}\left[\chi_{(0 \rightarrow 2)}\right](k) .
$$

The real and imaginary parts of the resummed result are defined as above,

$$
\begin{aligned}
& \delta_{\mathbb{R e}} F_{3, k}^{(0,0)}+\mathrm{i} \delta_{\mathbb{I m}} F_{3, k}^{(0,0)} \equiv F_{k}^{(0,0)} \frac{2 \pi \mathrm{i} A^{k}}{\Gamma(k)}-\mathrm{SF}_{(0 \rightarrow 1)} \mathcal{S}_{\mathbb{R e}} \mathrm{BP}_{N}\left[\chi_{(0 \rightarrow 1)}\right](k)- \\
& -\mathrm{iSF}_{(0 \rightarrow 1)} \mathcal{S}_{\mathbb{I m}} \mathrm{BP}_{N}\left[\chi_{(0 \rightarrow 1)}\right](k)-\mathrm{SF}_{(0 \rightarrow 2)} \mathcal{S}_{\mathbb{R e}_{\mathrm{e}}} \mathrm{BP}_{N}\left[\chi_{(0 \rightarrow 2)}\right](k)-\mathrm{iSF}_{(0 \rightarrow 2)} \mathcal{S}_{\mathbb{I m}} \mathrm{BP}_{N}\left[\chi_{(0 \rightarrow 2)}\right](k)= \\
& =F_{k}^{(0,0)} \frac{2 \pi \mathrm{i} A^{k}}{\Gamma(k)}+2 \mathcal{S}_{\mathbb{R e}} \mathrm{BP}_{N}\left[\chi_{(0 \rightarrow 1)}\right](k)+2 \mathrm{i} \mathcal{S}_{\mathbb{I m}} \mathrm{BP}_{N}\left[\chi_{(0 \rightarrow 1)}\right](k)-
\end{aligned}
$$




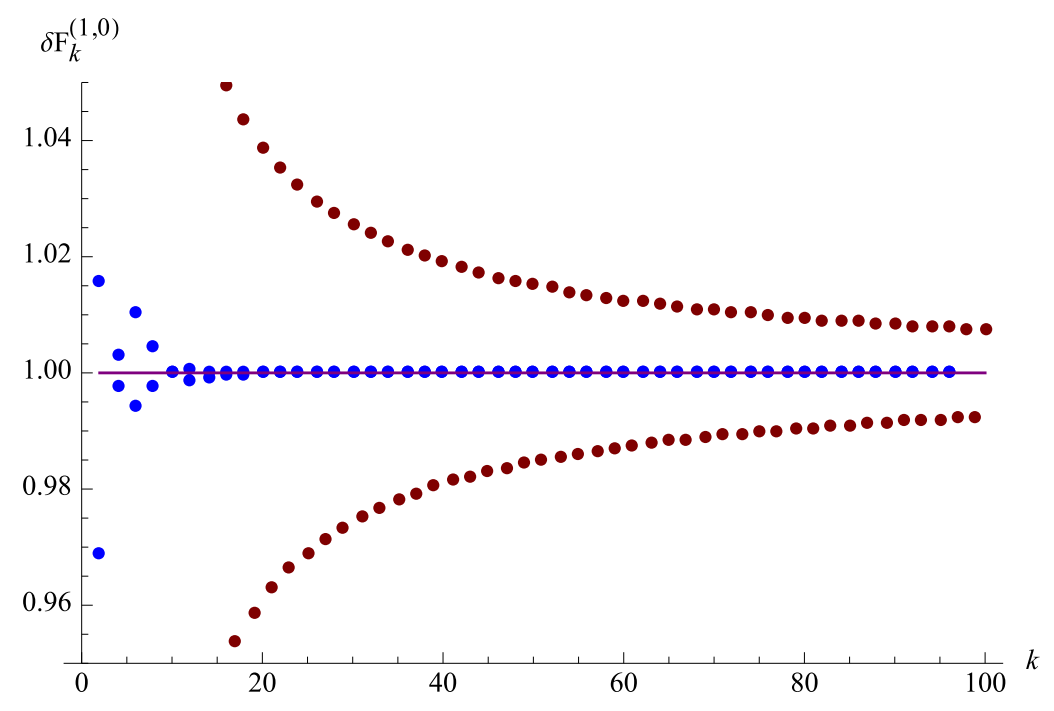

Figure 15. Plot of the large-order behaviour of the coefficients $\delta F_{k}^{(1,0)}$. The original sequence is shown in red, while in blue we plot its $\mathrm{RT}_{1}(0, k, 5)$ Richardson transform. The purple line shows the constant value towards which this sequence is converging, $\widetilde{S}_{-1} F_{0}^{(0,1)}=1$.

$$
-(-2)^{2} \mathcal{S}_{\mathbb{R e}} \mathrm{BP}_{N}\left[\chi_{(0 \rightarrow 2)}\right](k)-(-2)^{2} \mathrm{i} \mathcal{S}_{\mathbb{I m}} \mathrm{BP}_{N}\left[\chi_{(0 \rightarrow 2)}\right](k) .
$$

It turns out that this time around it will be the imaginary part, which was earlier of order $3^{-k}$, which will yield the leading behaviour at this order; while the real part will now be of order $4^{-k}$ and only contribute at next order, i.e.,

$$
\begin{aligned}
\mathrm{i} \delta_{\mathbb{I m}} F_{3, k}^{(0,0)} & \simeq 3^{-k} \mathrm{SF}_{(0 \rightarrow 3)}\left(F_{0}^{(3,0)}+\cdots\right)+\mathcal{O}\left(4^{-k}\right), \\
\delta_{\mathbb{R e}} F_{3, k}^{(0,0)} & \simeq \mathcal{O}\left(4^{-k}\right) .
\end{aligned}
$$

Herein, the coefficients $\mathrm{i} \delta_{\mathbb{I m}} F_{3, k}^{(0,0)}$ are expected to converge, at leading order in $1 / k$, towards $\mathrm{SF}_{(0 \rightarrow 3)} F_{0}^{(3,0)}=-\frac{2 \sqrt{2} \mathrm{i}}{3}$. This is tested in figure 14 for a BP of $N=60$, with the usual extremely small error,

$$
\frac{\mathrm{RT}_{0,3}(0,95,5)-\left(-\frac{2 \sqrt{2} \mathrm{i}}{3}\right)}{\left(-\frac{2 \sqrt{2} \mathrm{i}}{3}\right)} \approx 9.017 \times 10^{-7} .
$$

Having thoroughly tested the resurgent structure of the perturbative sector, let us now turn to the analysis of the large-order behaviour of higher (multi) instanton sectors. In particular, we shall next focus upon the one and two-instanton sectors. Starting with the asymptotic expansion around the one-instanton sector, $\Phi_{(1,0)}$, we proceed in complete analogy with what was done for the perturbative sector. Using both Stokes discontinuities ${ }^{54}$ (2.101) and (2.102) alongside

\footnotetext{
${ }^{54}$ Once again, Stokes coefficients and Borel residues are trivially related, via (2.68).
} 
Cauchy's theorem (2.149), one immediately obtains the large-order relation ${ }^{55}$

$$
\begin{gathered}
F_{k}^{(1,0)} \frac{2 \pi \mathrm{i} A^{k}}{\Gamma(k)} \simeq 2 S_{1}\left(F_{0}^{(2,0)}+\frac{A}{k-1} F_{1}^{(2,0)}+\frac{A^{2}}{(k-1)(k-2)} F_{2}^{(2,0)}+\cdots\right)+\frac{\widetilde{S}_{-1}}{(-1)^{k}} F_{0}^{(0,1)}+ \\
+2^{-k} 3 S_{1}^{2}\left(F_{0}^{(3,0)}+\frac{2 A}{k-1} F_{1}^{(3,0)}+\frac{(2 A)^{2}}{(k-1)(k-2)} F_{2}^{(3,0)}+\cdots\right)+\mathcal{O}\left(3^{-k}\right) .
\end{gathered}
$$

The main novelty as compared back to (2.151) is the appearance of "sideways" resurgence, via the Stokes constant $\widetilde{S}_{-1}$, which we analyze in the following. In any case, the whole procedure is very similar to what we did before. We first divide this large-order relation into its leading asymptotics and the exponentially-suppressed contributions at order $2^{-k}$ and smaller. Then, we plot the leading $1 / k$ coefficients, and their respective RTs, to numerically determine the Stokes constant $\widetilde{S}_{-1}$. This is shown in figure 15 , where the coefficients

$$
\delta F_{k}^{(1,0)} \equiv(-1)^{k}\left(F_{k}^{(1,0)} \frac{2 \pi \mathrm{i} A^{k}}{\Gamma(k)}-2 S_{1} F_{0}^{(2,0)}\right)
$$

are plotted, alongside their fifth RT. From (2.184) above, these coefficients are predicted to converge to $\widetilde{S}_{-1} F_{0}^{(0,1)}=1$ (where we use the predicted value for the Stokes constant $\widetilde{S}_{-1}=1$ ). The figure very clearly shows how this is indeed the case, with the usual extremely small error

$$
\frac{\mathrm{RT}_{1}(0,95,5)-1}{1} \approx 8.870 \times 10^{-10} .
$$

The procedure is entirely analogous to what we did earlier for the perturbative sector (only the resurgent structure of the large-order relations changes), and one could thus proceed into performing BP resummations of the leading behaviour and eventually test exponentially-suppressed predictions in the exact same fashion. We leave such tests as an exercise for the reader.

The one final test we still wish to discuss in here deals with the large-order behaviour of the two-instanton sector. Using the Stokes discontinuities (2.101) and (2.103), the large-order relation of this sector is:

$$
\begin{aligned}
F_{k}^{(2,0)} \frac{2 \pi \mathrm{i} A^{k}}{\Gamma(k)} \simeq & 3 S_{1}\left(F_{0}^{(3,0)}+\frac{A}{k-1} F_{1}^{(3,0)}+\frac{A^{2}}{(k-1)(k-2)} F_{2}^{(3,0)}+\cdots\right)+ \\
& +\frac{1}{(-1)^{k}} S_{-1}\left(F_{0}^{(1,0)}+\frac{(-A)}{k-1} F_{1}^{(1,0)}+\frac{(-A)^{2}}{(k-1)(k-2)} F_{2}^{(1,0)}+\cdots\right)+ \\
& +\frac{1}{2^{k}} 6 S_{1}^{2}\left(F_{0}^{(4,0)}+\frac{(2 A)}{k-1} F_{1}^{(4,0)}+\frac{(2 A)^{2}}{(k-1)(k-2)} F_{2}^{(4,0)}+\cdots\right)+ \\
& +\frac{1}{(-2)^{k}}\left(\widetilde{S}_{-2}+\frac{1}{2} \widetilde{S}_{-1} S_{-1}\right) F_{0}^{(0,1)}+\mathcal{O}\left(3^{-k}\right)
\end{aligned}
$$

${ }^{55}$ For completeness, let us also include the respective closed-form expression, valid for $k>h$,

$$
F_{k}^{(1,0)} \simeq \sum_{n=1}^{+\infty} \frac{\Gamma(k)}{(n A)^{k}}(n+1) \frac{S_{1}^{n}}{2 \pi \mathrm{i}} \sum_{h=0}^{+\infty} \frac{\Gamma(k-h)}{\Gamma(k)} F_{h}^{(n+1,0)}(n A)^{h}+\frac{\Gamma(k)}{(-A)^{k}} \frac{\widetilde{S}_{-1}}{2 \pi \mathrm{i}} F_{0}^{(0,1)} .
$$




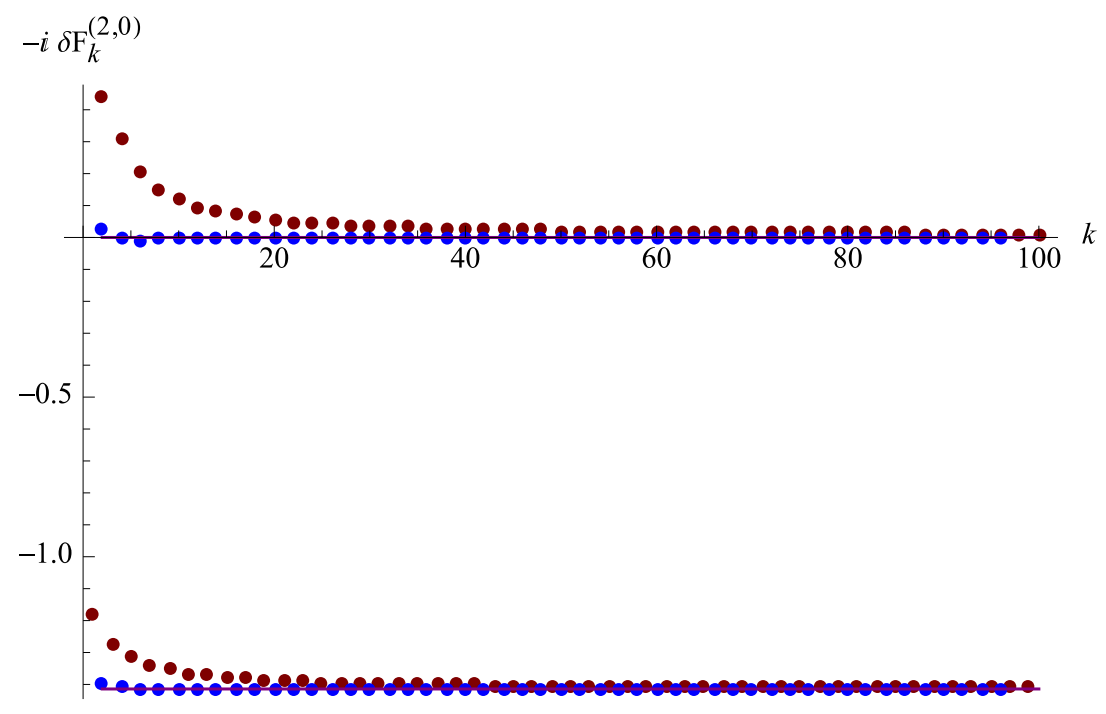

Figure 16. Plot of the large-order behaviour of the two-instanton coefficients $-\mathrm{i} \delta F_{k}^{(2,0)} \equiv F_{k}^{(2,0)} \frac{2 \pi A^{k}}{\Gamma(k)}$. As usual the original sequence is shown in red, while in blue we plot the Richardson transforms for each branch, $k$ even or odd, $\mathrm{RT}_{2 \text {,even/odd }}(0, k, 5)$. The purple lines show the constant values towards which each of these sequences is converging to, depending on parity, which is either 0 or $-\sqrt{2}$.

or, equivalently but with slightly simpler combinatorial factors, with Borel residues:

$$
\begin{aligned}
F_{k}^{(2,0)} \frac{2 \pi \mathrm{i} A^{k}}{\Gamma(k)} \simeq & -\mathrm{S}_{2 \rightarrow 3}\left(F_{0}^{(3,0)}+\frac{A}{k-1} F_{1}^{(3,0)}+\frac{A^{2}}{(k-1)(k-2)} F_{2}^{(3,0)}+\cdots\right)- \\
& -\frac{1}{(-1)^{k}} \mathrm{~S}_{2 \rightarrow 1}\left(F_{0}^{(1,0)}+\frac{(-A)}{k-1} F_{1}^{(1,0)}+\frac{(-A)^{2}}{(k-1)(k-2)} F_{2}^{(1,0)}+\cdots\right)- \\
& -\frac{1}{2^{k}} \mathrm{~S}_{2 \rightarrow 4}\left(F_{0}^{(4,0)}+\frac{(2 A)}{k-1} F_{1}^{(4,0)}+\frac{(2 A)^{2}}{(k-1)(k-2)} F_{2}^{(4,0)}+\cdots\right)- \\
& -\frac{1}{(-2)^{k}} \widetilde{\mathrm{S}}_{2 \rightarrow 0} F_{0}^{(0,1)}+\mathcal{O}\left(3^{-k}\right) .
\end{aligned}
$$

This two-instanton sector is particularly interesting as it is the first large-order relation which displays all types of resurgence in this problem; "forward", via $S_{+}$, "backward", via $S_{-}$, and "sideway", via $\widetilde{S}_{-}$. Much of the analysis of this relation now follows the same steps as before. For example, one may firstly check the leading large-order behaviour in the same manner as we did earlier with RTs. Then one can also re-sum the exponentially leading sectors, associated to the motions $\chi_{(2 \rightarrow 3)}$ and $\chi_{(2 \rightarrow 1)}$, in order to study the exponentially suppressed $2^{-k}$ contribution and, in this way, check the value of the Stokes constant $\widetilde{S}_{-2}$. Let us first say a few words about the leading large-order behaviour. From the expression above a novelty readily emerges, as this leading behaviour will now depend upon the parity of $k$. In fact, one has at leading order in $1 / k$

$$
F_{k}^{(2,0)} \frac{2 \pi \mathrm{i} A^{k}}{\Gamma(k)} \approx 3 S_{1} F_{0}^{(3,0)}+(-1)^{k} S_{-1} F_{0}^{(1,0)}+\mathcal{O}(1 / k) .
$$


Making use of $F_{0}^{(3,0)}=\frac{\mathrm{i}}{6 \sqrt{2}}$, it then follows that when $k$ is odd the sequence above converges towards $3 S_{1} F_{0}^{(3,0)}-S_{-1} F_{0}^{(1,0)}=-\mathrm{i} \sqrt{2}$; while when $k$ is even the sequence converges towards $3 S_{1} F_{0}^{(3,0)}+S_{-1} F_{0}^{(1,0)}=0$ instead. It should be rather evident that one may actually perform convergence acceleration for either $k$ even or odd, separately, by simple application of RTs on these sub-sequences. This is illustrated in figure 16, where we can perfectly see the convergence towards each of the above values separately. The error associated with the convergence is extremely small, as before; it is about $10^{-9}$. Moving onwards, resumming the sectors $\chi_{(2 \rightarrow 3)}$ and $\chi_{(2 \rightarrow 1)}$ and subtracting them from the original two-instanton sequence will then allow us to directly access the $2^{-k}$ behaviour of the large-order relations. In particular, this exercise (which we leave for the reader) directly confirms that $\widetilde{S}_{-2}=0$ as expected. Of course we could keep on analyzing higher and higher sectors, and given enough time and patience numerically check all the predicted Stokes constants alongside the transseries resurgent structure.

After all this, we may finally conclude that all these tests allowed us to very precisely verify the structure of the resurgent transseries we had put forward earlier, as a solution to the quartic free energy. In general, in harder problems (and without a linear "companion-problem" to help us bypass some of the nonlinear difficulties), the procedure develops in the opposite direction, or as a mix in-between. One puts forward a reasonable ansatz for a transseries solution, then checks it via large-order relations as above. Some aspects may be validated, others not-and require for extra structure to be added to the transseries ansatz. So one does, and repeats the different analyses and checks. Iteratively, one eventually constructs the full resurgent transseries solution to the problem at hand (also sometimes involving numerical determination of many Stokes constants). Recent examples where such procedure was applied include, e.g., the cases of the first Painlevé equations relating to $2 \mathrm{~d}$ (super) gravity and addressed in $[179,175,180,130$, 16, 205, 17]. Again, we have here chosen such a simple example as the quartic integral in order to introduce and develop resurgence and transseries in a "friendly environment", for pedagogical purposes and the ease of the first-time reader. Let us say that one issue we have not covered concerns transseries resummation, i.e., how to use the transseries to obtain nonperturbative results (numbers!) to whatever problem at hand. Such a discussion-alongside an analysis of strong-coupling expansions (as opposed to the present weak-coupling asymptotic expansions) - is briefly addressed in appendix C. We have nonetheless decided not to include this topic in the main part of this review (where we chose to focus more on asymptotics), and rather refer the interested reader to the general theory of median resummation as described in, e.g., [15], and to the (numerical) examples of, e.g., $[175,135,80,12,79,66,64]$, which very nicely illustrate the procedure of resummation and Stokes phenomena within the contexts of string theory and the large $N$ approximation.

\section{$3 \quad$ Lefschetz Thimbles and Linear Problems}

Having understood most of the basics concerning resurgence - within a very simple toy-model given by an ordinary one-dimensional integral - one would next like to bring these powerful techniques into the realm of quantum field theory. Following in analogy with what was done at the beginning of section 2, let us first consider the generalization of the saddle-point method into higher (but still finite) dimensions, which will provide a basis for a more systematic approach. This generalization entails higher-dimensional analogues of steepest-descent contours, which go by the name of Lefschetz thimbles (essentially, steepest-descent hypersurfaces), and the whole mathematical machinery surrounding it is known as Picard-Lefschetz theory (which has been 
considered since long ago within the mathematical-physics literature [196, 115, 89]). These methods relate to the theory of resurgence via hyperasymptotics, see, e.g., [43, 44, 41, 153, 50, 154, 191, 59]. Further, given their natural use in the evaluation of multi-dimensional integrals, they have also entailed recent developments and activity with the hope of opening a door towards a better understanding of both quantum-theoretic functional integrals, e.g., [229, 230, 142, 61, 218, 217, 160, 31, 219, 30, 125, 104, 220], and lattice field theory, e.g., [82, 187, 121, 3, 4, 6, 5, 190]. For most of this section we shall restrict to finite-dimensional integrals.

Let us begin with a $d$-dimensional "partition function", given by the integral

$$
Z(\hbar)=\frac{1}{(2 \pi)^{d}} \int_{\Gamma} \mathrm{d} z^{1} \wedge \cdots \wedge \mathrm{d} z^{d} \mathrm{e}^{-\frac{1}{\hbar} V(\boldsymbol{z})} .
$$

Herein, the potential $V(\boldsymbol{z})$ is a polynomial ${ }^{56}$ in the $d$ complex variables which we have collectively denoted by $\boldsymbol{z} \equiv\left(z^{1}, \ldots, z^{d}\right) \in \mathbb{C}^{d}$, and $\hbar \in \mathbb{C}$ as before. We shall further assume that the critical points of the potential, $V$, are isolated and non-degenerate, i.e., the determinant of the Hessian matrix of $V$, Hess $\equiv \operatorname{det}\left(\partial_{a} \partial_{b} V\right)$, at these critical points is non-zero.

Now, in order to give a precise meaning to the partition function (3.1), we still need to know over which integration cycle(s), $\boldsymbol{\Gamma}$, is the integral convergent. Further, we would like to know how this cycle changes as one changes the phase of $\hbar$, a feature which was already discussed in detail in the simpler context of section 2 (recall figure 2). Clearly, a cycle over which (3.1) is convergent for, say, $\hbar>0$, will be different from a convergent cycle with $\hbar<0$. Without surprise, we shall see how the change which the integration cycle undergoes, as one varies the phase of $\hbar$, is intimately related to Stokes phenomenon. Finally, we also want to learn how to explicitly evaluate the partition function, which usually can only be done as an asymptotic expansion of $Z(\hbar)$ for small coupling $\hbar$. As already advertised, we shall use the mathematical machinery of Picard-Lefschetz theory to answer these questions, which by generalizing the notion of steepestdescent contours to higher complex-dimensions further gives a topological interpretation to the possible integration cycles which ensure convergence of the partition-function integral.

\section{Constructing the Lefschetz Thimbles}

Our first goal is thus to classify the set of integration cycles over which the integral (3.1) is convergent. Convergence can be achieved by simply requiring $\mathbb{R e}\left(-\frac{1}{\hbar} V(\boldsymbol{z})\right) \rightarrow-\infty$ as $|\boldsymbol{z}| \rightarrow+\infty$. Of course that, depending on the form of the potential $V(\boldsymbol{z})$, there may be distinct regions in $\mathbb{C}^{d}$ which satisfy this requirement. To be more more precise, let us introduce the set

$$
\mathcal{X}_{\epsilon}=\left\{\boldsymbol{z} \in \mathbb{C}^{d} \mid \mathbb{R e}\left(-\frac{1}{\hbar} V(\boldsymbol{z})\right)=-\frac{1}{\epsilon}\right\},
$$

with $\epsilon$ a small (but positive) number. Generically this set will be made of disjoint subsets, with these different subsets corresponding to the different asymptotic regions that a convergent integration cycle may have. Recalling the example of the quartic potential, discussed in section 2 , the set $\mathcal{X}_{\epsilon}$ then had four disjoint subsets, each of which a region far away from the origin inside each of the four sectors associated to the four brown regions in figures 1 and 2. The non-trivial and convergent integration contours thus became those which join two distinct such regions. In fact, the integral will depend solely on which regions does the integration cycle join, and not on the specific (local) details of the contour itself (as long as, along its path, this contour does not

\footnotetext{
${ }^{56}$ In section 6 we shall discuss an example involving a meromorphic function rather than a polynomial potential. Nevertheless, most of the constructions in this section will apply to that example, with only slight modifications.
} 
cross any other critical point than the one which was initially attached to it). This is the key point which will assign a topological interpretation to our multi-dimensional integration cycles.

In fact, the main result we now wish to discuss is that any integration cycle, $\boldsymbol{\Gamma}$, over which (3.1) is convergent, is a $d$-cycle with integer coefficients. Furthermore, the partition-function integral will subsequently only depend upon the homology class of $\boldsymbol{\Gamma}$ in the relative ${ }^{57}$ homology group $H_{d}\left(\mathbb{C}^{d}, \mathcal{X}_{\epsilon} ; \mathbb{Z}\right)[196,115,229]$. Now, the properties of this relative homology group depend only upon the local properties of the potential, $V$, near its critical points. Moreover, a basis for this homology group may be identified with the equivalence classes of integration cycles which intersect only one $e^{58}$ critical point, $\boldsymbol{z}_{i}^{*}$, and which we shall denote by $\left\{\mathcal{J}_{i}\right\}$. It then turns out that, in this case, the original cycle $\boldsymbol{\Gamma}$ can be decomposed as

$$
\boldsymbol{\Gamma}=\sum_{i=1}^{N} n_{i} \mathcal{J}_{i}
$$

leading to

$$
\int_{\Gamma} \mathrm{d} z^{1} \wedge \cdots \wedge \mathrm{d} z^{d}=\sum_{i=1}^{N} n_{i} \int_{\mathcal{J}_{i}} \mathrm{~d} z^{1} \wedge \cdots \wedge \mathrm{d} z^{d},
$$

where the $n_{i}$ 's are integers and $N$ is the total number of critical points. The cycles $\mathcal{J}_{i}$ are what is known as the Lefschetz thimbles. Let us justify this construction in the following.

In order to construct the thimbles, start by labeling the critical points of the potential $V$ $\operatorname{as}^{59} \boldsymbol{z}_{i}^{*}$ with $i \in\{1,2, \ldots, N\}$; satisfying

$$
\left.\frac{\partial V}{\partial z^{a}}\right|_{\boldsymbol{z}=\boldsymbol{z}_{i}^{*}}=0
$$

Intuitively, if we start integrating out from a critical point $\boldsymbol{z}_{i}^{*}$, convergence of the partitionfunction integral can be ensured by requiring

$$
\rho(\boldsymbol{z}) \equiv \mathbb{R e}\left(-\frac{1}{\hbar}\left(V(\boldsymbol{z})-V\left(\boldsymbol{z}_{i}^{*}\right)\right)\right) \leq 0
$$

along the integration cycle. Moreover, this convergence will be fastest along that collection of curves (parameterized by $\tau$ ) which flow along the gradient of the above function, i.e., along

$$
\frac{\mathrm{d} \boldsymbol{z}}{\mathrm{d} \tau}=-2 \frac{\partial \rho}{\partial \overline{\boldsymbol{z}}}
$$

This set of equations (3.7) is known as the gradient flow equations [115, 159]. It is then straightforward to show that $\rho(\boldsymbol{z}(\tau))$ decreases along any curve with increasing $\tau$ :

$$
\frac{\mathrm{d} \rho(\boldsymbol{z}(\tau))}{\mathrm{d} \tau}=\frac{\partial \rho}{\partial \boldsymbol{z}} \cdot \frac{\mathrm{d} \boldsymbol{z}}{\mathrm{d} \tau}=-2\left|\frac{\partial \rho}{\partial \boldsymbol{z}}\right|^{2} \leq 0
$$

\footnotetext{
${ }^{57} \mathrm{~A} d$-cycle $\gamma$ in $\mathbb{C}^{d}$ is called a relative cycle $\bmod \mathcal{X}$ if its boundary, $\partial \gamma$, lies in $\mathcal{X}$ (if we think of $\mathbb{C}^{d}$ as a $2 d$ dimensional real manifold, then $\mathcal{X}$ is a $(2 d-1)$-dimensional real manifold). A natural equivalence class of relative $d$-cycles is simply constructed by saying that two cycles $\gamma$ and $\gamma^{\prime}$ are equivalent iff $\gamma-\gamma^{\prime}$ is homologous to zero. Then the relative homology group $H_{d}\left(\mathbb{C}^{d}, \mathcal{X} ; \mathbb{Z}\right)$ is the set of these equivalence classes with integer coefficients.

${ }^{58}$ The violation of this requirement concerning critical points leads to Stokes phenomenon, to be discussed later.

${ }^{59}$ In order to avoid notational confusion, we shall denote the critical points with a subscript $\{i, j, \ldots\}$, and the coordinate vector index, whenever present, with a superscript $\{a, b, \ldots\}$.
} 
Such solutions define downward flows out from a given critical point. In this context, the function $\rho(\boldsymbol{z})<\rho\left(\boldsymbol{z}_{i}^{*}\right)$ may be further interpreted as a height function, in the Morse-theory sense.

It is the set of all points that may be reached out from a given critical point, $\boldsymbol{z}_{i}^{*}$, via downward flow as above, which is called a Lefschetz thimble (associated with the critical point ${ }^{60}$ $\boldsymbol{z}_{i}^{*}$ ). Expanding the potential around the critical point, one can use Morse theory to show that there are $d$ real, orthogonal directions along which one can flow downwards. Therefore, the thimble generated by the collection of all such flows is a $d$-real-dimensional cycle. To be more precise, consider a $(d-1)$-sphere encircling the critical point, and pick any point on this sphere. Following the trajectory of such point along the gradient flow (3.7) will generate a curve in $\mathbb{C}^{d}$. The collection of all curves that originate from all points on the $(d-1)$-sphere is the $d$ dimensional real manifold which is the Lefschetz thimble. This $(d-1)$-sphere is known as the vanishing cycle; vanishing at the critical point (for $d=1$, the vanishing cycle is just two points).

Another important feature of the gradient flow curves is that the imaginary part of the potential is constant along them, and hence remains unchanged on the whole thimble. This implies that the thimble may be viewed as the natural higher-dimensional generalization of the stationary-phase contour (recall (2.11)), with

$$
\operatorname{Im}\left(-\frac{1}{\hbar}\left(V(\boldsymbol{z})-V\left(\boldsymbol{z}_{i}^{*}\right)\right)\right)=0
$$

holding true everywhere on the thimble ${ }^{61}$. To see how this comes about, let us split the complex coordinates $\boldsymbol{z}$ into their real and imaginary parts, $\boldsymbol{z}=\boldsymbol{x}+\mathrm{i} \boldsymbol{y}$, and use the Cauchy-Riemann equations to obtain

$$
\begin{aligned}
& \frac{\mathrm{d} \boldsymbol{x}}{\mathrm{d} \tau}=-\frac{\partial \rho}{\partial \boldsymbol{x}}=-\frac{\partial}{\partial \boldsymbol{y}} \operatorname{Im}\left(-\frac{1}{\hbar} V(\boldsymbol{z})\right), \\
& \frac{\mathrm{d} \boldsymbol{y}}{\mathrm{d} \tau}=-\frac{\partial \rho}{\partial \boldsymbol{y}}=\frac{\partial}{\partial \boldsymbol{x}} \operatorname{Im}\left(-\frac{1}{\hbar} V(\boldsymbol{z})\right),
\end{aligned}
$$

from where it follows

$$
\frac{\mathrm{d}}{\mathrm{d} \tau} \operatorname{Im}\left(-\frac{1}{\hbar} V(\boldsymbol{z})\right)=\frac{\mathrm{d} \boldsymbol{x}}{\mathrm{d} \tau} \cdot \frac{\partial}{\partial \boldsymbol{x}} \operatorname{Im}\left(-\frac{1}{\hbar} V(\boldsymbol{z})\right)+\frac{\mathrm{d} \boldsymbol{y}}{\mathrm{d} \tau} \cdot \frac{\partial}{\partial \boldsymbol{y}} \operatorname{Im}\left(-\frac{1}{\hbar} V(\boldsymbol{z})\right)=0 .
$$

In fact the gradient flow equations, combined with the Cauchy-Riemann equations (3.10) and (3.11), define a Hamiltonian flow where the imaginary part $\operatorname{Im}\left(-\frac{1}{\hbar} V(\boldsymbol{z})\right)$ is the conserved quantity (the "Hamiltonian") associated with this flow. One can combine the gradient flow equations for the real part, and the Hamiltonian flow equations for the imaginary part, into a single equation in complex variables (where we also make explicit the phase of $\hbar$, as in $\hbar=|\hbar| \mathrm{e}^{\mathrm{i} \theta}$ )

$$
\frac{\mathrm{d} z^{a}}{\mathrm{~d} \tau}=\frac{\mathrm{e}^{\mathrm{i} \theta}}{|\hbar|} \frac{\partial \bar{V}}{\partial \bar{z}^{a}}
$$

with the boundary condition $z^{a}(\tau \rightarrow-\infty)=\left(z_{i}^{*}\right)^{a}$. This is the complex gradient flow equation, also known as Picard-Lefschetz equation, whose solutions then naturally satisfy (3.9).

\footnotetext{
${ }^{60}$ Following our assumption that there is only one critical point associated with a given thimble - and deferring the discussion of Stokes phenomenon, which occurs when this assumption is violated, to the end of this section.

${ }^{61}$ Of course that generically (3.9) alone is not enough to define the thimble, unlike in our earlier one-dimensional stationary-phase contours. This is essentially because (3.9) defines a real-codimension-one surface, whereas the thimble is in fact a real-codimension- $d$ surface, defined via the collection of gradient-flow curves described above.
} 
In complete analogy to the downward flow, one may likewise define upward flow as originating from a critical point and defined by the equations

$$
\frac{\mathrm{d} z^{a}}{\mathrm{~d} \tau}=-\frac{\mathrm{e}^{\mathrm{i} \theta}}{|\hbar|} \frac{\partial \bar{V}}{\partial \bar{z}^{a}} .
$$

Along the upward flow the imaginary part of the potential still remains constant, but the real part $\rho(\boldsymbol{z})$ now increases. Morse theory again tells us that there will be $d$ real directions along which one can flow upwards. As such, and akin to the Lefschetz thimble construction, all the points in $\mathbb{C}^{d}$ which may be reached via upward flow originating on an $(d-1)$-sphere surrounding the critical point $\boldsymbol{z}_{i}^{*}$, will construct a $d$-dimensional cycle which we denote by $\mathcal{K}_{i}$. The set of all these cycles (upon adequate equivalence classes) constitute a basis for a dual homology class $H_{d}\left(\mathbb{C}^{d}, \mathcal{Y}_{\epsilon} ; \mathbb{Z}\right)$, where

$$
\mathcal{Y}_{\epsilon}=\left\{\boldsymbol{z} \in \mathbb{C}^{d} \mid \mathbb{R e}\left(-\frac{1}{\hbar} V(\boldsymbol{z})\right)=+\frac{1}{\epsilon}\right\} .
$$

Note that since the imaginary part of the potential associated with each critical point is a conserved quantity, and generically the imaginary parts of $V$ at different critical points are distinct, this implies that the downward/upward flows originating from different critical points do not generically intersect each other. However, when this does happen to be the case, these cycles form a good basis for the relative homology groups we started off with, above. Given two cycles $\boldsymbol{\Gamma}$ and $\boldsymbol{\Gamma}^{\prime}$, one can define an inner product via the intersection number

$$
\left\langle\mathcal{K}_{n}, \mathcal{J}_{m}\right\rangle=\delta_{n m}
$$

This inner product is a map $H_{d}\left(\mathbb{C}^{d}, \mathcal{Y}_{\epsilon} ; \mathbb{Z}\right) \otimes H_{d}\left(\mathbb{C}^{d}, \mathcal{X}_{\epsilon} ; \mathbb{Z}\right) \rightarrow \mathbb{Z}$ which tells us when does a given downward-flowing thimble intersects some upward-flowing thimble. In the generic case described above, where all the imaginary parts of the potential at different critical points are distinct, such an intersection never happens - except at the critical point itself. Moreover, this intersection occurs between the upward and downward flows originating from that same critical point leading to (3.16). Consequently, any integration cycle $\boldsymbol{\Gamma}$ such that (3.1) is convergent, can be decomposed as a chain $[196]$

$$
\boldsymbol{\Gamma}=\sum_{i=1}^{N} n_{i} \mathcal{J}_{i},
$$

where $n_{i}=\left\langle\mathcal{K}_{i}, \boldsymbol{\Gamma}\right\rangle$. This conclusion finally justifies our starting main equations, (3.3) and (3.4).

\section{Evaluation of the Asymptotic Expansion}

Having understood the integration cycles (3.4), let us now try to explicitly evaluate the partitionfunction integral (3.1). Generically, this will entail an asymptotic series expansion in powers of $\hbar$. To start off with, let us impose a constraint regarding the critical points; which is that no pair of critical values has the same imaginary part, i.e.,

$$
\operatorname{Im}\left(\frac{1}{\hbar} V\left(\boldsymbol{z}_{i}^{*}\right)\right) \neq \mathbb{I m}\left(\frac{1}{\hbar} V\left(\boldsymbol{z}_{j}^{*}\right)\right) \quad \text { for } \quad i \neq j .
$$

Since the imaginary part is a conserved quantity along the thimble, this assumption guarantees that there are no intersections between thimbles associated with different critical points, and the decomposition of the integration cycle (3.17) is thus well defined. In fact, this condition 
generically holds except for a finite set of particular values that $\theta=\arg (\hbar)$ takes. These are the points where Stokes phenomenon occurs, to be discussed a bit later on. But for the moment let us simply assume a generic value of $\theta$ for which there is no Stokes phenomenon (and thus also suppress the dependence on $\theta$ from our notation).

Let us consider the partition-function integral over some particular thimble, $\mathcal{J}_{n}$, associated with the critical point $\boldsymbol{z}_{n}^{*}$. Introducing the variable $s \equiv V(\boldsymbol{z})-V\left(\boldsymbol{z}_{n}^{*}\right)$, we can write this integral as

$$
Z^{(n)}(\hbar)=\frac{1}{(2 \pi)^{d}} \int_{\mathcal{J}_{n}} \mathrm{e}^{-\frac{1}{\hbar} V(\boldsymbol{z})} \Omega=\mathrm{e}^{-\frac{1}{\hbar} V_{n}^{*}} \int_{0}^{\mathrm{e}^{\mathrm{i} \theta} \infty} \mathrm{d} s \mathcal{B}\left[\Psi_{n}\right](s) \mathrm{e}^{-\frac{s}{\hbar}},
$$

where $\Omega=\mathrm{d} z^{1} \wedge \cdots \wedge \mathrm{d} z^{d}$ is the volume form and $V_{n}^{*} \equiv V\left(\boldsymbol{z}_{n}^{*}\right)$ is the value of the potential at the critical point $\boldsymbol{z}_{n}^{*}$. Remarkably, this change of variables is actually converting our original integral into a Borel-type integral, as in (2.3) (hence the notation!). In fact, it will turn out that the function

$$
\left.\mathcal{B}\left[\Psi_{n}\right](s) \equiv \frac{1}{(2 \pi)^{d}} \int_{\mathcal{V}_{n}(s)} \frac{\Omega}{\mathrm{d} s}\right|_{V(\boldsymbol{z})=V_{n}^{*}+s}
$$

is precisely a generalized Borel transform, of the type we shall discuss in the upcoming section 4, and later denoted by $\mathcal{B}\left[\Psi_{[d / 2]}\right](s)$ (but herein let us keep denoting it by just $\mathcal{B}[\Psi](s)$ ). The final integral in (3.19), over $s$, is along a ray which we shall refer to as $\Sigma_{n}$, such that the exponent $s / \hbar$ is positive and bounded from below ensuring convergence. The remaining $(d-1)$-dimensional integral in (3.20) is over a $(d-1)$-cycle, $\mathcal{V}_{n}(s)$, which is nothing but the vanishing cycle transported to the point $s$ along $\Sigma_{n}$. As such, the thimble $\mathcal{J}_{n}$ can be thought of as a $d$-dimensional cycle generated by the orbit of the vanishing cycle along $\Sigma_{n}$.

Around the origin, $\mathcal{B}\left[\Psi_{n}\right](s)$ has a convergent series expansion ${ }^{62}$ of the form

$$
\mathcal{B}\left[\Psi_{n}\right](s)=s^{\frac{d}{2}-1} \sum_{k=0}^{+\infty} B_{k}^{(n)} s^{k} .
$$

Notice that for odd dimensions there is a square root branch-cut, whereas for even dimensions the function is analytic near the origin. The above leading term is easy to calculate. Around the critical point (i.e., $s=0$ ), we can expand $V\left(\boldsymbol{z}_{n}^{*}+\boldsymbol{z}\right) \approx V_{n}^{*}+\frac{1}{2} H_{a b}^{n} z^{a} z^{b} \equiv V_{n}^{*}+s$, where $H_{a b}^{n} \equiv \operatorname{Hess}[V]\left(\boldsymbol{z}_{n}^{*}\right)$ is the Hessian matrix of the potential, $V$, at the critical point $\boldsymbol{z}_{n}^{*}$. Therefore, the vanishing cycle $\mathcal{V}_{n}(s)$ is a $(d-1)$-sphere whose radius is $\sqrt{|s|}$ and (3.20) reduces to a surface integral over this sphere, yielding

$$
\mathcal{B}\left[\Psi_{n}\right](s) \approx \frac{1}{\sqrt{(2 \pi)^{d} \operatorname{det}\left(\operatorname{Hess}[V]\left(\boldsymbol{z}_{n}^{*}\right)\right)}} \frac{s^{\frac{d}{2}-1}}{\Gamma\left(\frac{d}{2}\right)} .
$$

\footnotetext{
${ }^{62}$ This expansion can be generalized to include the cases where the critical points are degenerate and/or the integration cycles have boundaries. For the degenerate case, one "Morsifies" the critical point by perturbing it away from the degenerate case. The perturbation deforms the degenerate critical point into a $\mu_{n}$ number of nondegenerate critical points, where $\mu_{n}$ is the Milnor number of the original critical point. In this case the Lefschetz thimble $\mathcal{J}_{n}$ develops $\mu_{n}$ foldings in the limit where the perturbation vanishes. Furthermore, $\mathcal{B}\left[\Psi_{n}\right](s)$ is analytic over the universal covering of the punctured disk $\mathbb{C} \backslash\{0\}$ and satisfies an ordinary differential equation, known as a Picard-Fuchs equation or a Gauss-Manin connection, which has at most a regular singularity at the origin. The order of this Gauss-Manin connection can be greater than one, but is bounded by $\mu_{n}$ and the expansion of $\mathcal{B}\left[\Psi_{n}\right]$ near the origin will have powers of $\log s$ in addition to $s$. For more details, we refer the reader to [173, 196, 197, 89].
} 
The rest of the coefficients may be computed depending on the specific details of the potential $V$. Evaluating the remaining $s$-integral then leads to an asymptotic ${ }^{63}$ expansion for $Z^{(n)}(\hbar)$

$$
\begin{aligned}
Z^{(n)}(\hbar) & \simeq \mathrm{e}^{-\frac{1}{\hbar} V_{n}^{*}} \hbar^{\frac{d}{2}} \sum_{k=0}^{+\infty} \Gamma\left(k+\frac{d}{2}\right) B_{k}^{(n)} \hbar^{k} \\
& \simeq \frac{\mathrm{e}^{-\frac{1}{\hbar} V_{n}^{*}}}{\sqrt{(2 \pi)^{d} \operatorname{det}\left(\operatorname{Hess}[V]\left(\boldsymbol{z}_{n}^{*}\right)\right)}} \hbar^{\frac{d}{2}}+\mathrm{e}^{-\frac{1}{\hbar} V_{n}^{*}} \hbar^{\frac{d}{2}} \sum_{k=1}^{+\infty} Z_{k}^{(n)} \hbar^{k}
\end{aligned}
$$

where we have redefined $Z_{k}^{(n)} \equiv \Gamma(k+d / 2) B_{k}^{(n)}$.

Now that we have all the basic building blocks of the partition function (3.1)-i.e., the asymptotic expansions of the thimble integrations (3.19) as written above - we may construct the final asymptotic expansion of $Z(\hbar)$ over the cycle $\boldsymbol{\Gamma}$. Using the decomposition of $\boldsymbol{\Gamma}$ into thimbles (3.17), we find that the partition function in fact has a transseries form, as

$$
Z(\hbar)=\frac{1}{(2 \pi)^{d}} \int_{\Gamma} \mathrm{e}^{-\frac{1}{\hbar} V(\boldsymbol{z})} \Omega=\hbar^{\frac{d}{2}} \sum_{i=1}^{N} n_{i} \mathrm{e}^{-\frac{1}{\hbar} V_{i}^{*}} \Phi_{i}(\hbar),
$$

where each $\Phi_{i}(\hbar)$ is an asymptotic series

$$
\Phi_{i}(\hbar) \simeq \sum_{k=0}^{+\infty} Z_{k}^{(i)} \hbar^{k}
$$

whose coefficients are obtained by the local expansion of the vanishing-cycle integral (3.20) around the critical point $\boldsymbol{z}_{i}^{*}$, which is a solution of the Gauss-Manin connection. To sum up, each term $\mathrm{e}^{-\frac{1}{\hbar} V_{i}^{*}} \Phi_{i}(\hbar)$ in the transseries is associated with an element of a basis of a homology group, where the topology is induced by restricting the allowed integration cycles into regions in $\mathbb{C}^{n}$ that ensure the convergence of the integral $Z(\hbar)$. The asymptotic expansion of a given term is constructed locally by the geometric properties of the vanishing cycle.

With the total number of critical points $N$ a finite number, our final solution (3.25) also has a finite number of multi-instanton sectors and is thus an appropriate solution to some linear problem (in the distinction already discussed in section 2). One could now be tempted to think that extending this formalism to situations with an infinite number of multi-instanton sectors simply amounts to taking the limit $N \rightarrow+\infty$ in (3.25). This would cover nonlinear problems and eventually quantum field theoretic functional-integrals. But while for the former there may not necessarily exist any adequate integral representation of solutions, invalidating the whole procedure, for the latter one needs to be careful with subtleties due to renormalization [162].

Indeed, let us consider the partition function of some scalar quantum field theory,

$$
Z(\lambda)=\int[\mathrm{d} \varphi] \mathrm{e}^{-S(\varphi)},
$$

with action $S(\varphi)$ and coupling-constant $\lambda$. In order to use the toolbox described in this section, one would first want to factor out the coupling constant, as in

$$
S(\varphi) \rightarrow \frac{1}{\lambda} S(\widetilde{\varphi})
$$

\footnotetext{
${ }^{63}$ This is actually made explicit in the formula, due to the $\Gamma(k+d / 2)$ factor.
} 
and then compute (functional) saddle points for the functional-integral partition function (3.27). But this factoring can only be done when considering bare quantities. As renormalization kicks in, the coupling runs as $\lambda \equiv \lambda(\mu)$ at energy-scale $\mu$, further inducing mass renormalization and wave-function renormalization. For our purposes, the overall effect of such renormalization procedure is to invalidate the above factoring. One rather finds (very schematically - see [162] for a full discussion)

$$
S(\varphi) \rightarrow \frac{1}{\lambda} S_{-1}(\widetilde{\varphi})+S_{0}(\widetilde{\varphi})+\lambda S_{1}(\widetilde{\varphi})+\lambda^{2} S_{2}(\widetilde{\varphi})+\cdots
$$

This automatically implies that in order to address renormalizable quantum field theories, one will need more sophisticated saddle-point methods and answers than just (3.25), and likely more complicated transseries structures than the ones we have been addressing so far (see section 5 or, e.g., [110], for some such structures; and [2] for recent results pointing towards the need to include some of them within Yang-Mills/QCD theories). Do notice that this caveat is very much attached to any renormalization needs. In quantum field theoretic contexts where such needs do not arise, the transseries structures addressed so far are certainly enough; see, e.g., [14, 148150, 152] for supersymmetric gauge theories, e.g., [179, 175, 180, 163, 97, 16, 205, 80] for large $N$ gauge theories, and, e.g., $[174,195,178,130,77,135,78,76,79,65,66]$ for string theories.

\section{Dealing with Stokes Phenomena}

The transseries construction of the partition function in (3.25) arises from a local analysis on the Lefschetz thimbles, near their corresponding critical points. But, as we have been postponing for a while, there are also global aspects on the thimbles (and hence of the transseries) which need to be considered; most notably those leading to Stokes phenomena. In order to understand how global issues may come about, let us go back to the evaluation of the partition-function integral over some specific thimble $\mathcal{J}_{n}$, denoted by $Z^{(n)}(\hbar)$ in (3.19). Its asymptotic expansion was generated by a Laplace-type integration of $\mathcal{B}\left[\Psi_{n}\right](s)$ (defined in (3.20)) along some ray $\Sigma_{n}$ of direction $\theta$ on the complex $s$-plane. However, the function $\mathcal{B}\left[\Psi_{n}\right](s)$ may, in general, have singularities, which one can hit by varying the ray of integration as one varies $\theta$. These singularities of $\mathcal{B}\left[\Psi_{n}\right](s)$ occur precisely at the critical points; which follows from the fact that

$$
\mathrm{d} s=\sum_{a=1}^{d} \frac{\partial V}{\partial z^{a}} \mathrm{~d} z^{a}
$$

vanishes at a critical point. Previously, we have required that no two critical values of the potential had the same imaginary part; recall (3.18). It was this assumption which saved us so far from hitting a singularity along the $s$-integration: because the imaginary part of the potential is conserved on a thimble, integration along a fixed thimble could not hit another critical pointwith the same holding true for the projections of the Lefschetz thimbles onto rays of integration as in (3.19). Let us now relax this assumption and discuss what happens as we cross a singularity.

For simplicity, let us begin by assuming that there is only one critical point to be crossed, which we shall label by $m$. The location of this singularity is $s=V\left(\boldsymbol{z}_{m}^{*}\right)-V\left(\boldsymbol{z}_{n}^{*}\right)=V_{m}^{*}-V_{n}^{*} \equiv$ $V_{n m}^{*}$, which is nothing but the relative potential of the critical points $n$ and $m$. In order for the integration in (3.19) to be well defined, the integration contour needs to avoid this singularity. This can be done by avoiding it either from above or from below, by simply shifting to either $\theta^{+}$ or $\theta^{-}$. Each of these shifts will yield a different result for the partition function $Z^{(n)}(\hbar)$, which we shall denote by $Z_{ \pm}^{(n)}(\hbar)$, respectively. Their difference, $Z_{+}^{(n)}(\hbar)-Z_{-}^{(n)}(\hbar)$, essentially yields 
the monodromy that $\mathcal{B}\left[\Psi_{n}\right](s)$ acquires as one moves from $\theta^{-}$to $\theta^{+}$. This monodromy is induced by the monodromy of the vanishing cycle, $\mathcal{V}_{n}(s)$, as ones goes around the singularity $V_{n m}^{*}$ in a counter-clockwise fashion, and which is captured by the Picard-Lefschetz formula [170]

$$
\mathcal{V}_{n}\left(\mathrm{r}_{n m} \mathrm{e}^{\mathrm{i}(\phi+2 \pi)}\right)=\mathcal{V}_{n}\left(\mathrm{r}_{n m} \mathrm{e}^{\mathrm{i} \phi}\right)+(-1)^{\frac{d(d-1)}{2}} \mathrm{~S}_{n m} \mathcal{V}_{m}\left(\mathrm{r}_{n m} \mathrm{e}^{\mathrm{i} \phi}\right) .
$$

Here, $\mathbf{r}_{n m} \mathrm{e}^{\mathrm{i} \phi}$ parameterizes an infinitesimal circle around $V_{n m}^{*}$, and the coefficient $\mathrm{S}_{n m}$ is the intersection number of the two vanishing cycles, $\mathrm{S}_{n m} \equiv\left\langle\mathcal{V}_{n}, \mathcal{V}_{m}\right\rangle \in \mathbb{Z}$. Formula (3.31) shows how when the vanishing cycle $\mathcal{V}_{n}$, associated to the critical point $n$, is moved along a counterclockwise closed loop around the projection of the critical point $m$ (i.e., around the singular point at $s=V_{n m}^{*}$ ), then the resulting cycle will receive an additional contribution given by $\mathcal{V}_{m}$, the vanishing cycle associated with the critical point $m$.

From the Picard-Lefschetz expression (3.31), one can further deduce the monodromy of $\mathcal{B}\left[\Psi_{n}\right](s)$ around the singularity $V_{n m}^{*}$. Simply plugging the monodromy (3.31) in (3.20), one obtains

$$
\mathcal{B}_{-}\left[\Psi_{n}\right](s)=\mathcal{B}_{+}\left[\Psi_{n}\right](s)+(-1)^{\frac{d(d-1)}{2}} \mathrm{~S}_{n m} \mathcal{B}_{+}\left[\Psi_{m}\right](s) .
$$

Note that, since the $s$-integration in (3.19) basically corresponds to a Borel resummation, what we are describing here by tilting $\theta$ to $\theta^{ \pm}$in order to avoid the singularity is essentially the lateral Borel resummations which were discussed at the beginning of section 2 (recall (2.4)). The resulting monodromy between the different lateral Borel resummations describes the Stokes phenomenon (2.7), where the intersection numbers $S_{n m}$ relate to the Stokes constants. The computation of Stokes constants within this framework is a technically challenging problem, and for an extensive discussion along the present context we refer the reader to, e.g., [153, 43, 44].

It is worth mentioning that the resurgent relations take a standard form in this setting [89]

$$
\begin{aligned}
& \left.\mathcal{B}\left[\Psi_{n}\right](s)\right|_{s=V_{n m}^{*}}=\mathrm{S}_{n m} \mathcal{B}\left[\Psi_{m}\right]\left(s-V_{n m}^{*}\right) \frac{\log \left(s-V_{n m}^{*}\right)}{2 \pi \mathrm{i}}+\text { holomorphic, } \quad d=\text { even } \\
& \left.\mathcal{B}\left[\Psi_{n}\right](s)\right|_{s=V_{n m}^{*}}=-\frac{1}{2} \mathrm{~S}_{n m} \mathcal{B}\left[\Psi_{m}\right]\left(s-V_{n m}^{*}\right)+\text { holomorphic }, \quad d=\text { odd }
\end{aligned}
$$

As comparing to (2.62) - where we were dealing with $\Phi$-sectors, which relate to the $\Psi$-sectors herein as $\Psi=\hbar^{\frac{d}{2}} \Phi$ - one should match $V_{n m}^{*} \leftrightarrow k A$ and $(-1)^{d} \mathrm{~S}_{n m} \leftrightarrow-\mathrm{S}_{n \rightarrow n+k}+\cdots$ (so that the $\mathrm{S}_{n m}$ intersection numbers essentially are Borel residues). An example illustrating how to carry through this match will be discussed in the upcoming section 4. Furthermore, the generalization of the above discussion to the case of multiple singularities is conceptually straightforward. One just has to use (3.32), but where one should now sum over all the critical points $m$ that are found along the path. Finally, all the critical points whose singularities show up in the path of $n$, are below $n$ in the Morse-theory sense, i.e., $\mathbb{R e}\left(-\frac{1}{\hbar} V_{n}^{*}\right)>\mathbb{R e}\left(-\frac{1}{\hbar} V_{m}^{*}\right)>\cdots$.

It is also interesting to understand the relation between Stokes phenomenon and the thimble decomposition. The rays of integration in (3.19), $\Sigma_{n}$, are the projections of the Lefschetz thimbles upon the $s$-plane. Therefore, the aforementioned monodromy structure on the $s$-plane can be lifted onto a monodromy structure for the thimbles. In particular from (3.32) and (3.19) one gets $^{64}$

$$
\mathcal{J}_{n}\left(\theta^{-}\right)=\mathcal{J}_{n}\left(\theta^{+}\right)+(-1)^{\frac{d(d-1)}{2}} \sum_{m=1}^{N} \mathrm{~S}_{n m} \mathcal{J}_{m}\left(\theta^{+}\right) .
$$

\footnotetext{
${ }^{64}$ At this stage, the reader may want to review the steepest-descent discussion of Stokes phenomena from subsection 2.2, which took place in the paragraph after equation (2.38).
} 
As a result, our original thimble decomposition (3.17) ceases to be well-defined at $\theta$, precisely because the intersection numbers for the thimbles, the $n_{i}$ 's, are not well defined at these values. Instead, the intersection numbers acquire a $\theta$-dependence, as in $n_{i} \equiv n_{i}(\theta)$, and become piecewiseconstant functions of $\theta$. Integer-valued jumps occur for certain values of $\theta$, which are encoded by the intersection numbers of the vanishing cycles, or "Stokes constants", $\mathrm{S}_{n m}$. Consequently we have to refine the decomposition of the chain as

$$
\Gamma(\theta)=\sum_{i=1}^{N} n_{i}(\theta) \mathcal{J}_{i}(\theta),
$$

which eventually finds its way back onto our transseries construction of the partition function, in (3.25). This finally becomes

$$
Z\left(|\hbar| \mathrm{e}^{\mathrm{i} \theta}\right)=\hbar^{\frac{d}{2}} \sum_{i=1}^{N} n_{i}(\theta) \mathrm{e}^{-\frac{1}{\hbar} V_{i}^{*}} \Phi_{i}(\hbar) .
$$

When Stokes phenomenon occurs, the intersection numbers $n_{i}(\theta)$ jump in integer amounts. These jumps are precisely balanced by the jumps that arise from the lateral Borel resummations of $\Phi_{i}(\hbar)$, rendering (3.37) unambiguous. With the knowledge of the Stokes constants, we can therefore give a precise meaning to this expansion as a well-defined, continuous function of $\hbar$.

\section{Borel Transforms and Nonlinear Problems}

Let us now go back to Borel analysis, as discussed throughout section 2, in order to give it a more systematic basis. This is a more general and powerful tool than steepest-descent analysis (and its generalizations): all it requires to start-off is some asymptotic series, regardless of where it came from or how was it computed originally ${ }^{65}$. Its blindness to the origin of the asymptotic sequences at play implies that it addresses linear and nonlinear problems equally well (as introduced in section 2). Furthermore, from a quantum field theoretic standpoint, it always works for any observable, not being limited to the partition function, or to functional integrals or functional differential equations, or whatever else; and not caring if the nonperturbative effects at hand are multi-instantons, or multi-renormalons, or whatever else. Following a pragmatic point-ofview, one need not dwell much on the origins of any given (perturbative) factorially-divergent sequences, but rather simply focus on how to use them as an input in order to start the resurgence program, building towards finding their (nonperturbative) transseries completions.

In fact, the Borel transform of any such asymptotic sequence is the first step towards implementing the resurgence program: as discussed in section 2, resurgence emerges out of an analysis of Borel singularities, with its algebraic formulation following from an abstraction of the behaviour of these very same Borel transforms near their respective singularities. How Borel transforms are defined, and how resurgence is already evident from their singular structure on the Borel plane, is what we shall discuss in the following and place on firmer grounds. A better understanding of resurgence, as we wish to discuss in the upcoming section 5, implies that we first need to understand Borel analysis in a bit more detail than we have done up to this point.

\footnotetext{
${ }^{65}$ It even works for sequences with rather abstract origins, as for example sequences of enumerative invariants in algebraic geometry [81], or even more abstract combinatorial sequences [48].
} 


\section{The Borel Integral Representation of a Partition Function}

In retrospect, our definition of a Borel transform in (2.2) might now seem a bit vague or imprecise: why were the powers of the $x$ and $s$ variables related the way they were?, or why was the argument of the Gamma-function chosen the way it was? In order to properly define a Borel transform, and understand what kind of freedom such definition will nonetheless still entail, we shall start by going back to our earlier discussion on the Borel-like rewriting of a partition function, in (3.19) and (3.20) (albeit for the present purposes it will be enough to restrict to one-dimensional integrals). This will illustrate the aforementioned freedom in a (by now) familiar setting.

Consider as usual a "partition function", described by a one-dimensional integral ${ }^{66}$

$$
Z^{(n)}(\lambda)=\frac{1}{2 \pi} \int_{\Gamma_{n}} \mathrm{~d} z \mathrm{e}^{-V_{\lambda}(z)} .
$$

Herein we are considering a general analytic potential $V_{\lambda}(z)$, depending on some coupling constant $\lambda$, with a set of saddle-points labelled by $z_{n}^{*}(\lambda)$ and given by its critical points. The integration contour $\Gamma_{n}$ is the steepest descent-contour, or Lefschetz thimble, through the saddle $z_{n}^{*}$. Thus, the above integration defines the contribution to the partition function from each saddle. To make it a bit more explicit, let us expand the potential around this $n$th saddle as ${ }^{67}$

$$
V_{\lambda}(z)=V_{\lambda}\left(z_{n}^{*}\right)+\left.\sum_{k=1}^{N} \frac{\left(z-z_{n}^{*}\right)^{k}}{k !} \frac{\mathrm{d}^{k} V_{\lambda}}{\mathrm{d} z^{k}}\right|_{z=z_{n}^{*}} \equiv V_{n}^{*}(\lambda)+V_{\lambda}^{(n)}(z) .
$$

Rewriting the integral above, (4.1), we then have

$$
Z^{(n)}(\lambda)=\frac{\mathrm{e}^{-V_{n}^{*}(\lambda)}}{2 \pi} \int_{\Gamma_{n}} \mathrm{~d} z \mathrm{e}^{-V_{\lambda}^{(n)}(z)} .
$$

The coupling constant can take any complex value, $\lambda=|\lambda| \mathrm{e}^{\mathrm{i} \theta}$, which implies that the steepest-descent contour itself will depend on its phase, $\theta$, as $\Gamma_{n} \equiv \Gamma_{n}(\theta)$. Having fixed $\theta$, one can then perform the familiar (asymptotic expansion) evaluation of the integral, in powers of the coupling. This results in the usual

$$
Z^{(n)}(\lambda)=\mathrm{e}^{-V_{n}^{*}(\lambda)} \Phi_{n}(\lambda),
$$

where $^{68}$

$$
\Phi_{n}(\lambda) \simeq \lambda^{\beta_{n}} \sum_{k=0}^{+\infty} Z_{k}^{(n)} \lambda^{k}
$$

with the coefficients $Z_{k}^{(n)}$ given by

$$
\left.Z_{k}^{(n)} \equiv \frac{1}{k !} \int_{\Gamma_{n}} \mathrm{~d} z \frac{\mathrm{d}^{k}}{\mathrm{~d} \lambda^{k}} \mathrm{e}^{-V_{\lambda}^{(n)}(z)}\right|_{\lambda=0} .
$$

Of course that in all cases of interest to us, this expansion is asymptotic with the standard large-order behaviour

$$
Z_{k}^{(n)} \sim k !
$$

\footnotetext{
${ }^{66}$ Throughout this section we shall suppress $\hbar$ and only focus on the coupling constant, for the sake of notational simplicity. Of course it can be added back-in straightforwardly, or attached to the coupling as in section 2.

${ }^{67}$ Herein, $N$ could in principle be infinite.

${ }^{68}$ In this expression we are allowing for the leading term to start at some general power, $\beta_{n}$, of the coupling.
} 
$\Phi_{n}(\lambda)$ is thus the asymptotic perturbative expansion of the original partition function around the $z_{n}^{*}$ saddle; an analysis which may be repeated for all saddle-points of the potential.

Following the discussion at the beginning of section 2, one should now Borel transform the asymptotic expansion (4.5) by means of (2.2). This uses the (still not fully justified) assignment where $\lambda^{\alpha} \rightarrow \frac{s^{\alpha-1}}{\Gamma(\alpha)}$ (with the exception of the residual coefficient $Z_{0}^{(n)}$ when there is one, i.e., the leading constant term when $\beta_{n}=0$; and while assuming $\beta_{n} \geq 0$ ). The Borel transform is thus written as

$$
\mathcal{B}\left[\Phi_{n}\right](s)=\sum_{k=0}^{+\infty}\left(1-\delta_{k, 0} \delta_{\beta_{n}, 0}\right) Z_{k}^{(n)} \frac{s^{k+\beta_{n}-1}}{\Gamma\left(k+\beta_{n}\right)} .
$$

This series has a finite, non-zero radius of convergence, whose analytic-continuation yields a structure of singularities and branch-cuts which will give rise to the resurgence properties of the partition function. This partition function can then be properly defined via Borel resummation, (2.3), which is given by a Laplace-type ${ }^{69}$ integral as (for the moment considering the direction of integration along the positive real axis)

$$
Z^{(n)}(\lambda)=\mathrm{e}^{-V_{n}^{*}(\lambda)}\left(Z_{0}^{(n)} \delta_{\beta_{n}, 0}+\int_{0}^{+\infty} \mathrm{d} s \mathcal{B}\left[\Phi_{n}\right](s) \mathrm{e}^{-\frac{s}{\lambda}}\right) .
$$

This is not the only way to find a Borel-like integral-representation of our partition function. For example, one could have instead followed the strategy outlined in the previous section 3, changing the variable of integration from $z$ to the potential as in (3.19). Let us next check whether this leads to the same result as (4.9). First, one needs to assume that there exists a change of variables, from our current integration variable $z$ to a new one $x$, as in $z=z(x, \lambda)$, such that the potential can be rewritten factoring out the coupling constant,

$$
V_{\lambda}(z) \equiv \frac{1}{\lambda} v(x)
$$

In this case, the partition function may be written as

$$
Z^{(n)}(\lambda)=\frac{\mathrm{e}^{-\frac{1}{\lambda} v_{n}^{*}}}{2 \pi} \int_{\Gamma_{n}} \mathrm{~d} x \frac{\mathrm{d} z(x, \lambda)}{\mathrm{d} x} \mathrm{e}^{-\frac{1}{\lambda} v^{(n)}(x)} .
$$

Of course that the contour $\Gamma_{n}$ also changes, as to become a steepest-descent contour in the new variable $x$. We shall proceed by following the discussion in [44], addressing the slightly more general integration problem

$$
Z^{(n)}(\lambda)=\frac{\mathrm{e}^{-\frac{1}{\lambda} v_{n}^{*}}}{2 \pi} \int_{\Gamma_{n}} \mathrm{~d} x g(x, \lambda) \mathrm{e}^{-\frac{1}{\lambda} v^{(n)}(x)},
$$

for some function $g(x, \lambda)$ with an explicit coupling dependence. But we shall nonetheless focus on the situation of interest to the present discussion, where this coupling dependence may be factored out in the form

$$
g(x, \lambda)=\frac{\widetilde{g}(x)}{\sqrt{\lambda}}
$$

Having this in mind, the adequate rewriting of the partition function becomes

$$
Z^{(n)}(\lambda)=\mathrm{e}^{-\frac{1}{\lambda} v_{n}^{*}} \frac{1}{\sqrt{\lambda}} \Psi_{n}(\lambda),
$$

\footnotetext{
${ }^{69}$ In fact, the map $\lambda^{\alpha} \rightarrow \frac{s^{\alpha-1}}{\Gamma(\alpha)}$ is an inverse Laplace transform on each term of the asymptotic series.
} 
where

$$
\Psi_{n}(\lambda)=\frac{1}{2 \pi} \int_{\Gamma_{n}} \mathrm{~d} x \widetilde{g}(x) \mathrm{e}^{-\frac{1}{\lambda} v^{(n)}(x)} .
$$

First note that (4.15) is already of similar form to our earlier Borel resummation in (4.9). To try to make it equivalent, and identify the Borel transform in the process, one is tempted to define the Borel plane as

$$
s \equiv v^{(n)}(x)
$$

Given that the integration contour $\Gamma_{n}$ in (4.15) is a steeepest-descent contour, this seems to guarantee that the corresponding integration upon the $s$-plane, via (4.16), is in fact an integration over the interval $(0,+\infty)$ as one would expect from a Borel resummation (recall (2.3)). However, this change of variables is not without a few caveats, which have to be taken into account in order to find the correct integration contour and corresponding Borel transform. The first thing to notice is that upon inversion of (4.16), to find $x \equiv x(s)$, and due to the general nature of the potential, there can in fact be many roots $\left\{x_{\alpha}(s)\right\}$ which solve this equation. In particular, for a general polynomial potential there will be as many roots as the order of the potential, all of them seemingly resulting in the same integration contour $s \in(0,+\infty)$. Closer inspection reveals that this is not quite so [44]: in fact, it turns out that only two of these roots will pass through the saddle-point $x\left(z_{n}^{*}\right)$ when $s=0$, while having the required limits at infinity as $s \rightarrow+\infty$ (and which are determined by the steepest-descent contour $\Gamma_{n}$ ). These two solutions will be labeled as $x_{+}(s)$, corresponding to the half of the contour $\Gamma_{n}$ which emerges from the saddle point and goes off to infinity; and $x_{-}(s)$, which corresponds to the second half of the contour $\Gamma_{n}$ which comes in from infinity and into the saddle point. The resulting integration (4.15), after the change of variables (4.16), then becomes

$$
\Psi_{n}(\lambda)=\frac{1}{2 \pi} \int_{0}^{+\infty} \mathrm{d} s\left\{\frac{\widetilde{g}\left(x_{+}(s)\right)}{\frac{\mathrm{d} v^{(n)}}{\mathrm{d} x}\left(x_{+}(s)\right)}-\frac{\widetilde{g}\left(x_{-}(s)\right)}{\frac{\mathrm{d} v^{(n)}}{\mathrm{d} x}\left(x_{-}(s)\right)}\right\} \mathrm{e}^{-\frac{s}{\lambda}} .
$$

The integrand inside the wavy-brackets is the Borel transform of $\Psi_{n}(\lambda)$. Following [44], it can also be written as a contour integral ${ }^{70}$,

$$
\mathcal{B}\left[\Psi_{n}\right](s)=\frac{1}{2 \pi}\left\{\frac{\widetilde{g}\left(x_{+}(s)\right)}{\frac{\mathrm{d} v^{(n)}}{\mathrm{d} x}\left(x_{+}(s)\right)}-\frac{\widetilde{g}\left(x_{-}(s)\right)}{\frac{\mathrm{d} v^{(n)}}{\mathrm{d} x}\left(x_{-}(s)\right)}\right\}=\frac{1}{4 \pi^{2} \mathrm{i}} \frac{1}{\sqrt{s}} \oint_{\Gamma_{\circlearrowleft}} \mathrm{d} x \frac{\sqrt{v^{(n)}(x)}}{v^{(n)}(x)-s} \widetilde{g}(x) .
$$

The contour $\Gamma_{\circlearrowleft}$ is an anti-clockwise contour enclosing the original steepest descent contour $\Gamma_{n}$. This expression makes explicit the appearance of the $\sqrt{s}$ factor, crucial to understanding the origin of the double-valuedness of the map between the $s$ and $x$ variables. One may then think of this map as defined on a two-sheeted Riemann surface, connected at the saddle-point $s=0$, and where it has zero-phase on the contour associated to $x_{+}(s)$ and acquiring a phase $\pi$ on the contour associated to $x_{-}(s)$ (see [44] for figures and further details).

At this point, we have described two routes towards computing the Borel transform of our original partition function (4.1). The first approach directly uses the asymptotic expansion of $\Phi_{n}(\lambda)$, alongside our initial definition of a Borel transform, (2.2). The second approach changes variables inside the integral for $\Psi_{n}(\lambda)$, following the strategy in section 3, in particular (3.19) and (3.20). The results are (4.8) and (4.18), respectively, but these two functions are distinct.

\footnotetext{
${ }^{70}$ Notice that this calculation also helps making (3.20) a bit more explicit.
} 
This is rather obvious once one notices that the starting asymptotic expansions differ by a factor of $\sqrt{\lambda}$,

$$
\sqrt{\lambda} \Phi_{n}(\lambda) \simeq \Psi_{n}(\lambda)
$$

Of course their respective Borel transforms might still be related. Using the asymptotic expansion of $\Phi_{n}$ in (4.5), and rewriting it with this extra $\sqrt{\lambda}$ factor, essentially only changes $\beta_{n}$ as $\beta_{n} \rightarrow$ $\beta_{n}+\frac{1}{2}$. Then the power-series definition for the Borel transform of $\Psi_{n}(\lambda)$ is

$$
\mathcal{B}\left[\Psi_{n}\right](s)=\mathcal{B}\left[\sqrt{\lambda} \Phi_{n}\right](s)=\sum_{k=0}^{+\infty}\left(1-\delta_{k, 0} \delta_{\beta_{n},-\frac{1}{2}}\right) Z_{k}^{(n)} \frac{s^{k+\beta_{n}-\frac{1}{2}}}{\Gamma\left(k+\beta_{n}+\frac{1}{2}\right)}
$$

As such - and in spite of the fact that $\mathcal{B}\left[\Phi_{n}\right]$ and $\mathcal{B}\left[\Psi_{n}\right]$ are different functions, possibly with different singularity structure upon the complex plane - the question we have left to answer is whether the resurgence information encoded in $\mathcal{B}\left[\Phi_{n}\right],(4.8)$, is the same as that encoded in $\mathcal{B}\left[\Psi_{n}\right],(4.20)$, and how can it be read-off easily in these two different cases. We shall carry out this analysis in our favourite (and simplest!) example so far, the quartic partition-function (2.8).

But before we do that, let us make two comments. First, as already mentioned, Borel transforms are naturally attached to any asymptotic (perturbative) expansions, being completely independent of partition functions, or integrals, or steepest-descent methods. We just chose the above example of a partition-function integral, evaluated via saddle-points, in order to illustrate this apparent ambiguity concerning the definition of a Borel transform in a simple and familiar setting. Second, expecting that $\mathcal{B}\left[\Phi_{n}\right]$ and $\mathcal{B}\left[\Psi_{n}\right]$ yield the same resurgence information, i.e., that if the initial asymptotic sequences are somehow related then so should their Borel transforms be, is an extremely natural expectation: as the inverse Borel transform is essentially a Laplace transform, under reasonable assumptions the basic properties of this linear integral operator imply elementary, straightforward relations such as, e.g.,

$$
\mathcal{B}\left[\lambda^{-1} \Phi\right](s)=\frac{\partial}{\partial s} \mathcal{B}[\Phi](s) .
$$

Relations such as this strongly hint that very likely also the resurgence structure will be translated along, and we shall make the required dictionary precise in the following.

\section{Constructing Borel Transforms: Some Examples}

Now that we have realized that there is some ambiguity, or freedom, in the definition of what a Borel transform is, the reader might be asking: then what is the correct definition? As we shall see, this question is somewhat irrelevant for one should ask beforehand: does this freedom matter? To anticipate the upcoming conclusion, the answer is that in fact it does not matter, as the resurgence properties may be read-off from any "representative" of the Borel transform.

The quartic partition-function (2.10) was thoroughly analyzed in section 2, with Borel transforms computed straight out from the (perturbative) asymptotic expansions (2.16) and (2.17); recall the resulting (2.23) and (2.24). Let us next try to obtain these same Borel transforms directly out from the partition function, as described above. Recall that the quartic potential (2.9) has three saddle points, located at $z_{0}^{*}=0, z_{ \pm}^{*}= \pm \sqrt{\frac{6}{\lambda}}$. Rescaling out the coupling con$\operatorname{stant}^{71}$ in (2.8) by changing variables inside the integral $\operatorname{as}^{72} z=\frac{x}{\sqrt{\lambda}}$, one can rewrite the quartic

\footnotetext{
${ }^{71}$ Further recall that, as compared to section 2 , we have now set $\hbar=1$ and only use $\lambda$ for the overall coupling.

${ }^{72}$ Do not mistake the (silent) integrand variable $x$ in this section with the (overall) coupling $x$ of section 2 !
} 
partition-function around each saddle, following (4.12) and (4.14), as

$$
\begin{aligned}
Z^{(0)}(\lambda) & =\frac{1}{2 \pi \sqrt{\lambda}} \int_{\Gamma_{0}} \mathrm{~d} x \mathrm{e}^{-\frac{v^{(0)}(x)}{\lambda}} \equiv \frac{1}{\sqrt{\lambda}} \Psi_{0}(\lambda), \\
Z^{( \pm)}(\lambda) & =\frac{\mathrm{e}^{-\frac{3}{2 \lambda}}}{2 \pi \sqrt{\lambda}} \int_{\Gamma_{ \pm}} \mathrm{d} x \mathrm{e}^{-\frac{v^{( \pm)}(x)}{\lambda}} \equiv \mathrm{e}^{-\frac{3}{2 \lambda}} \frac{1}{\sqrt{\lambda}} \Psi_{ \pm}(\lambda),
\end{aligned}
$$

where the rescaled saddles are now $x_{0}^{*}=0$ and $x_{ \pm}^{*}= \pm \sqrt{6}$. The "local" potentials above are

$$
v^{(0)}(x)=\frac{1}{2} x^{2}-\frac{1}{24} x^{4} \quad \text { and } \quad v^{( \pm)}(x)=-\frac{3}{2}+\frac{1}{2} x^{2}-\frac{1}{24} x^{4},
$$

while the steepest-descent contours of integration can be read-off from figure 2 ; which for $\arg \lambda=$ 0 are

$$
\begin{aligned}
& \Gamma_{0}=\left(-\infty \mathrm{e}^{\mathrm{i} \frac{\pi}{4}},+\infty \mathrm{e}^{\mathrm{i} \frac{\pi}{4}}\right), \\
& \Gamma_{-}=\left(-\infty \mathrm{e}^{\mathrm{i} \frac{\pi}{4}},+\infty \mathrm{e}^{\mathrm{i} \frac{3 \pi}{4}}\right), \\
& \Gamma_{+}=\left(-\infty \mathrm{e}^{\mathrm{i} \frac{5 \pi}{4}},+\infty \mathrm{e}^{\mathrm{i} \frac{7 \pi}{4}}\right) .
\end{aligned}
$$

Do note that each of these contours naturally passes through its respective saddle.

We proceed with the change of variables (4.16), defining the Borel plane as

$$
s=v^{(n)}(x) \quad \text { for } \quad n=0, \pm .
$$

Inverting this map yields $x \equiv x(s)$. As we are dealing with a polynomial potential of fourth degree, one finds four different solutions to this equation. They are:

$$
\begin{array}{ll}
\text { Saddle } x_{0}^{*}: & x_{ \pm}^{( \pm)}(s)=( \pm) \sqrt{6\left(1 \pm \sqrt{1-\frac{2 s}{3}}\right)}, \\
\text { Saddle } x_{ \pm}^{*}: & x_{ \pm}^{( \pm)}(s)=( \pm) \sqrt{6\left(1 \pm \sqrt{-\frac{2 s}{3}}\right)} .
\end{array}
$$

Note that, with a slight abuse of notation, in here the \pm and $( \pm)$ signs in the solutions are labeling the solutions for each saddle, and are not related to the \pm signs labeling the saddleswhich hopefully will be clear from context. All these maps will result in a variable $s$ which is varying in $(0,+\infty)$, but, as we discussed earlier, only two for each saddle will correspond to the original steepest-descent contours $\Gamma_{n}$. After some careful examination of the signs of the square roots, one finds that as $s$ varies from zero to positive infinity for the saddle $x_{0}^{*}$ one needs to choose

$$
x_{-}^{(-)}(s) \in\left(0,+\infty \mathrm{e}^{\mathrm{i} \frac{\pi}{4}}\right), \quad x_{-}^{(+)}(s) \in\left(0,-\infty \mathrm{e}^{\mathrm{i} \frac{\pi}{4}}\right) .
$$

In this way $x_{-}^{(-)}$corresponds to the half-contour leaving the saddle-point off to infinity, while $x_{-}^{(+)}$ corresponds to the half-contour coming into the saddle-point from infinity. In a self-explanatory notation, we can thus write $\Gamma_{0} \equiv \Gamma_{-}^{(-)}-\Gamma_{-}^{(+)}$. A completely analogous analysis may be done 
for the other saddles, $x_{ \pm}^{*}$, where from the four solutions previously found, two go through one saddle, the other two through the other. In particular, as one varies $s \in(0,+\infty)$, one has:

$$
\begin{array}{ll}
\text { Saddle } x_{+}^{*}: & \Gamma_{+}=\Gamma_{+}^{(+)}-\Gamma_{-}^{(+)}, \\
\text {Saddle } x_{-}^{*}: & \Gamma_{-}=\Gamma_{-}^{(-)}-\Gamma_{+}^{(-)} .
\end{array}
$$

One can proceed by using the Laplace integral-representation for $\Psi_{n}(\lambda)$ in (4.17) in order to compute the Borel transforms of $\Psi_{0}(\lambda)$ and $\Psi_{ \pm}(\lambda)$, via (4.18). For the "perturbative saddle",

$$
\begin{aligned}
\Psi_{0}(\lambda) & =\frac{1}{2 \pi} \int_{0}^{+\infty} \mathrm{d} s\left\{\frac{1}{\frac{\mathrm{d} v^{(0)}}{\mathrm{d} x}\left(x_{-}^{(-)}(s)\right)}-\frac{1}{\frac{\mathrm{d} v^{(0)}}{\mathrm{d} x}\left(x_{-}^{(+)}(s)\right)}\right\} \mathrm{e}^{-\frac{s}{\lambda}}= \\
& =\frac{1}{2 \pi} \int_{0}^{+\infty} \mathrm{d} s\left\{\frac{2}{\frac{\mathrm{d} v^{(0)}}{\mathrm{d} x}\left(x_{-}^{(-)}(s)\right)}\right\} \mathrm{e}^{-\frac{s}{\lambda}},
\end{aligned}
$$

as $\frac{\mathrm{d} v^{(0)}}{\mathrm{d} x}\left(x_{-}^{(-)}(s)\right)=-\frac{\mathrm{d} v^{(0)}}{\mathrm{d} x}\left(x_{-}^{(+)}(s)\right)=\sqrt{6(1-\sqrt{1-2 s / 3})} \sqrt{1-2 s / 3}$. It then follows:

$$
\mathcal{B}\left[\Psi_{0}\right](s)=\frac{1}{2 \pi \sqrt{s}} \frac{\sqrt{1+\sqrt{1-\frac{2 s}{3}}}}{\sqrt{1-\frac{2 s}{3}}} .
$$

In much the same way we can calculate

$$
\Psi_{+}(\lambda)=-\Psi_{-}(\lambda) \equiv \int_{0}^{+\infty} \mathrm{d} s \mathcal{B}\left[\Psi_{1}\right](s) \mathrm{e}^{-\frac{s}{\lambda}}
$$

leading to the Borel transform

$$
\mathcal{B}\left[\Psi_{1}\right](s)=-\frac{\mathrm{i}}{2 \pi \sqrt{2 s}} \frac{\sqrt{1+\sqrt{1+\frac{2 s}{3}}}}{\sqrt{1+\frac{2 s}{3}}} .
$$

At first sight the Borel transforms we have just computed, $\mathcal{B}\left[\Psi_{0}\right](s)$ and $\mathcal{B}\left[\Psi_{1}\right](s)$, look very different from the Borel transforms computed in subsection 2.2, (2.23) and (2.24). This should not come as a surprise: indeed, as discussed earlier in this section, the asymptotic expansions for the $\Phi$ 's and the $\Psi$ 's differ by a factor of $\sqrt{\lambda}$. But as a consistency check on our calculations, we may now show how to obtain $\mathcal{B}\left[\Psi_{0}\right](s)$ and $\mathcal{B}\left[\Psi_{1}\right](s)$ directly from the asymptotic expansions for $\Phi_{0}$ and $\Phi_{1}$, written in (2.16) and (2.17). Recalling that $\Phi_{0}$ is the expansion around the saddle $x_{0}^{*}$, and that $\Phi_{1}$ the expansion around either saddle $x_{ \pm}^{*}$, we are expecting to find:

$$
\begin{aligned}
& \mathcal{B}\left[\Psi_{0}\right](s)=\mathcal{B}\left[\sqrt{\lambda} \Phi_{0}\right](s), \\
& \mathcal{B}\left[\Psi_{1}\right](s)=\mathcal{B}\left[\sqrt{\lambda} \Phi_{1}\right](s) .
\end{aligned}
$$

Let us check that this is in fact verified. Start with the asymptotic expansion for $\Phi_{0}(\lambda)$, in (2.16), which we reproduce in here

$$
\Phi_{0}(\lambda) \simeq \sum_{k=0}^{+\infty} Z_{k}^{(0)} \lambda^{k}, \quad Z_{k}^{(0)}=\left(\frac{2}{3}\right)^{k} \frac{(4 k) !}{2^{6 k}(2 k) ! k !} Z_{0}^{(0)},
$$


and where in the present conventions $Z_{0}^{(0)}=\frac{1}{\sqrt{2 \pi}}$. Computing the Borel transform above, using (4.20), it follows

$$
\mathcal{B}\left[\sqrt{\lambda} \Phi_{0}\right](s)=\sum_{k=0}^{+\infty} Z_{k}^{(0)} \frac{s^{k-\frac{1}{2}}}{\Gamma\left(k+\frac{1}{2}\right)}=\frac{1}{2 \pi \sqrt{s}} \frac{\sqrt{1+\sqrt{1-\frac{2 s}{3}}}}{\sqrt{1-\frac{2 s}{3}}}
$$

which is precisely $\mathcal{B}\left[\Psi_{0}\right](s)$ as expected. Performing the same analysis using the asymptotic expansion for $\Phi_{1}(\lambda)$, in (2.17), one likewise obtains

$$
\mathcal{B}\left[\sqrt{\lambda} \Phi_{1}\right](s)=\sum_{k=0}^{+\infty} Z_{k}^{(1)} \frac{s^{k-\frac{1}{2}}}{\Gamma\left(k+\frac{1}{2}\right)}=-\frac{\mathrm{i}}{2 \pi \sqrt{2 s}} \frac{\sqrt{1+\sqrt{1+\frac{2 s}{3}}}}{\sqrt{1+\frac{2 s}{3}}},
$$

which precisely matches $\mathcal{B}\left[\Psi_{1}\right](s)$. This explicitly checks our calculations, verifying how there is more than one way to compute a Borel transform, leading to distinct, albeit related results.

At this stage we are back to our main question. The Borel transforms computed in section 2, (2.23) and (2.24), had a singularity structure with poles and logarithms, (2.25) and (2.26), which immediately made explicit the resurgence properties of the quartic partition-function. In fact, the abstraction of these resurgence properties out from their associated Borel singularities, realized in the resurgence relations (2.30) and (2.31), or in the (finite) alien chain (2.32), was what then allowed to jump-start the whole resurgence program. But will the same remain true with the Borel transforms we have just computed, (4.35) and (4.37)? How can we recover the (algebraic) resurgence structure encoded in (2.32) out of these "new" Borel transforms?

The first thing to notice is that the Borel transforms (4.35) and (4.37) both have a squareroot singularity at the origin, i.e., $\sim 1 / \sqrt{s}$ near $s=0$. This is a somewhat artificial singularity from the resurgence standpoint, and is essentially related to the coupling square-root in the initial asymptotic expansion. In order to infer whether there is any clean resurgent behaviour in our "new" Borel transforms, we need to analyze them away from this singularity. In fact, we shall need to focus on the singularity at $s=\frac{3}{2}$ of the Borel transform $\mathcal{B}\left[\Psi_{0}\right](s)$, and on the singularity at $s=-\frac{3}{2}$ of the Borel transform $\mathcal{B}\left[\Psi_{1}\right](s)$. If resurgence is also at work in here, near each of these singularities we should see the "reappearance" of the other sector.

Expanding the Borel transforms (4.35) and (4.37) near their singularities at $s= \pm A \equiv \pm \frac{3}{2}$, we find

$$
\begin{array}{ll}
\mathcal{B}\left[\Psi_{0}\right](s)=\sum_{k=-1}^{+\infty} B_{k}^{(0)}(s-A)^{\frac{k}{2}}, & B_{k}^{(0)}=\frac{\mathrm{e}^{\mathrm{i} \frac{k \pi}{2}}}{2 \pi^{3 / 2}} \frac{\Gamma\left(k+\frac{3}{2}\right)}{\Gamma(k+2)} A^{-\frac{k+1}{2}}, \\
\mathcal{B}\left[\Psi_{1}\right](s)=\sum_{k=-1}^{+\infty} B_{k}^{(1)}(s+A)^{\frac{k}{2}}, & B_{k}^{(1)}=\frac{\mathrm{e}^{\mathrm{i} \pi}}{(2 \pi)^{3 / 2}} \frac{\Gamma\left(k+\frac{3}{2}\right)}{\Gamma(k+2)} A^{-\frac{k+1}{2}} .
\end{array}
$$

Recall that back in (2.25) and (2.26) the singularities were restricted to a simple pole and a logarithm branch-cut. Things here are a bit "messier", as there is now an infinite number of branch-cuts, one per fractional power in the above expansions (i.e., whenever $k$ is odd). Thus, at first sight, it now seems much harder to find the resurgence of, say, the $\Psi_{1}$ sector inside the expansion of $\mathcal{B}\left[\Psi_{0}\right](s)$ around $s=A$, due to this plethora of branch-cuts. This scenario quickly 
becomes more amenable once one computes the expansion of $\mathcal{B}\left[\Psi_{1}\right](s-A)$ near $s=A$ (which anyways is what we hope to find inside $\mathcal{B}\left[\Psi_{0}\right](s)$ near $s=A$ in the first place). One easily obtains

$$
\mathcal{B}\left[\Psi_{1}\right](s-A)=\sum_{k=-1}^{+\infty} \widehat{B}_{k}^{(1)}(s-A)^{k+\frac{1}{2}}, \quad \widehat{B}_{k}^{(1)}=\frac{\mathrm{i} \mathrm{e}^{\mathrm{i} \pi k}}{2 \pi^{3 / 2}} \frac{\Gamma\left(2 k+\frac{5}{2}\right)}{\Gamma(2 k+3)} A^{-(k+1)} .
$$

Comparing the coefficients $\widehat{B}_{k}^{(1)}$ with those particular coefficients $B_{k}^{(0)}$ which were attached to a singular fractional-power, i.e., with the coefficients $B_{2 k+1}^{(0)}$, one finds

$$
B_{2 k+1}^{(0)}=\widehat{B}_{k}^{(1)} .
$$

In this way, one may split the power-series expansion (4.43) into its regular holomorphic half and its singular half, both of which are infinite series on their own. Then, the singular series will match (4.45) above in such a way that one can write the resurgence-like relation

$$
\mathcal{B}\left[\Psi_{0}\right](s)=(+2) \times \frac{1}{2} \mathcal{B}\left[\Psi_{1}\right](s-A)+\text { holomorphic. }
$$

The exact same analysis equally applies to $\mathcal{B}\left[\Psi_{1}\right](s)$. This time around

$$
\mathcal{B}\left[\Psi_{0}\right](s+A)=\sum_{k=-1}^{+\infty} \widehat{B}_{k}^{(0)}(s+A)^{k+\frac{1}{2}}, \quad \widehat{B}_{k}^{(0)}=\frac{1}{2^{1 / 2} \pi^{3 / 2}} \frac{\Gamma\left(2 k+\frac{5}{2}\right)}{\Gamma(2 k+3)} A^{-(k+1)},
$$

in such a way that

$$
B_{2 k+1}^{(1)}=-\frac{1}{2} \widehat{B}_{k}^{(0)}
$$

from where the resurgence-like relation emerges

$$
\mathcal{B}\left[\Psi_{1}\right](s)=(-1) \times \frac{1}{2} \mathcal{B}\left[\Psi_{0}\right](s+A)+\text { holomorphic. }
$$

One may compare these results, (4.47) and (4.50), to (2.25) and (2.26), respectively. Recall that one needs to use the "dictionary" from (3.34) to read Stokes constants. In this way, via (3.34), (4.47) yields the Stokes constant $S_{1}=-2$, which is the precise constant obtained in (2.30). Likewise, (4.50) yields the Stokes constant $S_{-1}=1$, which matches the one obtained in (2.31).

We may finally conclude that the resurgence structure is seen both by the $\Psi$ - "representative" which was used above, as well as by the $\Phi$ - "representative" used in section 2 . Further, as expected, both these descriptions lead to the exact same structure, i.e., they are in the same "class". This means that, starting-off from our "new" resurgence relations (4.47) and (4.50), we may once again forget about any functional details concerning Borel singularities, and express resurgence solely at the level of the asymptotic series $\Psi_{0}(\lambda)$ and $\Psi_{1}(\lambda)$ (our transseries building blocks). Herein, resurgence is likewise formalized at an abstract level using alien derivatives and bridge equations in the exact same fashion as before, i.e., using the same bridge equations (2.30) and (2.31) and the same (finite) alien chain (2.32) with its associated allowed motions. The main difference, of course, is in the calculation process leading up to (4.47) and (4.50). Unlike (2.25) and (2.26), we no longer have just a finite number of singular structures (in fact, two singular contributions, one pole and one logarithmic branch-cut), but rather an infinite number of singular fractional powers, with the "reappearance" properties intertwined inside this series. While in the former case resurgence is seen directly in the functional coefficients of the singularities, in the latter case 
resurgence is only seen once the numerical coefficients of all singular powers are hold together. In other words, although (as before) we still need to take into account all singular structures, now this amounts to an infinite number of terms - just a few will not be enough!

This implies that using the $\Phi$ - "representative" rather than the $\Psi$ - "representative" allows for a more transparent reading of the encoded resurgence structure. For convenience, one should thus select the $\Phi$ - "representative" as the "cleanest" representative, leading up to the definition of a simple resurgent function, see, e.g., [109, 55, 56, 207]. A simple resurgent function is a resurgent function (asymptotic series) for which its Borel singularities solely reduce to a simple pole alongside a logarithmic branch-cut. To be fully precise, we should not say "simple resurgent function", but rather "simple resurgent Borel representative" —out of the (possibly) infinite number of distinct but related Borel representatives, for the (possibly) infinite number of distinct organizations of the asymptotic series we started off with. This will be made precise below.

Do note that our example above corresponds to a linear problem, where we were able to compute everything exactly. In general, nonlinear problems this will not be the case. This implies that in such cases one can only access partial information concerning Borel singularities and, if not dealing with a simple representative, the resurgence properties of our asymptotic sequence will be masked and may be very difficult to retrieve. It then becomes of utmost importance to know how to reach a simple Borel representative, independent of our starting point. As we shall discuss next, there is a very precise way of relating Borel transforms associated to asymptotic series which differ by factors of the coupling, and all members of such a family of sequences will be equally well described by their single simple representative.

This analysis makes two things clear. The first is that the definition of a Borel transform does not need to be very precise, and may allow for some freedom as discussed. It does not really matter, as at the end of the day one reaches the same resurgence structure. The second is that precisely because one always ends up with the same algebraic structure (upon abstraction from the functional nature of Borel singularities), the true fundamental structure to work with is that of abstract bridge equations and alien chains, from where all nonperturbative results may follow.

\section{On the Class of Simple Resurgent Functions}

Let us consider any observable $\Phi$, typically arising from some nonlinear problem, with an asymptotic perturbative expansion in the coupling $\lambda$ given by

$$
\Phi(\lambda) \simeq \sum_{k=0}^{+\infty} \Phi_{k} \lambda^{\alpha k+\beta},
$$

with $\alpha, \beta \in \mathbb{R}$. Let us further assume that the large-order behaviour of the (factorially divergent) coefficients above is of the form

$$
\Phi_{k} \sim(\alpha k+\delta-1) !
$$

with some other $\delta \in \mathbb{R}$. Given this data, we wish to compute the Borel transform of $\Phi, \mathcal{B}[\Phi](s)$, in such a way as to obtain a simple representative, i.e., in such a way that $\mathcal{B}[\Phi](s)$ only has simple poles and logarithmic branch-points as singularities (this is known as a simple singularity ${ }^{73}$ ). That is to say

$$
\mathcal{B}[\Phi](s)=\frac{\mathrm{S}_{\omega}^{\prime}}{2 \pi \mathrm{i}(s-\omega)}+\mathrm{S}_{\omega} \times \mathcal{B}\left[\Psi_{\omega}\right](s-\omega) \frac{\log (s-\omega)}{2 \pi \mathrm{i}}+\text { holomorphic }
$$

\footnotetext{
${ }^{73}$ All which is required is that the function in front of the logarithm is holomorphic. This requirement allows this function to be rewritten - or understood - as the Borel transform of some other asymptotic series (evaluated around the origin of its own complex Borel plane), as given in the expression above.
} 
near each singular point $\omega$, with $\mathrm{S}_{\omega}^{\prime}, \mathrm{S}_{\omega} \in \mathbb{C}$ and $\Psi_{\omega}$ some other asymptotic sector (and such that $\mathcal{B}\left[\Psi_{\omega}\right]$ is holomorphic near the origin, i.e., it introduces no further singularities in (4.53) above).

When all that is available to us is an asymptotic sequence as in (4.51), one might be tempted to use our initial definition of Borel transform, (2.2), and thus write

$$
\mathcal{B}[\Phi](s)=\sum_{k=0}^{+\infty} \Phi_{k} \frac{s^{\alpha k+\beta-1}}{\Gamma(\alpha k+\beta)} .
$$

The problem, of course, is the off-set $\delta$. If $\delta \neq \beta$, we are not removing the precise factorial growth and this will result in more intricate Borel transforms than one would wish for. The solution is nonetheless simple. Introducing

$$
\Phi_{[\gamma]}(\lambda) \equiv \lambda^{\gamma} \Phi(\lambda) \simeq \sum_{k=0}^{+\infty} \Phi_{k} \lambda^{\alpha k+\beta+\gamma}
$$

and choosing $\gamma=\delta-\beta$, then the Borel transform of $\Phi_{[\gamma]}$ will remove the precise factorial growth leading to the simple representative,

$$
\mathcal{B}\left[\Phi_{[\delta-\beta]}\right](s)=\sum_{k=0}^{+\infty} \Phi_{k} \frac{s^{\alpha k+\delta-1}}{\Gamma(\alpha k+\delta)} .
$$

All asymptotic expansions of the type $\Phi_{[\gamma]}(\lambda)=\lambda^{\gamma} \Phi(\lambda)$, with $\gamma \in \mathbb{R}$, are said to be in the same resurgence class (or family), meaning that their resurgence structure is equivalent for all values of $\gamma$ and that they may all be related to the one particular choice $\bar{\gamma}$ where the Borel transform $\mathcal{B}\left[\Phi_{[\bar{\gamma}]}\right](s)$ will have the singularity structure (4.53) of a simple resurgent function. We shall denote this particular choice, $\Phi_{[\bar{\gamma}]}$, as the standard representative, the representative where resurgent analysis is most straightforward. One may reach this standard representative directly at the level of the Borel transforms: in appendix $\mathrm{E}$ we show how to relate Borel transforms of distinct members in the family $\left\{\Phi_{[\gamma]}\right\}$, leading up to ${ }^{74}$

$$
\mathcal{B}\left[\Phi_{\left[\gamma^{\prime}\right]}\right](s)=D_{s}^{\gamma-\gamma^{\prime}} \mathcal{B}\left[\Phi_{[\gamma]}\right](s) .
$$

In here, $D_{s}^{\alpha}$ is the order- $\alpha$ (fractional) derivative with respect to $s$, when $\alpha>0$; and it is the (fractional) $|\alpha|$-integration with respect to $s$, when $\alpha<0$ (but see appendix E and, e.g., [166] for further details). Having this relation in mind, once one computes the Borel transform of some non-standard representative $\Phi_{[\gamma]}$, the only difficulty in subsequently finding the standardrepresentative Borel transform $\mathcal{B}\left[\Phi_{[\bar{\gamma}]}\right](s)$ is how to find $\bar{\gamma}$. At the level of Borel transforms this is done via an analysis of the original $\mathcal{B}\left[\Phi_{[\gamma]}\right](s)$ around its singular points, which however is not necessarily always straightforward as we shall illustrate next.

\section{Another Look at the Quartic Partition-Function}

Let us illustrate how (4.57) works in practice, back in the example of the quartic partitionfunction. We shall focus on the saddle $x_{0}^{*}$ and, for the sake of notational simplicity, suppress the 0 subscript in the asymptotic series around this saddle, say $\Phi_{0}(\lambda)$ in $(2.16)$ or $\Psi_{0}(\lambda)$ in (4.34), from now on. In this example we already know which is the standard representative; it is the

\footnotetext{
${ }^{74}$ Residual coefficients, which need to be removed upon Borel transformation, must be considered separately.
} 
original asymptotic series (2.16) with (simple) Borel transform (2.25), which we shall denote by $\Phi_{[0]}$. All other family members within this resurgence class may be obtained via

$$
\Phi_{[\gamma]}(\lambda)=\lambda^{\gamma} \Phi_{[0]}(\lambda)
$$

For example, $\Psi=\sqrt{\lambda} \Phi$ in (4.34) may now be written as $\Phi_{[1 / 2]}(\lambda)$. Equation (4.57) then explicitly relates both these Borel transforms (which we computed earlier) as

$$
\mathcal{B}\left[\Phi_{[0]}\right](s)=D_{s}^{1 / 2} \mathcal{B}\left[\Phi_{[1 / 2]}\right](s) .
$$

Here we have to deal with a non-local fractional operator, the semi-derivative $D_{s}^{1 / 2}$, which is defined by (see, e.g., [166] or appendix E)

$$
D_{s}^{1 / 2} f(s)=\frac{1}{\Gamma(1 / 2)} \frac{\mathrm{d}}{\mathrm{d} s} \int_{0}^{s} \mathrm{~d} t \frac{f(t)}{\sqrt{s-t}} .
$$

It is this non-locality of the semi-derivative which explains why all the resurgence information which is "localized", as functional coefficients, in the two singularities of $\mathcal{B}\left[\Phi_{[0]}\right](s)$ becomes "spread-out" over the infinite number of singular branch-cuts in $\mathcal{B}\left[\Phi_{[1 / 2]}\right](s)$, as discussed earlier. The formal derivation that (4.59) above holds, at the level of series expansions, may be found in appendix E. Here, we shall instead focus on this relation at the level of Borel singularities, i.e., we shall analyze the validity of (4.59) around $s=A$ (we are addressing the perturbative saddle).

Recall that the expansion of $\mathcal{B}\left[\Phi_{[1 / 2]}\right](s)$ around $s=A$ is given by (4.43), which we (partially) recall in here as

$$
\mathcal{B}\left[\Phi_{[1 / 2]}\right](s)=\sum_{k=-1}^{+\infty} B_{k}^{(0)}(s-A)^{\frac{k}{2}}
$$

In order to find the action of the semi-derivative on this function, let us first compute the action of the semi-derivative on each fractional-power term. Using its definition, one finds ${ }^{75}$

$$
\begin{aligned}
D_{s}^{1 / 2}(s-A)^{\frac{n}{2}} & =\frac{(-A)^{\frac{n}{2}+1}}{(n-1) \sqrt{\pi s^{3}}}-\frac{(-A)^{\frac{n}{2}}(s-A)}{(n-1) \sqrt{\pi s^{3}}}{ }_{2} F_{1}\left(1,-\frac{n}{2},-\frac{1}{2} \mid \frac{s}{A}\right), \quad n \geq 2 \\
D_{s}^{1 / 2}(s-A)^{\frac{1}{2}} & =-\mathrm{i} \sqrt{\frac{A}{\pi s}}+\frac{\mathrm{i}}{\sqrt{\pi}} \log (\sqrt{s}+\sqrt{A})-\frac{\mathrm{i}}{2 \sqrt{\pi}} \log (s-A)+\frac{\sqrt{\pi}}{2} \\
D_{s}^{1 / 2}(s-A)^{0} & =\frac{1}{\sqrt{\pi s}} \\
D_{s}^{1 / 2}(s-A)^{-\frac{1}{2}} & =\sqrt{\frac{A}{\pi s}} \frac{(-\mathrm{i})}{s-A}
\end{aligned}
$$

The hypergeometric function is easily expanded using its standard properties; see, e.g., [194]. In particular, this directly shows how when $n \geq 2$ and $n$ is even there are no logarithmic contributions; while when $n \geq 3$ and $n$ is odd, there will be. In the latter case, for $z \equiv \frac{s}{A} \rightarrow 1^{-}$,

$$
{ }_{2} F_{1}\left(1,-\frac{n}{2},-\frac{1}{2} \mid z\right)=\frac{\frac{3}{2}}{\Gamma\left(\frac{n-1}{2}\right) \Gamma\left(-\frac{n}{2}\right)}(z-1)^{\frac{n-3}{2}} \log (1-z) \times
$$

\footnotetext{
${ }^{75}$ Below, the $(-1)$ factor multiplying $A$ is actually $\mathrm{e}^{-\mathrm{i} \pi}$, while we change signs as $(A-s)=\mathrm{e}^{\mathrm{i} \pi}(s-A)$.
} 


$$
\times \sum_{k=0}^{+\infty} \frac{\Gamma\left(k-\frac{3}{2}\right)}{\Gamma(k+1)}(1-z)^{k}+\text { regular (non-logarithmic) terms. }
$$

Using this expansion alongside the semi-derivatives above, one can compute, term by term,

$$
\begin{aligned}
D_{s}^{1 / 2} \mathcal{B}\left[\Phi_{[1 / 2]}\right](s)= & \sum_{k=-1}^{+\infty} B_{k}^{(0)} D_{s}^{1 / 2}(s-A)^{\frac{k}{2}}=-\frac{\mathrm{i} B_{-1}^{(0)}}{\sqrt{\pi}} \frac{1}{s-A}-\frac{\mathrm{i} B_{1}^{(0)}}{2 \sqrt{\pi}} \log (s-A)+ \\
& +\mathrm{i} \log (s-A) \sum_{m=1}^{+\infty} \frac{(-1)^{m} B_{2 m+1}^{(0)}}{\Gamma(m+1) \Gamma\left(-m-\frac{1}{2}\right)}(s-A)^{m}+\text { regular terms. }
\end{aligned}
$$

Recall how we are only interested in the singular terms. Next, in order to compare to the expansion of $\mathcal{B}\left[\Phi_{[0]}\right](s)$ around the same point and check if we do obtain the same result, we also need to expand (2.25). Using the exact information encoded in (2.24) and expanding the hypergeometric function akin to what was done above, it follows

$$
\begin{aligned}
\mathcal{B}\left[\Phi_{0}\right](s)= & -\frac{1}{2 \pi^{\frac{3}{2}}} \frac{1}{s-A}+\frac{1}{16 \pi^{\frac{3}{2}}} \log (s-A)+ \\
& +\frac{1}{3 \sqrt{2} \pi^{\frac{5}{2}}} \log (s-A) \sum_{m=1}^{+\infty} \frac{(-1)^{m}}{A^{m}} \frac{\Gamma\left(m+\frac{5}{4}\right) \Gamma\left(m+\frac{7}{4}\right)}{\Gamma(m+1) \Gamma(m+2)}(s-A)^{m}+\text { regular terms. }
\end{aligned}
$$

It is now a matter of some simple algebra to check that we have obtained the very same result and thus checked the validity of (4.59). A completely analogous comparison may be done for the nonperturbative saddles, matching the Borel transforms of $\Phi_{1}$ and $\Psi_{1}$ close to the singularity at $s=-A$. In this way, these Borel transforms encode the same resurgence information and thus belong to the same resurgence family. Further, it should be clear that the resurgence information which is somewhat "locally" encoded in $\mathcal{B}[\Phi](s)$, becomes "non-locally" encoded in $\mathcal{B}[\Psi](s)$ due to the non-local nature of the (fractional) semi-derivative.

\section{Physical Resurgence: From Lattices to Virasoro Algebras}

Transseries structures are rather simple yet very general constructions. They prove extremely useful in finding or (iteratively) constructing explicit solutions to a wide range of linear and nonlinear problems. For instance, they can give rise to general solutions of differential equations, algebraic equations, finite difference equations, integrals or integral equations, functional equations, and so on (see, e.g., [110] for a review with many such explicit examples). Yet, so far we have been solely constructing transseries within very specific and simple examples. We shall now be more precise on how they come about, and how general their building-blocks might be.

In this process, it is important to have two points in mind. The first point was already discussed in the previous section, where explicit Borel calculations taught us how, at the end of the day, all one should care about are the abstract resurgence relations in-between the different

transseries building-blocks, or nodes. In other words, the specific details concerning which kind of Borel singularities show-up are somewhat irrelevant, as any "representative" will essentially yield the very same resurgence relations. But these nodes are asymptotic series which are "attached" to specific (and distinct) non-analytic transmonomials, which begs for some sort of classification of different types of transmonomials. Such structures will surely play a role in generalizing the alien chains we studied in section 2 to more general (and higher-dimensional) lattices. 
The second point has to do with the resurgent nature of a general transseries. Resurgence induces relations between the many nodes that build-up a transseries, as was already schematically depicted early-on, in (1.1) and (1.2). One can imagine an example where these resurgence relations directly connect the perturbative expansion to all other possible nonperturbative sectors. In this case, one may ask how exactly should (1.2) be fully exploited to decode all multi-instanton towers - and thus construct the complete transseries solution - out of the (also complete!) perturbative expansion. For this strategy to be fully implemented, one must first understand what is the full set of all allowed motions in-between transseries nodes, in the case of general, higherdimensional alien lattices. Let us explore these several issues in the following.

\section{General Transseries from Ordinary Differential Equations}

A natural and illustrative set-up where transseries appear is that of ordinary differential equations (ODEs). Suppose we are given a $k$ th-order nonlinear ODE, for the (yet unknown) function $u(x)$ in the variable $x$ (for instance, recall the second-order ODE for the free energy $F(x),(2.52)$, which was our main example back in subsection 2.4). If there are no singularities at $x=0$ one may first try to solve this ODE for $u(x)$ perturbatively, in powers of the monomial $x$ (again, as we did back in subsection 2.4, where a perturbative ansatz for $F(x)$ led to the recursion (2.53), yielding the perturbative coefficients $\left.F_{n}^{(0)}\right)$. Now, if the resulting perturbative coefficients $u_{n}^{(0)}$ grow at most exponentially with $n$, then the perturbative series has a finite, non-zero radius of convergence and our power-series solution may be studied with standard tools from classical analysis. Unfortunately, this is generically not the case, with the perturbative coefficients $u_{n}^{(0)}$ instead diverging factorially fast. What this divergence essentially means is that $x=0$ is sitting right next to a branch-cut of the solution, and thus it cannot possibly be made of powers of $x$ alone - one needs extra non-analytic contributions at $x=0$ in order to properly characterize $u(x)$. Such contributions, which add to the monomial $M \equiv x$, will be denoted by transmonomials, $T$, and different sets of transmonomials may be required for different problems.

One basic transmonomial we have already found several times before is $\exp \left(-\frac{1}{x}\right)$; but we might naturally ask what other types of transmonomials may one usually encounter? To address this question in a concrete example, let us go back to our $k$ th-order nonlinear ODE and try to solve it. The first (rather general) step is to rewrite it vectorially, as a rank- $k$ system of first-order (nonlinear) ODEs; schematically:

$$
\frac{\mathrm{d} \boldsymbol{u}}{\mathrm{d} x}(x)=\varphi(x, \boldsymbol{u}(x)) .
$$

Under broad conditions, equations of this type may be further rewritten in a rather useful form, known as the prepared form ${ }^{76}$ (see, e.g., [176] for an introduction to such procedure, and, e.g.,

\footnotetext{
${ }^{76}$ This prepared form (5.3) might look a bit odd at first. This is because we are working in the variable $x$, with perturbative expansions constructed around $x \sim 0$. This, however, is not the most standard variable in the resurgence literature. Therein one frequently uses $z=\frac{1}{x}$, with perturbative expansions built around $z \sim \infty$ and with the usual non-analytic transmonomial now being $\mathrm{e}^{-z}$ (non-analytic around $z \sim \infty$ ). In this variable, (5.3) looks a bit more "prepared", as

$$
\frac{\mathrm{d} \boldsymbol{u}}{\mathrm{d} z}(z)=-\mathbb{A} \cdot \boldsymbol{u}(z)-\frac{1}{z} \mathbb{B} \cdot \boldsymbol{u}(z)+\mathcal{O}\left(z^{-2} \boldsymbol{u},\|\boldsymbol{u}\|^{2}\right) .
$$

Appendix A also presents a "notational bridge" from our present discussions to the standard mathematical literature on resurgence, and many resurgence formulae in this variable $z$ will appear in there.
} 
$[69,70]$ for further details), which in this case is ${ }^{77}$ :

$$
\frac{\mathrm{d} \boldsymbol{u}}{\mathrm{d} x}(x)=\frac{1}{x^{2}} \mathbb{A} \cdot \boldsymbol{u}(x)+\frac{1}{x} \mathbb{B} \cdot \boldsymbol{u}(x)+\mathcal{O}\left(\boldsymbol{u}, x^{-2}\|\boldsymbol{u}\|^{2}\right),
$$

where the $k \times k$ matrices $\mathbb{A}$ and $\mathbb{B}$ are diagonal; $\mathbb{A}=\operatorname{diag}\left(A_{1}, \cdots, A_{k}\right)$ and $\mathbb{B}=\operatorname{diag}\left(\beta_{1}, \cdots, \beta_{k}\right)$. The reason why this prepared form (or, sometimes denoted, normal form) is useful is that if we neglect the higher-order and nonlinear terms, and just focus on the "linearized" version of (5.3), then the solution is immediate, as, component wise,

$$
u_{i}(x)=\sigma_{i} x^{\beta_{i}} \mathrm{e}^{-\frac{A_{i}}{x}},
$$

with $\boldsymbol{\sigma}$ a $k$-dimensional vector of coefficients (the boundary conditions). It is now very explicit how the transmonomial exp $\left(-\frac{1}{x}\right)$ first appears, and how it must play a central role in the construction of possible solutions. In fact, the next step in constructing a solution to (5.1) will be to try using a transseries ansatz precisely based upon the solution (5.4). In other words, in the same way that powers of the monomial $M=x$ were used as building blocks of the (incomplete) perturbative, power-series solution to the original nonlinear equation; one may now use powers of the transmonomials $T_{i}=\mathrm{e}^{-\frac{A_{i}}{x}}$ as new building blocks for the (eventually complete) nonperturbative, transseries solution to our ODE. Then, the typical transseries ansatz for such a solution is, without surprise,

$$
\boldsymbol{u}(x, \boldsymbol{\sigma})=\sum_{\boldsymbol{n} \in \mathbb{N}_{0}^{k}} \boldsymbol{\sigma}^{\boldsymbol{n}} x^{\boldsymbol{n} \cdot \boldsymbol{\beta}} \mathrm{e}^{-\frac{n \cdot \boldsymbol{A}}{x}} \boldsymbol{u}^{(\boldsymbol{n})}(x),
$$

where $\boldsymbol{\sigma}^{\boldsymbol{n}}=\prod_{i=1}^{k} \sigma_{i}^{n_{i}}$ are the $k$ independent transseries parameters. Further, there still are asymptotic series in powers of $x$ "attached" to each transmonomial, as

$$
\boldsymbol{u}^{(\boldsymbol{n})}(x) \simeq \sum_{g=0}^{+\infty} \boldsymbol{u}_{g}^{(\boldsymbol{n})} x^{g} .
$$

What other transmonomials could one find? Imagining we were forced to go beyond prepared form, it is simple to glare into the diversity of some of these new structures. Let us focus on the one-dimensional case for simplicity. Then, including for instance different powers of the monomial $x$ into the ODE (5.3), one finds terms in, e.g.,

$$
\frac{\mathrm{d} u}{\mathrm{~d} x}+\alpha n x^{n-1} u=0 \quad \Rightarrow \quad u(x)=\sigma \mathrm{e}^{-\alpha x^{n}} .
$$

When $n>0$ (these would be higher-order regular terms in (5.3)) the corresponding solutions are analytic. But when $n<-1$ (these would be higher-order irregular terms in (5.3), going beyond prepared form) the corresponding solutions yield new transmonomials. One could further envisage finding known transmonomials already in the ODE. For instance,

$$
\begin{array}{lll}
\frac{\mathrm{d} u}{\mathrm{~d} x}-\frac{1}{x^{2}} \mathrm{e}^{\frac{1}{x}} u(x)=0 & \Rightarrow & u(x)=\sigma \mathrm{e}^{-\mathrm{e}^{\frac{1}{x}},} \\
\frac{\mathrm{d} u}{\mathrm{~d} x}+\frac{\log x}{x^{2}} u(x)=0 & \Rightarrow & u(x)=\sigma \mathrm{e}^{-\frac{1}{x}(1+\log x),}
\end{array}
$$

\footnotetext{
${ }^{77}$ We are slightly abusing notation, as the prepared form is obtained after possibly more than one change-ofvariables; while in this expression we are using the exact same notation as in the previous equation, (5.1).
} 
which immediately leads to yet some new transmonomials as solutions. The diversity is enormous.

Let us next be more precise on definitions and nomenclature associated to transseries (now following [110]). A monomial is typically a term of the form

$$
M \equiv x^{\alpha},
$$

where $\alpha \in \mathbb{R}$. In this context, one defines a power-series $\mathcal{S}$ as a (possibly infinite) formal ${ }^{78}$ sum of monomials (and their respective powers). Monomials near $x \sim 0$ are easily ordered as

$$
x^{\alpha} \ll x^{\beta} \quad \text { iff } \quad \alpha>\beta,
$$

where the power-series $\mathcal{S}$ then collects monomials ordered in this way. In a similar fashion, a transmonomial is typically a term of the form ${ }^{79}$

$$
T \equiv x^{\alpha} \mathrm{e}^{\mathcal{S}(x)},
$$

where $\alpha \in \mathbb{R}$ and $\mathcal{S}(x)$ may be a function - it may be a (convergent) power-series, or it may even be a transseries itself (now we are running the risk of falling upon a slightly circular definition, so we refer the interested reader to [110] for further details). These transmonomials may be either analytic (in which case they essentially lead back to our earlier power-series) or non-analytic (where they finally lead to transseries!). In this language, it is straightforward to define a (logfree) transseries $\mathcal{T}$ as a (possibly infinite) formal sum of transmonomials (and their respective powers). Ordering transmonomials near $x \sim 0$ is now done lexicographically, i.e.,

$$
x^{\alpha} \mathrm{e}^{-A(x)} \ll x^{\beta} \mathrm{e}^{-B(x)} \quad \text { iff } \quad A(x) \gg B(x) \quad \text { or } \quad\{A(x)=B(x) \text { and } \alpha>\beta\},
$$

and the transseries $\mathcal{T}$ then collects transmonomials ordered in this way. The fact that the definitions above only require "formal sums" naturally leads to rather simple constructions, as we are now allowed to do essentially any formal (nonlinear) operations on transseries we may wish, from algebraic to differential to integral, without worrying about any convergence issues (we nonetheless refer back to [110] for precise definitions of all such basic manipulations).

In order to define a general transmonomial and a general transseries, one further needs to include logarithms. This is done quite straightforwardly. Define

$$
\log _{m} x:=\underbrace{\log \log \cdots \log x}_{m}
$$

with $m \in \mathbb{N}$. A general transmonomial is then obtained by replacing some of the $x$ 's in a logfree transmonomial by $\log _{m} x$. The exact same definition holds for a general transseries. These compositions also lead to a natural classification of transmonomials and transseries. On one side, composing exponentials as in

$$
\mathrm{e}^{\mathrm{e}^{\mathrm{e}}}
$$

one can define the (positive) exponential height of a transmonomial (or of a transseries) in the obvious way. Likewise, this is easily generalized to negative exponential height, more commonly denoted by exponential depth (or even "logarithmic depth" if to make it more transparent), where a transmonomial is obtained by composing a log-free transmonomial with $\log _{m}$ (on the right). So far, most fully worked-out physical problems seem to remain within height one and

\footnotetext{
${ }^{78}$ As we shall discuss shortly, issues of convergence need not concern us.

${ }^{79}$ To be precise this is a so-called log-free transmonomial; we will include logarithms shortly.
} 
depth one ${ }^{80}$, but there are recent results pointing towards the need to include (at the very least) height-two contributions in the Sachdev-Ye-Kitaev model describing AdS black holes [75], and in Yang-Mills/QCD theories [2]. These are very interesting venues for future study.

One rather interesting consequence of all these constructions is the following. Whenever our transseries construction (as a solution to some problem) is associated to convergent series, then we are essentially assembling elementary functions. But whenever these transseries constructions become associated to divergent series, then this means that upon (Borel and Écalle!) resummation we are obtaining functions which are not elementary functions [110].

\section{Multi-Parameter Transseries and their Resurgence Relations}

Having discussed some basic generalities on transseries, we may now turn to their resurgence properties. For the discussion that follows we shall go back to the original $k$ th-order nonlinear ODE (i.e., go back to addressing the original unknown function $u(x)$, rather than its vectorial rewriting $\boldsymbol{u}(x)$ from (5.1)), and we shall further assume a simple $k$-parameter transseries with only transmonomials of the type $\sim \mathrm{e}^{-\frac{A_{i}}{x}}$. This obviously implies there are $k$ distinct instanton actions, which we may again assemble vectorially as $\boldsymbol{A} \equiv\left(A_{1}, \ldots, A_{k}\right)$. We shall further assume the so-called non-resonant case $^{81}$, i.e., that these instanton actions are $\mathbb{Z}$-linearly independent,

$$
\nexists \boldsymbol{n} \neq \mathbf{0} \in \mathbb{Z}^{k} \mid \boldsymbol{n} \cdot \boldsymbol{A}=0 .
$$

In this case, and much like (5.5), the typical transseries ansatz for our solution is

$$
u(x, \boldsymbol{\sigma})=\sum_{\boldsymbol{n} \in \mathbb{N}_{0}^{k}} \boldsymbol{\sigma}^{\boldsymbol{n}} \mathrm{e}^{-\frac{\boldsymbol{n} \cdot \boldsymbol{A}}{x}} \Phi_{\boldsymbol{n}}(x),
$$

with the $\Phi_{\boldsymbol{n}}(x)$ asymptotic series ${ }^{82}$ in $x$

$$
\Phi_{\boldsymbol{n}}(x) \simeq \sum_{g=0}^{+\infty} u_{g}^{(\boldsymbol{n})} x^{g} .
$$

For example, the one-parameter transseries studied in subsection 2.4 is just the particular case where $k=1$, while the "1.5"-parameter transseries also studied in subsection 2.4 just corresponds to taking $k=2$, but with $\boldsymbol{A}=(A, 0)$ and where the only surviving sectors are $\Phi_{(n, 0)}, n \geq 0$, and $\Phi_{(0,1)}$ (this one not asymptotic; in fact it was just a constant).

The $\Phi_{\boldsymbol{n}}$ sectors in (5.17) are labeled by $\boldsymbol{n} \in \mathbb{N}_{0}^{k}$ and thus live on a $k$-dimensional (semipositive) lattice. What should we expect concerning their Borel singularities? If the structure is to be similar to the one-dimensional case, then they are located on the complex plane but must also be related to the aforementioned transseries sectors living on this $k$-dimensional lattice. Recalling the one-dimensional case, described by (2.62), it is reasonable to expect that the singularities of $\mathcal{B}\left[\Phi_{\boldsymbol{n}}\right](s)$ will be determined by the argument of the (non-analytic) exponential transmonomials in the transseries, i.e., they will be located at $s=\boldsymbol{\ell} \cdot \boldsymbol{A}$. This certainly satisfies the previous requirement. Further, due to possible "backwards" motions out from $\Phi_{\boldsymbol{n}}$, their

\footnotetext{
${ }^{80} \mathrm{It}$ is not hard to find contributions with logarithmic depth greater than one in several (higher-loop) Feynman diagrammatic calculations in field theory. But those are just contributions appearing at fixed loop-order, not contributions of higher logarithmic depth in the coupling constant (which would change the transseries nature).

${ }^{81}$ Resonance will be thoroughly discussed in the upcoming section 6 .

${ }^{82}$ As before, we could consider $\Phi_{\boldsymbol{n}}(x) \simeq x^{\beta_{\boldsymbol{n}}} \sum_{g=0}^{+\infty} u_{g}^{(\boldsymbol{n})} x^{g}$, but this will not influence the discussion below.
} 
location should be said more precisely as $s=\boldsymbol{\ell} \cdot \boldsymbol{A}$ and with $\boldsymbol{\ell} \in \mathbb{Z}^{k}$. Finally, we must certify that $\Phi_{n+\ell}$ is still a valid transseries sector, and thus can "resurge" at this $s=\boldsymbol{\ell} \cdot \boldsymbol{A}$ Borel singularity.

Let us make these requirements more precise. When starting off at $\Phi_{\boldsymbol{n}}$, asking whether $\Phi_{\boldsymbol{n}+\boldsymbol{\ell}}$ is still a transseries sector is a question on a $\mathbb{Z}^{k}$ transseries grid. But if it is still a sector, and the transseries is resurgent, this may generically imply a possible Borel singularity of $\mathcal{B}\left[\Phi_{\boldsymbol{n}}\right](s)$ at $s=\boldsymbol{\ell} \cdot \boldsymbol{A}$, which effectively projects the $\mathbb{Z}^{k}$ transseries grid into the complex Borel plane $\mathbb{C}$. This defines a projection map ${ }^{83}$

$$
\begin{aligned}
\mathfrak{P}: \mathbb{Z}^{k} & \rightarrow \mathbb{C} \\
\boldsymbol{\ell} & \mapsto \boldsymbol{A} \cdot \boldsymbol{\ell}
\end{aligned}
$$

which specifies singularity locations and resurgent "motions" upon the Borel plane. In this way, the natural $k$-dimensional generalization of $(2.62)$ becomes $^{84}$

$$
\left.\mathcal{B}\left[\Phi_{\boldsymbol{n}}\right](s)\right|_{s=\ell \cdot \boldsymbol{A}}=\mathrm{S}_{\boldsymbol{n} \rightarrow \boldsymbol{n}+\boldsymbol{\ell}} \times \mathcal{B}\left[\Phi_{\boldsymbol{n}+\boldsymbol{\ell}}\right](s-\boldsymbol{\ell} \cdot \boldsymbol{A}) \frac{\log (s-\boldsymbol{\ell} \cdot \boldsymbol{A})}{2 \pi \mathrm{i}}, \quad \boldsymbol{\ell} \neq \mathbf{0}
$$

where each singular point $s=\boldsymbol{\ell} \cdot \boldsymbol{A}$ is uniquely ${ }^{85}$ determined by the vector $\boldsymbol{\ell} \in \mathbb{Z}^{k}$.

As already extensively discussed throughout these lectures, resurgence is best expressed at an algebraic level of abstraction, based on the alien derivative $\Delta_{\omega}$. In this way, we may now extract the algebraic structure encoded in (5.20) and write it in terms of $\Delta_{\omega}$. One may proceed very much in analogy with the reasoning that led to (2.64) (or one may also easily generalize the corresponding bridge-equation calculations in appendix A to the present $k$-dimensional setting). In any case, the natural extension of the resurgence relations is found to be:

$$
\Delta_{\ell \cdot A} \Phi_{n}=S_{\ell} \cdot(\boldsymbol{n}+\ell) \Phi_{n+\ell},
$$

where we now have to deal with a $k$-dimensional vector of Stokes coefficients $\boldsymbol{S}_{\boldsymbol{\ell}} \equiv\left(S_{\ell}^{(1)}, \ldots, S_{\boldsymbol{\ell}}^{(k)}\right)$. Notice that, much like in (2.64), we labeled these Stokes vectors with subscripts $\boldsymbol{\ell}$, and think of them as living on lattice sites. We could have equally labeled them with subscripts $\boldsymbol{\ell} \cdot \boldsymbol{A}$, in which case they would be living on the complex Borel plane, attached to each singular point. As long as the projection (5.19) is one-to-one, as it presently is, this choice does not really matter. Further, of course not every point of the form $s=\boldsymbol{\ell} \cdot \boldsymbol{A}$ should correspond to a new alienderivative singularity (this was certainly not the case back in (2.64)), and, as such, at this stage one must still allow for some possible extra constraints or "limitations" either on the vector $\ell$ or on the Stokes vectors (e.g., this was the requirement that $k \leq 1$ in (2.64)). These are probably best understood at the level of motions on the "transseries lattice", to which we turn next. Akin to what happened in subsection 2.4, (5.21) leads to a set of allowed motions, this time around defined on an alien lattice. Again, this interpretation will naturally lead to a "statistical mechanical" approach to $k$-dimensional resurgence. To understand these motions, first recall the one-dimensional case illustrated in figures 9 and 10. In that case there was a single type of forward motion, essentially dictated by the constraint that the only non-vanishing "positive"

\footnotetext{
${ }^{83}$ In the present non-resonant case this linear map is one-to-one, i.e., $\operatorname{ker} \mathfrak{P}=\mathbf{0}$.

${ }^{84}$ Recalling the discussion in section 4 , we only need to consider the standard representative, i.e., we only need to focus upon the singularity structure of a simple resurgent function. Further, this expression may be derived (rather than motivated, as done here for pedagogical reasons) by starting-off with the bridge equations, and the interested reader may consult our appendix A on these technicalities.

${ }^{85}$ Again, this is due to presently addressing the non-resonant case.
} 
Stokes constant was $S_{1}$. At the same time, there were many different types of backward motions. Let us consider $k=2$ to illustrate the higher-dimensional cases in the following.

In the two-dimensional case the nodes $\Phi_{\boldsymbol{n}}$ live on a two-dimensional lattice, as $\boldsymbol{n} \in \mathbb{N}_{0}^{2}$. The alien derivative (5.21) will then induce different motions on this lattice, as illustrated in figure 17. Let $\boldsymbol{e}_{1} \equiv(1,0)$ and $\boldsymbol{e}_{2} \equiv(0,1)$ be the canonical basis of $\mathbb{Z}^{2}$ and write $\boldsymbol{\ell}=\ell_{1} \boldsymbol{e}_{1}+\ell_{2} \boldsymbol{e}_{2}$ and $\boldsymbol{S}_{\boldsymbol{\ell}}=S_{\boldsymbol{\ell}}^{(1)} \boldsymbol{e}_{1}+S_{\boldsymbol{\ell}}^{(2)} \boldsymbol{e}_{2}$. Starting-off at the lattice node $\Phi_{\boldsymbol{n}}$, strictly-backward motions-described by vectors with components $\ell_{1}, \ell_{2} \leq 0$-are allowed, and lead to corresponding singularities of $\mathcal{B}\left[\Phi_{\boldsymbol{n}}\right](s)$ at $s=\boldsymbol{\ell} \cdot \boldsymbol{A}$, as long as $\left|\ell_{i}\right| \leq n_{i}$ (recall that transseries sectors $\Phi_{\boldsymbol{m}}$ are set to vanish - do not exist - whenever one $m_{j}<0$ ), i.e., one can move backwards up to the boundary of the transseries $\mathbb{N}_{0}^{2}$ semi-positive grid. This is a familiar scenario also from the one-dimensional case, e.g., recall the backward motions in figure 10. On the other hand, one expects forward motions to be more restricted. In the one-dimensional case, the only ${ }^{86}$ allowed forward motion on the $\mathbb{Z}$-chain was the one generated by $\boldsymbol{\ell}=\boldsymbol{e}_{1}$ (here $\boldsymbol{e}_{1}$ the canonical basis of $\mathbb{Z}$ ). A similar two-dimensional scenario would entail that the only allowed strictly-forward motions, $\ell_{1}, \ell_{2} \geq 0$, would be associated to either $\boldsymbol{\ell}=\boldsymbol{e}_{1}$ (setting $\boldsymbol{S}_{\boldsymbol{\ell}}=\left(S_{\boldsymbol{e}_{1}}^{(1)}, 0\right)$ to be as close to the one-dimensional example as possible) or $\boldsymbol{\ell}=\boldsymbol{e}_{2}$ (now setting $\boldsymbol{S}_{\boldsymbol{\ell}}=\left(0, S_{\boldsymbol{e}_{2}}^{(2)}\right)$ ). This statement may be generalized to $k$ dimensions by only allowing strictly-forward motions which are elementary, i.e., given by some basis vector $\boldsymbol{e}_{i}$ within the canonical basis of $\mathbb{Z}^{k}$. In this way, an elementary forward motion $\boldsymbol{\ell}=\boldsymbol{e}_{i}$ would create a singularity at $s=\boldsymbol{\ell} \cdot \boldsymbol{A}=A_{i}$ with Stokes vector $\boldsymbol{S}_{\boldsymbol{e}_{i}}=\left(0, \ldots, 0, S_{\boldsymbol{e}_{i}}^{(i)}, 0, \ldots, 0\right)$, and the resurgent structure (5.21) becomes

$$
\Delta_{A_{i}} \Phi_{\boldsymbol{n}}=S_{\boldsymbol{e}_{i}}^{(i)}\left(n_{i}+1\right) \Phi_{\boldsymbol{n}+\boldsymbol{e}_{i}} .
$$

Consequentially, note that for forward motions along orthogonal directions, say $\boldsymbol{e}_{i}$ and $\boldsymbol{e}_{j}$ with $i \neq j$, the action of the alien derivatives commutes:

$$
\left[\Delta_{A_{i}}, \Delta_{A_{j}}\right] \Phi_{n}=0 .
$$

The above general expectations may be written as general constraints ${ }^{87}$ on Stokes vectors,

$$
S_{\ell}^{(j)}=0 \quad \text { if } \quad \ell_{i} \geq 1+\delta_{i j}, \quad \forall_{i \in\{1, \ldots, k\}}
$$

i.e., the $k$-dimensional extension of (2.64), or, the complete formulation of (5.21), is

$$
\Delta_{\ell \cdot \boldsymbol{A}} \Phi_{\boldsymbol{n}}=\boldsymbol{S}_{\boldsymbol{\ell}} \cdot(\boldsymbol{n}+\boldsymbol{\ell}) \Phi_{\boldsymbol{n}+\boldsymbol{\ell}}, \quad \ell_{i} \leq \delta_{i j} \quad \text { and } \quad \boldsymbol{\ell} \neq \mathbf{0} .
$$

Besides many strictly-backwards and few strictly-forward motions, of course one may also find a couple of mixed forward/backward motions on the lattice; where along one (and only one) direction one has an elementary forward motion, and along all others one has either no motion or some backwards motion. A few of these possible motions are illustrated in figure 17. Note that the "statistical mechanical" language from section 2 essentially holds in here as well, with the straightforward $k$-dimensional adaptations (instead of motions on a chain we now have motions on a lattice, with transseries nodes at each grid-site, and so on). In this way, the weight $w$ associated to a step $\mathcal{S}$ is defined analogously to (2.80), and as read directly from (5.25). For a step connecting the lattice nodes $\Phi_{\boldsymbol{n}}$ and $\Phi_{\boldsymbol{m}}$ one has

$$
w(\mathcal{S}(\boldsymbol{n} \rightarrow \boldsymbol{m}))=\boldsymbol{m} \cdot \boldsymbol{S}_{\boldsymbol{m}-\boldsymbol{n}} .
$$

\footnotetext{
${ }^{86}$ As mentioned, this was the requirement that $k \leq 1$ back in (2.64).

${ }^{87}$ Again, these conditions may be derived rather than just motivated; see footnote 84 .
} 


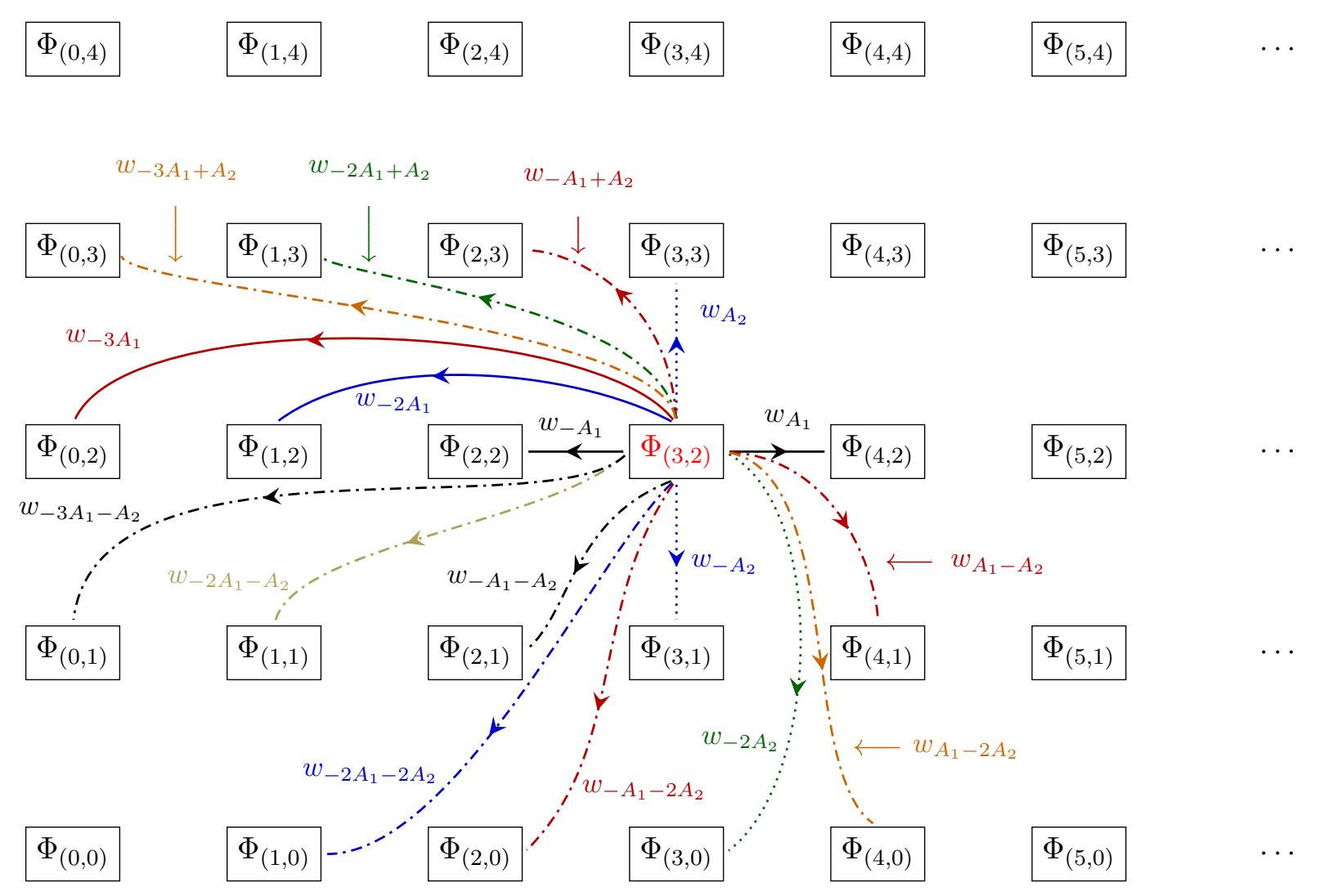

Figure 17. The $2 \mathrm{~d}$ alien lattice: a pictorial representation of the action of the alien derivative upon the sector $\Phi_{\boldsymbol{n}}$, with lattice-node $\boldsymbol{n}=(3,2)$ (now regarded as the origin for the $\mathbb{Z}^{2}$ lattice of Stokes vectors, thus dividing the alien lattice into quadrants). Different single arrows correspond to different single steps, and each step has an associated weight, as dictated by the right-hand-side of the resurgence relations (5.25). The weight of each step - written next to the corresponding arrow - is given in terms of Stokes vectors as explained in the text. The solid links refer to the action of the alien derivative along the grid direction $\boldsymbol{e}_{1}$ (with instanton action $A_{1}$ ), depicting both forward and backwards motions. The dotted links correspond to the action along the grid direction $\boldsymbol{e}_{2}$ (now with instanton action $A_{2}$ ). The mixed dashed/dotted links are motions which mix both directions. In the plot, colors are used solely to help differentiate distinct motions. The steps shown are all the single steps which are allowed starting at the $(3,2)$ lattice-site (where paths can then be constructed with different middle nodes, and corresponding products of weights).

In figure 17 we illustrate all motions out from $\boldsymbol{n} \equiv\left(n_{1}, n_{2}\right)=(3,2)$. There are two strictlyforward motions, with $\boldsymbol{\ell}=\boldsymbol{e}_{1}, \boldsymbol{e}_{2}$. One can use them to either reach the $(4,2)$ node, in which case $^{88} w_{A_{1}}=(4,2) \cdot \boldsymbol{S}_{(4,2)-(3,2)}=(4,2) \cdot \boldsymbol{S}_{\boldsymbol{e}_{1}}=4 S_{\boldsymbol{e}_{1}}^{(1)}$; or to reach the node $(3,3)$, in which case $w_{A_{2}}=(3,3) \cdot \boldsymbol{S}_{(3,3)-(3,2)}=(3,3) \cdot \boldsymbol{S}_{\boldsymbol{e}_{2}}=3 S_{\boldsymbol{e}_{2}}^{(2)}$. There are also many strictly-backwards motions,

\footnotetext{
${ }^{88}$ To make notation clearer, we are labeling the weights with the subscript $s=\boldsymbol{\ell} \cdot \boldsymbol{A}$.
} 
for example the one that takes us back to node $(1,1)$. In this case, $w_{-2 A_{1}-A_{2}}=(1,1) \cdot \boldsymbol{S}_{(1,1)-(3,2)}=$ $(1,1) \cdot \boldsymbol{S}_{(-2,-1)}=S_{-2 \boldsymbol{e}_{1}-\boldsymbol{e}_{2}}^{(1)}+S_{-2 \boldsymbol{e}_{1}-\boldsymbol{e}_{2}}^{(2)}$. Finally, there are a few "mixed" motions; consider for example the one taking us to node $(2,3)$. In this case, $w_{-A_{1}+A_{2}}=(2,3) \cdot \boldsymbol{S}_{(2,3)-(3,2)}=$ $(2,3) \cdot \boldsymbol{S}_{(-1,1)}=2 S_{-\boldsymbol{e}_{1}+\boldsymbol{e}_{2}}^{(1)}+3 S_{-\boldsymbol{e}_{1}+\boldsymbol{e}_{2}}^{(2)}$. All these, and many other, are illustrated in figure 17.

The $k$-dimensional case follows in a straightforward fashion. The alien geometry induced on the lattice based upon a given fixed node, $\boldsymbol{n}$, naturally splits $\mathbb{Z}^{k}$ (with origin at $\boldsymbol{n}$ ) into $k$ orthants (i.e., $k$-dimensional orthants or $k$-hyperoctants, generalizing the 2 -orthants or quadrants in figure 17). These $k$-orthants have distinct features on what concerns distributions of Stokes vectors - in fact, generically, most $k$-orthants will be empty of any Stokes vectors. The $\mathbb{Z}^{k}$ axes will be populated with Stokes vectors much like the one-dimensional case was, with Stokes constants. The "all-negative" $(-, \cdots,-) k$-orthant will be fully populated (at least up to the boundary of the original transseries semi-positive grid-now shifted by $\boldsymbol{n}$ ). The "single-plus" $(-, \cdots,-,+,-, \cdots,-) k$-orthants will only be partially populated, much like the second and fourth quadrants in figure 17 are. But any "double-plus" $(\cdots,+, \cdots,+, \cdots)$ or higher $k$-orthant will be empty of any Stokes vectors. This split of the $\mathbb{Z}^{k}$ alien lattice into distinct orthants is solely dictated by the constraint $\ell_{i} \leq \delta_{i j}$ on geometric motions given in (5.25).

As in the one-dimensional case, the structures encoded in (5.20) and (5.25) must be the same, upon relating the proportionality factors to each other. The $k$-dimensional novelty is that while Borel residues $\boldsymbol{S}_{\boldsymbol{n} \rightarrow \boldsymbol{n}+\boldsymbol{\ell}}$ are still scalars, the Stokes coefficients $\boldsymbol{S}_{\boldsymbol{\ell}}$ have now been "upgraded" to vectors. This obviously implies that relating them to each other will become a bit more intricate. Generalizing the one-dimensional calculations in appendix A, a few of these results follow. Let us split them according to the $k$-orthant classification we discussed above:

- Forward Axes: Let us pick any axis, generated by the $\mathbb{Z}^{k}$ canonical-basis vector $\boldsymbol{e}_{i}$, and consider its forward direction. This projects into a direction angle of $\theta_{i} \equiv \arg A_{i}$ on the complex Borel plane. For this forward direction, the $k$-dimensional generalization of (2.65) through (2.67) is, very naturally,

$$
\begin{aligned}
\mathrm{S}_{\boldsymbol{n} \rightarrow \boldsymbol{n}+\boldsymbol{e}_{i}} & =-\left(\boldsymbol{n}+\boldsymbol{e}_{i}\right) \cdot \boldsymbol{S}_{\boldsymbol{e}_{i}}, \\
\mathrm{~S}_{\boldsymbol{n} \rightarrow \boldsymbol{n}+2 \boldsymbol{e}_{i}} & =-\frac{1}{2}\left(\boldsymbol{n}+\boldsymbol{e}_{i}\right) \cdot \boldsymbol{S}_{\boldsymbol{e}_{i}}\left(\boldsymbol{n}+2 \boldsymbol{e}_{i}\right) \cdot \boldsymbol{S}_{\boldsymbol{e}_{i}}, \\
\mathrm{~S}_{\boldsymbol{n} \rightarrow \boldsymbol{n}+3 \boldsymbol{e}_{i}} & =-\frac{1}{6}\left(\boldsymbol{n}+\boldsymbol{e}_{i}\right) \cdot \boldsymbol{S}_{\boldsymbol{e}_{i}}\left(\boldsymbol{n}+2 \boldsymbol{e}_{i}\right) \cdot \boldsymbol{S}_{\boldsymbol{e}_{i}}\left(\boldsymbol{n}+3 \boldsymbol{e}_{i}\right) \cdot \boldsymbol{S}_{\boldsymbol{e}_{i}} .
\end{aligned}
$$

A closed-form expression is also simple to obtain (and comparing back to (2.68), it should now be clear why we wrote that equation without the binomial coefficient, back in section 2),

$$
\mathrm{S}_{\boldsymbol{n} \rightarrow \boldsymbol{n}+\ell \boldsymbol{e}_{i}}=-\frac{1}{\ell !} \prod_{m=1}^{\ell}\left(\boldsymbol{n}+m \boldsymbol{e}_{i}\right) \cdot \boldsymbol{S}_{\boldsymbol{e}_{i}} .
$$

Now, along the positive direction $\boldsymbol{e}_{i}$ there is a single Stokes vector, with components $\boldsymbol{S}_{\boldsymbol{e}_{i}}=$ $S_{\boldsymbol{e}_{i}}^{(i)} \boldsymbol{e}_{i}$, in which case the inverse map follows immediately from (5.27) above,

$$
S_{\boldsymbol{e}_{i}}^{(i)}=-\frac{1}{n_{i}+1} \mathrm{~S}_{\boldsymbol{n} \rightarrow \boldsymbol{n}+\boldsymbol{e}_{i}} .
$$

Finally, the fact that there are no further Stokes vectors along this positive direction results in the set of constraints generalizing (2.78) to the $k$-dimensional setting,

$$
\mathrm{S}_{\boldsymbol{n} \rightarrow \boldsymbol{n}+\ell \boldsymbol{e}_{i}}=\frac{(-1)^{\ell-1}}{\ell !} \prod_{m=0}^{\ell-1} \mathrm{~S}_{\boldsymbol{n}+m \boldsymbol{e}_{i} \rightarrow \boldsymbol{n}+(m+1) \boldsymbol{e}_{i}} .
$$


- Backward Axes: Let us remain along the same axis as in the previous case, but now consider its backward direction, i.e., the one associated to the direction vector $-\boldsymbol{e}_{i}$ and projecting into a direction angle of $\theta_{i}+\pi$ on the complex Borel plane. For this backward direction, the $k$-dimensional generalization of (2.69) through (2.71) is

$$
\begin{aligned}
\mathrm{S}_{\boldsymbol{n} \rightarrow \boldsymbol{n}-\boldsymbol{e}_{i}}= & -\left(\boldsymbol{n}-\boldsymbol{e}_{i}\right) \cdot \boldsymbol{S}_{-\boldsymbol{e}_{i}} \\
\mathrm{~S}_{\boldsymbol{n} \rightarrow \boldsymbol{n}-2 \boldsymbol{e}_{i}}= & -\left(\boldsymbol{n}-2 \boldsymbol{e}_{i}\right) \cdot\left\{\boldsymbol{S}_{-2 \boldsymbol{e}_{i}}+\frac{1}{2}\left(\boldsymbol{n}-\boldsymbol{e}_{i}\right) \cdot \boldsymbol{S}_{-\boldsymbol{e}_{i}} \boldsymbol{S}_{-\boldsymbol{e}_{i}}\right\} \\
\mathrm{S}_{\boldsymbol{n} \rightarrow \boldsymbol{n}-3 \boldsymbol{e}_{i}}= & -\left(\boldsymbol{n}-3 \boldsymbol{e}_{i}\right) \cdot\left\{\boldsymbol{S}_{-3 \boldsymbol{e}_{i}}+\frac{1}{2}\left(\boldsymbol{n}-\boldsymbol{e}_{i}\right) \cdot \boldsymbol{S}_{-\boldsymbol{e}_{i}} \boldsymbol{S}_{-2 \boldsymbol{e}_{i}}+\right. \\
& \left.+\frac{1}{2}\left(\boldsymbol{n}-2 \boldsymbol{e}_{i}\right) \cdot\left(\boldsymbol{S}_{-2 \boldsymbol{e}_{i}}+\frac{1}{3}\left(\boldsymbol{n}-\boldsymbol{e}_{i}\right) \cdot \boldsymbol{S}_{-\boldsymbol{e}_{i}} \boldsymbol{S}_{-\boldsymbol{e}_{i}}\right) \boldsymbol{S}_{-\boldsymbol{e}_{i}}\right\} .
\end{aligned}
$$

A closed-form expression is now a bit more intricate to obtain than in the earlier forwardcase. But, when comparing back to (2.72), it should also be clear how the present vectorial form is somewhat cleaner than the one-dimensional (scalar-like) case:

$$
\begin{aligned}
\mathrm{S}_{\boldsymbol{n} \rightarrow \boldsymbol{n}-\ell \boldsymbol{e}_{i}}= & -\sum_{s=1}^{\ell} \frac{1}{s !} \sum_{\substack{\left\{\ell_{1}, \ldots, \ell_{s} \geq 1\right\} \\
\sum \ell_{i}=\ell}}\left(\boldsymbol{n}-\ell_{1} \boldsymbol{e}_{i}\right) \cdot \boldsymbol{S}_{-\ell_{1} \boldsymbol{e}_{i}}\left(\boldsymbol{n}-\left(\ell_{1}+\ell_{2}\right) \boldsymbol{e}_{i}\right) \cdot \boldsymbol{S}_{-\ell_{2} \boldsymbol{e}_{i}} \cdots \\
& \cdots\left(\boldsymbol{n}-\left(\ell_{1}+\ell_{2}+\cdots+\ell_{s-1}\right) \boldsymbol{e}_{i}\right) \cdot \boldsymbol{S}_{-\ell_{s-1} \boldsymbol{e}_{i}}\left(\boldsymbol{n}-\ell \boldsymbol{e}_{i}\right) \cdot \boldsymbol{S}_{-\ell_{s} \boldsymbol{e}_{i}} \cdot
\end{aligned}
$$

Inverse maps follow as usual. A few examples, generalizing (2.73) through (2.75) to the present $k$-dimensional setting, are

$$
\begin{aligned}
\left(\boldsymbol{n}-\boldsymbol{e}_{i}\right) \cdot \boldsymbol{S}_{-\boldsymbol{e}_{i}}= & -\mathrm{S}_{\boldsymbol{n} \rightarrow \boldsymbol{n}-\boldsymbol{e}_{i}}, \\
\left(\boldsymbol{n}-2 \boldsymbol{e}_{i}\right) \cdot \boldsymbol{S}_{-2 \boldsymbol{e}_{i}}= & -\mathrm{S}_{\boldsymbol{n} \rightarrow \boldsymbol{n}-2 \boldsymbol{e}_{i}}-\frac{1}{2} \mathrm{~S}_{\boldsymbol{n} \rightarrow \boldsymbol{n}-\boldsymbol{e}_{i}} \mathrm{~S}_{\boldsymbol{n}-\boldsymbol{e}_{i} \rightarrow \boldsymbol{n}-2 \boldsymbol{e}_{i}}, \\
\left(\boldsymbol{n}-3 \boldsymbol{e}_{i}\right) \cdot \boldsymbol{S}_{-3 \boldsymbol{e}_{i}}= & -\mathrm{S}_{\boldsymbol{n} \rightarrow \boldsymbol{n}-3 \boldsymbol{e}_{i}}-\frac{1}{2} \mathrm{~S}_{\boldsymbol{n} \rightarrow \boldsymbol{n}-\boldsymbol{e}_{i}} \mathrm{~S}_{\boldsymbol{n}-\boldsymbol{e}_{i} \rightarrow \boldsymbol{n}-3 \boldsymbol{e}_{i}}-\frac{1}{2} \mathrm{~S}_{\boldsymbol{n} \rightarrow \boldsymbol{n}-2 \boldsymbol{e}_{i}} \mathrm{~S}_{\boldsymbol{n}-2 \boldsymbol{e}_{i} \rightarrow \boldsymbol{n}-3 \boldsymbol{e}_{i}}- \\
& -\frac{1}{3} \mathrm{~S}_{\boldsymbol{n} \rightarrow \boldsymbol{n}-\boldsymbol{e}_{i}} \mathrm{~S}_{\boldsymbol{n}-\boldsymbol{e}_{i} \rightarrow \boldsymbol{n}-2 \boldsymbol{e}_{i}} \mathrm{~S}_{\boldsymbol{n}-2 \boldsymbol{e}_{i} \rightarrow \boldsymbol{n}-3 \boldsymbol{e}_{i}},
\end{aligned}
$$

leading up to the vectorial closed-form expression (in fact very similar to its one-dimensional analogue (2.76))

$$
\left(\boldsymbol{n}-\ell \boldsymbol{e}_{i}\right) \cdot \boldsymbol{S}_{-\ell \boldsymbol{e}_{i}}=-\sum_{s=1}^{\ell} \frac{1}{s} \sum_{\substack{\left\{\ell_{1}, \ldots, \ell_{s} \geq 1\right\} \\ \sum \ell_{i}=\ell}} \mathrm{S}_{\boldsymbol{n} \rightarrow \boldsymbol{n}-\ell_{1} \boldsymbol{e}_{i}} \mathrm{~S}_{\boldsymbol{n}-\ell_{1} \boldsymbol{e}_{i} \rightarrow \boldsymbol{n}-\left(\ell_{1}+\ell_{2}\right) \boldsymbol{e}_{i}} \cdots \mathrm{S}_{\boldsymbol{n}-\left(\ell-\ell_{s}\right) \boldsymbol{e}_{i} \rightarrow \boldsymbol{n}-\ell \boldsymbol{e}_{i}} .
$$

This equation is valid as long as $1 \leq \ell<n_{i}$. Together with what we have just discussed in the forward-axes case, this tells us that for $\boldsymbol{\ell}=\boldsymbol{\ell} \boldsymbol{e}_{i}$ essentially the familiar one-dimensional constraints on the resurgence relations (from (2.64)) applies; i.e., $\ell \leq 1, \ell \neq 0$.

- "Single-Plus" k-Orthant: We shall illustrate this case with the $(+,-, \cdots,-) k$-orthant (this is the so-called "last", or $2^{k}$-th $k$-orthant), but the results are completely generic. In 
this $k$-orthant, a specific direction vector on the alien grid is given by, e.g.,

$$
\boldsymbol{v}=\boldsymbol{e}_{1}-\sum_{i=2}^{k} \nu_{i} \boldsymbol{e}_{i}, \quad \nu_{i} \in \mathbb{N}
$$

which projects into a direction angle of $\theta_{\boldsymbol{v}} \equiv \arg \boldsymbol{A} \cdot \boldsymbol{v}$ on the complex Borel plane. Any other directions are not allowed as they would lead to vanishing Stokes vectors. For these directions, the $k$-dimensional generalization of (2.65) through (2.67) is in fact completly equivalent to the forward-axes case, (5.27) through (5.29),

$$
\begin{aligned}
\mathrm{S}_{n \rightarrow n+v} & =-(\boldsymbol{n}+\boldsymbol{v}) \cdot \boldsymbol{S}_{\boldsymbol{v}} \\
\mathrm{S}_{n \rightarrow n+2 v} & =-\frac{1}{2}(\boldsymbol{n}+\boldsymbol{v}) \cdot \boldsymbol{S}_{\boldsymbol{v}}(\boldsymbol{n}+2 \boldsymbol{v}) \cdot \boldsymbol{S}_{\boldsymbol{v}} \\
\mathrm{S}_{\boldsymbol{n} \rightarrow \boldsymbol{n}+3 \boldsymbol{v}} & =-\frac{1}{6}(\boldsymbol{n}+\boldsymbol{v}) \cdot \boldsymbol{S}_{\boldsymbol{v}}(\boldsymbol{n}+2 \boldsymbol{v}) \cdot \boldsymbol{S}_{\boldsymbol{v}}(\boldsymbol{n}+3 \boldsymbol{v}) \cdot \boldsymbol{S}_{\boldsymbol{v}}
\end{aligned}
$$

leading up to the expression which generalizes (2.68) and (5.30),

$$
\mathrm{S}_{\boldsymbol{n} \rightarrow \boldsymbol{n}+\ell \boldsymbol{v}}=-\frac{1}{\ell !} \prod_{m=1}^{\ell}(\boldsymbol{n}+m \boldsymbol{v}) \cdot \boldsymbol{S}_{\boldsymbol{v}} \quad \text { for } \quad 1 \leq \ell<\min \left\{\left\lfloor\frac{n_{i}}{\nu_{i}}\right\rfloor\right\}_{i=2,3, \ldots, k}
$$

and zero otherwise. Except for the constraint of reaching the transseries-grid boundary, this is essentially the same result as in the forward-axes case. Further, because along the direction $\boldsymbol{v}$ there is a single non-vanishing Stokes vector $\boldsymbol{S}_{\boldsymbol{v}}$, the inverse map is already implicit in (5.42) above. The fact that there are no further Stokes vectors along this direction, results in the set of constraints generalizing (2.78) and (5.32),

$$
\mathrm{S}_{\boldsymbol{n} \rightarrow \boldsymbol{n}+\ell \boldsymbol{v}}=\frac{(-1)^{\ell-1}}{\ell !} \prod_{m=0}^{\ell-1} \mathrm{~S}_{\boldsymbol{n}+m \boldsymbol{v} \rightarrow \boldsymbol{n}+(m+1) \boldsymbol{v}} .
$$

Again, this is essentially the same result as in the earlier forward-axes case.

- "All-Negative" $k$-Orthant: This is the $(-, \cdots,-) k$-orthant. In this $k$-orthant, a specific direction vector on the alien grid is conventionally given by $-\boldsymbol{v}$, where, e.g.,

$$
\boldsymbol{v}=\sum_{i=1}^{k} \nu_{i} \boldsymbol{e}_{i}, \quad \nu_{i} \in \mathbb{N},
$$

so that $\boldsymbol{v}$ belongs to the "all-positive" $(+, \cdots,+) k$-orthant. Notice that the choice of coefficients $\nu_{i}$ is not completely arbitrary, as $\boldsymbol{v}$ must also be a lattice versor. This does not imply that this vector is normalized to unit length (it would not be on the lattice), but that it is the smallest lattice vector defining the said direction. The vector $\boldsymbol{v}$ projects into a direction angle of $\theta_{\boldsymbol{v}} \equiv \arg \boldsymbol{A} \cdot \boldsymbol{v}$, and thus the "all-negative" $k$-orthant projection yields a direction angle of $\theta_{\boldsymbol{v}}+\pi$ on the complex Borel plane. For this direction, the $k$ dimensional generalization of equations (2.69), (2.70) and (2.71), is completely equivalent to the backward-axes case, (5.33), (5.34) and (5.35), as

$$
\mathrm{S}_{n \rightarrow n-v}=-(\boldsymbol{n}-\boldsymbol{v}) \cdot S_{-\boldsymbol{v}}
$$




$$
\begin{aligned}
\mathrm{S}_{\boldsymbol{n} \rightarrow \boldsymbol{n}-2 \boldsymbol{v}}= & -(\boldsymbol{n}-2 \boldsymbol{v}) \cdot\left\{\boldsymbol{S}_{-2 \boldsymbol{v}}+\frac{1}{2}(\boldsymbol{n}-\boldsymbol{v}) \cdot \boldsymbol{S}_{-\boldsymbol{v}} \boldsymbol{S}_{-\boldsymbol{v}}\right\} \\
\mathrm{S}_{\boldsymbol{n} \rightarrow \boldsymbol{n}-3 \boldsymbol{v}}= & -(\boldsymbol{n}-3 \boldsymbol{v}) \cdot\left\{\boldsymbol{S}_{-3 \boldsymbol{v}}+\frac{1}{2}(\boldsymbol{n}-\boldsymbol{v}) \cdot \boldsymbol{S}_{-\boldsymbol{v}} \boldsymbol{S}_{-2 \boldsymbol{v}}+\right. \\
& \left.+\frac{1}{2}(\boldsymbol{n}-2 \boldsymbol{v}) \cdot\left(\boldsymbol{S}_{-2 \boldsymbol{v}}+\frac{1}{3}(\boldsymbol{n}-\boldsymbol{v}) \cdot \boldsymbol{S}_{-\boldsymbol{v}} \boldsymbol{S}_{-\boldsymbol{v}}\right) \boldsymbol{S}_{-\boldsymbol{v}}\right\} .
\end{aligned}
$$

It is tempting to notice how the content inside the curly brackets can be thought-of as a projection into the linear subspace spanned by $\mathcal{L}\left\{\boldsymbol{S}_{-\ell \boldsymbol{v}}, \boldsymbol{S}_{-(\ell-1) \boldsymbol{v}}, \ldots, \boldsymbol{S}_{-\boldsymbol{v}}\right\}$ (where, however, no statement may be made concerning dimension as, in general, nothing is said about the linear (in)dependence of these Stokes vectors). However, it turns out that this reasoning is not the best way to write a general formula (it leads to rather intricate coefficients at higher orders). Instead, the good generalization of (2.72) is essentially the one already used in the backward-axes case (5.36),

$$
\begin{aligned}
\mathrm{S}_{\boldsymbol{n} \rightarrow \boldsymbol{n}-\ell \boldsymbol{v}}= & -\sum_{s=1}^{\ell} \frac{1}{s !} \sum_{\substack{\left\{\ell_{1}, \ldots, \ell_{s} \geq 1\right\} \\
\sum \ell_{i}=\ell}}\left(\boldsymbol{n}-\ell_{1} \boldsymbol{v}\right) \cdot \boldsymbol{S}_{-\ell_{1} \boldsymbol{v}}\left(\boldsymbol{n}-\left(\ell_{1}+\ell_{2}\right) \boldsymbol{v}\right) \cdot \boldsymbol{S}_{-\ell_{2} \boldsymbol{v}} \cdots \\
& \cdots\left(\boldsymbol{n}-\left(\ell_{1}+\ell_{2}+\cdots+\ell_{s-1}\right) \boldsymbol{v}\right) \cdot \boldsymbol{S}_{-\ell_{s-1} \boldsymbol{v}}(\boldsymbol{n}-\ell \boldsymbol{v}) \cdot \boldsymbol{S}_{-\ell_{s} \boldsymbol{v}}
\end{aligned}
$$

The inverse mapping also follows in a similar fashion to the backward-axes case. A few examples, generalizing (2.73) through (2.75) and completely equivalent to (5.37) through (5.39), are

$$
\begin{aligned}
(\boldsymbol{n}-\boldsymbol{v}) \cdot \boldsymbol{S}_{-\boldsymbol{v}}= & -\mathrm{S}_{\boldsymbol{n} \rightarrow \boldsymbol{n}-\boldsymbol{v}} \\
(\boldsymbol{n}-2 \boldsymbol{v}) \cdot \boldsymbol{S}_{-2 \boldsymbol{v}}= & -\mathrm{S}_{\boldsymbol{n} \rightarrow \boldsymbol{n}-2 \boldsymbol{v}}-\frac{1}{2} \mathrm{~S}_{\boldsymbol{n} \rightarrow \boldsymbol{n}-\boldsymbol{v}} \mathrm{S}_{\boldsymbol{n}-\boldsymbol{v} \rightarrow \boldsymbol{n}-2 \boldsymbol{v}} \\
(\boldsymbol{n}-3 \boldsymbol{v}) \cdot \boldsymbol{S}_{-3 \boldsymbol{v}}= & -\mathrm{S}_{\boldsymbol{n} \rightarrow \boldsymbol{n}-3 \boldsymbol{v}}-\frac{1}{2} \mathrm{~S}_{\boldsymbol{n} \rightarrow \boldsymbol{n}-\boldsymbol{v}} \mathrm{S}_{\boldsymbol{n}-\boldsymbol{v} \rightarrow \boldsymbol{n}-3 \boldsymbol{v}}-\frac{1}{2} \mathrm{~S}_{\boldsymbol{n} \rightarrow \boldsymbol{n}-2 \boldsymbol{v}} \mathrm{S}_{\boldsymbol{n}-2 \boldsymbol{v} \rightarrow \boldsymbol{n}-3 \boldsymbol{v}}- \\
& -\frac{1}{3} \mathrm{~S}_{\boldsymbol{n} \rightarrow \boldsymbol{n}-\boldsymbol{v}} \mathrm{S}_{\boldsymbol{n}-\boldsymbol{v} \rightarrow \boldsymbol{n}-2 \boldsymbol{v}} \mathrm{S}_{\boldsymbol{n}-2 \boldsymbol{v} \rightarrow \boldsymbol{n}-3 \boldsymbol{v}}
\end{aligned}
$$

For the closed-form expression one finds

$$
(\boldsymbol{n}-\ell \boldsymbol{v}) \cdot \boldsymbol{S}_{-\ell \boldsymbol{v}}=-\sum_{s=1}^{\ell} \frac{1}{s} \sum_{\substack{\left\{\ell_{1}, \ldots, \ell_{s} \geq 1\right\} \\ \sum \ell_{i}=\ell}} \mathrm{S}_{\boldsymbol{n} \rightarrow \boldsymbol{n}-\ell_{1} \boldsymbol{v}} \mathrm{S}_{\boldsymbol{n}-\ell_{1} \boldsymbol{v} \rightarrow \boldsymbol{n}-\left(\ell_{1}+\ell_{2}\right) \boldsymbol{v}} \cdots \mathrm{S}_{\boldsymbol{n}-\left(\ell-\ell_{s}\right) \boldsymbol{v} \rightarrow \boldsymbol{n}-\ell \boldsymbol{v}}
$$

which generalizes (2.76) to the present $k$-dimensional setting, in complete analogy to (5.40). In both (5.51) and (5.55) one has to constrain

$$
1 \leq \ell<\min \left\{\left\lfloor\frac{n_{i}}{\nu_{i}}\right\rfloor\right\}_{i=1, \ldots, k},
$$

in order not to go past beyond the transseries-grid boundary.

So far the above considerations were rather generic. Furthermore, they were all motivated, none was really derived. Short of checking the derivation of these results (e.g., in appendix A), 
how can we test if these considerations are actually correct, and realized in examples? If the reader recalls subsection 2.4, one way to (numerically) probe the complex Borel plane of some specific example was via Padé approximants to the Borel transforms, as in (2.169). Back then, while addressing the nonlinear second-order ODE (2.52), we used this method to (numerically) learn about the nature of Borel singularities associated to different asymptotic sectors in its transseries solution (2.58). In particular, we found out about the existence of the "1.5"-parameter transseries due to the structure and accumulation of Borel (pole) singularities for the one-instanton sector; recall figure 7 . Within our present context, is it also possible to carry through an analogous procedure which, eventually, could validate the previous general expectations, equations (5.20) and (5.25)? This is what we shall investigate next, numerically analyzing the singular structure of the Borel transforms (5.20) via Padé approximants, within one example with $k>1$.

For simplicity and clarity of comparison, we shall choose an example with $k=2$ and such that it reproduces figure 17 as much as possible (in order to validate - or not - the structure we have put forward). In other words, we want the projection map (5.19), from $\mathbb{Z}^{2}$ to $\mathbb{C}$, to be as close as possible to the identity map (but without any risk to induce resonance!). In this way, we chose the nonlinear second-order Riccati-type ODE from [193], which is solved by a two-parameter transseries, but we modified it such that one of the instanton actions, $A_{1}$, is purely real, while the other, $A_{2}$, is purely imaginary. We also made sure their ratio is irrational. Following along similar steps to the ones in section 2, one now puts forward a two-parameter transseries ansatz and after some work eventually finds ${ }^{89}$ recursive equations for the coefficients in the asymptotic sectors $\Phi_{(n, m)}$. Once these coefficients have been computed, one can write down Borel transforms for each of the asymptotic sectors, determine their Padé approximants, and thus finally analyze their singularity (pole) structure. These numerical results are summarized in the plots of figure 18, for the cases of perturbative, $\Phi_{(0,0)}$, and $(3,2)$-instanton, $\Phi_{(3,2)}$, sectors. Let us first consider the singularity structure of $\mathcal{B}\left[\Phi_{(0,0)}\right](s)$, depicted on the left plot of figure 18 . It is simple to identify the expected branch-cuts starting at both $A_{1}=1$ and $A_{2}=\mathrm{i} \sqrt{2}$; these correspond to the Stokes single-step elementary forward motions $\boldsymbol{e}_{1}$ and $\boldsymbol{e}_{2}$ from figure 17, and are plotted in green. Apart from these branch-cuts, we further find the appearance of cuts at $2 A_{1}$ and $3 A_{1}$, as well as $2 A_{2}$ and $3 A_{2}$, plotted in orange. This is also precisely as expected, now corresponding to multi-step paths (not plotted in figure 17). In fact, recall that while figure 17 plotted (single-step) locations of (non-vanishing) Stokes vectors, figure 18 is now (numerically) identifying locations of all possible Borel residues, via the projection (5.19). So while in the former case the only non-vanishing possibility was, e.g., $\boldsymbol{S}_{\boldsymbol{e}_{1}}=S_{\boldsymbol{e}_{1}}^{(1)} \boldsymbol{e}_{1}$; in the latter case we have an infinite array of possibilities as listed in (5.30) and, e.g., now translating to

$$
\mathrm{S}_{0 \rightarrow \ell e_{1}}=-\left(S_{\boldsymbol{e}_{1}}^{(1)}\right)^{\ell}
$$

The same structure appears when addressing the singularity structure of $\mathcal{B}\left[\Phi_{(3,2)}\right](s)$ (shown on

${ }^{89}$ Our modified Ricatti-type ODE is (but see also [193] for the original, unmodified version):

$$
u^{\prime \prime}(z)+(1+\mathrm{i} \sqrt{2})\left(u^{\prime}(z)+u^{2}(z)\right)+\mathrm{i} \sqrt{2} u(z)+\mathrm{i} \sqrt{2} u(z) u^{\prime}(z)+u^{2}(z)+\frac{1}{z}=0,
$$

where we are now using the variable $z$, instead of the usual $x=\frac{1}{z}$ from the main text (but see appendix A). The solution to this differential equation, for $z \gg 1$, is a two-parameter transseries with instanton actions $A_{1}=1$ and $A_{2}=\mathrm{i} \sqrt{2}$. We are not going to give any further details on how to solve this equation, or on how to plot figure 18 . Instead, we leave it as an exercise to the reader to use the techniques learnt in section 2, alongside some elementary Mathematica coding, in order to iteratively construct the transseries solution to the above equation, and reproduce the numerical plots in figure 18. Hopefully this will be a very illustrative exercise! 

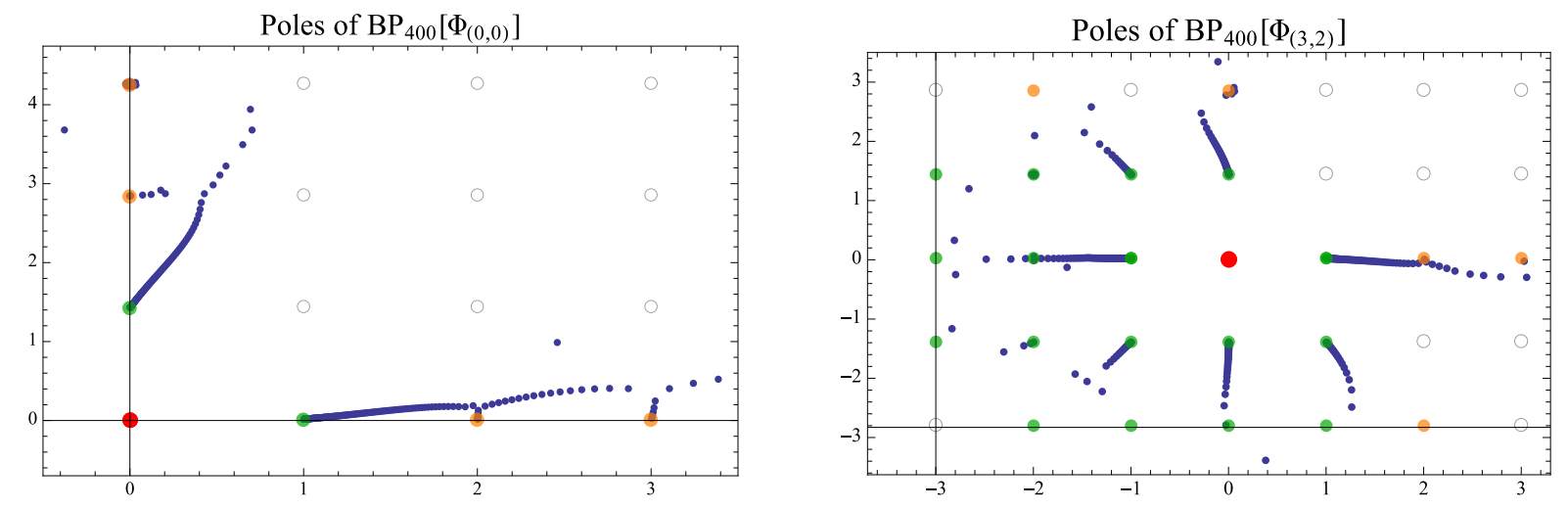

Figure 18. Poles of the (diagonal) order-400 Padé approximants for the Borel transforms of perturbative $\left(\mathrm{BP}_{400}\left[\Phi_{(0,0)}\right]\right)$ and $(3,2)$-instanton $\left(\mathrm{BP}_{400}\left[\Phi_{(3,2)}\right]\right)$ sectors in a modified, second-order nonlinear Riccati ODE. The particular perturbative (left image) or $(3,2)$-instanton (right image) sector which is under analysis is plotted with a red disk, whereas its "basic" singularities (i.e., the ones corresponding to the single-steps of figure 17) are plotted by green disks. Singularities corresponding to multi-step paths are then plotted as orange disks. The grey circles mark points on the Borel plane where no singularities are to be found (see the corresponding discussion in the main text). The condensation of poles to form branch-cuts is very clear - in fact, close to many of the singular points - and the more data available the more visible this would become in other (i.e., further "far away") singularities.

the right plot of figure 18). On one side we find the branch-cuts corresponding to the expected Stokes single-step backwards and forward motions depicted in figure 17; they are plotted in green. Apart from these, we further find poles gathering around the remaining multi-step singularities (and plotted in orange). The first quadrant is a "double-plus" 2-orthant and, as such, it is without surprise that we find it empty of any Borel singularities. All these results are exactly what was expected, based on our earlier $k$-orthant classification of singularities, thus yielding an excellent numerical support for the resurgent structure of Borel singularities which we have put forward in equations (5.20) and (5.25).

In conclusion, this time around numerical tests did not produce any new features as compared to our original expectations - unlike what happened back in subsection 2.4, where indeed they led to new results. Instead, on second thought, they helped us realize how the surprisingly simple resurgence relations (5.25) encode a vast amount of non-trivial information (the one displayed in figures 17 and 18). This should give the reader a glimpse into the fundamental role that alien derivatives play in this game, and the associated power of working at an algebraic level.

\section{Stokes Discontinuities and Transseries Asymptotics}

Following along the steps from subsection 2.4, our next goal is to compute Stokes discontinuities, $\operatorname{Disc}_{\theta}=\mathbf{1}-\underline{\mathfrak{S}}_{\theta}$, having in sight obtaining large-order formulae for the asymptotics of general transseries such as (5.17). These are given in terms of the Stokes automorphism (2.33), but where $\theta$ may now take multiple values, depending on the projections (5.19) of the several possible lattice directions (it was essentially restricted to being either 0 or $\pi$ back in subsection 2.4). Recall:

$$
\underline{\mathfrak{S}}_{\theta}=\exp \left(\sum_{\left\{\omega_{\theta}\right\}} \mathrm{e}^{-\frac{\omega_{\theta}}{x}} \Delta_{\omega_{\theta}}\right) \text {. }
$$


Although we are labeling this operator by $\theta$, on the complex Borel plane, it will be computed out of information on the alien lattice via the alien derivatives. This implies the "statistical mechanical" language we have used before holds generically. In particular, all concepts from subsection 2.4 pertaining to a step, $\mathcal{S}$, depicted in $(2.79)$; the weight of a step $\mathcal{S}, w(\mathcal{S})$, now given by (5.26); a path, $\mathcal{P}$, defined by (2.81); the length of a path, $\mathcal{P}, \ell(\mathcal{P})$, defined in (2.82); the weight of a path, $\mathcal{P}, w(\mathcal{P})$, defined in (2.83); and the combinatorial factor of a path $\mathcal{P}, \operatorname{CF}(\mathcal{P})$, defined in (2.84); they all have completely clear analogues in the present higher-dimensional case.

Let us illustrate the general principles by computing Stokes discontinuities within a twodimensional example, as usual, where $k=2$ and where we assume there is no resonance. We shall denote the two instanton actions by $A_{1}, A_{2}$, and will illustrate the computation of Stokes discontinuities along different singular directions by focusing on the $\Phi_{(3,2)}$ asymptotic sector shown in figure 17. The first question to address is how many different singular directions does this sector have, on the complex Borel plane (also have figure 18 in mind in the following). This question was already answered in full generality when we classified the distribution of Stokes vectors along $k$-orthants, but let us now see it in practice. For the moment, we simply split the set of singular directions into directions including one forward motion (including both the "forward axes" and "single-plus $k$-orthant" cases), and directions consisting only of backward motions (including both the "backward axes" and "all-negative $k$-orthant" cases). This implies the following, momentary, classification of singular directions (and their singular branch-points):

- Forward motions: forward resurgence motions which connect the original sector $\Phi_{(3,2)}$ with the sectors $\Phi_{\left(3-\ell_{1}, 3\right)}, \ell_{1}=0,1,2,3$ (the alien-lattice vectors defining these motions are $\boldsymbol{\ell}=$ $\left(-\ell_{1}, 1\right)$, i.e., the "upwards" motions of figure 17 ); and iterations thereof (meaning we can still reach sectors $\Phi_{(1,4)}$ and $\Phi_{(0,5)}$ by iterating the $\boldsymbol{\ell}=(-1,1)$ motion, and indefinitely reach sectors $\Phi_{(3, m)}$ with $m>3$ by iterating the $\boldsymbol{\ell}=(0,1)$ motion). Or else, forward resurgence motions which connect the original sector $\Phi_{(3,2)}$ with the sectors $\Phi_{\left(4,2-\ell_{2}\right)}, \ell_{2}=0,1,2$ (the alien-lattice vectors defining these motions are $\boldsymbol{\ell}=\left(1,-\ell_{2}\right)$, i.e., the "rightwards" motions of figure 17); and iterations thereof (meaning we can still reach sector $\Phi_{(5,0)}$ by iterating the $\boldsymbol{\ell}=(1,-1)$ motion, and indefinitely reach sectors $\Phi_{(n, 2)}$ with $n>4$ by iterating the $\boldsymbol{\ell}=(1,0)$ motion). The angle $\theta_{\ell}$ on the complex Borel plane, associated to each of the corresponding projected directions, is simply $\theta_{\ell}=\arg (\boldsymbol{\ell} \cdot \boldsymbol{A})$.

- Backward motions: all other resurgence motions from figure 17, now connecting the original sector $\Phi_{(3,2)}$ with the sectors $\Phi_{\left(3-\ell_{1}, 2-\ell_{2}\right)}$, where $\boldsymbol{\ell}=\left(-\ell_{1},-\ell_{2}\right)$ with $0 \leq \ell_{1} \leq 3,0 \leq \ell_{2} \leq 2$ but $\boldsymbol{\ell} \neq \mathbf{0}$ and $\boldsymbol{\ell} \neq(3,2)$; and iterations thereof (in this case, only the motions $\boldsymbol{\ell}=$ $(-1,0), \boldsymbol{\ell}=(0,-1)$, and $\boldsymbol{\ell}=(-1,-1)$ may be iterated, at least up to the boundary of the transseries grid). Again, the angle $\theta_{\ell}$ on the complex Borel plane, associated to each of the corresponding projected directions, is $\theta_{\boldsymbol{\ell}}=\arg (\boldsymbol{\ell} \cdot \boldsymbol{A})$.

Let us proceed with some examples, and first focus on the above class of forward motions. Fix a lattice direction $\boldsymbol{\ell}$, corresponding to a singular direction $\theta_{\boldsymbol{\ell}}=\arg (\boldsymbol{\ell} \cdot \boldsymbol{A})$ on the complex Borel plane. In this case, what will the Stokes automorphism (5.59) look like? It should be clear by now that we need to sum over all singular points along this fixed direction $\theta_{\ell}$, which may be reachable via (lattice) trajectories along this very same (projected) fixed direction $\theta_{\ell}$ (recall from figure 5 that the Stokes automorphism, being the operator behind Stokes phenomena as in, e.g., (2.7), is local in the singular direction of the complex Borel plane). It should also be clear that there is a single Stokes vector associated to each of these forward directions. This means that the Stokes automorphism for forward motions will be very similar to (2.88): akin to back then, 
there is basically one single forward motion which needs to be iterated in order to reach each of the other singular points along the chosen fixed direction. The straightforward $k$-dimensional generalization of (2.88) is then, very simply ${ }^{90}$,

$$
\underline{\mathfrak{S}}_{\theta_{\ell}} \Phi_{\boldsymbol{n}}=\exp \left(\mathrm{e}^{-\frac{\ell \cdot A}{x}} \Delta_{\ell \cdot A}\right) \Phi_{\boldsymbol{n}}
$$

Considering our "example-sector" $\boldsymbol{n}=(3,2)$, one finds ${ }^{91}$

$$
\begin{aligned}
\underline{\mathfrak{S}}_{\theta_{\ell}} \Phi_{(3,2)}= & \left(1+\mathrm{e}^{-\frac{\ell \cdot \boldsymbol{A}}{x}} \Delta_{\ell \cdot \boldsymbol{A}}+\frac{1}{2 !} \mathrm{e}^{-2 \frac{\ell \cdot \boldsymbol{A}}{x}} \Delta_{\boldsymbol{\ell} \cdot \boldsymbol{A}}^{2}+\frac{1}{3 !} \mathrm{e}^{-3 \frac{\boldsymbol{\ell} \cdot \boldsymbol{A}}{x}} \Delta_{\ell \cdot \boldsymbol{A}}^{3}+\cdots\right) \Phi_{(3,2)}= \\
= & \Phi_{(3,2)}+\boldsymbol{S}_{\boldsymbol{\ell}} \cdot\left(3-\ell_{1}, 3\right) \mathrm{e}^{-\frac{\boldsymbol{\ell} \cdot \boldsymbol{A}}{x}} \Phi_{\left(3-\ell_{1}, 3\right)}+\frac{1}{2 !}\left(\boldsymbol{S}_{\boldsymbol{\ell}} \cdot\left(3-\ell_{1}, 3\right)\right)\left(\boldsymbol{S}_{\boldsymbol{\ell}} \cdot\left(3-2 \ell_{1}, 4\right)\right) \times \\
& \times \mathrm{e}^{-2 \frac{\boldsymbol{\ell} \cdot \boldsymbol{A}}{x}} \Phi_{\left(3-2 \ell_{1}, 4\right)}+\frac{1}{3 !} \prod_{m=1}^{3}\left(\boldsymbol{S}_{\boldsymbol{\ell}} \cdot\left(3-m \ell_{1}, 2+m\right)\right) \mathrm{e}^{-3 \frac{\boldsymbol{\ell} \cdot \boldsymbol{A}}{x}} \Phi_{\left(3-3 \ell_{1}, 5\right)}+\cdots \\
= & \Phi_{(3,2)}-\mathrm{S}_{(3,2) \rightarrow\left(3-\ell_{1}, 3\right)} \mathrm{e}^{-\frac{\boldsymbol{\ell} \cdot \boldsymbol{A}}{x}} \Phi_{\left(3-\ell_{1}, 3\right)}-\mathrm{S}_{(3,2) \rightarrow\left(3-2 \ell_{1}, 4\right)} \mathrm{e}^{-2 \frac{\boldsymbol{\ell} \cdot \boldsymbol{A}}{x}} \Phi_{\left(3-2 \ell_{1}, 4\right)}- \\
& -\mathrm{S}_{(3,2) \rightarrow\left(3-3 \ell_{1}, 5\right)} \mathrm{e}^{-3 \frac{\boldsymbol{\ell} \cdot \boldsymbol{A}}{x}} \Phi_{\left(3-3 \ell_{1}, 5\right)}-\cdots,
\end{aligned}
$$

where we chose $\boldsymbol{\ell}=\left(-\ell_{1}, 1\right)$ and, as we already said above, this can be $\ell_{1} \in\{0,1,2,3\}$. But now we explicitly see the aforementioned interesting novelty as compared to the one-dimensional case. If we consider the strictly vertical direction, $\ell_{1}=0$, then the above expansion continues indefinitely, pretty much as in the one-dimensional case. But if otherwise, i.e., if $\ell_{1} \in\{1,2,3\}$ mixing vertical and horizontal lattice motions, then the expansion will truncate, which in the one-dimensional case only happened for backward motions. It is without surprise that the same features we uncovered when discussing Stokes vectors now reappear when discussing Stokes automorphisms: as already explained, this just means we have reached the boundary of the $\mathbb{N}_{0}^{2}$ semi-positive grid, and in this regard is somewhat similar to the one-dimensional truncation. Quantitatively, the $n$th term in the expansion above is proportional to the sector $\Phi_{\left(3-n \ell_{1}, 2+n\right)}$, which is non-zero only if $3-n \ell_{1} \geq 0$. Let us understand this in terms of motions and data on the alien lattice from figure 17, using the "statistical mechanical" language we used back in section 2. Similar to what happened in the one-dimensional case, this should yield a "physical" explanation behind the coefficients (Borel residues) in (5.61) above. In turns, let us consider a case where the expansion (5.61) does not truncate, and one where it does truncate:

- What is the Stokes automorphism along the strictly vertical direction $\boldsymbol{\ell}=(0,1)$, when acting upon the node $\Phi_{(3,2)}$ ? It is simple to compare the action of $\underline{\mathfrak{S}}_{\theta_{(0,1)}}$ on $\Phi_{(3,2)}$ from (5.61), with the allowed strictly-vertical resurgence motions out of the node $\Phi_{(3,2)}$ on the alien lattice of figure 17. The coefficients of the several terms in the expansion above then have the following "statistical mechanical" origin:

$-\Phi_{(3,2)} \rightarrow \Phi_{(3,2)}$ : this motion simply leaves the node invariant;

$-\Phi_{(3,2)} \rightarrow \Phi_{(3,3)}$ : there is a single path, of length $\ell=1$ and weight ${ }^{92} w=3 S_{\boldsymbol{e}_{2}}^{(2)}$, leading to a combinatorial factor $\mathrm{CF}=\frac{1}{1 !}$ and nonperturbative contribution $\mathrm{e}^{-\frac{A_{2}}{x}}$;

\footnotetext{
${ }^{90}$ Recall that here, when selecting directions, $\boldsymbol{\ell}$ is lattice versor.

${ }^{91}$ Just like in the one-dimensional case, if we did not know equation (5.45), one could also take each exponential term in the equality (5.61) as the defining equations for the Borel residues as functions of the Stokes constants.

${ }^{92}$ This is $w=(3,3) \cdot \boldsymbol{S}_{(0,1)}=3 S_{\boldsymbol{e}_{2}}^{(2)}$. It is interesting to compare this case to the one following $(2.90)$.
} 
$-\Phi_{(3,2)} \rightarrow \Phi_{(3,4)}$ : there is a single path (made of two steps), with length $\ell=2$ and weight $w=12\left(S_{\boldsymbol{e}_{2}}^{(2)}\right)^{2}$, leading to $\mathrm{CF}=\frac{1}{2 !}$ and nonperturbative contribution $\mathrm{e}^{-2 \frac{A_{2}}{x}}$;

$-\Phi_{(3,2)} \rightarrow \Phi_{(3,5)}$ : again there is a single path (with three steps), of length $\ell=3$ and weight $w=60\left(S_{\boldsymbol{e}_{2}}^{(2)}\right)^{3}$, leading to $\mathrm{CF}=\frac{1}{3 !}$ and nonperturbative contribution $\mathrm{e}^{-3 \frac{A_{2}}{x}}$.

- What is the Stokes automorphism along the diagonal "northwest" direction $\boldsymbol{\ell}=(-1,1)$, when acting upon the node $\Phi_{(3,2)}$ ? The procedure is essentially the same, only now we compare the action of $\underline{\mathfrak{S}}_{\theta_{(-1,1)}}$ on $\Phi_{(3,2)}$, with the allowed lattice diagonal-motions out of $\Phi_{(3,2)}$ and along $\boldsymbol{\ell}=(-1,1)$. Again, our coefficients have a "statistical mechanical" origin:

$-\Phi_{(3,2)} \rightarrow \Phi_{(3,2)}$ : this motion leaves the node invariant;

$-\Phi_{(3,2)} \rightarrow \Phi_{(2,3)}$ : there is one single path, of length $\ell=1$ and weight $w=\boldsymbol{S}_{(-1,1)} \cdot(2,3)$, leading to a combinatorial factor $\mathrm{CF}=\frac{1}{1 !}$ and nonperturbative contribution $\mathrm{e}^{-\frac{A_{2}-A_{1}}{x}}$;

$-\Phi_{(3,2)} \rightarrow \Phi_{(1,4)}$ : there is one single path (made of two steps), of length $\ell=2$ and weight $w=\left(\boldsymbol{S}_{(-1,1)} \cdot(2,3)\right)\left(\boldsymbol{S}_{(-1,1)} \cdot(1,4)\right)$, leading to $\mathrm{CF}=\frac{1}{2 !}$ and nonperturbative contribution $\mathrm{e}^{-2 \frac{A_{2}-A_{1}}{x}}$;

$-\Phi_{(3,2)} \rightarrow \Phi_{(0,5)}$ : again there is one single path (with three steps), of length $\ell=3$ and weight $w=\prod_{m=1}^{3} \boldsymbol{S}_{(-1,1)} \cdot(3-m, 2+m)$, leading to $\mathrm{CF}=\frac{1}{3 !}$ and nonperturbative contribution $\mathrm{e}^{-3 \frac{A_{2}-A_{1}}{x}}$. Further, one cannot move diagonally "northwest" from this node, in which case the expansion necessarily truncates here.

The general pattern should now be clear. Furthermore, we see how our initial class of forward motions - motivated by (5.60) - is actually best addressed if split in two, where one class is closer to the one-dimensional example (where there is no truncation of the exponential action) and the other somewhat closer to the backward motions due to the above truncation. Happily, this was precisely the original split we made when classifying $k$-orthants according to Stokes vectors.

Next, one can turn to the class of backward motions. Let us now fix the lattice direction as $\boldsymbol{\ell}=\left(-\ell_{1},-\ell_{2}\right)$, corresponding to the singular direction $\theta_{\boldsymbol{\ell}}=\arg (\boldsymbol{\ell} \cdot \boldsymbol{A})$ on the complex Borel plane. In order to understand what will the Stokes automorphism (5.59) look like in this case, we only need to recall the discussion that led up to (5.60). One quickly realizes that now the Stokes automorphism for backward motions must be very similar to (2.89): akin to back then, there may be several backward motions, some of which may even be iterated, but a truncation will always occur as soon as one hits the transseries-grid boundary. The straightforward $k$-dimensional generalization of (2.89) is then, very simply ${ }^{93}$,

$$
\underline{\mathfrak{S}}_{\theta_{\ell}} \Phi_{\boldsymbol{n}}=\exp \left(\sum_{m=1}^{\mathrm{n}} \mathrm{e}^{-m \frac{\ell \cdot \boldsymbol{A}}{x}} \Delta_{m \boldsymbol{\ell} \cdot \boldsymbol{A}}\right) \Phi_{\boldsymbol{n}}, \quad \mathrm{n}=\min \left\{\left\lfloor\frac{n_{i}}{\ell_{i}}\right\rfloor\right\}_{i=1, \ldots, k},
$$

where $\mathrm{n}$ locates the transseries-grid boundary. Considering our usual "example-sector" $\boldsymbol{n}=$ $(3,2)$, one may move backwards to $\left(-\ell_{1},-\ell_{2}\right)$, with $\ell_{1} \in\{0,1,2,3\}$ and $\ell_{2} \in\{0,1,2\}$ (obviously excluding $(0,0)$ and $(3,2)$ ). Let us then understand the resulting Stokes automorphism using the familiar "statistical mechanical" language to describe motions and data on the alien lattice.

\footnotetext{
${ }^{93}$ Recall that here, when selecting directions, $\boldsymbol{\ell}$ is lattice versor.
} 
- What is the Stokes automorphism along the diagonal "southwest" direction $\boldsymbol{\ell}=(-1,-1)$, when acting upon the node $\Phi_{(3,2)}$ ? In this case $\mathrm{n}=2$ and $\boldsymbol{\ell} \cdot \boldsymbol{A}=-A_{1}-A_{2}$. Having said this, the procedure is essentially the same as in the previous case. First expand the exponential (5.62), and use the resurgence relations to compute:

$$
\begin{aligned}
\underline{\mathfrak{S}}_{\theta \boldsymbol{\ell}} \Phi_{(3,2)}= & \left(1+\sum_{m=1}^{2} \mathrm{e}^{-m \frac{\boldsymbol{\ell} \cdot \boldsymbol{A}}{x}} \Delta_{m \boldsymbol{\ell} \cdot \boldsymbol{A}}+\frac{1}{2 !}\left(\sum_{m=1}^{2} \mathrm{e}^{-m \frac{\boldsymbol{\ell} \cdot \boldsymbol{A}}{x}} \Delta_{m \boldsymbol{\ell} \cdot \boldsymbol{A}}\right)^{2}+\right. \\
& \left.+\frac{1}{3 !}\left(\sum_{m=1}^{2} \mathrm{e}^{-m \frac{\boldsymbol{\ell} \cdot \boldsymbol{A}}{x}} \Delta_{m \boldsymbol{\ell} \cdot \boldsymbol{A}}\right)^{3}+\cdots\right) \Phi_{(3,2)}= \\
= & \Phi_{(3,2)}+\boldsymbol{S}_{(-1,-1)} \cdot(2,1) \mathrm{e}^{\frac{A_{1}+A_{2}}{x}} \Phi_{(2,1)}+\left(\boldsymbol{S}_{(-2,-2)} \cdot(1,0)+\right. \\
& \left.+\frac{1}{2 !}\left(\boldsymbol{S}_{(-1,-1)} \cdot(2,1)\right)\left(\boldsymbol{S}_{(-1,-1)} \cdot(1,0)\right)\right) \mathrm{e}^{2 \frac{A_{1}+A_{2}}{x}} \Phi_{(1,0)} \\
= & \Phi_{(3,2)}-\mathrm{S}_{(3,2) \rightarrow(2,1)} \mathrm{e}^{\frac{A_{1}+A_{2}}{x}} \Phi_{(2,1)}-\mathrm{S}_{(3,2) \rightarrow(1,0)} \mathrm{e}^{2 \frac{A_{1}+A_{2}}{x}} \Phi_{(1,0)} .
\end{aligned}
$$

The result may be written with either Stokes constants or, equivalently, Borel residues, $\operatorname{via}^{94}(5.48)$ and (5.49) (or, more generally, via (5.51)). It includes all the backward motions contributing to the Stokes automorphism along the direction defined by $\boldsymbol{\ell}=(-1,-1)$ and starting-off at node $\Phi_{(3,2)}$. Comparing this action of $\underline{\mathfrak{S}}_{\theta_{\ell}}$ on $\Phi_{(3,2)}$ with the allowed backward resurgence motions out of the $(3,2)$ node on the alien lattice of figure 17 , its "physical" interpretation easily follows:

- $\Phi_{(3,2)} \rightarrow \Phi_{(3,2)}$ : this simply leaves the node invariant;

$-\Phi_{(3,2)} \rightarrow \Phi_{(2,1)}$ : there is a single path of length $\ell=1$ and weight $w=2 S_{(-1,-1)}^{(1)}+$ $S_{(-1,-1)}^{(2)}$, leading to $\mathrm{CF}=\frac{1}{1 !}$ and nonperturbative contribution $\mathrm{e}^{+\frac{A_{1}+A_{2}}{x}}$;

$-\Phi_{(3,2)} \rightarrow \Phi_{(1,0)}$ : there are now two paths. One consists of a single step, with length $\ell=1$, weight $w=S_{(-2,-2)}^{(1)}$ and $\mathrm{CF}=\frac{1}{1 !}$; the other consists of two steps, with length $\ell=2$, weight $w=\left(2 S_{(-1,-1)}^{(1)}+S_{(-1,-1)}^{(2)}\right) S_{(-1,-1)}^{(1)}$ and CF $=\frac{1}{2 !}$. They both lead to a nonperturbative contribution $\mathrm{e}^{+2 \frac{A_{1}+A_{2}}{x}}$.

From these examples a general pattern should be easily recognizable, which we shall spell out in the two-dimensional case. In line with our earlier constructions, one may now define the Stokes discontinuities, $\operatorname{Disc}_{\theta_{\ell}}$, purely in terms of motions and data on the alien chain ${ }^{95}$ :

\footnotetext{
${ }^{94}$ Again, just like in the one-dimensional case, if we did not know equation (5.51), one could also take each exponential term in (5.63) as the defining equations for the Borel residues as functions of the Stokes constants.

${ }^{95}$ One may also think of the statistical component below, $\mathrm{SF}_{(n \rightarrow m)}$, as a "statistical-mechanical" definition of the Borel residues (up to a sign).
} 
Classification of Stokes directions: $\theta_{\boldsymbol{\ell}}=\arg (\boldsymbol{\ell} \cdot \boldsymbol{A})$, labeled by $\boldsymbol{\ell}=\left(\ell_{1}, \ell_{2}\right)$ and thus naturally divided into three types:

- Solely forward: $\boldsymbol{\ell}=(1,0)$ or $\boldsymbol{\ell}=(0,1)$;

- Mixed forward/backward: $\ell=\left(1,-\ell_{2}\right)$ or $\ell=\left(-\ell_{1}, 1\right)$, with $\ell_{1}, \ell_{2}>0$;

- Solely backwards: $\boldsymbol{\ell}=m\left(-\ell_{1},-\ell_{2}\right)$ or $\boldsymbol{\ell}=m(-1,0)$ or $\boldsymbol{\ell}=m(0,-1)$, with $\ell_{1}, \ell_{2}, m>0$, and $\ell_{1} / \ell_{2}$ an irreducible fraction.

Stokes discontinuity on direction $\theta_{\ell}: \operatorname{Disc}_{\theta_{\ell}} \Phi_{n}$ is given by a sum over all paths, linking to nodes along the same direction, $\Phi_{\boldsymbol{n}+m \boldsymbol{\ell}}$ (with $m>0$ ).

For all cases, each term in this $\operatorname{sum}\left(\Phi_{\boldsymbol{n}} \rightarrow \Phi_{\boldsymbol{n}+m \ell}\right)$ can be decomposed in two factors:

- Functional component, dictated solely by beginning and end nodes:

$$
-\mathrm{e}^{-m \frac{\ell \cdot \boldsymbol{A}}{x}} \Phi_{\boldsymbol{n}+m \ell} \cdot
$$

- Statistical component, sum over all the allowed paths $\mathcal{P}(\boldsymbol{n} \rightarrow \boldsymbol{n}+m \boldsymbol{\ell})$ linking the nodes as in figure 17 :

$$
\mathrm{SF}_{(\boldsymbol{n} \rightarrow \boldsymbol{n}+m \boldsymbol{\ell})} \equiv \sum_{\mathcal{P}(\boldsymbol{n} \rightarrow \boldsymbol{n}+m \boldsymbol{\ell})} \mathrm{CF}(\mathcal{P}) w(\mathcal{P}) .
$$

Having arrived at a complete description of all Stokes discontinuities, at this stage we shall not further pursue the general analysis of asymptotics and large-order relations within the context of multi-parameter transseries. While most formulae may be obtained somewhat straightforwardly ${ }^{96}$ by following our general reasonings in section 2 -now based upon the above results for the Stokes discontinuities - a particular example of a multi-parameter transseries (further displaying resonance) will be thoroughly studied in the upcoming section 6 , and addressing it should be enough. As such, let us pause for a moment in order to address Stokes phenomena.

We have mentioned Stokes phenomenon in the one-dimensional setting, (2.7), but we are still short of a proof for such statement (another simple proof, based on the bridge equations, may be found in appendix A). This is actually very simple, in light of the combinatorics of the Borel residues. Consider the one-dimensional setting with transseries (2.5) and (2.6),

$$
\Phi(x, \sigma)=\sum_{n=0}^{+\infty} \sigma^{n} \mathrm{e}^{-n \frac{A}{x}} \Phi_{n}(x),
$$

and forward $(\theta=0)$ Stokes automorphism (2.88), or, equivalently for our present purposes, forward Borel residues (2.68) — which we shall now write binomially as

$$
\mathrm{S}_{n \rightarrow n+\ell}=-\left(\begin{array}{c}
n+\ell \\
\ell
\end{array}\right) S_{1}^{\ell}
$$

\footnotetext{
${ }^{96}$ This is in itself a very good and challenging exercise for the reader.
} 
Instead of focusing solely on the action of the Stokes automorphism upon a transseries node, as we have been doing up to now, we may ask what is the action of the Stokes automorphism on the full transseries. The calculation is elementary, but illustrative:

$$
\begin{aligned}
\underline{S}_{0} \Phi(x, \sigma) & =\Phi(x, \sigma)-\sum_{n=0}^{+\infty} \sigma^{n} \mathrm{e}^{-n \frac{A}{x}} \operatorname{Disc}_{0} \Phi_{n}(x)=-\sum_{n=0}^{+\infty} \sum_{\ell=0}^{+\infty} \mathrm{S}_{n \rightarrow n+\ell} \sigma^{n} \mathrm{e}^{-(n+\ell) \frac{A}{x}} \Phi_{n+\ell} \\
= & \sum_{n=0}^{+\infty} \sum_{\ell=0}^{+\infty}\left(\begin{array}{c}
n+\ell \\
\ell
\end{array}\right) S_{1}^{\ell} \sigma^{n} \mathrm{e}^{-(n+\ell) \frac{A}{x}} \Phi_{n+\ell}=\sum_{m=0}^{+\infty}\left(\sigma+S_{1}\right)^{m} \mathrm{e}^{-m \frac{A}{x}} \Phi_{m}(x)=\Phi\left(x, \sigma+S_{1}\right),
\end{aligned}
$$

where the key step occurs at the beginning of the second line, when we make use of the binomial formulation (5.65). It should be clear that this trivially generalizes to the $k$-dimensional setting, when and only when in the forward-axes case: this is essentially due to the fact that the Stokes vector only has one component, $\boldsymbol{S}_{\boldsymbol{e}_{i}}=S_{\boldsymbol{e}_{i}}^{(i)} \boldsymbol{e}_{i}$, in which case (2.68) and (5.30) are basically the same and the calculation above follows unchanged. In such a purely forward direction, $\boldsymbol{e}_{i}$, one thus finds that the action of the Stokes automorphism (5.60) upon the full multi-parameter transseries (5.17) is given by

$$
\underline{\mathfrak{S}}_{i} u(x, \boldsymbol{\sigma})=u\left(x, \boldsymbol{\sigma}+\boldsymbol{S}_{\boldsymbol{e}_{i}}\right) \text {, }
$$

generalizing (2.7) for rather broad transseries classes.

On the other hand, in a mixed forward/backward direction, while the combinatorics remain similar the action of the Stokes automorphism will truncate; and in a solely backwards direction the combinatorics get much more involved while the action of the Stokes automorphism keeps truncating. This results in much more intricate formulae (and although we briefly mention them in appendix A, we also refer the reader to [15] for some such expressions). In this way, an alternative and perhaps easier path to address Stokes automorphisms along these backward directions is to first notice the following key fact concerning the above, purely forward direction: the action of the Stokes automorphism (5.67) is a translation along $\boldsymbol{e}_{i}$ in $\boldsymbol{\sigma}$-space. In other words, we can write

$$
\underline{\mathfrak{S}}_{i} u(x, \boldsymbol{\sigma})=u\left(x, \boldsymbol{\sigma}+\boldsymbol{S}_{\boldsymbol{e}_{i}}\right) \equiv \exp \left(\boldsymbol{S}_{\boldsymbol{e}_{i}} \cdot \frac{\partial}{\partial \boldsymbol{\sigma}}\right) u(x, \boldsymbol{\sigma}) .
$$

But, from (5.60), the Stokes automorphism in this case is

$$
\underline{\mathfrak{S}}_{\theta_{i}}=\exp \left(\mathrm{e}^{-\frac{e_{i} \cdot \boldsymbol{A}}{x}} \Delta_{\boldsymbol{e}_{i} \cdot \boldsymbol{A}}\right)
$$

which implies that the alien derivative has a $\boldsymbol{\sigma}$-space vector-field representation given by

$$
\mathrm{e}^{-\frac{\boldsymbol{e}_{i} \cdot \boldsymbol{A}}{x}} \Delta_{\boldsymbol{e}_{i} \cdot \boldsymbol{A}}=\boldsymbol{S}_{\boldsymbol{e}_{i}} \cdot \frac{\partial}{\partial \boldsymbol{\sigma}}
$$

The reader should recall that this is not the first time such an expression appears; we have seen this very same feature back in section 2, equations (2.117) and (2.118), or (2.122) and (2.143).

This vector-field representation is, essentially, the famous bridge equation we already alluded to several times before. In fact, such representation establishes a bridge between alien derivations and ordinary calculus - albeit in $\boldsymbol{\sigma}$-space. Recall that, e.g., in the ODE context, the transseries parameters encode the boundary conditions needed to specify a particular solution, which means that $\boldsymbol{\sigma}$-space is essentially describing the moduli space of solutions to this given ODE. But the take-home message from this discussion is that such vector-field representations, or bridge equations, may allow for a more geometrical viewpoint on the analysis of Stokes phenomena along more complicated (i.e., non-elementary) directions [15]. This is undissociated from the algebraic structures encoded in the alien derivations, to which we need to turn next. 


\section{Algebras of Alien Derivatives and Virasoro-Like Algebras}

The alert reader may have already noticed two "teasers" earlier in the text, pointing towards what is to come now. These were, first, the non-commutativity of alien derivatives along the same (one-dimensional) direction in (2.87), and, second, the commutativity of alien derivatives along orthogonal ( $k$-dimensional) directions in (5.23). In particular, equation (2.87), which we rewrite in here as

$$
\left[\frac{\Delta_{-m A}}{S_{-m}}, \frac{\Delta_{-n A}}{S_{-n}}\right]=(m-n) \frac{\Delta_{-(m+n) A}}{S_{-(m+n)}} .
$$

is already making a very strong case for the appearance of the $c=0$ (classical) Virasoro algebra in the present context,

$$
\left[L_{m}, L_{n}\right]=(m-n) L_{m+n} .
$$

The similarity is striking, but one should proceed with caution in order to understand exactly how much of the algebra of alien derivatives is equatable to the Virasoro algebra (and, thus, to the use of eventual benefits from its well-studied representation theory). One immediately notices that there cannot be an isomorphism as not all Virasoro generators $L_{n}$-where one has $n \in \mathbb{Z}$ will have an alien derivative counterpart $\Delta_{n A}$-where now $n \leq 1$ with $n \neq 0$. It is precisely this lack of a $L_{0}$ counterpart which prevents the use of the Virasoro representation theory in the present context. In order to delve on this a bit further, let us first make a very short detour in order to summarize basic ideas of conformal transformations and the representation theory of the Virasoro algebra (but see also, e.g., [120, 133], which we follow below).

Within the setting of conformal field theory (CFT), the aforementioned Virasoro operators $L_{n}$ with $n \in \mathbb{Z}$ (and their complex conjugates $\bar{L}_{n}$ ), generate local conformal transformations acting on two-dimensional conformal fields, with given conformal weight $(h, \bar{h})$ (in particular implying that these conformal fields will have associated scaling dimension $\Delta=h+\bar{h}$ and spin $s=h-\bar{h}$ ). These operators $L_{n}$ are generators of the (full) Virasoro algebra, with central charge $c$, given by

$$
\left[L_{m}, L_{n}\right]=(m-n) L_{m+n}+\frac{c}{12}\left(m^{3}-m\right) \delta_{m+n} .
$$

However, due to a possibly non-vanishing central charge $c$, global conformal transformations are only generated by the subset $\left\{L_{-1}, L_{0}, L_{1}\right\}$ (alongside their complex conjugates).

Constructing representations of the Virasoro algebra follows standard steps ${ }^{97}$. It is based upon the existence of eigenstates $|\psi\rangle$ of $L_{0}$,

$$
L_{0}|\psi\rangle=h_{\psi}|\psi\rangle
$$

for which the $L_{n}$ may act as either creation or annihilation operators. In fact, because $\left[L_{0}, L_{n}\right]=$ $-n L_{n}$, operators $L_{n}$ with $n>0$ will act as lowering or annihilation operators for $L_{0}$,

$$
L_{0}\left(L_{n}|\psi\rangle\right)=\left(h_{\psi}-n\right)\left(L_{n}|\psi\rangle\right) .
$$

Likewise, operators $L_{n}$ with $n<0$ will act as raising or creation operators for $L_{0}$. In this setting, it is then rather natural to define a highest-weight state, denoted by $|h\rangle$, as an eigenvector of the $L_{0}$ operator (with eigenvalue $h$ ) and which is further annihilated by all the lowering operators $L_{n>0}$, i.e.,

$$
L_{0}|h\rangle=h|h\rangle, \quad L_{n>0}|h\rangle=0 .
$$

\footnotetext{
${ }^{97}$ As we shall see in the following, the representation theory of the Virasoro algebra is quite similar to the one of $\mathrm{SU}(2)$, with $L_{0}$ playing the role of $J_{3}$ and with the several $L_{n>0}$ and $L_{n<0}$ playing the role of an infinite number of copies of the (single) $\mathrm{SU}(2)$ pair $J_{ \pm}$.
} 
If one is to act instead with the raising operators $L_{n<0}$ on the highest-weight state $|h\rangle$, this then creates a tower of descendent states associated to $|h\rangle$. These descendent states will also be eigenstates of $L_{0}$, and one may thus group them into different levels in such a way that the $N$ th level is spanned by the states

$$
L_{-k_{1}} L_{-k_{2}} \cdots L_{-k_{q}}|h\rangle \quad \text { with } \quad k_{1} \geq \cdots \geq k_{q}>0 \quad \text { and } \quad \sum_{i=1}^{q} k_{i}=N,
$$

with corresponding $L_{0}$ eigenvalue $h+N$. The set of descendant states constructed from a highestweight state $|h\rangle$ is known as a Verma module, which summarizes a Virasoro representation.

The conclusion is thus quite swift: due to the lack of a $L_{0}$ counterpart (i.e., $\Delta_{0}$ does not exist), there is no sense of a highest-weight state in the alien derivative context, and one cannot make use of the Virasoro representation theory in this context. Nonetheless, there is one thing that might be learnt from the (partial) match between (5.71) and (5.72): this is yet another (and, actually, rather non-standard) glimpse at the origin of the bridge equations; further supporting and extending our previous (5.70). The (classical) Virasoro algebra (5.72) has rather well-know representations using vector fields (this algebra describes diffeomorphisms of the circle), and thus so will (5.71). One such representation using homogeneous vector fields (of degree or weight $m$, $n$, and $m+n$, respectively) is precisely given by

$$
\left[\left(-\sigma^{m+1} \frac{\partial}{\partial \sigma}\right),\left(-\sigma^{n+1} \frac{\partial}{\partial \sigma}\right)\right]=(m-n)\left(-\sigma^{m+n+1} \frac{\partial}{\partial \sigma}\right) .
$$

Upon comparison with (5.71), this immediately implies:

$$
\Delta_{-n A} \propto S_{-n} \sigma^{n+1} \frac{\partial}{\partial \sigma} .
$$

Notice how this yields a standard derivative differential representation for the alien derivative. The identification of the present $\sigma$-variable with the transseries parameter is done based upon (5.70), which unfolded a bridge equation for forward motions. The above result, (5.79), now unfolds a completion of the bridge equation (5.70) also for backward motions. This bridge between standard and alien calculus is herein established via the Virasoro-like vector-field representation of the alien-derivative algebra (5.71), although we also refer the reader to appendix A for a more standard (and complete!) derivation of this equation.

Having started a "CFT description" of alien chains, based upon the Virasoro-like algebraic structure found in (5.71), we may ask whether this description extends to alien lattices (recall our standard example in figure 17). In this case, the multi-dimensional resurgence relations (5.25) lead us to first introduce the more general operators $G_{\boldsymbol{n}}(\boldsymbol{v})$, depending on an arbitrary vector field, $\boldsymbol{v}$, and defined as

$$
G_{\boldsymbol{n}}(\boldsymbol{v}) \Phi_{\boldsymbol{k}}=\boldsymbol{v} \cdot(\boldsymbol{k}+\boldsymbol{n}) \Phi_{\boldsymbol{k}+\boldsymbol{n}} .
$$

Of course that when the vector field $\boldsymbol{v}$ is chosen to be $\boldsymbol{v}=\boldsymbol{S}_{\boldsymbol{n}}$ we recover the alien derivative operators (at least when $\boldsymbol{n}$ obeys its required constraints in (5.25)). This implies that the algebraic structure of the alien derivatives, acting on a multi-dimensional alien lattice, essentially sits inside the general algebraic structure of the above $G_{n}(\boldsymbol{v})$ operators. This general structure 
is itself given by ${ }^{98}$ the commutation relations ${ }^{99}$

$$
\left[G_{\boldsymbol{n}}(\boldsymbol{v}), G_{\boldsymbol{m}}(\boldsymbol{u})\right]=G_{\boldsymbol{n}+\boldsymbol{m}}((\boldsymbol{v} \cdot \boldsymbol{m}) \boldsymbol{u}-(\boldsymbol{u} \cdot \boldsymbol{n}) \boldsymbol{v}) .
$$

The algebra of alien-lattice alien-derivatives then follows as the subalgebra of (5.83) where we constrain ourselves to operators of the form $G_{\boldsymbol{\ell}}\left(\boldsymbol{S}_{\boldsymbol{\ell}}\right) \equiv \Delta_{\boldsymbol{\ell} \cdot \boldsymbol{A}}$. In this case, one finds:

$$
\left[G_{n}\left(S_{n}\right), G_{m}\left(S_{m}\right)\right]=G_{n+m}\left(\left(S_{n} \cdot \boldsymbol{m}\right) S_{m}-\left(S_{m} \cdot \boldsymbol{n}\right) \boldsymbol{S}_{\boldsymbol{n}}\right) .
$$

Two facts are immediately noticeable concerning this final result. The first is that this multidimensional alien-lattice algebraic structure is a multi-dimensional generalization of the Virasoro algebra (albeit, as we shall see in the following, the standard Virasoro algebra (5.73) also sits inside this structure). The second, more problematic issue, is that this multi-dimensional alienlattice algebraic structure is generically open, i.e., the commutator of two alien derivatives is not an alien derivative. This is unlike the previous (more general) algebraic structure (5.83) (and, in fact, it is the reason why we started-off with that structure in the first place; because it is algebraically closed). Closure of the alien-lattice algebraic structure may nonetheless be achieved when one has

$$
\left(\boldsymbol{S}_{\boldsymbol{n}} \cdot \boldsymbol{m}\right) \boldsymbol{S}_{\boldsymbol{m}}-\left(\boldsymbol{S}_{\boldsymbol{m}} \cdot \boldsymbol{n}\right) \boldsymbol{S}_{\boldsymbol{n}} \propto \boldsymbol{S}_{\boldsymbol{n}+\boldsymbol{m}}
$$

This is a non-trivial requirement on Stokes vectors, and it would be interesting to classify what types of linear or nonlinear problems satisfy this constraint. In the following, however, we shall limit ourselves to a couple of simple exercises where the alien-lattice algebra does close, and where we may further rediscover Virasoro-like structures as in (5.71).

One simple exercise to return to is that of the commutator of two, orthogonal, forward motions, as in (5.23). Choosing $\boldsymbol{n}=\boldsymbol{e}_{n}$ and $\boldsymbol{m}=\boldsymbol{e}_{m}$, with $n \neq m$, we already know that this implies corresponding Stokes vectors $\boldsymbol{S}_{\boldsymbol{e}_{n}}=S_{\boldsymbol{e}_{n}}^{(n)} \boldsymbol{e}_{n}$ and $\boldsymbol{S}_{\boldsymbol{e}_{m}}=S_{\boldsymbol{e}_{m}}^{(m)} \boldsymbol{e}_{m}$. Then it immediately follows that $\left(\boldsymbol{S}_{\boldsymbol{e}_{n}} \cdot \boldsymbol{e}_{m}\right) \boldsymbol{S}_{\boldsymbol{e}_{m}}-\left(\boldsymbol{S}_{\boldsymbol{e}_{m}} \cdot \boldsymbol{e}_{n}\right) \boldsymbol{S}_{\boldsymbol{e}_{n}}=\mathbf{0}$; in which case, with $G_{\boldsymbol{\ell}}(\mathbf{0})=\mathbf{0}$ due to linearity in the vectorial argument, one finds the expected result, i.e., (5.84) translates to

$$
\left[G_{\boldsymbol{e}_{n}}\left(\boldsymbol{S}_{\boldsymbol{e}_{n}}\right), G_{\boldsymbol{e}_{m}}\left(\boldsymbol{S}_{\boldsymbol{e}_{m}}\right)\right]=G_{\boldsymbol{e}_{n}+\boldsymbol{e}_{m}}(\mathbf{0})=0 .
$$

Without surprise, this recovers the commutation relation we had already found.

The other simple exercise to discuss is one of the cleanest scenarios where (5.85) is indeed verified and the alien-lattice algebra (5.84) closes: this is the case where all Stokes vectors are parallel. Let us pick some direction, defined by the versor $\boldsymbol{u}$, and let us suppose that all Stokes vectors, $\boldsymbol{S}_{\boldsymbol{n}}, \boldsymbol{S}_{\boldsymbol{m}}$, and $\boldsymbol{S}_{\boldsymbol{m}+\boldsymbol{n}}$, are parallel, i.e.,

$$
S_{n}\left\|S_{m}\right\| S_{m+n} \quad \Rightarrow \quad\left\{\begin{array}{l}
S_{n}=S_{n} u \\
S_{m}=S_{m} u \\
S_{m+n}=S_{m+n} u
\end{array}\right.
$$

\footnotetext{
${ }^{98}$ Finding this result is a straightforward exercise which we leave for the reader. Just use (5.80) twice to compute the consecutive action of two $G_{\boldsymbol{n}}$-operators on a fixed sector,

$$
G_{\boldsymbol{n}}(\boldsymbol{v}) G_{\boldsymbol{m}}(\boldsymbol{u}) \Phi_{\boldsymbol{k}}=\boldsymbol{u} \cdot(\boldsymbol{k}+\boldsymbol{m}) \boldsymbol{v} \cdot(\boldsymbol{k}+\boldsymbol{m}+\boldsymbol{n}) \Phi_{\boldsymbol{k}+\boldsymbol{m}+\boldsymbol{n}},
$$

and then compute the commutator by expressing it back as the action of (5.80) on the chosen fixed sector.

${ }^{99}$ These may also be written component-wise, as

$$
\left[G_{\boldsymbol{n}}^{i}, G_{\boldsymbol{m}}^{j}\right]=\left(m^{i} \delta_{\ell}^{j}-n^{j} \delta_{\ell}^{i}\right) G_{\boldsymbol{n}+\boldsymbol{m}}^{\ell} .
$$


This immediately implies that, in this case,

$$
\left(S_{n} \cdot \boldsymbol{m}\right) \boldsymbol{S}_{m}-\left(\boldsymbol{S}_{m} \cdot \boldsymbol{n}\right) \boldsymbol{S}_{n}=\boldsymbol{u} \cdot(\boldsymbol{m}-\boldsymbol{n}) S_{n} S_{m} \boldsymbol{u}
$$

and thus the alien-lattice algebra closes as

$$
\left[\frac{G_{n}\left(S_{n}\right)}{S_{n}}, \frac{G_{m}\left(S_{m}\right)}{S_{m}}\right]=u \cdot(\boldsymbol{m}-\boldsymbol{n}) \frac{G_{m+n}\left(S_{m+n}\right)}{S_{m+n}}
$$

This is essentially the same result as in (5.71). In the present multi-dimensional case one thus finds underlying Virasoro-like algebraic structures along directions of parallel Stokes vectors.

In short, the algebraic structure of multi-dimensional alien derivatives clearly extends beyond the realm of the Virasoro algebra. It is properly defined as the more general algebraic structure (5.83), as the particular alien-derivative subset (5.84) does not necessarily form a subalgebra (i.e., does not necessarily close). In cases where it does closed, such as the above (5.87), then we see how Virasoro-like structures reappear along special directions in the space of Stokes vectors.

Let us finish this section with a multi-dimensional generalization of the rather non-standard (albeit partial) derivation of the bridge equations, which we discussed earlier in the one-dimensional case (5.79) (as mentioned many times before, the standard derivation of the bridge equations is done in appendix A). A differential representation of (5.83), with homogeneous vector fields, may be achieved via (with no implicit summations)

$$
\left[\left(-\boldsymbol{\sigma}^{-\boldsymbol{n}} \sigma_{i} \frac{\partial}{\partial \sigma_{i}}\right),\left(-\boldsymbol{\sigma}^{-\boldsymbol{m}} \sigma_{j} \frac{\partial}{\partial \sigma_{j}}\right)\right]=\left(m^{i} \delta_{j}^{\ell}-n^{j} \delta_{i}^{\ell}\right)\left(-\boldsymbol{\sigma}^{-\boldsymbol{n}-\boldsymbol{m}} \sigma_{\ell} \frac{\partial}{\partial \sigma_{\ell}}\right),
$$

where under $\sigma_{i} \rightarrow \lambda \sigma_{i}$ the degree or weight of each vector field is

$$
\boldsymbol{\sigma}^{n} \sigma_{j} \frac{\partial}{\partial \sigma_{j}} \rightarrow \lambda^{n_{i}} \boldsymbol{\sigma}^{n} \sigma_{j} \frac{\partial}{\partial \sigma_{j}}
$$

Upon comparison with (5.82) and (5.84), this immediately implies:

$$
\Delta_{\ell \cdot \boldsymbol{A}} \propto \boldsymbol{\sigma}^{-\ell} \sum_{i=1}^{k} S_{\ell}^{(i)} \sigma_{i} \frac{\partial}{\partial \sigma_{i}} .
$$

Again, this yields a standard-derivative differential representation for the alien derivative, with the expected identification of the present $\boldsymbol{\sigma}$-variables with the transseries parameters following from (5.70). This equation gives further support to the existence of a multi-dimensional bridge equation, also building upon (5.70) and (5.79), and appendix A should make a good departure point into these technical issues for the interested reader.

\section{Resurgent Analysis of an Elliptic-Potential Integral}

Having arrived this far, the reader has by now acquired a fairly complete knowledge on the main ideas of resurgence, transseries, and their asymptotics. There is, however, one final topic we still need to address in order to complete the basic toolset: this is the phenomenon of resonance. Resonance is a new feature which only occurs within the realm of multi-parameter transseries, and which we shall introduce in the present section as it appears in a simple example (much along the spirit of what was done back in section 2 , when introducing generalities on resurgence 
and transseries via the quartic potential). Note how on the road to resonance we will necessarily drive by familiar locations (e.g., the asymptotics of non-resonant resurgent-transseries roughly occupies the first half of the present section). When doing so we shall be less detailed than before (leaving the filling of the details as exercises), but obviously will still keep our higher pedagogical standards from previous sections as soon as any discussions involving resonance begin.

In parallel with what was done in section 2 , let us consider a partition-function toy-model described by a one-dimensional integral, as in (2.8), but where the potential is now given by an elliptic function. This "elliptic partition function" is given by

$$
Z(\hbar \mid m)=\frac{1}{\sqrt{\pi \hbar}} \int_{\Gamma} \mathrm{d} z \exp \left(-\frac{1}{\hbar} \operatorname{sd}^{2}(z \mid m)\right)
$$

where $\operatorname{sd}(z \mid m)$ is a subsidiary Jacobian elliptic function with (squared) modulus $m$ (see, e.g., [194] for further details). The potential, $\operatorname{sd}^{2}(z \mid m)$, is then a doubly-periodic complex function, along both real and imaginary axes with respective periods ${ }^{100} 2 \mathbb{K}(m)$ and $2 \mathrm{i} \mathbb{K}^{\prime}(m) \equiv 2 \mathrm{i} \mathbb{K}(1-m)$. In particular, this elliptic potential has a zero at the origin and a double pole at $z=\mathbb{K}+\mathrm{i} \mathbb{K}^{\prime}$. Due to the double periodicity the potential is naturally defined over a torus, which we may usually take as the fundamental parallelogram $\left\{0,2 \mathbb{K}, 2 \mathrm{i} \mathbb{K}^{\prime}, 2\left(\mathbb{K}+\mathrm{i} \mathbb{K}^{\prime}\right)\right\}$ in $\mathbb{C}$, with the appropriate congruent identifications (see figure 19). The (squared) modulus $m \in[0,1]$ sets the ratio of the orthogonal periods of this torus. In particular, at the limiting value $m=0$ the imaginary period diverges, whereas at $m=1$ it is the real period which diverges. For (standard) notational simplicity, we also define $m^{\prime} \equiv 1-m$. This example, (6.1), was originally introduced in [27] as a toy model to illustrate the appearance of complex saddles leading to negative-real instanton actions within a simple ${ }^{101}$ setting. The choice of such an elliptic potential also had a very natural physical justification, being the sole Jacobian-elliptic combination interpolating between the familiar sineGordon $\left(\sin ^{2}(z)\right.$ as $\left.m \rightarrow 0\right)$ and $\sinh -G o r d o n\left(\sinh ^{2}(z)\right.$ as $\left.m \rightarrow 1\right)$ potentials. Herein, we will extend the large-order analysis of [27] into a complete resurgent analysis and further use this elliptic potential as a toy model to introduce the phenomenon of resonance.

In order to specify the contours of integration, $\Gamma$, and understand what are their admissible choices on the present compact domain (this is in contrast with what happened in section 2), let us begin with naïve steepest-descent analysis. There are three critical points of the potential

$$
V(z)=\operatorname{sd}^{2}(z \mid m),
$$

given by the zeroes of the $\operatorname{sd}(z), \operatorname{cd}(z)$, and $\operatorname{nd}(z)$ subsidiary Jacobian elliptic functions. They are located at, respectively,

$$
z_{0}^{*}=0, \quad z_{1}^{*}=\mathbb{K}, \quad \text { and } \quad z_{2}^{*}=\mathrm{i} \mathbb{K}^{\prime} .
$$

These critical points lead to $V\left(z_{0}^{*}\right)=0$ and to the (real) instanton actions

$$
V\left(z_{1}^{*}\right)=\frac{1}{m^{\prime}} \equiv A_{1} \quad \text { and } \quad V\left(z_{2}^{*}\right)=-\frac{1}{m} \equiv A_{2} .
$$

In this way, the critical point at the origin, $z_{0}^{*}$, corresponds to the standard perturbative saddle; the critical point which sits on the positive real axis, $z_{1}^{*}$, leads to a positive-real instanton action; and the critical point which sits on the positive imaginary axis, $z_{2}^{*}$, finally leads to a negative-real

\footnotetext{
${ }^{100}$ In here, $\mathbb{K}(m)$ denotes the complete elliptic-integral of the first kind, $\mathbb{K}(m)=\int_{0}^{\frac{\pi}{2}} \frac{\mathrm{d} \theta}{\sqrt{1-m \sin ^{2} \theta}}$.

${ }^{101}$ Such negative-real instanton actions first appeared within the harder Painlevé realm [130]; see also [16, 205].
} 


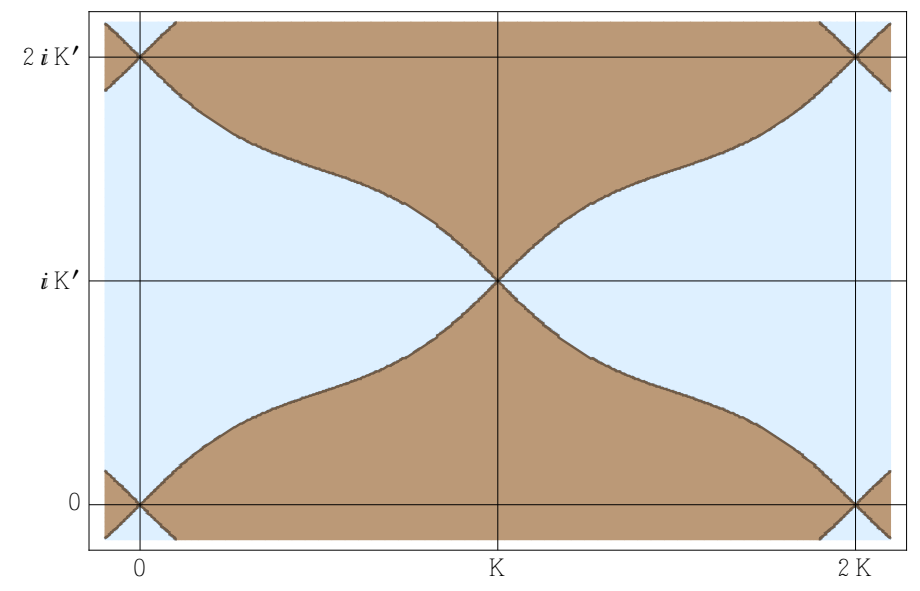

Figure 19. The elliptic potential in (6.1) in the complex $z$-plane, with $\hbar \in \mathbb{R}^{+}$and $m=0.9$ in the plot. The brown (darker) region is where $\mathbb{R e} V(z)>0$, while the blue (lighter) region is where $\mathbb{R e} V(z)<0$.

instanton action (at least when $\hbar \in \mathbb{R}^{+}$). Further, due to their dependence upon the (squared) modulus $m$, both these instanton actions are tunable (i.e., they vary with $m$ ). We will later see how specific choices of $m$ will either display, or not, a resonant structure.

At this stage we may already guess the form of the partition-function transseries as (compare with $(2.22))$

$$
\begin{aligned}
\mathcal{Z}\left(\hbar, \sigma_{0}, \sigma_{1}, \sigma_{2}\right) & =\sigma_{0} \Phi_{0}(\hbar)+\sigma_{1} \mathrm{e}^{-\frac{A_{1}}{\hbar}} \Phi_{1}(\hbar)+\sigma_{2} \mathrm{e}^{-\frac{A_{2}}{\hbar}} \Phi_{2}(\hbar) \\
& =\sigma_{0} \Phi_{0}(\hbar)+\sigma_{1} \mathrm{e}^{-\frac{1}{\hbar m^{\prime}}} \Phi_{1}(\hbar)+\sigma_{2} \mathrm{e}^{+\frac{1}{\hbar m}} \Phi_{2}(\hbar) .
\end{aligned}
$$

The $\Phi_{i}(\hbar)$ are formal asymptotic series in $\hbar$ and the $\sigma_{i}$ are the transseries parameters. Recalling the discussion in section 2 , it is natural to assume that each of these asymptotic series is associated to specific - and distinct - steepest-descent contours. In order to understand which are these admissible choices for $\Gamma$ in (6.1), we will next follow the ideas introduced in subsection 2.1, and formalised in section 3 in terms of Picard-Lefschetz theory, in order to construct the steepestdescent contours, or Lefschetz thimbles, associated to each saddle. This will further provide a geometrical perspective on the Stokes phenomenon.

\subsection{Phase Diagram from Stokes Phenomena}

The Picard-Lefschetz theory outlined in section 3 was built upon a polynomial potential, $V(z)$, which made it easy to identify the set of (steepest-descent) integration cycles over which the integral (3.1) was convergent. In fact, therein, convergence was simply achieved by requiring $\mathbb{R e}\left(-\frac{1}{\hbar} V(z)\right) \rightarrow-\infty$ as $|z| \rightarrow+\infty$ along some appropriate region within $\mathbb{C}$. Identifying the set of integration cycles over which the integral (6.1) is convergent is now necessarily different as the potential is no longer polynomial (it is in fact meromorphic) neither is its domain unbounded (the torus $\mathbb{T}^{2}$ is compact). Happily, however, the potential (6.2) has a single (double) pole on $\mathbb{T}^{2}$ (located at $z=\mathbb{K}+\mathrm{i} \mathbb{K}^{\prime}$ in the fundamental parallelogram), in which case it turns out that we can set-up Picard-Lefschetz theory in the present setting in a completely analogous fashion to what we did earlier in section 3. All one has to note is that the Lefschetz thimbles are now curves (cycles) which originate and end at this double-pole, wrapping around the torus and passing 
through the relevant critical point(s). In this particular example things are even simpler as the thimbles amount to oriented paths of steepest descent, just like in (2.11); recall it:

$$
\operatorname{Im}\left(V(z)-V\left(z^{*}\right)\right)=0 .
$$

For the perturbative saddle at the origin, $z_{0}^{*}$, and for both positive-real and negative-real instanton saddles, $z_{1}^{*}$ and $z_{2}^{*}$, steepest-descent contours are depicted in figure 20 for different values of $\theta=\arg \hbar$ (in the following we use $x \equiv \hbar$ in order to make it easier to compare present formulae with the ones in section 2). In this figure, we have also plotted the regions where the elliptic potential is positive (negative): comparing with figure 19, we now see how these regions change with $\theta$. Let us explain the plotting details of figure 20: for each value of $\theta$ we have plotted the leading saddle(s) with a solid disk, while the subleading saddle(s) were plotted with a circle. Regardless of dominance, the perturbative saddle is always plotted in black, with the positive-real instanton saddle in red and the negative-real instanton saddle in blue. In this way, solid contours are steepest-descent contours associated with the leading saddle(s), while dashed contours are steepest-descent contours associated with the subleading saddle(s). All steepest-descent contours originate and end at the double-poles, which are plotted with solid stars. Note how the plots extend over a couple of fundamental parallelograms, but this is for drawing clarity (i.e., so that the three steepest-descent contours may be plotted without falling on top of each other). As such, the period lattice must still be modded-out in order to obtain the corresponding final picture on $\mathbb{T}^{2}$. Finally, as expected, generically a steepest-descent contour goes through a single saddle - but this is not always the case due to Stokes phenomenon.

In the above set-up, the analysis carried out in section 3 holds. In such Picard-Lefschetz framework, let us first analyze the structure of steepest-descent contours for the elliptic partitionfunction (6.1) as the phase of $\hbar$ changes (subsequently denoted by $x$ ). Associated to the three critical points $z_{i}^{*}$ there will be three corresponding thimbles $\mathcal{J}_{i}$. For generic values of $\theta=\arg x$ all exponential arguments $\sim A / x$ from (6.4) have different imaginary parts, in which case there is a well-defined thimble decomposition of the integration contour as

$$
\Gamma=\sum_{i=0}^{2} n_{i} \mathcal{J}_{i} .
$$

However, when $\arg x=0, \pi$, the imaginary parts of all the critical values coincide - in fact they all vanish. These values of $\theta$ describe the Stokes lines, and the intersection numbers $n_{i}$ encounter monodromies as we cross them. These results are also summarised in figure 20, for different values of $\theta \in[0,2 \pi)$ (where the Stokes lines are very distinctive, with different thimbles crossing more than one saddle). In-between this interchange of exponential dominance, one also identifies the corresponding anti-Stokes lines, at $\theta=\frac{\pi}{2}, \frac{3 \pi}{2}$; the directions where all saddles are of the same magnitude. These are the turning points where saddles which used to be leading become subleading, and vice-versa. Let us next discuss the Stokes jumps, at both Stokes lines $\theta=0, \pi$.

\section{Monodromy and Stokes Phenomenon at $\theta=0$}

Comparing the last and second plots in figure 20 it is clear how some thimbles are jumping at the $\theta=0$ Stokes line. This may also be simply checked by evaluating (6.7) at either $\theta=0^{-}$or $\theta=0^{+}$. What is happening is that the asymptotic series in (6.6), obtained by integrations along the corresponding cycles, must remain continuous across Stokes rays. As we already discussed back in section 2, this can only be guaranteed as long as each thimble has the same asymptotics for 

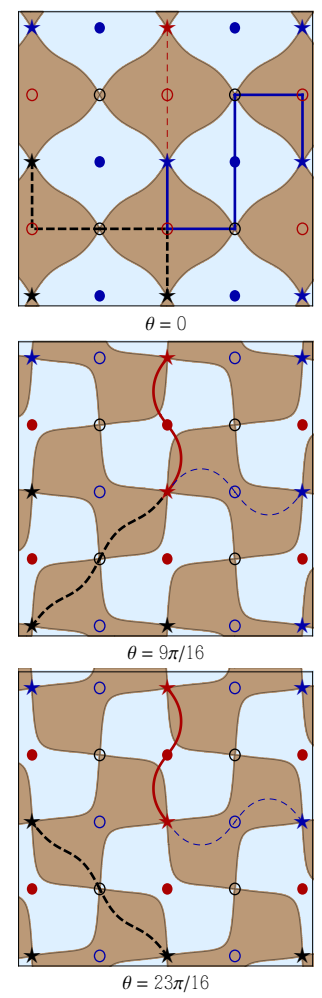
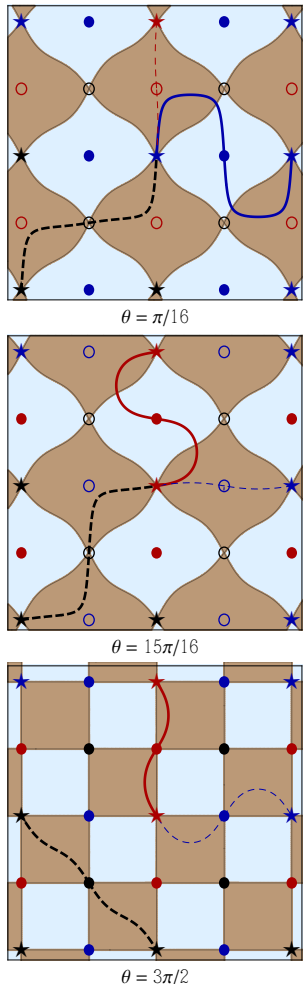
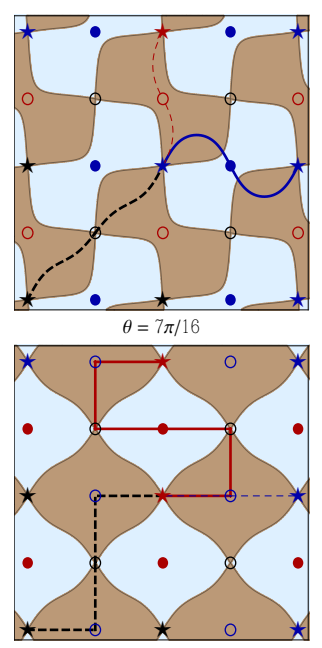

$\theta=\pi$

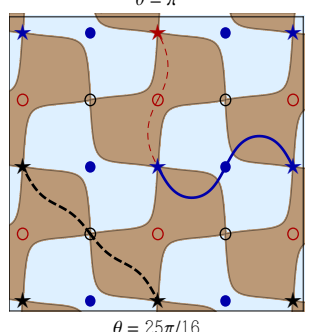

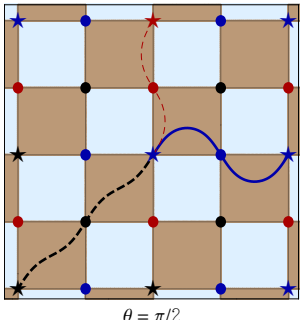

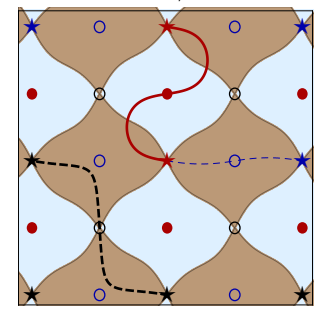

$\theta=17 \pi / 1$

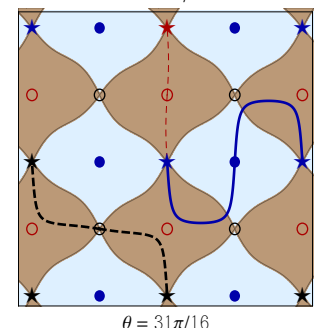

Figure 20. Steepest-descent contours for the elliptic integral, through the several saddles, along with positivity of the potential, for different values of $\theta=\arg x(|x|=1, m=0.9$ in the plot). The saddles and their respective thimbles are depicted as follows: $z_{0}^{*}$ in black, $z_{1}^{*}$ in red, and $z_{2}^{*}$ in blue. The leading saddles are plotted as solid lines/filled circles, while the subleading saddles are plotted as empty circles/dashed lines (the "lighter" dashed-lines being the most suppressed). The paths start and end at the double-poles, represented by filled stars. Stokes lines are at $\arg x=0, \pi$ when the steepest-descent contours hit more than one saddle; anti-Stokes lines are at $\arg x=\frac{\pi}{2}, \frac{3 \pi}{2}$. See the main text for a full discussion.

$\theta=0^{-}$and $\theta=0^{+}$. This continuity condition then implies that, as one moves counterclockwise from $\theta=0^{-}$to $\theta=0^{+}$, the cycles $\mathcal{J}_{i}$ must acquire the monodromy ("jumps")

$$
\left(\begin{array}{c}
\mathcal{J}_{2} \\
\mathcal{J}_{0} \\
\mathcal{J}_{1}
\end{array}\right) \rightarrow S_{\circlearrowleft}^{(0)}\left(\begin{array}{c}
\mathcal{J}_{2} \\
\mathcal{J}_{0} \\
\mathcal{J}_{1}
\end{array}\right)=\left(\begin{array}{ccc}
1 & -2 & -2 \\
0 & 1 & 2 \\
0 & 0 & 1
\end{array}\right)\left(\begin{array}{c}
\mathcal{J}_{2} \\
\mathcal{J}_{0} \\
\mathcal{J}_{1}
\end{array}\right),
$$

where $S_{\circlearrowleft}^{(0)}$ is the "Stokes matrix" associated to $\theta=0$.

In more geometric terms, this non-trivial monodromy may also be understood as a result of the continuity of the integration cycle itself, $\Gamma$, across $\theta=0$. In other words, the thimble decomposition (6.8) of a given cycle $\Gamma$ is different for $\theta=0^{-}$and $\theta=0^{+}$, and thus the same cycle $\Gamma$ is expressed as different linear combination of thimbles for $\theta=0^{ \pm}$(recall (3.36)). Let us elucidate this point starting with the particular "perturbative" cycle $\Gamma=\mathcal{J}_{0}$, illustrated in the two middle plots of figure 21 (and start with the middle-left plot). As $\theta$ changes from $0^{-}$ to $0^{+}$, the tails of the cycle $\mathcal{J}_{0}$ (i.e., the neighbourhood of $\mathcal{J}_{0}$ around the pole) flip. This flip is necessary in order to keep the orientation of $\mathcal{J}_{0}$ around the saddle point, which itself is required to preserve continuity of integration along such contour. Now, at the level of the integration cycle 

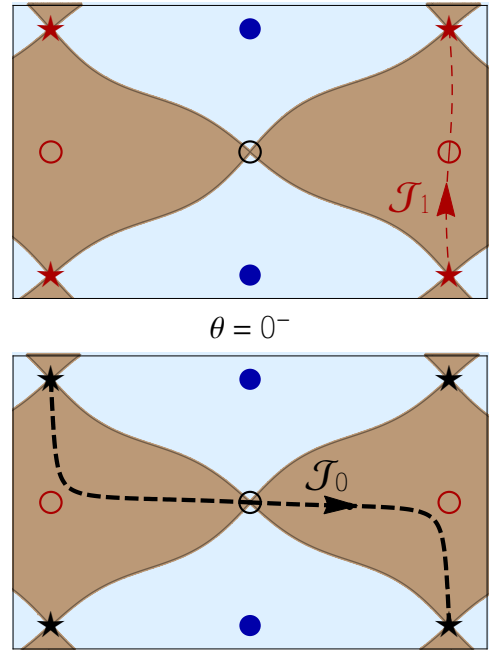

$\theta=0^{-}$

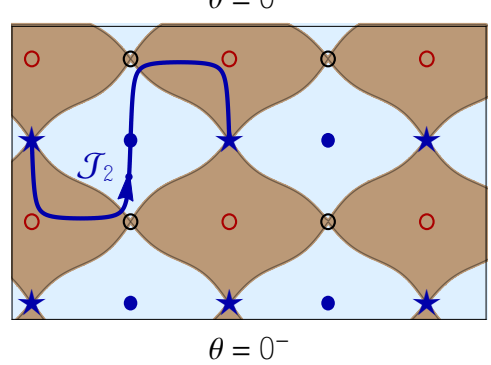

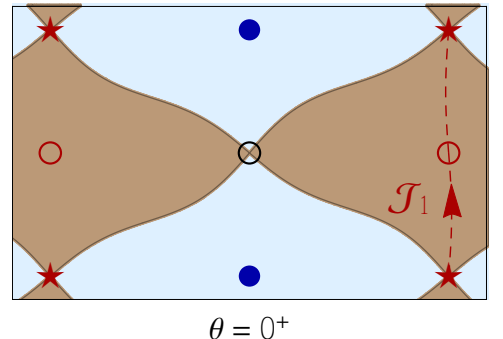

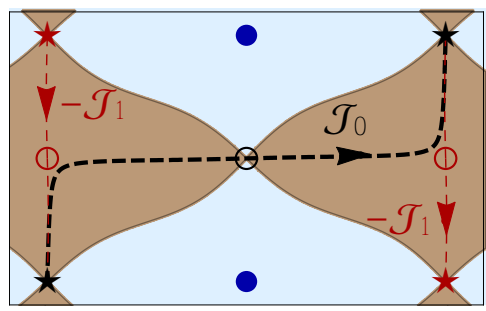

$\theta=0^{+}$

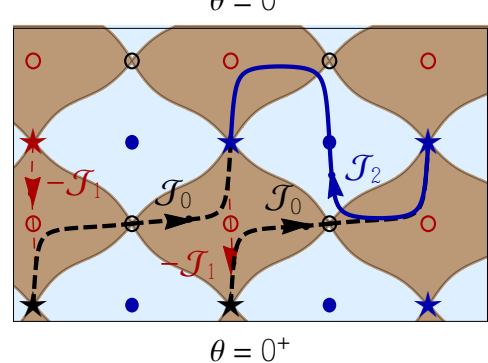

Figure 21. Stokes phenomenon around $\theta=0$, from $\theta=0^{-}$to $\theta=0^{+}$, and where $-A_{2} / x>0>-A_{1} / x$. At this particular angle, nothing really happens with the $\mathcal{J}_{1}$ thimble (top plots). On the other hand, as explained in the main text, both thimbles $\mathcal{J}_{0}$ and $\mathcal{J}_{2}$ do acquire discontinuities which occur at their tails. In order to preserve the continuity of the final integration contours, these discontinuities have to be balanced by addition of other thimbles, as pictured (middle and bottom plots, respectively).

$\Gamma$, continuity is kept as long as one compensates for this flip of $\mathcal{J}_{0}$ at $\theta=0^{+}$by adding $-2 \mathcal{J}_{1}$ to $\mathcal{J}_{0}\left(0^{+}\right)$, as illustrated in the middle-right plot of figure 21 (note how $\Gamma=\mathcal{J}_{1}$ is unchanged; see top plots of figure 21). In fact, without this addition there would be an exponentially suppressed discontinuity (of order $\sim \mathrm{e}^{-A_{1} / x}$; see (6.11) below) in the integral over $\Gamma$. In short, the monodromy arises as a result of demanding continuity of the integration cycle $\Gamma$ across a Stokes line - which also amounts to a description of the Stokes phenomenon. Indeed, from the identification between the transseries and cycle decomposition, equations (3.36) and (3.37), we can conclude that

$$
\int_{\mathcal{J}_{0}\left(0^{-}\right)} \mathrm{d} z \mathrm{e}^{-\frac{1}{x} V(z)}=\int_{\mathcal{J}_{0}\left(0^{+}\right)-2 \mathcal{J}_{1}\left(0^{+}\right)} \mathrm{d} z \mathrm{e}^{-\frac{1}{x} V(z)}
$$

translates to

$$
\lim _{\theta \rightarrow 0^{-}} \Phi_{0}=\lim _{\theta \rightarrow 0^{+}}\left(\Phi_{0}-2 \mathrm{e}^{-\frac{A_{1}}{x}} \Phi_{1}\right) .
$$

The case of $\mathcal{J}_{2}$ is similar, albeit slightly more intricate. This time around, in order to maintain the continuity of the "instanton" cycle $\Gamma=\mathcal{J}_{2}$, we will need the addition of terms proportional to both $\mathcal{J}_{0}$ and $\mathcal{J}_{1}$. This is illustrated in the bottom two plots of figure 21. As expected from the structure of the transseries in (6.6), both these extra terms are exponentially 
suppressed relative to the contribution from the critical point $z_{2}^{*}$. Following the contour plots in figure 21 , the reader should be able to arrive at the end result

$$
\int_{\mathcal{J}_{2}\left(0^{-}\right)} \mathrm{d} z \mathrm{e}^{-\frac{1}{x} V(z)}=\int_{\mathcal{J}_{2}\left(0^{+}\right)+2 \mathcal{J}_{0}\left(0^{+}\right)-2 \mathcal{J}_{1}\left(0^{+}\right)} \mathrm{d} z \mathrm{e}^{-\frac{1}{x} V(z)},
$$

which translates to

$$
\lim _{\theta \rightarrow 0^{-}} \mathrm{e}^{-\frac{A_{2}}{x}} \Phi_{2}=\lim _{\theta \rightarrow 0^{+}}\left(\mathrm{e}^{-\frac{A_{2}}{x}} \Phi_{2}+2 \Phi_{0}-2 \mathrm{e}^{-\frac{A_{1}}{x}} \Phi_{1}\right) .
$$

Note how the exponential hierarchy at play, $-A_{2} / x>0>-A_{1} / x$, dictates the form of the monodromy matrix to be upper-triangular, since all the Stokes jumps have to be exponentially suppressed compared to the main contribution to the integral.

\section{Monodromy and Stokes Phenomenon at $\theta=\pi$}

The analysis of the monodromies and Stokes jumps at $\theta=\pi$ is analogous to what we just did at $\theta=0$. Since $x=-|x|$, the exponential hierarchy is now reversed as

$$
-\frac{A_{1}}{x}>0>-\frac{A_{2}}{x}, \quad \text { for } \quad \theta=\pi .
$$

Therefore there is no Stokes jump for the $\Gamma=\mathcal{J}_{2}$ cycle; the jump in the "perturbative" $\Gamma=$ $\mathcal{J}_{0}$ cycle is now proportional to $\mathcal{J}_{2}$; and the jump in the "instanton" cycle $\Gamma=\mathcal{J}_{1}$ now has contributions from both $\mathcal{J}_{0}$ and $\mathcal{J}_{1}$. All these jumps are encoded in the "Stokes matrix" associated to $\theta=\pi, S_{\circlearrowleft}^{(\pi)}$, where:

$$
\left(\begin{array}{c}
\mathcal{J}_{2} \\
\mathcal{J}_{0} \\
\mathcal{J}_{1}
\end{array}\right) \rightarrow S_{\circlearrowleft}^{(\pi)}\left(\begin{array}{c}
\mathcal{J}_{2} \\
\mathcal{J}_{0} \\
\mathcal{J}_{1}
\end{array}\right)=\left(\begin{array}{ccc}
1 & 0 & 0 \\
2 & 1 & 0 \\
-2 & -2 & 1
\end{array}\right)\left(\begin{array}{l}
\mathcal{J}_{2} \\
\mathcal{J}_{0} \\
\mathcal{J}_{1}
\end{array}\right)
$$

Finally, we can write the monodromy matrix $\mathfrak{M}=S_{\circlearrowleft}^{(\pi)} \cdot S_{\circlearrowleft}^{(0)}$, and check that $\mathfrak{M}^{2}=\mathbf{1}$.

\section{Complex Phase Diagram}

Before proceeding with the resurgence properties of this problem, let us briefly comment on the possible phase diagrams associated to the elliptic partition function (6.1). If we set $x=|x| \mathrm{e}^{\mathrm{i} \theta}$, and vary $\theta$ as we did earlier, then the phase diagram of our model is the very same as the phase diagram for the quartic partition function (2.8), which was plotted back in figure 3 (herein holding true for any modulus $m \in(0,1)$, and leading to the structure of Stokes and anti-Stokes lines just discussed). On the other hand, as we shall see in the following (and in parallel with what was done back in subsection 2.2), the elliptic partition function satisfies a third-order linear ODE. From this standpoint, each of the three linearly-independent solutions to the said ODE is actually better thought-of as a one-parameter family of solutions, parametrized by the modulus $m$. As an ODE parameter, it is then natural to let $m$ be complex; $m=|m| \mathrm{e}^{\mathrm{i} \varphi}$. In this way, as long as $\varphi \neq 0, \pi$, the actions $A_{1}$ and $A_{2}$ will also be complex and no longer collinear. One is then led to address another phase diagram associated to the elliptic partition function, this time around keeping $\theta$ fixed and varying $\varphi$ instead - as shown in figure 22. This turns out to be an example of parametric, or co-equational resurgence (a topic not addressed in these lectures; see, e.g., [13]) where the actions and the coefficients of the asymptotic series depend on a parameter, and where varying this parameter will change the position and shape of the Stokes lines. 


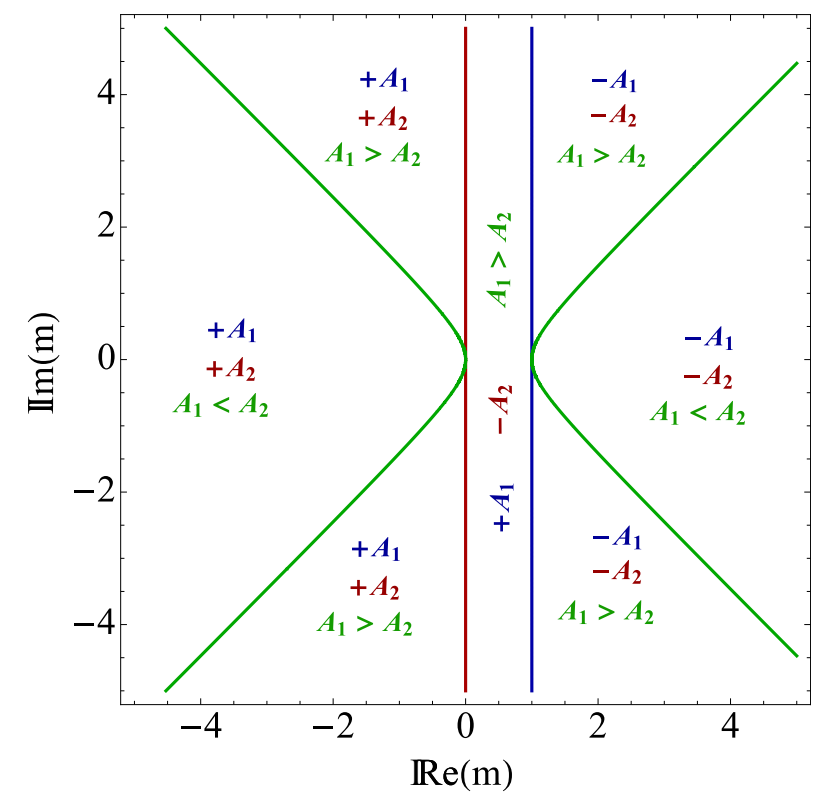

Figure 22. Phase diagram for the elliptic partition function, in the complex $m$-plane. Only the antiStokes lines are plotted, satisfying $\mathbb{R e}\left(A_{i}(m)-A_{j}(m)\right)=0$ (we are now considering $x \in \mathbb{R}^{+}$). Different colors represent different pairs of actions: in blue $i=1, j=0$; in red $i=2, j=0$; in green $i=1, j=2$. The relative sign of $\mathbb{R e}\left(A_{i}(m)-A_{j}(m)\right)$ is also shown in the plots (recall that $\left.A_{0}=0\right)$.

\subsection{Basic Formulae for Resurgent Analysis}

In order to explicitly evaluate the different asymptotic series appearing in (6.6), it is simpler to proceed differentially rather than integrally. This is straightforward once one notices that the elliptic partition function (6.1) satisfies a third-order linear $\mathrm{ODE}^{102}[27,62]$

$$
\begin{aligned}
& 4 m m^{\prime} x^{4} Z^{\prime \prime \prime}(x)-4\left(m-m^{\prime}-6 m m^{\prime} x\right) x^{2} Z^{\prime \prime}(x)-\left(4-\left(m-m^{\prime}\right) x+\right. \\
& \left.+9\left(m-m^{\prime}-3 m m^{\prime} x\right) x\right) Z^{\prime}(x)-\left(m-m^{\prime}-3 m m^{\prime} x\right) Z(x)=0 .
\end{aligned}
$$

It is simple to check that this differential equation is invariant under the simultaneous interchange

$$
m \rightarrow m^{\prime}=1-m \text { and } \quad x \rightarrow-x .
$$

This symmetry is a special case of the full modular symmetry associated with the underlying torus: it first exchanges the two periods of the torus, which accompanied with a change in the sign of $x$ ends up leaving the partition function unchanged. We shall use it later on.

Running in parallel with subsection 2.2, let us try to solve the above differential equation with the usual "exponential times asymptotic-power-series" ansatz,

$$
Z^{(i)}(x)=x^{\beta_{i}} \mathrm{e}^{-\frac{A_{i}}{x}} \Phi_{i}(x) \simeq x^{\beta_{i}} \mathrm{e}^{-\frac{A_{i}}{x}} \sum_{n=0}^{+\infty} Z_{n}^{(i)} x^{n} .
$$

\footnotetext{
${ }^{102}$ Recall that we have defined $x \equiv \hbar$ and $m^{\prime}=1-m$.
} 
This is now slightly more intricate than for the quartic partition function back in (2.14). Indeed, plugging (6.18) into the ODE (6.16) leads to a more complicated recursion for the coefficients $Z_{n}^{(i)}$; namely

$$
\begin{aligned}
& 4 A_{i}\left(1+\left(m-m^{\prime}\right) A_{i}-m m^{\prime} A_{i}^{2}\right) Z_{n}^{(i)}+ \\
& +4\left(\beta_{i}+n-1\right)\left(1+2\left(m-m^{\prime}\right)-3 m m^{\prime} A_{i}^{2}\right) Z_{n-1}^{(i)}+ \\
& +\left(m-m^{\prime}-3 m m^{\prime} A_{i}\right)\left(1+4\left(\beta_{i}+n-1\right)\left(\beta_{i}+n-2\right)\right) Z_{n-2}^{(i)}- \\
& -m m^{\prime}\left(3+\left(\beta_{i}+n-3\right)\left(3+4\left(\beta_{i}+n-1\right)\left(\beta_{i}+n-2\right)\right)\right) Z_{n-3}^{(i)}=0,
\end{aligned}
$$

where we set $Z_{n}^{(i)} \equiv 0$ whenever $i, n<0$ as usual. In particular, if we set $n=0$ and $n=1$ in (6.19) we learn that there are three sectors, $Z^{(i)}(x), i=0,1,2$, with, respectively,

$$
A_{i} \in\left\{0, \frac{1}{m^{\prime}},-\frac{1}{m}\right\} \quad \text { and } \quad \beta_{i}=0, \quad i=0,1,2 .
$$

This matches our earlier saddle-point expectations, and is now a straightforward consequence of the fact that $Z(x)$ satisfies a linear, third-order ODE. As mentioned, we shall be particularly interested in the fact that $A_{1}$ and $A_{2}$ are such that they can be linearly combined to vanish, i.e., that specific nonperturbative sectors can end up with the same exponential weight as the perturbative sector, and subsequently mix with one another - one of the hallmarks of resonance. But we leave that discussion to the upcoming subsections 6.7 and on.

Focusing for the moment in the non-resonant case, one can use the above recursion relation (6.19) to compute the coefficients $Z_{n}^{(i)}$ associated to each sector. In spite of being a more intricate relation, akin to the results for quartic potential in (2.16) and (2.17) one can herein also compute such coefficients in closed form. For the perturbative sector $Z^{(0)}(x)$, nonperturbative sector $Z^{(1)}(x)$ with action $A_{1}=1 / m^{\prime}$, and nonperturbative sector $Z^{(2)}(x)$ with action $A_{2}=-1 / m$, we find, respectively,

$$
\begin{aligned}
& \Phi_{0}(x) \simeq \sum_{n=0}^{+\infty} Z_{n}^{(0)}(m) x^{n}, \quad Z_{n}^{(0)}(m)=\frac{\Gamma^{2}\left(n+\frac{1}{2}\right)}{\pi \Gamma(n+1)}{ }_{2} F_{1}\left(-n,-n, \frac{1}{2}-n \mid m\right) \\
& \Phi_{1}(x) \simeq \sum_{n=0}^{+\infty} Z_{n}^{(1)}(m) x^{n}, \quad Z_{n}^{(1)}(m)=Z_{0}^{(1)} \frac{(-1)^{n}}{A_{1}^{n}} \frac{\Gamma^{2}\left(n+\frac{1}{2}\right)}{\pi \Gamma(n+1)}{ }_{2} F_{1}\left(\frac{1}{2},-n, \frac{1}{2}-n \mid m\right), \\
& \Phi_{2}(x) \simeq \sum_{n=0}^{+\infty} Z_{n}^{(2)}(m) x^{n}, \quad Z_{n}^{(2)}(m)=Z_{0}^{(2)} \frac{(-1)^{n}}{A_{2}^{n}} \frac{\Gamma^{2}\left(n+\frac{1}{2}\right)}{\pi \Gamma(n+1)}{ }_{2} F_{1}\left(\frac{1}{2},-n, \frac{1}{2}-n \mid m^{\prime}\right)
\end{aligned}
$$

The instanton actions are evident in the growth of the several coefficients $\sim A^{-n} n !$. Further, the coefficients in the nonperturbative sectors relate to each other as $Z_{n}^{(2)}(m)=(-1)^{n} Z_{n}^{(1)}\left(m^{\prime}\right)$, which follows from the modular symmetry (6.17). Finally, the residual coefficients were set to $Z_{0}^{(1)} \equiv Z_{0}^{(1)}(m)=\mathrm{i} \sqrt{m^{\prime}}$ and $Z_{0}^{(2)} \equiv Z_{0}^{(2)}(m)=\mathrm{i} \sqrt{m}$, which will be used throughout.

Having constructed three linearly independent solutions - given by (6.18), with asymptotic series (6.21), (6.22), and (6.23) - to the linear third-order ODE (6.16), the general solution follows as their linear combination. This introduces three integration constants, parameterizing boundary conditions, and leading to the three-parameter transseries (6.6), which we now rewrite as

$$
\mathcal{Z}\left(x, \sigma_{0}, \sigma_{1}, \sigma_{2}\right)=\sigma_{0} Z^{(0)}(x)+\sigma_{1} Z^{(1)}(x)+\sigma_{2} Z^{(2)}(x) .
$$


Proceeding to analyze the resurgent properties of this example, let us turn to the computation of the Borel transforms (2.2) for each asymptotic series above, $\Phi_{i}(x)$. Unlike in subsection 2.2, however, we shall now do this in two different steps: we will first address general resurgence expectations (based on what we have learnt so far), and only then will we compute explicit expressions. On very general grounds we expect the Borel transform of any given sector to have non-zero but finite radius of convergence. For instance, $\mathcal{B}\left[\Phi_{0}\right](s)$ will converge for $s<$ $\min \left(\left|A_{1}\right|,\left|A_{2}\right|\right)=\min \left(1 / m^{\prime}, 1 / m\right)$ (recall that the leading singularity on the Borel plane is determined by the closest-to-the-origin relative-action). Considering the simple representative, the singularities at $s=-1 / m$ and at $s=1 / m^{\prime}$ will be logarithmic branch-points (where the branch cuts emerging from these points can be tested numerically as accumulations of Padéapproximant poles; recall, e.g., figures 7 or 18). When further considering the nonperturbative sectors, generically $\mathcal{B}\left[\Phi_{i}\right](s)$ will have singularities located at $\omega_{i j}=A_{j}-A_{i}$, for $i \neq j \in\{0,1,2\}$ and with $A_{0}:=0$, and near each of these singular points we find the simple singularity structure $(4.53)$,

$$
\mathcal{B}\left[\Phi_{i}\right](s)=\mathrm{S}_{i \rightarrow j} \frac{Z_{0}^{(j)}}{2 \pi \mathrm{i}\left(s-\omega_{i j}\right)}+\mathrm{S}_{i \rightarrow j} \times \mathcal{B}\left[\Phi_{j}\right]\left(s-\omega_{i j}\right) \frac{\log \left(s-\omega_{i j}\right)}{2 \pi \mathrm{i}}+\text { holomorphic. }
$$

The resurgence of the sector $\Phi_{j}$ within the singular behaviour of the Borel transform for $\Phi_{i}$, $i \neq j$, is quite explicit as expected. The proportionality constant $S_{i \rightarrow j}$ is the "Borel residue", which has already appeared back in, e.g., (2.62) or (5.20).

Let us next rewrite the above Borel expression at the usual resurgence algebraic-level-ofabstraction, based upon alien derivations $\Delta_{\omega}$. Given the three transseries sectors, there are six possible singularities, $\omega_{i j}$ and $\omega_{j i}=-\omega_{i j}$, of the form (6.25),

$$
\omega_{01}=A_{1}, \quad \omega_{21}=A_{1}-A_{2}=A_{1}+\left|A_{2}\right|, \quad \omega_{02}=A_{2}=-\left|A_{2}\right| .
$$

Each action $A_{i}$ is defined in (6.4); $A_{0}:=0, A_{1}>0$ and $A_{2}<0$. Continuing our parallel with subsection 2.2, we may now use the definition (2.29) appropriate for a linear problem - or even the general formalism discussed in appendix A - in order to write all possible alien derivatives. One finds (compare with (2.30) and (2.31)):

$$
\begin{aligned}
\Delta_{\omega_{01}} \Phi_{0} & =S_{\omega_{01}} \Phi_{1}, & \Delta_{\omega_{02}} \Phi_{0} & =S_{\omega_{02}} \Phi_{2}, \\
\Delta_{\omega_{10}} \Phi_{1} & =S_{\omega_{10}} \Phi_{0}, & \Delta_{\omega_{12}} \Phi_{1} & =S_{\omega_{12}} \Phi_{2}, \\
\Delta_{\omega_{20}} \Phi_{2} & =S_{\omega_{20}} \Phi_{0}, & \Delta_{\omega_{21}} \Phi_{2} & =S_{\omega_{21}} \Phi_{1} .
\end{aligned}
$$

In these expressions the $S_{\omega_{i j}}$ are the Stokes coefficients, related to the Borel residues $S_{i \rightarrow j}$ appearing in the Borel transforms, and whose values may be determined, e.g., from an analysis of Stokes phenomena (as discussed later in the present subsection), or from an analysis of largeorder behaviour (as discussed in the next subsection). The algebraic structure written above closes upon itself, much like what happened for the partition function of the quartic potential back in (2.32), albeit now with a few more allowed motions. The algebraic structure encoded in (6.27)-(6.29) is depicted in figure 23, where we have assumed $m \in(0,1)$ and where we plot motions along the positive-real direction as solid lines, and motions along the negative-real direction as dashed lines. Note that while for $m \in(0,1)$ the three actions $A_{i}$ are all real and we will only have two singular directions, along $\theta=0$ and $\pi$, if one allows $m \in \mathbb{C}$ the actions $A_{1}$ and $A_{2}$ become complex and might not fall along the same direction (this was already noticed in the discussion associated to figure 22). Also for this reason we are now plotting the sectors 


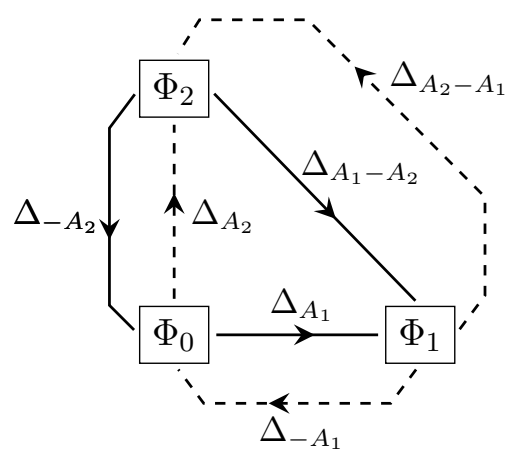

Figure 23. Algebraic structure associated to the behaviour of Borel transforms or alien derivatives for the $\Phi_{i}$ sectors, at their respective singularities. When $m \in(0,1)$ the motions fall along the real axis on the Borel plane: solid lines correspond to motions along the $\theta=0$ direction; dashed lines along $\theta=\pi$.

corresponding to each of these actions as orthogonal; in order to depict the general independence between the two actions (in fact, as occurs for the completely general case; recall figure 17).

The relation between the Stokes coefficients in (6.27)-(6.29) and the Borel residues in (6.25) can be easily determined following the example ${ }^{103}$ outlined in appendix A; or else along the very general lines discussed earlier in sections 2 and 5 . It is simple to find:

$$
\begin{array}{ll}
\mathrm{S}_{0 \rightarrow 1}=-S_{\omega_{01}}, & \mathrm{~S}_{1 \rightarrow 0}=-S_{\omega_{10}} \\
\mathrm{~S}_{0 \rightarrow 2}=-S_{\omega_{02}}, & \mathrm{~S}_{2 \rightarrow 0}=-S_{\omega_{20}}, \\
\mathrm{~S}_{1 \rightarrow 2}=-S_{\omega_{12}}-\frac{1}{2} S_{\omega_{10}} S_{\omega_{02}}, & \mathrm{~S}_{2 \rightarrow 1}=-S_{\omega_{21}}-\frac{1}{2} S_{\omega_{20}} S_{\omega_{01}} .
\end{array}
$$

The next step is to determine the Stokes automorphism, given by (2.4) and explicitly in (2.33), along all possible singular directions. This essentially follows from the alien derivatives (6.27)-(6.29). Possible singular directions may depend on the value of the modular parameter $m$, and to make things simpler we will only have in mind the case of $m \in(0,1)$ already depicted in figure 23. The Stokes automorphism at $\theta=0$ is

$$
\underline{\mathfrak{S}}_{0}=\exp \left(\mathrm{e}^{-\frac{\omega_{01}}{x}} \Delta_{\omega_{01}}+\mathrm{e}^{-\frac{\omega_{20}}{x}} \Delta_{\omega_{20}}+\mathrm{e}^{-\frac{\omega_{21}}{x}} \Delta_{\omega_{21}}\right) .
$$

As expected, only the alien derivatives whose associated relative actions are positive (i.e., $\omega_{01}$, $\left.\omega_{20}, \omega_{21}\right)$ contribute to the discontinuity along $\theta=0$, albeit not all will act non-trivially on each sector $\Phi_{i}$. Similarly, for $\theta=\pi$ we get

$$
\underline{\mathfrak{S}}_{\pi}=\exp \left(\mathrm{e}^{-\frac{\omega_{10}}{x}} \Delta_{\omega_{10}}+\mathrm{e}^{-\frac{\omega_{02}}{x}} \Delta_{\omega_{02}}+\mathrm{e}^{-\frac{\omega_{12}}{x}} \Delta_{\omega_{12}}\right)
$$

with only the negative relative actions $\omega_{10}, \omega_{02}, \omega_{12}$ contributing. Acting on each asymptotic sector $\Phi_{i}$, via expansion of the exponentials in (6.33) and (6.34) (which collapse to finite sums), and further using (6.27)-(6.29), it simply follows:

$$
\underline{\mathfrak{S}}_{0} \Phi_{0}=\Phi_{0}+S_{\omega_{01}} \mathrm{e}^{-\frac{A_{1}}{x}} \Phi_{1}=\Phi_{0}-\mathrm{S}_{0 \rightarrow 1} \mathrm{e}^{-\frac{A_{1}}{x}} \Phi_{1},
$$

\footnotetext{
${ }^{103}$ This was dubbed the "explicit (linear) example" in appendix A.
} 


$$
\begin{aligned}
\underline{\mathfrak{S}}_{\pi} \Phi_{0} & =\Phi_{0}+S_{\omega_{02}} \mathrm{e}^{-\frac{A_{2}}{x}} \Phi_{2}=\Phi_{0}-\mathrm{S}_{0 \rightarrow 2} \mathrm{e}^{-\frac{A_{2}}{x}} \Phi_{2}, \\
\underline{\mathfrak{S}}_{\pi} \Phi_{1} & =\Phi_{1}+S_{\omega_{10}} \mathrm{e}^{+\frac{A_{1}}{x}} \Phi_{0}+\left(S_{\omega_{12}}+\frac{1}{2} S_{\omega_{02}} S_{\omega_{10}}\right) \mathrm{e}^{+\frac{A_{1}-A_{2}}{x}} \Phi_{2} \\
& =\Phi_{1}-\mathrm{S}_{1 \rightarrow 0} \mathrm{e}^{+\frac{A_{1}}{x}} \Phi_{0}-\mathrm{S}_{1 \rightarrow 2} \mathrm{e}^{+\frac{A_{1}-A_{2}}{x}} \Phi_{2}, \\
\underline{\mathfrak{S}}_{0} \Phi_{2} & =\Phi_{2}+S_{\omega_{20}} \mathrm{e}^{+\frac{A_{2}}{x}} \Phi_{0}+\left(S_{\omega_{21}}+\frac{1}{2} S_{\omega_{01}} S_{\omega_{20}}\right) \mathrm{e}^{-\frac{A_{1}-A_{2}}{x}} \Phi_{1} \\
& =\Phi_{2}-\mathrm{S}_{2 \rightarrow 0} \mathrm{e}^{+\frac{A_{2}}{x}} \Phi_{0}-\mathrm{S}_{2 \rightarrow 1} \mathrm{e}^{-\frac{A_{1}-A_{2}}{x}} \Phi_{1},
\end{aligned}
$$

where we made use of the relations (6.30)-(6.32) to display all results with either Stokes coefficients or Borel residues. These expressions encode Stokes phenomena across all Stokes lines. The lateral resummations across these lines, (2.4), applied to the transseries (6.24) can be easily written down, from where one obtains the Stokes transitions

$$
\begin{aligned}
& \mathcal{S}_{0^{+}} \mathcal{Z}\left(x, \sigma_{0}, \sigma_{1}, \sigma_{2}\right)=\mathcal{S}_{0^{-}} \mathcal{Z}\left(x, \sigma_{0}-\mathrm{S}_{2 \rightarrow 0} \sigma_{2}, \sigma_{1}-\mathrm{S}_{0 \rightarrow 1} \sigma_{0}-\mathrm{S}_{2 \rightarrow 1} \sigma_{2}, \sigma_{2}\right), \\
& \mathcal{S}_{\pi^{+}} \mathcal{Z}\left(x, \sigma_{0}, \sigma_{1}, \sigma_{2}\right)=\mathcal{S}_{\pi^{-}} \mathcal{Z}\left(x, \sigma_{0}-\mathrm{S}_{1 \rightarrow 0} \sigma_{1}, \sigma_{1}, \sigma_{2}-\mathrm{S}_{0 \rightarrow 2} \sigma_{0}-\mathrm{S}_{1 \rightarrow 2} \sigma_{1}\right) .
\end{aligned}
$$

For simplicity, we have written the Stokes transitions in terms of the Borel residues. The same expressions in terms of the Stokes coefficients may be easily obtained from (6.30)-(6.32).

By comparing the Stokes transitions with the jumps of the steepest-descent contours encoded in the Stokes matrices (6.9) and (6.15), one can easily determine the value of the Borel residues and Stokes coefficients. The jump of a given contour $\mathcal{J}_{i}$ across a Stokes ray will correspond to the jump of the corresponding asymptotic sector $Z^{(i)}$ in the Stokes transition. For $\theta=0$ one has (compare with (6.9))

$$
\left(\begin{array}{l}
Z^{(2)} \\
Z^{(0)} \\
Z^{(1)}
\end{array}\right) \rightarrow S_{\circlearrowleft}^{(0)}\left(\begin{array}{l}
Z^{(2)} \\
Z^{(0)} \\
Z^{(1)}
\end{array}\right)=\left(\begin{array}{ccc}
1 & -\mathrm{S}_{2 \rightarrow 0} & -\mathrm{S}_{2 \rightarrow 1} \\
0 & 1 & -\mathrm{S}_{0 \rightarrow 1} \\
0 & 0 & 1
\end{array}\right)\left(\begin{array}{l}
Z^{(2)} \\
Z^{(0)} \\
Z^{(1)}
\end{array}\right),
$$

from where we can read the Borel residues $\mathrm{S}_{2 \rightarrow 0}=\mathrm{S}_{2 \rightarrow 1}=-\mathrm{S}_{0 \rightarrow 1}=2$. The exact same reasoning applied at $\theta=\pi$ yields $S_{0 \rightarrow 2}=-S_{1 \rightarrow 2}=-S_{1 \rightarrow 0}=-2$. Finally, using (6.30)-(6.32), one obtains the complete set of Stokes coefficients,

$$
S_{\omega_{01}}=2, \quad S_{\omega_{10}}=-2, \quad S_{\omega_{02}}=2, \quad S_{\omega_{20}}=-2, \quad S_{\omega_{12}}=0=S_{\omega_{21}} .
$$

With these numbers in hand, computing monodromies is now straightforward. In fact, moving twice around the origin, i.e., acting with $\left(\underline{\mathfrak{S}}_{\pi} \underline{\mathfrak{S}}_{0}\right)^{2}$ upon the transseries, the parameters $\sigma_{i}$ get mapped onto themselves. In other words,

$$
\left(\underline{\mathfrak{S}}_{\pi} \underline{\mathfrak{S}}_{0}\right)^{2}=\mathbf{1},
$$

which encompasses the monodromy encoded in the transseries solution for the elliptic partition function; once again leading to a multi-sheeted Riemann surface (compare with (2.38)).

It is also very instructive to analyze the resurgent relations between the different sectors $\Phi_{i}$ purely from the perspective of the Picard-Lefschetz theory introduced in section 3 (and further using the main discussion from section 4 , as will be clear momentarily). Each asymptotic series in (6.21) through (6.23) is basically dictated by the small $x \equiv \hbar$ expansion of the integral (6.1) 
evaluated on the appropriate thimble $\Gamma_{i}$, i.e., the one associated with the critical point $z_{i}^{*}$. The calculation is rather straightforward thanks to the elliptic identities

$$
\begin{aligned}
\frac{\mathrm{d}}{\mathrm{d} z} \operatorname{sd}(z \mid m) & =\operatorname{nd}(z \mid m) \operatorname{cd}(z \mid m), \\
\operatorname{nd}^{2}(z \mid m) & =1+m \operatorname{sd}^{2}(z \mid m) \\
\operatorname{cd}^{2}(z \mid m) & =1-m^{\prime} \operatorname{sd}^{2}(z \mid m)
\end{aligned}
$$

Using these identities and the change of variables $s \equiv \operatorname{sd}^{2}(z \mid m)$ - which is of the type discussed in section 4 ; recall (4.16) - the integration over the cycle $\mathcal{J}_{0}(\theta)$ may be expressed as

$$
\frac{1}{\sqrt{\pi x}} \int_{\mathcal{J}_{0}(\theta)} \mathrm{d} z \mathrm{e}^{-\frac{1}{x} \mathrm{sd}^{2}(z \mid m)}=\frac{1}{\sqrt{\pi x}} \int_{0}^{\mathrm{e}^{\mathrm{i} \theta} \infty} \mathrm{d} s \frac{\mathrm{e}^{-\frac{s}{x}}}{\sqrt{s\left(1-m^{\prime} s\right)(1+m s)}} \simeq \mathrm{e}^{-\frac{A_{0}}{x}} \sum_{n=0}^{+\infty} Z_{n}^{(0)}(m) x^{n},
$$

where, in the third step, we formally Taylor-expanded the denominator outside its radius of convergence and carried out the resulting $s$-integration, leading to the asymptotic perturbative expansion $\Phi_{0}(x)$. In this way, it should not come as a great surprise that the coefficients of the above asymptotic series are found as

$$
\begin{aligned}
Z_{n}^{(0)}(m) & =\frac{\Gamma\left(n+\frac{1}{2}\right)}{\pi^{\frac{3}{2}}} \sum_{k=0}^{n}(-1)^{k} \frac{\Gamma\left(k+\frac{1}{2}\right) \Gamma\left(n-k+\frac{1}{2}\right)}{\Gamma(k+1) \Gamma(n-k+1)} m^{k} m^{\prime n-k} \\
& =\frac{\Gamma^{2}\left(n+\frac{1}{2}\right)}{\pi \Gamma(n+1)}{ }_{2} F_{1}\left(-n,-n, \frac{1}{2}-n \mid m\right),
\end{aligned}
$$

in precise agreement with expression (6.21), which was derived from the recursion relations (6.19). Notice that, as expected from the discussion of section 4, the form of the second integral in (6.47) resembles the resummation of the Borel transform $\mathcal{B}\left[\Phi_{0}\right](s)$, up to the extra factor of $1 / \sqrt{x}$ in front. To deal with these extra factors and "odd-looking" Borel transforms, in section 4 we introduced sectors of the form $\Phi_{i,[\alpha]}(x)=x^{\alpha} \Phi_{i,[0]}(x)$ (recall (4.55)) where herein $\Phi_{i,[0]}(x)$ is any of the original asymptotic sectors $\Phi_{i}(x)$ whose Borel transforms have (simple) logarithmic singularities. Form (6.47) one can now immediately identify

$$
\Phi_{0,[1 / 2]}(x)=\frac{1}{\sqrt{\pi}} \int_{\mathcal{J}_{0}(\theta)} \mathrm{d} z \mathrm{e}^{-\frac{1}{x} \mathrm{sd}^{2}(z \mid m)},
$$

whose Borel transform is given by

$$
\mathcal{B}\left[\Phi_{0,[1 / 2]}\right](s)=\frac{1}{\sqrt{\pi s\left(1-m^{\prime} s\right)(1+m s)}} .
$$

The Borel transforms associated to sectors $\Phi_{i,[\alpha]}(x)$ for different $\alpha$ are related by a fractional derivative, via (4.57). As discussed in section 4, and due to the involved fractional derivatives, these Borel transforms are related in highly non-local fashions. Although alien calculus might give preference to the choice $\alpha=0$, we shall demonstrate below that it may also be illuminating to work with $\alpha=1 / 2$. In fact, herein we will find a surprisingly simple relation between the Borel transforms of the series associated with the different critical points.

Similarly to $(6.47)$, the integration over the thimble $\mathcal{J}_{1}(\theta)$ may be expressed as

$$
\frac{1}{\sqrt{\pi x}} \int_{\mathcal{J}_{1}(\theta)} \mathrm{d} z \mathrm{e}^{-\frac{1}{x} \operatorname{sd}^{2}(z \mid m)}=-\frac{1}{\sqrt{\pi x}} \int_{\frac{1}{m^{\prime}}}^{\mathrm{e}^{\mathrm{i} \theta} \infty} \mathrm{d} s \frac{\mathrm{e}^{-\frac{s}{x}}}{\sqrt{s\left(1-m^{\prime} s\right)(1+m s)}}
$$




$$
\begin{aligned}
& =\mathrm{i}^{-\frac{1}{m^{\prime} x}} \frac{\sqrt{m^{\prime}}}{\sqrt{\pi x}} \int_{0}^{\mathrm{e}^{\mathrm{i} \theta} \infty} \mathrm{d} s \frac{\mathrm{e}^{-\frac{s}{x}}}{\sqrt{s\left(1+m m^{\prime} s\right)\left(1+m^{\prime} s\right)}} \\
& \equiv \mathrm{e}^{-\frac{A_{1}}{x}} \frac{1}{\sqrt{x}} \mathcal{S}_{\theta} \Phi_{1,[1 / 2]}(x) .
\end{aligned}
$$

Notice that the integrand of the integral on the right-hand-side of the first line is precisely the Borel transform of $\Phi_{0,[1 / 2]}(x)$ (up to a shift $1 / m^{\prime}$ ), while in the last line we obtain instead a resummation of $\Phi_{1,[1 / 2]}(x)$. Furthermore, the shift in question is precisely the relative action $A_{0}-A_{1}$. As we shall see next, this is a manifestation of resurgence: the two asymptotic series, attached to different critical points, are related by a simple shift in the Borel plane, where the shift is precisely the difference between the actions at those critical points. We can moreover write

$$
\mathcal{B}\left[\Phi_{1,[1 / 2]}\right](s)=\frac{\mathrm{i} \sqrt{m^{\prime}}}{\sqrt{\pi s\left(1+m m^{\prime} s\right)\left(1+m^{\prime} s\right)}} .
$$

Finally, the integration over the thimble $\mathcal{J}_{2}(\theta)$ can be expressed in a totally similar fashion as

$$
\begin{aligned}
\frac{1}{\sqrt{\pi x}} \int_{\mathcal{J}_{2}(\theta)} \mathrm{d} z \mathrm{e}^{-\frac{1}{x} s \mathrm{~d}^{2}(z \mid m)} & =-\frac{1}{\sqrt{\pi x}} \int_{-\frac{1}{m}}^{\mathrm{e}^{\mathrm{i} \theta} \infty} \mathrm{d} s \frac{\mathrm{e}^{-\frac{s}{x}}}{\sqrt{s\left(1-m^{\prime} s\right)(1+m s)}} \\
& =\mathrm{i} \mathrm{e}^{\frac{1}{m x}} \frac{\sqrt{m}}{\sqrt{\pi x}} \int_{0}^{\mathrm{e}^{\mathrm{i} \theta} \infty} \mathrm{d} s \frac{\mathrm{e}^{-\frac{s}{x}}}{\sqrt{s\left(1-m m^{\prime} s\right)(1-m s)}} \\
& \equiv \mathrm{e}^{-\frac{A_{2}}{x}} \frac{1}{\sqrt{x}} \mathcal{S}_{\theta} \Phi_{2,[1 / 2]}(x),
\end{aligned}
$$

where

$$
\mathcal{B}\left[\Phi_{2,[1 / 2]}\right](s)=\frac{\mathrm{i} \sqrt{m}}{\sqrt{\pi s\left(1-m m^{\prime} s\right)(1-m s)}} .
$$

It is now clear that the Borel transforms of the series associated with each critical point are related to each other in a strikingly simple way,

$$
\mathcal{B}\left[\Phi_{i,[1 / 2]}\right](s)= \pm \mathcal{B}\left[\Phi_{j,[1 / 2]}\right]\left(s-\left(A_{j}-A_{i}\right)\right), \quad i, j=0,1,2,
$$

where the plus-sign occurs when $j=0$. In words: on the Borel plane, and up to a sign ${ }^{104}$, these Borel transforms are expressed by the exact same function, only shifted by the relative action $A_{j}-A_{i}$. An immediate consequence of this relation is that, having access to, say, the perturbative asymptotic series around the vacuum, one can then reconstruct the expansions around all other nonperturbative sectors without much effort. Further recalling the expected general relations between such Borel transforms, (3.34), we can proceed to read-off the values of the Borel residues and then of the Stokes coefficients $S_{\omega_{i j}}$ in (6.27)-(6.29). These numbers agree with the ones obtained in (6.42). These very same values can also be obtained from a large-order analysis of the asymptotic behaviour of the coefficients $Z^{(i)_{n}}$, to which we turn next.

\subsection{Asymptotics and Large-Order Behaviour: Partition Function}

Let us now turn to the analysis of the large-order behaviour of the partition-function coefficients $Z_{n}^{(i)}$. For concreteness, let us start with the perturbative sector. Using the connection formula

\footnotetext{
${ }^{104}$ This sign is governed by the branch of the square-root one goes through when performing the shift.
} 
for the hypergeometric function, one can write

$$
Z_{n}^{(0)}(m)=\frac{\Gamma(n+1)}{\pi\left(n+\frac{1}{2}\right)}\left\{\left(m^{\prime}\right)^{n+\frac{1}{2}}{ }_{2} F_{1}\left(\frac{1}{2}, \frac{1}{2}, \frac{3}{2}+n \mid m^{\prime}\right)+(-1)^{n} m^{n+\frac{1}{2}}{ }_{2} F_{1}\left(\frac{1}{2}, \frac{1}{2}, \frac{3}{2}+n \mid m\right)\right\} .
$$

This decomposition of the perturbative coefficients already hints that they must somehow relate to nonperturbative content, since $A_{1}=\left(m^{\prime}\right)^{-1}$ and $A_{2}=-m^{-1}$. Of course such relations may be made very precise via resurgence. But before we do that, let us proceed by expanding the above hypergeometric functions in both $m^{\prime}$ and $m$,

$$
\begin{aligned}
Z_{n}^{(0)}(m) \simeq \frac{\Gamma(n+1)}{\pi(2 n+1)}\{ & \left(m^{\prime}\right)^{n+\frac{1}{2}}\left(2+\frac{m^{\prime}}{2 n+3}+\frac{9\left(m^{\prime}\right)^{2}}{4(2 n+3)(2 n+5)}+\cdots\right)+ \\
& \left.+(-1)^{n} m^{n+\frac{1}{2}}\left(2+\frac{m}{2 n+3}+\frac{9(m)^{2}}{4(2 n+3)(2 n+5)}+\cdots\right)\right\} .
\end{aligned}
$$

This expansion is valid for any $n$; in particular one can take the limit $n \gg 1$ and reorganize the several terms as

$$
\begin{aligned}
Z_{n}^{(0)}(m) & \simeq \frac{\left(m^{\prime}\right)^{n+\frac{1}{2}} \Gamma(n)}{\pi}\left(1-\frac{1}{2 n}+\frac{1}{4 n^{2}}+\cdots+\frac{m^{\prime}}{4 n}-\frac{m^{\prime}}{2 n^{2}}+\cdots+\frac{9\left(m^{\prime}\right)^{2}}{32 n^{2}}+\cdots\right)+ \\
& +\frac{(-1)^{n} m^{n+\frac{1}{2}} \Gamma(n)}{\pi}\left(1-\frac{1}{2 n}+\frac{1}{4 n^{2}}+\cdots+\frac{m}{4 n}-\frac{m}{2 n^{2}}+\cdots+\frac{9 m^{2}}{32 n^{2}}+\cdots\right) \\
& \approx \frac{\left(m^{\prime}\right)^{n} \Gamma(n)}{\pi}\left(\sqrt{m^{\prime}}-\sqrt{m^{\prime}} \frac{m^{\prime}-2}{4(n-1)}+\sqrt{m^{\prime}} \frac{9\left(m^{\prime}\right)^{2}-24 m^{\prime}+24}{32(n-1)(n-2)}+\cdots\right)+ \\
& +\frac{(-1)^{n} m^{n} \Gamma(n)}{\pi}\left(\sqrt{m}-\sqrt{m} \frac{m-2}{4(n-1)}+\sqrt{m} \frac{9 m^{2}-24 m+24}{32(n-1)(n-2)}+\cdots\right) .
\end{aligned}
$$

Interestingly enough, this form already is precisely the expected large-order behaviour for the perturbative series, usually written as

$$
\begin{aligned}
Z_{n}^{(0)}(m) & \simeq \frac{2}{2 \pi \mathrm{i}} \frac{\Gamma(n)}{A_{1}^{n}}\left(Z_{0}^{(1)}+\frac{A_{1}}{n-1} Z_{1}^{(1)}+\frac{A_{1}^{2}}{(n-1)(n-2)} Z_{2}^{(1)}+\cdots\right)+ \\
& +\frac{2}{2 \pi \mathrm{i}} \frac{\Gamma(n)}{A_{2}^{n}}\left(Z_{0}^{(2)}+\frac{A_{2}}{n-1} Z_{1}^{(2)}+\frac{A_{2}^{2}}{(n-1)(n-2)} Z_{2}^{(2)}+\cdots\right) .
\end{aligned}
$$

The standard approach - which in fact allows for a determination of the large-order relations associated to all (perturbative and nonperturbative) sectors - is to follow the same steps as in subsection 2.3. From Cauchy's theorem,

$$
\Phi_{i}(x)=-\frac{1}{2 \pi \mathrm{i}} \sum_{\theta=0, \pi} \int_{0}^{\mathrm{e}^{\mathrm{i} \theta} \infty} \mathrm{d} w \frac{\operatorname{Disc}_{\theta} \Phi_{i}(w)}{w-x},
$$

where the discontinuity is defined by $(2.45)$ and the relevant Stokes automorphisms are given in (6.35)-(6.38). For $\Phi_{0}$, it follows

$$
\sum_{n=0}^{+\infty} Z_{n}^{(0)} x^{n} \simeq \frac{S_{\omega_{01}}}{2 \pi \mathrm{i}} \int_{0}^{+\infty} \mathrm{d} w \frac{1}{w-x} \mathrm{e}^{-\frac{A_{1}}{w}} \sum_{k=0}^{+\infty} Z_{k}^{(1)} w^{k}+\frac{S_{\omega_{02}}}{2 \pi \mathrm{i}} \int_{0}^{-\infty} \mathrm{d} w \frac{1}{w-x} \mathrm{e}^{-\frac{A_{2}}{w}} \sum_{k=0}^{+\infty} Z_{k}^{(2)} w^{k},
$$


leading to

$$
Z_{n}^{(0)} \simeq \frac{S_{\omega_{01}}}{2 \pi \mathrm{i}} \frac{\Gamma(n)}{A_{1}^{n}} \sum_{k=0}^{+\infty} \frac{\Gamma(n-k)}{\Gamma(n)} A_{1}^{k} Z_{k}^{(1)}+\frac{S_{\omega_{02}}}{2 \pi \mathrm{i}} \frac{\Gamma(n)}{A_{2}^{n}} \sum_{k=0}^{+\infty} \frac{\Gamma(n-k)}{\Gamma(n)} A_{2}^{k} Z_{k}^{(2)} .
$$

This exactly matches (6.60), once the Stokes coefficients are identified as $S_{\omega_{01}}=S_{\omega_{02}}=2$.

Let us take another look at (6.60) or (6.63). It is clear that the large-order coefficients in the perturbative expansion are dominated at next-to-leading order by the nearest action to the origin, which, depending on whether $m<1 / 2$ or $m>1 / 2$, is either $A_{1}$ or $A_{2}$. However, at the special point where $m=1 / 2=m^{\prime}$ both instanton actions will contribute symmetrically. In particular, this will cause all the odd powers $Z_{2 \mathbb{N}+1}^{(0)}$ to vanish (which can already be seen directly from (6.56)). This is a particular case of resonant behaviour, where $A_{1}+A_{2}=0$.

The large-order behaviours of the series $\Phi_{1}$ and $\Phi_{2}$ can be determined in the exact same way. Repeating such familiar procedure, the large-order growth of $\Phi_{1}$ is found to be

$$
\begin{aligned}
& Z_{n}^{(1)}(m) \simeq \frac{S_{\omega_{10}}}{2 \pi \mathrm{i}} \frac{\Gamma(n)}{\left(-A_{1}\right)^{n}}\left(Z_{0}^{(0)}+\frac{-A_{1}}{n-1} Z_{1}^{(0)}+\frac{\left(-A_{1}\right)^{2}}{(n-1)(n-2)} Z_{2}^{(0)}+\cdots\right)+ \\
& +\frac{S_{\omega_{12}}+\frac{1}{2} S_{\omega_{02}} S_{\omega_{10}}}{2 \pi \mathrm{i}} \frac{\Gamma(n)}{\left(A_{2}-A_{1}\right)^{n}}\left(Z_{0}^{(2)}+\frac{A_{2}-A_{1}}{n-1} Z_{1}^{(2)}+\frac{\left(A_{2}-A_{1}\right)^{2}}{(n-1)(n-2)} Z_{2}^{(2)}+\cdots\right)
\end{aligned}
$$

Recall that $S_{\omega_{10}}=-2$ and $S_{\omega_{12}}+\frac{1}{2} S_{\omega_{02}} S_{\omega_{10}}=-2$. Further recall that $-A_{1}$ is actually the relative action $A_{0}-A_{1}$, where $A_{0}=0$ is the "perturbative action". Both relative actions $A_{0}-A_{1}$ and $A_{2}-A_{1}$ are negative, in which case $\Phi_{1}$ only has Borel singularities along the negative real axis. Similarly, the large-order growth of $\Phi_{2}$ is

$$
\begin{aligned}
Z_{n}^{(2)}(m) & \simeq \frac{S_{\omega_{20}}}{2 \pi \mathrm{i}} \frac{\Gamma(n)}{\left(-A_{2}\right)^{n}}\left(Z_{0}^{(0)}+\frac{-A_{2}}{n-1} Z_{1}^{(0)}+\frac{\left(-A_{2}\right)^{2}}{(n-1)(n-2)} Z_{2}^{(0)}+\cdots\right)+ \\
& +\frac{S_{\omega_{21}}+\frac{1}{2} S_{\omega_{01}} S_{\omega_{20}}}{2 \pi \mathrm{i}} \frac{\Gamma(n)}{\left(A_{1}-A_{2}\right)^{n}}\left(Z_{0}^{(1)}+\frac{A_{1}-A_{2}}{n-1} Z_{1}^{(1)}+\frac{\left(A_{1}-A_{2}\right)^{2}}{(n-1)(n-2)} Z_{2}^{(1)}+\cdots\right) .
\end{aligned}
$$

Herein one should recall that $S_{\omega_{20}}=-2$ and $S_{\omega_{21}}+\frac{1}{2} S_{\omega_{01}} S_{\omega_{20}}=-2$. Further, and in contrast to $\Phi_{1}$, the Borel singularities of $\Phi_{2}$ are now located along the positive real axis. In summary, we have made it rather explicit how the large-order behaviour of each formal series $\Phi_{i}$ is controlled by the relative actions with respect to the other critical points. The most dominant contribution always comes from the nearest critical point $j$ such that $\left|A_{j}-A_{i}\right|$ is the smallest. Furthermore, for $x \in \mathbb{R}^{+}$, if $A_{j}>A_{i}$ then the contribution of the critical point $j$ to the large-order growth of $i$ is non-alternating whereas for $A_{j}<A_{i}$ it is alternating.

In linear examples we are fortunate to have closed-form expressions for the asymptotic-series coefficients, from which one may analyze large-order behaviours without resorting to numerical calculations. In most nonlinear examples, however, this is not the case and asymptotic-series coefficients can only be computed recursively up to some fixed given order. Nevertheless, we have learnt in section 2 how to fit the large-order behaviour predicted by resurgence to such recursive (numerical) data; and in this way extract the values of the Stokes coefficients. In the present (simple) linear scenario, figure 24 illustrates such fit. On the left image, we plot the coefficients $Z_{n}^{(0)}$ weighted by the growth factor $2 \pi A_{1}^{n} / \Gamma(n)$, and show the convergence towards 

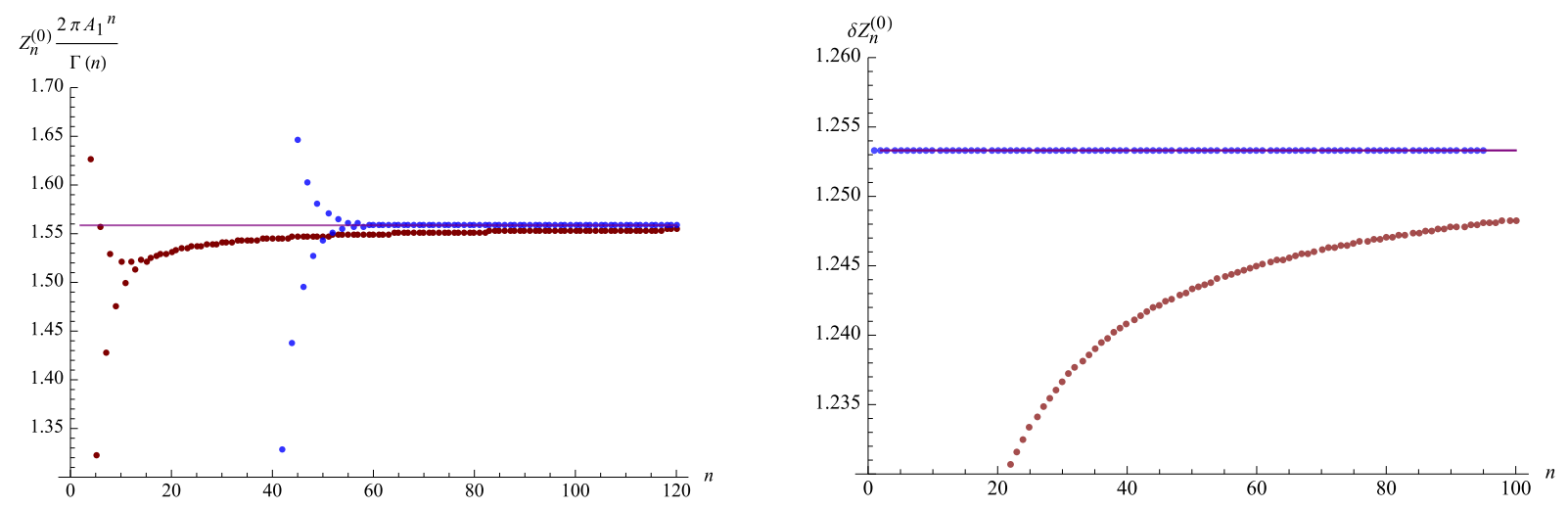

Figure 24. Numerical plots of the asymptotic large-order growth of the perturbative series coefficients, $Z_{n}^{(0)}$ (left), and its subleading exponentially-suppressed growth (right), as predicted by (6.60) (with $m=$ $\pi / 8$ in the plots). On the left we plot the coefficients $Z_{n}^{(0)}$ weighted by their growth factor (in red), together with the corresponding fifth Richardson transform (in blue). The sequence is seen to converge to the predicted value $-S_{\omega_{01}}$ i $Z_{0}^{(1)}=2 \sqrt{1-m}$ (in purple). On the right we plot the weighted resummation (6.67) (in red), the corresponding fifth Richardson transform (in blue), and the predicted value of convergence, $-S_{\omega_{02}}$ i $Z_{0}^{(2)}=2 \sqrt{m}$ (in purple). The errors associated with this numerical calculation are $\sim \mathcal{O}\left(10^{-13}\right)$.

the predicted value (6.60), given by $-S_{\omega_{01}} \mathrm{i} Z_{0}^{(1)}=2 \sqrt{1-m}$ (where in the plot we set $m=\pi / 8$ ). For our choice of $m$, the large-order growth is dominated by the critical point $z_{1}^{*}$ since $A_{1}<\left|A_{2}\right|$. In order to numerically check the exponentially-suppressed contributions associated with $A_{2}$ in (6.60), we first need to resum the asymptotic large-order series ${ }^{105}$

$$
\chi_{0 \rightarrow 1}(n) \simeq \sum_{k=0}^{+\infty} \frac{\Gamma(n-k)}{\Gamma(n)} A_{1}^{k} Z_{k}^{(1)} .
$$

With this result in hand one may then plot the weighted resummation

$$
\delta Z_{n}^{(0)} \equiv\left(Z_{n}^{(0)} \frac{2 \pi A_{1}^{n}}{\Gamma(n)}-\left(-\mathrm{i} S_{\omega_{01}}\right) \mathcal{S}_{0} \mathrm{BP}_{100}\left[\chi_{0 \rightarrow 1}(n)\right]\right)\left(\frac{A_{2}}{A_{1}}\right)^{n},
$$

which is done on the right-hand plot of figure 24 (and where $\mathrm{BP}_{100}\left[\chi_{0 \rightarrow 1}(n)\right]$ is the resummed Borel-Padé approximant associated to the series $\left.\chi_{0 \rightarrow 1}(n)\right)$. We can very clearly see the convergence towards the expected value, $-S_{\omega_{02}} \mathrm{i} Z_{0}^{(2)}=2 \sqrt{m}$.

\subsection{First Steps Towards Nonlinear Resurgent Analysis}

Having studied the linear problem associated with the partition function, let us turn to its closely related nonlinear problem - in parallel with subsection 2.4-namely, the "elliptic free energy". Our motivation is dual. On the one hand this is a more intricate nonlinear problem than the one addressed in section 2, and it will help the reader gain further insight into the general structure of transseries with infinitely many instanton sectors; their asymptotics and their large-order behaviour. But, on the other hand and more importantly, the present non-resonant set-up will lay the ground to later address resonance within nonlinear problems which is the main goal of the present section (to be addressed in the upcoming subsections 6.7 and on).

\footnotetext{
${ }^{105}$ See subsection 2.6 for definitions and a thorough introduction to this whole procedure.
} 

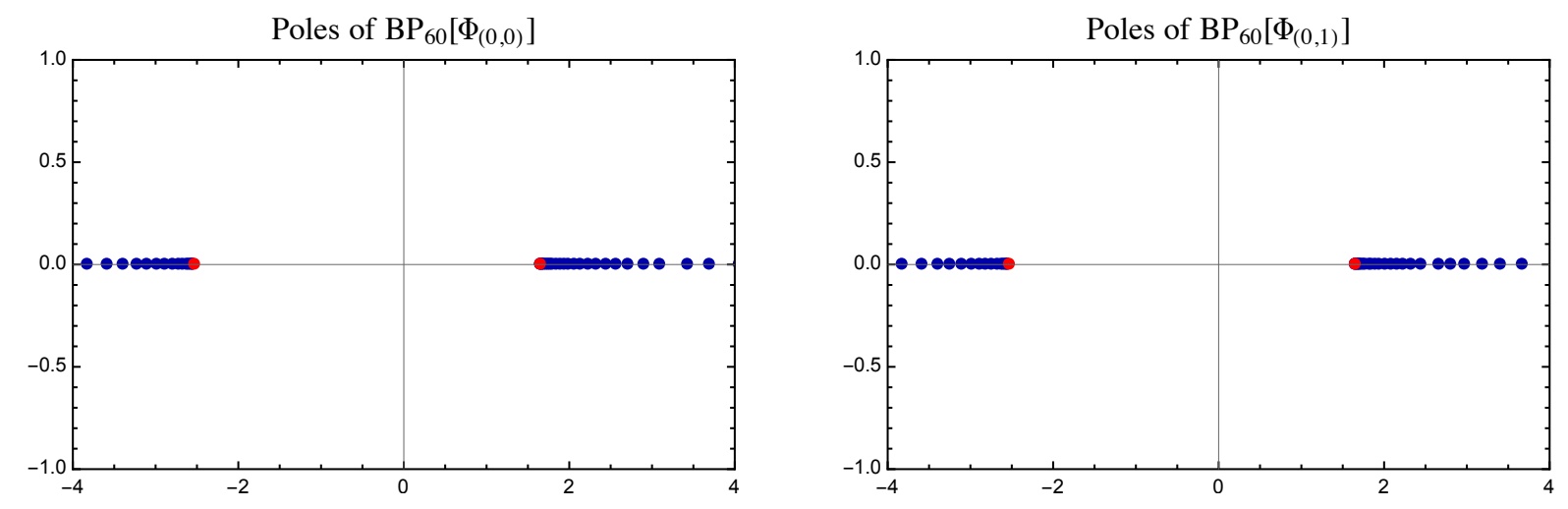

Figure 25. Poles of the (diagonal) order-60 Padé approximants for the Borel transforms of perturbative $\left(\mathrm{BP}_{60}\left[\Phi_{(0,0)}\right]\right.$; left image) and $(0,1)$-instanton $\left(\mathrm{BP}_{60}\left[\Phi_{(0,1)}\right]\right.$; right image) sectors in the free energy of the elliptic potential, with the asymptotic series truncated at $N=120$. The poles (shown in blue) are all on the positive real line, condensing into branch-cuts which start at either $A_{1}=1 /(1-m)$ and $A_{2}=-1 / m$ (instanton actions shown in red). In these plots we have set $m=\pi / 8$.

The elliptic-potential free energy, $F=\log Z$, satisfies a nonlinear third-order ODE (compare with the quartic-potential example back in (2.52)),

$$
\begin{aligned}
& 4 m m^{\prime} x^{4}\left(F^{\prime \prime \prime}(x)+3 F^{\prime}(x) F^{\prime \prime}(x)+\left(F^{\prime}(x)\right)^{3}\right)-4 x^{2}\left(m-m^{\prime}-6 m m^{\prime} x\right)\left(F^{\prime \prime}(x)+\left(F^{\prime}(x)\right)^{2}\right)- \\
& -\left(4+8\left(m-m^{\prime}\right) x-27 m m^{\prime} x^{2}\right) F^{\prime}(x)-\left(m-m^{\prime}-3 m m^{\prime} x\right)=0 .
\end{aligned}
$$

Due to the nonlinearity of this equation, there will be infinitely many instanton sectors in the resulting transseries. Before diving into these nonperturbative sectors let us address the asymptotic perturbative solution, with standard ansatz

$$
F(x) \simeq x^{\beta} \sum_{k=0}^{+\infty} F_{k}^{(0)} x^{k} .
$$

Plugging this perturbative ansatz into (6.68) yields a recursion relation ${ }^{106}$ for the perturbative coefficients $F_{k}^{(0)}$. The reader may check that this leads to an asymptotic series whose Borel transform has singularities along the real axis. Without surprise, these singularities are branch points, located at $A_{1}=1 /(1-m)$ and $A_{2}=-1 / m$. This is depicted in the left plot of figure 25 , where we used a symmetric $N=60$ Padé approximant (recall (2.169)) for the Borel transform of $F(x) \equiv \Phi_{(0,0)}(x)$. The sequence of poles which accumulate at $A_{1} \approx 1.65$ and $A_{2} \approx-2.55$ reflects the underlying singularities of the Borel transform which are closest to the origin (we chose $m=\pi / 8$ in the plots). The fact that there are two distinct branch points implies the existence of at least two separate instanton sectors, with actions $A_{1}$ and $A_{2}$. Indeed, considering a slightly more general ansatz for the solution of (6.68), of the form (recall (2.56))

$$
F(x) \simeq \mathrm{e}^{-\frac{\ell A}{x}} x^{\ell \beta} \sum_{n=0}^{+\infty} F_{n}^{(\ell)} x^{n},
$$

\footnotetext{
${ }^{106}$ This recursion relation can be found in appendix D, by simply setting $\boldsymbol{n}=\left(n_{1}, n_{2}\right)=\mathbf{0}$ in the general recursion.
} 
one easily finds that a solution exists only if either $A=0, A=1 / m^{\prime}$, or $A=-1 / m$, and $\beta=0$. The existence of these three sectors - apparently one perturbative and two nonperturbative - is expected given that the free energy satisfies a third-order ODE, and at first one might expect that the general solution is a two-parameter transseries (i.e., one per nonperturbative sector). However, and in the exact same fashion as it happened for the quartic-potential starting in (2.92) through (2.97), this is not the full story: the above $A=0$ sector is not quite the perturbative sector. In other words, in addition to the nonperturbative sectors associated with the nonzero instanton actions $A_{1}$ and $A_{2}$, there is one extra sector whose "action" vanishes, but which is distinct from the perturbative sector. Furthermore, there are no perturbative fluctuations associated with this sector; it is merely a constant. Of course that the reader is already familiar with this phenomenon, given the experience acquired in section 2 and comparing (6.68) back to (2.52). Also now, the existence of such a non-asymptotic, or "silent" sector-henceforth denoted by $\widetilde{\Phi}_{(0,0)}$-is associated with the fact that the third-order ODE (6.68) only depends on derivatives of the free energy, and hence it can be trivially integrated into a second-order ODE by a redefinition $\mathfrak{F}(x) \equiv F^{\prime}(x)$. In this way, this new sector can then be identified with the integration constant of the redefinition. Consequently, one effectively has a "two-and-a-half" transseries: a three-parameter transseries where one of the sectors, $\widetilde{\Phi}_{(0,0)}$ associated to the "instanton action" $A=0$, is not asymptotic and essentially decouples from the asymptotic sectors associated to the non-zero instanton actions $A_{1}$ and $A_{2}$ (which themselves give rise to a bona fide two-parameter transseries). Labelling these two truly nonperturbative sectors as $\Phi_{\left(0, n_{1}, n_{2}\right)}$ and denoting the constant sector by $\Phi_{(1,0,0)} \equiv \widetilde{\Phi}_{(0,0)}$, this structure leads to the transseries ansatz

$$
F\left(x, \sigma_{0}, \sigma_{1}, \sigma_{2}\right)=\sigma_{0} \Phi_{(1,0,0)}+\sum_{n_{1}=0}^{+\infty} \sum_{n_{2}=0}^{+\infty} \sigma_{1}^{n_{1}} \sigma_{2}^{n_{2}} \mathrm{e}^{-\frac{n_{1} A_{1}+n_{2} A_{2}}{x}} \Phi_{\left(0, n_{1}, n_{2}\right)}(x),
$$

where

$$
\Phi_{\left(0, n_{1}, n_{2}\right)}(x) \simeq \sum_{k=0}^{+\infty} F_{k}^{\left(n_{1}, n_{2}\right)} x^{k} .
$$

Note how this structure is completely analogous to the free energy of the quartic potential discussed in section 2. Because of the simple form of the "third" transseries sector (i.e., the one related to the transseries parameter $\left.\sigma_{0}\right)$, one is led to further denote $\Phi_{\left(0, n_{1}, n_{2}\right)}(x) \equiv \Phi_{\left(n_{1}, n_{2}\right)}(x)$, and finally rewrite the full transseries for the free energy as

$$
F\left(x, \sigma_{0}, \boldsymbol{\sigma}\right)=\sigma_{0} \widetilde{\Phi}_{(0,0)}+\sum_{\boldsymbol{n} \in \mathbb{N}_{0}^{2}} \boldsymbol{\sigma}^{\boldsymbol{n}} \mathrm{e}^{-\frac{\boldsymbol{n} \cdot \boldsymbol{A}}{x}} \Phi_{\boldsymbol{n}}(x) .
$$

We follow the multi-dimensional ${ }^{107}$ notation of section 5, defining vectors $\boldsymbol{A}=\left(A_{1}, A_{2}\right), \boldsymbol{\sigma}=$ $\left(\sigma_{1}, \sigma_{2}\right)$ and $\boldsymbol{n}=\left(n_{1}, n_{2}\right)$, and products $\boldsymbol{\sigma}^{\boldsymbol{n}}=\sigma_{1}^{n_{1}} \sigma_{2}^{n_{2}}$ and $\boldsymbol{n} \cdot \boldsymbol{A}=n_{1} A_{1}+n_{2} A_{2}$.

Plugging the final transseries ansatz (6.73) into the free-energy ODE (6.68) leads to recursion relations for all asymptotic coefficients $F_{k}^{\left(n_{1}, n_{2}\right)}$ (as usual, by simply keeping track of equal powers of $\mathrm{e}^{-\frac{n \cdot \boldsymbol{A}}{x}}$ and of $x^{k}$ ). Even though these recursion relations are relatively straightforward to obtain, they are rather lengthy and not particularly illuminating - as such, we have included them in appendix D rather than presenting them explicitly in the main body of the text. Note, however, that all the coefficients $F_{k}^{(\boldsymbol{n})} \equiv F_{k}^{(\boldsymbol{n})}(m)$ are polynomials in the modular parameter $m$.

\footnotetext{
${ }^{107}$ Recall from section 5 that the sum over $\boldsymbol{n} \in \mathbb{N}_{0}^{2}$ corresponds to summing over $n_{1} \geq 0$ and $n_{2} \geq 0$.
} 
The next step to address concerns resurgence relations in-between different sectors $\Phi_{\boldsymbol{n}}$. This is best done starting with the pictorial language of alien lattices introduced in section 5 . With the experience accumulated throughout section 2 , where we were dealing with a " 1.5 dimensional" alien chain as in figure 11, it is clear from (6.73) that we are now dealing with a "2.5 dimensional" alien lattice which should similarly extend figure 17 . One can then start by identifying each sector $\Phi_{\boldsymbol{n}}$ with a lattice node labeled by the two-dimensional lattice vector $\boldsymbol{n}=\left(n_{1}, n_{2}\right)$. The extra "half dimension" follows as an additional lattice-site which corresponds to the constant $\widetilde{\Phi}_{(0,0)}$ sector. Having this comparison between "1.5" and "2.5" transseries in mind - and recalling how in the "1.5" case the structure of Borel singularities (2.98) was a straightforward extension of (2.62) - , uncovering the local structure of Borel singularities in the "2.5" case should be equally straightforward. In parallel with (2.98), we expect only mild changes to (5.20) and find ${ }^{108}$

$$
\mathcal{B}\left[\Phi_{\boldsymbol{n}}\right](s+\boldsymbol{\ell} \cdot \boldsymbol{A})=\left(\mathrm{S}_{\boldsymbol{n} \rightarrow \boldsymbol{n}+\boldsymbol{\ell}} \times \mathcal{B}\left[\Phi_{\boldsymbol{n}+\boldsymbol{\ell}}\right](s)+\delta_{\boldsymbol{n}+\boldsymbol{\ell}} \mathrm{S}_{\boldsymbol{n} \rightarrow \widetilde{\mathbf{0}}} \times \mathcal{B}\left[\widetilde{\Phi}_{(0,0)}\right](s)\right) \frac{\log s}{2 \pi \mathrm{i}}, \quad \boldsymbol{\ell} \neq \mathbf{0} .
$$

Moving towards the usual alien-derivative algebraic level of abstraction should by now also be quite straightforward, and the process is essentially the same which led to (5.21) and (5.25). The resurgence relations associated to (6.74) are thus given by

$$
\Delta_{\ell \cdot \boldsymbol{A}} \Phi_{\boldsymbol{n}}=\boldsymbol{S}_{\ell} \cdot(\boldsymbol{n}+\ell) \Phi_{\boldsymbol{n}+\ell}+\delta_{\boldsymbol{n}+\ell} S_{\ell}^{(0)} \widetilde{\Phi}_{(0,0)} .
$$

Let us make a couple of remarks about this equation. By definition, $\Delta_{\ell \cdot A} \Phi_{\boldsymbol{n}}=0$ whenever any component is $\ell_{i}<-n_{i}$ (since $\Phi_{\boldsymbol{m}}$ vanishes whenever one $m_{j}$ is negative). Further, $\Delta_{\ell \cdot \boldsymbol{A}} \widetilde{\Phi}_{(0,0)}=0$ for any $\boldsymbol{\ell}$, as the sector $\widetilde{\Phi}_{(0,0)}$ is not asymptotic. The Stokes vectors in (6.75) are two-dimensional, $\boldsymbol{S}_{\boldsymbol{\ell}}=\left(S_{\boldsymbol{\ell}}^{(1)}, S_{\boldsymbol{\ell}}^{(2)}\right)$, but-as thoroughly discussed in section 5-many of their coefficients are zero. In particular, writing $\boldsymbol{\ell}=\left(\ell_{1}, \ell_{2}\right)$,

$$
\begin{array}{ll}
S_{\mathbf{0}}^{(i)}=0, & \text { for } i=0,1,2, \\
S_{\ell}^{(0)}=0, & \text { if any } \ell_{i}>0, \\
S_{\ell}^{(1)}=0, & \text { if } \ell_{1}>1 \text { or } \ell_{2}>0, \\
S_{\ell}^{(2)}=0, & \text { if } \ell_{1}>0 \text { or } \ell_{2}>1 .
\end{array}
$$

This is a simple generalization ${ }^{109}$ from the two-parameter case encoded in (5.24) or (5.25) and illustrated in figure 17. Of course that once again the Stokes vectors appearing in the resurgence

\footnotetext{
${ }^{108}$ Recall that $\widetilde{\Phi}_{(0,0)}$ is a constant and Borel transforms are not defined for constant terms. This caveat is not a big problem: one simply needs to define them as an identity on the Borel plane in order to conduct calculations.

${ }^{109}$ As usual, these results may be derived rather than just motivated by starting-off with the bridge equations, and we included such technical alien-calculus results in appendix A. For the reader who rather stay mid-way between motivation and derivation, let us just flash the argument. At the level of the full transseries (6.73), the bridge equations of this problem have the form
}

$$
\mathrm{e}^{-\frac{\ell \cdot \boldsymbol{A}}{x}} \Delta_{\ell \cdot \mathbf{A}} F\left(x, \sigma_{0}, \boldsymbol{\sigma}\right)=\sum_{i=0}^{2} S_{\ell}^{(i)}\left(\sigma_{0}, \boldsymbol{\sigma}\right) \frac{\partial F}{\partial \sigma_{i}}\left(x, \sigma_{0}, \boldsymbol{\sigma}\right),
$$

where, via the arguments of degree and homogeneity of appendix A (also addressed in section 5), the proportionality Stokes coefficients must be monomials of the form (and with a slight abuse of notation)

$$
S_{\ell}^{(0)}\left(\sigma_{0}, \boldsymbol{\sigma}\right) \equiv S_{\ell}^{(0)} \sigma_{1}^{-\ell_{1}} \sigma_{2}^{-\ell_{2}}, \quad S_{\ell}^{(1)}\left(\sigma_{0}, \boldsymbol{\sigma}\right) \equiv S_{\ell}^{(1)} \sigma_{1}^{1-\ell_{1}} \sigma_{2}^{-\ell_{2}}, \quad S_{\ell}^{(2)}\left(\sigma_{0}, \boldsymbol{\sigma}\right) \equiv S_{\ell}^{(2)} \sigma_{1}^{-\ell_{1}} \sigma_{2}^{1-\ell_{2}} .
$$

The allowed values of $\boldsymbol{\ell}$ are then such that the bridge equations are regular in $\boldsymbol{\sigma}$ (which is our result above). 
relations (6.75) are directly related to the Borel residues appearing in the Borel transforms (6.74). This occurs in the exact same manner as was explained in section 5 . The extra residue, $S_{n \rightarrow \widetilde{\mathbf{0}}}$, simply appears due to the existence of the extra (non-asymptotic) sector $\widetilde{\Phi}_{(0,0)}$.

The " $2.5 \mathrm{~d}$ " alien lattice where the alien derivative (6.75) induces different motions is illustrated in figure 26 (in particular, it describes the action of $\Delta_{\boldsymbol{\ell} \cdot \boldsymbol{A}}$ on the same $(3,2)$-sector of figure 17). The range of (single step) depicted motions follows directly from the discussion in section 5. In particular, strictly-forward motions can only occur in unit jumps, $(1,0)$ or $(0,1)$, implying that the only non-vanishing alien-derivative actions along these directions arise from either $\Delta_{A_{1}}$ or $\Delta_{A_{2}}$. This translates into the above vanishing of Stokes coefficients, i.e., $S_{\ell}^{(i)}=0$ for any $\ell_{1}>0$ and $\ell_{2}>0$ except for $S_{(1,0)}^{(1)}$ and $S_{(0,1)}^{(2)}$. These two elementary forward motions are depicted in figure 26 with weights $w_{A_{1}}$ and $w_{A_{2}}$. Cases where $\boldsymbol{\ell}$ only has non-positive coefficients characterize strictly-backward motions: these are the links which connect $\Phi_{(3,2)}$ to any sector to the left and/or below it, e.g., the link in lighter red connecting to the sector $\Phi_{(2,0)}$ with weight $w_{-A_{1}-2 A_{2}}$. Finally, if one of the components of $\boldsymbol{\ell}$ is negative and the other one positive (equal to 1), we find mixed forward/backward motions. An example of such a mixed motion is the link in orange connecting to sector $\Phi_{(4,0)}$, with weight $w_{A_{1}-2 A_{2}}$. The present lattice is the natural extension of the " $1.5 \mathrm{~d}$ " alien chain, associated to the free energy of the quartic potential and illustrated in figure 11, into the realm of $2 \mathrm{~d}$ alien lattices illustrated in figure 17 . One final and important point to have in mind is the following: in this example, the lattice notion of forward directions does not correlate to singularities along the positive real axis on the Borel plane. In fact, because $A_{1}>0$ and $A_{2}<0$, the projection map (5.19) is such that, say, the lattice "forward" direction connecting $\Phi_{(3,2)}$ to $\Phi_{(4,2)}$ does fall on the positive real axis, while, say, the lattice "forward" direction connecting $\Phi_{(3,2)}$ to $\Phi_{(3,3)}$ instead falls on the negative real axis.

The "statistical mechanical" language of section 2, with its multi-dimensional generalization of section 5, obviously still holds. The weight $w$ associated to a step $\mathcal{S}$ is defined as in (5.26). For a step connecting lattice sites $\boldsymbol{n}$ and $\boldsymbol{n}+\boldsymbol{\ell}$, one has the familiar

$$
w(\mathcal{S}(\boldsymbol{n} \rightarrow \boldsymbol{n}+\ell))=(\boldsymbol{n}+\boldsymbol{\ell}) \cdot \boldsymbol{S}_{\boldsymbol{\ell}}
$$

where this expression holds as long as $\Phi_{\boldsymbol{n}+\boldsymbol{\ell}} \neq \widetilde{\Phi}_{(0,0)}$. In the special case where a step links some sector $\Phi_{\boldsymbol{n}}$ to the sector $\widetilde{\Phi}_{(0,0)}$, which will be denoted by $\widetilde{\mathcal{S}}(\boldsymbol{n} \rightarrow \mathbf{0})$, the corresponding weight is given by

$$
w(\widetilde{\mathcal{S}}(\boldsymbol{n} \rightarrow \mathbf{0}))=S_{-\boldsymbol{n}}^{(0)} .
$$

These weights are shown in figure 26, where the departing node is $\boldsymbol{n}=(3,2)$, and where we use the standard notation introduced for figure 17, i.e., $w(\mathcal{S}(\boldsymbol{n} \rightarrow \boldsymbol{n}+\boldsymbol{\ell}))) \equiv w_{\ell_{1} A_{1}+\ell_{2} A_{2}}$.

Equipped with a geometric picture and an associated "statistical mechanical" language, we may now turn to the standard discussion of Stokes discontinuities. Since there is one positive-real $\left(A_{1}\right)$ and one negative-real $\left(A_{2}\right)$ instanton actions, the projection map (5.19) implies that the expected singularities on the Borel plane are along $\theta=0$ and $\theta=\pi$. These lead to discontinuities which may be determined from (2.33) - and which in the multi-dimensional case this can lead to more explicit equations, such as (5.60) or (5.62) - and (2.45),

$$
\operatorname{Disc}_{0} \Phi_{n}=\Phi_{n}-\underline{\mathfrak{S}}_{0} \Phi_{n}=\left\{1-\exp \left(\sum_{\ell \in \mathbb{L}^{+}} \mathrm{e}^{-\frac{\ell \cdot \boldsymbol{A}}{x}} \Delta_{\ell \cdot \boldsymbol{A}}\right)\right\} \Phi_{n}
$$




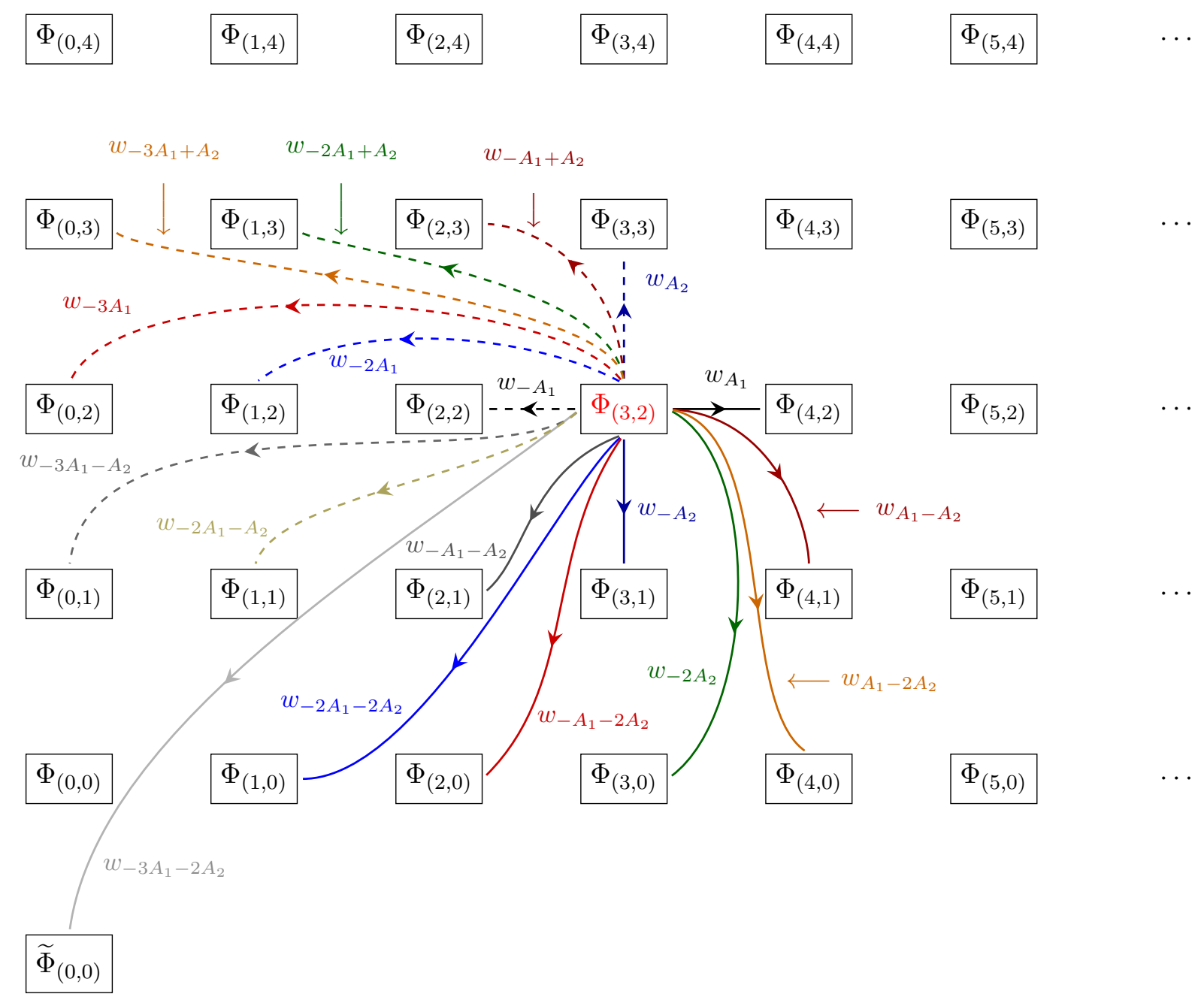

Figure 26. The "2.5d" alien lattice: a pictorial representation of the action of the alien derivative upon the $(3,2)$-sector (compare with figure 17). Different single arrows correspond to different single steps, where the weight of each step is dictated by the right-hand-side of the resurgence relations (6.75). This weight is written next to the corresponding arrow, and is given in terms of Stokes vectors as explained in the main text. The solid and dashed lines represent steps with $\boldsymbol{\ell} \cdot \boldsymbol{A}>0$ and $\boldsymbol{\ell} \cdot \boldsymbol{A}<0$, respectively, for the choice $m=\pi / 8$ (and which will later correspondingly appear in the discontinuities along $\theta=0$ and $\theta=\pi$, respectively). Different colors represent different magnitudes $|\boldsymbol{\ell} \cdot \boldsymbol{A}|$ which dictate exponential dominance. The steps shown are all the single steps which are allowed starting at the $\Phi_{(3,2)}$ lattice-site (and where paths can then be constructed with different middle nodes, and corresponding products of weights).

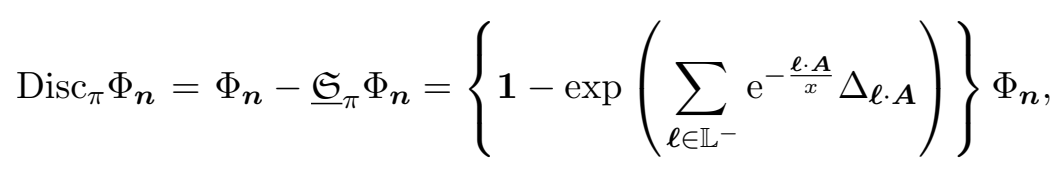


where $\mathbb{L}^{+}=\left\{\forall \boldsymbol{\ell} \in \mathbb{Z}^{2} \mid \boldsymbol{\ell} \cdot \boldsymbol{A}>0\right\}$ and $\mathbb{L}^{-}=\left\{\forall \boldsymbol{\ell} \in \mathbb{Z}^{2} \mid \boldsymbol{\ell} \cdot \boldsymbol{A}<0\right\}$ (of course that many of the lattice vectors chosen in this way lead to vanishing alien derivatives due to the constraints on the Stokes vectors, but, as long as the reader has this in mind, for the moment it is simpler to label the discontinuities in this way). With all this in hand, computing the discontinuities follows the same procedure we already used several times in these lectures, since back in subsection 2.4. Let us quickly run through it as it applies to the present example. The "statistical mechanical" algorithmic procedure directly follows from a simple expansion of the above exponentials and subsequent use of the resurgence relations (6.75). It leads to a sum over all allowed paths $\mathcal{P}$, defined in (2.81), that connect a given fixed node $\Phi_{\boldsymbol{n}}$ to all possible other nodes $\left\{\Phi_{\boldsymbol{m}}\right\}$ such that $(\boldsymbol{m}-\boldsymbol{n}) \cdot \boldsymbol{A}$ is positive (negative) for $\operatorname{Disc}_{0}\left(\operatorname{Disc}_{\pi}\right)$. Each such path $\mathcal{P}$ is made out of steps $\mathcal{S}_{i}$, defined in (2.79), where each step corresponds to a lattice shift $\boldsymbol{\ell}_{i}$ (with $\boldsymbol{\ell}_{i} \cdot \boldsymbol{A}>0$ for Disc $_{0}$, and $\ell_{i} \cdot \boldsymbol{A}<0$ for $\operatorname{Disc}_{\pi}$ ) such that the set of these shifts builds ${ }^{110}$ the corresponding path, i.e., $\sum_{i} \ell_{i}=\boldsymbol{m}-\boldsymbol{n}$. The length of a path, $\ell(\mathcal{P})$, is then naturally defined as the number of its constituent steps, as in (2.82). In the aforementioned sum over all allowed paths, each contribution corresponds to the weight of each path, as defined in (2.83),

$$
w(\mathcal{P})=\prod_{i=1}^{\ell(\mathcal{P})} w\left(\mathcal{S}_{i}\right),
$$

and where the weights of each individual step were defined above; see (6.82) and (6.83). This sum over all allowed paths is further weighted by adequate combinatorial factors; recall (2.84)

$$
\mathrm{CF}(\mathcal{P})=\frac{1}{(\ell(\mathcal{P})) !}
$$

This is a straightforward generalization of the analogous procedure discussed in section 5 .

As concrete examples, let us now explicitly work out the Disc $c_{0}$ and $\operatorname{Disc}_{\pi}$ discontinuities for a couple of sectors. Our first example is the perturbative $(0,0)$ sector. Located at the boundary of the semi-positive transseries grid, any lattice motion which originates from this sector has to move forward. Therefore the only term that contributes to the sum inside the exponential in (6.84), for the $\theta=0$ discontinuity, is $\Delta_{\boldsymbol{\ell} \cdot \boldsymbol{A}}$ with $\boldsymbol{\ell}=\boldsymbol{e}_{1} \equiv(1,0)$. Expanding this exponential and using (6.75) one obtains

$$
\operatorname{Disc}_{0} \Phi_{(0,0)}=-\sum_{n=1}^{+\infty}\left(S_{\boldsymbol{e}_{1}}^{(1)}\right)^{n} \mathrm{e}^{-\frac{n A_{1}}{x}} \Phi_{(n, 0)} .
$$

Similarly, for the $\theta=\pi$ discontinuity the only contributing term comes from $\boldsymbol{\ell}=\boldsymbol{e}_{2} \equiv(0,1)$, which leads to

$$
\operatorname{Disc}_{\pi} \Phi_{(0,0)}=-\sum_{n=1}^{+\infty}\left(S_{\boldsymbol{e}_{2}}^{(2)}\right)^{n} \mathrm{e}^{\frac{n\left|A_{2}\right|}{x}} \Phi_{(0, n)}
$$

A slightly more complicated example is, e.g., the sector $\Phi_{(0,1)}$. In this case, and in addition to the standard forward motions, it is also possible to move strictly backwards to $\widetilde{\Phi}_{(0,0)}$. Taking

\footnotetext{
${ }^{110}$ Each step links different intermediate nodes between $\boldsymbol{n}$ and $\boldsymbol{m}$. For example, consider a path contributing to the discontinuity along $\theta=0$, divided into 3 steps $\mathcal{S}_{i}, i=1,2,3$, and where step $\mathcal{S}_{1}$ connects $\boldsymbol{n} \rightarrow \boldsymbol{n}+\boldsymbol{\ell}_{1}$, step $\mathcal{S}_{2}$ connects $\boldsymbol{n}+\boldsymbol{\ell}_{1} \rightarrow \boldsymbol{n}+\boldsymbol{\ell}_{1}+\boldsymbol{\ell}_{2}$, and finally step $\mathcal{S}_{3}$ connects $\boldsymbol{n}+\boldsymbol{\ell}_{1}+\boldsymbol{\ell}_{2} \rightarrow \boldsymbol{m}$. As such, $\boldsymbol{m}-\boldsymbol{n}=\sum_{i=1}^{3} \boldsymbol{\ell}_{i}$.
} 
$m=\pi / 8$, the $\theta=0$ discontinuity will then include contributions such as $\boldsymbol{\ell}=(0,-1)=-\boldsymbol{e}_{2}$ and $^{111} \ell=(-1,-1)=-\boldsymbol{e}_{1}-\boldsymbol{e}_{2}$. Expanding the exponential in (6.84) and using (6.75) leads to

$$
\begin{aligned}
\operatorname{Disc}_{0} \Phi_{(0,1)}= & -\left(S_{-\boldsymbol{e}_{2}}^{(0)}+\frac{1}{2} S_{\boldsymbol{e}_{1}}^{(1)} S_{-\boldsymbol{e}_{1}-\boldsymbol{e}_{2}}^{(0)}\right) \mathrm{e}^{-\frac{\left|A_{2}\right|}{x}} \widetilde{\Phi}_{(0,0)}-S_{\boldsymbol{e}_{1}-\boldsymbol{e}_{2}}^{(1)} \mathrm{e}^{-\frac{A_{1}+\left|A_{2}\right|}{x}} \Phi_{(1,0)}- \\
& -S_{\boldsymbol{e}_{1}}^{(1)} \mathrm{e}^{-\frac{A_{1}}{x}} \Phi_{(1,1)}-\left(S_{\boldsymbol{e}_{1}}^{(1)}\right)^{2} \mathrm{e}^{-\frac{2 A_{1}}{x}} \Phi_{(2,1)}-\frac{1}{2 !}\left(S_{\boldsymbol{e}_{1}}^{(1)}\right)^{3} \mathrm{e}^{-\frac{3 A_{1}}{x}} \Phi_{(3,1)}-\cdots \\
= & \mathrm{S}_{(0,1) \rightarrow \widetilde{\mathbf{0}}} \mathrm{e}^{-\frac{\left|A_{2}\right|}{x}} \widetilde{\Phi}_{(0,0)}+\mathrm{S}_{(0,1) \rightarrow(1,0)} \mathrm{e}^{-\frac{A_{1}+\left|A_{2}\right|}{x}} \Phi_{(1,0)}+ \\
& +\mathrm{S}_{(0,1) \rightarrow(1,1)} \mathrm{e}^{-\frac{A_{1}}{x}} \Phi_{(1,1)}+\mathrm{S}_{(0,1) \rightarrow(2,1)} \mathrm{e}^{-\frac{2 A_{1}}{x}} \Phi_{(2,1)}+\mathrm{S}_{(0,1) \rightarrow(3,1)} \mathrm{e}^{-\frac{3 A_{1}}{x}} \Phi_{(3,1)}+\cdots
\end{aligned}
$$

As usual, in the above expression we show the discontinuity written both in terms of Stokes coefficients as well as Borel residues. As for the discontinuity at $\theta=\pi$, a similar exercise yields

$$
\begin{aligned}
\operatorname{Disc}_{\pi} \Phi_{(0,1)} & =-2 S_{\boldsymbol{e}_{2}}^{(2)} \mathrm{e}^{\frac{\left|A_{2}\right|}{x}} \Phi_{(0,2)}-3\left(S_{\boldsymbol{e}_{2}}^{(2)}\right)^{2} \mathrm{e}^{\frac{2\left|A_{2}\right|}{x}} \Phi_{(0,3)}-4\left(S_{\boldsymbol{e}_{2}}^{(2)}\right)^{3} \mathrm{e}^{\frac{3\left|A_{2}\right|}{x}} \Phi_{(0,4)}-\cdots \\
& =\mathrm{S}_{(0,1) \rightarrow(0,2)} \mathrm{e}^{\frac{\left|A_{2}\right|}{x}} \Phi_{(0,2)}+\mathrm{S}_{(0,1) \rightarrow(0,3)} \mathrm{e}^{\frac{2\left|A_{2}\right|}{x}} \Phi_{(0,3)}+\mathrm{S}_{(0,1) \rightarrow(0,4)} \mathrm{e}^{\frac{3\left|A_{2}\right|}{x}} \Phi_{(0,4)}+\cdots
\end{aligned}
$$

As one final example let us consider the sector $\Phi_{(3,2)}$, which was the sector used to illustrate figure 26 (recall, depicted in the case where $m=\pi / 8$ ). The discontinuity at $\theta=0$ will involve lattice sites labeled by $(3,2)+\boldsymbol{\ell}$, with $\boldsymbol{\ell} \cdot \boldsymbol{A}>0$ (further recall how steps between the $(3,2)$ node and these sites were plotted with solid lines back in figure 26$)$. The factor $\mathrm{e}^{-\frac{\boldsymbol{\ell} \cdot \boldsymbol{A}}{x}}$ in $(6.84)$ determines the exponential magnitude of the contributions from different sectors, and different magnitudes were represented by different colors in figure 26. The smaller $\boldsymbol{\ell} \cdot \boldsymbol{A}$ is, the more dominant (i.e., less exponentially suppressed) the contribution of the sector $\Phi_{(3,2)+\ell}$ will be (of course, provided it remains $(3,2)+\boldsymbol{\ell} \geq \mathbf{0}$, since otherwise $\left.\Delta_{\boldsymbol{\ell} \cdot \boldsymbol{A}} \Phi_{(3,2)}=0\right)$. The smallest such value is simply reached when $\ell=-(3,2)$. Always assuming $m=\pi / 8$, the ordering of dominances then goes as $\boldsymbol{\ell} \in\{(-3,-2),(-1,-1),(1,0),(-2,-2), \cdots\}$, and the Disc 0 discontinuity is

$$
\begin{aligned}
\operatorname{Disc}_{0} \Phi_{(3,2)}= & -\mathrm{SF}_{((3,2) \rightarrow \widetilde{\mathbf{0}})} \mathrm{e}^{\frac{3 A_{1}+2 A_{2}}{x}} \widetilde{\Phi}_{(0,0)}-\mathrm{SF}_{((3,2) \rightarrow(2,1))} \mathrm{e}^{\frac{A_{1}+A_{2}}{x}} \Phi_{(2,1)}- \\
& -\mathrm{SF}_{((3,2) \rightarrow(4,2))} \mathrm{e}^{-\frac{A_{1}}{x}} \Phi_{(4,2)}-\mathrm{SF}_{((3,2) \rightarrow(1,0))} \mathrm{e}^{\frac{2 A_{1}+2 A 2}{x}} \Phi_{(1,0)}-\cdots
\end{aligned}
$$

The statistical factors $\mathrm{SF}_{(\boldsymbol{n} \rightarrow \boldsymbol{m})}$ appearing in (6.93) are just the Borel residues $\mathrm{S}_{\boldsymbol{n} \rightarrow \boldsymbol{m}}=-\mathrm{SF}_{(\boldsymbol{n} \rightarrow \boldsymbol{m})}$. As thoroughly discussed, they are combinations of Stokes coefficients $S_{\ell}^{(i)}$ which arise from all the different ways of decomposing the motion $(3,2) \rightarrow(3,2)+\boldsymbol{\ell}$ in different paths, i.e., different collections of steps ${ }^{112} \boldsymbol{\ell}=\sum_{i} \boldsymbol{\ell}_{i}$, with $\boldsymbol{\ell}_{i} \cdot \boldsymbol{A}>0$. In other words, there are different ways to go from $\Phi_{(3,2)}$ to any other fixed sector of figure 26 . For instance, consider the contribution associated to connecting $\Phi_{(3,2)} \rightarrow \Phi_{(4,1)}$. There are three possible paths, with weights and combinatorial factors given by (6.86) and (6.87) (and where the step-weights are (6.82) and (6.83)):

- The first path, $\mathcal{P}_{1}$, consists of a single step connecting the two nodes directly, with pathlength $\ell=1$, combinatorial factor $\mathrm{CF}=\frac{1}{1 !}$, and weight $w=w_{A_{1}-A_{2}}=4 S_{\boldsymbol{e}_{1}-\boldsymbol{e}_{2}}^{(1)}+S_{\boldsymbol{e}_{1}-\boldsymbol{e}_{2}}^{(2)}$;

\footnotetext{
${ }^{111}$ If we choose a value $m<1 / 2$, then one has $A_{1}+A_{2}=(2 m-1) /\left(m-m^{2}\right)<0$-which is the case for our numerical demonstrations below. But when $m>1 / 2$, the $\boldsymbol{\ell}=(-1,-1)$ term would show up in the discontinuity along $\theta=\pi$ instead of the one along $\theta=0$, since then $\boldsymbol{\ell} \cdot \boldsymbol{A}<0$ for $m>1 / 2$.

${ }^{112}$ Arising from the expansion of the exponentials in (6.84) or (6.85), and applying $\cdots \Delta_{\ell_{2} \cdot \boldsymbol{A}} \Delta_{\ell_{1} \cdot \boldsymbol{A}}$ to $\Phi_{\boldsymbol{n}}$.
} 
- The second path, $\mathcal{P}_{2}$, consists of two steps, $\mathcal{S}((3,2) \rightarrow(3,1))$ followed by $\mathcal{S}((3,1) \rightarrow(4,1))$, with path-length $\ell=2$, combinatorial factor $\mathrm{CF}=\frac{1}{2 !}$, and path-weight

$$
w\left(\mathcal{P}_{2}\right)=w(\mathcal{S}((3,2) \rightarrow(3,1))) w(\mathcal{S}((3,1) \rightarrow(4,1)))=\left(3 S_{-\boldsymbol{e}_{2}}^{(1)}+S_{-\boldsymbol{e}_{2}}^{(2)}\right)\left(4 S_{\boldsymbol{e}_{1}}^{(1)}+S_{\boldsymbol{e}_{1}}^{(2)}\right) ;
$$

- The final path, $\mathcal{P}_{3}$, also consists of two steps, which are now $\mathcal{S}((3,2) \rightarrow(4,2))$ followed by $\mathcal{S}((4,2) \rightarrow(4,1))$, with path-length $\ell=2$, combinatorial factor $\mathrm{CF}=\frac{1}{2 !}$, and path-weight

$$
w\left(\mathcal{P}_{3}\right)=w(\mathcal{S}((3,2) \rightarrow(4,2))) w(\mathcal{S}((4,2) \rightarrow(4,1)))=\left(4 S_{\boldsymbol{e}_{1}}^{(1)}+2 S_{\boldsymbol{e}_{1}}^{(2)}\right)\left(4 S_{-\boldsymbol{e}_{2}}^{(1)}+S_{-\boldsymbol{e}_{2}}^{(2)}\right) .
$$

The statistical factor $\mathrm{SF}_{((3,2) \rightarrow(4,1))}$ will then be

$$
\mathrm{SF}_{((3,2) \rightarrow(4,1))}=\sum_{i=1}^{3} \mathrm{CF}\left(\mathcal{P}_{i}\right) w\left(\mathcal{P}_{i}\right) .
$$

Similarly, the discontinuity at $\theta=\pi$ involves lattice sites labeled by $(3,2)+\boldsymbol{\ell}$, now with $\boldsymbol{\ell} \cdot \boldsymbol{A}<0$ (and whose steps were plotted with dashed lines back in figure 26). This time around, the exponential magnitude of the different contributions will be ordered starting with smaller $|\boldsymbol{\ell} \cdot \boldsymbol{A}|$, so as to correspond to successively more dominant (i.e., more exponentially enhanced) contributions, and such difference of magnitudes was again represented by different colors in figure 26. Always assuming $m=\pi / 8$, this ordering of dominances goes as $\boldsymbol{\ell} \in$ $\{(-2,-1),(-1,0),(-3,-1),(0,1), \cdots\}$. Repeating the same exercise as above now leads to

$$
\begin{aligned}
\operatorname{Disc}_{\pi} \Phi_{(3,2)}= & -\mathrm{SF}_{((3,2) \rightarrow(1,1))} \mathrm{e}^{\frac{2 A_{1}+A_{2}}{x}} \Phi_{(1,1)}-\mathrm{SF}_{((3,2) \rightarrow(2,2))} \mathrm{e}^{\frac{A_{1}}{x}} \Phi_{(2,2)}- \\
& -\mathrm{SF}_{((3,2) \rightarrow(0,1))} \mathrm{e}^{\frac{3 A_{1}+A_{2}}{x}} \Phi_{(0,1)}-\mathrm{SF}_{((3,2) \rightarrow(3,3))} \mathrm{e}^{\frac{\left|A_{2}\right|}{x}} \Phi_{(3,3)}-\cdots
\end{aligned}
$$

where once again the statistical factors are identified with the Borel residues, $\mathrm{S}_{\boldsymbol{n} \rightarrow \boldsymbol{m}}=-\mathrm{SF}_{(\boldsymbol{n} \rightarrow \boldsymbol{m})}$, and are given by the combinations of Stokes coefficients which arise as a result of decomposing each contribution labeled by $\boldsymbol{\ell}$ into all different ways (different paths) $\boldsymbol{\ell}=\sum_{i} \boldsymbol{\ell}_{i}$ (now with $\boldsymbol{\ell}_{i} \cdot \boldsymbol{A}<0$ ), and then applying the associated alien derivatives to $\Phi_{\boldsymbol{n}}$.

Just like what happened throughout section 2, when dealing with the quartic-potential free energy, the results derived in the present subsection will now also allow us to perform very accurate numerical checks in upcoming subsections. These checks will verify that the transseries solution we are considering is indeed the complete solution to the nonlinear problem of the elliptic-potential free energy. But, akin to what we did back in subsection 2.5, before proceeding towards these large-order checks we shall first focus our attention on actually determining the Stokes coefficients of the elliptic free energy - via a direct comparison with the ones already determined for the partition function - and this is what we shall turn to next.

\subsection{Stokes Constants of Partition Function versus Free Energy}

Given that the free-energy nonlinear problem may actually be derived from the partition-function linear problem (i.e., the one for which we do know Stokes coefficients), then the Stokes coefficients for the nonlinear problem should be explicitly computable simply by formally expanding $\mathcal{F}=$ 
$\log \mathcal{Z}$ (this is exactly the same strategy as the one used in subsection 2.5). In order to do this, start by rewriting the transseries for the partition function (6.24) as

$$
\mathcal{Z}\left(x, \sigma_{0}, \sigma_{1}, \sigma_{2}\right)=\sigma_{0} \Phi_{0}(x)\left(1+\zeta_{1} \mathrm{e}^{-\frac{A_{1}}{x}} \frac{\Phi_{1}(x)}{\Phi_{0}(x)}+\zeta_{2} \mathrm{e}^{-\frac{A_{2}}{x}} \frac{\Phi_{2}(x)}{\Phi_{0}(x)}\right),
$$

where we have introduced $\zeta_{i} \equiv \frac{\sigma_{i}}{\sigma_{0}}, i=1,2$. Next, as the free energy is the logarithm of the partition function, we determine it as the formal expansion of the function $\log (1+z)$. It follows:

$$
\mathcal{F}\left(x, \sigma_{0}, \sigma_{1}, \sigma_{2}\right)=\log \sigma_{0}+\log \Phi_{0}+\sum_{n_{1}=0}^{+\infty} \sum_{n_{2}=0}^{+\infty}\left(1-\delta_{n_{1}, 0} \delta_{n_{2}, 0}\right) \zeta_{1}^{n_{1}} \zeta_{2}^{n_{2}} \mathrm{e}^{-\frac{n_{1} A_{1}+n_{2} A_{2}}{x}} F^{\left(n_{1}, n_{2}\right)}(x),
$$

where the free-energy nonperturbative sectors $F^{\left(n_{1}, n_{2}\right)}$ may be written in terms of the original sectors in the partition function as

$$
F^{\left(n_{1}, n_{2}\right)}:=(-1)^{n_{1}+n_{2}+1} \frac{\left(n_{1}+n_{2}-1\right) !}{n_{1} ! n_{2} !}\left(\frac{\Phi_{1}}{\Phi_{0}}\right)^{n_{1}}\left(\frac{\Phi_{2}}{\Phi_{0}}\right)^{n_{2}} .
$$

On the other hand, from the discussions in the previous subsection, we know that the free energy will be given by a three-parameter ${ }^{113}$ transseries, namely,

$$
F(x, \boldsymbol{\tau})=\sum_{n \in \mathbb{N}_{0}^{3}} \tau^{n} \mathrm{e}^{-\frac{n \cdot A}{x}} \Phi_{n}(x)
$$

Here we used the notation introduced in section 5 , in particular defined ${ }^{114} \boldsymbol{\tau}=\left(\tau_{0}, \tau_{1}, \tau_{2}\right)$, $\boldsymbol{n}=\left(n_{0}, n_{1}, n_{2}\right)$ and $\boldsymbol{A}=\left(0, A_{1}, A_{2}\right)$; and the free-energy nonperturbative sectors $\Phi_{\boldsymbol{n}}$ may be directly read from (6.71) and (6.72). Comparing this expression for the free energy with the previous one, (6.99), which follows directly from the partition function, we immediately identify

$$
\begin{aligned}
\tau_{0} & =\log \sigma_{0}, \quad \tau_{i}=\frac{\sigma_{i}}{\sigma_{0}}, i=1,2, \\
\Phi_{(0,0,0)} & =\log \Phi_{0}, \\
\Phi_{\left(0, n_{1}, n_{2}\right)} & =F^{\left(n_{1}, n_{2}\right)} \\
\Phi_{(1,0,0)} & =1, \\
\Phi_{\left(n_{1}, n_{2}, n_{3}\right)} & =0, \quad \text { if } n_{1}>1 \vee\left(n_{1}=1 \wedge\left(n_{2}, n_{3}\right) \neq(0,0)\right) .
\end{aligned}
$$

In particular, this identification is now validating our "2.5"-parameter transseries ansatz in (6.73).

Following subsection 2.5, let us next see if we can make similar identifications at the level of resurgence relations and, eventually, at the level of Stokes data. In particular, let us follow the discussion at the very end of subsection 2.5, where we were able to relate the Stokes coefficients of the partition function to those of the free energy via a direct comparison of Stokes automorphisms. Recall that the main idea behind that discussion was to use the fact that $\Delta_{\omega}$ is a derivation, in order to then show how the Stokes automorphisms for the free energy have to be the exact same

\footnotetext{
${ }^{113}$ We have renamed the transseries parameters of the free energy as $\tau_{i}$, in order to avoid confusion with the transseries parameters of the partition function $\sigma_{i}$.

${ }^{114}$ Note that, for the purposes of this subsection, we are using three-dimensional vectors within a three-parameter transseries, although we already know that $\tau_{0}$ is associated to a single (non-asymptotic) sector.
} 
operators as those acting at the level of the partition function, once the identifications (6.102) between transseries parameters are taken into account. For the partition function, the Stokes coefficients $^{115}$ were already determined in $(6.42)$; recall

$$
S_{\omega_{01}}^{Z}=2, \quad S_{\omega_{10}}^{Z}=-2, \quad S_{\omega_{02}}^{Z}=2, \quad S_{\omega_{20}}^{Z}=-2, \quad S_{\omega_{12}}^{Z}=0=S_{\omega_{21}}^{Z} .
$$

With these coefficients, one may completely determine the Stokes automorphisms (6.33) and (6.34), and subsequently the Stokes transitions for the partition function, (6.39) and (6.40). But in order to carry through our present goal, one must first rewrite all these expressions with a slightly different flavor (albeit one we have already seen back in subsection 2.5).

Starting along $\theta=0$, one easily finds ${ }^{116}$

$$
\begin{aligned}
\underline{\mathfrak{S}}_{0} \mathcal{Z}\left(x, \sigma_{0}, \sigma_{1}, \sigma_{2}\right) & =\exp \left(\mathrm{e}^{-\frac{\omega_{01}}{x}} \Delta_{\omega_{01}}+\mathrm{e}^{-\frac{\omega_{21}}{x}} \Delta_{\omega_{21}}+\mathrm{e}^{-\frac{\omega_{20}}{x}} \Delta_{\omega_{20}}\right) \mathcal{Z}\left(x, \sigma_{0}, \sigma_{1}, \sigma_{2}\right)= \\
& =\mathcal{Z}\left(x, \sigma_{0}+S_{\omega_{20}}^{Z} \sigma_{2}, \sigma_{1}+S_{\omega_{01}}^{Z} \sigma_{0}+\left(S_{\omega_{21}}^{Z}+\frac{1}{2} S_{\omega_{01}}^{Z} S_{\omega_{20}}^{Z}\right) \sigma_{2}, \sigma_{2}\right)= \\
& \equiv \exp \left\{S_{\omega_{20}}^{Z} \sigma_{2} \frac{\partial}{\partial \sigma_{0}}+\left(S_{\omega_{01}}^{Z} \sigma_{0}+S_{\omega_{21}}^{Z} \sigma_{2}\right) \frac{\partial}{\partial \sigma_{1}}\right\} \mathcal{Z}\left(x, \sigma_{0}, \sigma_{1}, \sigma_{2}\right) .
\end{aligned}
$$

If one now uses the relations (6.102), between partition-function and free-energy transseries parameters, the above differential-representation of the Stokes automorphism may be rewritten for the $\tau_{i}$ variables rather than the $\sigma_{i}$ variables. Using

$$
\begin{aligned}
\sigma_{2} \frac{\partial}{\partial \sigma_{0}} & =\tau_{2}\left(\frac{\partial}{\partial \tau_{0}}-\tau_{1} \frac{\partial}{\partial \tau_{1}}-\tau_{2} \frac{\partial}{\partial \tau_{2}}\right), \\
\sigma_{0} \frac{\partial}{\partial \sigma_{1}} & =\frac{\partial}{\partial \tau_{1}}, \\
\sigma_{2} \frac{\partial}{\partial \sigma_{1}} & =\tau_{2} \frac{\partial}{\partial \tau_{1}},
\end{aligned}
$$

it immediately follows

$$
\underline{\mathfrak{S}}_{0}=\exp \left\{S_{\omega_{20}}^{Z} \tau_{2} \frac{\partial}{\partial \tau_{0}}+\left(S_{\omega_{01}}^{Z}+S_{\omega_{21}}^{Z} \tau_{2}-S_{\omega_{20}}^{Z} \tau_{1} \tau_{2}\right) \frac{\partial}{\partial \tau_{1}}-S_{\omega_{20}}^{Z} \tau_{2}^{2} \frac{\partial}{\partial \tau_{2}}\right\}
$$

When acting upon the free energy, this operator must exactly match the one we already computed when addressing the $\theta=0$ discontinuity, i.e., (6.112) must exactly match the Stokes automorphism in (6.84). In other words, the (differential operator) argument of the above exponential must equal $\sum_{\ell} \mathrm{e}^{-\frac{\ell \cdot \boldsymbol{A}}{x}} \Delta_{\ell \cdot \boldsymbol{A}}$ for $\boldsymbol{\ell} \cdot \boldsymbol{A}>0$. This simple relation between the Stokes automorphisms of linear and nonlinear problems essentially stems from the fact that the free energy ends up inheriting the Borel singularities of the partition function, as explained in detail in subsection 2.5.

\footnotetext{
${ }^{115}$ Akin to subsection 2.5, also in this subsection we shall label Stokes coefficients associated with the partition function with a superscript $Z$, while those associated with the free energy will be labeled with a superscript $F$.

${ }^{116}$ The last line can be simply checked by expanding the exponential and applying the required derivatives. In this process, it is interesting to note how the alien derivatives (acting upon asymptotic series) from the first line ended up being translated into regular derivatives (now acting upon transseries parameters) in the last line. As repeated many times before, this is the essence of the bridge equations, which is now being made explicit: to establish a "bridge" between alien and ordinary calculus - see appendix A for further details.
} 
Now, to match (6.112) and (6.84) we need to dig further into the bridge equations, relating alien to regular calculus. In the present case it can be shown that they imply ${ }^{117}$

$$
\mathrm{e}^{-\frac{\ell \cdot \boldsymbol{A}}{x}} \Delta_{\ell \cdot \boldsymbol{A}}=S_{\ell}^{F(0)} \tau_{1}^{-\ell_{1}} \tau_{2}^{-\ell_{2}} \frac{\partial}{\partial \tau_{0}}+S_{\ell}^{F(1)} \tau_{1}^{1-\ell_{1}} \tau_{2}^{-\ell_{2}} \frac{\partial}{\partial \tau_{1}}+S_{\ell}^{F(2)} \tau_{1}^{-\ell_{1}} \tau_{2}^{1-\ell_{2}} \frac{\partial}{\partial \tau_{2}},
$$

where $\boldsymbol{\ell}=\left(\ell_{1}, \ell_{2}\right)$ satisfies $\boldsymbol{\ell} \cdot \boldsymbol{A}>0$. Comparing (6.113) with the argument of the exponential in (6.112) results in that only three steps, namely $\boldsymbol{\ell}=(1,0), \boldsymbol{\ell}=(1,-1)$, and $\boldsymbol{\ell}=(0,-1)$, respectively related to $\omega_{01}, \omega_{21}$, and $\omega_{20}$, contribute to the Stokes automorphism. Further, this immediately leads to the Stokes identifications:

$$
\begin{array}{ll}
S_{(1,0)}^{F(1)}=S_{\omega_{01}}^{Z} & (=2), \\
S_{(1,-1)}^{F(1)}=S_{\omega_{21}}^{Z} & (=0), \\
S_{(0,-1)}^{F(0)}=S_{\omega_{20}}^{Z} & (=-2), \\
S_{(0,-1)}^{F(1)}=-S_{\omega_{20}}^{Z} & (=2), \\
S_{(0,-1)}^{F(2)}=-S_{\omega_{20}}^{Z} & (=2) ;
\end{array}
$$

all other coefficients vanishing.

The exact same procedure may be applied along $\theta=\pi$, where one now simply starts from (6.34) rather than (6.33). In this case, the Stokes transition (6.40) implies

$$
\begin{aligned}
\underline{\mathfrak{S}}_{\pi} \mathcal{Z}\left(x, \sigma_{0}, \sigma_{1}, \sigma_{2}\right) & =\exp \left(\mathrm{e}^{-\frac{\omega_{02}}{x}} \Delta_{\omega_{02}}+\mathrm{e}^{-\frac{\omega_{10}}{x}} \Delta_{\omega_{10}}+\mathrm{e}^{-\frac{\omega_{12}}{x}} \Delta_{\omega_{12}}\right) \mathcal{Z}\left(x, \sigma_{0}, \sigma_{1}, \sigma_{2}\right)= \\
& =\mathcal{Z}\left(x, \sigma_{0}+S_{\omega_{10}}^{Z} \sigma_{1}, \sigma_{1}, \sigma_{2}+S_{\omega_{02}}^{Z} \sigma_{0}+\left(S_{\omega_{12}}^{Z}+\frac{1}{2} S_{\omega_{02}}^{Z} S_{\omega_{10}}^{Z}\right) \sigma_{1}\right)= \\
& \equiv \exp \left\{S_{\omega_{10}}^{Z} \sigma_{1} \frac{\partial}{\partial \sigma_{0}}+\left(S_{\omega_{02}}^{Z} \sigma_{0}+S_{\omega_{12}}^{Z} \sigma_{1}\right) \frac{\partial}{\partial \sigma_{2}}\right\} \mathcal{Z}\left(x, \sigma_{0}, \sigma_{1}, \sigma_{2}\right) .
\end{aligned}
$$

Using

$$
\begin{aligned}
\sigma_{1} \frac{\partial}{\partial \sigma_{0}} & =\tau_{1}\left(\frac{\partial}{\partial \tau_{0}}-\tau_{1} \frac{\partial}{\partial \tau_{1}}-\tau_{2} \frac{\partial}{\partial \tau_{2}}\right), \\
\sigma_{0} \frac{\partial}{\partial \sigma_{2}} & =\frac{\partial}{\partial \tau_{2}} \\
\sigma_{1} \frac{\partial}{\partial \sigma_{2}} & =\tau_{1} \frac{\partial}{\partial \tau_{2}}
\end{aligned}
$$

it immediately follows

$$
\underline{\mathfrak{S}}_{\pi}=\exp \left\{S_{\omega_{10}}^{Z} \tau_{1} \frac{\partial}{\partial \tau_{0}}-S_{\omega_{10}}^{Z} \tau_{1}^{2} \frac{\partial}{\partial \tau_{1}}+\left(S_{\omega_{02}}^{Z}-S_{\omega_{10}}^{Z} \tau_{1} \tau_{2}-S_{\omega_{12}}^{Z} \tau_{1}\right) \frac{\partial}{\partial \tau_{2}}\right\}
$$

Comparing the (differential operator) argument of the exponential in (6.123) above with (6.113) (where now $\boldsymbol{\ell}=\left(\ell_{1}, \ell_{2}\right)$ satisfies $\left.\boldsymbol{\ell} \cdot \boldsymbol{A}<0\right)$, results in that only three steps, namely $\boldsymbol{\ell}=(0,1)$,

\footnotetext{
${ }^{117}$ This is basically the content of footnote 109 - or else we refer the reader to the discussion in appendix A.
} 
$\boldsymbol{\ell}=(-1,0)$, and $\boldsymbol{\ell}=(-1,1)$, respectively related to $\omega_{02}, \omega_{10}$, and $\omega_{12}$, contribute to the Stokes automorphism. Further, this immediately leads to the Stokes identifications:

$$
\begin{array}{ll}
S_{(0,1)}^{F(2)}=S_{\omega_{02}}^{Z} & (=2), \\
S_{(-1,1)}^{F(2)}=-S_{\omega_{12}}^{Z} & (=0), \\
S_{(-1,0)}^{F(0)}=S_{\omega_{10}}^{Z} & (=-2), \\
S_{(-1,0)}^{F(1)}=-S_{\omega_{10}}^{Z} & (=2), \\
S_{(-1,0)}^{F(2)}=-S_{\omega_{10}}^{Z} & (=2) ;
\end{array}
$$

all other coefficients vanishing.

\subsection{Asymptotics and Large-Order Behaviour: Free Energy}

Having arrived at this point, it is immediate to acknowledge our construction of a transseries solution for the elliptic-potential free energy, as well as the computation of its complete set of Stokes data. This means we can access (at least in principle, and recursively) all the free-energy (multi) instanton nonperturbative sectors, as well as explicitly write down the associated Stokes discontinuities for all these sectors. However, some assumptions were made along the way, and short of a rigorous proof that we have achieved the correct and complete solution, the nextbest thing to do (and in complete analogy with the procedure of section 2; in particular of subsection 2.6) is to test our constructions via resurgent large-order (numerical) analyses.

These upcoming tests (which will completely vindicate our constructions and calculations), will follow very closely what was done in subsection 2.6 for the quartic free-energy, and to where we refer the reader if in need of a reminder on numerical methods and techniques. Without further ado, first note that - similarly to the case of the quartic potential - also in the current example the constant part of the transseries $(6.73), F_{0}^{(0,0)}+\sigma_{0} \widetilde{\Phi}_{(0,0)}$, is an integration constant which does not get fixed by the recursion relations (and does not change the numerical analyses performed below). Of course it is fixed by a choice of initial conditions, corresponding to some particular solution of the ODE (6.68). It may also be fixed upon comparison of free-energy against partition-function; i.e., taking (6.103) and (6.105) into account it is natural to set $F_{0}^{(0,0)}=0$ and $\widetilde{\Phi}_{(0,0)}=1$. But this is not all: as $(6.68)$ is a third-order ODE, there will still be two other coefficients which need to be fixed from initial conditions, namely, $F_{0}^{(1,0)}$ and $F_{0}^{(0,1)}$. Comparing again with the partition function, this time via (6.104), we easily find ${ }^{118}$

$$
F_{0}^{(1,0)}=\frac{Z_{0}^{(1)}}{Z_{0}^{(0)}}=\mathrm{i} \sqrt{1-m}, \quad F_{0}^{(0,1)}=\frac{Z_{0}^{(2)}}{Z_{0}^{(0)}}=\mathrm{i} \sqrt{m} .
$$

We shall use these values in the numerical analyses addressed below.

Let us begin by discussing the general large-order pattern we find, and how it may be succinctly written-down based upon "statistical mechanical" rules (this is essentially the higherdimensional analogue of (2.153) and (2.154)). The generalization of (2.153), the large-order

\footnotetext{
${ }^{118}$ Recall from subsection 6.2 that $Z_{0}^{(0)}=1, Z_{0}^{(1)}(m)=\mathrm{i} \sqrt{1-m}$, and $Z_{0}^{(2)}(m)=\mathrm{i} \sqrt{m}$.
} 
factor for a path on the alien lattice connecting the nodes $\Phi_{n}$ and $\Phi_{\boldsymbol{m}}$, is straightforward ${ }^{119}$

$$
\chi_{(\boldsymbol{n} \rightarrow \boldsymbol{m})}(k) \equiv \sum_{h=0}^{+\infty} \frac{\Gamma(k-h)}{\Gamma(k)} F_{h}^{(\boldsymbol{m})}((\boldsymbol{m}-\boldsymbol{n}) \cdot \boldsymbol{A})^{h} .
$$

In here, we are assuming that the starting node can be any node except the non-asymptotic sector $\widetilde{\Phi}_{(0,0)}$. The end node, on the other hand, can be any node except the perturbative sector $\Phi_{(0,0)}$. As both these sectors have $\boldsymbol{m}=(0,0)$, let us distinguish them by introducing the notation $\boldsymbol{m}=\widetilde{\mathbf{0}}$ for the sector $\Phi_{\widetilde{\mathbf{0}}} \equiv \widetilde{\Phi}_{(0,0)}$, while $\boldsymbol{m}=\mathbf{0}$ naturally remains attached to $\Phi_{(0,0)}$ (recall ${ }^{120}$ (6.73)). In this way, the starting node will have $\boldsymbol{n} \geq \mathbf{0}$, while the end node will obey either $\boldsymbol{m} \equiv\left(m_{1}, m_{2}\right)>\mathbf{0}$ (i.e., $m_{i} \geq 0$ and $\left.\boldsymbol{m} \neq(0,0)\right)$ or $\boldsymbol{m}=\widetilde{\mathbf{0}}$. Finally, recall that $\widetilde{\mathbf{0}}$ is not asymptotic: we have only one non-zero coefficient $F_{0}^{(\widetilde{\mathbf{0}})}=1$ (all others vanish; $F_{h>0}^{(\widetilde{\mathbf{0}})}=0$ ). Having all this in mind, a straightforward re-run of the analysis in subsection 2.6 allows us to write down generic large-order relations for the "two-and-a-half" transseries of the elliptic potential, purely in terms of motions and data on the alien lattice:

Large-order relations: The large-order (large $k$ ) behaviour of the coefficients $F_{k}^{(\boldsymbol{n})}$, associated to the node $\Phi_{\boldsymbol{n}}$, is given by a sum over all paths, linking to nodes $\Phi_{\boldsymbol{m}}$ with $\boldsymbol{m}>\mathbf{0}$, in addition to a sum over paths to the extra "orthogonal" node in the lattice, $\widetilde{\Phi}_{(0,0)}$ (when $\boldsymbol{m}=\mathbf{0}$ ).

Each term in this sum $\left(\Phi_{\boldsymbol{n}} \rightarrow \Phi_{\boldsymbol{m}}\right)$ can be decomposed into three factors:

- Leading growth-factor:

$$
\frac{\Gamma(k)}{2 \pi \mathrm{i}((\boldsymbol{m}-\boldsymbol{n}) \cdot \boldsymbol{A})^{k}} .
$$

- Large-order factor (6.130), dictated solely by beginning and end nodes:

$$
\chi_{(\boldsymbol{n} \rightarrow \boldsymbol{m})}(k) .
$$

- Statistical factor, sum over all the allowed paths $\mathcal{P}(\boldsymbol{n} \rightarrow \boldsymbol{m})$ linking the nodes as in figure 26 :

$$
\mathrm{SF}_{(\boldsymbol{n} \rightarrow \boldsymbol{m})} \equiv \sum_{\mathcal{P}(\boldsymbol{n} \rightarrow \boldsymbol{m})} \mathrm{CF}(\mathcal{P}) w(\mathcal{P})
$$

Spelled out in writing, the result is:

$$
F_{k}^{(\boldsymbol{n})} \simeq \frac{\Gamma(k)}{2 \pi \mathrm{i}((\boldsymbol{m}-\boldsymbol{n}) \cdot \boldsymbol{A})^{k}} \sum_{\boldsymbol{m} \neq \boldsymbol{n}} \operatorname{SF}_{(\boldsymbol{n} \rightarrow \boldsymbol{m})} \chi_{(\boldsymbol{n} \rightarrow \boldsymbol{m})}(k) .
$$

Further proceeding along the lines of subsection 2.6, let us next address the (resurgent)

\footnotetext{
${ }^{119} \mathrm{Up}$ to the "multi-instanton-number factorization" which is now no longer possible.

${ }^{120}$ If one were to use the notation of a full three-parameter transseries, as in (6.71), one should really have $\boldsymbol{m}=(0,0,0) \equiv \mathbf{0}$ and $\boldsymbol{m}=(1,0,0) \equiv \widetilde{\mathbf{0}}$; and with all other sectors labeled by $\boldsymbol{m}=\left(0, m_{1}, m_{2}\right)$.
} 
large-order behaviour of the perturbative sector, $\Phi_{(0,0)}$. The analogue of $(2.151)$ will now be

$$
\begin{aligned}
F_{k}^{(0,0)} \simeq & \frac{S_{\boldsymbol{e}_{1}}^{(1)}}{2 \pi \mathrm{i}} \frac{\Gamma(k)}{A_{1}^{k}}\left(F_{0}^{(1,0)}+\frac{A_{1}}{k-1} F_{1}^{(1,0)}+\frac{A_{1}^{2}}{(k-1)(k-2)} F_{2}^{(1,0)}+\cdots\right)+ \\
& +\frac{\left(S_{\boldsymbol{e}_{1}}^{(1)}\right)^{2}}{2 \pi \mathrm{i}} \frac{\Gamma(k)}{\left(2 A_{1}\right)^{k}}\left(F_{0}^{(2,0)}+\frac{2 A_{1}}{k-1} F_{1}^{(2,0)}+\frac{\left(2 A_{1}\right)^{2}}{(k-1)(k-2)} F_{2}^{(2,0)}+\cdots\right)+\cdots \\
& +\frac{S_{\boldsymbol{e}_{2}}^{(2)}}{2 \pi \mathrm{i}} \frac{\Gamma(k)}{A_{2}^{k}}\left(F_{0}^{(0,1)}+\frac{A_{2}}{k-1} F_{1}^{(0,1)}+\frac{A_{2}^{2}}{(k-1)(k-2)} F_{2}^{(0,1)}+\cdots\right)+ \\
& +\frac{\left(S_{\boldsymbol{e}_{2}}^{(2)}\right)^{2}}{2 \pi \mathrm{i}} \frac{\Gamma(k)}{\left(2 A_{2}\right)^{k}}\left(F_{0}^{(0,2)}+\frac{2 A_{2}}{k-1} F_{1}^{(0,2)}+\frac{\left(2 A_{2}\right)^{2}}{(k-1)(k-2)} F_{2}^{(0,2)}+\cdots\right)+\cdots
\end{aligned}
$$

where the two instanton actions $A_{1}$ and $A_{2}=-\left|A_{2}\right|$ both come into play at large order, albeit generically with distinct weights (a novelty as compared to the quartic potential). In particular, these relative contributions get exponentially-suppressed as $\left(A_{1} /\left|A_{2}\right|\right)^{k}$ when $A_{1}<\left|A_{2}\right|$, and $\left(\left|A_{2}\right| / A_{1}\right)^{k}$ when $A_{1}>\left|A_{2}\right|$; and such that the sector with smallest action is always the dominant one. We shall see how to "disentangle" them in the following. One similarity with the quartic potential is that contributions to the large-order growth of the perturbative series are exclusively associated to forward motions (either along $\boldsymbol{e}_{1}, \Phi_{(0,0)} \rightarrow \Phi_{(1,0)} \rightarrow \Phi_{(2,0)} \rightarrow \Phi_{(3,0)} \cdots$, or along $\boldsymbol{e}_{2}$, $\left.\Phi_{(0,0)} \rightarrow \Phi_{(0,1)} \rightarrow \Phi_{(0,2)} \rightarrow \Phi_{(0,3)} \cdots\right)$. However, since $A_{2}<0$, the motion along the $\boldsymbol{e}_{2}$-direction now yields oscillatory contributions to the large-order behaviour, since $A_{2}^{k}=(-1)^{k}\left|A_{2}\right|^{k}$.

Let us next illustrate how one can decode nonperturbative information (say, the one-instanton sector $\left.\Phi_{(1,0)}\right)$ solely out of the above large-order behaviour of the perturbative coefficients. As usual, we set the non-resonant value of $m=\pi / 8$. For this choice of $m, A_{1}<\left|A_{2}\right|$ and the dominant contribution to the large-order growth of the perturbative sector thus comes from the $\Phi_{(1,0)}$ nonperturbative sector, i.e., the first line of equation (6.132). At leading (exponential) order we have

$$
\begin{aligned}
F_{k}^{(0,0)} \frac{2 \pi \mathrm{i} A_{1}^{k}}{\Gamma(k)} & \simeq S_{\boldsymbol{e}_{1}}^{(1)}\left(F_{0}^{(1,0)}+\frac{F_{1}^{(1,0)} A_{1}}{k}+\frac{F_{1}^{(1,0)} A_{1}+F_{2}^{(1,0)} A_{1}^{2}}{k^{2}}+\cdots\right)+\mathcal{O}\left(2^{-k}\right) \\
& \equiv \sum_{r=0}^{+\infty} \frac{s_{r}}{k^{r}}+\mathcal{O}\left(2^{-k}\right)
\end{aligned}
$$

where the coefficients $\left\{s_{r}\right\}$ in the second line are simply defined to match the expansion of the first line. If we plot the left-hand side of (6.133), it should asymptote to $s_{0} \equiv S_{\boldsymbol{e}_{1}}^{(1)} F_{0}^{(1,0)}=2 \mathrm{i} \sqrt{1-m}$. Figure 27 (left) shows how this is indeed the case, albeit rather slowly. In order to increase the rate of convergence, let us use Richardson extrapolation akin to what was done in subsection 2.6 (recall that the Richardson transform effectively removes the subleading $1 / k$ corrections that appear on the right-hand side of (6.133), leading to much faster convergence). As such, in the left image of figure 27 we have plotted the left-hand side of (6.133) (multiplied by i) in red, whereas in blue we have plotted its corresponding fifth Richardson transform (5-RT) $\mathrm{RT}_{(0,0)}(0, k, 5)$. The solid purple line shows the constant value $s_{0}$ to which this Richardson transform is converging to. Note that if at this stage we did not know the value of the Stokes coefficient $S_{\boldsymbol{e}_{1}}^{(1)}$, but only of 

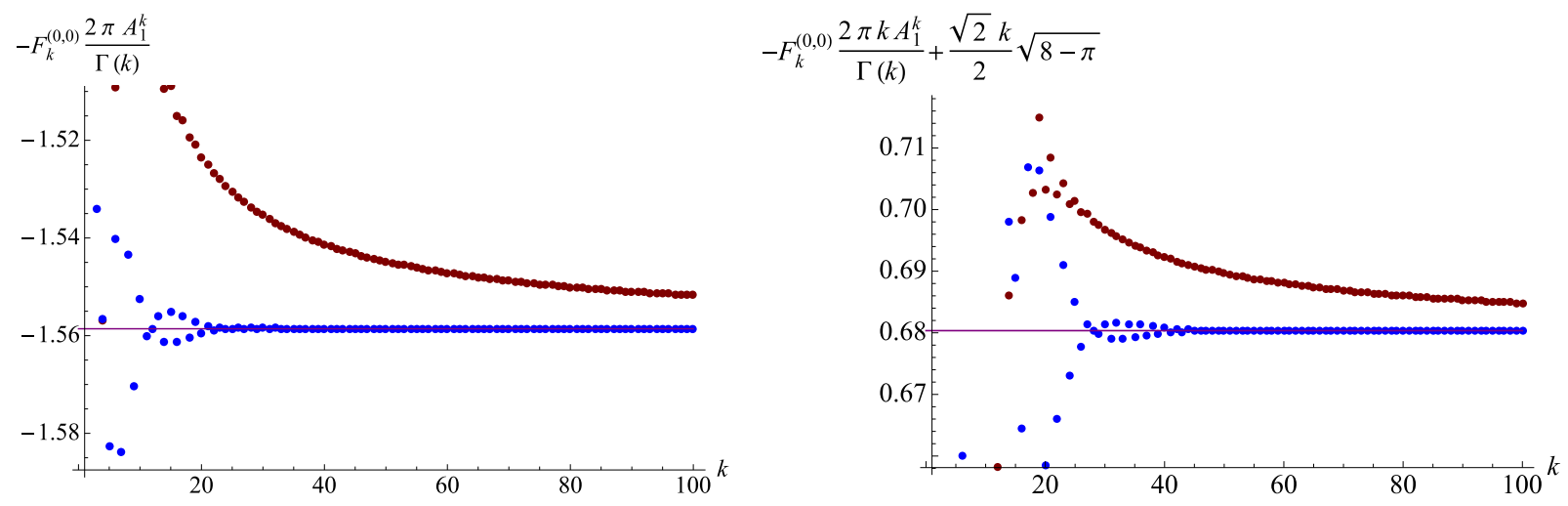

Figure 27. The leading (left) and subleading $1 / k$ (right) large-order behaviour of the perturbative sector $F_{k}^{(0,0)}$, weighted by the leading growth-factor. In red we plot the original sequences, while in blue we plot the Richardson transforms $\mathrm{RT}_{(0,0)}(r, k, 5)(r=0$ for the left plot and $r=1$ for the right plot). The purple lines show the constant values to which these sequences converge: on the left, $\mathrm{i} S_{\boldsymbol{e}_{1}}^{(1)} F_{0}^{(1,0)}=-2 \sqrt{1-m}$; while on the right, is $A_{1} S_{\boldsymbol{e}_{1}}^{(1)} F_{1}^{(1,0)}=\frac{2}{\sqrt{1-m}}$ (and where we have set $m=\frac{\pi}{8}$ ).

$F_{0}^{(1,0)}$, then this large-order result would allow us to further determine ${ }^{121} S_{\boldsymbol{e}_{1}}^{(1)}=2$. Comparing the exact value of $s_{0}$ with the value of the Richardson transform $\operatorname{RT}_{(0,0)}(0,100,5)$, one finds the usual, remarkably small relative errors

$$
\frac{\mathrm{RT}_{(0,0)}(0,100,5)-\mathrm{i} s_{0}}{\mathrm{i} s_{0}} \approx 2.7372 \times 10^{-10} .
$$

Having computed the value of $F_{0}^{(1,0)}$ from the partition function, large order then allowed us to determine (or, more precisely, to check) the value of the Stokes coefficient $S_{\boldsymbol{e}_{1}}^{(1)}$. But from here on one can proceed to compute all subleading terms $F_{r}^{(1,0)}$, with $r \geq 1$, without any further input. In the right image of figure 27 we have plotted the sequence $-F_{k}^{(0,0)} \frac{2 \pi k A_{1}^{k}}{\Gamma(k)}-\mathrm{i} s_{0} k$ in red, whereas in blue we have plotted its corresponding 5 -RT, $\operatorname{RT}_{(0,0)}(1, k, 5)$. These two sequences converge to i $s_{1}=\mathrm{i} A_{1} S_{\boldsymbol{e}_{1}}^{(1)} F_{1}^{(1,0)}=\frac{2}{\sqrt{1-m}} \approx 0.6804$ (whose value is shown as the solid purple line), in complete agreement with the value of $F_{1}^{(1,0)}$ which we directly computed from the recursion relations. One may then continue this process and subtract $s_{0}+\frac{s_{1}}{k}$ from (6.133), multiply the result by $k^{2}$, and thus obtain $F_{2}^{(1,0)}$. Iterate the procedure and one obtains $F_{3}^{(1,0)}, F_{4}^{(1,0)}$, and so on. At all stages, comparing exact coefficients with the numerical Richardson extrapolations one finds the familiar extremely small relative errors; e.g.,

$$
\frac{\mathrm{RT}_{(0,0)}(1,100,5)-\mathrm{i} s_{1}}{\mathrm{i} s_{1}} \approx-1.3120 \times 10^{-11} .
$$

\footnotetext{
${ }^{121}$ This procedure of computing Stokes coefficients might seem somehow redundant since we already know their values from the previous subsection. However, in general we do not have the luxury of independent computations of Stokes coefficients and this is essentially the only way to determine them. Of course that if neither Stokes coefficient nor the first term of the instanton series are known, then the best one can do is to determine their product; see [16] on this point.
} 
Moving on, let us focus on the terms which are exponentially suppressed, and for starts let us focus on the second and third lines of (6.132). In particular, note that since $\left|A_{2}\right| \approx 2.54<$ $2 A_{1} \approx 3.29$ the leading exponentially-suppressed-sector contributing to the large-order growth of the perturbative series will be $\Phi_{(0,1)}$. In order to isolate the contribution of this sector, the contribution from the leading sector $\Phi_{(1,0)}$ needs to be removed, as a whole. Such procedures were thoroughly discussed in subsection 2.6 via the use of Borel-Padé (BP) resummation (e.g., recall $(2.169))$, which may now be applied to the asymptotic series $\chi_{((0,0) \rightarrow(1,0))}(k)$, that may then be subtracted-out from the original sequence $F_{k}^{(0,0)} \frac{2 \pi \mathrm{i} A_{1}^{k}}{\Gamma(k)}$. From $(6.132)$, it then simply follows ${ }^{122}$ (compare with (2.171))

$$
\begin{aligned}
F_{k}^{(0,0)} & \frac{2 \pi \mathrm{i} A_{1}^{k}}{\Gamma(k)}-\mathrm{SF}_{((0,0) \rightarrow(1,0))} \times \mathcal{S}_{0^{-}} \mathrm{BP}_{N}\left[\chi_{((0,0) \rightarrow(1,0))}\right](k) \simeq \\
& \simeq\left(\frac{A_{1}}{A_{2}}\right)^{k} \mathrm{SF}_{((0,0) \rightarrow(0,1))}\left(F_{0}^{(0,1)}+\frac{F_{1}^{(0,1)} A_{2}}{k}+\frac{F_{1}^{(0,1)} A_{2}+F_{2}^{(0,1)} A_{2}^{2}}{k^{2}}+\cdots\right)+\mathcal{O}\left(2^{-k}\right) .
\end{aligned}
$$

The above Laplace transform yielding the resummed series, $\mathcal{S}_{0}-\mathrm{BP}_{N}\left[\chi_{((0,0) \rightarrow(1,0))}\right](k)$, is a lateral resummation evaluated along $\theta=0^{-}$(i.e., just below the real line). This is essentially the same formulae that we have already seen in subsection 2.6-e.g., recall the discussion following (2.171) - and such lateral resummation is needed in order to avoid the poles of the BP approximant to $\chi_{((0,0) \rightarrow(1,0))}$ which lie on the positive real line. Exactly as it happened in subsection 2.6, this will produce a result which has both real and imaginary parts (compare with (2.172))

$$
\mathcal{S}_{0^{-}} \mathrm{BP}_{N}\left[\chi_{((0,0) \rightarrow(1,0))}\right](k)=\mathcal{S}_{\mathbb{R e}} \mathrm{BP}_{N}\left[\chi_{((0,0) \rightarrow(1,0))}\right](k)+\mathrm{i} \mathcal{S}_{\mathbb{I m}} \mathrm{BP}_{N}\left[\chi_{((0,0) \rightarrow(1,0))}\right](k) .
$$

We find that the imaginary component is the leading one, of the same magnitude as the original sequence; while the real component is exponentially suppressed, as $\mathcal{O}\left(2^{-k}\right)$. Once the subtraction in (6.137) is carried through (do recall that the perturbative coefficients $F_{k}^{(0,0)}$ are real), one finds the following magnitudes ${ }^{123}$ :

$$
\begin{aligned}
\mathrm{i} \delta_{\mathbb{I m}} F_{k}^{(0,0)} & \equiv F_{k}^{(0,0)} \frac{2 \pi \mathrm{i} A_{1}^{k}}{\Gamma(k)}-\mathrm{iSF}_{((0,0) \rightarrow(1,0))} \mathcal{S}_{\mathbb{I m}} \mathrm{BP}_{N}\left[\chi_{((0,0) \rightarrow(1,0))}\right](k) \simeq \mathcal{O}\left(\left(A_{2} / A_{1}\right)^{-k}\right), \\
\delta_{\mathbb{R e}} F_{k}^{(0,0)} & \equiv-\mathrm{SF}_{((0,0) \rightarrow(1,0))} \mathcal{S}_{\mathbb{R e}} \mathrm{BP}_{N}\left[\chi_{((0,0) \rightarrow(1,0))}\right](k) \simeq \mathcal{O}\left(2^{-k}\right)
\end{aligned}
$$

In this way, the large-order sequence, and corresponding behaviour, we wish to address is, finally,

$$
\left(\frac{A_{2}}{A_{1}}\right)^{k} \delta_{\mathbb{I m}} F_{k}^{(0,0)} \simeq-\mathrm{iSF}_{((0,0) \rightarrow(0,1))}\left(F_{0}^{(0,1)}+\frac{F_{1}^{(0,1)} A_{2}}{k}+\frac{F_{1}^{(0,1)} A_{2}+F_{2}^{(0,1)} A_{2}^{2}}{k^{2}}+\cdots\right) .
$$

The overall factor $\left(\frac{A_{2}}{A_{1}}\right)^{k}$ on the left-hand side of (6.141) removes any exponential dependence, and we are left with a simple sequence which should converge to $-\mathrm{i} S_{\boldsymbol{e}_{2}}^{(2)} F_{0}^{(0,1)}=\sqrt{\pi / 2}$ (where we are using $S_{\boldsymbol{e}_{2}}^{(2)}=2$ and $\left.F_{0}^{(0,1)}=\mathrm{i} \frac{\sqrt{\pi}}{2 \sqrt{2}}\right)$. Indeed, such convergence is very explicitly seen

\footnotetext{
${ }^{122}$ Recall that $\mathrm{SF}_{((0,0) \rightarrow(1,0))}=S_{\boldsymbol{e}_{1}}^{(1)}$ and that $\mathrm{SF}_{((0,0) \rightarrow(0,1))}=S_{\boldsymbol{e}_{2}}^{(2)}$.

${ }^{123}$ As $\left|A_{2}\right|<2 A_{1}$ it follows $\left|A_{2}\right| / A_{1}<2$ and the imaginary contribution is the exponentially leading one. This is different from what happened back with the quartic potential, wherein the imaginary part was the exponentially suppressed one-recall, e.g., figure 13.
} 


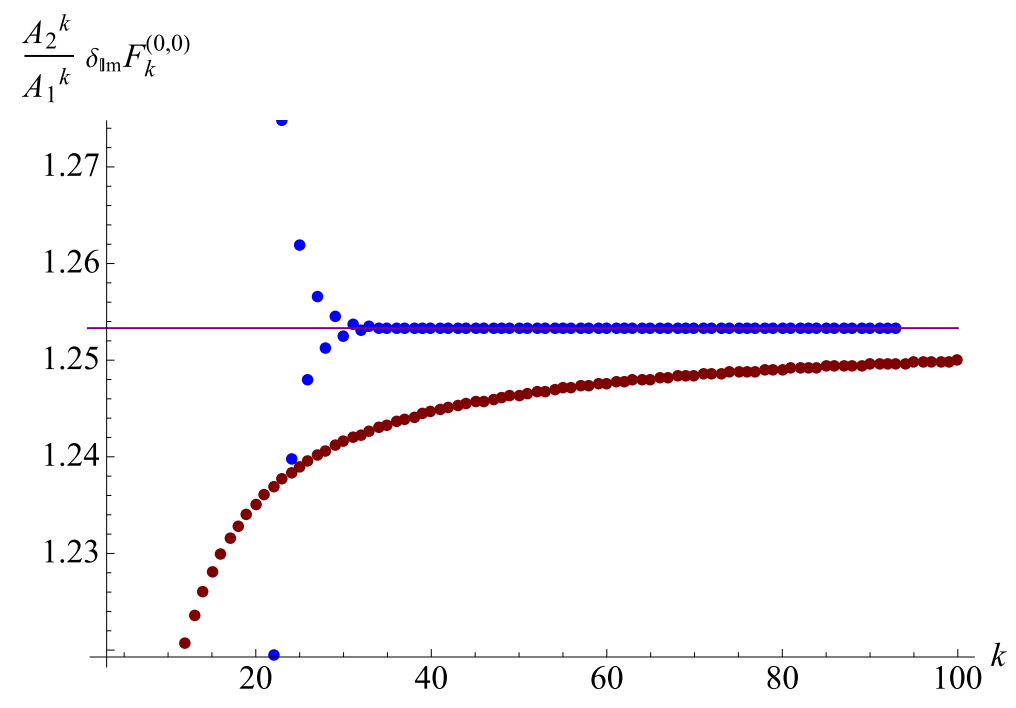

Figure 28. Illustration of the subleading, exponentially-suppressed contributions to the large-order behaviour of the perturbative sector. The plot shows the sequence $\left(\frac{A_{2}}{A_{1}}\right)^{k} \delta_{\mathbb{I m}} F_{k}^{(0,0)}$, defined in (6.139), and corresponding to a numerical $\mathrm{BP}$ resummation of order $N=50$. The original sequence is plotted in red, while in blue we plot its corresponding $\mathrm{RT}_{(0,0), A_{2}}(0, k, 5)$ Richardson transform. The purple line shows the constant value that this sequence is converging to, $-\mathrm{i} S_{\boldsymbol{e}_{2}}^{(2)} F_{0}^{(0,1)}=\sqrt{\pi / 2} \approx 1.2533$.

in figure 28 (corresponding to a numerical BP resummation of order $N=50$ ). Let us recall the notation $\mathrm{RT}_{n, \ell}(0, k, N)$, used to denote the $N^{\text {th }}$-RT associated to the $\ell^{-k}$ exponentiallysuppressed behaviour of the large-order relation obeyed by the $\boldsymbol{n}$-instanton coefficients $F_{k}^{(\boldsymbol{n})}$. For our current case, i.e., for the sequence $\left(\frac{A_{2}}{A_{1}}\right)^{k} \delta_{\mathbb{I m}} F_{k}^{(0,0)}$ obtained via BP resummation, we find the usual extremely-small convergence ${ }^{124}$ error

$$
\frac{\mathrm{RT}_{(0,0), \frac{A_{2}}{A_{1}}}(0,95,5)-\sqrt{\frac{\pi}{2}}}{\sqrt{\frac{\pi}{2}}} \approx 4.0376 \times 10^{-10} .
$$

Via continued BP resummations of the subleading large-order contributions, one could go on analysing the subsequent exponentially-suppressed sequences within (6.132), much along the spirit of what was done in subsection 2.6- a laborious exercise left for the interested reader.

Let us now turn to the analysis of the large-order behaviour of higher instanton sectors, in particular let us focus upon the $\Phi_{(0,1)}$ sector (which in itself will include most relevant features of multi-instantonic large-orders). Using the expressions for both Stokes discontinuities (6.90) and (6.92) into Cauchy's theorem (2.149), one obtains the large-order relation ${ }^{125}$

$$
F_{k}^{(0,1)} \frac{2 \pi \mathrm{i} A_{1}^{k}}{\Gamma(k)} \simeq S_{\boldsymbol{e}_{1}}^{(1)}\left(F_{0}^{(1,1)}+\frac{A_{1}}{k-1} F_{1}^{(1,1)}+\frac{A_{1}^{2}}{(k-1)(k-2)} F_{2}^{(1,1)}+\cdots\right)+
$$

\footnotetext{
${ }^{124} \mathrm{Had}$ we not previously known the separate values of $S_{\boldsymbol{e}_{2}}^{(2)}$ and $F_{0}^{(0,1)}$, then we could now use this convergence to compute their product, $S_{\boldsymbol{e}_{2}}^{(2)} F_{0}^{(0,1)}$, to such small error.

${ }^{125}$ The ordering of the contributions is organized according to their exponential dominance, which follows from the ordered sequence $1<\left|A_{2}\right| / A_{1} \approx 1.546<2<\left(A_{1}+\left|A_{2}\right|\right) / A_{1} \approx 2.546\left(=\left|A_{2}\right|\right)<3<\cdots$.
} 


$$
\begin{aligned}
& +\left(-\frac{A_{1}}{\left|A_{2}\right|}\right)^{k} 2 S_{\boldsymbol{e}_{2}}^{(2)}\left(F_{0}^{(0,2)}+\frac{-\left|A_{2}\right|}{k-1} F_{1}^{(0,2)}+\frac{\left|A_{2}\right|^{2}}{(k-1)(k-2)} F_{2}^{(0,2)}+\cdots\right)+ \\
& +\left(\frac{A_{1}}{\left|A_{2}\right|}\right)^{k}\left(S_{-\boldsymbol{e}_{2}}^{(0)}+\frac{1}{2} S_{\boldsymbol{e}_{1}}^{(1)} S_{-\boldsymbol{e}_{1}-\boldsymbol{e}_{2}}^{(0)}\right) F_{0}^{(\widetilde{\mathbf{0}})}+ \\
& +\frac{1}{2^{k}}\left(S_{\boldsymbol{e}_{1}}^{(1)}\right)^{2}\left(F_{0}^{(2,1)}+\frac{2 A_{1}}{k-1} F_{1}^{(2,1)}+\frac{\left(2 A_{1}\right)^{2}}{(k-1)(k-2)} F_{2}^{(2,1)}+\cdots\right)+ \\
& +\left(\frac{A_{1}}{A_{1}+\left|A_{2}\right|}\right)^{k} S_{\boldsymbol{e}_{1}-\boldsymbol{e}_{2}}^{(1)}\left(F_{0}^{(1,0)}+\frac{A_{1}+\left|A_{2}\right|}{k-1} F_{1}^{(1,0)}+\frac{\left(A_{1}+\left|A_{2}\right|\right)^{2}}{(k-1)(k-2)} F_{2}^{(1,0)}+\cdots\right)+ \\
& +\mathcal{O}\left(3^{-k}\right) .
\end{aligned}
$$

This large-order relation is particularly interesting as it displays the many different types of resurgence which appear within this problem. In particular, the third line above corresponds to the "orthogonal" resurgence motion in the alien lattice, akin to the analogous term which appeared back in (2.184). The large-order relation (6.143) may be equivalently written with slightly simpler combinatorial factors, using Borel residues instead of Stokes coefficients. One has:

$$
\begin{aligned}
F_{k}^{(0,1)} & \frac{2 \pi \mathrm{i} A_{1}^{k}}{\Gamma(k)} \simeq-\mathrm{S}_{(0,1) \rightarrow(1,1)}\left(F_{0}^{(1,1)}+\frac{A_{1}}{k-1} F_{1}^{(1,1)}+\frac{A_{1}^{2}}{(k-1)(k-2)} F_{2}^{(1,1)}+\cdots\right)- \\
& -\left(-\frac{A_{1}}{\left|A_{2}\right|}\right)^{k} \mathrm{~S}_{(0,1) \rightarrow(0,2)}\left(F_{0}^{(0,2)}+\frac{-\left|A_{2}\right|}{k-1} F_{1}^{(0,2)}+\frac{\left|A_{2}\right|^{2}}{(k-1)(k-2)} F_{2}^{(0,2)}+\cdots\right)- \\
& -\left(\frac{A_{1}}{\left|A_{2}\right|}\right)^{k} \mathrm{~S}_{(0,1) \rightarrow \widetilde{\mathbf{0}}} F_{0}^{(\widetilde{\mathbf{0}})}- \\
& -\frac{1}{2^{k}} \mathrm{~S}_{(0,1) \rightarrow(2,1)}\left(F_{0}^{(2,1)}+\frac{2 A_{1}}{k-1} F_{1}^{(2,1)}+\frac{\left(2 A_{1}\right)^{2}}{(k-1)(k-2)} F_{2}^{(2,1)}+\cdots\right)- \\
& -\left(\frac{A_{1}}{A_{1}+\left|A_{2}\right|}\right)^{k} \mathrm{~S}_{(0,1) \rightarrow(1,0)}\left(F_{0}^{(1,0)}+\frac{A_{1}+\left|A_{2}\right|}{k-1} F_{1}^{(1,0)}+\frac{\left(A_{1}+\left|A_{2}\right|\right)^{2}}{(k-1)(k-2)} F_{2}^{(1,0)}+\cdots\right)- \\
& -\mathcal{O}\left(3^{-k}\right) .
\end{aligned}
$$

Large-order analysis now follows as usual. Let us start with a quick check of the leading sequence (the first line above). On the left image of figure 29 we plot the sequence $F_{k}^{(0,1)} \frac{2 \pi \mathrm{i} A_{1}^{k}}{\Gamma(k)}$ (red) alongside its fifth Richardson transform (blue), $\mathrm{RT}_{(0,1)}(0, k, 5)$, and compare these with the expected result $S_{\boldsymbol{e}_{1}}^{(1)} F_{0}^{(1,1)}$ (purple) (using the explicit values $S_{\boldsymbol{e}_{1}}^{(1)}=2$ and $F_{0}^{(1,1)}=\sqrt{m-m^{2}} \approx$ 0.488). The convergence is excellent, as always, with a relative error given by

$$
\frac{\mathrm{RT}_{(0,1)}(0,95,5)-2 \sqrt{\frac{\pi}{8}\left(1-\frac{\pi}{8}\right)}}{2 \sqrt{\frac{\pi}{8}\left(1-\frac{\pi}{8}\right)}} \approx 5.0594 \times 10^{-10} .
$$

Moving towards exponentially-suppressed contributions (the second and following lines above), let us first address how to "disentangle" the second and third lines of (6.143). These correspond to contributions from the sectors $\Phi_{(0,2)}$ and $\widetilde{\Phi}_{(0,0)}$, which have the same weight but are exponentially suppressed as compared to the contribution of the $\Phi_{(1,1)}$ sector. As such, in order to 

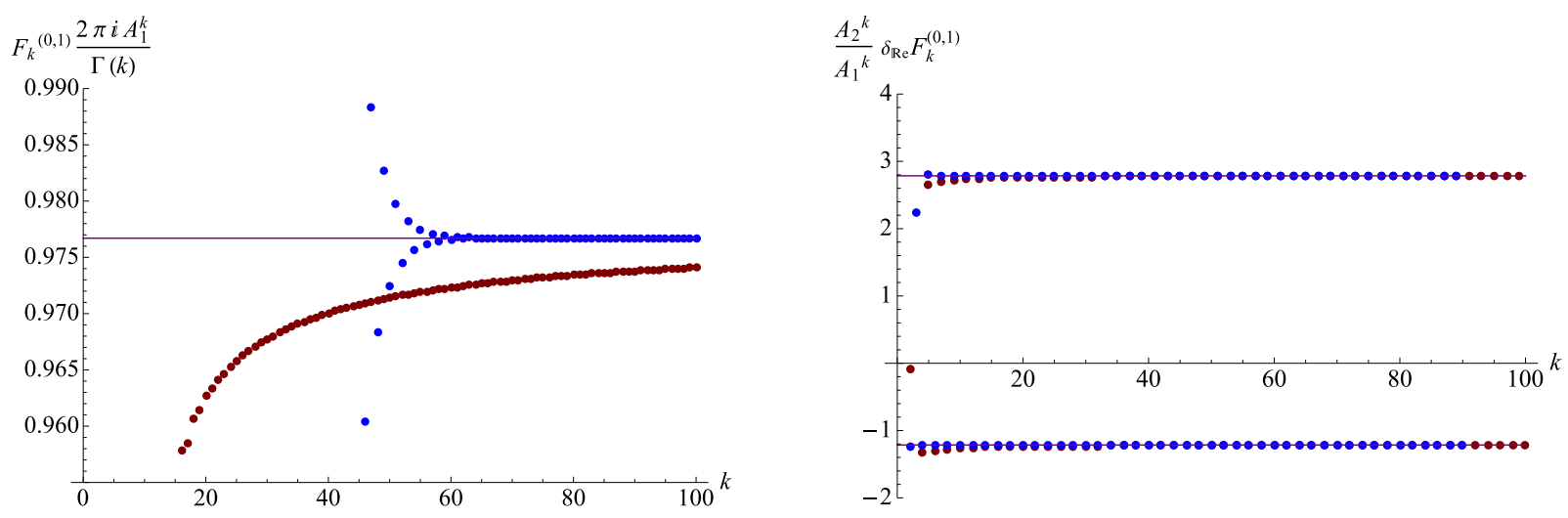

Figure 29. Leading (left) and exponentially suppressed (right; via BP resummation of order $N=50$ ) large-order behaviour of the coefficients $F_{k}^{(0,1)}$, weighted by the leading growth factor. In red we plot the original sequences, while their corresponding 5 -RTs are plotted in blue (these are $\mathrm{RT}_{(0,1)}(0, k, 5)$ for the left plot and $\mathrm{RT}_{(0,1), A_{2}}$,even/odd $(0, k, 5)$ for the right plot). Purple (solid) lines denote the constants to which the sequences converge: $S_{\boldsymbol{e}_{1}}^{(1)} F_{0}^{(1,1)}=2 \sqrt{\frac{\pi}{8}\left(1-\frac{\pi}{8}\right)}$ (left) and $2 S_{\boldsymbol{e}_{2}}^{(2)} F_{0}^{(0,2)} \pm S_{-\boldsymbol{e}_{2}}^{(0)} F_{0}^{(\widetilde{\mathbf{o}})}=\frac{\pi}{4} \mp 2$ (right).

isolate the contribution of these subleading sectors, the whole contribution of $\Phi_{(1,1)}$ has to be resummed and subtracted from the large-order growth of the coefficients $F_{k}^{(0,1)}$. This is basically the same which was just done within the analysis of the perturbative sector: the contribution to be subtracted is in itself an asymptotic series, and one first needs to calculate its BP resummation. This resummation will once again have both real and imaginary parts, and given that the coefficients $F_{k}^{(0,1)}$ are purely imaginary, one ends up working with the combinations ${ }^{126}$

$$
\begin{aligned}
\delta_{\mathbb{R e}} F_{k}^{(0,1)} & \equiv F_{k}^{(0,1)} \frac{2 \pi \mathrm{i} A_{1}^{k}}{\Gamma(k)}-\mathrm{SF}_{((0,1) \rightarrow(1,1))} \mathcal{S}_{\mathbb{R e}} \mathrm{BP}_{N}\left[\chi_{((0,1) \rightarrow(1,1))}\right](k) \simeq \mathcal{O}\left(\left(A_{2} / A_{1}\right)^{-k}\right), \\
\mathrm{i} \delta_{\mathbb{I m}} F_{k}^{(0,1)} & \equiv-\mathrm{iSF}_{((0,1) \rightarrow(1,1))} \mathcal{S}_{\mathbb{I m}} \mathrm{BP}_{N}\left[\chi_{((0,1) \rightarrow(1,1))}\right](k) \simeq \mathcal{O}\left(2^{-k}\right) .
\end{aligned}
$$

Clearly the relevant sequence is $\delta_{\mathbb{R e}} F_{k}^{(0,1)}$, whose leading large-order growth will be dictated by the second and third lines of $(6.143)$ (while $\delta_{\mathbb{I m}} F_{k}^{(0,1)}$ is exponentially suppressed with respect to $\left.\delta_{\mathbb{R e}} F_{k}^{(0,1)}\right)$. This large order is:

$$
\begin{aligned}
\left(\frac{A_{2}}{A_{1}}\right)^{k} \delta_{\mathbb{R e}} F_{k}^{(0,1)} \simeq & \operatorname{SF}_{((0,1) \rightarrow(0,2))}\left(F_{0}^{(0,2)}+\frac{F_{1}^{(0,2)} A_{2}}{k}+\frac{F_{1}^{(0,2)} A_{2}+F_{2}^{(0,2)} A_{2}^{2}}{k^{2}}+\cdots\right)+ \\
& +(-1)^{k} \operatorname{SF}_{((0,1) \rightarrow \widetilde{\mathbf{0}})} F_{0}^{(\widetilde{\mathbf{0}})} .
\end{aligned}
$$

The statistical factors in the above expression are given by $\mathrm{SF}_{((0,1) \rightarrow(0,2))}=2 S_{\boldsymbol{e}_{2}}^{(2)}=4$ and $\mathrm{SF}_{((0,1) \rightarrow \widetilde{\mathbf{0}})}=S_{-\boldsymbol{e}_{2}}^{(0)}+\frac{1}{2} S_{\boldsymbol{e}_{1}}^{(1)} S_{-\boldsymbol{e}_{1}-\boldsymbol{e}_{2}}^{(0)}=-2$ (using the values for the Stokes coefficients previously determined in $(6.124),(6.116)$, and $(6.114))$. Further, we have $F_{0}^{(0,2)}=\frac{1}{2} m$ and $F_{0}^{(\widetilde{\mathbf{0}})}=1$. Now, the leading large-order behaviour of the sequence on the left-hand side of (6.148) has a fixed

\footnotetext{
${ }^{126}$ The statistical factor here is $\mathrm{SF}_{((0,1) \rightarrow(1,1))}=S_{\boldsymbol{e}_{1}}^{(1)}$.
} 
contribution (coming from the first line) alongside an alternating contribution (coming from the second line). As such, this sequence is composed of two (even and odd) sub-sequences, which respectively converge to either $\mathrm{SF}_{((0,1) \rightarrow(0,2))} F_{0}^{(0,2)}+\mathrm{SF}_{((0,1) \rightarrow \widetilde{\mathbf{0}})} F_{0}^{(\widetilde{\mathbf{0}})}=2 m-2 \approx-1.215$ or $\mathrm{SF}_{((0,1) \rightarrow(0,2))} F_{0}^{(0,2)}-\mathrm{SF}_{((0,1) \rightarrow \widetilde{\mathbf{0}})} F_{0}^{(\widetilde{\mathbf{0}})}=2 m+2 \approx 2.785$. These two sub-sequences, together with their respective 5-RTs, are plotted on the right image of figure 29 (the label even/odd on the RTs denotes the Richardson transform of the even/odd sequence, respectively). Comparing the limits of these sub-sequences - obtained from their 5-RT-with the exact coefficients - obtained from the recursion relations - we find a relative numerical error of the order $\sim 10^{-10}$. As always, the numerical precision obtained from large-order analysis is quite remarkable.

This concludes our analysis of the resurgent structure of the transseries solution we constructed for the elliptic-potential free energy. The numerical checks we performed thoroughly verified the large-order predictions which were obtained from resurgence, and in this way completely vindicated our earlier transseries constructions and calculations. It is worth noting that up to now the analysis was quite similar to the case of the quartic potential, once one upgrades the underlying structure from the one-dimensional alien chain to a two-dimensional alien lattice. This in itself is an important extension to learn and have in mind, but, as alluded to earlier, there is more to this example than just a higher-dimensional generalization of section 2 . This is in fact the final topic we wish to discuss, as we finally turn to the study of resonance.

\subsection{Nonlinear Resonance and Transseries Structures}

As already pointed out in section 5, multiple instanton actions may give rise to new phenomena. Back then, we limited ourselves to discuss what we dubbed as the "non-resonant case", where the instanton actions in $\boldsymbol{A}=\left(A_{1}, \ldots, A_{k}\right)$ were $\mathbb{Z}$-linearly independent; recall equation (5.16)

$$
\nexists \boldsymbol{n} \neq \mathbf{0} \in \mathbb{Z}^{k} \mid \boldsymbol{n} \cdot \boldsymbol{A}=0 .
$$

In particular, this condition implied that the projection map (5.19) had vanishing kernel, i.e., ker $\mathfrak{P}=\mathbf{0}$. It so happens that in our present example the instanton actions (6.4) do depend on a parameter (they are "tunable") and, depending on this choice of $m$, the above condition may be violated. In this case, we have to deal with resonance, to which we turn in the following.

One way to understand the origin of this phenomenon is to think of the inverse of the coupling-constant variable, $1 / x$, as a (imaginary) "time variable"; to think of the instanton actions, $A_{i}$, as "frequencies"; and to think of the transmonomials as "plane-wave contributions". In such a familiar physical scenario, resonance usually occurs for special choices of the different frequencies at play (e.g., at special values of the driving frequencies). It turns out that the relevant concept of (nonlinear) resonance within the transseries setting finds its origin within perturbed Hamiltonian dynamics and its associated KAM theory; see, e.g., [138, 200]. In the following we shall discuss resonance within the present transseries language, but will follow such discussion in parallel with the one one might have if discussing Hamiltonian dynamics.

Consider the scenario of section 5 , where we are dealing with a multi-parameter (specifically, $k$-dimensional) transseries of the form (5.17) and (5.18), and where the $k$ instanton actions assemble vectorially as $\boldsymbol{A}=\left(A_{1}, \ldots, A_{k}\right)$. The instanton actions are said to be rationally independent or non-resonant, when they are linearly independent over the rationals:

$$
\forall \boldsymbol{n} \in \mathbb{Z}^{k}, \quad \boldsymbol{n} \cdot \boldsymbol{A}=0 \Rightarrow \boldsymbol{n}=\mathbf{0} .
$$



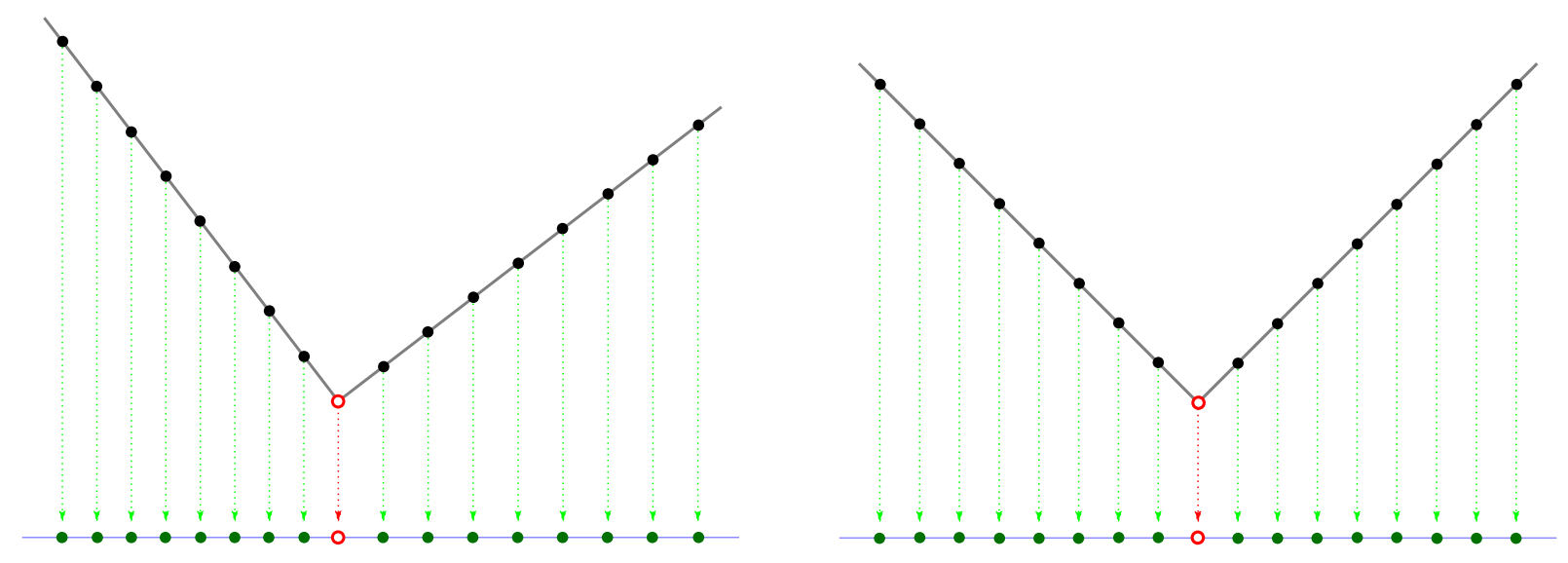

Figure 30. Non-vanishing nodes of the $(0,0)$ alien lattice, and their projection upon the complex Borel plane. The left plot shows a non-resonant example, where $A_{1}$ and $A_{2}$ are rationally independent (but both still real and symmetric), while the right plot shows the resonant example we are considering, with $A_{2}=-A_{1}$. For the perturbative sector, nothing special really happens as we approach the resonant case.

Otherwise, the instanton actions are said to be rationally dependent or resonant, and one has:

$$
\exists \mathfrak{n} \neq \mathbf{0} \in \mathbb{Z}^{k} \mid \mathfrak{n} \cdot \boldsymbol{A}=0 .
$$

Each such vector $\mathfrak{n}$ is denoted as an action correlation. The set of all action correlations, $\left\{\mathfrak{n}_{i} \neq \mathbf{0} \in \mathbb{Z}^{k} \mid \mathfrak{n}_{i} \cdot \boldsymbol{A}=0\right\}$, alongside the standard vector addition, is a subgroup of $\left(\mathbb{Z}^{k},+\right)$. This group of action-correlations can generically have $r$ generators, with $1 \leq r \leq k-1$, which are also linearly-independent $\mathbb{Z}^{k}$-vectors. This number of generators equals the number of truly independent correlations one can write down, in-between the $k$ instanton actions.

In the Hamiltonian dynamics formulation of integrable systems, set-up in action-angle variables, the above instanton actions correspond to the frequencies of the periodic motion taking place in phase space. More precisely, the trajectory of motion in phase space generically takes place over a $k$-dimensional torus, $\mathbb{T}^{k}$. But if there are, say, $r$ frequency correlations, then the trajectory "collapses" to having support on the smaller torus $\mathbb{T}^{k-r} \subset \mathbb{T}^{k}$. As long as the frequencies are rationally independent (non-resonant), the phase-curves on the torus $\mathbb{T}^{k}$ are everywhere dense. But when they are rationally dependent (resonant), each orbit is only dense in the lowerdimensional torus $\mathbb{T}^{k-r}$ (i.e., on the original torus $\mathbb{T}^{k}$ the trajectories are now only closed). This split between resonant and non-resonant tori ${ }^{127}$ is crucial for KAM theory. Start off with an integrable system, whose motion is confined to an invariant torus. Upon an arbitrarily small but nonlinear perturbation, resonant tori are generically destroyed by this perturbation (eventually giving rise to chaotic behaviour). However, non-resonant tori (which end-up being the majority) do survive (thus constraining the possible chaotic motions near each resonance). This fascinating story is developed at length within the setting of KAM theory, to which we refer the reader to $[138,200]$ and references therein - the goal of this paragraph was simply to point out how there are countless interesting links between the theory of resurgence and that of dynamical systems.

\footnotetext{
${ }^{127}$ Note how resonant tori are the analogue of the rational numbers, while non-resonant tori are the analogue of the irrational numbers; which extends to their respective cardinalities.
} 

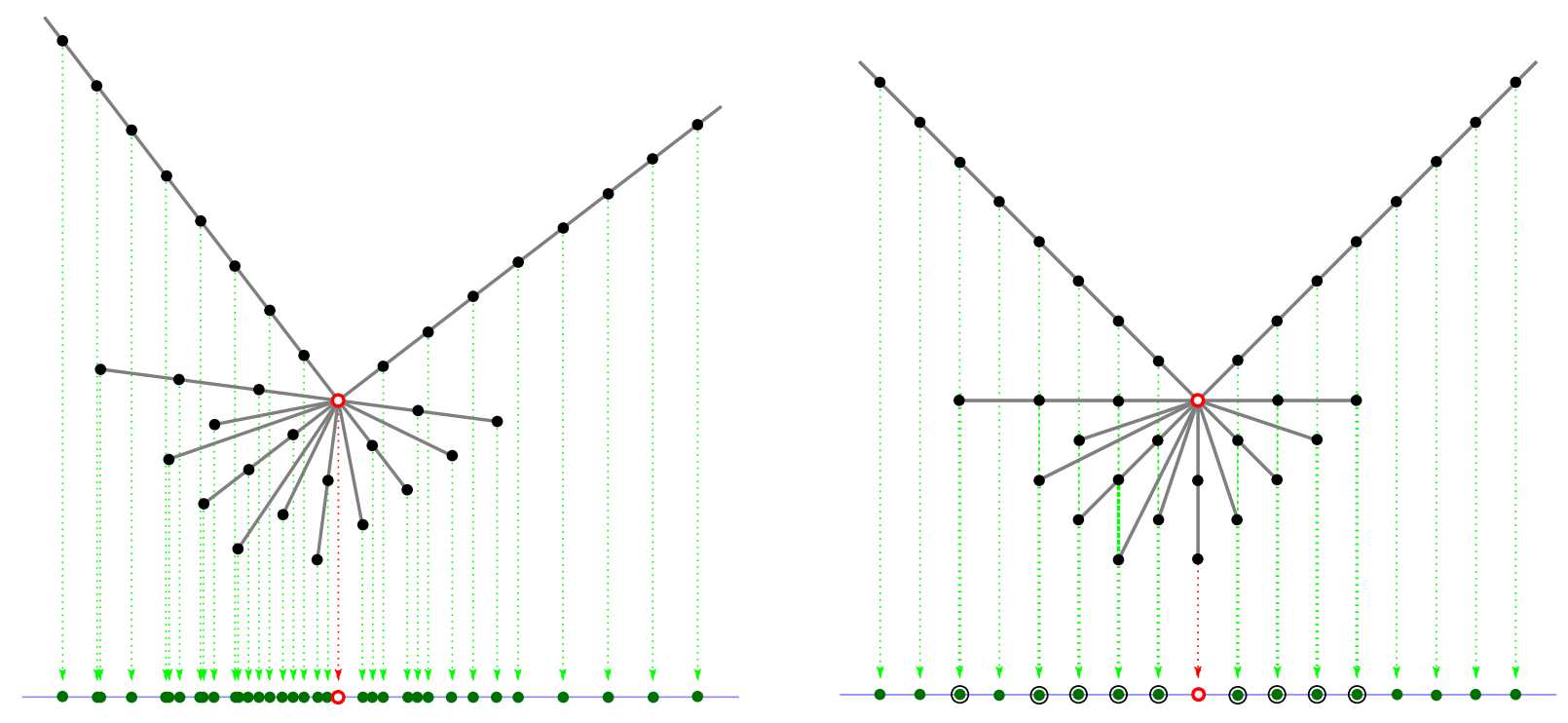

Figure 31. Non-vanishing nodes of the $(3,2)$ alien lattice, and their projection upon the complex Borel plane. The left plot shows a non-resonant example, where the projection map is one-to-one, while the right plot shows the resonant example we are considering, with $\mathfrak{n}_{0}=(1,1)$ and where the projection map is now many-to-one. It is clear that when considering multi-instanton sectors, the approach to resonance creates new phenomena implying new non-trivial relations between Stokes vectors and Borel residues.

Perhaps one of the simplest possible examples to consider ${ }^{128}$ is when $k=2$ and the instanton actions are such that $\boldsymbol{A}=(A,-A)$, with $A \in \mathbb{R}^{+}$. In this case, the group of action correlations is basically $\{(n, n),+\}$, with $n \in \mathbb{Z}$, and it is thus generated by $\mathfrak{n}_{0}=(1,1)$ (i.e., there is a single independent correlation between the actions). Let us analyze how the transseries and its resurgence relations are affected, as compared to the discussion in section 5 . The first change occurs when considering the projection map (5.19), as now $\operatorname{ker} \mathfrak{P}=\mathcal{L}\left\{\mathfrak{n}_{i}\right\}$ is a $r$-dimensional linear space, and the projection is no longer one-to-one. What will happen to Stokes vectors and Borel residues in this case? Clearly, Stokes vectors remain unchanged as long as we think of them as living on alien-lattice sites. But their projection into Borel residues must necessarily change. Let us illustrate how this occurs remaining within the "Painlevé-like" example and focusing on the perturbative and $(3,2)$ transseries-nodes of figures 17 and 18, as usual. In our present example, the projection (5.19) results on a line on the complex Borel plane; more specifically its real axis. In figures 30 and 31 we illustrate the corresponding alien lattices (and their projections) for perturbative and $(3,2)$ transseries-nodes, both at resonance and slightly way from resonance (where we perturb our present example with a small irrational non-resonant content). It is clear how nothing significant really changes for the perturbative sector, but how the projection is now many-to-one for the instanton sectors. In fact, whenever they exist, lattice nodes of the form $\boldsymbol{n}+\mathcal{L}_{\mathbb{Z}}\left\{\mathfrak{n}_{0}\right\}$ are all projected to the same point. It is this new aspect that changes the relations between Stokes vectors and Borel residues which were discussed at length in section 5 . In the following subsections, we shall discuss the exact nature of these required changes.

It is important to realize that resonance is a property of the particular equation we might be dealing with (e.g., the equations for partition function (6.16) and free energy (6.68) in the present section are resonant; while the equations for partition function (2.14) and free energy

\footnotetext{
${ }^{128}$ This is the relevant set-up for addressing resonance in both Painlevé I [130, 16] and Painlevé II [205] examples.
} 
(2.52) back in section 2 were not). Of course it is always possible to consider special solutions which themselves do not display resonance, e.g., solutions where a single instanton action has been turned on. Nonetheless, in order to construct the whole respective transseries, resonance must already have been taken into consideration. In fact, when using power-series and transseries to solve differential equations, the latter are turned into recursion equations for the coefficients in these power-series or transseries ansätze (see appendix D). At the level of these recursion equations, resonance then translates into the cancelation of the leading (corresponding to the unknown) coefficient term, at each step in the recursion. But if such a cancelation occurs, then the recursion is not solving for any unknown coefficients, and is thus not allowing for an iterative construction of the solution in the first place. One way around this problem is when resonance is "tunable", as in the example in the present section. In this case, one first solves the recursion for arbitrary values of the "tunable parameter" (which is $m$, in this case) and only sets for its resonant values at the very end (this was done in appendix D). Generically, however, resonance need not be "tunable". For instance, it may be associated to special integrability properties, as in the Painlevé-type examples addressed in [130, 16, 205]. In these cases, the aforementioned cancelation of unknown coefficients is simply telling us that the (power-series or transseries) solution ansatz we are using is inconsistent. Instead, it must be modified, in such a way that the resulting recursion will work again. For instance, in the Painlevé-type examples addressed in $[130,16,205]$, the transseries needed to be modified so as to include logarithmic sectors. Once this is done, the recursion equations get modified just enough as to no longer display cancelation of the unknown coefficients and, instead, allow for a fully iterative construction of the transseries solution. It should be clear that, in general, more intricate modifications of the transseries ansatz may be required, than the aforementioned simple inclusion of logarithms.

\subsection{Linear Resonant-Asymptotics: Partition Function}

Our simplest illustration of resonance occurs within the linear problem, i.e., when considering the case of the partition function of the elliptic potential. The alien lattice of this system is so simple (recall figure 23) that it is almost blind to any resonant effects - except in the case where $m=\frac{1}{2}$. For this value of the modulus, the manifestation of resonance actually becomes rather clear. The magnitude of positive and negative instanton actions becomes the same, $A_{1}=-A_{2} \equiv A=2$; with the associated alien lattice illustrated in figure 32. In particular, this causes all the odd $n$ terms in the perturbative expansion $Z_{n}^{(0)}$ to cancel, and the resulting resonant perturbative series is in even powers, $x^{2}$, alone (instead of all powers of $x$, as for any other value of $m$ ).

This can also be seen from the large-order behaviour of the perturbative coefficients in (6.60) (and illustrated in figure 33). Recall that, due to the modular symmetry (6.17), the coefficients in the partition-function nonperturbative sectors relate to each other as $Z_{n}^{(2)}(m)=(-1)^{n} Z_{n}^{(1)}\left(m^{\prime}\right)$. Thus, for $m=m^{\prime}=\frac{1}{2}$ and consequentially $A_{1}=-A_{2}$, one can immediately see that all the odd $n$ terms cancel in (6.60). This phenomenon is a straightforward consequence of the fact that both nonperturbative saddles contribute to the large-order growth of the perturbative series with the same magnitude but with symmetric values (i.e., the contribution of saddle (2) is alternating while that of saddle (1) is not). This leads to the "destructive interference" which causes all odd terms in the perturbative series to vanish. Note that this is an extremely simple yet quite important phenomenon: given an asymptotic perturbative-series in even powers of the coupling $x^{2}$, one might naïvely assume that its associated nonperturbative terms should be of the form $\sim \exp \left(-A / x^{2}\right)$. However, our example illustrates how this need not be the case. When our system is resonant, nonperturbative terms of the form $\sim \exp (-A / x)$ do lead to perturbative series in even powers $x^{2}$. For example, these resonance phenomena occur in 


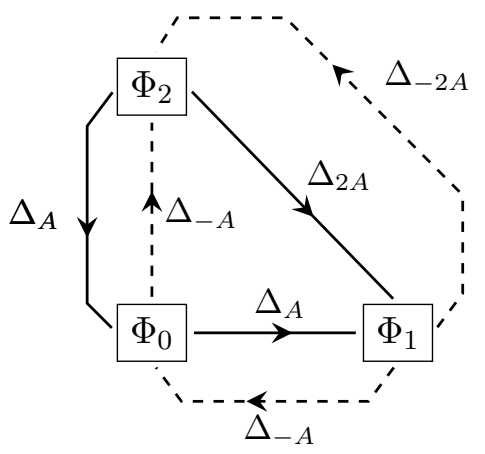

Figure 32. Algebraic structure associated to the behaviour of Borel transforms or alien derivatives for the $\Phi_{i}$ sectors, at their respective singularities, for the resonant case with $m=\frac{1}{2}$. Motions fall along the real axis on the Borel plane: solid lines correspond to motions along the $\theta=0$ direction; dashed lines along $\theta=\pi$. The steps which connect either $\Phi_{1}$ or $\Phi_{2}$ to the perturbative sector are of equal size, $A=2$, which implies that their singularities are equidistant to the origin on the Borel plane.

the asymptotics of Painlevé I and Painlevé II equations [130, 16, 205], describing non-critical strings; as well as in topological string theory [195, 77, 78, 76]. This seems to point to the fact that resonance might play a central role in the asymptotics of string theory, as it explains how open and closed string sectors play with each other at large order ${ }^{129}$ : the closed-string perturbative expansion is asymptotic, in powers of string-coupling-squared, but, via resonance, its large-order behaviour is still controlled by D-branes (open strings) with nonperturbative weight $\sim \exp (-1 /$ string-coupling $)[210,199]$.

\subsection{First Steps Towards Nonlinear Resonant Analysis}

Having discussed the simpler aspects of linear resonance, let us turn to resonance within our freeenergy nonlinear problem. Without surprise, the nonlinear case will turn out to be much richer than the linear one. Let us first recall that our two non-zero instanton actions, $A_{1}=\frac{1}{1-m}>0$ and $A_{2}=-\frac{1}{m}<0$, depend on a modulus $m \in(0,1)$. This implies, as per definition (6.151), that these actions are resonant for any rational value of $m$. In fact, given any $m \in \mathbb{Q}$, one can always find a non-vanishing $\boldsymbol{n}=\left(n_{1}, n_{2}\right) \in \mathbb{Z}^{2}$ satisfying $n_{1} / n_{2}=(1-m) / m$, which will thus imply $\boldsymbol{n} \cdot \boldsymbol{A}=0$. For concreteness, let us tune $m$ to a particular fixed resonant value for the remainder of this section; say:

$$
m=\frac{1}{3} .
$$

With this choice of $m$, we now have an explicit realization of a resonant transseries, with two rationally dependent instanton actions,

$$
A_{1}=\frac{3}{2} \equiv A \quad \text { and } \quad A_{2}=-2 A=-3,
$$

defining the action-vector $\boldsymbol{A}=(A,-2 A)$. Following the discussion in subsection 6.7, in this case the group of action correlations is generated by $\mathfrak{n}_{0}=(2,1)$ (there is a single independent correlation between the actions), and the kernel of the projection map (5.19) is thus given by

$$
\operatorname{ker} \mathfrak{P}=\mathcal{L}\left\{\mathfrak{n}_{0}\right\} .
$$

\footnotetext{
${ }^{129} \mathrm{~A}$ more technical discussion of this point may be found in subsection 4.3 of [16].
} 


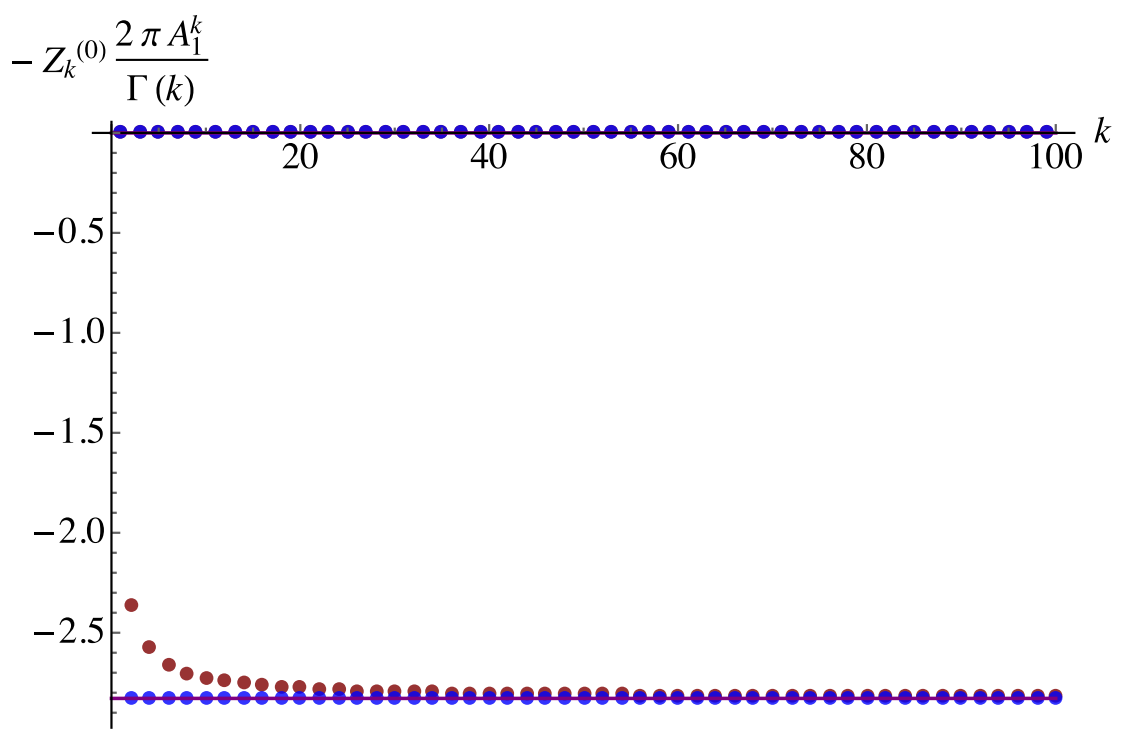

Figure 33. Illustration of the asymptotic large-order behaviour of the perturbative-series coefficients, $Z_{k}^{(0)}$; via (6.60) for the resonant value $m=1 / 2$. In red we plot the original weighted sequence $-Z_{k}^{(0)} \frac{2 \pi A_{1}^{k}}{\Gamma(k)}$, while in blue we plot its corresponding 5-RT. The solid purple lines denote the constant values to which these sequences converge, $2 \mathrm{i} Z_{0}^{(1)} \pm 2 \mathrm{i} Z_{0}^{(2)}$. For odd values of $k$ the coefficients vanish as a result of resonance since $Z_{0}^{(1)}=Z_{0}^{(2)}$; for even values of $k$ the sequence converges to $-2 \sqrt{2}$ as expected.

One canonical choice we shall use for these generators is simply to pick the "smallest" ones, i.e., a basis of the kernel whose vector-entries are the smallest-possible non-negative integers.

As discussed in section 5, generic transseries $\mathcal{T}$ are defined as ordered formal-sums of transmonomials. For a $k$-dimensional transseries with exponential transmonomials (5.17) this ordering is simply organized according to the respective exponential weights (either growing or decaying). The arguments of these ordered exponential-transmonomials then determine the distinct locations of Borel singularities, leading - upon projection (5.19) - to the singular structure (5.20). But for resonant systems - and as explained in subsection 6.7; in particular illustrated in figure 31-many different alien-lattice nodes may be associated to the same exponential weight and thus project to the same point on the Borel plane, contributing simultaneously. We shall thus need to reorganize the non-resonant transseries ordering in (5.17) in order to better understand how does resonance change the singular structure encoded in (5.20) (and, eventually, how does it change the resulting resurgence relations, (5.21) or (5.25)).

Consider two distinct nonperturbative sectors, $\boldsymbol{n}$ and $\boldsymbol{n}^{\prime}$, which differ by an element in the projection kernel (6.154), i.e.,

$$
\boldsymbol{n}-\boldsymbol{n}^{\prime} \in \operatorname{ker} \mathfrak{P} \text {. }
$$

Both these sectors will have the same exponential grading in the transseries, and, in fact, generically there will be infinitely many sectors in these conditions. The required reordering of the transseries should then be naturally organized according to the equivalence classes in the resulting quotient defined by (6.155), i.e., in $\mathbb{N}^{2} /$ ker $\mathfrak{P}$. In our on-going example, there is an infinite number of such equivalence classes which may be neatly organized into four basic families: the "perturbative" contribution $\mathbb{F}_{0}:=[(0,0)]$, the "positive instanton" family $\mathbb{F}_{+}(n):=[(n, 0)]$, the "negative-even instanton" family $\mathbb{F}_{-, \mathrm{e}}(n):=[(0, n)]$, and the "negative-odd instanton" family 
$\mathbb{F}_{-, \mathrm{o}}(n):=[(1, n)]$. The exponential grading associated to this decomposition is, respectively, 0 , $n A,-2 n A$, and $-(2 n-1) A$ (herein we are always considering $\left.n \in \mathbb{N}^{+}\right)$. The choice of representative element $\boldsymbol{\rho}(n) \in \mathbb{N}^{2} /$ ker $\mathfrak{P}$ for each family follows our canonical choice of picking the "smallest" vectors, i.e., the ones whose entries are the smallest-possible non-negative integers. In this way, any transseries alien-lattice node $\boldsymbol{n} \in \mathbb{Z}^{2}$ can be decomposed as the sum of a representative in one of the four families $\boldsymbol{\rho}_{\alpha}(n) \in \mathbb{F}_{\alpha}(n)$ together with an element of the kernel of the form $\mathcal{L}_{\mathbb{N}^{+}}\left\{\mathfrak{n}_{0}\right\}$. The end result is that the resonant free-energy transseries may be finally reorganized $^{130}$ as (compare with (6.73))

$$
F\left(x, \sigma_{0}, \boldsymbol{\sigma}\right)=\sigma_{0} \widetilde{\Phi}_{(0,0)}+\sum_{\boldsymbol{\rho} \in \mathbb{N}^{2} / \operatorname{ker} \mathfrak{P}} \mathrm{e}^{-\frac{\rho \cdot \boldsymbol{A}}{x}} \sum_{\ell=0}^{+\infty} \boldsymbol{\sigma}^{\boldsymbol{\rho}+\ell \mathfrak{n}_{0}} \Phi_{\boldsymbol{\rho}+\ell \mathfrak{n}_{0}}(x) .
$$

This reordering has implications for the large-order behaviour, as we shall see in subsection 6.11.

The $\Phi_{\boldsymbol{\rho}+\ell \mathfrak{n}_{0}}$ sectors in (6.156) are labeled by an element $\boldsymbol{\rho}$ in the quotient $\mathbb{N}^{2} / \operatorname{ker} \mathfrak{P}$ plus an element in the positive-integer kernel $\mathcal{L}_{\mathbb{N}^{+}}\left\{\mathfrak{n}_{0}\right\}$, and thus continue living on the original 2dimensional (semi-positive) lattice. But if their Borel singularities will still be determined by the argument of their corresponding exponential-transmonomials, then their locations are at $s=\boldsymbol{\rho} \cdot \boldsymbol{A}$. These locations only depend on $\boldsymbol{\rho} \in \mathbb{N}^{2} /$ ker $\mathfrak{P}$, in which case the whole (integer) kernel $\mathcal{L}\left\{\mathfrak{n}_{0}\right\}$ must thus contribute in the same way, at the same point. As such, the natural resonant generalization of $(5.20)$ becomes

$$
\left.\mathcal{B}\left[\Phi_{\boldsymbol{n}}\right](s)\right|_{s=\boldsymbol{\rho} \cdot \boldsymbol{A}}=\left\{\sum_{\ell \in \mathbb{Z}} S_{\boldsymbol{n} \rightarrow \boldsymbol{n}+\boldsymbol{\rho}+\ell \mathfrak{n}_{0}} \times \mathcal{B}\left[\Phi_{\boldsymbol{n}+\boldsymbol{\rho}+\ell \mathfrak{n}_{0}}\right](s-\boldsymbol{\rho} \cdot \boldsymbol{A})\right\} \frac{\log (s-\boldsymbol{\rho} \cdot \boldsymbol{A})}{2 \pi \mathrm{i}}, \quad \boldsymbol{\rho} \neq \mathbf{0} .
$$

Notice, however, that the above sum generically truncates and becomes finite, as most $k$-orthants are empty of Stokes vectors (one might recall the discussion in section 5, but this is actually very clearly illustrated in figure 31). For our precise transseries (6.156), the structure of Borel singularities is slightly more intricate than just (6.157), and one finds (compare with (6.74)):

$$
\mathcal{B}\left[\Phi_{\boldsymbol{n}}\right](s+\boldsymbol{\rho} \cdot \boldsymbol{A})=\sum_{\left\{\boldsymbol{\ell} \in \mathbb{Z}^{2} \mid \boldsymbol{\ell}=\boldsymbol{\rho}+\operatorname{ker} \mathfrak{P}\right\}}\left(\mathrm{S}_{\boldsymbol{n} \rightarrow \boldsymbol{n}+\boldsymbol{\ell}} \times \mathcal{B}\left[\Phi_{\boldsymbol{n}+\boldsymbol{\ell}}\right](s)+\delta_{\boldsymbol{n}+\boldsymbol{\ell}} \mathrm{S}_{\boldsymbol{n} \rightarrow \widetilde{\mathbf{0}}} \times \mathcal{B}\left[\widetilde{\Phi}_{(0,0)}\right](s)\right) \frac{\log s}{2 \pi \mathrm{i}} .
$$

Addressing the familiar alien-derivative algebraic level of abstraction is by-now straightforward. Let us first readdress the arguments which led to (6.156) and (6.158) from the standpoint of (alien-derivative induced) motions on the alien lattice. Due to resonance, the infinitely many nodes which lie along each foliation of the $\mathbb{Z}^{2}$-lattice by the action correlation $\mathfrak{n}_{0}$ have the exact same exponential grading - these are just the equivalence classes (6.155). As such, the alien derivatives should induce no motions whatsoever along these directions, i.e., if $\boldsymbol{n}-\boldsymbol{n}^{\prime} \in \operatorname{ker} \mathfrak{P}$ then

$$
\Delta_{n \cdot \boldsymbol{A}}=\Delta_{n^{\prime} \cdot \boldsymbol{A}}
$$

All one is left with are motions in-between distinct equivalence classes, which in this case imply that the original two-dimensional lattice effectively collapses to an one-dimensional alien chain. The resulting one-dimensional forward or backward motions, jumping from equivalence class to equivalence class, are organized by our aforementioned four basic families, $\mathbb{F}_{\alpha}(n)$. In particular,

\footnotetext{
${ }^{130}$ Although, as we shall see next, this transseries reordering is key to understanding the resonant structure of Borel singularities, it need not be the most efficient way to do resonant-transseries analytic resummation; see [17].
} 
nodes in the "perturbative" contribution $\mathbb{F}_{0}$ project to the origin of the Borel plane and must have vanishing alien derivative, i.e., are associated with no motions on the alien chain. On the other hand, vectors in the "positive instanton" family $\mathbb{F}_{+}(n)$ will induce forward motions, while vectors in both "negative instanton" families, even $\mathbb{F}_{-, \mathrm{e}}(n)$ and odd $\mathbb{F}_{-, \mathrm{o}}(n)$, will induce all possible backward motions. Furthermore, all our steps have the exact same size, given by $A$. On the Borel plane, singularities are thus located at positions $s=n A$, with $n \in \mathbb{Z} \backslash\{0\}$; falling upon both positive $(n>0)$ and negative $(n<0)$ real axes. One would thus be tempted to think that the alien derivative should be only labeled by a non-zero integer $n$, as $\Delta_{n A}$, and equivalent to the one-dimensional alien chain case (2.64). But this problem is more intricate than that one-dimensional case. In fact, the one subtlety still to take into consideration is that the many different nodes on the two-dimensional alien lattice belonging to the same equivalence class have ended-up being projected into the exact same position on the Borel plane. This infinite degeneracy of each step, and which is the hallmark of resonance, must still be present somewhere - and the information about this resonant degeneracy precisely appears at the level of a rearrangement of the Stokes data, much like in (6.158). The resonant resurgence relations generalizing (6.75) are then given by

$$
\Delta_{\boldsymbol{\rho} \cdot \boldsymbol{A}} \Phi_{\boldsymbol{n}}=\sum_{\left\{\boldsymbol{\ell} \in \mathbb{Z}^{2} \mid \ell=\boldsymbol{\rho}+\mathrm{ker} \mathfrak{P}\right\}}\left(\boldsymbol{S}_{\boldsymbol{\ell}} \cdot(\boldsymbol{n}+\boldsymbol{\ell}) \Phi_{\boldsymbol{n}+\boldsymbol{\ell}}+\delta_{\boldsymbol{n}+\boldsymbol{\ell}} S_{\boldsymbol{\ell}}^{(0)} \widetilde{\Phi}_{(0,0)}\right) .
$$

In this expression, $\boldsymbol{\rho}$ is a (canonical) representative of one of the equivalence classes, and elements in the right-hand side vanish whenever $\boldsymbol{n}+\boldsymbol{\ell}$ has a negative entry. Moreover, the above sum further truncates (and is in fact generically finite) as most $k$-orthants are empty of Stokes vectors. The Stokes coefficients obey the same conditions as in the non-resonant case, (6.76) through (6.79), which we reproduce in here for completeness:

$$
\begin{array}{ll}
S_{0}^{(i)}=0, & \text { for } i=0,1,2, \\
S_{\ell}^{(0)}=0, & \text { if any } \ell_{i}>0, \\
S_{\ell}^{(1)}=0, & \text { if } \ell_{1}>1 \text { or } \ell_{2}>0, \\
S_{\ell}^{(2)}=0, & \text { if } \ell_{1}>0 \text { or } \ell_{2}>1 .
\end{array}
$$

The resonant alien lattice where the alien derivative (6.160) induces different motions is illustrated in figure 34 (it describes the action of $\Delta_{\boldsymbol{\ell} \cdot \boldsymbol{A}}$ on the same $(3,2)$-sector of figures 17 and 26). It is particularly illuminating to compare the present resonant case of figure 34 to the exact same but non-resonant problem of figure 26. As usual, solid lines indicate motions associated to singularities which lie on the positive real axis of the Borel plane, while dashed lines indicate motions associated to singularities which fall upon the negative real axis. Throughout, we have been labelling weights $w$ with subscript $s=\boldsymbol{\ell} \cdot \boldsymbol{A}$, but in the resonant case this is no longer enough. It is simple to see from figure 34 how weights with this very same label organize according to each foliation of the alien-lattice by $\mathfrak{n}_{0}=(2,1)$. In particular the foliation associated to the $(3,2)$-node itself cannot have any associated motions (it projects to the origin on the Borel plane). In the figure this includes nodes $(1,1)$ and $(5,3)$, which are "dotted out". But along other foliations, the label is always the same although the weight might be distinct; depending on the end-nodes according to (6.160). This is simply solved by adding one extra label as in ${ }^{131}$ $\boldsymbol{\ell}=\boldsymbol{\rho}+\kappa \mathfrak{n}_{0}$ and $w_{\boldsymbol{\ell} \cdot \boldsymbol{A}} \equiv w_{\boldsymbol{\rho} \cdot \boldsymbol{A}, \kappa}$ (for instance, the (3,2)-node itself is in this way decomposed as

\footnotetext{
${ }^{131}$ Note that when addressing the $\mathbb{Z}^{2}$ lattice rather than the $\mathbb{N}^{2}$ lattice, i.e., when addressing motions starting-off
} 
$\left.(3,2)=(1,1)+\mathfrak{n}_{0}\right)$. In this set-up, the "statistical mechanical" rules for Stokes data are basically the same as in the non-resonant case starting in (6.82) and (6.83). The weight $w$ associated to the step $\mathcal{S}$ connecting lattice sites $\boldsymbol{n}$ and $\boldsymbol{n}+\boldsymbol{\ell}$ is given by the standard expressions

$$
w(\mathcal{S}(\boldsymbol{n} \rightarrow \boldsymbol{n}+\boldsymbol{\ell}))=(\boldsymbol{n}+\boldsymbol{\ell}) \cdot \boldsymbol{S}_{\boldsymbol{\ell}}
$$

and

$$
w(\widetilde{\mathcal{S}}(\boldsymbol{n} \rightarrow \mathbf{0}))=S_{-\boldsymbol{n}}^{(0)} .
$$

These weights are shown in figure 34, where the departing node is $\boldsymbol{n}=(3,2)$, and where we use the notation explained above, i.e., $w(\mathcal{S}(\boldsymbol{n} \rightarrow \boldsymbol{n}+\boldsymbol{\ell}))) \equiv w_{\boldsymbol{\rho} \cdot \boldsymbol{A}, \kappa}$.

Equipped with the (resonant) resurgence relations (6.160) and their geometrical picture, and with the associated "statistical mechanical" language essentially unchanged from what was used in subsection 6.4, we may now turn to the discussion of resonant Stokes discontinuities. Let us start from definition (2.33). For the discontinuity at $\theta=0$, the relevant singularities on the Borel plane are those which lie along the positive real axis, i.e., one needs to consider the forward steps generated by $\Delta_{n A}$ with $n>0$. For the discontinuity at $\theta=\pi$, we instead need to consider all the backward steps generated by $\Delta_{-n A}$ with $n>0$. This leads to the standard picture where these discontinuities arise as sums over all paths $\mathcal{P}$ which connect a given fixed sector $\Phi_{n}$ to any other possible sectors $\Phi_{\boldsymbol{n}^{\prime}}$ such that $\left(\boldsymbol{n}^{\prime}-\boldsymbol{n}\right) \cdot \boldsymbol{A}= \pm n A$; positive or negative for either Disc 0 or Disc ${ }_{\pi}$, respectively. Each such path $\mathcal{P}$ is a collection of steps $\mathcal{S}_{i}$, where in this case each step is associated to a jump $\pm n_{i} A$, such that $\sum_{i} n_{i}=n$. Further, each path $\mathcal{P}$ contributes with an associated weight, given by (6.86), and an associated combinatorial factor, given by (6.87). For the perturbative sector, it is very easy to check that we get the following discontinuities:

$$
\begin{aligned}
& \operatorname{Disc}_{0} \Phi_{(0,0)}=-\sum_{n=1}^{+\infty}\left(S_{e_{1}}^{(1)}\right)^{n} \mathrm{e}^{-\frac{n A}{x}} \Phi_{(n, 0)} \\
& \operatorname{Disc}_{\pi} \Phi_{(0,0)}=-\sum_{n=1}^{+\infty}\left(S_{e_{2}}^{(2)}\right)^{n} \mathrm{e}^{\frac{2 n A}{x}} \Phi_{(0, n)} .
\end{aligned}
$$

These are none others than (6.88) and (6.89), once we appropriatly select $A_{1}$ and $A_{2}$. No surprise.

For a more non-trivial comparison with the non-resonant case, addressed earlier in subsection 6.4, let us next work out the $\Phi_{(0,1)}$ sector. The resurgence relations (6.160) for this sector read

$$
\begin{aligned}
\Delta_{A} \Phi_{(0,1)} & =S_{\boldsymbol{e}_{1}}^{(1)} \Phi_{(1,1)}, \\
\Delta_{-2 A} \Phi_{(0,1)} & =2 S_{\boldsymbol{e}_{2}}^{(2)} \Phi_{(0,2)}, \\
\Delta_{2 A} \Phi_{(0,1)} & =S_{-\boldsymbol{e}_{2}}^{(0)} \widetilde{\Phi}_{(0,0)}, \\
\Delta_{3 A} \Phi_{(0,1)} & =S_{\boldsymbol{e}_{1}-\boldsymbol{e}_{2}}^{(1)} \Phi_{(1,0)} .
\end{aligned}
$$

These are all the allowed single-step motions starting-out on sector $\Phi_{(0,1)}$. Up to now, other than appropriate selecting $A_{1}$ and $A_{2}$ there are still no novelties due to resonance. The discontinuities

at some transseries node $\boldsymbol{n}$ rather than the transseries itself which starts at $\mathbf{0}$, our canonical choice for $\boldsymbol{\rho}$ needs to be adapted (so as to remain in the same "minimal" spirit). This choice is now the vector with the most negative entries, such that it still ends-up in an allowed (i.e., non-vanishing) node. 


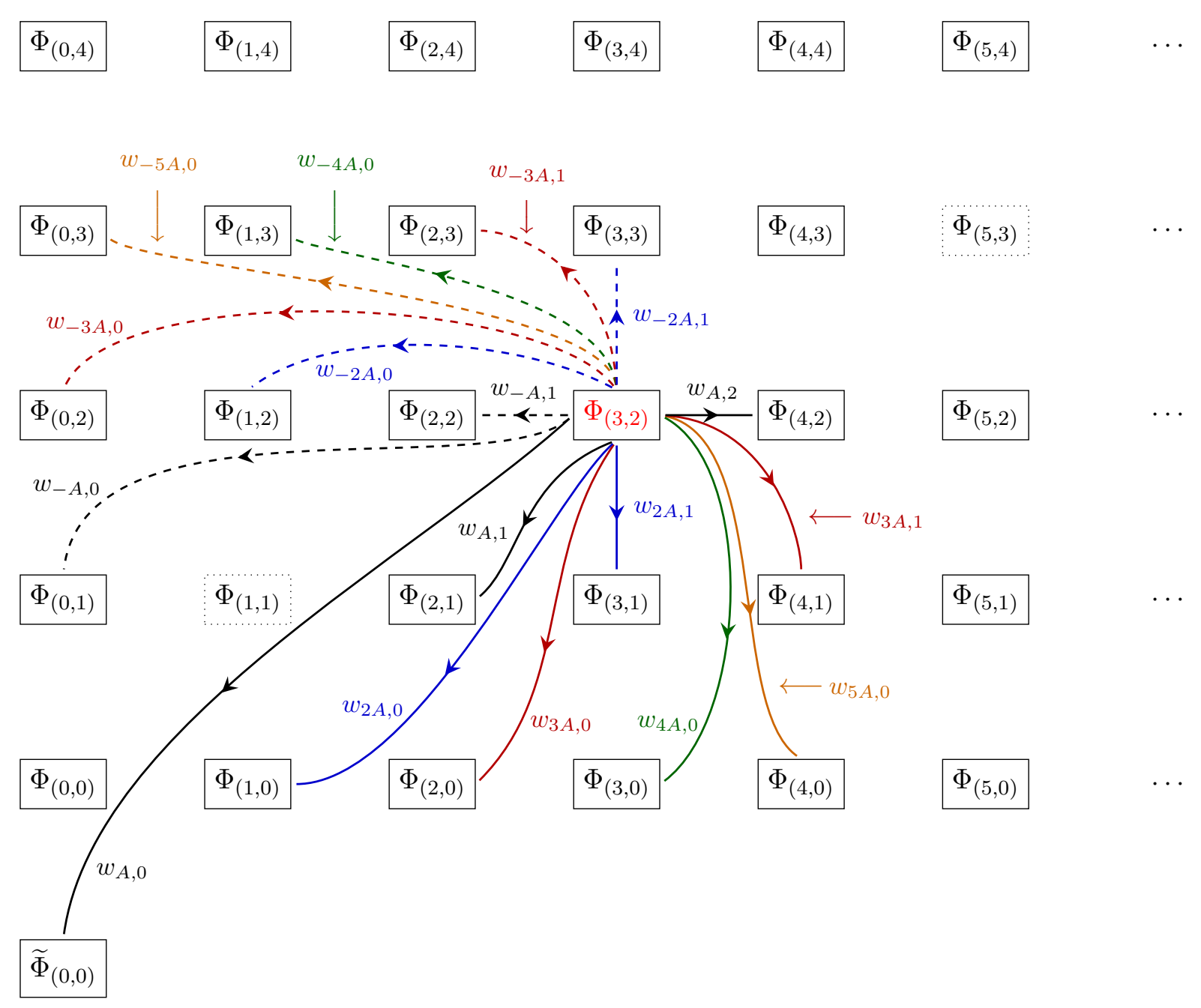

Figure 34. The resonant alien lattice: a pictorial representation of the action of the resonant alien derivative upon the $(3,2)$-sector (compare with the non-resonant figure 26). Different single arrows correspond to different single steps, where the weight of each step is dictated by the right-hand-side of the resonant resurgence relations (6.160) (and where we are using the $\boldsymbol{w}_{\boldsymbol{\rho} \cdot \boldsymbol{A}, \kappa}$ labelling explained in the text). Solid and dashed lines represent steps with positive and negative actions, respectively. Different colors represent different magnitudes in exponential grading. In particular, note how equal colors are organized according to foliations of the lattice by the resonance $\mathfrak{n}_{0}=(2,1)$. For example, nodes $(1,1)$ and $(5,3)$ are in the same equivalence class of our $(3,2)$-sector (they have been singled-out by their dotted squares), and project to the origin of the Borel plane. As a result, there are no steps between them or with anyone else. The steps shown are all the single steps which are allowed starting at the $\Phi_{(3,2)}$ lattice-site (and where paths can then be constructed with different middle nodes, and corresponding products of weights). 
for $\theta=0, \pi$ follow from definition, (2.33) and (2.45). Let us start with the $\theta=0$ discontinuity,

$$
\begin{aligned}
& \operatorname{Disc}_{0} \Phi_{(0,1)}=\left\{\boldsymbol{1}-\exp \left(\sum_{n=1}^{+\infty} \mathrm{e}^{-\frac{n A}{x}} \Delta_{n A}\right)\right\} \Phi_{(0,1)}= \\
& =-\left(\mathrm{e}^{-\frac{A}{x}} \Delta_{A}+\mathrm{e}^{-\frac{2 A}{x}} \Delta_{2 A}+\frac{1}{2 !}\left(\mathrm{e}^{-\frac{A}{x}} \Delta_{A}\right)^{2}\right) \Phi_{(0,1)}+\mathcal{O}\left(\mathrm{e}^{-3 A / x}\right) .
\end{aligned}
$$

Having seen how the single-step motions bear no novelties due to resonance, let us consider multi-step paths. Up to the exponential order we are considering, there is a single factor, $\left(\Delta_{A}\right)^{2}$, associated to such a multi-step path, as can be seen in the expression above. Let us compare its evaluation in both resonant and non-resonant scenarios. One begins with (6.169), which is the same is both cases (6.75) and (6.160) (up to the appropriate identification of $A_{1}=A$ ),

$$
\begin{aligned}
\Delta_{A} \Phi_{(0,1)} & =S_{\boldsymbol{e}_{1}}^{(1)} \Phi_{(1,1)}, & \text { (resonant) } \\
\Delta_{A_{1}} \Phi_{(0,1)} & =S_{\boldsymbol{e}_{1}}^{(1)} \Phi_{(1,1)} . & \text { (non-resonant) }
\end{aligned}
$$

Resonance then manifests itself when considering the second alien-derivative, as

$$
\begin{aligned}
\Delta_{A} \Phi_{(1,1)} & =2 S_{\boldsymbol{e}_{1}}^{(1)} \Phi_{(2,1)}+S_{-\boldsymbol{e}_{1}-\boldsymbol{e}_{2}}^{(0)} \widetilde{\Phi}_{(0,0)}, & \text { (resonant) } \\
\Delta_{A_{1}} \Phi_{(1,1)} & =2 S_{\boldsymbol{e}_{1}}^{(1)} \Phi_{(2,1)} . & \text { (non-resonant) }
\end{aligned}
$$

In the resonant case it is clear how the $\Phi_{(2,1)}$ and $\widetilde{\Phi}_{(0,0)}$ sectors fall upon the same equivalence class, and thus both appear in the right-hand side of (6.160). But in the non-resonant case there seems to be no trace of the $\widetilde{\Phi}_{(0,0)}$ contribution, even after the appropriate identification of $A_{1}=A$. Of course even in the non-resonant case one may reach $\widetilde{\Phi}_{(0,0)}$ out of $\Phi_{(1,1)}$, only with a different motion:

$$
\Delta_{-A_{1}-A_{2}} \Phi_{(1,1)}=S_{-\boldsymbol{e}_{1}-\boldsymbol{e}_{2}}^{(0)} \widetilde{\Phi}_{(0,0)} \quad \text { (non-resonant) }
$$

This apparent mismatch is now made clear: upon appropriately selecting $A_{1}=A$ and $A_{2}=-2 A$, then also $-A_{1}-A_{2}=A$ and the two non-resonant contributions are joint together upon resonance (as it happens!). The resonant resurgence relations (6.160) precisely take this into account.

Having understood the different resonant contributions, alongside their comparison to the earlier non-resonant case, let us proceed with the discontinuity $\operatorname{Disc}_{0} \Phi_{(0,1)}$. At leading exponential order, $\mathrm{e}^{-\frac{A}{x}}$, only one sector may be reached, and this occurs via the single forward step generated by $\Delta_{A}$. This is $\Phi_{(1,1)}$ as in (6.169), corresponding to the step $\mathcal{S}((0,1) \rightarrow(1,1))$. The corresponding path has length $\ell=1$, weight $w=S_{\boldsymbol{e}_{1}}^{(1)}$, and combinatorial factor $\mathrm{CF}=\frac{1}{1 !}$. This resulting resonant-contribution is essentially the same as in the non-resonant case (6.90). At next-to-leading exponential order, $\mathrm{e}^{-\frac{2 A}{x}}$, there are two sectors within reach: $\Phi_{(2,1)}$ and $\widetilde{\Phi}_{(0,0)}$, as discussed above. The first of these, sector $\Phi_{(2,1)}$, is reached via the two-step path $\Phi_{(0,1)} \rightarrow \Phi_{(1,1)} \rightarrow \Phi_{(2,1)}$ (with length $\ell=2$ ), which is generated by the $\left(\Delta_{A}\right)^{2}$ factor via $(6.174)$ and (6.176), has weight $w=2\left(S_{\boldsymbol{e}_{1}}^{(1)}\right)^{2}$, and combinatorial factor $\mathrm{CF}=\frac{1}{2 !}$. Again, the resulting resonant-contribution is essentially the same as in the non-resonant case (6.90). As to the second sector, $\widetilde{\Phi}_{(0,0)}$, it is reached by two distinct paths: a single step path (length $\left.\ell=1\right) \Phi_{(0,1)} \rightarrow \widetilde{\Phi}_{(0,0)}$, 
generated by $\Delta_{2 A}$ via (6.171), with weight $w=S_{-\boldsymbol{e}_{2}}^{(0)}$ and combinatorial factor $\mathrm{CF}=\frac{1}{1 !}$; and a two-step path $\Phi_{(0,1)} \rightarrow \Phi_{(1,1)} \rightarrow \widetilde{\Phi}_{(0,0)}$ (with length $\ell=2$ ), which is generated by the $\left(\Delta_{A}\right)^{2}$ factor via the very same (6.174) and (6.176) which led us to $\Phi_{(2,1)}$, with weight $w=S_{\boldsymbol{e}_{1}}^{(1)} S_{-\boldsymbol{e}_{1}-\boldsymbol{e}_{2}}^{(0)}$ and combinatorial factor $\mathrm{CF}=\frac{1}{2 !}$. As should be now clear, its resulting resonant-contribution is essentially the same as in the non-resonant case (6.90), but it was now reached with just the two $\Delta_{A}$ motions (6.174) and (6.176), while the non-resonant case further required a distinct motion (6.178). This is a straightforward consequence of the resonant reorganization (6.160). All in all, this results in the discontinuity

$$
\begin{aligned}
\operatorname{Disc}_{0} \Phi_{(0,1)} & =-S_{\boldsymbol{e}_{1}}^{(1)} \mathrm{e}^{-\frac{A}{x}} \Phi_{(1,1)}-\left(S_{\boldsymbol{e}_{1}}^{(1)}\right)^{2} \mathrm{e}^{-\frac{2 A}{x}} \Phi_{(2,1)}-\left(S_{-\boldsymbol{e}_{2}}^{(0)}+\frac{1}{2} S_{\boldsymbol{e}_{1}}^{(1)} S_{-\boldsymbol{e}_{1}-\boldsymbol{e}_{2}}^{(0)}\right) \mathrm{e}^{-\frac{2 A}{x}} \widetilde{\Phi}_{(0,0)}-\cdots \\
& =\mathrm{S}_{(0,1) \rightarrow(1,1)} \mathrm{e}^{-\frac{A}{x}} \Phi_{(1,1)}+\mathrm{e}^{-\frac{2 A}{x}}\left(\mathrm{~S}_{(0,1) \rightarrow(2,1)} \Phi_{(2,1)}+\mathrm{S}_{(0,1) \rightarrow \widetilde{\mathbf{0}}} \widetilde{\Phi}_{(0,0)}\right)+\cdots
\end{aligned}
$$

Comparing with (6.90), we see that upon identification of the actions $A_{1}$ and $A_{2}$ the result is the same. The difference lies in the resonant organization of the alien-derivative-induced motions (6.160), which made us arrive at the same result via a different route. Nevertheless, because of the resonant structure of the actions, this result will have a very clear impact on the large-order behaviour, as we shall see in a subsequent subsection.

The discontinuity at $\theta=\pi$ follows via a completely similar reasoning (compare with (6.92))

$$
\begin{aligned}
\operatorname{Disc}_{\pi} \Phi_{(0,1)} & =-2 S_{\boldsymbol{e}_{2}}^{(2)} \mathrm{e}^{\frac{2 A}{x}} \Phi_{(0,2)}-3\left(S_{\boldsymbol{e}_{2}}^{(2)}\right)^{2} \mathrm{e}^{\frac{4 A}{x}} \Phi_{(0,3)}-4\left(S_{\boldsymbol{e}_{2}}^{(2)}\right)^{3} \mathrm{e}^{\frac{6 A}{x}} \Phi_{(0,4)}-\cdots \\
& =\mathrm{S}_{(0,1) \rightarrow(0,2)} \mathrm{e}^{\frac{2 A}{x}} \Phi_{(0,2)}+\mathrm{S}_{(0,1) \rightarrow(0,3)} \mathrm{e}^{\frac{4 A}{x}} \Phi_{(0,3)}+\mathrm{S}_{(0,1) \rightarrow(0,4)} \mathrm{e}^{\frac{6 A}{x}} \Phi_{(0,4)}+\cdots
\end{aligned}
$$

Our final example in this subsection is, of course, to consider our favourite nonperturbative sector, namely, $\Phi_{(3,2)}$ (illustrated in figure 34 ). In particular, we shall sketch the resonant resurgence-relations based upon this sector, using (6.160), which would then allow us to compute the resonant versions of (6.93) and (6.97) (which we leave as a straightforward exercise for the reader). Along the $\theta=0$ direction, we find the contributions

$$
\begin{aligned}
\Delta_{A} \Phi_{(3,2)} & =w_{A, 0} \widetilde{\Phi}_{(0,0)}+w_{A, 1} \Phi_{(2,1)}+w_{A, 2} \Phi_{(4,2)}, \\
\Delta_{2 A} \Phi_{(3,2)} & =w_{2 A, 0} \Phi_{(1,0)}+w_{2 A, 1} \Phi_{(3,1)}, \\
\Delta_{3 A} \Phi_{(3,2)} & =w_{3 A, 0} \Phi_{(2,0)}+w_{3 A, 1} \Phi_{(4,1)}, \\
\Delta_{4 A} \Phi_{(3,2)} & =w_{4 A, 0} \Phi_{(3,0)}, \\
\Delta_{5 A} \Phi_{(3,2)} & =w_{5 A, 0} \Phi_{(4,0)} .
\end{aligned}
$$

These are all the allowed (forward) single-step motions starting out at our (3,2)-node, which are also represented with appropriate matching colors in figure 34. It is simple to see how the right-hand side of the above expressions is arranged according to the foliations of the alien-lattice by ker $\mathfrak{P}$. The values of the different weights in these equations are:

$$
\begin{array}{rlrl}
w_{A, 0} & =S_{(-3,-2)}^{(0)}, & w_{A, 1} & =2 S_{(-1,-1)}^{(1)}+S_{(-1,-1)}^{(2)}, \\
w_{2 A, 0}=S_{(-2,-2)}^{(1)}, & w_{2 A, 1}=3 S_{(0,-1)}^{(1)}+S_{(0,-1)}^{(2)}, & \\
w_{3 A, 0}=2 S_{(-1,-2)}^{(1)}, & w_{3 A, 1}=4 S_{(1,-1)}^{(1)}+S_{(1,-1)}^{(2)},
\end{array}
$$




$$
\begin{aligned}
& w_{4 A, 0}=3 S_{(0,-2)}^{(1)}, \\
& w_{5 A, 0}=4 S_{(1,-2)}^{(1)} .
\end{aligned}
$$

For completeness we also list all the allowed backward (along the $\theta=\pi$ direction) single-step motions starting out at our $(3,2)$-node, now represented by dashed lines in figure 34 . One finds:

$$
\begin{aligned}
\Delta_{-A} \Phi_{(3,2)} & =w_{-A, 0} \Phi_{(0,1)}+w_{-A, 1} \Phi_{(2,2)}, \\
\Delta_{-2 A} \Phi_{(3,2)} & =w_{-2 A, 0} \Phi_{(1,2)}+w_{-2 A, 1} \Phi_{(3,3)}, \\
\Delta_{-3 A} \Phi_{(3,2)} & =w_{-3 A, 0} \Phi_{(0,2)}+w_{-3 A, 1} \Phi_{(2,3)} \\
\Delta_{-4 A} \Phi_{(3,2)} & =w_{-4 A, 0} \Phi_{(1,3)}, \\
\Delta_{-5 A} \Phi_{(3,2)} & =w_{-5 A, 0} \Phi_{(0,3)},
\end{aligned}
$$

where the weights are given by

$$
\begin{array}{rlrl}
w_{-A, 0} & =S_{(-3,-1)}^{(2)}, & w_{-A, 1} & =2 S_{(-1,0)}^{(1)}+2 S_{(-1,0)}^{(2)}, \\
w_{-2 A, 0} & =S_{(-2,0)}^{(1)}+2 S_{(-2,0)}^{(2)}, & w_{-2 A, 1}=3 S_{(0,1)}^{(2)}, \\
w_{-3 A, 0}=2 S_{(-3,0)}^{(2)}, & w_{-3 A, 1}=2 S_{(-1,1)}^{(1)}+3 S_{(-1,1)}^{(2)}, \\
w_{-4 A, 0}=S_{(-2,1)}^{(1)}+3 S_{(-2,1)}^{(2)}, & \\
w_{-5 A, 0}=3 S_{(-3,1)}^{(2)} . &
\end{array}
$$

So far we have seen how resonance ended-up yielding a reorganization of transseries structures (6.156), Borel singularities (6.158), and, equivalently, alien-derivative resurgence relations (6.160). These apparently mild reorganizations will have dire consequences in the large-order behaviour, as we shall see in the following. But first, let us take our usual pause to consider the relation between partition-function and free-energy Stokes coefficients, which will be required for the numerical verifications in the upcoming asymptotic large-order checks.

\subsection{Stokes Constants of Partition Function versus Free Energy: Resonance}

In the present resonant case, Stokes data for the free energy may also be equated with that for the partition function. Indeed, such relations follow in the exact same way as in the non-resonant case, earlier addressed in subsection 6.5. This is essentially because the fact that the free-energy inherits the fundamental Borel singularities of the partition-function still holds in the presence of resonance. Given the similarity of the argument, we will not repeat the full calculation. Instead, we shall limit ourselves to pointing out the differences due to resonance, and then list the values for the free-energy Stokes coefficients as they relate to partition-function Stokes-data.

The analysis in subsection 6.5 is valid up to the determination of the Stokes automorphism along $\theta=0$ with respect to the free-energy transseries parameters $\tau_{i}$, starting in (6.112) (equivalently, along $\theta=\pi$, starting in (6.123)). As stated many times before, the bridge equations implement a bridge between alien-operators $\Delta$ and regular-derivatives $\partial$, which in the non-resonant case is implemented by the map (6.113). Applying the exact same such procedure to the present 
resonant case, this implies we now need to match (6.112) with ${ }^{132}$

$$
\mathrm{e}^{-\frac{n A}{x}} \Delta_{n A}=\sum_{\left\{\boldsymbol{\ell} \in \mathbb{Z}^{2} \mid \ell \cdot \boldsymbol{A}=n A\right\}}\left(S_{\ell}^{F(0)} \tau_{1}^{-\ell_{1}} \tau_{2}^{-\ell_{2}} \frac{\partial}{\partial \tau_{0}}+S_{\ell}^{F(1)} \tau_{1}^{1-\ell_{1}} \tau_{2}^{-\ell_{2}} \frac{\partial}{\partial \tau_{1}}+S_{\ell}^{F(2)} \tau_{1}^{-\ell_{1}} \tau_{2}^{1-\ell_{2}} \frac{\partial}{\partial \tau_{2}}\right) .
$$

The sum is over $\boldsymbol{\ell} \cdot \boldsymbol{A}=n A$ (where $n \in \mathbb{N}^{+}$for the $\theta=0$ direction), which implies that the vector-entries of $\ell=\left(\ell_{1}, \ell_{2}\right)$ obey the constraint $\ell_{1}=n+2 \ell_{2}$. The differential-operator in the right-hand side of (6.201) may thus be rewritten as ${ }^{133}$

$$
S_{\left(n+2 \ell_{2}, \ell_{2}\right)}^{F(0)} \tau_{1}^{-n-2 \ell_{2}} \tau_{2}^{-\ell_{2}} \frac{\partial}{\partial \tau_{0}}+S_{\left(n+2 \ell_{2}, \ell_{2}\right)}^{F(1)} \tau_{1}^{1-n-2 \ell_{2}} \tau_{2}^{-\ell_{2}} \frac{\partial}{\partial \tau_{1}}+S_{\left(n+2 \ell_{2}, \ell_{2}\right)}^{F(2)} \tau_{1}^{-n-2 \ell_{2}} \tau_{2}^{1-\ell_{2}} \frac{\partial}{\partial \tau_{2}} .
$$

The required match now easily follows. Using (6.201) with (6.202), and then comparing against (6.112) is quite straightforward. For instance, consider the terms which are proportional to $\frac{\partial}{\partial \tau_{0}}$. Their matching requires

$$
\sum_{n=1}^{+\infty} \sum_{\ell_{2}=-\infty}^{-\left\lfloor\frac{n}{2}\right\rfloor} S_{\left(n+2 \ell_{2}, \ell_{2}\right)}^{F(0)} \tau_{1}^{-n-2 \ell_{2}} \tau_{2}^{-\ell_{2}}=S_{\omega_{20}}^{Z} \tau_{2}
$$

This implies there is a single (free energy) non-zero Stokes coefficient, associated to the $\tau_{0}$-action. One must set $\ell_{2}=-1$-and subsequently $n=2$-in which case this Stokes coefficient is

$$
S_{(0,-1)}^{F(0)}=S_{\omega_{20}}^{Z} \quad(=-2) .
$$

As another example, let us consider the terms which are proportional to $\frac{\partial}{\partial \tau_{1}}$. Their matching now requires

$$
\sum_{n=1}^{+\infty} \sum_{\ell_{2}=-\infty}^{-\left\lfloor\frac{n-1}{2}\right\rfloor} S_{\left(n+2 \ell_{2}, \ell_{2}\right)}^{F(1)} \tau_{1}^{1-n-2 \ell_{2}} \tau_{2}^{-\ell_{2}}=S_{\omega_{01}}^{Z}+S_{\omega_{21}}^{Z} \tau_{2}-S_{\omega_{20}}^{Z} \tau_{1} \tau_{2}
$$

For example, if $\ell_{2}=0$ we must further have $n=1$ in which case one obtains the first term on the right-hand-side, resulting in $S_{(1,0)}^{F(1)}=S_{\omega_{01}}^{Z}$. Repeating the same procedure for the other terms in $(6.205)$, and for the matching associated to terms proportional to $\frac{\partial}{\partial \tau_{2}}$, yields:

$$
\begin{array}{cc}
S_{(1,0)}^{F(1)}=S_{\omega_{01}}^{Z} & (=2), \\
S_{(1,-1)}^{F(1)}=S_{\omega_{21}}^{Z} & (=0), \\
S_{(0,-1)}^{F(1)}=-S_{\omega_{20}}^{Z} & (=2), \\
S_{(0,-1)}^{F(2)}=-S_{\omega_{20}}^{Z} & (=2) .
\end{array}
$$

Interestingly enough, these are the exact same formulae as in subsection 6.5.

Having computed all non-vanishing Stokes coefficients associated to the free-energy Stokes discontinuities along $\theta=0$, let us address the same problem along $\theta=\pi$. The matching is now

\footnotetext{
${ }^{132}$ Recall that the entries $\ell_{i}$ are assumed to be such that all powers of the $\tau_{i}$ are non-negative.

${ }^{133}$ Where $\ell_{2}$ must still satisfy the $\tau_{i}$-regularity requirements, which are now $n$-dependent.
} 
done against (6.123), and the procedure is completely analogous to the one above (just recall that in $(6.201) n$ now is $n<0)$. The final result is:

$$
\begin{array}{cc}
S_{(0,1)}^{F(2)}=S_{\omega_{02}}^{Z} & (=2), \\
S_{(-1,1)}^{F(2)}=-S_{\omega_{12}}^{Z} & (=0), \\
S_{(-1,0)}^{F(0)}=S_{\omega_{10}}^{Z} & (=-2), \\
S_{(-1,0)}^{F(1)}=-S_{\omega_{10}}^{Z} & (=2), \\
S_{(-1,0)}^{F(2)}=-S_{\omega_{10}}^{Z} & (=2) .
\end{array}
$$

Again, these end-up being the exact same formulae as in subsection 6.5.

Having matched Stokes data from the partition function with that for the resonant freeenergy associated to the elliptic potential, all we have left to do is to proceed with the numerical checks of nonlinear resonant resurgence, via our usual large-order analyses.

\subsection{Nonlinear Resonant-Asymptotics: Free Energy}

Our final task in these lectures is to address the nonlinear resonant asymptotics of the elliptic free-energy, and, in particular, to compare against its non-resonant asymptotics discussed in subsection 6.6. Equipped with the Stokes data computed in the previous subsection, obtaining the associated discontinuities and subsequent large-order relations is by-now straightforward. Most of the construction occurs in parallel with what was done in subsection 6.6 and, as such, herein we shall mostly focus on the manifestations of resonance.

As usual, let us start with the perturbative sector. Its large-order behaviour can be derived from (6.167) and (6.168), such that the resonant version of (6.132) is now

$$
\begin{aligned}
F_{k}^{(0,0)} \frac{2 \pi \mathrm{i} A^{k}}{\Gamma(k)} \simeq & S_{\boldsymbol{e}_{1}}^{(1)}\left(F_{0}^{(1,0)}+\frac{A}{k-1} F_{1}^{(1,0)}+\frac{A^{2}}{(k-1)(k-2)} F_{2}^{(1,0)}+\cdots\right)+ \\
& +2^{-k}\left(S_{\boldsymbol{e}_{1}}^{(1)}\right)^{2}\left(F_{0}^{(2,0)}+\frac{2 A}{k-1} F_{1}^{(2,0)}+\frac{(2 A)^{2}}{(k-1)(k-2)} F_{2}^{(2,0)}+\cdots\right)+ \\
& +(-2)^{-k} S_{\boldsymbol{e}_{2}}^{(2)}\left(F_{0}^{(0,1)}+\frac{-2 A}{k-1} F_{1}^{(0,1)}+\frac{(-2 A)^{2}}{(k-1)(k-2)} F_{2}^{(0,1)}+\cdots\right)+\mathcal{O}\left(3^{-k}\right) .
\end{aligned}
$$

As compared to the non-resonant case in (6.132), we see that now the instanton action $A$ may be factored-out to the left-hand-side (similarly to what happened in the one-dimensional case (2.151)). But, other than that, at leading exponential order - the first line of (6.215) - the resonant large-order growth is exactly the same as the non-resonant one ${ }^{134}$. However, at next-toleading exponential-order resonance kicks-in and the resonant large-order growth becomes rather distinct from the non-resonant one. In the non-resonant case of subsection 6.6 , with $m=\pi / 8$, the large-order relation (6.132) shows how the contribution of the $\Phi_{(2,0)}$-sector was exponentiallysuppressed by $\left(A_{2} /\left(2 A_{1}\right)\right)^{k}$ as compared to $\Phi_{(0,1)}$, and these two sectors could not play with each other at large-order. But in the present resonant case the contributions of sectors $\Phi_{(2,0)}$ and $\Phi_{(0,1)}$ are now of the same order, and, furthermore, the $\Phi_{(0,1)}$ contribution is oscillatory.

\footnotetext{
${ }^{134}$ In fact, given that it is the same, we shall obviously not readdress it in the present subsection.
} 
Let us then illustrate the specificities of this resonant behaviour at order $\sim \mathcal{O}\left(2^{-k}\right)$, and how it differs from what was discussed back in subsection 6.6. Making use of our standard methods, subleading exponential growths are isolated by resumming and then subtracting any leading-order growth. Recalling the definition of large-order factor in (6.130), the exponentiallysubleading large-order growth inside (6.215) may then be numerically observed by plotting the sequence (note the differences from (6.137))

$$
\begin{aligned}
F_{k}^{(0,0)} & \frac{2 \pi \mathrm{i} A^{k}}{\Gamma(k)}-\mathrm{SF}_{((0,0) \rightarrow(1,0))} \times \mathcal{S}_{0^{-}} \mathrm{BP}_{N}\left[\chi_{((0,0) \rightarrow(1,0)}\right](k) \simeq \\
& \simeq 2^{-k}\left(\mathrm{SF}_{((0,0) \rightarrow(2,0))} \times \chi_{((0,0) \rightarrow(2,0))}+(-1)^{k} \mathrm{SF}_{((0,0) \rightarrow(0,1))} \times \chi_{((0,0) \rightarrow(0,1))}\right)+\mathcal{O}\left(3^{-k}\right) .
\end{aligned}
$$

The notation is always the same. $\mathcal{S}_{0^{-}} \mathrm{BP}_{N}\left[\chi_{((0,0) \rightarrow(1,0))}\right](k)$ denotes the lateral resummation of the large-order factor along $\theta=0^{-}$(avoiding the poles which lie on the positive real axis). All present statistical factors were defined in the box of page 129 (and are now evaluated with respect to the likes of figure 34). Finally, weights and combinatorial factors of each path were defined in (6.86) and (6.87); while the weights of each step are now given by (6.165) and (6.166). In particular, making use of the results in the previous subsection, $\mathrm{SF}_{((0,0) \rightarrow(n, 0))}=\left(S_{\boldsymbol{e}_{1}}^{(1)}\right)^{n}=2^{n}$, with $n \geq 1$, and $\mathrm{SF}_{((0,0) \rightarrow(0,1))}=S_{\boldsymbol{e}_{2}}^{(2)}=2$. The lateral resummation follows the exact same calculation as in subsection 6.6, recall (6.138), with both real and imaginary parts:

$$
\mathcal{S}_{0^{-}} \mathrm{BP}_{N}\left[\chi_{((0,0) \rightarrow(1,0))}\right](k)=\mathcal{S}_{\mathbb{R e}} \mathrm{BP}_{N}\left[\chi_{((0,0) \rightarrow(1,0))}\right](k)+\mathrm{i} \mathcal{S}_{\mathbb{I m}} \mathrm{BP}_{N}\left[\chi_{((0,0) \rightarrow(1,0))}\right](k) .
$$

The first imprint of resonance appears once the above subtraction is carried out, as both real and imaginary components are now of the same order (unlike in the non-resonant case, recall (6.139) and (6.140)),

$$
\begin{aligned}
\mathrm{i} \delta_{\mathbb{I m}} F_{k}^{(0,0)} & \equiv F_{k}^{(0,0)} \frac{2 \pi \mathrm{i} A^{k}}{\Gamma(k)}-\mathrm{iSF}_{((0,0) \rightarrow(1,0))} \mathcal{S}_{\mathbb{I m}} \mathrm{BP}_{N}\left[\chi_{((0,0) \rightarrow(1,0))}\right](k) \simeq \mathcal{O}\left(2^{-k}\right), \\
\delta_{\mathbb{R e}} F_{k}^{(0,0)} & \equiv-\mathrm{SF}_{((0,0) \rightarrow(1,0))} \mathcal{S}_{\mathbb{R e}} \mathrm{BP}_{N}\left[\chi_{((0,0) \rightarrow(1,0))}\right](k) \simeq \mathcal{O}\left(2^{-k}\right)
\end{aligned}
$$

Having the same magnitude, it would be natural to expect these two contributions to intertwine, with the large-order matching then occurring against the growth of the sum of second and third lines in (6.215) - with possibly yet another intertwine of their own. This particular case actually turns out to be simpler than that, as ${ }^{135}$ all coefficients $F_{m}^{(2,0)}$ are real, and all $F_{m}^{(0,1)}$ are purely imaginary. As such, one finds the simpler identifications (in particular, the first line below is essentially the same as (6.141)):

$$
\begin{aligned}
(-2)^{k} \delta_{\mathbb{I m}} F_{k}^{(0,0)} & \simeq-\operatorname{iSF}_{((0,0) \rightarrow(0,1))}\left(F_{0}^{(0,1)}-\frac{2 A}{k} F_{1}^{(0,1)}+\cdots\right), \\
2^{k} \delta_{\mathbb{R e}} F_{k}^{(0,0)} & \simeq \operatorname{SF}_{((0,0) \rightarrow(2,0))}\left(F_{0}^{(2,0)}+\frac{2 A}{k} F_{1}^{(2,0)}+\cdots\right) .
\end{aligned}
$$

The imaginary-component of our (exponentially-weighted) sequence, $(-2)^{k} \delta_{\mathbb{I m}} F_{k}^{(0,0)}$, is predicted to converge to $-\operatorname{iSF}_{((0,0) \rightarrow(0,1))} F_{0}^{(0,1)} \equiv 2 \sqrt{m}$, whereas ${ }^{136}$ the real component, $2^{k} \delta_{\mathbb{R e}} F_{k}^{(0,0)}$, is

\footnotetext{
${ }^{135}$ This can be seen directly, e.g., from the relation of free-energy sectors $\Phi_{(2,0)}$ and $\Phi_{(0,1)}$ with the original sectors in the partition function, as related via (6.100).

${ }^{136}$ From (6.104) and (6.100) we can read-off $F_{0}^{(0,1)}=\mathrm{i} \sqrt{m}$ and $F_{0}^{(2,0)}=\frac{1}{2}(1-m)$.
} 

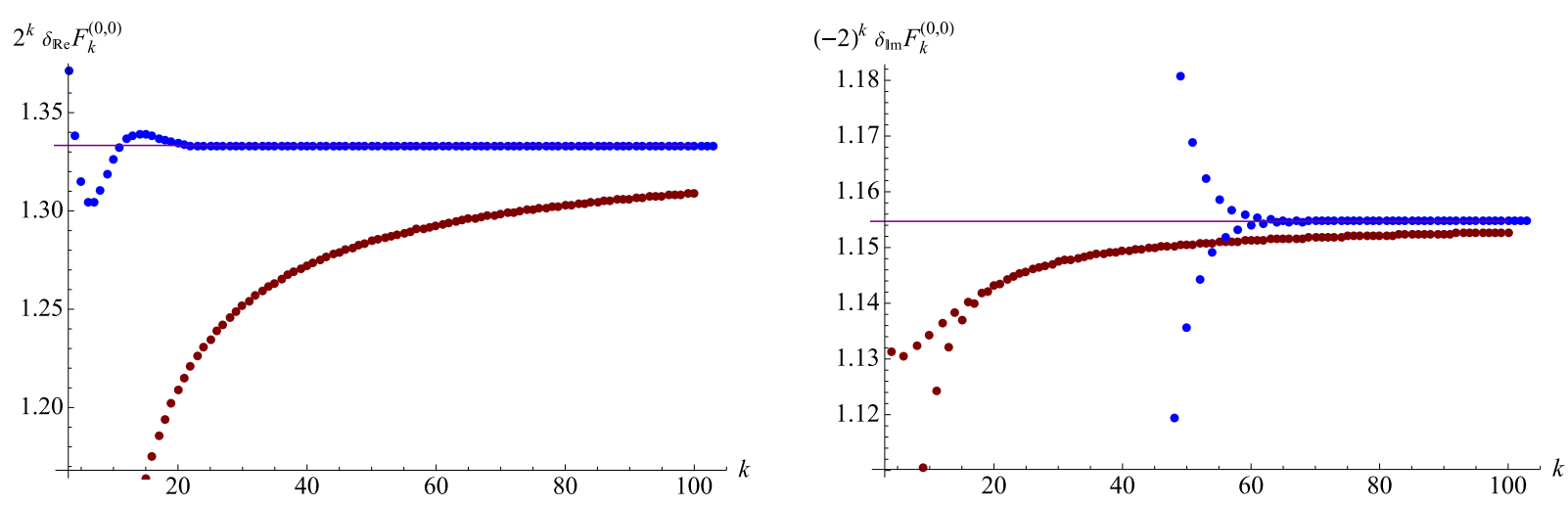

Figure 35. Exponentially-suppressed contributions to the large-order behaviour of the resonant perturbative sector. On the left we plot the leading behaviour of the real sequence $2^{k} \delta_{\mathbb{R e}} F_{k}^{(0,0)}$, defined in (6.219), while on the right we plot the leading behaviour of the imaginary sequence $(-2)^{k} \delta_{\mathbb{I m}} F_{k}^{(0,0)}$, defined in (6.218). In both cases the original sequences are plotted in red, while in blue we plot their 5-RTs. Furthermore, both cases involve a BP resummation of order $N=50$. Purple (solid) lines denote the expected (exact) convergence values: $4 F_{0}^{(2,0)}=\frac{4}{3} \approx 1.3333$ (left) and $-2 \mathrm{i} F_{0}^{(0,1)}=\frac{2}{\sqrt{3}} \approx 1.1547$ (right).

predicted to converge to $\mathrm{SF}_{((0,0) \rightarrow(2,0))} F_{0}^{(2,0)}=2(1-m)$. Note how the contribution from the $\Phi_{(0,1)}$-sector to the imaginary sequence is alternating, thus accounting for the minus sign in the exponential growth. These convergences are shown very explicitly in figure 35 (recall we have fixed $m=\frac{1}{3}$ to illustrate the resonant example). Comparing the fifth Richardson transforms of the corresponding sequences with their expected exact results, we find the typical vanishinglysmall relative errors ${ }^{137}$

$$
\begin{gathered}
\frac{\mathrm{RT}_{(0,0), 2, \mathbb{R e}}(0,95,5)-\frac{4}{3}}{\frac{4}{3}} \approx 1.4877 \times 10^{-8}, \\
\frac{\operatorname{RT}_{(0,0), 2, \mathbb{I m}}(0,95,5)-\frac{2}{\sqrt{3}}}{\frac{2}{\sqrt{3}}} \approx 5.425 \times 10^{-9} .
\end{gathered}
$$

As the final example, let us consider an instanton sector; e.g., let us revisit the $\Phi_{(0,1)}$-sector (also analyzed in the non-resonant case). Its large-order follows directly from (6.179) and (6.180),

$$
\begin{aligned}
F_{k}^{(0,1)} \frac{2 \pi \mathrm{i} A^{k}}{\Gamma(k)} \simeq & S_{\boldsymbol{e}_{1}}^{(1)}\left(F_{0}^{(1,1)}+\frac{A}{k-1} F_{1}^{(1,1)}+\frac{A^{2}}{(k-1)(k-2)} F_{2}^{(1,1)}+\cdots\right)+ \\
& +\frac{1}{(-2)^{k}} 2 S_{\boldsymbol{e}_{2}}^{(2)}\left(F_{0}^{(0,2)}+\frac{-2 A}{k-1} F_{1}^{(0,2)}+\frac{(-2 A)^{2}}{(k-1)(k-2)} F_{2}^{(0,2)}+\cdots\right)+ \\
& +\frac{1}{2^{k}}\left(S_{-\boldsymbol{e}_{2}}^{(0)}+\frac{1}{2} S_{\boldsymbol{e}_{1}}^{(1)} S_{-\boldsymbol{e}_{1}-\boldsymbol{e}_{2}}^{(0)}\right) F_{0}^{(\widetilde{\mathbf{0}})}+ \\
& +\frac{1}{2^{k}}\left(S_{\boldsymbol{e}_{1}}^{(1)}\right)^{2}\left(F_{0}^{(2,1)}+\frac{2 A}{k-1} F_{1}^{(2,1)}+\frac{(2 A)^{2}}{(k-1)(k-2)} F_{2}^{(2,1)}+\cdots\right)+ \\
& +\mathcal{O}\left(3^{-k}\right) .
\end{aligned}
$$

\footnotetext{
${ }^{137}$ We have added labels $\mathbb{R e}$ and $\mathbb{I m}$ to the RTs, in order to distinguish real and imaginary sequences.
} 


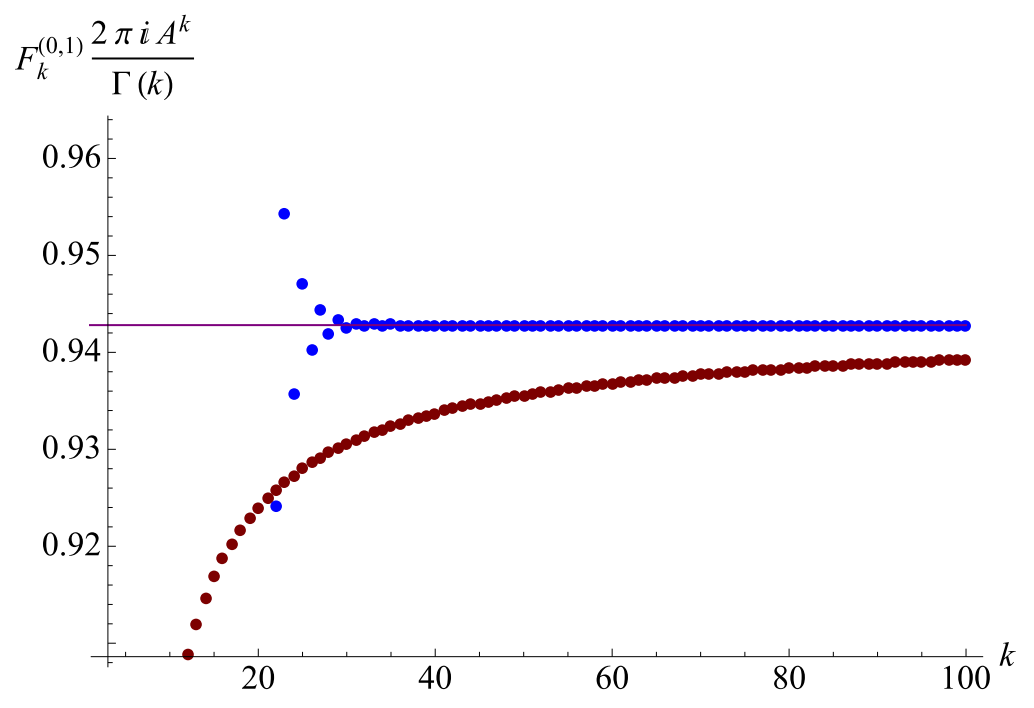

Figure 36. Leading large-order growth of the $\Phi_{(0,1)}$-sector in the resonant free-energy. The original weighted-sequence $F_{k}^{(0,1)} \frac{2 \pi \mathrm{i} A^{k}}{\Gamma(k)}$ is plotted in red, while in blue we plot its corresponding $\operatorname{RT}_{(0,1)}(0, k, 5)$. The purple line shows the value to which these sequences converge, $S_{\boldsymbol{e}_{1}}^{(1)} F_{0}^{(1,1)}=\frac{2 \sqrt{2}}{3} \approx 0.9428$.

Upon comparison with the non-resonant case (6.143), it is clear that the overall structure is the same. Furthermore, at leading exponential-order the result is exactly the same. However, at next-to-leading exponential-order, resonance kicks-in as many sectors will now talk to each other at large order, and the resonant asymptotics is now distinct from the non-resonant one. It is interesting to realize how, so far, resonance has not lead to any qualitative changes in the exponentially-leading large-order growth: similarly to what happened for the perturbative sector in (6.215), there is now only one sector which contributes at leading exponential-order, $\Phi_{(1,1)}$, and this is the exact same as in the non-resonant case (6.143). This leading behaviour is illustrated in figure 36, where the leading growth of the (weighted) sequence $F_{k}^{(0,1)} \frac{2 \pi \mathrm{i} A^{k}}{\Gamma(k)}$ is shown to converge to its predicted value, $S_{\boldsymbol{e}_{1}}^{(1)} F_{0}^{(1,1)}=\frac{2 \sqrt{2}}{3}$, with remarkable accuracy (and this is in fact basically the same as in the non-resonant case, in the left image of figure 29).

At next-to-leading exponential-order, the effects of resonance start materializing in the asymptotics. Sectors $\widetilde{\Phi}_{(0,0)}$ (third line of (6.224)) and $\Phi_{(2,1)}$ (fourth line of (6.224)) contribute to the large-order growth on equal footing, as they are both reached via forward motions of the same length (whereas in the non-resonant case the contribution of the $\Phi_{(2,1)}$ sector was exponentiallysuppressed $^{138}$ with respect to that of $\left.\widetilde{\Phi}_{(0,0)}\right)$. This can be understood pictorially: figure 34 illustrates the same phenomenon (albeit now centered on sector $\Phi_{(3,2)}$ ), where in the plot it is sectors $\Phi_{(3,1)}$ and $\Phi_{(1,0)}$ which are both reached by a forward motion of length $2 A$. Back to our $\Phi_{(0,1)}$-sector; the large-order contribution of $\Phi_{(0,2)}$ (second line of (6.224)) also contributes at the same exponential-order, altough it arises from a backwards path (in the same way as in the non-resonant case), thus its alternating-sign within the growth. In order to numerically analyse all these exponentially-suppressed contributions, we perform the usual BP resummation of the leading growth (first line of (6.224)), split it into real and imaginary parts, and define (as in

\footnotetext{
${ }^{138}$ At least for our choice of non-resonant modulus $m$.
} 
(6.146) and (6.147))

$$
\begin{aligned}
\delta_{\mathbb{R e}} F_{k}^{(0,1)} & \equiv F_{k}^{(0,1)} \frac{2 \pi \mathrm{i} A^{k}}{\Gamma(k)}-\mathrm{SF}_{((0,1) \rightarrow(1,1))} \mathcal{S}_{\mathbb{R e}} \mathrm{BP}_{N}\left[\chi_{((0,1) \rightarrow(1,1))}\right](k) \simeq \mathcal{O}\left(2^{-k}\right), \\
\mathrm{i} \delta_{\mathbb{I m}} F_{k}^{(0,1)} & \equiv-\mathrm{iSF}_{((0,1) \rightarrow(1,1))} \mathcal{S}_{\mathbb{I m}} \mathrm{BP}_{N}\left[\chi_{((0,1) \rightarrow(1,1))}\right](k) \simeq \mathcal{O}\left(2^{-k}\right) .
\end{aligned}
$$

In these expressions we used the fact that the coefficients $F_{k}^{(0,1)}$ are purely imaginary. As expected, both real and imaginary sequences are of the same exponential order. Finally, because the coefficients $F_{k}^{(0,2)}$ and $F_{0}^{(\widetilde{\mathbf{0}})} \equiv 1$ are real, while the $F_{k}^{(2,1)}$ are purely imaginary, we can write the respective predicted growths as

$$
\begin{aligned}
(-2)^{k} \delta_{\mathbb{R e}} F_{k}^{(0,1)} & \simeq(-1)^{k} \mathrm{SF}_{((0,1) \rightarrow \widetilde{\mathbf{0}})} F_{0}^{(\widetilde{\mathbf{0}})}+\mathrm{SF}_{((0,1) \rightarrow(0,2))}\left(F_{0}^{(0,2)}-\frac{2 A}{k} F_{1}^{(0,2)}+\cdots\right), \\
2^{k} \delta_{\mathbb{I m}} F_{k}^{(0,1)} & \simeq-\mathrm{iSF}_{((0,1) \rightarrow(2,1))}\left(F_{0}^{(2,1)}+\frac{2 A}{k} F_{1}^{(2,1)}+\cdots\right) .
\end{aligned}
$$

Using the values for the Stokes coefficients from the previous subsection, namely, (6.206), (6.204), and (6.210), the required statistical-factors ${ }^{139}$ above are:

$$
\begin{aligned}
\mathrm{SF}_{((0,1) \rightarrow(1,1))} & \equiv S_{\boldsymbol{e}_{1}}^{(1)}=2, \\
\mathrm{SF}_{((0,1) \rightarrow \widetilde{\mathbf{0}})} & \equiv S_{-\boldsymbol{e}_{2}}^{(0)}+\frac{1}{2} S_{\boldsymbol{e}_{1}}^{(1)} S_{-\boldsymbol{e}_{1}-\boldsymbol{e}_{2}}^{(0)}=-2, \\
\mathrm{SF}_{((0,1) \rightarrow(0,2))} & \equiv 2 S_{\boldsymbol{e}_{2}}^{(2)}=4, \\
\mathrm{SF}_{((0,1) \rightarrow(2,1))} & \equiv\left(S_{\boldsymbol{e}_{1}}^{(1)}\right)^{2}=4 .
\end{aligned}
$$

The large-order tests are shown in figure 37. The left image plots the leading growth of the real sequence, $(-2)^{k} \delta_{\mathbb{R e}} F_{k}^{(0,1)}$, defined in (6.225), and its convergence to the predicted alternating values $^{140} \pm \mathrm{SF}_{((0,1) \rightarrow \widetilde{\mathbf{0}})} F_{0}^{(\widetilde{\mathbf{0}})}+\mathrm{SF}_{((0,1) \rightarrow(0,2))} F_{0}^{(0,2)}=\frac{2}{3} \mp 2$. The right image plots the leading growth of the imaginary sequence, $2^{k} \delta_{\mathbb{I m}} F_{k}^{(0,1)}$, defined in (6.226), and its convergence to the predicted value ${ }^{141}-\mathrm{iSF}_{((0,1) \rightarrow(2,1))} F_{0}^{(2,1)}=-\frac{8}{3 \sqrt{3}}$. The fifth-order Richardson transforms of both these real and imaginary sequences (the blue sequences in figure 37) converge very clearly to their predicted values, with vanishingly-small relative errors of the order of $\sim 10^{-10}$ for the imaginary sequence, and $\sim 10^{-8}$ for the real ${ }^{142}$ one.

This concludes our analysis of the resonant resurgent-structure of the transseries solution we constructed for the elliptic-potential free energy. The many numerical checks clearly validate all large-order predictions, which were now obtained from resonant resurgence. This example was particularly illuminating as resonance was tunable, and, in this way, allowed us to compare differences and similarities between resonant and non-resonant cases. In general, in harder problems, resonance need not be tunable and the asymptotics may be much more intricate than illustrated

\footnotetext{
${ }^{139}$ These are just the Borel residues associated to the singularities of $\mathcal{B}\left[\Phi_{(0,1)}\right](s) ; \mathrm{SF}_{((0,1) \rightarrow \boldsymbol{m})}=-\mathrm{S}_{(0,1) \rightarrow \boldsymbol{m}}$.

${ }^{140}$ Using $F_{0}^{(\widetilde{\mathbf{0}})}=1$ and $F_{0}^{(0,2)}=\frac{1}{6}$.

${ }^{141}$ Using $F_{0}^{(2,1)}=-\frac{2 \mathrm{i}}{3 \sqrt{3}}$.

${ }^{142}$ Once again, due to its alternating nature, one needs to separate and independently address even and odd terms in order to perform the Richardson transforms for the real sequence.
} 

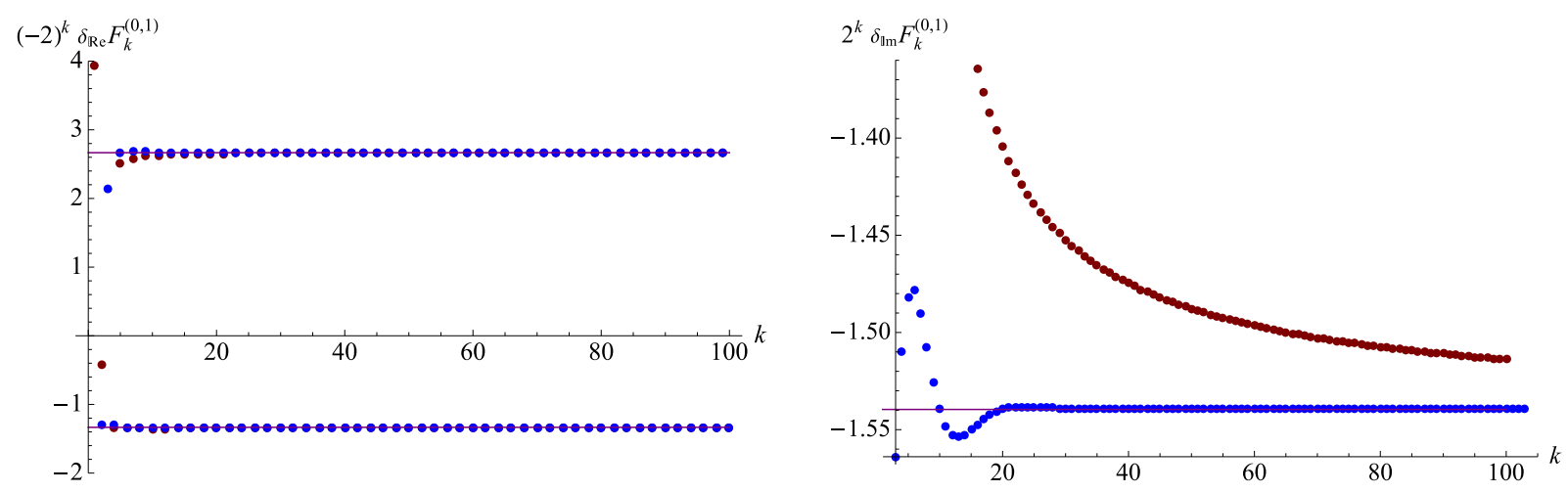

Figure 37. Exponentially-suppressed contributions to the large-order behaviour of the resonant $\Phi_{(0,1)}$ sector. We plot the leading behaviour of both real $(-2)^{k} \delta_{\mathbb{R e}} F_{k}^{(0,1)}$ (left) and imaginary $2^{k} \delta_{\mathbb{I m}} F_{k}^{(0,1)}$ (right) sequences, as defined in (6.225) and (6.226), respectively. In red we plot the original sequences, with their 5-RTs in blue $\left(\operatorname{RT}_{(0,1), 2, \mathbb{R e} \text {,even/odd }}(0, k, 5)\right.$ for the left plot and $\operatorname{RT}_{(0,1), 2, \mathbb{I m}}(0, k, 5)$ for the right plot). Purple (solid) lines denote the values to which these sequences converge, namely, $\pm \mathrm{SF}_{((0,1) \rightarrow \widetilde{\mathbf{0}}} F_{0}^{(\widetilde{\mathbf{0}})}+$ $\mathrm{SF}_{((0,1) \rightarrow(0,2))} F_{0}^{(0,2)}=\frac{2}{3} \mp 2$ (left) and $-\mathrm{iSF}_{((0,1) \rightarrow(2,1))} F_{0}^{(2,1)}=-\frac{8}{3 \sqrt{3}}$ (right).

here. Recent such examples include different string-theoretic scenarios, as are the cases of the first Painlevé equations describing $2 \mathrm{~d}$ (super) gravity [130, 16, 205] and topological string theory $[195,77,78,76]$. This introduction to resonance should hopefully allow the interested reader to easily understand the resurgent properties of those examples.

As we reach the end, hopefully we have contributed to helping the reader enlarge their toolbox of methods for addressing nonperturbative questions in modern theoretical and mathematical physics. It should be clear that resurgence and transseries have a built-in very natural power to tackle truly nonperturbative questions, both at numerical and analytical levels. Further, they are explicitly constructed in a physically-intuitive semiclassical language. The range of problems where they may be applicable - from quantum mechanics, field theory, gauge theory, through string theory - is enormous, and we hope this contribution will help opening new nonperturbative windows into so many fascinating problems. Closer to our own research interests, while these methods by themselves may not tell us why string perturbation theory diverges (we think of the "why" as a question concerning the physics), very likely they will tell us exactly how string perturbation theory diverges (as these lectures hopefully made clear, the "how" is, in fact, a very sharp and answerable mathematical question): seemingly in a very specific mixture of both resonant and parametric/co-equational resurgences. While the former has, at this stage, become well-known to the reader, the latter will have to wait for future work.

\section{Acknowledgments}

We would like to thank Aleksey Cherman, Santiago Codesido, Ovidiu Costin, Ricardo CousoSantamaría, Gerald Dunne, José Edelstein, Frédéric Fauvet, Tiago Dinis da Fonseca, Stavros Garoufalidis, Alba Grassi, Chris Howls, Marcos Mariño, Sara Pasquetti, Jorge Russo, David Sauzin, Mithat Ünsal, Ricardo Vaz, Marcel Vonk, André Voros, Marlene Weiss, Niclas Wyllard and Szabolcs Zakany, for very valuable collaborations, discussions, comments and/or correspondence; since the early days we started working on these topics, and then over the slow process 
across the years that it took for these lectures to finally become concluded. IA would further like to thank the Kavli Institute for Theoretical Physics for extended hospitality, where parts of this work were conducted. GB would further like to thank the Kavli Institute for Theoretical Physics for hospitality, where parts of this work were conducted. RS would further like to thank CERN TH-Division, the Kavli Institute for Theoretical Physics, and the University of Geneva, for extended hospitality, where parts of this work were conducted. IA's research was partially supported by the NCN grant 2012/06/A/ST2/00396. GB is supported by the U.S. Department of Energy under Contract No. DE FG02-01ER41195. The research of RS was partially supported by the FCT-Portugal grants PTDC/MAT/119689/2010, EXCL/MAT-GEO/0222/2012, and UID/MAT/04459/2013; and the Swiss-NSF grant NCCR 51NF40-141869 "The Mathematics of Physics" (SwissMAP). This research was supported in part by the National Science Foundation under Grant No. NSF PHY-1125915. 


\section{A Further Details on Alien Calculus}

The goal of this appendix is to present a mathematical (albeit much shorter) introduction to alien calculus. This will hopefully lay down a notational bridge between the contents of the main text and the mathematical literature; thus guiding the interested reader within the general framework described in, e.g., [109, 55, 56, 69-71, 91, 207, 203, 204, 84, 185, 167, 85], and from then on. We shall begin with general definitions and results, and then proceed to an example involving two instanton actions but with no multi-instanton corrections, i.e., a strictly "linear" example ${ }^{143}$. This example will nonetheless have enough structure so as to illustrate all the required material.

Note that, when compared against the main text, the asymptotic expansions in this appendix are in the variable (coupling constant) $z=1 / x$, with $z \sim+\infty$. The analogue of $(2.1)$ is now simply

$$
\Phi^{(0)}(z) \simeq \sum_{g=0}^{+\infty} \frac{\Phi_{g}^{(0)}}{z^{g+1}} .
$$

While this is the standard notation within the mathematical literature - and we shall follow it in this appendix-, as one compares against formulae in the main text - and, in particular, largeorder formulae - it is important to notice that there might be some overall minus-sign differences.

\section{Asymptotic Gevrey Series}

Up to this point we have been dealing with asymptotic series whose coefficients grow as $\Phi_{g} \sim g$ !, but there are many other possibilities. One very useful classification of different possible types of asymptotic behaviour is due to Gevrey (see, e.g., [155] for an introduction). Herein, one says that the formal series (A.1) is a Gevrey-p series, iff $\exists M, C \in \mathbb{R}^{+}$such that

$$
\left|\Phi_{g}\right| \leq M C^{g}(g !)^{p}
$$

In particular, a convergent series is Gevrey- 0 while the asymptotic series we have been considering in the main text are Gevrey-1. The set of all Gevrey- $p$ formal series, which we shall denote by $\mathbb{C}_{(p)}[[z]]$, is a differential algebra. One can make sense out of these general, divergent series by following a rather straightforward generalization of the Borel resummation procedure outlined at the beginning of section 2. Let $\Phi(z)$ in (A.1) be a Gevrey-p series, i.e., $\Phi(z) \in \mathbb{C}_{(p)}[[z]]$. Further, define the index- $m$ Borel transform as

$$
\mathcal{B}_{m}\left[\frac{1}{z^{\alpha+1}}\right](s)=\frac{s^{\alpha}}{\Gamma\left(\frac{\alpha}{m}+1\right)},
$$

which, in particular, generalizes (2.2). In this set-up, the index- $\frac{1}{p}$ Borel transform

$$
\mathcal{B}_{1 / p}[\Phi](s)
$$

has positive radius of convergence and may thus by analytically extended throughout $s \in \mathbb{C}$, albeit with the resulting function having singularities and branch cuts, as expected. Once this is done, the index- $\frac{1}{p}$ Borel resummation ${ }^{144}$ (generalizing the standard Borel resummation (2.3))

$$
\mathcal{S}_{1 / p} \Phi(z)=z^{\frac{1}{p}-1} \int_{0}^{+\infty} \mathrm{d} s \mathcal{B}_{1 / p}[\Phi]\left(s^{p}\right) \mathrm{e}^{-z^{\frac{1}{p}} s}
$$

\footnotetext{
${ }^{143}$ This is, of course, an example inspired by a partition function already computed in section 6 .

${ }^{144}$ This resummation is defined via the index- $m$ Laplace transform, itself given by

$$
\mathcal{L}_{m}[f(s)](z):=z^{m-1} \int_{0}^{+\infty} \mathrm{d} s f\left(s^{1 / m}\right) \mathrm{e}^{-z^{m} s} .
$$


finally defines a function out from the Gevrey- $p$ series (A.1) (see, e.g., [155] for further details). Of course that, as usual, this integral only makes sense as long as the integration contour does not cross any singularities of the integrand. If it does, Stokes automorphisms must come into play and the resurgence framework necessarily makes its way onto the realm of general Gevrey series. Albeit very interesting, we shall not further pursue the question of multi-summability in this appendix, but refer the interested reader to, e.g., [167]. Having briefly unveiled the general set-up of Borel multi-summability, we shall next return to our analysis of Gevrey-1 series.

\section{Resurgent Functions and Simple Resurgent Functions}

Under the above set-up, let us introduce the definition of resurgence as due to Écalle [109]. The Gevrey-1 formal series (A.1) is said to be resurgent, or, more generically, to define a resurgent function, if its Borel transform has endless analytic continuation. This means that, for any chosen path emanating from some neighborhood of the origin, and which avoids a fixed countable set ${ }^{145}$ on the complex plane, the Borel transform must have analytic continuation along this path. This "forbidden" set is, of course, the set of Borel singularities. This definition leads to a very general class of functions, from which a particularly useful subclass is that of simple resurgent functions (also as already discussed in section 4). This subclass is still rather large so as to include many examples, but a bit more under control on what concerns Borel singularities. Recall from section 4 that a simple resurgent function is a resurgent function whose Borel singularities restrict to simple poles and logarithmic branch-points. If we recall (4.53), the precise statement is ${ }^{146}$

$$
\mathcal{B}[\Phi](s)=\mathrm{S}_{\omega} \times \mathcal{B}\left[\Psi_{\omega}\right](s-\omega) \frac{\log (s-\omega)}{2 \pi \mathrm{i}}+\text { holomorphic }
$$

near each singular point $\omega$, with $\mathrm{S}_{\omega} \in \mathbb{C}$ and $\Psi_{\omega}$ some other asymptotic sector (but such that $\mathcal{B}\left[\Psi_{\omega}\right]$ is holomorphic near the origin, i.e., it introduces no further singularities near $s \approx \omega$ ).

For simple resurgent functions, one may actually use the above explicit expression (A.7) in order to be precise upon what happens as the Borel resummation (A.6) crosses a Stokes line (recall figure 5 in section 2). Using the lateral Borel resummations

$$
\mathcal{S}_{\theta^{ \pm}} \Phi(z)=\int_{0}^{\mathrm{e}^{\mathrm{i} \theta^{ \pm}} \infty} \mathrm{d} s \mathcal{B}[\Phi](s) \mathrm{e}^{-z s}
$$

and labeling the (possibly infinite) singular points on the Stokes line along $\theta$ by $\left\{\omega_{n}\right\}$ (i.e., $\left.\forall n \in \mathbb{N}, \arg \omega_{n}=\theta\right)$, it is straightforward to obtain

$$
\mathcal{S}_{\theta^{+}} \Phi(z)=\mathcal{S}_{\theta^{-}} \Phi(z)-\sum_{\left\{\omega_{n}\right\}} \mathrm{S}_{\omega_{n}} \mathrm{e}^{-\omega_{n} z} \mathcal{S}_{\theta^{-}} \Psi_{\omega_{n}}(z)
$$

This expression encodes the Stokes automorphism $\underline{\mathfrak{S}}_{\theta}$ in $(2.4), \mathcal{S}_{\theta^{+}}=\mathcal{S}_{\theta^{-}} \circ \underline{\mathfrak{S}}_{\theta}$, or, equivalently, the discontinuity across the Stokes line,

$$
\mathcal{S}_{\theta^{+}}-\mathcal{S}_{\theta^{-}}=-\mathcal{S}_{\theta^{-}} \circ \operatorname{Disc}_{\theta}
$$

\footnotetext{
${ }^{145}$ This countable set may still be allowed to be everywhere dense, making the definition rather broad.

${ }^{146}$ In this appendix we are dropping the simple pole in (4.53), associated to a residual coefficient, without loss of generality. It should be straightforward for the reader to include it back at any stage in the present analysis.
} 
where we recall from (2.45) that $\operatorname{Disc}_{\theta}=\mathbf{1}-\underline{\mathfrak{S}}_{\theta}$. One concludes that, for simple resurgent functions with Borel singularities (A.7), the Stokes discontinuities take the general form ${ }^{147}$

$$
\operatorname{Disc}_{\theta} \Phi(z)=\sum_{\left\{\omega_{n}\right\}} \mathrm{S}_{\omega_{n}} \mathrm{e}^{-\omega_{n} z} \Psi_{\omega_{n}}(z)
$$

This directional discontinuity is easily decomposable as $\operatorname{Disc}_{\theta} \Phi=\sum_{\left\{\omega_{n}\right\}} \operatorname{Disc}_{\omega_{n}} \Phi$, where the discontinuity at each specific singularity $\omega$ is given by

$$
\operatorname{Disc}_{\omega} \Phi \equiv \mathrm{S}_{\omega} \mathrm{e}^{-\omega z} \Psi_{\omega}
$$

This "discontinuity operator at $\omega$ " is linear by construction. It may be equally defined as either acting on the asymptotic series or on the corresponding Borel transforms. It also relates in a completely direct way to the Borel singularity structure in (A.7). As we shall see next, this state of affairs may be further improved by introducing a closely related operator: the alien derivation.

\section{Alien Derivatives and Alien Calculus}

The alien derivative at $\omega$, denoted by $\Delta_{\omega} \Phi$, improves upon the discontinuity operator, $\operatorname{Disc}_{\omega} \Phi$, precisely by further being a derivation (and thus, a vector field). One way to approach it, starting from (A.7), is to define the alien derivative of $\mathcal{B}[\Phi]$ at the singularity $\omega$ (of $\Phi$, at $\omega$ ), denoted by $\Delta_{\omega} \mathcal{B}[\Phi]$ (by $\left.\Delta_{\omega} \Phi\right)$, as a specific weighted-average of analytic continuations of $\mathcal{B}\left[\Psi_{\omega}\right]$ along every possible path from the origin up to $\omega$ along $\theta$ (where $\arg \omega=\theta$ ), which circumvent the previous singularities on the Stokes line in every possible way (e.g., recall figure 8). The alien derivative thus constructed is linear and may be equally defined as either acting on the Borel transforms or on the corresponding asymptotic series. But, in contrast with the discontinuity, it is also a derivation in the sense that it satisfies the Leibniz rule with respect to either the convolution product of Borel transforms or the standard product of asymptotic series. Thus its usefulness over (A.12). The precise definition is too technical for this short appendix and we shall next approach it in a slightly detoured way (see, e.g., [109, 203, 204] for rigorous definitions).

Within the usual picture of figures 5 and 8 in section 2, and our on-going discussion, let us consider a Stokes line along $\theta$ with singularities $\left\{\omega_{n}\right\}$. Let us further introduce the pointed (or dotted) alien derivative, simply defined by the combination $\dot{\Delta}_{\omega}:=\mathrm{e}^{-\omega z} \Delta_{\omega}$. Then, akin to (A.11), one defines the directional pointed alien-derivative as

$$
\dot{\Delta}_{\theta}=\sum_{\left\{\omega_{n}\right\}} \dot{\Delta}_{\omega_{n}}
$$

The main relation between all these concepts (and which, for the purposes of this appendix, we shall take as the definition of alien derivative) is now [109]

$$
\underline{\underline{\Delta}}_{\theta}:=\log \underline{\mathfrak{S}}_{\theta}=\log \left(\mathbf{1}-\operatorname{Disc}_{\theta}\right)=-\operatorname{Disc}_{\theta}-\frac{1}{2} \operatorname{Disc}_{\theta} \circ \operatorname{Disc}_{\theta}-\frac{1}{3} \operatorname{Disc}_{\theta} \circ \operatorname{Disc}_{\theta} \circ \operatorname{Disc}_{\theta}-\cdots .
$$

In this way, via the logarithm, the proof that $\Delta_{\omega}$ is a derivation becomes equivalent to the proof that $\underline{\mathfrak{S}}_{\theta}$ is an automorphism [109]. As it stands, (A.14) is a surprisingly simple relation. But once we insert (A.13), and iteratively (A.11), it will result in intricate ${ }^{148}$ expressions $^{149}$ for

\footnotetext{
${ }^{147}$ This also shows how $\Phi$ alone cannot be the complete answer to whatever problem one is addressing: all $\left\{\Psi_{\omega_{n}}\right\}$ contributions found by probing the singularity structure of $\mathcal{B}[\Phi]$ must also be appropriately taken into account.

${ }^{148}$ Except, of course, if there is a single singularity $\omega$ along $\theta$, in which case $\dot{\Delta}_{\theta}=-\operatorname{Disc}_{\theta}$ as $\operatorname{Disc}_{\theta}^{2} \Phi \sim \operatorname{Disc}_{\theta} \Psi_{\omega}=0$.

${ }^{149}$ See [203] for some very explicit expressions and examples, obtained by following this sort of analysis.
} 
the alien derivatives $\Delta_{\omega_{n}}$ (resulting in the aforementioned weighted averages). Nevertheless, the main point is that although Disc $\omega$ connects to the Borel transform in a very direct way (as we have seen above), it is not a derivation - thus the usefulness of introducing $\Delta_{\omega}$ instead.

\section{Transseries and the Bridge Equation}

The natural question which follows from the above discussion is: is there an alternative (meaning simpler) way to compute $\Delta_{\omega}$ ? The answer is "yes", and it will be achieved by the use of the bridge equation. One of the cleanest ways to introduce it is to follow the historical development of resurgence, going back to its origins within the context of (first order) nonlinear ODEs. Herein, one constructs formal solutions to the said ODE via the use of transseries, just like the one back in $(2.5)$,

$$
\Phi(z, \sigma)=\sum_{n=0}^{+\infty} \sigma^{n} \Phi^{(n)}(z) .
$$

Under appropriate conditions, $\Phi^{(0)}(z)$ is a Gevrey-1 series of the form (A.1) and the $\Phi^{(n)}(z)$ are $n$-instanton contributions ${ }^{150}$, analogues of $(2.6)$,

$$
\Phi^{(n)}(z) \simeq \mathrm{e}^{-n A z} \sum_{g=1}^{+\infty} \frac{\Phi_{g}^{(n)}}{z^{g+\beta_{n}}} \equiv \mathrm{e}^{-n A z} \Phi_{n}(z),
$$

with $\Phi_{n}$ also Gevrey-1 series. The transseries parameter $\sigma$ parameterizes different choices of initial (boundary) conditions, and one can write an infinite sequence of linear ODEs (homogeneous for $n=1$; inhomogeneous for $n \geq 2$ ) for the $\Phi_{n}$, starting from the original nonlinear equation.

The construction of the bridge equation now follows from a simple observation. The pointed alien derivative which we introduced earlier, $\dot{\Delta}_{\omega}$, turns out to commute with the ordinary derivative, i.e., $\left[\dot{\Delta}_{\omega}, \partial_{z}\right]=0$ (in particular, this implies that the standard alien derivative $\Delta_{\omega}$ does not commute with the ordinary derivative), see, e.g., [109, 203, 204]. Further, a partial derivative with respect to the transseries parameter, $\partial_{\sigma}$, obviously also commutes with $\partial_{z}$. This implies that both $\dot{\Delta}_{\omega} \Phi(z, \sigma)$ and $\partial_{\sigma} \Phi(z, \sigma)$ will satisfy the very same, first-order ODE. In particular, this will be a linear, homogeneous ODE in the variable $z$, which implies that these two quantities must be proportional to each other. In formulae:

$$
\dot{\Delta}_{\omega} \Phi(z, \sigma)=S_{\omega}(\sigma) \frac{\partial \Phi}{\partial \sigma}(z, \sigma)
$$

where $S_{\omega}(\sigma)$ is the (z-independent) proportionality coefficient, and this equation is precisely the bridge equation, establishing a "bridge" between alien and ordinary derivatives. The argument may be generalized to higher-order ODEs, to PDEs, to finite-difference equations, multidimensional integrals and so on, which is to say that the bridge equation is quite general and somewhat independent from the context where it was derived [109, 203, 204].

One may be more specific about the proportionality coefficient $S_{\omega}(\sigma)$. The transmonomial building blocks of (A.15) are of the form $\sigma^{n} \mathrm{e}^{m A z}$, to which we may assign weight $\mathrm{w}=n+m$. Now, the derivative $\dot{\Delta}_{k A}$ acts on these transmonomials by lowering ${ }^{151}$ their weight as $m \rightarrow m-k$ and the derivative $\partial_{\sigma}$ acts upon them by lowering weight as $n \rightarrow n-1$. Since the transseries

\footnotetext{
${ }^{150}$ If by no other way, all sectors in the transseries (A.15) may be found by probing the singularity structure of $\mathcal{B}\left[\Phi^{(0)}\right]$, as in (A.11) (and, if necessary, successively the singularity structures of the thus uncovered $\mathcal{B}\left[\Phi^{(n)}\right]$ ).

${ }^{151}$ For positive $k$ and $A$, which is implicitly assumed when saying "lowering".
} 
(A.15) has overall weight $w=0$, the bridge equation (A.17) can only hold ${ }^{152}$ at $\omega=k A$ if $S_{k A}(\sigma)$ has weight $\mathrm{w}=1-k$, i.e.,

$$
S_{k A}(\sigma) \equiv S_{k} \sigma^{1-k}
$$

is a monomial in $\sigma$. Notice that one must further have $k \leq 1$, as both sides of (A.17) are regular. In this case, the bridge equation is finally written as

$$
\dot{\Delta}_{k A} \Phi(z, \sigma)=S_{k} \sigma^{1-k} \frac{\partial \Phi}{\partial \sigma}(z, \sigma), \quad k \leq 1, \quad k \neq 0,
$$

up to the as-yet-unknown complex numbers $\left\{S_{1}, S_{-1}, S_{-2}, \cdots\right\}$; the Écalle analytic invariants ${ }^{153}$ for the original ODE. This bridge equation that the transseries formal-solution satisfies, (A.19), translates to an infinite sequence of resurgence relations for the transseries components $\Phi_{n}$ (by simple replacement) as

$$
\Delta_{k A} \Phi_{n}=S_{k}(n+k) \Phi_{n+k}, \quad k \leq 1, \quad k \neq 0 .
$$

These expressions compute all alien derivatives, up to the analytic invariants. It is also simple to realize that, via (A.14), the invariants $S_{\omega}$ are determined by the "Borel residues" $\mathrm{S}_{\omega}$ in (A.7).

This discussion also illustrates the advantage of working with the alien operator $\Delta_{\omega}$ rather than the discontinuity Disc $\omega$ : as $\Delta_{\omega}$ is a derivation, it is also a vector field, and it is precisely such a vector field realization that the bridge equation is implementing.

\section{Stokes Phenomena and its Stokes Constants}

The framework described above now allows for a very elegant reformulation of Stokes phenomena (which we first explained back in (2.7)). Let us consider the action of the Stokes automorphism $\underline{\mathfrak{S}}_{\theta}$ on the transseries (A.15), along $\theta=0$. In this direction, the bridge equation (A.19) yields a single singularity at $\omega=A$, in which case it follows

$$
\underline{\mathfrak{S}}_{0} \Phi(z, \sigma)=\mathrm{e}^{\underline{\dot{\Delta}}_{0}} \Phi(z, \sigma)=\mathrm{e}^{\dot{\Delta}_{A}} \Phi(z, \sigma)=\mathrm{e}^{S_{1} \frac{\partial}{\partial \sigma}} \Phi(z, \sigma)=\Phi\left(z, \sigma+S_{1}\right),
$$

where we made use of the relation between the Stokes automorphism and the alien derivative (A.14) in the first equality, and of the bridge equation (A.19) in the third equality. This expression shows why the Stokes automorphism is indeed an automorphism: it acts upon transseries (via the bridge equation) as inducing a translation along the $\sigma$-direction. It also shows why this automorphism is named Stokes. But, interestingly enough, this is not the only type of Stokes phenomena that must be considered. The bridge equation (A.19) also predicts a Stokes line along $\theta=\pi$, where we now find nonlinear Stokes phenomena. In fact, along this direction there is an infinite number of singularities, and the Stokes automorphism acts as

$$
\begin{aligned}
\underline{\mathfrak{S}}_{\pi} \Phi(z, \sigma) & =\mathrm{e}^{\underline{\dot{\Delta}}_{\pi}} \Phi(z, \sigma)=\exp \left(\sum_{n=1}^{+\infty} \dot{\Delta}_{-n A}\right) \Phi(z, \sigma)= \\
& =\exp \left(\sum_{n=1}^{+\infty} S_{-n} \sigma^{n+1} \frac{\partial}{\partial \sigma}\right) \Phi(z, \sigma) \equiv \mathrm{e}^{\underline{S}}(\sigma) \frac{\partial}{\partial \sigma} \Phi(z, \sigma)=\Phi\left(z, \underline{\mathbb{S}}_{\pi}(\sigma)\right),
\end{aligned}
$$

\footnotetext{
${ }^{152}$ These concepts of degree and homogeneity were further discussed, in a slightly different context, in section 5 .

${ }^{153}$ These numbers are invariants of the ODE in the following sense: two (apparently distinct) ODEs with the same set of analytic invariants, must in fact be the same equation up to a (possibly complicated) change of variables.
} 
where $\underline{\mathbb{S}}_{\pi}(\sigma)$ essentially generalizes the above translation $\sigma \mapsto \mathbb{S}_{0}(\sigma) \equiv \sigma+S_{1}$ to a more generic automorphism $\sigma \mapsto \underline{\mathbb{S}}_{\pi}(\sigma)$ generated by the one-parameter flow of the vector field $\underline{S}_{\pi}(\sigma) \frac{\partial}{\partial \sigma}$. A more complete discussion of Stokes phenomena and the Stokes automorphism, along different types of Stokes lines and for transseries with multiple parameters, may be found in, e.g., [15].

The above discussion further shows how the analytic invariants are in fact the Stokes constants of the problem; which themselves must relate to the Borel residues. In this way, one may equally denote the $S_{k}$ constants as Écalle analytic invariants, Stokes constants, or Écalle-Stokes invariants. But perhaps the most interesting point here is that, because they relate to the Borel residues $\mathrm{S}_{\omega}$ in (A.7) or (A.12) via (A.14), such relation gives us, at least in principle, a way to compute them. Let us understand how this occurs in our present setting.

Rewriting (A.7) for any given sector of our specific transseries (A.15), one finds

$$
\left.\mathcal{B}\left[\Phi_{n}\right](s)\right|_{s=k A}=\mathrm{S}_{n \rightarrow n+k} \times \mathcal{B}\left[\Phi_{n+k}\right](s-k A) \frac{\log (s-k A)}{2 \pi \mathrm{i}}+\text { holomorphic }, \quad k \neq 0,
$$

where the enlarged labeling of the Borel residues, including departure and arrival nodes, will become clear shortly. Further rewriting (A.11) for any of the transseries sectors, one finds

$$
\operatorname{Disc}_{\theta} \Phi_{n}=\sum_{\{k\}} \mathrm{S}_{n \rightarrow n+k} \mathrm{e}^{-k A z} \Phi_{n+k}
$$

where the sum in taken over all singularities $\omega=k A$ appearing along ${ }^{154} \theta$. We may now expand (A.14) in the present setting, and compare equal exponential terms in order to obtain the alien derivative acting on a sector $\Phi_{n}$ as a combination of Borel residues. Along $\theta=0$ one finds (with $k>0)$

$$
\Delta_{k A} \Phi_{n}=-\sum_{s=1}^{k} \frac{1}{s} \sum_{\substack{\left\{\ell_{1}, \ldots, \ell_{s} \geq 1\right\} \\ \sum \ell_{i}=k}} \mathrm{~S}_{n \rightarrow n+\ell_{1}} \mathrm{~S}_{n+\ell_{1} \rightarrow n+\ell_{1}+\ell_{2}} \cdots \mathrm{S}_{n+k-\ell_{s} \rightarrow n+k} \Phi_{n+k} .
$$

But now the constraint $k \leq 1$ in (A.20) implies that there is only one non-vanishing term, i.e., $\Delta_{k A} \Phi_{n}=0$ for $k>1$. Comparing (A.28) with the resurgence relations (A.20), this non-vanishing term yields

$$
S_{1}=-\frac{1}{n+1} \mathrm{~S}_{n \rightarrow n+1},
$$

which relates the $k=1$ Stokes constant with the Borel residues of a "single-step" (and vice-versa). All the remaining vanishing terms in (A.28) then yield a series of constraints which essentially build the "multi-step" Borel residues as products of "single-step" ones. One finds

$$
\mathrm{S}_{n \rightarrow n+k}=\frac{(-1)^{k-1}}{k !} \prod_{\ell=0}^{k-1} \mathrm{~S}_{n+\ell \rightarrow n+\ell+1},
$$

\footnotetext{
${ }^{154}$ For the two directions of interest in the one-parameter transseries setting, $\theta=0$ and $\theta=\pi$, this results in:

$$
\begin{aligned}
\operatorname{Disc}_{0} \Phi_{n} & =\sum_{k=1}^{+\infty} \mathrm{S}_{n \rightarrow n+k} \mathrm{e}^{-k A z} \Phi_{n+k}, \\
\operatorname{Disc}_{\pi} \Phi_{n} & =\sum_{k=1}^{n} \mathrm{~S}_{n \rightarrow n-k} \mathrm{e}^{+k A z} \Phi_{n-k} .
\end{aligned}
$$


or, explicitly ${ }^{155}$,

$$
\mathrm{S}_{n \rightarrow n+k}=-\frac{1}{k !} \frac{(n+k) !}{n !} S_{1}^{k} .
$$

Along $\theta=\pi$ the story is more intricate. One now finds a very similar result to the case of $\theta=0$ (with $k>0$ )

$$
\Delta_{-k A} \Phi_{n}=-\sum_{s=1}^{k} \frac{1}{s} \sum_{\substack{\left\{\ell_{1}, \ldots, \ell_{s} \geq 1\right\} \\ \sum \ell_{i}=k}} \mathrm{~S}_{n \rightarrow n-\ell_{1}} \mathrm{~S}_{n-\ell_{1} \rightarrow n-\ell_{1}-\ell_{2}} \cdots \mathrm{S}_{n-k+\ell_{s} \rightarrow n-k} \Phi_{n-k}
$$

but where there are no vanishing constraints. Comparing (A.32) with the resurgence relations (A.20) finally yields a closed-form expression for the Stokes constants in terms of the Borel residues $^{156}$ [203]

$$
S_{-k}=-\frac{1}{n-k} \sum_{s=1}^{k} \frac{1}{s} \sum_{\substack{\left\{\ell_{1}, \ldots, \ell_{s} \geq 1\right\} \\ \sum \ell_{i}=k}} \mathrm{~S}_{n \rightarrow n-\ell_{1}} \mathrm{~S}_{n-\ell_{1} \rightarrow n-\ell_{1}-\ell_{2}} \cdots \mathrm{S}_{n-k+\ell_{s} \rightarrow n-k}, \quad 1 \leq k<n .
$$

Before finding the (equivalent) inverse mapping, let us first notice a simple consequence of the resurgence relations (A.20). They yield $\Delta_{-n A} \Phi_{n}=0$, which, in terms of Borel residues, translates to ${ }^{157}$

$$
\mathrm{S}_{n \rightarrow 0}=0, \quad n \geq 1 .
$$

Now, the complete inverse mapping corresponding to (A.33) can be determined for each $k \geq 1$. It is given $b y^{158}$ :

$$
\mathrm{S}_{n \rightarrow n-k}=-\sum_{s=1}^{k} \frac{1}{s !} \sum_{\substack{\left\{\ell_{1}, \ldots, \ell_{s} \geq 1\right\} \\ \sum \ell_{i}=k}} \prod_{a=1}^{s}\left(n-\sum_{j=1}^{a} \ell_{j}\right) \prod_{b=1}^{s} S_{-\ell_{b}}, \quad 1 \leq k<n .
$$

The above formulae also show how the analytic invariants $S_{k}$ are in some sense more "fundamental" than the Borel residues $\mathrm{S}_{n \rightarrow n+k}$. In fact, the analytic invariants only depend on the "distance vector" $k$ between transseries nodes $n$ and $n+k$ (they are "alien-chain translational invariant"), while the Borel residues need to have specified "departure", $n$, and "arrival", $n+k$, nodes in their labels (thus also implicitly depending on this "distance vector" $k$ ). In this way, the analytic invariants depend on less data, and there are less of them. On the other hand, as we shall see next, in spite of less "fundamental" the Borel residues do turn out to appear in simpler combinations than the Stokes constants in most formulae, thus still being rather useful.

\footnotetext{
${ }^{155}$ This also serves as a consistency check on the $n$-independence of $S_{1}$ in (A.29).

${ }^{156}$ Note that although the right-hand sides of these relations have an explicit $n$-dependence, the specific combinations which appear in these formulae are such that the left-hand sides turn out to be $n$-independent. This relates back to the resurgence relations (A.20), where the $n$-dependence of their proportionality coefficient $S_{k}(n+k)$ appears only (and explicitly) in the second factor $(n+k)$. In other words, the Stokes constants $S_{k}$ do not depend on departure or arrival transseries nodes; they are "alien-chain translational-invariant" (unlike the Borel residues).

${ }^{157}$ This restriction was lifted in the example studied in section 2, as it dealt with a two-parameter transseries.

${ }^{158}$ This type of expressions may be written as sums over partitions, and many such closed-form formulae may be found in $[16,15]$. However, the vectorial generalization of this type of expressions - which follows straightforwardly from the above reasoning and was presented in section 5-is perhaps more illuminating.
} 


\section{An Explicit (Linear) Example}

In order to make the above mathematical framework more explicit, let us consider an example based on the partition function addressed in section 6, i.e., a linear example involving a "perturbative" sector and two, distinct "instanton" sectors. The transseries for such partition function will be chosen of the form

$$
\mathcal{Z}(z, \boldsymbol{\sigma})=\sigma_{0} \Phi_{0}(z)+\sigma_{1} \mathrm{e}^{-A_{1} z} \Phi_{1}(z)+\sigma_{2} \mathrm{e}^{+A_{2} z} \Phi_{2}(z),
$$

with $A_{1}, A_{2}>0$, seemingly leading to Borel singularities located at $+A_{1}$ and $-A_{2}$ (being a linear problem, there will be no multi-instanton Borel singularities). Each sector above is, of course, asymptotic (as in (A.1) and (A.16)),

$$
\Phi_{n}(z) \simeq \sum_{g=1}^{+\infty} \frac{\Phi_{g}^{(n)}}{z^{g+\beta_{n}}} .
$$

Let us start by computing the alien derivatives of this example. The bridge equation (A.17) in this problem is

$$
\dot{\Delta}_{\omega} \mathcal{Z}(z, \boldsymbol{\sigma})=\boldsymbol{S}_{\omega}(\boldsymbol{\sigma}) \cdot \frac{\partial \mathcal{Z}}{\partial \boldsymbol{\sigma}}(z, \boldsymbol{\sigma}),
$$

where $\boldsymbol{S}_{\omega}(\boldsymbol{\sigma})$ is now a ( $z$-independent) proportionality vector, which in principle may be built out of monomials of the form $\sigma_{0}^{n} \sigma_{1}^{m} \sigma_{2}^{k}$. However, in this linear example things turn out to be much simpler. In fact, due to linearity, the right-hand side of (A.38) includes the very simple

$$
\boldsymbol{S}_{\omega}(\boldsymbol{\sigma}) \cdot \frac{\partial \mathcal{Z}}{\partial \boldsymbol{\sigma}}=\boldsymbol{S}_{\omega}(\boldsymbol{\sigma}) \cdot\left(\Phi_{0}, \mathrm{e}^{-A_{1} z} \Phi_{1}, \mathrm{e}^{+A_{2} z} \Phi_{2}\right),
$$

whereas the left-hand side may be written as

$$
\dot{\Delta}_{\omega} \mathcal{Z}=\boldsymbol{\sigma} \cdot\left(\mathrm{e}^{-\omega z} \Delta_{\omega} \Phi_{0}, \mathrm{e}^{-\left(\omega+A_{1}\right) z} \Delta_{\omega} \Phi_{1}, \mathrm{e}^{-\left(\omega-A_{2}\right) z} \Delta_{\omega} \Phi_{2}\right) .
$$

This immediately implies that

$$
\boldsymbol{S}_{\omega}(\boldsymbol{\sigma})=\left(\begin{array}{ccc}
S_{\omega}^{00} & S_{\omega}^{01} & S_{\omega}^{02} \\
S_{\omega}^{10} & S_{\omega}^{11} & S_{\omega}^{12} \\
S_{\omega}^{20} & S_{\omega}^{21} & S_{\omega}^{22}
\end{array}\right) \boldsymbol{\sigma} \equiv \mathbf{S}_{\omega} \cdot \boldsymbol{\sigma},
$$

where we have organized the Stokes analytic-invariants into a matrix. Matching equal powers of the transseries parameters in (A.38) leads to the three equations

$$
\mathrm{e}^{-\left(\omega+A_{\ell}\right) z} \Delta_{\omega} \Phi_{\ell}=S_{\omega}^{0 \ell} \Phi_{0}+S_{\omega}^{1 \ell} \mathrm{e}^{-A_{1} z} \Phi_{1}+S_{\omega}^{2 \ell} \mathrm{e}^{+A_{2} z} \Phi_{2},
$$

with $A_{\ell}=0, A_{1},-A_{2}$, respectively, and from where the resurgence relations may be found (alongside the vanishing Stokes constants). Indeed, now matching equal powers of the transmonomials $\mathrm{e}^{-z}$ finally leads to the resurgence relations ${ }^{159}$

$$
\begin{aligned}
\Delta_{A_{1}} \Phi_{0} & =S_{A_{1}}^{10} \Phi_{1}, & \Delta_{-A_{2}} \Phi_{0} & =S_{-A_{2}}^{20} \Phi_{2}, \\
\Delta_{-A_{1}} \Phi_{1} & =S_{-A_{1}}^{01} \Phi_{0}, & \Delta_{-A_{1}-A_{2}} \Phi_{1} & =S_{-A_{1}-A_{2}}^{21} \Phi_{2},
\end{aligned}
$$

\footnotetext{
${ }^{159}$ Recall that Borel transforms cannot have singularities at the origin; this would contradict their definition based on holomorphicity around some neighborhood of the origin.
} 


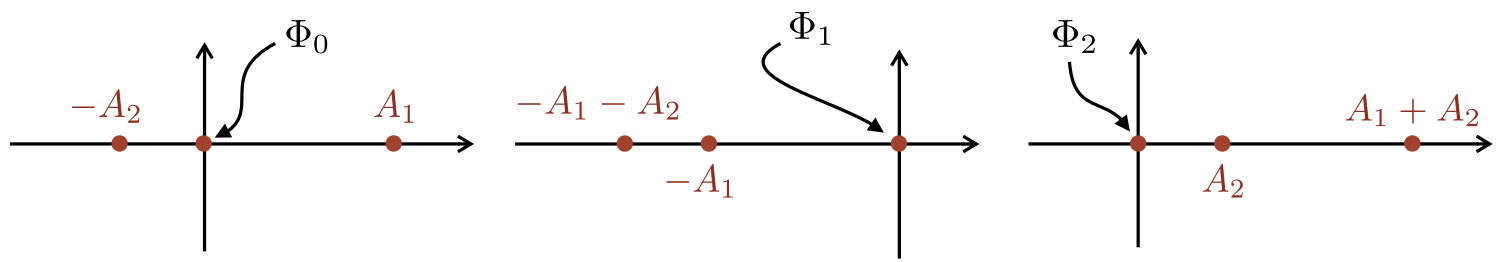

Figure 38. Singularities on the Borel plane, for each of the three sectors $\Phi_{n}$.

$$
\Delta_{A_{2}} \Phi_{2}=S_{A_{2}}^{02} \Phi_{0}, \quad \Delta_{A_{1}+A_{2}} \Phi_{2}=S_{A_{1}+A_{2}}^{12} \Phi_{1}
$$

or, equivalently, to the analytic-invariant matrices ${ }^{160}$

$$
\begin{aligned}
\mathbf{S}_{A_{1}}=\left(\begin{array}{ccc}
0 & 0 & 0 \\
S_{A_{1}}^{10} & 0 & 0 \\
0 & 0 & 0
\end{array}\right), & \mathbf{S}_{A_{2}}=\left(\begin{array}{ccc}
0 & 0 & S_{A_{2}}^{02} \\
0 & 0 & 0 \\
0 & 0 & 0
\end{array}\right), & \mathbf{S}_{A_{1}+A_{2}}=\left(\begin{array}{ccc}
0 & 0 & 0 \\
0 & 0 & S_{A_{1}+A_{2}}^{12} \\
0 & 0 & 0
\end{array}\right) \\
\mathbf{S}_{-A_{1}}=\left(\begin{array}{ccc}
0 & S_{-A_{1}}^{01} & 0 \\
0 & 0 & 0 \\
0 & 0 & 0
\end{array}\right), & \mathbf{S}_{-A_{2}}=\left(\begin{array}{ccc}
0 & 0 & 0 \\
0 & 0 & 0 \\
S_{-A_{2}}^{20} & 0 & 0
\end{array}\right), & \mathbf{S}_{-A_{1}-A_{2}}=\left(\begin{array}{lll}
0 & 0 & 0 \\
0 & 0 & 0 \\
0 & S_{-A_{1}-A_{2}}^{21} & 0
\end{array}\right) .
\end{aligned}
$$

Besides having computed all alien derivatives in the problem (up to the Stokes constants), this shows how there are indeed two Stokes lines: one along $\theta=0$ and another along $\theta=\pi$. The Borel singularity structure is slightly more intricate than one had naïvely guessed from the original transseries, and is fully illustrated in figure 38. Furthermore, Stokes phenomena is now straightforward to implement. Proceeding stepwise, one first writes the directional alien derivatives $\dot{\Delta}_{\theta}$ along $\theta=0$,

$$
\dot{\Delta}_{0}=\mathrm{e}^{-A_{1} z} \Delta_{A_{1}}+\mathrm{e}^{-A_{2} z} \Delta_{A_{2}}+\mathrm{e}^{-\left(A_{1}+A_{2}\right) z} \Delta_{A_{1}+A_{2}}
$$

and along $\theta=\pi$,

$$
\dot{\Delta}_{\pi}=\mathrm{e}^{A_{1} z} \Delta_{-A_{1}}+\mathrm{e}^{A_{2} z} \Delta_{-A_{2}}+\mathrm{e}^{\left(A_{1}+A_{2}\right) z} \Delta_{-A_{1}-A_{2}}
$$

Next, these expansions lead to their corresponding Stokes automorphisms ${ }^{161}$

$$
\begin{aligned}
& \underline{\mathfrak{S}}_{0} \Phi_{0}=\Phi_{0}+S_{A_{1}} \mathrm{e}^{-A_{1} z} \Phi_{1}, \\
& \underline{\mathfrak{S}}_{0} \Phi_{1}=\Phi_{1}, \\
& \underline{\mathfrak{S}}_{0} \Phi_{2}=\Phi_{2}+S_{A_{2}} \mathrm{e}^{-A_{2} z} \Phi_{0}+\left(S_{A_{1}+A_{2}}+\frac{1}{2 !} S_{A_{1}} S_{A_{2}}\right) \mathrm{e}^{-\left(A_{1}+A_{2}\right) z} \Phi_{1},
\end{aligned}
$$

and

$$
\underline{\mathfrak{S}}_{\pi} \Phi_{0}=\Phi_{0}+S_{-A_{2}} \mathrm{e}^{A_{2} z} \Phi_{2}
$$

\footnotetext{
${ }^{160}$ Because each matrix has a single non-vanishing entry, this shows very explicitly how the Stokes constants only depend on their subscript (the "distance vector" between nodes) and not on their superscripts (labeling "departure" and "arrival" transseries nodes), as discussed earlier. In the following we will drop these superscripts and label the Stokes constants only by their "translational invariant" subscripts.

${ }^{161}$ Note how there is only one type of second-order alien derivative along each direction, but no higher-order ones.
} 


$$
\begin{aligned}
& \underline{\mathfrak{S}}_{\pi} \Phi_{1}=\Phi_{1}+S_{-A_{1}} \mathrm{e}^{A_{1} z} \Phi_{0}+\left(S_{-A_{1}-A_{2}}+\frac{1}{2 !} S_{-A_{2}} S_{-A_{1}}\right) \mathrm{e}^{\left(A_{1}+A_{2}\right) z} \Phi_{2}, \\
& \underline{\mathfrak{S}}_{\pi} \Phi_{2}=\Phi_{2} .
\end{aligned}
$$

These operators act on the full partition-function transseries as

$$
\underline{\mathfrak{S}}_{0} \mathcal{Z}(z, \boldsymbol{\sigma})=\mathcal{Z}\left(z, \underline{\mathbb{S}}_{\mathbf{0}} \cdot \boldsymbol{\sigma}\right) \quad \text { and } \quad \underline{\mathfrak{S}}_{\pi} \mathcal{Z}(z, \boldsymbol{\sigma})=\mathcal{Z}\left(z, \underline{\mathbb{S}}_{\boldsymbol{\pi}} \cdot \boldsymbol{\sigma}\right)
$$

where, finally, the flows acting on the transseries parameters $\boldsymbol{\sigma} \mapsto \underline{\mathbb{S}}_{\boldsymbol{\theta}} \cdot \boldsymbol{\sigma}$ are implemented by the Stokes matrices

$$
\underline{S}_{0}=\left(\begin{array}{ccc}
1 & 0 & S_{A_{2}} \\
S_{A_{1}} & 1 & S_{A_{1}+A_{2}}+\frac{1}{2} S_{A_{1}} S_{A_{2}} \\
0 & 0 & 1
\end{array}\right) \quad \text { and } \quad \underline{\mathbb{S}}_{\boldsymbol{\pi}}=\left(\begin{array}{ccc}
1 & S_{-A_{1}} & 0 \\
0 & 1 & 0 \\
S_{-A_{2}} & S_{-A_{1}-A_{2}}+\frac{1}{2} S_{-A_{2}} S_{-A_{1}} & 1
\end{array}\right) \text {. }
$$

It is also interesting to rewrite these matrices using the Borel residues instead of the analytic invariants, via (A.33), where one finds the much neater:

$$
\underline{S}_{0}=\left(\begin{array}{ccc}
1 & 0 & -S_{2 \rightarrow 0} \\
-S_{0 \rightarrow 1} & 1 & -S_{2 \rightarrow 1} \\
0 & 0 & 1
\end{array}\right), \quad \underline{\mathbb{S}}_{\boldsymbol{\pi}}=\left(\begin{array}{ccc}
1 & -S_{1 \rightarrow 0} & 0 \\
0 & 1 & 0 \\
-S_{0 \rightarrow 2} & -S_{1 \rightarrow 2} & 1
\end{array}\right)
$$

\section{Large-Order Behaviour and Transseries Asymptotics}

The one question that remains is: how do the above concepts relate to asymptotics?, which was a central topic throughout these lectures. As discussed at length in the main body of this text, the connection arises via the (Stokes) discontinuity (A.11), and how it may be used within the Cauchy theorem. This was well explained in the main text; here we shall limit ourselves to very briefly readdressing this issue within the alien-calculus mathematical framework outlined above.

As first explained back in subsection 2.3-but now within the example of the partition function (A.36) with Stokes lines along $\theta=0, \pi$-, straightforward application of the Cauchy theorem to each of its asymptotic components (A.37) yields (this is very similar to (2.149))

$$
\Phi(z)=\frac{1}{2 \pi \mathrm{i}} \int_{0}^{+\infty} \mathrm{d} w \frac{\operatorname{Disc}_{0} \Phi(w)}{w-z}+\frac{1}{2 \pi \mathrm{i}} \int_{0}^{-\infty} \mathrm{d} w \frac{\operatorname{Disc}_{\pi} \Phi(w)}{w-z} .
$$

where Disc $\theta=\mathbf{1}-\underline{\mathfrak{S}}_{\theta}$ and $\underline{\mathfrak{S}}_{\theta}$ was computed earlier for each $\Phi_{n}$-sector via alien calculus. Using the asymptotic expansions (A.37) and expanding the above integrands around large $z$, it follows:

- Discontinuities of the perturbative sector $\Phi_{0}$ :

$$
\begin{aligned}
& \operatorname{Disc}_{0} \Phi_{0}(w)=-S_{A_{1}} \mathrm{e}^{-A_{1} w} \Phi_{1}(w), \\
& \operatorname{Disc}_{\pi} \Phi_{0}(w)=-S_{-A_{2}} \mathrm{e}^{A_{2} w} \Phi_{2}(w) .
\end{aligned}
$$

- Asymptotics of the perturbative sector $\Phi_{0}$ (with analytic invariants):

$$
\begin{gathered}
\Phi_{g \gg 1}^{(0)} \simeq \frac{S_{A_{1}}}{2 \pi \mathrm{i}} \frac{\Gamma\left(g-\beta_{1}\right)}{A_{1}^{g-\beta_{1}}} \sum_{h=1}^{+\infty} \frac{\Gamma\left(g-\beta_{1}-h+1\right)}{\Gamma\left(g-\beta_{1}\right)} \Phi_{h}^{(1)} A_{1}^{h-1}+ \\
+\frac{S_{-A_{2}}}{2 \pi \mathrm{i}} \frac{\Gamma\left(g-\beta_{2}\right)}{\left(-A_{2}\right)^{g-\beta_{2}}} \sum_{h=1}^{+\infty} \frac{\Gamma\left(g-\beta_{2}-h+1\right)}{\Gamma\left(g-\beta_{2}\right)} \Phi_{h}^{(2)}\left(-A_{2}\right)^{h-1} .
\end{gathered}
$$


- Asymptotics of the perturbative sector $\Phi_{0}$ (with Borel residues):

$$
\begin{aligned}
\Phi_{g \gg 1}^{(0)} \simeq-\frac{\mathrm{S}_{0 \rightarrow 1}}{2 \pi \mathrm{i}} \frac{\Gamma\left(g-\beta_{1}\right)}{A_{1}^{g-\beta_{1}}} \sum_{h=1}^{+\infty} \frac{\Gamma\left(g-\beta_{1}-h+1\right)}{\Gamma\left(g-\beta_{1}\right)} \Phi_{h}^{(1)} A_{1}^{h-1}- \\
-\frac{\mathrm{S}_{0 \rightarrow 2}}{2 \pi \mathrm{i}} \frac{\Gamma\left(g-\beta_{2}\right)}{\left(-A_{2}\right)^{g-\beta_{2}}} \sum_{h=1}^{+\infty} \frac{\Gamma\left(g-\beta_{2}-h+1\right)}{\Gamma\left(g-\beta_{2}\right)} \Phi_{h}^{(2)}\left(-A_{2}\right)^{h-1} .
\end{aligned}
$$

- Leading large-order behaviour:

$$
\begin{aligned}
\Phi_{g \gg 1}^{(0)} \simeq \frac{S_{A_{1}}}{2 \pi \mathrm{i}} \frac{\Gamma\left(g-\beta_{1}\right)}{A_{1}^{g-\beta_{1}}}\left(\Phi_{1}^{(1)}+\frac{A_{1}}{g-\beta_{1}-1} \Phi_{2}^{(1)}+\cdots\right)+ \\
\quad+\frac{S_{-A_{2}}}{2 \pi \mathrm{i}} \frac{\Gamma\left(g-\beta_{2}\right)}{\left(-A_{2}\right)^{g-\beta_{2}}}\left(\Phi_{1}^{(2)}-\frac{A_{2}}{g-\beta_{2}-1} \Phi_{2}^{(2)}+\cdots\right) .
\end{aligned}
$$

- Discontinuities of the nonperturbative sector $\Phi_{1}$ :

$$
\begin{aligned}
& \operatorname{Disc}_{0} \Phi_{1}(w)=0 \\
& \operatorname{Disc}_{\pi} \Phi_{1}(w)=-S_{-A_{1}} \mathrm{e}^{A_{1} w} \Phi_{0}(w)-\left(S_{-A_{1}-A_{2}}+\frac{1}{2} S_{-A_{2}} S_{-A_{1}}\right) \mathrm{e}^{\left(A_{1}+A_{2}\right) w} \Phi_{2}(w \nmid \text { A.66) }
\end{aligned}
$$

- Asymptotics of the nonperturbative sector $\Phi_{1}$ (with analytic invariants):

$$
\begin{aligned}
& \Phi_{g \gg 1}^{(1)} \simeq \frac{S_{-A_{1}}}{2 \pi \mathrm{i}} \frac{\Gamma\left(g+\beta_{1}\right)}{\left(-A_{1}\right)^{g+\beta_{1}}} \sum_{h=0}^{+\infty} \frac{\Gamma\left(g+\beta_{1}-h-1\right)}{\Gamma\left(g+\beta_{1}\right)} \Phi_{h}^{(0)}\left(-A_{1}\right)^{h+1}+ \\
& +\frac{S_{-A_{1}-A_{2}}+\frac{1}{2} S_{-A_{2}} S_{-A_{1}}}{2 \pi \mathrm{i}} \frac{\Gamma\left(g+\beta_{1}-\beta_{2}\right)}{\left(-A_{1}-A_{2}\right)^{g+\beta_{1}-\beta_{2}}} \sum_{h=1}^{+\infty} \frac{\Gamma\left(g+\beta_{1}-\beta_{2}-h\right)}{\Gamma\left(g+\beta_{1}-\beta_{2}\right)} \Phi_{h}^{(2)}\left(-A_{1}-A_{2}\right)^{h} .
\end{aligned}
$$

- Asymptotics of the nonperturbative sector $\Phi_{1}$ (with Borel residues):

$$
\begin{aligned}
\Phi_{g \gg 1}^{(1)} \simeq-\frac{\mathrm{S}_{1 \rightarrow 0}}{2 \pi \mathrm{i}} & \frac{\Gamma\left(g+\beta_{1}\right)}{\left(-A_{1}\right)^{g+\beta_{1}}} \sum_{h=0}^{+\infty} \frac{\Gamma\left(g+\beta_{1}-h-1\right)}{\Gamma\left(g+\beta_{1}\right)} \Phi_{h}^{(0)}\left(-A_{1}\right)^{h+1}- \\
- & \frac{\mathrm{S}_{1 \rightarrow 2}}{2 \pi \mathrm{i}} \frac{\Gamma\left(g+\beta_{1}-\beta_{2}\right)}{\left(-A_{1}-A_{2}\right)^{g+\beta_{1}-\beta_{2}}} \sum_{h=1}^{+\infty} \frac{\Gamma\left(g+\beta_{1}-\beta_{2}-h\right)}{\Gamma\left(g+\beta_{1}-\beta_{2}\right)} \Phi_{h}^{(2)}\left(-A_{1}-A_{2}\right)^{h} .
\end{aligned}
$$

- Leading large-order behaviour:

$$
\begin{aligned}
\Phi_{g \gg 1}^{(1)} \simeq & \frac{S_{-A_{1}}}{2 \pi \mathrm{i}} \frac{\Gamma\left(g+\beta_{1}\right)}{\left(-A_{1}\right)^{g+\beta_{1}}}\left(-\frac{A_{1}}{g+\beta_{1}-1} \Phi_{0}^{(0)}+\cdots\right)+ \\
& +\frac{S_{-A_{1}-A_{2}}+\frac{1}{2} S_{-A_{2}} S_{-A_{1}}}{2 \pi \mathrm{i}} \frac{\Gamma\left(g+\beta_{1}-\beta_{2}\right)}{\left(-A_{1}-A_{2}\right)^{g+\beta_{1}-\beta_{2}}}\left(-\frac{A_{1}+A_{2}}{g+\beta_{1}-\beta_{2}-1} \Phi_{1}^{(2)}+\cdots\right) .
\end{aligned}
$$


- Discontinuities of the nonperturbative sector $\Phi_{2}$ :

$$
\begin{aligned}
& \operatorname{Disc}_{0} \Phi_{2}(w)=-S_{A_{2}} \mathrm{e}^{-A_{2} w} \Phi_{0}(w)-\left(S_{A_{1}+A_{2}}+\frac{1}{2} S_{A_{1}} S_{A_{2}}\right) \mathrm{e}^{-\left(A_{1}+A_{2}\right) w} \Phi_{1}(w),( \\
& \operatorname{Disc}_{\pi} \Phi_{2}(w)=0 .
\end{aligned}
$$

- Asymptotics of the nonperturbative sector $\Phi_{2}$ (with analytic invariants):

$$
\begin{aligned}
\Phi_{g \gg 1}^{(2)} & \simeq \frac{S_{A_{2}}}{2 \pi \mathrm{i}} \frac{\Gamma\left(g+\beta_{2}\right)}{A_{2}^{g+\beta_{2}}} \sum_{h=0}^{+\infty} \frac{\Gamma\left(g+\beta_{2}-h-1\right)}{\Gamma\left(g+\beta_{2}\right)} \Phi_{h}^{(0)} A_{2}^{h+1}+ \\
& +\frac{S_{A_{1}+A_{2}}+\frac{1}{2} S_{A_{1}} S_{A_{2}}}{2 \pi \mathrm{i}} \frac{\Gamma\left(g+\beta_{2}-\beta_{1}\right)}{\left(A_{1}+A_{2}\right)^{g+\beta_{2}-\beta_{1}}} \sum_{h=1}^{+\infty} \frac{\Gamma\left(g+\beta_{2}-\beta_{1}-h\right)}{\Gamma\left(g+\beta_{2}-\beta_{1}\right)} \Phi_{h}^{(1)}\left(A_{1}+A_{2}\right)^{h} .
\end{aligned}
$$

- Asymptotics of the nonperturbative sector $\Phi_{2}$ (with Borel residues):

$$
\begin{aligned}
\Phi_{g \gg 1}^{(2)} \simeq & -\frac{\mathrm{S}_{2 \rightarrow 0}}{2 \pi \mathrm{i}} \frac{\Gamma\left(g+\beta_{2}\right)}{A_{2}^{g+\beta_{2}}} \sum_{h=0}^{+\infty} \frac{\Gamma\left(g+\beta_{2}-h-1\right)}{\Gamma\left(g+\beta_{2}\right)} \Phi_{h}^{(0)} A_{2}^{h+1}- \\
& -\frac{\mathrm{S}_{2 \rightarrow 1}}{2 \pi \mathrm{i}} \frac{\Gamma\left(g+\beta_{2}-\beta_{1}\right)}{\left(A_{1}+A_{2}\right)^{g+\beta_{2}-\beta_{1}}} \sum_{h=1}^{+\infty} \frac{\Gamma\left(g+\beta_{2}-\beta_{1}-h\right)}{\Gamma\left(g+\beta_{2}-\beta_{1}\right)} \Phi_{h}^{(1)}\left(A_{1}+A_{2}\right)^{h} .
\end{aligned}
$$

- Leading large-order behaviour:

$$
\begin{aligned}
\Phi_{g \gg 1}^{(2)} \simeq & \frac{S_{A_{2}}}{2 \pi \mathrm{i}} \frac{\Gamma\left(g+\beta_{2}\right)}{A_{2}^{g+\beta_{2}}}\left(\frac{A_{2}}{g+\beta_{2}-1} \Phi_{0}^{(0)}+\cdots\right)+ \\
& +\frac{S_{A_{1}+A_{2}}+\frac{1}{2} S_{A_{2}} S_{A_{1}}}{2 \pi \mathrm{i}} \frac{\Gamma\left(g+\beta_{2}-\beta_{1}\right)}{\left(A_{1}+A_{2}\right)^{g+\beta_{2}-\beta_{1}}}\left(\frac{A_{1}+A_{2}}{g+\beta_{2}-\beta_{1}-1} \Phi_{1}^{(1)}+\cdots\right) .
\end{aligned}
$$

Note how the above formulae become invalid and must necessarily change under conditions for resonance, i.e., if $A_{1}+A_{2}=0$. This issue was discussed at length in section 6. Many other closed-form large-order relations, both resonant and non-resonant, may be found in [16].

\section{B Stokes Lines Within the WKB Expansion}

Both Stokes and anti-Stokes lines were discussed at length in the main body of this work, illustrated via, e.g., figures 2 and 3 for the example discussed in section 2, and figures 20, 21 and 22 for the example discussed in section 6 . These examples were associated to one-dimensional integrals for (simplified) partition functions. For the sake of completeness, let us also illustrate how Stokes or anti-Stokes lines appear when considering problems based upon differential equations. Addressing a simple and standard setting (but perhaps also one of the most popular ones), let us briefly discuss the WKB analysis ${ }^{162}$ of quantum mechanical potentials.

Start with the one-dimensional time-independent Schrödinger equation,

$$
\left(-\frac{\hbar^{2}}{2 m} \frac{\mathrm{d}^{2}}{\mathrm{~d} q^{2}}+V(q)\right) \psi(q)=E \psi(q)
$$

\footnotetext{
${ }^{162}$ WKB is textbook material. For a modern introduction see, e.g., [164] and references therein.
} 
and rewrite it (adequately for what follows) as

$$
\hbar^{2} \psi^{\prime \prime}(q)+p^{2}(q) \psi(q)=0
$$

where $p$ is the "classical momentum" $p=\sqrt{2 m(E-V(q))}$ (at least when this result is real). WKB analysis now follows via its familiar ansatz for the wavefunction solution,

$$
\psi(q)=\exp \left(\frac{\mathrm{i}}{\hbar} \int_{q_{0}}^{q} \mathrm{~d} z \mathrm{~V}(z, \hbar)\right)
$$

where $\mathrm{V}(q, \hbar)$ satisfies the Riccati equation $\mathrm{V}^{2}-\mathrm{i} \hbar \mathrm{V}^{\prime}=p^{2}$ via (B.2) and should be thought of as a power series in $\hbar$,

$$
\mathrm{V}(q, \hbar)=\mathrm{V}_{0}(q)+\hbar \mathrm{V}_{1}(q)+\hbar^{2} \mathrm{~V}_{2}(q)+\cdots
$$

In other words, a (perturbative) WKB solution to the above one-dimensional time-independent Schrödinger equation is determined by the recursion:

$$
\begin{aligned}
& \mathrm{V}_{0}= \pm p \\
& \mathrm{~V}_{n}=\frac{1}{2 \mathrm{~V}_{0}}\left(\mathrm{i} \frac{\mathrm{d}}{\mathrm{d} q} \mathrm{~V}_{n-1}-\sum_{k=1}^{n-1} \mathrm{~V}_{k} \mathrm{~V}_{n-k}\right)
\end{aligned}
$$

A simplification of the WKB ansatz (B.3) occurs when one splits even and odd powers of $\hbar$ in the expansion (B.4), as $\mathrm{V}_{\text {even }}(q, \hbar)=\mathrm{V}_{0}(q)+\cdots$ and $\mathrm{V}_{\text {odd }}(q, \hbar)=\hbar \mathrm{V}_{1}(q)+\cdots$. One then immediately notices that the odd coefficients are determined by the even ones,

$$
\mathrm{V}_{\text {odd }}=\frac{\mathrm{i} \hbar}{2} \frac{\mathrm{d}}{\mathrm{d} q} \log \mathrm{V}_{\text {even }}
$$

Replacing this in the WKB ansatz (B.3) and considering the two options implied by the recursion in (B.5), one finally obtains the expected two linearly-independent solutions (in fact forming a basis for the space of solutions)

$$
\psi_{ \pm}(q)=\frac{1}{\sqrt{\mathrm{V}_{\text {even }}(q, \hbar)}} \exp \left( \pm \frac{\mathrm{i}}{\hbar} \int_{q_{0}}^{q} \mathrm{~d} z \mathrm{~V}_{\text {even }}(z, \hbar)\right)
$$

Of course that the WKB solution was perturbative to start-off with, in which case one should still expand these wavefunctions as (asymptotic) series (see, e.g., [65] for some explicit expressions)

$$
\psi_{ \pm}(q) \simeq \exp \left( \pm \frac{\mathrm{i}}{\hbar} \int_{q_{0}}^{q} \mathrm{~d} z p(z)\right) \sum_{k=0}^{+\infty} \hbar^{k} \psi_{ \pm, k}(q) .
$$

These WKB solutions are local, i.e., valid in specific regions of the complex plane. In order to construct a global solution for the wavefunction one needs "connection formulae", in order to stepwise match WKB solutions of the type (B.9), valid in adjacent regions. Further, this match only works if the corresponding WKB solutions have similar (exponential) magnitudes, and thus the relevance of Stokes and anti-Stokes lines in the present framework.

In this way, our focus of interest in this appendix is precisely the above exponential pre-factor. Let us normalize it by setting $q_{0}$ to be a classical turning point, i.e., separating a classically 

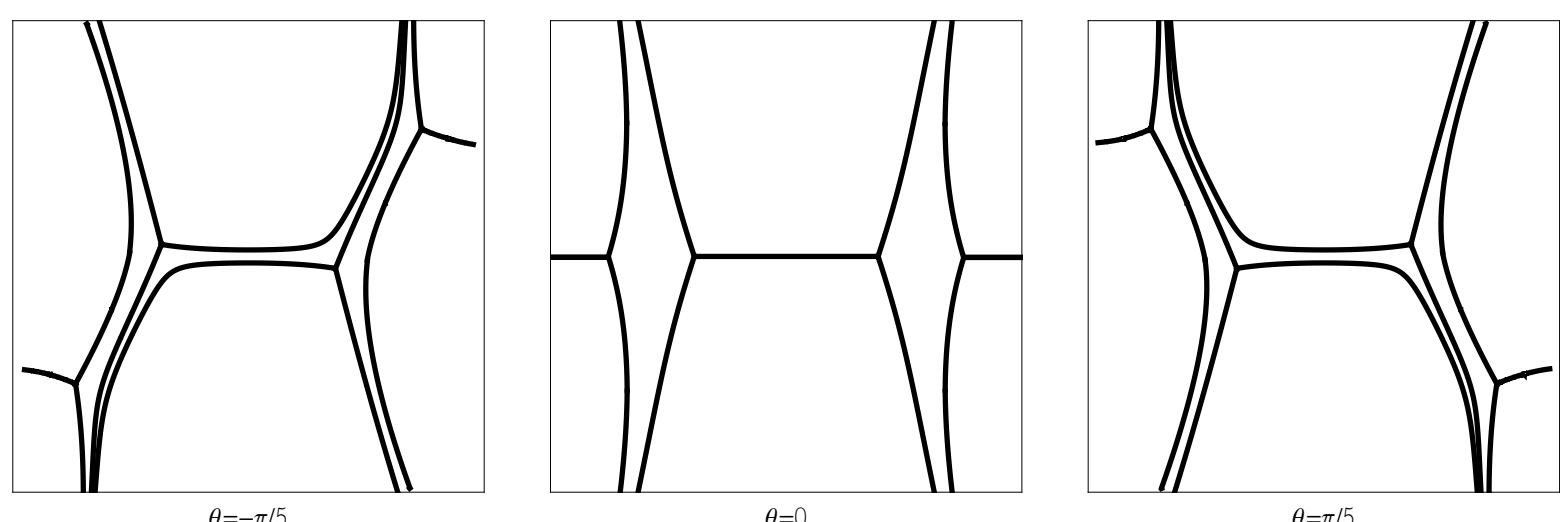

Figure 39. Anti-Stokes lines for the quartic quantum-mechanical potential (B.12). Wall-crossing is very clear across $\theta=0$, when the topology of the network effectively changes.

allowed region from a classically forbidden one. These turning points are the zeroes of the "classical momentum", $p(q)$, which also signal the associated square-root branch points. In this context, at real $\hbar$, Stokes lines of (B.9) are defined as ${ }^{163}$

$$
\mathbb{R e} \int_{q_{0}}^{q} \mathrm{~d} z \sqrt{2 m(E-V(z))}=0
$$

while anti-Stokes lines are (where WKB solutions will have similar exponential weights)

$$
\operatorname{Im} \int_{q_{0}}^{q} \mathrm{~d} z \sqrt{2 m(E-V(z))}=0 .
$$

Note how at simple zeroes of the argument of the square-root, one has $\int \sqrt{z} \sim z^{3 / 2}$, in which case (anti) Stokes lines will form networks with trivalent nodes at the turning points.

Let us illustrate how this WKB matching might work by plotting anti-Stokes lines (B.11) for the quartic potential (which may then be compared to figure 2 in section 2). Recall that in our conventions this potential is (2.9),

$$
V(z)=\frac{1}{2} z^{2}-\frac{\lambda}{24} z^{4} .
$$

Then, at fixed energy $E$, the four turning points are $E=V\left(q_{0}\right)$, i.e.,

$$
q_{0}^{4}-\frac{12}{\lambda} q_{0}^{2}+\frac{24}{\lambda} E=0 \quad \Leftrightarrow \quad q_{0}^{2}=\frac{2 \sqrt{3}}{\lambda}(\sqrt{3} \pm \sqrt{3-2 \lambda E})
$$

The interesting aspect here is that, at fixed energy $E$, these turning points (and, subsequently, the structure of anti-Stokes lines) still depend on the quartic coupling $\lambda$ (a phenomenon which was well addressed in the main text). As such, the whole structure of anti-Stokes lines changes as this parameter is changed. This is illustrated in figure 39 for our quartic potential. In particular, it is quite clear how at $\theta=\arg \lambda=0$ the topology of the network "jumps", a phenomenon sometimes going by the names of "wall crossing" or "parametric Stokes phenomena". See, e.g., [128] for an excellent overview of these WKB spectral networks.

\footnotetext{
${ }^{163}$ Recall the extra factor of $\mathrm{i}$ in the exponential (B.9), as compared to what was discussed in the main text.
} 
Let us finally mention that these techniques are not exclusive to quantum mechanics, and have found applications in many other problems. For instance, they have even been applied in the study of black hole physics (see, e.g., $[186,189,57,188,143]$ ) and, in fact, it would be very interesting to further apply resurgent analysis to this large class of gravitational problems.

\section{On the Nature of Strong-Coupling Expansions}

Our main concern in these lectures dealt with making perturbation theory complete, in the sense of extracting exact information out of initially divergent or asymptotic perturbative expansions. But, of course, not all perturbative expansions need to be divergent or asymptotic. One very interesting feature of many quantum theoretic problems is that while, on the one hand, their weak-coupling perturbative expansions are indeed asymptotic, one finds that instead, on the other hand, their strong-coupling perturbative expansions lead to convergent expansions. Note that this is not a solution to the problems of perturbation theory, as it is not common to have access to such strong-coupling expansions. But, when such a regime is indeed available, this is a rather interesting phenomena which we shall now illustrate.

Let us consider our original example of the "quartic partition function" from section 2, as written in (2.10). In the main text we have addressed the calculation of this partition function using a weak-coupling (small $x \ll 1$ ) perturbative analysis. The (opposite) strong-coupling regime corresponds to large $x \gg 1$ and proceeds as follows ${ }^{164}$. Let us first change variables in the integrand by a simple rescaling $z \rightarrow y=\frac{z}{x^{1 / 4}}$, and let us momentarily work with the now more convenient coupling $\mathrm{x}=x^{1 / 4}$. In this case, the partition function becomes (compare with $(2.10))$

$$
Z(\mathrm{x})=\frac{\sqrt{\hbar}}{2 \pi \mathrm{x}} \int_{\Gamma} \mathrm{d} y \exp \left(-\frac{1}{2 \mathrm{x}^{2}} y^{2}+\frac{1}{24} y^{4}\right)
$$

In the limit where $x \rightarrow+\infty$, one can use the (convergent) power-series expansion of the exponential to write

$$
Z_{\infty}(\mathrm{x})=\frac{\sqrt{\hbar}}{2 \pi \mathrm{x}} \sum_{n=0}^{+\infty} \frac{(-1)^{n}}{2^{n} n !} \frac{1}{\mathrm{x}^{2 n}} \int_{\Gamma} \mathrm{d} y y^{2 n} \mathrm{e}^{\frac{1}{24} y^{4}} .
$$

Of course that the path of integration $\Gamma$ still needs to be chosen such that all integrals above converge. This simply amounts to selecting $\Gamma=\left(-\infty \mathrm{e}^{\mathrm{i} \frac{\pi}{4}},+\infty \mathrm{e}^{\mathrm{i} \frac{\pi}{4}}\right)$, in which case we can evaluate these integrals and obtain

$$
Z_{\infty}(\mathrm{x})=\frac{\sqrt{\hbar}}{2 \pi \mathrm{x}}\left(-\frac{3}{2}\right)^{1 / 4} \sum_{n=0}^{+\infty} \frac{\Gamma\left(\frac{2 n+1}{4}\right)}{n !}\left(-\frac{(-24)^{1 / 2}}{2 \mathrm{x}^{2}}\right)^{n} .
$$

The main point now is that this is a convergent expansion for $x \gg 1$. In fact, one may write

$$
\frac{Z_{\infty}(\mathrm{x})}{\frac{\sqrt{\hbar}}{2 \pi \times}\left(-\frac{3}{2}\right)^{1 / 4}}=\sum_{n=0}^{+\infty} \frac{\Gamma\left(\frac{2 n+1}{4}\right)}{n !}(-w)^{n} \equiv \sum_{n=0}^{+\infty} \zeta_{n} w^{n},
$$

with $w=\frac{(-24)^{1 / 2}}{2 \mathrm{x}^{2}}$, where the radius of convergence of this power series around $w=0(\mathbf{x}=\infty)$ is actually infinite, i.e., the above resulting function has no singularities on the complex plane

\footnotetext{
${ }^{164}$ This is rather standard in the literature. See, e.g., [68] for a recent very similar analysis.
} 
except perhaps at $w=\infty(\mathbf{x}=0)$. These results are not a big surprise, as the sum of this series is actually well-known (see, e.g., [194]):

$$
\sum_{n=0}^{+\infty} \zeta_{n} w^{n}=\mathrm{e}^{\frac{w^{2}}{8}} \sqrt{w} K_{\frac{1}{4}}\left(\frac{w^{2}}{8}\right),
$$

where $K_{\nu}(z)$ is a modified Bessel function of the second kind. Going back to the original variable $x$, this implies that ${ }^{165}$

$$
\begin{aligned}
Z_{\infty}(x) & =\mathrm{e}^{\mathrm{i} \frac{\pi}{2}} \sqrt{\frac{\hbar}{2 \pi}} \sqrt{\frac{3}{2 \pi x}} \mathrm{e}^{-\frac{3}{4 x}} K_{\frac{1}{4}}\left(\mathrm{e}^{\mathrm{i} \pi} \frac{3}{4 x}\right)= \\
& =\mathrm{e}^{\mathrm{i} \frac{\pi}{4}} \sqrt{\frac{\hbar}{2}} \sqrt{\frac{3}{4 x}} \mathrm{e}^{-\frac{3}{4 x}}\left(I_{-\frac{1}{4}}\left(\frac{3}{4 x}\right)-\mathrm{i} I_{\frac{1}{4}}\left(\frac{3}{4 x}\right)\right) .
\end{aligned}
$$

It is interesting to see how exponentials with the "instanton action" naturally appear in our end result even though there is no notion of a Borel plane in this calculation (recall that, in the appropriate regime, there is still an exponential piece inside the Bessel function which, together with the exponential in the expression above, will give rise to the instanton action (2.15)).

In summary, we have managed to compute the partition function (C.1) by summing the convergent expansion at strong coupling $x \gg 1$. But a couple of remarks on how to arrive at this final result are still in order. First, note that the function $K_{1 / 4}(w)$ has two branch points, located at $w=0$ and $w=\infty$. It is the factor $\sqrt{w}$ multiplying the Bessel function which guarantees a convergent behaviour at $w=0$, as $K_{1 / 4}(w)$ behaves like $\log w$ close to the origin. Finally, to obtain the end result above, we used the familiar connection formula/analytic continuation for the modified Bessel functions ${ }^{166}$ (see, e.g., [194]):

$$
\begin{aligned}
K_{\nu}(z) & =\frac{\pi}{2 \sin (\nu \pi)}\left(I_{-\nu}(z)-I_{\nu}(z)\right), \\
K_{\nu}\left(\mathrm{e}^{\mathrm{i} \pi m} \cdot z\right) & =\mathrm{e}^{-\mathrm{i} \nu m \pi} K_{\nu}(z)-\mathrm{i} \pi \frac{\sin (m \nu \pi)}{\sin (\nu \pi)} I_{\nu}(z), \quad m \in \mathbb{Z} .
\end{aligned}
$$

As a consistency check, it would be interesting to compare this result against the one that follows from the transseries solution to the quartic partition-function (2.22) addressed in the main text (for some fixed values of the transseries parameters, $\sigma_{0}, \sigma_{1}$ ). This is particularly interesting in the light of the fact that while the above results were obtained via strong-coupling convergent power-series expansions, the ones in the main text were obtained by working with weak-coupling asymptotic expansions. If we perform this comparison along the positive real axis $\arg x=0$, we must first recall that this line is a Stokes line. Nevertheless, performing the Borel resummation above the real axis (in the direction $\theta=0^{+}$), or, alternatively, choosing the steepest-descent contour passing through the origin and with coupling with a small, positive imaginary part, we can easily obtain the result (C.7). Thus, for $x=|x| \mathrm{e}^{\mathrm{i} \theta}$, with $0<\theta<\pi$, one finds

$$
Z_{\infty}(x)=\mathcal{S}_{\theta} \Phi_{0}(x)
$$

\footnotetext{
${ }^{165}$ As already briefly mentioned in the discussion surrounding (2.43).

${ }^{166}$ In particular, for $\nu=\frac{1}{4}$ we have:

$$
K_{\frac{1}{4}}\left(\mathrm{e}^{\mathrm{i} \pi m} \cdot z\right)=\frac{\pi}{\sqrt{2}} \mathrm{e}^{-\mathrm{i} m \frac{\pi}{4}}\left(I_{-\frac{1}{4}}(z)-\mathrm{e}^{\mathrm{i} m \frac{\pi}{2}} I_{\frac{1}{4}}(z)\right) .
$$
}




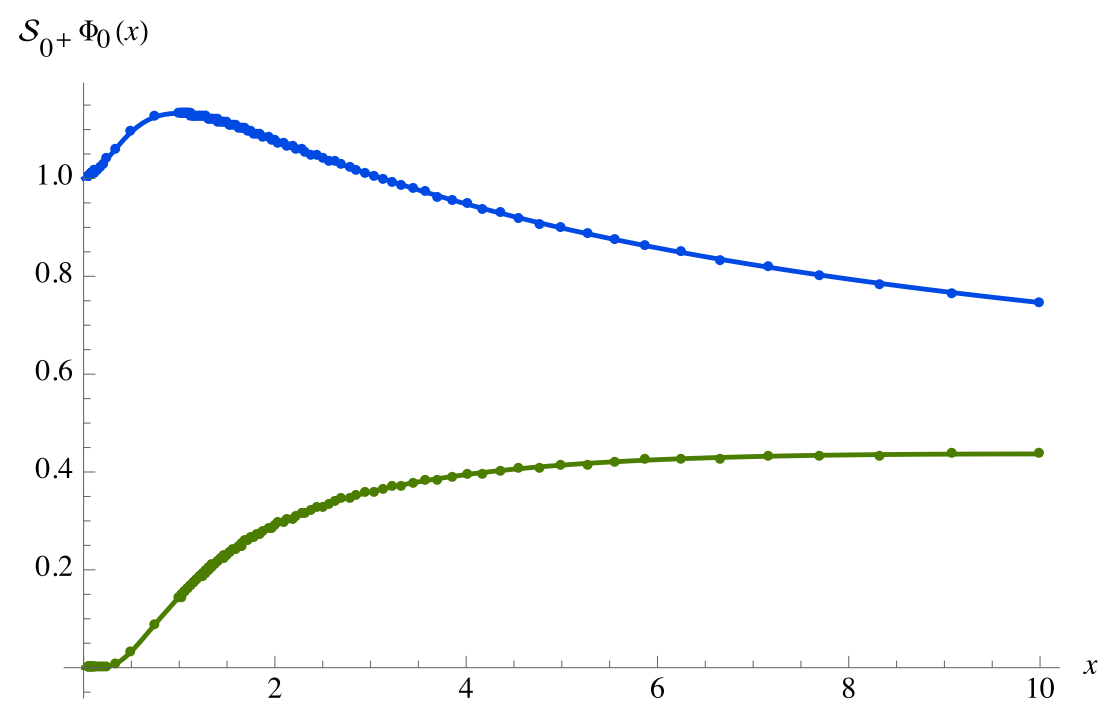

Figure 40. Strong-weak coupling interpolation out from the weak-coupling asymptotic series $\Phi_{0}$, herein Borel resummed along the $\theta=0^{+}$direction. The numerical Borel resummation is depicted by the blue (real part) and green dots (imaginary part). As is clearly seen in the plot, this matches to great accuracy against the corresponding analytic value (solid lines) determined from the strong-coupling result $Z_{\infty}(x)$.

In the specified angular region this result is valid for all values of $|x|$, i.e., the function $Z_{\infty}(x)$ may be analytically continued throughout the entire upper-half plane, always matching against the Borel resummation $\mathcal{S}_{\theta} \Phi_{0}(x)$. We have checked this match numerically by choosing values for the coupling ranging from small $|x| \ll 1$ up to large $|x| \gtrsim 10$. This is actually rather simple to implement numerically in the present case, as there is no need for Padé approximants in order to perform a numerical Borel resummation. In fact, we know the exact Borel transform associated to the perturbative sector, earlier computed in (2.23). Focusing on the ray just above the real axis, with $\theta=0^{+}$, we have computed the corresponding Borel resummation for a sample of values $|x|=\frac{1}{19}, \cdots, 10$. This is depicted by the dots in figure 40 (where the real part of the resummation is plotted in blue, while the imaginary part is plotted in green). These values are then compared against the (blue and green) solid lines, which correspond to the analytical value of $Z_{\infty}(x)$. The resulting perfect match ${ }^{167}$ is very clear in the figure. Effectively, this exercise also shows how to perform a strong-weak coupling (numerical) interpolation, along a particular direction $\theta$, starting only from data associated to the asymptotic expansion at weak coupling.

One may next ask what will happen were we to consider $\theta$ outside of the range $(0, \pi)$ ? The answer is again quite straightforward: all one has to do is to properly take Stokes phenomena into account on the transseries side, and then compare against the strong-coupling result $Z_{\infty}(x)$. In order to illustrate how this works, consider for example the ray just below the positive real line, $\theta=0^{-}$. Computing the Borel resummations $\mathcal{S}_{0^{-}} \Phi_{0}(x)$ and $\mathcal{S}_{0} \Phi_{1}(x)$ is again quite easy to implement (recall that we have previously computed the exact Borel transforms of both perturbative and one-instanton sectors, in equations (2.23) and (2.24)), and we have done so for

\footnotetext{
${ }^{167}$ Note that were we to plot the difference between this Borel resummation and the corresponding value of $Z_{\infty}(x)$, we would obtain an identically vanishing result up to the error of the numerical calculation. It so happens that in this case this error is extremely small, of order $\sim 10^{-200}$. In general, for nonlinear problems, this will not be the case as such a difference can then become of the order of higher, exponentially suppressed contributions.
} 


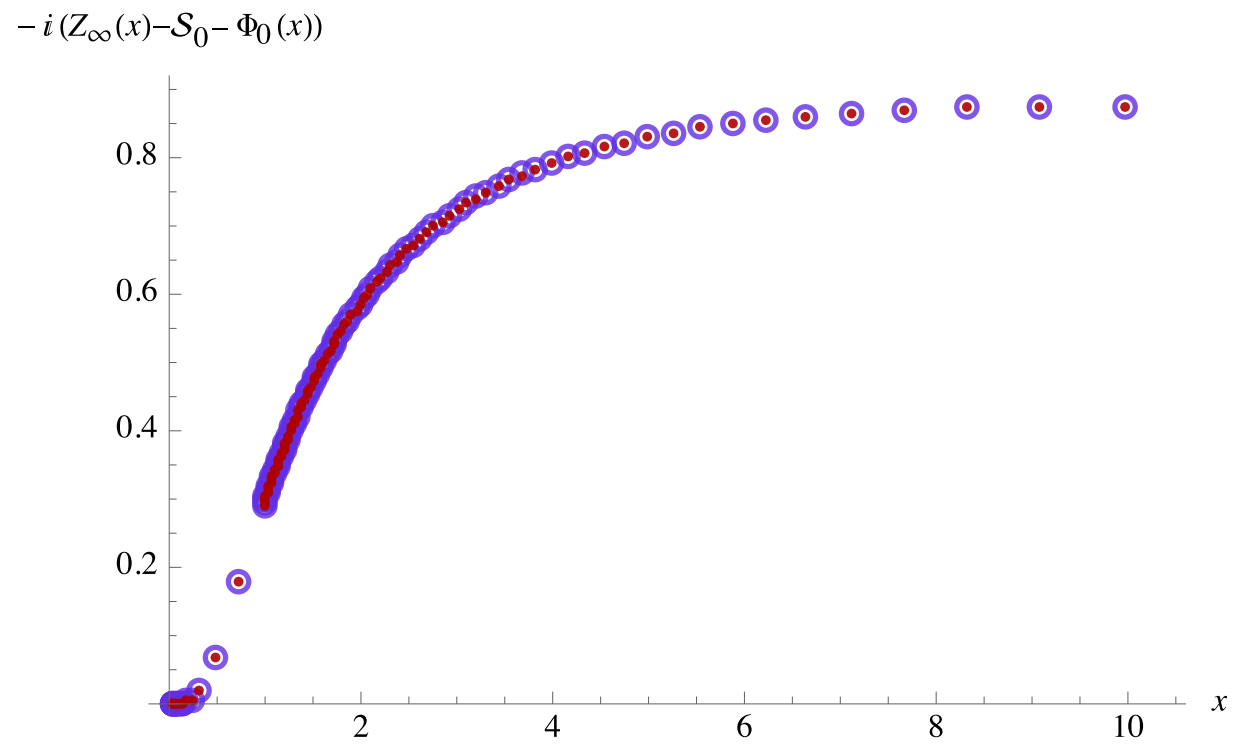

Figure 41. Comparison of the resummation $-\mathrm{i}\left(Z_{\infty}(x)-\mathcal{S}_{0^{-}} \Phi_{0}(x)\right)$, Borel resummed along $\theta=0^{-}$, against the Borel resummation 2i $\mathcal{S}_{0} \Phi_{1}(x)$ along $\theta=0$. The former is plotted as the large purple circles, while the latter is plotted as the (inner) small red dots. As usual, they show perfect agreement.

the same sample of values of the coupling as above, $|x|=\frac{1}{19}, \cdots, 10$. One finds

$$
Z_{\infty}(x)=\mathcal{S}_{0^{-}} \Phi_{0}(x)-2 \mathcal{S}_{0} \Phi_{1}(x)
$$

as expected from the Stokes transition at $\theta=0$. This agreement is shown in figure 41, where we have plotted ${ }^{168}$ both numerical resummations, $-\mathrm{i}\left(Z_{\infty}(x)-\mathcal{S}_{0^{-}} \Phi_{0}(x)\right)$ and $2 \mathrm{i} \mathcal{S}_{0} \Phi_{1}(x)$, for different values of the coupling in the ranges $0<x \ll 1$ and $x \gtrsim 1$. The match is spot on.

In this appendix we discussed the strong-coupling analysis for the partition function of section 2. Doing the same analysis for the free energy, i.e., for a nonlinear problem, would be much more involved: in nonlinear problems it is much harder to have direct access to the strongcoupling region. We have also shown how resummation may interpolate from weak to strong coupling, and such resurgence methods have recently been used in many different problems in order to explicitly connect weak to strong coupling regimes; see, e.g., [175, 135, 62, 80, 12, 79, 66].

\section{Recurrence Relations for Free Energies}

In the main text we dealt with a few nonlinear differential equations, and transseries ansätze were used in order to solve them recursively, i.e., incorporating both (perturbative) analytical and (nonperturbative) non-analytical contributions. This led to intricate recurrence equations for the multi-loop multi-instanton coefficients in the asymptotic expansions building up the transseries, and we present such recursive relations in this appendix. The set-up was already discussed in the main text, herein we shall restrict ourselves to presenting the recurrence relations.

\footnotetext{
${ }^{168}$ Once again, given the simplicity of this linear problem, the difference between the analytic result and the resummed one will vanish up to the extremely small error of the numerical calculation, of order $\sim 10^{-200}$.
} 


\section{Recurrence Relations for the Quartic Free Energies}

The one-parameter transseries (2.58),

$$
F(x, \sigma)=\sum_{\ell=0}^{+\infty} \sigma^{\ell} \mathrm{e}^{-\frac{\ell A}{x}} \Phi_{\ell}(x),
$$

is built out of asymptotic expansions (2.59),

$$
\Phi_{\ell}(x) \simeq \sum_{n=0}^{+\infty} F_{n}^{(\ell)} x^{n}
$$

with multi-loop multi-instanton coefficients $F_{n}^{(\ell)}$. If one is to plug-in this transseries ansatz (2.58) into the quartic nonlinear differential equation (2.52), while keeping track of equal powers of $\mathrm{e}^{-\frac{n A}{x}}$ and $x^{k}$ (recall that $A=\frac{3}{2}$ ), one immediately obtains a set of nonlinear, coupled recursive relations for these multi-loop multi-instanton coefficients $F_{n}^{(\ell)}$. The nonlinearity of the original equation implies that there will be an infinite number of both multi-loop and multi-instanton coefficients.

For $n=0$ and $k \geq 1$,

$$
\begin{aligned}
F_{1}^{(0)} & =\frac{1}{8} \\
F_{2}^{(0)} & =\frac{1}{12} \\
F_{k+1}^{(0)} & =\frac{2}{3}\left(k F_{k}^{(0)}+\frac{1}{k+1} \sum_{\ell=1}^{k-1}(k-\ell) \ell F_{k-\ell}^{(0)} F_{\ell}^{(0)}\right), \quad k \geq 2 .
\end{aligned}
$$

For $n=1$ and $k \geq 1$,

$$
\begin{aligned}
& F_{1}^{(1)}=-\frac{1}{4} F_{0}^{(1)}, \\
& F_{2}^{(1)}=\frac{1}{32} F_{0}^{(1)},
\end{aligned}
$$

$F_{k+1}^{(1)}=-\frac{1}{(k+1) A}\left(k(k+1) F_{k}^{(1)}+2 \sum_{\ell=1}^{k-1}(k-\ell) \ell F_{k-\ell}^{(1)} F_{\ell}^{(0)}+2 A \sum_{\ell=0}^{k}(k-\ell+1) F_{k-\ell+1}^{(0)} F_{\ell}^{(1)}\right)$.

For $n \geq 2$ and $k \geq 0$,

$$
\begin{aligned}
F_{k}^{(n)}= & -\frac{1}{n(n-1)} \sum_{m=1}^{n-1}(n-m) m \sum_{\ell=0}^{k} F_{k-\ell}^{(n-m)} F_{\ell}^{(m)}, \quad k=0,1, \\
F_{k}^{(n)}= & -\frac{1}{n(n-1) A^{2}}\left(A^{2} \sum_{m=1}^{n-1}(n-m) m \sum_{\ell=0}^{k} F_{k-\ell}^{(n-m)} F_{\ell}^{(m)}+(2 n-1)(k-1) A F_{k-1}^{(n)}+\right. \\
& +(k-2)(k-1) F_{k-2}^{(n)}+2 A \sum_{m=1}^{n} \sum_{\ell=0}^{k-2} m(k-\ell-1) F_{k-\ell-1}^{(n-m)} F_{\ell}^{(m)}+
\end{aligned}
$$




$$
\left.+\sum_{m=0}^{n} \sum_{\ell=1}^{k-3} \ell(k-\ell-2) F_{k-\ell-2}^{(n-m)} F_{\ell}^{(m)}\right), \quad k \geq 2 .
$$

Do note that the coefficients $F_{0}^{(0)}$ and $F_{0}^{(1)}$ are not fixed by any recurrence relations; they are unknown integration constants. Further recall that throughout this paper, we are using conventions where if the upper limit of a sum is less than its lower limit, that sum has no contribution.

\section{Recurrence Relations for the Elliptic Free Energies}

For the case of the elliptic nonlinear differential equation (6.68) one deals with a "two-anda-half" transseries (6.73). This may be turned into an effective two-parameter transseries by simply incorporating the sectors $\widetilde{\Phi}_{(0,0)} \equiv \Phi_{(1,0,0)}$ and $\Phi_{(0,0,0)}$ into a new, perturbative sector which, with some abuse of notation, we shall denote in this appendix by $\Phi_{(0,0)}$. As such, this effective two-parameter transseries is written as

$$
F(x, \boldsymbol{\sigma})=\sum_{\boldsymbol{n} \in \mathbb{N}_{0}^{2}} \boldsymbol{\sigma}^{\boldsymbol{n}} \mathrm{e}^{-\frac{\boldsymbol{n} \cdot \boldsymbol{A}}{x}} \Phi_{\boldsymbol{n}}(x),
$$

where we are using the usual notation from sections 5 and 6 , and it is built out of the asymptotic expansions

$$
\begin{gathered}
\Phi_{(0,0)}(x)=\sigma_{0} \Phi_{(1,0,0)}+\Phi_{(0,0,0)} \simeq \sum_{k=0}^{+\infty} F_{k}^{(0,0)} x^{k}, \\
\Phi_{\boldsymbol{n}}(x)=\Phi_{(0, \boldsymbol{n})} \simeq \sum_{k=0}^{+\infty} F_{k}^{(\boldsymbol{n})} x^{k}, \quad \boldsymbol{n} \neq(0,0) .
\end{gathered}
$$

Just as in the quartic case, if one is to plug-in the above transseries ansatz into the elliptic nonlinear differential equation (6.68), while keeping track of equal powers of $\mathrm{e}^{-\frac{n \cdot \boldsymbol{A}}{x}}$ and $x^{k}$ (recall that $\boldsymbol{A}=\left(\frac{1}{1-m},-\frac{1}{m}\right)$ ), one immediately obtains a set of nonlinear, coupled recursive relations for the multi-loop multi-instanton coefficients $F_{k}^{(\boldsymbol{n})}$. The nonlinearity of the original equation implies that there will be an infinite number of both multi-loop and multi-instanton coefficients.

Let us start by analyzing the non-resonant case. Define the useful combination:

$$
c_{k}^{(\boldsymbol{n})} \equiv(\boldsymbol{n} \cdot \boldsymbol{A}) F_{k}^{(\boldsymbol{n})}+(k-1) F_{k-1}^{(\boldsymbol{n})},
$$

where we are assuming, as usual, that $F_{k}^{(\boldsymbol{n})}=0$ when $k<0$. Then, these recursion relations may be written in a more-or-less compact form as

$$
\begin{aligned}
0= & -\left(m-m^{\prime}\right) \delta_{k, 2} \delta_{\boldsymbol{n}, \mathbf{0}}+3 m m^{\prime} \delta_{k, 3} \delta_{\boldsymbol{n}, \mathbf{0}}+4\left(m m^{\prime}(\boldsymbol{n} \cdot \boldsymbol{A})^{2}-\left(m-m^{\prime}\right)(\boldsymbol{n} \cdot \boldsymbol{A})-1\right) c_{k}^{(\boldsymbol{n})}+ \\
& +4(k-1)\left(2 m m^{\prime}(\boldsymbol{n} \cdot \boldsymbol{A})-\left(m-m^{\prime}\right)\right) c_{k-1}^{(\boldsymbol{n})}+m m^{\prime}(3+4(k-1)(k-2)) c_{k-2}^{(\boldsymbol{n})}- \\
& -4\left(m-m^{\prime}\right) \sum_{n_{1}^{\prime}=0}^{n_{1}} \sum_{n_{2}^{\prime}=0}^{n_{2}} \sum_{k^{\prime}=0}^{k} c_{k-k^{\prime}}^{\left(\boldsymbol{n}-\boldsymbol{n}^{\prime}\right)} c_{k^{\prime}}^{\left(\boldsymbol{n}^{\prime}\right)}+ \\
& +12 m m^{\prime} \sum_{n_{1}^{\prime}=0}^{n_{1}} \sum_{n_{2}^{\prime}=0}^{n_{2}} \sum_{k^{\prime}=0}^{k}\left(\left(\boldsymbol{n}^{\prime} \cdot \boldsymbol{A}\right) c_{k^{\prime}}^{\left(\boldsymbol{n}^{\prime}\right)}+\left(k^{\prime}-1\right) c_{k^{\prime}-1}^{\left(\boldsymbol{n}^{\prime}\right)}\right) c_{k-k^{\prime}}^{\left(\boldsymbol{n}-\boldsymbol{n}^{\prime}\right)}+
\end{aligned}
$$




$$
+4 m m^{\prime}\left(\sum_{n_{1}^{\prime}=0}^{n_{1}} \sum_{n_{1}^{\prime \prime}=0}^{n_{1}^{\prime}} \sum_{n_{2}^{\prime}=0}^{n_{2}} \sum_{n_{2}^{\prime \prime}=0}^{n_{2}^{\prime}} \sum_{k^{\prime}=0}^{k} \sum_{k^{\prime \prime}=0}^{k^{\prime}} c_{k-k^{\prime}}^{\left(\boldsymbol{n}-\boldsymbol{n}^{\prime}\right)} c_{k^{\prime}-k^{\prime \prime}}^{\left(\boldsymbol{n}^{\prime}-\boldsymbol{n}^{\prime \prime}\right)} c_{k^{\prime \prime}}^{\left(\boldsymbol{n}^{\prime \prime}\right)}\right),
$$

where we set $k \geq 0$ and used $\boldsymbol{n}-\boldsymbol{n}^{\prime}=\left(n_{1}-n_{1}^{\prime}, n_{2}-n_{2}^{\prime}\right)$. Note that for $\boldsymbol{n}=\mathbf{0}$ the coefficients are $c_{k}^{(\mathbf{0})}=0$ for $k=0,1$, which means that $F_{0}^{(\mathbf{0})}$ remains indeterminate from the recursion. Furthermore, the coefficients $c_{0}^{1,0}$ and $c_{0}^{0,1}$ also cannot be determined from the recursion relations.

In the resonant case, there are some extra subtleties. As discussed at length in section 6, resonance implies that different sectors $\boldsymbol{n}$ will end-up leading to the same exponential factor $\mathrm{e}^{-\frac{\boldsymbol{n} \cdot \boldsymbol{A}}{x}}$. For instance, when $m=1 / 3$ the exponential depends only on an element $\boldsymbol{\rho} \in \mathbb{N}^{2} / \operatorname{ker} \mathfrak{P}$. In this case, defining $\boldsymbol{n}=\left(n_{1}, n_{2}\right) \equiv \boldsymbol{\rho}_{\alpha}+\kappa \mathfrak{n}_{0}$, where $\mathfrak{n}_{0}=(2,1)$, will result in the same exponential factor for any $\kappa \in \mathbb{N}$. This sort of effects may naturally change the above recursion relations. One example where this phenomenon of resonance is particularly visible is when one tries to write the recursion relations for the sector $\boldsymbol{n}=(2,2)=\boldsymbol{\rho}_{\alpha}+\mathfrak{n}_{0}$ (or any other sectors with $\kappa \neq 0)$. Let us illustrate what happens by considering the equations for $c_{0}^{(2,2)}$ and $c_{1}^{(2,2)}$, as a function of the parameter $m$. They are:

$$
\begin{aligned}
& 0=4(9(m-1) m+2) c_{0}^{(2,2)}+12(2 m-1)(3 m-2)(3 m-1), \\
& 0=4(9(m-1) m+2) c_{1}^{(2,2)}+18(9(m-1) m+2)(2 m-1)^{2} .
\end{aligned}
$$

Setting $m=1 / 3$ in both these equations makes their right-hand side identically vanish, and we cannot solve for the unknown coefficients. An analogous situation may be found in the realm of Painlevé equations, e.g., the analysis of resonance for the Painlevé I [130, 16] and Painlevé II equations [205]. In those cases, resonance implied that the transseries itself needed to be modified in order to include logarithmic sectors (which lifted the indeterminacy created by the resonant behaviour). In the present case this is not necessary: one can study solutions to the elliptic nonlinear ODE as functions ${ }^{169}$ of the free parameter $m$. In this way, the solutions to the above (apparently indetermined) equations are simply $c_{0}^{(2,2)}=-3\left(m-m^{\prime}\right)$ and $c_{1}^{(2,2)}=-\frac{9}{2}\left(m-m^{\prime}\right)^{2}$, both of which have well-defined limits when $m=1 / 3$. In this way it is possible to determine every resonant sector, simply from the recursion relations for arbitrary $m$.

\section{E Further Details on Borel Transforms}

This appendix presents a formal derivation of the relation between Borel transforms within the same resurgence class; equation (4.57) in the main text. Let us consider a family of asymptotic series in the same resurgence class - with the class specified by the constants $\alpha$ and $\beta$ (different sets yielding different classes) and the family $\left\{\Phi_{[\gamma]}\right\}$ parameterized by $\gamma$ - which is given by

$$
\Phi_{[\gamma]}(\lambda)=\lambda^{\gamma} \Phi_{[0]}(\lambda) \simeq \sum_{k=0}^{+\infty} \Phi_{k} \lambda^{\alpha k+\beta+\gamma},
$$

and where we are taking $\Phi_{[0]}$ to be the standard representative. This essentially means that the coefficients $\Phi_{k}$ have the precise (large-order) factorial growth of

$$
\Phi_{k} \sim(\alpha k+\beta-1) !
$$

\footnotetext{
${ }^{169}$ This is not an option in the Painlevé cases, where resonance is not "tunable" as in the present example.
} 
and that the Borel transform $\mathcal{B}\left[\Phi_{[0]}\right](s)$ will have the singular structure (4.53). All other Borel transforms, associated with each other element in this family of functions, will be defined as

$$
\mathcal{B}\left[\widehat{\Phi}_{[\gamma]}\right](s)=\sum_{k=n_{0}(\gamma)}^{+\infty} \Phi_{k} \frac{s^{\alpha k+\beta+\gamma-1}}{\Gamma(\alpha k+\beta+\gamma)} .
$$

Let us explain the notation. In order to obtain the Borel transform of the generic element $\Phi_{[\gamma]}(\lambda)$, one first needs to remove the first $k<n_{0}(\gamma)$ terms within its asymptotic expansion, in such a way that $\alpha n_{0}(\gamma)+\beta+\gamma>0$ and the Borel transform is thus well-defined. This "truncated" asymptotic series is then denoted by $\widehat{\Phi}_{[\gamma]}(\lambda)$. As to the terms which get removed, they are handled separately, and can be added back-in at the very end, once we have Borel resummed and obtained $\mathcal{S}_{\theta} \widehat{\Phi}_{[\gamma]}$ following the standard procedures. In order to simplify our present discussion, we will not consider these terms as they are always straightforward to add at any stage.

For integer values of $\gamma$, the straightforward relation (with $n, m \in \mathbb{N}^{+}$)

$$
\frac{s^{n}}{\Gamma(n+1)}=\frac{\mathrm{d}^{m}}{\mathrm{~d} s^{m}} \frac{s^{n+m}}{\Gamma(n+m+1)}
$$

directly implies the relation between Borel transforms ${ }^{170}$

$$
\mathcal{B}\left[\widehat{\Phi}_{\left[\gamma^{\prime}\right]}\right](s)=D_{s}^{\gamma-\gamma^{\prime}} \mathcal{B}\left[\widehat{\Phi}_{[\gamma]}\right](s) .
$$

Assuming that $\gamma-\gamma^{\prime}$ is an integer and that $\gamma>\gamma^{\prime}$, the operator $D_{s}^{n}$ is herein defined as the $n$th derivative with respect to $s$. The relation (E.5) also holds if $\gamma<\gamma^{\prime}$, in which case one simply defines $D_{s}^{n}$ as the $n$th primitive $e^{171}$ with respect to $s$. If $\gamma=\gamma^{\prime}$ then $D_{s}^{0}$ is simply the identity.

The assumption that $\gamma-\gamma^{\prime}$ is an integer may be lifted, allowing (E.5) to be valid for a continuous parameter $\gamma \in \mathbb{R}$. Let us show how this is done when ${ }^{172} \gamma>\gamma^{\prime}$. In this case we have to deal with a fractional derivative, where $D_{s}^{\alpha}$ is defined, when $0<\alpha<1$, as (see, e.g., [166])

$$
D_{s}^{\alpha} f(s)=\frac{1}{\Gamma(1-\alpha)} \frac{\mathrm{d}}{\mathrm{d} s} \int_{0}^{s} \mathrm{~d} t \frac{f(t)}{(s-t)^{\alpha}} .
$$

This definition naturally extends to any real order $\gamma$ by setting $\gamma=\alpha+m$, with $\gamma \in \mathbb{R}, m \in \mathbb{N}_{+}$, and $\alpha$ as above, in which case

$$
D_{s}^{\gamma} f(s)=D_{s}^{\alpha} D_{s}^{m} f(s)=D_{s}^{\alpha} \frac{\mathrm{d}^{m} f}{\mathrm{~d} s^{m}}(s) .
$$

Applying the fractional derivative to each term in the definition of a Borel transform is straightforward; one only needs to know that, for $f(s)=s^{\rho}, \rho>-1$, one has

$$
D_{s}^{\alpha} s^{\rho}=\frac{\Gamma(\rho+1)}{\Gamma(\rho+1-\alpha)} s^{\rho-\alpha} .
$$

\footnotetext{
${ }^{170}$ As an historical aside, note that this sort of relations was probably first noticed in [24], but, unfortunately, the role of the standard representative - with its (simple) logarithmic ramified structure, and where resurgent analysis becomes most straightforward - was not emphasized enough to spark subsequent further analysis.

${ }^{171}$ Each primitive yields an integration constant, and upon multiple primitivation one obtains a polynomial in $s$ with maximum degree $\gamma^{\prime}-\gamma$. This polynomial is directly related to the first $n_{0}(\gamma)$ coefficients which were removed from the original asymptotic series $\Phi_{[\gamma]}(\lambda)$ in order to obtain the "Borel transformable" asymptotic series $\widehat{\Phi}_{[\gamma]}(\lambda)$.

${ }^{172}$ The case where $\gamma<\gamma^{\prime}$ follows in complete analogy by simply inverting (E.5) and having the operator $D_{s}^{\gamma^{\prime}-\gamma}$, which is now a derivative, acting on $\mathcal{B}\left[\widehat{\Phi}_{\left[\gamma^{\prime}\right]}\right](s)$.
} 
The action of the fractional derivative $D_{s}^{\gamma-\gamma^{\prime}}$ on the Borel transform $\mathcal{B}\left[\widehat{\Phi}_{[\gamma]}\right](s)$ in (E.5) can then be easily computed making use of the above expression order-by-order in the series definition of $\mathcal{B}\left[\widehat{\Phi}_{[\gamma]}\right](s)$. One obtains

$$
D_{s}^{\gamma-\gamma^{\prime}} \mathcal{B}\left[\widehat{\Phi}_{[\gamma]}\right](s)=\sum_{k=n_{0}(\gamma)}^{+\infty} \Phi_{k} \frac{s^{\alpha k+\beta+\gamma^{\prime}-1}}{\Gamma\left(\alpha k+\beta+\gamma^{\prime}\right)} \approx \mathcal{B}\left[\widehat{\Phi}_{\left[\gamma^{\prime}\right]}\right](s) .
$$

We have used the symbol $\approx$ as a reminder that this equality only holds up to the removal/addition of $\gamma-\gamma^{\prime}$ initial terms in the expansions (residual coefficients et al).

This shows how to relate Borel transforms of asymptotic series within the same resurgence class. Note that the derivation above was done at the level of the series expansions of the respective Borel transforms, around $s \sim 0$. One natural question is whether such relation still holds should we instead expand the Borel transforms around their singularities - and thus if generic resurgence structures may always be mapped back to those associated to the standard representative. In the main text we have illustrated how this holds in the example of the quartic partition-function, where this relation is upheld by a semi-derivative, $D_{s}^{1 / 2}$. This should be a generic feature, with the relation (E.5) holding around singularities of Borel transforms. 


\section{References}

[1] A. Ahmed and G.V. Dunne, Transmutation of a Trans-Series: The Gross-Witten-Wadia Phase Transition, JHEP 1711 (2017) 054, arXiv: 1710.01812 [hep-th].

[2] K. Aitken, A. Cherman, E. Poppitz and L.G. Yaffe, QCD on a Small Circle, Phys. Rev. D96 (2017) 096022, arXiv:1707.08971 [hep-th].

[3] A. Alexandru, G. Başar and P. Bedaque, Monte Carlo Algorithm for Simulating Fermions on Lefschetz Thimbles, Phys. Rev. D93 (2016) 014504, arXiv:1510.03258[hep-lat].

[4] A. Alexandru, G. Başar, P.F. Bedaque, G.W. Ridgway and N.C. Warrington, Sign Problem and Monte Carlo Calculations Beyond Lefschetz Thimbles, JHEP 1605 (2016) 053, arXiv: 1512.08764 [hep-lat].

[5] A. Alexandru, G. Başar, P.F. Bedaque, G.W. Ridgway and N.C. Warrington, Monte Carlo Calculations of the Finite Density Thirring Model, Phys. Rev. D95 (2017) 014502, arXiv: 1609.01730[hep-lat].

[6] A. Alexandru, G. Başar, P.F. Bedaque, S. Vartak and N.C. Warrington, Monte Carlo Study of Real Time Dynamics on the Lattice, Phys. Rev. Lett. 117 (2016) 081602, arXiv:1605.08040 [hep-lat].

[7] G. Álvarez, Langer-Cherry Derivation of the Multi-Instanton Expansion for the Symmetric Double Well, J. Math. Phys. 45 (2004) 3095.

[8] G. Álvarez and C. Casares, Uniform Asymptotic and JWKB Expansions for Anharmonic Oscillators, J. Phys. A: Math. Gen. 33 (2000) 2499.

[9] G. Álvarez and C. Casares, Exponentially Small Corrections in the Asymptotic Expansion of the Eigenvalues of the Cubic Anharmonic Oscillator, J. Phys. A: Math. Gen. 33 (2000) 5171.

[10] G. Álvarez, C.J. Howls and H.J. Silverstone, Anharmonic Oscillator Discontinuity Formulae Up To Second-Exponentially-Small Order, J. Phys. A: Math. Gen. 35 (2002) 4003.

[11] G. Álvarez and H.J. Silverstone, A New Method to Sum Divergent Power Series: Educated Match, J. Phys. Commun. 1 (2017) 025005, arXiv: 1706.00329 [math-ph].

[12] I. Aniceto, The Resurgence of the Cusp Anomalous Dimension, J. Phys. A49 (2016) 065403, arXiv: 1506.03388 [hep-th].

[13] I. Aniceto, F. Fauvet, D. Sauzin and R. Schiappa, Parametric Resurgence, to appear (2018) (http://online.kitp.ucsb.edu/online/resurgent_c17/sauzin).

[14] I. Aniceto, J.G. Russo and R. Schiappa, Resurgent Analysis of Localizable Observables in Supersymmetric Gauge Theories, JHEP 1503 (2015) 172, arXiv:1410.5834[hep-th].

[15] I. Aniceto and R. Schiappa, Nonperturbative Ambiguities and the Reality of Resurgent Transseries, Commun. Math. Phys. 335 (2015) 183, arXiv:1308.1115[hep-th].

[16] I. Aniceto, R. Schiappa and M. Vonk, The Resurgence of Instantons in String Theory, Commun. Number Theor. Phys. 6 (2012) 339, arXiv:1106.5922 [hep-th].

[17] I. Aniceto, R. Schiappa and M. Vonk, Painlevé Resurgent Transseries, to appear (2018) (http://online.kitp.ucsb.edu/online/resurgent_c17/vonk).

[18] I. Aniceto, R. Schiappa and M. Vonk, Large N Anti-Stokes Phases, to appear (2018) (http://online.kitp.ucsb.edu/online/resurgent_c17/aniceto).

[19] I. Aniceto and M. Spaliński, Resurgence in Extended Hydrodynamics, Phys. Rev. D93 (2016) 085008, arXiv: 1511.06358 [hep-th] .

[20] P.C. Argyres and M. Ünsal, A Semiclassical Realization of Infrared Renormalons, Phys. Rev. Lett. 109 (2012) 121601, arXiv:1204.1661 [hep-th]. 
[21] P.C. Argyres and M. Ünsal, The Semiclassical Expansion and Resurgence in Gauge Theories: New Perturbative, Instanton, Bion, and Renormalon Effects, JHEP 1208 (2012) 063, arXiv: 1206.1890 [hep-th].

[22] G. Arutyunov, D. Dorigoni and S. Savin, Resurgence of the Dressing Phase for $\operatorname{AdS}_{5} \times \mathbb{S}^{5}$, JHEP 1701 (2017) 055, arXiv:1608.03797 [hep-th].

[23] R. Balian, G. Parisi and A. Voros, Discrepancies from Asymptotic Series and their Relation to Complex Classical Trajectories, Phys. Rev. Lett. 41 (1978) 1141.

[24] R. Balian, G. Parisi and A. Voros, Quartic Oscillator, in "Marseille Workshop on Feynman Path Integrals" (1978).

[25] G. Başar and G.V. Dunne, Resurgence and the Nekrasov-Shatashvili Limit: Connecting Weak and Strong Coupling in the Mathieu and Lamé Systems, JHEP 1502 (2015) 160, arXiv: 1501.05671 [hep-th].

[26] G. Başar and G.V. Dunne, Hydrodynamics, Resurgence and Trans-Asymptotics, Phys. Rev. D92 (2015) 125011, arXiv: 1509.05046 [hep-th].

[27] G. Başar, G.V. Dunne and M. Ünsal, Resurgence Theory, Ghost-Instantons, and Analytic Continuation of Path Integrals, JHEP 1310 (2013) 041, arXiv:1308.1108[hep-th].

[28] G. Başar, G.V. Dunne and M. Ünsal, Quantum Geometry of Resurgent Perturbative/Nonperturbative Relations, JHEP 1705 (2017) 087, arXiv:1701.06572 [hep-th].

[29] A. Behtash, G.V. Dunne, T. Schäfer, T. Sulejmanpasic and M. Ünsal, Complexified Path Integrals, Exact Saddles and Supersymmetry, Phys. Rev. Lett. 116 (2016) 011601, arXiv:1510.00978[hep-th].

[30] A. Behtash, E. Poppitz, T. Sulejmanpasic and M. Ünsal, The Curious Incident of Multi-Instantons and the Necessity of Lefschetz Thimbles, JHEP 1511 (2015) 175, arXiv:1507.04063 [hep-th].

[31] A. Behtash, T. Sulejmanpasic, T. Schaefer and M. Ünsal, Hidden Topological Angles and Lefschetz Thimbles, Phys. Rev. Lett. 115 (2015) 041601, arXiv: 1502.06624 [hep-th].

[32] M.P. Bellon, Alien Calculus and Nonperturbative Effects in Quantum Field Theory, Front. Phys. 11 (2016) 113201, arXiv: 1701.02294 [hep-th].

[33] M.P. Bellon and P.J. Clavier, A Schwinger-Dyson Equation in the Borel Plane: Singularities of the Solution, Lett. Math. Phys. 105 (2015) 795, arXiv:1411.7190 [math-ph].

[34] M.P. Bellon and P.J. Clavier, Alien Calculus and a Schwinger-Dyson Equation: Two-Point Function with a Nonperturbative Mass Scale, Lett. Math. Phys. 108 (2018) 391, arXiv: 1612 .07813 [hep-th] .

[35] C.M. Bender and C. Heissenberg, Convergent and Divergent Series in Physics, in "22th Saalburg Summer School on Foundations and New Methods in Theoretical Physics" (2016), arXiv: 1703.05164 [math-ph].

[36] C.M. Bender, K. Olaussen and P.S. Wang, Numerological Analysis of the WKB Approximation in Large Order, Phys. Rev. D16 (1977) 1740.

[37] C.M. Bender and S.A. Orszag, Advanced Mathematical Methods for Scientists and Engineers, Mc-Graw Hill (1978), Springer-Verlag (1999).

[38] C.M. Bender and T.T. Wu, Anharmonic Oscillator, Phys. Rev. 184 (1969) 1231.

[39] C.M. Bender and T.T. Wu, Anharmonic Oscillator 2: A Study of Perturbation Theory in Large Order, Phys. Rev. D7 (1973) 1620.

[40] M. Beneke, Renormalons, Phys. Rept. 317 (1999) 1, arXiv:hep-ph/9807443.

[41] M. Berry, Asymptotics, Superasymptotics, Hyperasymptotics, in "Asymptotics Beyond All Orders", NATO ASI Series B284 (1991) 1. 
[42] M.V. Berry, Dingle's Self-Resurgence Formula, Nonlinearity 30 (2017) R25.

[43] M.V. Berry and C.J. Howls, Hyperasymptotics, Proc. R. Soc. Lond. A430 (1990) 653.

[44] M.V. Berry and C.J. Howls, Hyperasymptotics for Integrals with Saddles, Proc. R. Soc. Lond. A434 (1991) 657.

[45] E.B. Bogomolny, Calculation of Instanton-Anti-Instanton Contributions in Quantum Mechanics, Phys. Lett. B91 (1980) 431.

[46] G. Bonnet, F. David and B. Eynard, Breakdown of Universality in Multicut Matrix Models, J. Phys. A33 (2000) 6739, arXiv: cond-mat/0003324.

[47] D. Boito, I. Caprini, M. Golterman, K. Maltman and S. Peris, Hyperasymptotics and Quark-Hadron Duality Violations in QCD, arXiv:1711.10316 [hep-ph] .

[48] M. Borinsky, Generating Asymptotics for Factorially Divergent Sequences, in "Proceedings of the 29th Conference on Formal Power Series and Algebraic Combinatorics" (2017), arXiv: 1603.01236 [math.CO].

[49] M. Borinsky, Renormalized Asymptotic Enumeration of Feynman Diagrams, Annals Phys. 385 (2017) 95, arXiv: 1703.00840 [hep-th].

[50] J.P. Boyd, The Devil's Invention: Asymptotic, Superasymptotic and Hyperasymptotic Series, Acta Appl. Math. 56 (1999) 1.

[51] E. Brézin, E. Marinari and G. Parisi, A Nonperturbative Ambiguity Free Solution of a String Model, Phys. Lett. B242 (1990) 35.

[52] J. Brown, A. Cole, W. Cottrell and G. Shiu, Gravitational Decoupling and Picard-Lefschetz, Phys. Rev. D97 (2018) 025002, arXiv: 1710.04737 [hep-th].

[53] A. Buchel, M.P. Heller and J. Noronha, Beyond Adiabatic Approximation in Big Bang Cosmology: Hydrodynamics, Resurgence and Entropy Production in the Universe, Phys. Rev. D94 (2016) 106011, arXiv: 1603.05344 [hep-th].

[54] E. Caliceti, M. Meyer-Hermann, P. Ribeca, A. Surzhykov and U.D. Jentschura, From Useful Algorithms for Slowly Convergent Series to Physical Predictions Based on Divergent Perturbative Expansions, Phys. Rep. 446 (2007) 1, arXiv:0707.1596 [physics.comp-ph].

[55] B. Candelpergher, J.C. Nosmas and F. Pham, Premiers Pas en Calcul Étranger, Ann. Inst. Fourier 43 (1993) 201.

[56] B. Candelpergher, J.C. Nosmas and F. Pham, Approche de la Résurgence, Hermann Éditeurs (1993).

[57] V. Cardoso, J. Natário and R. Schiappa, Asymptotic Quasinormal Frequencies for Black Holes in Nonasymptotically Flat Spacetimes, J. Math. Phys. 45 (2004) 4698, arXiv:hep-th/0403132.

[58] J. Casalderrey-Solana, N.I. Gushterov and B. Meiring, Resurgence and Hydrodynamic Attractors in Gauss-Bonnet Holography, arXiv : 1712.02772 [hep-th].

[59] S.J. Chapman, C.J. Howls, J.R. King and A.B. Olde Daalhuis, Why is a Shock Not a Caustic? The Higher-Order Stokes Phenomenon and Smoothed Shock Formation, Nonlinearity 20 (2007) 2425.

[60] A. Cherman, D. Dorigoni, G.V. Dunne and M. Ünsal, Resurgence in Quantum Field Theory: Nonperturbative Effects in the Principal Chiral Model, Phys. Rev. Lett. 112 (2014) 021601, arXiv: 1308.0127 [hep-th].

[61] A. Cherman, D. Dorigoni and M. Ünsal, Decoding Perturbation Theory using Resurgence: Stokes Phenomena, New Saddle Points and Lefschetz Thimbles, JHEP 1510 (2015) 056, arXiv: 1403.1277 [hep-th].

[62] A. Cherman, P. Koroteev and M. Ünsal, Resurgence and Holomorphy: From Weak to Strong Coupling, J. Math. Phys. 56 (2015) 053505, arXiv: 1410.0388 [hep-th]. 
[63] S. Chun, A Resurgence Analysis of the SU(2) Chern-Simons Partition Functions on a Brieskorn Homology Sphere $\Sigma(2,5,7)$, arXiv:1701.03528 [hep-th] .

[64] S. Codesido, On the Resummation of the Lee-Yang Edge Singularity Coupled to Gravity, arXiv: 1712.02752 [hep-th].

[65] S. Codesido and M. Mariño, Holomorphic Anomaly and Quantum Mechanics, J. Phys. A51 (2018) 055402, arXiv: 1612.07687 [hep-th].

[66] S. Codesido, M. Mariño and R. Schiappa, Nonperturbative Quantum Mechanics from Nonperturbative Strings, arXiv:1712.02603 [hep-th].

[67] J.C. Collins and D.E. Soper, Large Order Expansion in Perturbation Theory, Annals Phys. 112 (1978) 209.

[68] C. Cordova, B. Heidenreich, A. Popolitov and S. Shakirov, Orbifolds and Exact Solutions of Strongly-Coupled Matrix Models, arXiv:1611.03142 [hep-th].

[69] O. Costin, Exponential Asymptotics, Transseries, and Generalized Borel Summation for Analytic Rank One Systems of ODE's, Inter. Math. Res. Notices 8 (1995) 377, arXiv:math/0608414.

[70] O. Costin, On Borel Summation and Stokes Phenomena for Rank-1 Nonlinear Systems of Ordinary Differential Equations, Duke Math. J. 93 (1998) 289, arXiv:math/0608408.

[71] O. Costin, Asymptotics and Borel Summability, Mono. Surv. Pure App. Math. 141 (2008).

[72] O. Costin and R. Costin, On the Formation of Singularities of Solutions of Nonlinear Differential Systems in Anti-Stokes Directions, Invent. Math. 145 (2001) 425, arXiv:math.CA/0202234.

[73] O. Costin, R.D. Costin and M. Huang, Tronquée Solutions of the Painlevé Equation PI, Constr. Approx. 41 (2015) 467, arXiv:1310.5330 [math.CA].

[74] O. Costin and G.V. Dunne, Convergence from Divergence, J. Phys. A51 (2018) 04LT01, arXiv: 1705.09687 [hep-th].

[75] J.S. Cotler, G. Gur-Ari, M. Hanada, J. Polchinski, P. Saad, S.H. Shenker, D. Stanford, A. Streicher and M. Tezuka, Black Holes and Random Matrices, JHEP 1705 (2017) 118, arXiv: 1611.04650 [hep-th]

[76] R. Couso-Santamaría, Universality of the Topological String at Large Radius and NS-Brane Resurgence, Lett. Math. Phys. 107 (2017) 343, arXiv: 1507.04013 [hep-th].

[77] R. Couso-Santamaría, J.D. Edelstein, R. Schiappa and M. Vonk, Resurgent Transseries and the Holomorphic Anomaly, Ann. Henri Poincaré 17 (2016) 331, arXiv:1308.1695 [hep-th].

[78] R. Couso-Santamaría, J.D. Edelstein, R. Schiappa and M. Vonk, Resurgent Transseries and the Holomorphic Anomaly: Nonperturbative Closed Strings in Local $\mathbb{C P}^{2}$, Commun. Math. Phys. 338 (2015) 285, arXiv: 1407.4821 [hep-th] .

[79] R. Couso-Santamaría, M. Mariño and R. Schiappa, Resurgence Matches Quantization, J. Phys. A50 (2017) 145402, arXiv: 1610.06782 [hep-th].

[80] R. Couso-Santamaría, R. Schiappa and R. Vaz, Finite $N$ from Resurgent Large N, Annals Phys. 356 (2015) 1, arXiv: 1501.01007 [hep-th].

[81] R. Couso-Santamaría, R. Schiappa and R. Vaz, On Asymptotics and Resurgent Structures of Enumerative Gromov-Witten Invariants, Commun. Number Theor. Phys. 11 (2017) 707, arXiv: 1605.07473 [math. AG] .

[82] M. Cristoforetti, F. Di Renzo, A. Mukherjee and L. Scorzato, Monte Carlo Simulations on the Lefschetz Thimble: Taming the Sign Problem, Phys. Rev. D88 (2013) 051501, arXiv: 1303.7204 [hep-lat].

[83] E. Delabaere, Effective Resummation Methods for an Implicit Resurgent Function, arXiv:math-ph/0602026. 
[84] E. Delabaere, Resurgent Methods and the First Painlevé Equation, CIMPA Lecture Notes (2014).

[85] E. Delabaere, Divergent Series, Summability and Resurgence III: Resurgent Methods and the First Painlevé Equation, Lec. Notes Math. 2155 (2016).

[86] E. Delabaere, H. Dillinger and F. Pham, Développements Semi-Classiques Exacts des Niveaux d'Énergie d'un Oscillateur à Une Dimension, Compt. Rend. Acad. Sci. 310 (1990) 141.

[87] E. Delabaere, H. Dillinger and F. Pham, Résurgence de Voros et Périodes des Courbes Hyperelliptiques, Ann. Inst. Fourier 43 (1993) 163.

[88] E. Delabaere, H. Dillinger and F. Pham, Exact Semiclassical Expansions for One-Dimensional Quantum Oscillators, J. Math. Phys. 38 (1997) 6126.

[89] E. Delabaere and C.J. Howls, Global Asymptotics for Multiple Integrals with Boundaries, Duke Math. J. 112 (2002) 199.

[90] E. Delabaere and F. Pham, Unfolding the Quartic Oscillator, Annals Phys. 261 (1997) 180.

[91] E. Delabaere and F. Pham, Resurgent Methods in Semi-Classical Asymptotics, Ann. Inst. Henri Poincaré 71 (1999) 1.

[92] S. Demulder, D. Dorigoni and D.C. Thompson, Resurgence in $\eta$-Deformed Principal Chiral Models, JHEP 1607 (2016) 088, arXiv: 1604.07851 [hep-th].

[93] D. Dorigoni, An Introduction to Resurgence, Trans-Series and Alien Calculus, arXiv: 1411.3585 [hep-th].

[94] D. Dorigoni and P. Glass, The Grin of Cheshire Cat Resurgence from Supersymmetric Localization, arXiv: 1711.04802 [hep-th]

[95] D. Dorigoni and Y. Hatsuda, Resurgence of the Cusp Anomalous Dimension, JHEP 1509 (2015) 138, arXiv: 1506.03763 [hep-th].

[96] J.D. Dorronsoro, J.J. Halliwell, J.B. Hartle, T. Hertog and O. Janssen, The Real No-Boundary Wave Function in Lorentzian Quantum Cosmology, Phys. Rev. D96 (2017) 043505, arXiv: 1705.05340 [gr-qc].

[97] N. Drukker, M. Mariño and P. Putrov, Nonperturbative Aspects of ABJM Theory, JHEP 1111 (2011) 141, arXiv:1103.4844 [hep-th].

[98] G.V. Dunne, M. Shifman and M. Ünsal, IR Renormalons vs. Operator Product Expansion in Supersymmetric and Related Gauge Theories, Phys. Rev. Lett. 114 (2015) 191601, arXiv: 1502.06680 [hep-th] .

[99] G.V. Dunne and M. Ünsal, Resurgence and Trans-Series in Quantum Field Theory: The $\mathbb{C P}^{N-1}$ Model, JHEP 1211 (2012) 170, arXiv:1210.2423 [hep-th].

[100] G.V. Dunne and M. Ünsal, Continuity and Resurgence: Towards a Continuum Definition of the $\mathbb{C P}^{N-1}$ Model, Phys. Rev. D87 (2013) 025015, arXiv:1210.3646 [hep-th].

[101] G.V. Dunne and M. Ünsal, Generating Nonperturbative Physics from Perturbation Theory, Phys. Rev. D89 (2014) 041701, arXiv: 1306.4405 [hep-th].

[102] G.V. Dunne and M. Ünsal, Uniform WKB, Multi-Instantons, and Resurgent Trans-Series, Phys. Rev. D89 (2014) 105009, arXiv:1401.5202 [hep-th].

[103] G.V. Dunne and M. Ünsal, Resurgence and Dynamics of $O(N)$ and Grassmannian Sigma Models, JHEP 1509 (2015) 199, arXiv: 1505.07803 [hep-th].

[104] G.V. Dunne and M. Ünsal, What is QFT? Resurgent Trans-Series, Lefschetz Thimbles, and New Exact Saddles, PoS LATTICE2015 (2016) 010, arXiv:1511.05977 [hep-lat].

[105] G.V. Dunne and M. Ünsal, New Methods in QFT and QCD: From Large-N Orbifold Equivalence to Bions and Resurgence, Ann. Rev. Nucl. Part. Sci. 66 (2016) 245, arXiv: 1601.03414 [hep-th]. 
[106] G.V. Dunne and M. Ünsal, WKB and Resurgence in the Mathieu Equation, in "Resurgence, Physics and Numbers", CRM Series 20 (2017) 249, arXiv:1603.04924 [math-ph].

[107] G.V. Dunne and M. Ünsal, Deconstructing Zero: Resurgence, Supersymmetry and Complex Saddles, JHEP 1612 (2016) 002, arXiv:1609.05770[hep-th].

[108] F.J. Dyson, Divergence of Perturbation Theory in Quantum Electrodynamics, Phys. Rev. 85 (1952) 631.

[109] J. Écalle, Les Fonctions Résurgentes, Prépub. Math. Université Paris-Sud 81-05 (1981), 81-06 (1981), 85-05 (1985).

[110] G.A. Edgar, Transseries for Beginners, Real Anal. Exchange 35 (2009) 253, arXiv:0801.4877 [math.RA].

[111] B. Eynard, Large N Expansion of Convergent Matrix Integrals, Holomorphic Anomalies, and Background Independence, JHEP 0903 (2009) 003, arXiv:0802.1788 [math-ph].

[112] B. Eynard and M. Mariño, A Holomorphic and Background Independent Partition Function for Matrix Models and Topological Strings, J. Geom. Phys. 61 (2011) 1181, arXiv:0810.4273 [hep-th] .

[113] B. Eynard and J. Zinn-Justin, The $O(n)$ Model on a Random Surface: Critical Points and Large Order Behaviour, Nucl. Phys. B386 (1992) 558, arXiv:hep-th/9204082.

[114] B. Eynard and J. Zinn-Justin, Large Order Behaviour of 2D Gravity Coupled to d $<1$ Matter, Phys. Lett. B302 (1993) 396, arXiv:hep-th/9301004.

[115] M.V. Fedoryuk, The Saddle-Point Method, Nauka Moscow (1977).

[116] J. Feldbrugge, J.-L. Lehners and N. Turok, Lorentzian Quantum Cosmology, Phys. Rev. D95 (2017) 103508, arXiv:1703.02076 [hep-th].

[117] J. Feldbrugge, J.-L. Lehners and N. Turok, No Smooth Beginning for Spacetime, Phys. Rev. Lett. 119 (2017) 171301, arXiv:1705.00192 [hep-th].

[118] J. Feldbrugge, J.-L. Lehners and N. Turok, No Rescue for the No Boundary Proposal, Phys. Rev. D97 (2018) 023509, arXiv: 1708.05104 [hep-th].

[119] P. Di Francesco, P.H. Ginsparg and J. Zinn-Justin, 2D Gravity and Random Matrices, Phys. Rept. 254 (1995) 1, arXiv:hep-th/9306153.

[120] D. Friedan, Z. Qiu and S. Shenker, Conformal Invariance, Unitarity and Critical Exponents in Two Dimensions, Phys. Rev. Lett. 52 (1984) 1575.

[121] H. Fujii, D. Honda, M. Kato, Y. Kikukawa, S. Komatsu and T. Sano, Hybrid Monte Carlo on Lefschetz thimbles - A Study of the Residual Sign Problem, JHEP 1310 (2013) 147, arXiv:1309.4371 [hep-lat].

[122] T. Fujimori, S. Kamata, T. Misumi, M. Nitta and N. Sakai, Non-Perturbative Contributions from Complexified Solutions in $\mathbb{C P}^{N-1}$ Models, Phys. Rev. D94 (2016) 105002, arXiv: 1607.04205 [hep-th].

[123] T. Fujimori, S. Kamata, T. Misumi, M. Nitta and N. Sakai, Exact Resurgent Trans-Series and Multi-Bion Contributions to All Orders, Phys. Rev. D95 (2017) 105001, arXiv: 1702.00589 [hep-th].

[124] T. Fujimori, S. Kamata, T. Misumi, M. Nitta and N. Sakai, Resurgence Structure to All Orders of Multi-bions in Deformed SUSY Quantum Mechanics, Prog. Theor. Exp. Phys. 2017 (2017) 083B02, arXiv: 1705.10483 [hep-th].

[125] K. Fukushima and Y. Tanizaki, Hamilton Dynamics for Lefschetz-Thimble Integration akin to the Complex Langevin Method, Prog. Theor. Exp. Phys. (2015) 111A01, arXiv:1507.07351 [hep-th].

[126] I. Gahramanov and K. Tezgin, Remark on the Dunne-Ünsal Relation in Exact Semi-Classics, Phys. Rev. D93 (2016) 065037, arXiv:1512.08466 [hep-th]. 
[127] I. Gahramanov and K. Tezgin, A Resurgence Analysis for Cubic and Quartic Anharmonic Potentials, Int. J. Mod. Phys. A32 (2017) 1750033, arXiv:1608.08119[hep-th].

[128] D. Gaiotto, G.W. Moore and A. Neitzke, Wall-Crossing, Hitchin Systems, and the WKB Approximation, Adv. Math. 234 (2013) 239, arXiv:0907. 3987 [hep-th].

[129] M. Garay, A. de Goursac, D. van Straten, Resurgent Deformation Quantization, Annals Phys. 342 (2014) 83, arXiv: 1309.0437 [math-ph].

[130] S. Garoufalidis, A. Its, A. Kapaev and M. Mariño, Asymptotics of the Instantons of Painlevé I, Int. Math. Res. Notices 2012 (2012) 561, arXiv:1002.3634 [math.CA].

[131] S. Garoufalidis, T.T.Q. Le and M. Mariño, Analyticity of the Free Energy of a Closed 3-Manifold, SIGMA 4 (2008) 080, arXiv:0809.2572 [math.GT].

[132] S. Garoufalidis and M. Mariño, Universality and Asymptotics of Graph Counting Problems in Nonorientable Surfaces, J. Comb. Theo. A117 (2010) 715, arXiv:0812.1195 [math.C0].

[133] P.H. Ginsparg, Applied Conformal Field Theory, in "Les Houches Summer School" (1988), arXiv:hep-th/9108028.

[134] P.H. Ginsparg and J. Zinn-Justin, Large Order Behaviour of Nonperturbative Gravity, Phys. Lett. B255 (1991) 189.

[135] A. Grassi, M. Mariño, S. Zakany, Resumming the String Perturbation Series, JHEP 1505 (2015) 038, arXiv: 1405. 4214 [hep-th].

[136] D.J. Gross and V. Periwal, String Perturbation Theory Diverges Phys. Rev. Lett. 60 (1988) 2105.

[137] J. Gu and T. Sulejmanpasic, High Order Perturbation Theory for Difference Equations and Borel Summability of Quantum Mirror Curves, JHEP 1712 (2017) 014, arXiv:1709.00854 [hep-th].

[138] J. Guckenheimer and P. Holmes, Nonlinear Oscillations, Dynamical Systems, and Bifurcations of Vector Fields, Applied Mathematical Sciences 42 (1983), Springer New York.

[139] S. Gukov, RG Flows and Bifurcations, Nucl. Phys. B919 (2017) 583, arXiv:1608.06638 [hep-th].

[140] S. Gukov, M. Mariño and P. Putrov, Resurgence in Complex Chern-Simons Theory, arXiv: 1605.07615 [hep-th].

[141] G. Guralnik and Z. Guralnik, Complexified Path Integrals and the Phases of Quantum Field Theory, Annals Phys. 325 (2010) 2486, arXiv:0710.1256 [hep-th].

[142] D. Harlow, J. Maltz and E. Witten, Analytic Continuation of Liouville Theory, JHEP 1112 (2011) 071, arXiv: 1108.4417 [hep-th].

[143] T. Harmark, J. Natário and R. Schiappa, Greybody Factors for d-Dimensional Black Holes, Adv. Theor. Math. Phys. 14 (2010) 727, arXiv:0708.0017 [hep-th].

[144] Y. Hatsuda, Perturbative/Nonperturbative Aspects of Bloch Electrons in a Honeycomb Lattice, arXiv: 1712 . 04012 [hep-th] .

[145] Y. Hatsuda and K. Okuyama, Resummations and Non-Perturbative Corrections, JHEP 1509 (2015) 051, arXiv: 1505.07460 [hep-th].

[146] M.P. Heller and M. Spalinski, Hydrodynamics Beyond the Gradient Expansion: Resurgence and Resummation, Phys. Rev. Lett. 115 (2015) 072501, arXiv: 1503.07514 [hep-th].

[147] N. Hoe, B. D'Etat, J. Grumberg, M. Caby, E. Leboucher and G. Coulaud, Stark Effect of Hydrogenic Ions, Phys. Rev. A25 (1982) 891.

[148] M. Honda, Borel Summability of Perturbative Series in $4 d \mathcal{N}=2$ and $5 d \mathcal{N}=1$ Theories, Phys. Rev. Lett. 116 (2016) 211601, arXiv:1603.06207 [hep-th].

[149] M. Honda, How to Resum Perturbative Series in $3 d \mathcal{N}=2$ Chern-Simons Matter Theories, Phys. Rev. D94 (2016) 025039, arXiv:1604.08653 [hep-th]. 
[150] M. Honda, Role of Complexified Supersymmetric Solutions, arXiv:1710.05010 [hep-th].

[151] M. Honda and D.P. Jatkar, Interpolating Function and Stokes Phenomena, Nucl. Phys. B900 (2015) 533, arXiv: 1504.02276 [hep-th].

[152] M. Honda and D. Yokoyama, Resumming Perturbative Series in the Presence of Monopole Bubbling Effects, arXiv:1711.10799 [hep-th].

[153] C.J. Howls, Hyperasymptotics for Multidimensional Integrals, Exact Remainders and the Global Connection Problem, Proc. R. Soc. Lond. A453 (1997) 2271.

[154] C.J. Howls, P.J. Langman and A.B. Olde Daalhuis, On the Higher-Order Stokes Phenomenon, Proc. R. Soc. Lond. A460 (2004) 2285.

[155] M.R. Jahnke, A Equação de Euler e a Análise Assintótica de Gevrey, arXiv:1404.2416 [math. CV].

[156] U.D. Jentschura, A. Surzhykov and J. Zinn-Justin, Multi-Instantons and Exact Results III: Unification of Even and Odd Anharmonic Oscillators, Annals Phys. 325 (2010) 1135.

[157] U.D. Jentschura and J. Zinn-Justin, Instantons in Quantum Mechanics and Resurgent Expansions, Phys. Lett. B596 (2004) 138, arXiv:hep-ph/0405279.

[158] U.D. Jentschura and J. Zinn-Justin, Multi-Instantons and Exact Results IV: Path Integral Formalism, Annals Phys. 326 (2011) 2186.

[159] D. Kaminski, Exponentially Improved Stationary Phase Approximations for Double Integrals, Methods Appl. Anal 1 (1994) 44.

[160] T. Kanazawa and Y. Tanizaki, Structure of Lefschetz Thimbles in Simple Fermionic Systems, JHEP 1503 (2015) 044, arXiv: 1412.2802 [hep-th].

[161] A.-K. Kashani-Poor and J. Troost, Pure $\mathcal{N}=2$ Super Yang-Mills and Exact WKB, JHEP 1508 (2015) 160, arXiv:1504.08324 [hep-th].

[162] L. Klaczynski, Resurgent Transseries \& 3 Dyson-Schwinger Equations, Annals Phys. 372 (2016) 397, arXiv: 1601.04140 [hep-th].

[163] A. Klemm, M. Mariño and M. Rauch, Direct Integration and Non-Perturbative Effects in Matrix Models, JHEP 1010 (2010) 004, arXiv:1002.3846 [hep-th].

[164] K. Konishi and G. Paffuti, Quantum Mechanics: A New Introduction, Oxford University Press (2009).

[165] C. Kozçaz, T. Sulejmanpasic, Y. Tanizaki and M. Ünsal, Cheshire Cat Resurgence, Self-Resurgence and Quasi-Exact Solvable Systems, arXiv:1609.06198 [hep-th].

[166] J.L. Lavoie, T.J. Osler and R. Tremblay, Fractional Derivatives and Special Functions, SIAM Rev. 18 (1976) 240.

[167] M. Loday-Richaud, Divergent Series, Summability and Resurgence II: Simple and Multiple Summability, Lec. Notes Math. 2154 (2016).

[168] C.N. Yang and T.D. Lee, Statistical Theory of Equations of State and Phase Transitions 1: Theory of Condensation, Phys. Rev. 87 (1952) 404.

[169] T.D. Lee and C.N. Yang, Statistical Theory of Equations of State and Phase Transitions 2: Lattice Gas and Ising Model, Phys. Rev. 87 (1952) 410.

[170] S. Lefschetz, L'Analysis Situs et la Géométrie Algébrique, Gauthier-Villars (1924).

[171] J.C. Le Guillou and J. Zinn-Justin (eds.), Large Order Behaviour of Perturbation Theory North-Holland Publishing (1990).

[172] A. Maiezza and J.C. Vasquez, Resummation and Renormalons in a General Quantum Field Theory, arXiv: 1802.06022 [hep-th] . 
[173] B. Malgrange, Intégrales Asymptotiques et Monodromie, Annales Sci. École Norm. Sup. 7 (1974) 405.

[174] M. Mariño, Open String Amplitudes and Large-Order Behaviour in Topological String Theory, JHEP 0803 (2008) 060, arXiv:hep-th/0612127.

[175] M. Mariño, Nonperturbative Effects and Nonperturbative Definitions in Matrix Models and Topological Strings, JHEP 0812 (2008) 114, arXiv:0805.3033[hep-th].

[176] M. Mariño, Lectures on Non-Perturbative Effects in Large N Gauge Theories, Matrix Models and Strings, Fortsch. Phys. 62 (2014) 455, arXiv:1206.6272 [hep-th].

[177] M. Mariño, Instantons and Large N: An Introduction to Non-Perturbative Methods in Quantum Field Theory, Cambridge University Press (2015).

[178] M. Mariño, S. Pasquetti and P. Putrov, Large N Duality Beyond the Genus Expansion, JHEP 1007 (2010) 074, arXiv:0911.4692 [hep-th].

[179] M. Mariño, R. Schiappa and M. Weiss, Nonperturbative Effects and the Large-Order Behaviour of Matrix Models and Topological Strings, Commun. Number Theor. Phys. 2 (2008) 349, arXiv:0711.1954 [hep-th].

[180] M. Mariño, R. Schiappa and M. Weiss, Multi-Instantons and Multi-Cuts, J. Math. Phys. 50 (2009) 052301, arXiv:0809.2619 [hep-th].

[181] H. Mera, T.G. Pedersen and B.K. Nikolic, Fast Summation of Divergent Series and Resurgent Transseries in Quantum Field Theories from Meijer-G Approximants, arXiv: 1802.06034 [hep-th].

[182] A. Milekhin, On Lagrangian Description of Borel Resummation, arXiv:1701.00805 [hep-th].

[183] T. Misumi, M. Nitta and N. Sakai, Resurgence in Sine-Gordon Quantum Mechanics: Exact Agreement between Multi-Instantons and Uniform WKB, JHEP 1509 (2015) 157, arXiv: 1507.00408 [hep-th] .

[184] T. Misumi, M. Nitta and N. Sakai, Non-BPS Exact Solutions and their Relation to Bions in $\mathbb{C P}^{N-1}$ Models, JHEP 1605 (2016) 057, arXiv:1604.00839 [hep-th].

[185] C. Mitschi and D. Sauzin, Divergent Series, Summability and Resurgence I: Monodromy and Resurgence, Lec. Notes Math. 2153 (2016).

[186] L. Motl and A. Neitzke, Asymptotic Black Hole Quasinormal Frequencies, Adv. Theor. Math. Phys. 7 (2003) 307, arXiv:hep-th/0301173.

[187] A. Mukherjee, M. Cristoforetti and L. Scorzato, Metropolis Monte Carlo Integration on the Lefschetz Thimble: Application to a One-Plaquette Model, Phys. Rev. D88 (2013) 051502, arXiv: 1308.0233 [physics. comp-ph].

[188] J. Natário and R. Schiappa, On the Classification of Asymptotic Quasinormal Frequencies for d-Dimensional Black Holes and Quantum Gravity, Adv. Theor. Math. Phys. 8 (2004) 1001, arXiv:hep-th/0411267.

[189] A. Neitzke, Greybody Factors at Large Imaginary Frequencies, arXiv:hep-th/0304080.

[190] J. Nishimura and S. Shimasaki, Combining the Complex Langevin Method and the Generalized Lefschetz-Thimble Method, JHEP 1706 (2017) 023, arXiv:1703.09409 [hep-lat].

[191] A.B. Olde Daalhuis, On Higher-Order Stokes Phenomena of an Inhomogeneous Linear Ordinary Differential Equation, J. Comp. App. Math. 169 (2004) 235.

[192] A.B. Olde Daalhuis, Hyperasymptotics for Nonlinear ODEs I: A Riccati Equation, Proc. R. Soc. A461 (2005) 2503.

[193] A.B. Olde Daalhuis, Hyperasymptotics for Nonlinear ODEs II: The First Painlevé Equation and a Second-Order Riccati Equation, Proc. R. Soc. A461 (2005) 3005. 
[194] F.W.J. Olver, D.W. Lozier, R.F. Boisvert and C.W. Clark, NIST Handbook of Mathematical Functions, Cambridge University Press (2010).

[195] S. Pasquetti and R. Schiappa, Borel and Stokes Nonperturbative Phenomena in Topological String Theory and $c=1$ Matrix Models, Ann. Henri Poincaré 11 (2010) 351, arXiv:0907.4082 [hep-th].

[196] F. Pham, Vanishing Homologies and the $n$ Variable Saddlepoint Method, Proc. Sympos. Pure Math. 40 (1983) 319.

[197] F. Pham, La Descente des Cols par les Onglets de Lefschetz, avec Vues sur Gauss-Manin, Syst. Diff. Singul. 130 (1985) 11.

[198] C. Pisani and E.R. Smith, Lee-Yang Zeros and Stokes Phenomenon in a Model with a Wetting Transition, J. Stat. Phys. 72 (1993) 51.

[199] J. Polchinski, Dirichlet Branes and Ramond-Ramond Charges, Phys. Rev. Lett. 75 (1995) 4724, arXiv:hep-th/9510017.

[200] J. Pöschel, A Lecture on the Classical KAM Theorem, Proc. Symp. Pure Math. 69 (2001) 707, arXiv:0908.2234[math.DS].

[201] J.G. Russo, A Note on Perturbation Series in Supersymmetric Gauge Theories, JHEP 1206 (2012) 038, arXiv:1203.5061 [hep-th].

[202] J.G. Russo and M. Tierz, Quantum Phase transition in Many-Flavor Supersymmetric $Q E D_{3}$, Phys. Rev. D95 (2017) 031901, arXiv: 1610.08527 [hep-th].

[203] D. Sauzin, Resurgent Functions and Splitting Problems, Res. Inst. Math. Sci. 1493 (2006) 48, arXiv:0706.0137 [math.DS].

[204] D. Sauzin, Introduction to 1-Summability and Resurgence, in "Divergent Series, Summability and Resurgence I: Monodromy and Resurgence", Lec. Notes Math. 2153 (2016), arXiv: 1405.0356 [math.DS].

[205] R. Schiappa and R. Vaz, The Resurgence of Instantons: Multi-Cut Stokes Phases and the Painlevé II Equation, Commun. Math. Phys. 330 (2014) 655, arXiv:1302.5138[hep-th].

[206] R. Schiappa and N. Wyllard, An Ar Threesome: Matrix Models, 2d CFTs and 4 d $\mathcal{N}=2$ Gauge Theories, J. Math. Phys. 51 (2010) 082304, arXiv:0911.5337 [hep-th].

[207] T.M. Seara and D. Sauzin, Resumació de Borel i Teoria de la Ressurgència, Butl. Soc. Catalana Mat. 18 (2003) 131.

[208] M. Serone, G. Spada and G. Villadoro, Instantons from Perturbation Theory, Phys. Rev. D96 (2017) 021701, arXiv: 1612.04376 [hep-th].

[209] M. Serone, G. Spada and G. Villadoro, The Power of Perturbation Theory, JHEP 1705 (2017) 056, arXiv: 1702.04148 [hep-th].

[210] S.H. Shenker, The Strength of Nonperturbative Effects in String Theory, in "The Large $N$ Expansion in Quantum Field Theory and Statistical Physics" (1990) 809.

[211] H.J. Silverstone, JWKB Connection-Formula Problem Revisited via Borel Summation, Phys. Rev. Lett. 55 (1985) 2523.

[212] H.J. Silverstone, J.G. Harris, J. Čížek and J. Paldus, Asymptotics of High-Order Perturbation Theory for the One-Dimensional Anharmonic Oscillator by Quasisemiclassical Methods, Phys. Rev. A32 (1985) 1965.

[213] M. Stingl, Field Theory Amplitudes as Resurgent Functions, arXiv:hep-ph/0207349.

[214] G.G. Stokes, On the Discontinuity of Arbitrary Constants which Appear in Divergent Developments, Trans. Camb. Phil. Soc. 10 (1864) 106. 
[215] M. Stone and J. Reeve, Late Terms in the Asymptotic Expansion for the Energy Levels of a Periodic Potential, Phys. Rev. D18 (1978) 4746.

[216] T. Sulejmanpasic and M. Ünsal, Aspects of Perturbation Theory in Quantum Mechanics: The BenderWu Mathematica Package, arXiv:1608.08256 [hep-th].

[217] Y. Tanizaki, Lefschetz-Thimble Techniques for Path Integral of Zero-Dimensional O(n) Sigma Models, Phys. Rev. D91 (2015) 036002, arXiv:1412.1891[hep-th].

[218] Y. Tanizaki and T. Koike, Real-Time Feynman Path Integral with Picard-Lefschetz Theory and its Applications to Quantum Tunneling, Annals Phys. 351 (2014) 250, arXiv:1406.2386 [math-ph].

[219] Y. Tanizaki, H. Nishimura and K. Kashiwa, Evading the Sign Problem in the Mean-Field Approximation through Lefschetz-Thimble Path Integral, Phys. Rev. D91 (2015) 101701, arXiv: 1504.02979 [hep-th].

[220] Y. Tanizaki, H. Nishimura and J.J.M. Verbaarschot, Gradient Flows without Blow-Up for Lefschetz Thimbles, JHEP 1710 (2017) 100, arXiv:1706.03822 [hep-lat].

[221] M. Vonk, Resurgence and Topological Strings, Proc. Symp. Pure Math. 93 (2015) 221, arXiv: 1502.05711 [hep-th].

[222] A. Voros, The Zeta Function of the Quartic Oscillator, Nucl. Phys. B165 (1980) 209.

[223] A. Voros, The Return of the Quartic Oscillator: The Complex WKB Method, Ann. Inst. Henri Poincaré 39 (1983) 211.

[224] A. Voros, Résurgence Quantique, Ann. Inst. Fourier 43 (1993) 1509.

[225] A. Voros, Exact Quantization Condition for Anharmonic Oscillators (in One Dimension), J. Phys. A: Math. Gen. 27 (1994) 4653.

[226] A. Voros, Aspects of Semiclassical Theory in the Presence of Classical Chaos, Prog. Theo. Phys. Supp. 116 (1994) 17.

[227] A. Voros, Exact Resolution Method for General 1D Polynomial Schrödinger Equation, J. Phys. A: Math. Gen. 32 (1999) 5993.

[228] A. Voros, Exercises in Exact Quantization, J. Phys. A: Math. Gen. 33 (2000) 7423.

[229] E. Witten, Analytic Continuation of Chern-Simons Theory, AMS/IP Stud. Adv. Math. 50 (2011) 347, arXiv: 1001.2933 [hep-th].

[230] E. Witten, A New Look at the Path Integral of Quantum Mechanics, Surv. Diff. Geo. XV (2011) 345, arXiv: 1009.6032 [hep-th].

[231] M. Yamazaki and K. Yonekura, From 4d Yang-Mills to $2 d \mathbb{C P}^{N-1}$ Model: IR Problem and Confinement at Weak Coupling, JHEP 1707 (2017) 088, arXiv:1704.05852 [hep-th].

[232] J. Zinn-Justin, Expansion Around Instantons in Quantum Mechanics, J. Math. Phys. 22 (1981) 511.

[233] J. Zinn-Justin, Perturbation Series at Large Orders in Quantum Mechanics and Field Theories: Application to the Problem of Resummation, Phys. Rept. 70 (1981) 109.

[234] J. Zinn-Justin, Multi-Instanton Contributions in Quantum Mechanics, Nucl. Phys. B192 (1981) 125.

[235] J. Zinn-Justin, Multi-Instanton Contributions in Quantum Mechanics 2, Nucl. Phys. B218 (1983) 333.

[236] J. Zinn-Justin, Instantons in Quantum Mechanics: Numerical Evidence for a Conjecture, J. Math. Phys. 25 (1984) 549.

[237] J. Zinn-Justin, From Multi-Instantons to Exact Results, Ann. Inst. Fourier 53 (2003) 1259. 
[238] J. Zinn-Justin and U.D. Jentschura, Multi-Instantons and Exact Results I: Conjectures, WKB Expansions, and Instanton Interactions, Annals Phys. 313 (2004) 197, arXiv:quant-ph/0501136.

[239] J. Zinn-Justin and U.D. Jentschura, Multi-Instantons and Exact Results II: Specific Cases, Higher-Order Effects, and Numerical Calculations, Annals Phys. 313 (2004) 269, arXiv:quant-ph/0501137. 